\title{
Evaluation of Climate Models
}

\author{
Coordinating Lead Authors: \\ Gregory Flato (Canada), Jochem Marotzke (Germany)
}

\section{Lead Authors:}

Babatunde Abiodun (South Africa), Pascale Braconnot (France), Sin Chan Chou (Brazil), William Collins (USA), Peter Cox (UK), Fatima Driouech (Morocco), Seita Emori (Japan), Veronika Eyring (Germany), Chris Forest (USA), Peter Gleckler (USA), Eric Guilyardi (France), Christian Jakob (Australia), Vladimir Kattsov (Russian Federation), Chris Reason (South Africa), Markku Rummukainen (Sweden)

\section{Contributing Authors:}

Krishna AchutaRao (India), Alessandro Anav (UK), Timothy Andrews (UK), Johanna Baehr (Germany), Nathaniel L. Bindoff (Australia), Alejandro Bodas-Salcedo (UK), Jennifer Catto (Australia), Don Chambers (USA), Ping Chang (USA), Aiguo Dai (USA), Clara Deser (USA), Francisco Doblas-Reyes (Spain), Paul J. Durack (USA/Australia), Michael Eby (Canada), Ramon de Elia (Canada), Thierry Fichefet (Belgium), Piers Forster (UK), David Frame (UK/New Zealand), John Fyfe (Canada), Emiola Gbobaniyi (Sweden/Nigeria), Nathan Gillett (Canada), Jesus Fidel González-Rouco (Spain), Clare Goodess (UK), Stephen Griffies (USA), Alex Hall (USA), Sandy Harrison (Australia), Andreas Hense (Germany), Elizabeth Hunke (USA), Tatiana llyina (Germany), Detelina Ivanova (USA), Gregory Johnson (USA), Masa Kageyama (France), Viatcheslav Kharin (Canada), Stephen A. Klein (USA), Jeff Knight (UK), Reto Knutti (Switzerland), Felix Landerer (USA), Tong Lee (USA), Hongmei Li (Germany/China), Natalie Mahowald (USA), Carl Mears (USA), Gerald Meehl (USA), Colin Morice (UK), Rym Msadek (USA), Gunnar Myhre (Norway), J. David Neelin (USA), Jeff Painter (USA), Tatiana Pavlova (Russian Federation), Judith Perlwitz (USA), Jean-Yves Peterschmitt (France), Jouni Räisänen (Finland), Florian Rauser (Germany), Jeffrey Reid (USA), Mark Rodwell (UK), Benjamin Santer (USA), Adam A. Scaife (UK), Jörg Schulz (Germany), John Scinocca (Canada), David Sexton (UK), Drew Shindell (USA), Hideo Shiogama (Japan), Jana Sillmann (Canada), Adrian Simmons (UK), Kenneth Sperber (USA), David Stephenson (UK), Bjorn Stevens (Germany), Peter Stott (UK), Rowan Sutton (UK), Peter W. Thorne (USA/Norway/UK), Geert Jan van Oldenborgh (Netherlands), Gabriel Vecchi (USA), Mark Webb (UK), Keith Williams (UK), Tim Woollings (UK), Shang-Ping Xie (USA), Jianglong Zhang (USA)

\section{Review Editors:}

Isaac Held (USA), Andy Pitman (Australia), Serge Planton (France), Zong-Ci Zhao (China)

This chapter should be cited as:

Flato, G., J. Marotzke, B. Abiodun, P. Braconnot, S.C. Chou, W. Collins, P. Cox, F. Driouech, S. Emori, V. Eyring, C. Forest, P. Gleckler, E. Guilyardi, C. Jakob, V. Kattsov, C. Reason and M. Rummukainen, 2013: Evaluation of Climate Models. In: Climate Change 2013: The Physical Science Basis. Contribution of Working Group I to the Fifth Assessment Report of the Intergovernmental Panel on Climate Change [Stocker, T.F., D. Qin, G.-K. Plattner, M. Tignor, S.K. Allen, J. Boschung, A. Nauels, Y. Xia, V. Bex and P.M. Midgley (eds.)]. Cambridge University Press, Cambridge, United Kingdom and New York, NY, USA. 


\section{Table of Contents}

Executive Summary

9.1 Climate Models and Their Characteristics. 746

9.1.1 Scope and Overview of this Chapter ......................... 746

9.1.2 Overview of Model Types to Be Evaluated ................ 746

9.1.3 Model Improvements ................................................ 748

Box 9.1: Climate Model Development and Tuning ................ 749

9.2 Techniques for Assessing Model Performance ....... 753

9.2.1 New Developments in Model Evaluation Approaches 753

9.2.2 Ensemble Approaches for Model Evaluation ............. 754

9.2.3 The Model Evaluation Approach Used in this Chapter and Its Limitations

\subsection{Experimental Strategies in Support of Climate} Model Evaluation

9.3.1 The Role of Model Intercomparisons 759

9.3.2 Experimental Strategy for Coupled Model Intercomparison Project Phase 5

\subsection{Simulation of Recent and Longer-Term Records} in Global Models

Atmosphere 760

Box 9.2: Climate Models and the Hiatus in Global Mean Surface Warming of the Past 15 Years 769

9.4.2 Ocean..................................................................... 777

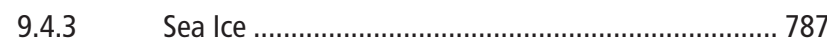

9.4.4 Land Surface, Fluxes and Hydrology......................... 790

9.4.5 Carbon Cycle......................................................... 792

9.4.6 Aerosol Burdens and Effects on Insolation................ 794

9.5 Simulation of Variability and Extremes ..................... 795

9.5.1 Importance of Simulating Climate Variability ............ 795

9.5.2 Diurnal-to-Seasonal Variability................................. 796

9.5.3 Interannual-to-Centennial Variability ........................ 799

9.5.4 Extreme Events ................................................. 806

Box 9.3: Understanding Model Performance 809
9.6 Downscaling and Simulation of Regional-Scale Climate

9.6.1 Global Models.......................................................... 810

9.6.2 Regional Climate Downscaling ................................ 814

9.6.3 Skill of Downscaling Methods................................. 814

9.6.4 Value Added through RCMs ..................................... 815

9.6.5 Sources of Model Errors and Uncertainties ............... 815

9.6.6 Relating Downscaling Performance to Credibility of Regional Climate Information ................................. 816

9.7 Climate Sensitivity and Climate Feedbacks 817

9.7.1 Equilibrium Climate Sensitivity, Idealized Radiative Forcing, and Transient Climate Response in the Coupled Model Intercomparison Project Phase 5 Ensemble

9.7.2 Understanding the Range in Model Climate Sensitivity: Climate Feedbacks 819

9.7.3 Climate Sensitivity and Model Performance 820

9.8 Relating Model Performance to Credibility of Model Applications 821

9.8.1 Synthesis Assessment of Model Performance............. 821

9.8.2 Implications of Model Evaluation for Climate Change Detection and Attribution

9.8.3 Implications of Model Evaluation for Model Projections of Future Climate.

References

Appendix 9.A: Climate Models Assessed

in Chapter 9

\section{Frequently Asked Questions}

FAQ 9.1 Are Climate Models Getting Better, and How Would We Know? 


\section{Executive Summary}

Climate models have continued to be developed and improved since the AR4, and many models have been extended into Earth System models by including the representation of biogeochemical cycles important to climate change. These models allow for policy-relevant calculations such as the carbon dioxide $\left(\mathrm{CO}_{2}\right)$ emissions compatible with a specified climate stabilization target. In addition, the range of climate variables and processes that have been evaluated has greatly expanded, and differences between models and observations are increasingly quantified using 'performance metrics'. In this chapter, model evaluation covers simulation of the mean climate, of historical climate change, of variability on multiple time scales and of regional modes of variability. This evaluation is based on recent internationally coordinated model experiments, including simulations of historic and paleo climate, specialized experiments designed to provide insight into key climate processes and feedbacks and regional climate downscaling. Figure 9.44 provides an overview of model capabilities as assessed in this chapter, including improvements, or lack thereof, relative to models assessed in the AR4. The chapter concludes with an assessment of recent work connecting model performance to the detection and attribution of climate change as well as to future projections. \{9.1.2, 9.8.1, Table 9.1, Figure 9.44\}

The ability of climate models to simulate surface temperature has improved in many, though not all, important aspects relative to the generation of models assessed in the AR4. There continues to be very high confidence ${ }^{1}$ that models reproduce observed large-scale mean surface temperature patterns (pattern correlation of $\sim 0.99$ ), though systematic errors of several degrees are found in some regions, particularly over high topography, near the ice edge in the North Atlantic, and over regions of ocean upwelling near the equator. On regional scales (sub-continental and smaller), the confidence in model capability to simulate surface temperature is less than for the larger scales; however, regional biases are near zero on average, with intermodel spread of roughly $\pm 3^{\circ} \mathrm{C}$. There is high confidence that regional-scale surface temperature is better simulated than at the time of the AR4. Current models are also able to reproduce the large-scale patterns of temperature during the Last Glacial Maximum (LGM), indicating an ability to simulate a climate state much different from the present. \{9.4.1, 9.6.1, Figures 9.2, 9.6, 9.39, 9.40\}

There is very high confidence that models reproduce the general features of the global-scale annual mean surface temperature increase over the historical period, including the more rapid warming in the second half of the 20th century, and the cooling immediately following large volcanic eruptions. Most simulations of the historical period do not reproduce the observed reduction in global mean surface warming trend over the last 10 to 15 years. There is medium confidence that the trend difference between models and observations during 1998-2012 is to a substantial degree caused by internal variability, with possible contributions from forcing error and some models overestimating the response to increasing greenhouse gas (GHG) forcing. Most, though not all, models overestimate the observed warming trend in the tropical troposphere over the last 30 years, and tend to underestimate the long-term lower stratospheric cooling trend. $\{9.4 .1$, Box 9.2, Figure 9.8\}

The simulation of large-scale patterns of precipitation has improved somewhat since the AR4, although models continue to perform less well for precipitation than for surface temperature. The spatial pattern correlation between modelled and observed annual mean precipitation has increased from 0.77 for models available at the time of the AR4 to 0.82 for current models. At regional scales, precipitation is not simulated as well, and the assessment remains difficult owing to observational uncertainties. $\{9.4 .1,9.6 .1$, Figure 9.6\}

The simulation of clouds in climate models remains challenging. There is very high confidence that uncertainties in cloud processes explain much of the spread in modelled climate sensitivity. However, the simulation of clouds in climate models has shown modest improvement relative to models available at the time of the AR4, and this has been aided by new evaluation techniques and new observations for clouds. Nevertheless, biases in cloud simulation lead to regional errors on cloud radiative effect of several tens of watts per square meter. $\{9.2 .1,9.4 .1,9.7 .2$, Figures 9.5, 9.43\}

Models are able to capture the general characteristics of storm tracks and extratropical cyclones, and there is some evidence of improvement since the AR4. Storm track biases in the North Atlantic have improved slightly, but models still produce a storm track that is too zonal and underestimate cyclone intensity. $\{9.4 .1\}$

Many models are able to reproduce the observed changes in upper ocean heat content from 1961 to 2005 with the multi-model mean time series falling within the range of the available observational estimates for most of the period. The ability of models to simulate ocean heat uptake, including variations imposed by large volcanic eruptions, adds confidence to their use in assessing the global energy budget and simulating the thermal component of sea level rise. \{9.4.2, Figure 9.17\}

The simulation of the tropical Pacific Ocean mean state has improved since the AR4, with a $30 \%$ reduction in the spurious westward extension of the cold tongue near the equator, a pervasive bias of coupled models. The simulation of the tropical Atlantic remains deficient with many models unable to reproduce the basic east-west temperature gradient. \{9.4.2, Figure 9.14$\}$

\footnotetext{
In this Report, the following summary terms are used to describe the available evidence: limited, medium, or robust; and for the degree of agreement: low, medium, or high. A level of confidence is expressed using five qualifiers: very low, low, medium, high, and very high, and typeset in italics, e.g., medium confidence. For a given evidence and agreement statement, different confidence levels can be assigned, but increasing levels of evidence and degrees of agreement are correlated with increasing confidence (see Section 1.4 and Box TS.1 for more details).
} 
Current climate models reproduce the seasonal cycle of Arctic sea ice extent with a multi-model mean error of less than about $10 \%$ for any given month. There is robust evidence that the downward trend in Arctic summer sea ice extent is better simulated than at the time of the AR4, with about one quarter of the simulations showing a trend as strong as, or stronger, than in observations over the satellite era (since 1979). There is a tendency for models to slightly overestimate sea ice extent in the Arctic (by about 10\%) in winter and spring. In the Antarctic, the multi-model mean seasonal cycle agrees well with observations, but inter-model spread is roughly double that for the Arctic. Most models simulate a small decreasing trend in Antarctic sea ice extent, albeit with large inter-model spread, in contrast to the small increasing trend in observations. $\{9.4 .3$, Figures $9.22,9.24\}$

Models are able to reproduce many features of the observed global and Northern Hemispher (NH) mean temperature variance on interannual to centennial time scales (high confidence), and most models are now able to reproduce the observed peak in variability associated with the El Niño (2- to 7-year period) in the Tropical Pacific. The ability to assess variability from millennial simulations is new since the AR4 and allows quantitative evaluation of model estimates of low-frequency climate variability. This is important when using climate models to separate signal and noise in detection and attribution studies (Chapter 10). \{9.5.3, Figures 9.33, 9.35\}

Many important modes of climate variability and intraseasonal to seasonal phenomena are reproduced by models, with some improvements evident since the AR4. The statistics of the global monsoon, the North Atlantic Oscillation, the El Niño-Southern Oscillation (ENSO), the Indian Ocean Dipole and the Quasi-Biennial Oscillation are simulated well by several models, although this assessment is tempered by the limited scope of analysis published so far, or by limited observations. There are also modes of variability that are not simulated well. These include modes of Atlantic Ocean variability of relevance to near term projections in Chapter 11 and ENSO teleconnections outside the tropical Pacific, of relevance to Chapter 14. There is high confidence that the multi-model statistics of monsoon and ENSO have improved since the AR4. However, this improvement does not occur in all models, and process-based analysis shows that biases remain in the background state and in the strength of associated feedbacks. \{9.5.3, Figures $9.32,9.35,9.36\}$

There has been substantial progress since the AR4 in the assessment of model simulations of extreme events. Based on assessment of a suite of indices, the inter-model range of simulated climate extremes is similar to the spread amongst observationally based estimates in most regions. In addition, changes in the frequency of extreme warm and cold days and nights over the second half of the 20th century are consistent between models and observations, with the ensemble global mean time series generally falling within the range of observational estimates. The majority of models underestimate the sensitivity of extreme precipitation to temperature variability or trends, especially in the tropics, which implies that models may underestimate the projected increase in extreme precipitation in the future. Some high-resolution atmospheric models have been shown to reproduce observed year-to-year variability of Atlantic hurricane counts when forced with observed sea surface temperatures, though so far only a few studies of this kind are available. $\{9.5 .4$, Figure 9.37$\}$

An important development since the AR4 is the more widespread use of Earth System models, which include an interactive carbon cycle. In the majority of these models, the simulated global land and ocean carbon sinks over the latter part of the 20th century fall within the range of observational estimates. However, the regional patterns of carbon uptake and release are less well reproduced, especially for $\mathrm{NH}$ land where models systematically underestimate the sink implied by atmospheric inversion techniques The ability of models to simulate carbon fluxes is important because these models are used to estimate 'compatible emissions' (carbon dioxide emission pathways compatible with a particular climate change target; see Chapter 6). \{9.4.5, Figure 9.27\}

The majority of Earth System models now include an interactive representation of aerosols, and make use of a consistent specification of anthropogenic sulphur dioxide emissions. However, uncertainties in sulphur cycle processes and natural sources and sinks remain and so, for example, the simulated aerosol optical depth over oceans ranges from 0.08 to 0.22 with roughly equal numbers of models over- and under-estimating the satellite-estimated value of 0.12. $\{9.1 .2,9.4 .6$, Table 9.1, Figure 9.29\}

Time-varying ozone is now included in the latest suite of models, either prescribed or calculated interactively. Although in some models there is only medium agreement with observed changes in total column ozone, the inclusion of time-varying stratospheric ozone constitutes a substantial improvement since the AR4 where half of the models prescribed a constant climatology. As a result, there is robust evidence that the representation of climate forcing by stratospheric ozone has improved since the AR4. \{9.4.1, Figure 9.10\}

Regional downscaling methods are used to provide climate information at the smaller scales needed for many climate impact studies, and there is high confidence that downscaling adds value both in regions with highly variable topography and for various small-scale phenomena. Regional models necessarily inherit biases from the global models used to provide boundary conditions. Furthermore, the ability to systematically evaluate regional climate models, and statistical downscaling schemes, is hampered because coordinated intercomparison studies are still emerging. However, several studies have demonstrated that added value arises from higher resolution of stationary features like topography and coastlines, and from improved representation of small-scale processes like convective precipitation. $\{9.6 .4\}$

Earth system Models of Intermediate Complexity (EMICs) provide simulations of millennial time-scale climate change, and are used as tools to interpret and expand upon the results of more comprehensive models. Although they are limited in the scope and resolution of information provided, EMIC simulations of global mean surface temperature, ocean heat content and carbon cycle response over the 20th century are consistent with the historical records and with more comprehensive models, suggesting that they can be used to provide calibrated projections of long-term transient climate response 
and stabilization, as well as large ensembles and alternative, policy-relevant, scenarios. $\{9.4 .1,9.4 .2,9.4 .5$, Figures $9.8,9.17,9.27\}$

The Coupled Model Intercomparison Project Phase 5 (CMIP5) model spread in equilibrium climate sensitivity ranges from $2.1^{\circ} \mathrm{C}$ to $4.7^{\circ} \mathrm{C}$ and is very similar to the assessment in the AR4. No correlation is found between biases in global mean surface temperature and equilibrium climate sensitivity, and so mean temperature biases do not obviously affect the modelled response to GHG forcing. There is very high confidence that the primary factor contributing to the spread in equilibrium climate sensitivity continues to be the cloud feedback. This applies to both the modern climate and the LGM. There is likewise very high confidence that, consistent with observations, models show a strong positive correlation between tropospheric temperature and water vapour on regional to global scales, implying a positive water vapour feedback in both models and observations. \{9.4.1, 9.7.2, Figures $9.9,9.42,9.43\}$

Climate and Earth System models are based on physical principles, and they reproduce many important aspects of observed climate. Both aspects contribute to our confidence in the models' suitability for their application in detection and attribution studies (Chapter 10) and for quantitative future predictions and projections (Chapters 11 to 14). In general, there is no direct means of translating quantitative measures of past performance into confident statements about fidelity of future climate projections. However, there is increasing evidence that some aspects of observed variability or trends are well correlated with inter-model differences in model projections for quantities such as Arctic summertime sea ice trends, snow albedo feedback, and the carbon loss from tropical land. These relationships provide a way, in principle, to transform an observable quantity into a constraint on future projections, but the application of such constraints remains an area of emerging research. There has been substantial progress since the AR4 in the methodology to assess the reliability of a multi-model ensemble, and various approaches to improve the precision of multi-model projections are being explored. However, there is still no universal strategy for weighting the projections from different models based on their historical performance. $\{9.8 .3$, Figure 9.45\} 


\subsection{Climate Models and Their Characteristics}

\subsubsection{Scope and Overview of this Chapter}

Climate models are the primary tools available for investigating the response of the climate system to various forcings, for making climate predictions on seasonal to decadal time scales and for making projections of future climate over the coming century and beyond. It is crucial therefore to evaluate the performance of these models, both individually and collectively. The focus of this chapter is primarily on the models whose results will be used in the detection and attribution Chapter 10 and the chapters that present and assess projections (Chapters 11 to 14; Annex I), and so this is necessarily an incomplete evaluation. In particular, this chapter draws heavily on model results collected as part of the Coupled Model Intercomparison Projects (CMIP3 and CMIP5) (Meehl et al., 2007; Taylor et al., 2012b), as these constitute a set of coordinated and thus consistent and increasingly well-documented climate model experiments. Other intercomparison efforts, such as those dealing with Regional Climate Models (RCMs) and those dealing with Earth System Models of Intermediate Complexity (EMICS) are also used. It should be noted that the CMIP3 model archive has been extensively evaluated, and much of that evaluation has taken place subsequent to the AR4. By comparison, the CMIP5 models are only now being evaluated and so there is less published literature available. Where possible we show results from both CMIP3 and CMIP5 models so as to illustrate changes in model performance over time; however, where only CMIP3 results are available, they still constitute a useful evaluation of model performance in that for many quantities, the CMIP3 and CMIP5 model performances are broadly similar.

The direct approach to model evaluation is to compare model output with observations and analyze the resulting difference. This requires knowledge of the errors and uncertainties in the observations, which have been discussed in Chapters 2 through 6. Where possible, averages over the same time period in both models and observations are compared, although for many quantities the observational record is rather short, or only observationally based estimates of the climatological mean are available. In cases where observations are lacking, we resort to intercomparison of model results to provide at least some quantification of model uncertainty via inter-model spread.

After a more thorough discussion of the climate models and methods for evaluation in Sections 9.1 and 9.2, we describe climate model experiments in Section 9.3, evaluate recent and longer-term records as simulated by climate models in Section 9.4, variability and extremes in Section 9.5, and regional-scale climate simulation including downscaling in Section 9.6. We conclude with a discussion of model performance and climate sensitivity in Section 9.7, and the relation between model performance and the credibility of future climate projections in Section 9.8 .

\subsubsection{Overview of Model Types to Be Evaluated}

The models used in climate research range from simple energy balance models to complex Earth System Models (ESMs) requiring state of the art high-performance computing. The choice of model depends directly on the scientific question being addressed (Held, 2005; Collins et al., 2006d). Applications include simulating palaeo or historical climate, sensitivity and process studies for attribution and physical understanding, predicting near-term climate variability and change on seasonal to decadal time scales, making projections of future climate change over the coming century or more and downscaling such projections to provide more detail at the regional and local scale. Computational cost is a factor in all of these, and so simplified models (with reduced complexity or spatial resolution) can be used when larger ensembles or longer integrations are required. Examples include exploration of parameter sensitivity or simulations of climate change on the millennial or longer time scale. Here, we provide a brief overview of the climate models evaluated in this chapter.

\subsubsection{Atmosphere-Ocean General Circulation Models}

Atmosphere-Ocean General Circulation Models (AOGCMs) were the 'standard' climate models assessed in the AR4. Their primary function is to understand the dynamics of the physical components of the climate system (atmosphere, ocean, land and sea ice), and for making projections based on future greenhouse gas (GHG) and aerosol forcing. These models continue to be extensively used, and in particular are run (sometimes at higher resolution) for seasonal to decadal climate prediction applications in which biogeochemical feedbacks are not critical (see Chapter 11). In addition, high-resolution or variable-resolution AOGCMs are often used in process studies or applications with a focus on a particular region. An overview of the AOGCMs assessed in this chapter can be found in Table 9.1 and the details in Table 9.A.1. For some specific applications, an atmospheric component of such a model is used on its own.

\subsubsection{Earth System Models}

ESMs are the current state-of-the-art models, and they expand on AOGCMs to include representation of various biogeochemical cycles such as those involved in the carbon cycle, the sulphur cycle, or ozone (Flato, 2011). These models provide the most comprehensive tools available for simulating past and future response of the climate system to external forcing, in which biogeochemical feedbacks play an important role. An overview of the ESMs assessed in this chapter can be found in Table 9.1 and details in Table 9.A.1.

\subsubsection{Earth System Models of Intermediate Complexity}

EMICs attempt to include relevant components of the Earth system, but often in an idealized manner or at lower resolution than the models described above. These models are applied to certain scientific questions such as understanding climate feedbacks on millennial time scales or exploring sensitivities in which long model integrations or large ensembles are required (Claussen et al., 2002; Petoukhov et al., 2005). This class of models often includes Earth system components not yet included in all ESMs (e.g., ice sheets). As computing power increases, this model class has continued to advance in terms of resolution and complexity. An overview of EMICs assessed in this chapter and in the AR5 WG1 is provided in Table 9.2 with additional details in Table 9.A.2. 
Table 9.1 Main features of the Atmosphere-Ocean General Circulation Models (AOGCMs) and Earth System Models (ESMs) participating in Coupled Model Intercomparison Project Phase 5 (CMIP5), and a comparison with Coupled Model Intercomparison Project Phase 3 (CMIP3), including components and resolution of the atmosphere and the ocean models. Detailed CMIP5 model description can be found in Table 9.A.1 ( ${ }^{*}$ refers to Table 9.A.1 for more details). Official CMIP model names are used. HT stands for High-Top atmosphere, which has a fully resolved stratosphere with a model top above the stratopause. AMIP stands for models with atmosphere and land surface only, using observed sea surface temperature and sea ice extent. A component is coloured when it includes at least a physically based prognostic equation and at least a two-way coupling with another component, allowing climate feedbacks. For aerosols, lighter shading means 'semi-interactive' and darker shading means 'fully interactive'. The resolution of the land surface usually follows that of the atmosphere, and the resolution of the sea ice follows that of the ocean. In moving from CMIP3 to CMIP5, note the increased complexity and resolution as well as the absence of artificial flux correction (FC) used in some CMIP3 models.

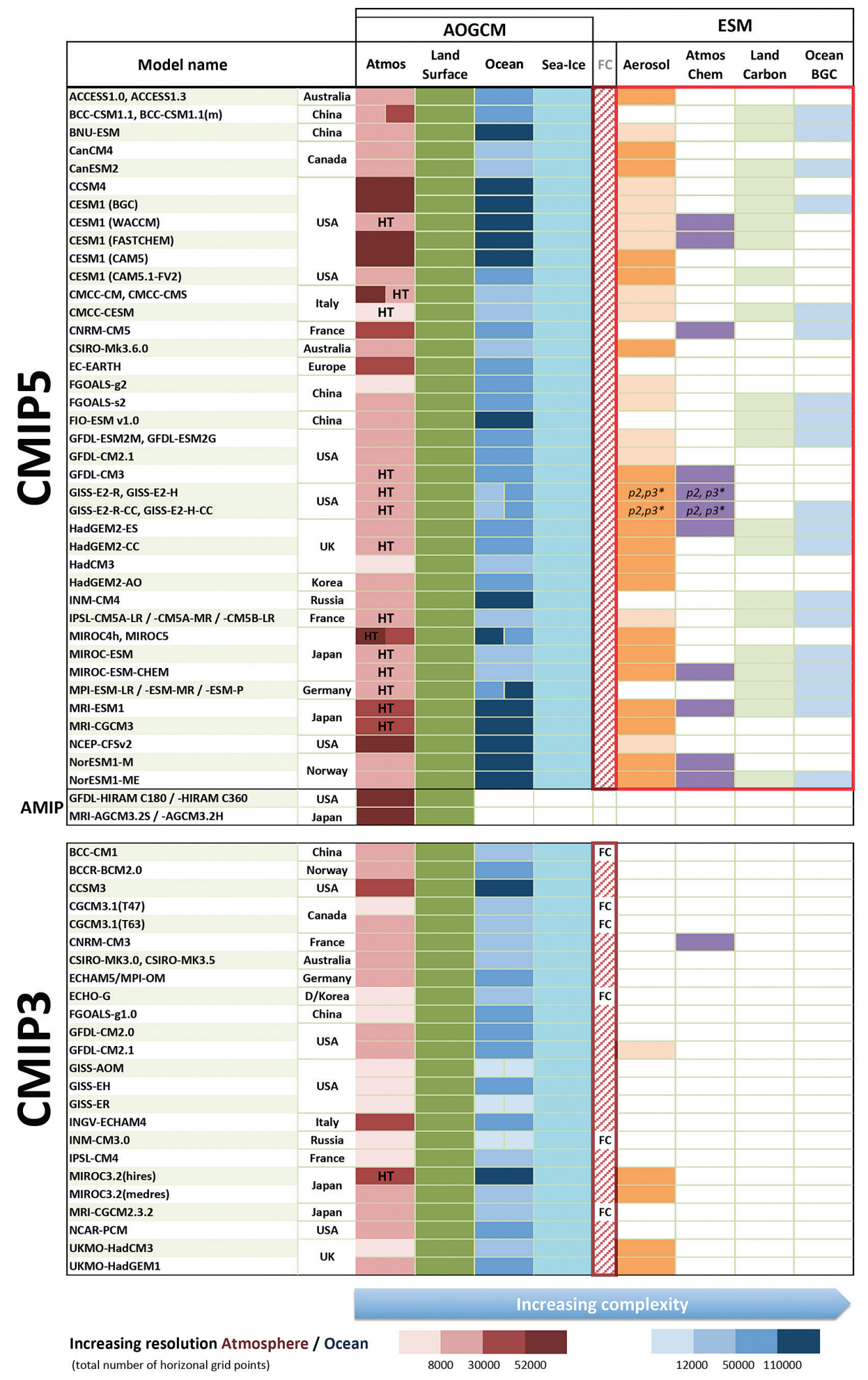


Table 9.2 | Main features of the EMICs assessed in the AR5, including components and complexity of the models. Model complexity for four components is indicated by colour shading. Further detailed descriptions of the models are contained in Table 9.A.2.

\begin{tabular}{|c|c|c|c|c|c|c|c|c|c|}
\hline \multicolumn{2}{|c|}{ Model name } & Atmos & Ocean & Land Surface & Sea Ice & Coupling & Biosphere & Ice & Sediment \& \\
\hline Bern3D & Switzerland & & & & & & & & \\
\hline CLIMBER2 & Germany & & & & & & & & \\
\hline CLIMBER3 & Germany & & & & & & & & \\
\hline DCESS & Denmark & & & & & & & & \\
\hline FAMOUS & UK & & & & & & & & \\
\hline GENIE & UK & & & & & & & & \\
\hline IAP RAS CM & Russia & & & & & & & & \\
\hline IGSM2 & USA & & & & & & & & \\
\hline LOVECLIM1.2 & Netherlands & & & & & & & & \\
\hline MESMO & USA & & & & & & & & \\
\hline MIROC-lite & Japan & & & & & & & & \\
\hline MIROC-lite-LCM & Japan & & & & & & & & \\
\hline SPEEDO & Netherlands & & & & & & & & \\
\hline UMD & USA & & & & & & & & \\
\hline Uvic & Canada & & & & & & & & \\
\hline
\end{tabular}

\begin{tabular}{|l|l|l|l|}
\hline \multicolumn{5}{|c|}{ Increasing Complexity (light to dark) } \\
\hline EMBM & 2-Box & NST/NSM & None \\
\hline SD & Q-flux ML & LST/NSM & BO \\
\hline QG & FG & LST/BSM & \\
\hline PE & PE & LST/CSM & BO,BT \\
\hline
\end{tabular}

Significant advances in EMIC capabilities are inclusion of ice sheets (UVic 2.9, Weaver et al., 2001; CLIMBER-2.4, Petoukhov et al., 2000; LOVECLIM, Goosse et al., 2010) and ocean sediment models (DCESS, Shaffer et al., 2008; UVic 2.9, Weaver et al., 2001; Bern3D-LPJ, Ritz et al., 2011). These additional interactive components provide critical feedbacks involved in sea level rise estimates and carbon cycle response on millennial time scales (Zickfeld et al., 2013). Further, the flexibility and efficiency of EMICs allow calibration to specific climate change events to remove potential biases.

\subsubsection{Regional Climate Models}

RCMs are limited-area models with representations of climate processes comparable to those in the atmospheric and land surface components of AOGCMs, though typically run without interactive ocean and sea ice. RCMs are often used to dynamically 'downscale' global model simulations for some particular geographical region to provide more detailed information (Laprise, 2008; Rummukainen, 2010). By contrast, empirical and statistical downscaling methods constitute a range of techniques to provide similar regional or local detail.

\subsubsection{Model Improvements}

The climate models assessed in this report have seen a number of improvements since the AR4. Model development is a complex and iterative task: improved physical process descriptions are developed, new model components are introduced and the resolution of the models is improved. After assembly of all model components, model parameters are adjusted, or tuned, to provide a stable model climate.
The overall approach to model development and tuning is summarized in Box 9.1.

\subsubsection{Parameterizations}

Parameterizations are included in all model components to represent processes that cannot be explicitly resolved; they are evaluated both in isolation and in the context of the full model. The purpose of this section is to highlight recent developments in the parameterizations employed in each model component. Some details for individual models are listed in Table 9.1.

\subsection{Atmosphere}

Atmospheric models must parameterize a wide range of processes, including those associated with atmospheric convection and clouds, cloud-microphysical and aerosol processes and their interaction, boundary layer processes, as well as radiation and the treatment of unresolved gravity waves. Advances made in the representation of cloud processes, including aerosol-cloud and cloud-radiation interactions, and atmospheric convection are described in Sections 7.2.3 and 7.4 .

Improvements in representing the atmospheric boundary layer since the AR4 have focussed on basic boundary layer processes, the representation of the stable boundary layer, and boundary layer clouds (Teixeira et al., 2008). Several global models have successfully adopted new approaches to the parameterization of shallow cumulus convection and moist boundary layer turbulence that acknowledge their 


\section{Box 9.1 | Climate Model Development and Tuning}

The Atmosphere-Ocean General Circulation Models, Earth System Models and Regional Climate Models evaluated here are based on fundamental laws of nature (e.g., energy, mass and momentum conservation). The development of climate models involves several principal steps:

1. Expressing the system's physical laws in mathematical terms. This requires theoretical and observational work in deriving and simplifying mathematical expressions that best describe the system.

2. Implementing these mathematical expressions on a computer. This requires developing numerical methods that allow the solution of the discretized mathematical expressions, usually implemented on some form of grid such as the latitude-longitude-height grid for atmospheric or oceanic models.

3. Building and implementing conceptual models (usually referred to as parameterizations) for those processes that cannot be represented explicitly, either because of their complexity (e.g., biochemical processes in vegetation) or because the spatial and/or temporal scales on which they occur are not resolved by the discretized model equations (e.g., cloud processes and turbulence). The development of parameterizations has become very complex (e.g., Jakob, 2010) and is often achieved by developing conceptual models of the process of interest in isolation using observations and comprehensive process models. The complexity of each process representation is constrained by observations, computational resources and current knowledge (e.g., Randall et al., 2007).

The application of state-of-the-art climate models requires significant supercomputing resources. Limitations in those resources lead to additional constraints. Even when using the most powerful computers, compromises need to be made in three main areas:

1. Numerical implementations allow for a choice of grid spacing and time step, usually referred to as 'model resolution'. Higher model resolution generally leads to mathematically more accurate models (although not necessarily more reliable simulations) but also to higher computational costs. The finite resolution of climate models implies that the effects of certain processes must be represented through parameterizations (e.g., the carbon cycle or cloud and precipitation processes; see Chapters 6 and 7).

2. The climate system contains many processes, the relative importance of which varies with the time scale of interest (e.g., the carbon cycle). Hence compromises to include or exclude certain processes or components in a model must be made, recognizing that an increase in complexity generally leads to an increase in computational cost (Hurrell et al., 2009).

3. Owing to uncertainties in the model formulation and the initial state, any individual simulation represents only one of the possible pathways the climate system might follow. To allow some evaluation of these uncertainties, it is necessary to carry out a number of simulations either with several models or by using an ensemble of simulations with a single model, both of which increase computational cost.

Trade-offs amongst the various considerations outlined above are guided by the intended model application and lead to the several classes of models introduced in Section 9.1.2.

Individual model components (e.g., the atmosphere, the ocean, etc.) are typically first evaluated in isolation as part of the model development process. For instance, the atmospheric component can be evaluated by prescribing sea surface temperature (SST) (Gates et al., 1999) or the ocean and land components by prescribing atmospheric conditions (Barnier et al., 2006; Griffies et al., 2009). Subsequently, the various components are assembled into a comprehensive model, which then undergoes a systematic evaluation. At this stage, a small subset of model parameters remains to be adjusted so that the model adheres to large-scale observational constraints (often global averages). This final parameter adjustment procedure is usually referred to as 'model tuning'. Model tuning aims to match observed climate system behaviour and so is connected to judgements as to what constitutes a skilful representation of the Earth's climate. For instance, maintaining the global mean top of the atmosphere (TOA) energy balance in a simulation of pre-industrial climate is essential to prevent the climate system from drifting to an unrealistic state. The models used in this report almost universally contain adjustments to parameters in their treatment of clouds to fulfil this important constraint of the climate system (Watanabe et al., 2010; Donner et al., 2011; Gent et al., 2011; Golaz et al., 2011; Martin et al., 2011; Hazeleger et al., 2012; Mauritsen et al., 2012; Hourdin et al., 2013).

With very few exceptions (Mauritsen et al., 2012; Hourdin et al., 2013) modelling centres do not routinely describe in detail how they tune their models. Therefore the complete list of observational constraints toward which a particular model is tuned is generally not 
Box 9.1 (continued)

available. However, it is clear that tuning involves trade-offs; this keeps the number of constraints that can be used small and usually focuses on global mean measures related to budgets of energy, mass and momentum. It has been shown for at least one model that the tuning process does not necessarily lead to a single, unique set of parameters for a given model, but that different combinations of parameters can yield equally plausible models (Mauritsen et al., 2012). Hence the need for model tuning may increase model uncertainty. There have been recent efforts to develop systematic parameter optimization methods, but owing to model complexity they cannot yet be applied to fully coupled climate models (Neelin et al., 2010).

Model tuning directly influences the evaluation of climate models, as the quantities that are tuned cannot be used in model evaluation. Quantities closely related to those tuned will provide only weak tests of model performance. Nonetheless, by focusing on those quantities not generally involved in model tuning while discounting metrics clearly related to it, it is possible to gain insight into model performance. Model quality is tested most rigorously through the concurrent use of many model quantities, evaluation techniques, and performance metrics that together cover a wide range of emergent (or un-tuned) model behaviour.

The requirement for model tuning raises the question of whether climate models are reliable for future climate projections. Models are not tuned to match a particular future; they are tuned to reproduce a small subset of global mean observationally based constraints. What emerges is that the models that plausibly reproduce the past, universally display significant warming under increasing greenhouse gas concentrations, consistent with our physical understanding.

close mutual coupling. One new development is the Eddy-Diffusivity-Mass-Flux (EDMF) approach (Siebesma et al., 2007; Rio and Hourdin, 2008; Neggers, 2009; Neggers et al., 2009; Rio et al., 2010). The EDMF approach, like the shallow cumulus scheme of Park and Bretherton (2009), determines the cumulus-base mass flux from the statistical distribution of boundary layer updraft properties, a conceptual advance over the ad hoc closure assumptions used in the past. Realistic treatment of the stable boundary layer remains difficult (Beare et al., 2006; Cuxart et al., 2006; Svensson and Holtslag, 2009) with implications for modelling of the diurnal cycle of temperature even under clear skies (Svensson et al., 2011).

Parameterizations of unresolved orographic and non-orographic gravity-wave drag (GWD) have seen only a few changes since the AR4 (e.g., Richter et al., 2010; Geller et al., 2011). In addition to new formulations, the estimation of the parameters used in the GWD schemes has recently been advanced through the availability of satellite and groundbased observations of gravity-wave momentum fluxes, high-resolution numerical modelling, and focussed process studies (Alexander et al., 2010). Evidence from the Numerical Weather Prediction community that important terrain-generated features of the atmospheric circulation are better represented at higher model resolution has recently been confirmed (Watanabe et al., 2008; Jung et al., 2012).

\subsection{Ocean}

Ocean components in contemporary climate models generally have horizontal resolutions that are too coarse to admit mesoscale eddies. Consequently, such models typically employ some version of the Redi (Redi, 1982) neutral diffusion and Gent and McWilliams (Gent and McWilliams, 1990) eddy advection parameterization (see also Gent et al., 1995; McDougall and McIntosh, 2001). Since the AR4, a focus has been on how parameterized mesoscale eddy fluxes in the ocean interior interact with boundary layer turbulence (Gnanadesikan et al.,
2007; Danabasoglu et al., 2008; Ferrari et al., 2008, 2010). Another focus concerns eddy diffusivity, with many CMIP5 models employing flow-dependent schemes. Both of these refinements are important for the mean state and the response to changing forcing, especially in the Southern Ocean (Hallberg and Gnanadesikan, 2006; Boning et al., 2008; Farneti et al., 2010; Farneti and Gent, 2011; Gent and Danabasoglu, 2011; Hofmann and Morales Maqueda, 2011).

In addition to mesoscale eddies, there has been a growing awareness of the role that sub-mesoscale eddies and fronts play in restratifying the mixed layer (Boccaletti et al., 2007; Fox-Kemper et al., 2008; Klein and Lapeyre, 2009), and the parameterization of Fox-Kemper et al. (2011) is now used in some CMIP5 models.

There is an active research effort on the representation of dianeutral mixing associated with breaking gravity waves (MacKinnon et al., 2009), with this work adding rigour to the prototype energetically consistent abyssal tidal mixing parameterization of Simmons et al. (2004) now used in several climate models (e.g., Jayne, 2009; Danabasoglu et al., 2012). The transport of dense water down-slope by gravity currents (e.g., Legg et al., 2008, 2009) has also been the subject of focussed efforts, with associated parameterizations making their way into some CMIP5 models (Jackson et al., 2008b; Legg et al., 2009; Danabasoglu et al., 2010).

\subsection{Land}

Land surface properties such as vegetation, soil type and the amount of water stored on the land as soil moisture, snow and groundwater all strongly influence climate, particularly through their effects on surface albedo and evapotranspiration. These climatic effects can be profound; for example, it has been suggested that changes in the state of the land surface may have played an important part in the severity and length of the 2003 European drought (Fischer et al., 2007), and 
that more than $60 \%$ of the projected increase in interannual summer temperature variability in Europe is due to soil moisture-temperature feedbacks (Seneviratne et al., 2006).

Land surface schemes calculate the fluxes of heat, water and momentum between the land and the atmosphere. At the time of the AR4, even the more advanced land surface schemes suffered from obvious simplifications, such as the need to prescribe rather than simulate the vegetation cover and a tendency to ignore lateral flows of water and sub-gridscale heterogeneity in soil moisture (Pitman, 2003). Since the AR4, a number of climate models have included some representation of vegetation dynamics (see Sections 9.1.3.2.5 and 9.4.4.3), landatmosphere $\mathrm{CO}_{2}$ exchange (see Section 9.4.5), sub-gridscale hydrology (Oleson et al., 2008b) and changes in land use (see Section 9.4.4.4).

\subsection{Sea ice}

Most large-scale sea ice processes, such as basic thermodynamics and dynamics, are well understood and well represented in models (Hunke et al., 2010). However, important details of sea ice dynamics and deformation are not captured, especially at small scales (Coon et al., 2007; Girard et al., 2009; Hutchings et al., 2011). Currently, sea ice model development is focussed mainly on (1) more precise descriptions of physical processes such as microstructure evolution and anisotropy and (2) including biological and chemical species. Many models now include some representation of sub-grid-scale thickness variations, along with a description of mechanical redistribution that converts thinner ice to thicker ice under deformation (Hunke et al., 2010).

Sea ice albedo has long been recognized as a critical aspect of the global heat balance. The average ice surface albedo on the scale of a climate model grid cell is (as on land) the result of a mixture of surface types: bare ice, melting ice, snow-covered ice, open water, etc. Many sea ice models use a relatively simple albedo parameterization that specifies four albedo values: cold snow; warm, melting snow; cold, bare ice; and warm, melting ice, and the specific values may be subject to tuning (e.g., Losch et al., 2010). Some parameterizations take into account the ice and snow thickness, spectral band and surface melt (e.g., Pedersen et al., 2009; Vancoppenolle et al., 2009). Solar radiation may be distributed within the ice column assuming exponential decay or via a more complex multiple-scattering radiative transfer scheme (Briegleb and Light, 2007).

Snow model development for sea ice has lagged behind terrestrial snow models. Lecomte et al. (2011) introduced vertically varying snow temperature, density and conductivity to improve vertical heat conduction and melting in a 1D model intended for climate simulation, but many physical processes affecting the evolution of the snow pack, such as redistribution by wind, moisture transport (including flooding and snow ice formation) and snow grain size evolution, still are not included in most climate models.

Salinity affects the thermodynamic properties of sea ice, and is used in the calculation of fresh water and salt exchanges at the ice-ocean interface (Hunke et al., 2011). Some models allow the salinity to vary in time (Schramm et al., 1997), while others assume a salinity profile that is constant (e.g., Bitz and Lipscomb, 1999). Another new thrust is the inclusion of chemistry and biogeochemistry (Piot and von Glasow, 2008; Zhao et al., 2008; Vancoppenolle et al., 2010; Hunke et al., 2011), with dependencies on the ice microstructure and salinity profile.

Melt ponds can drain through interconnected brine channels when the ice becomes warm and permeable. This flushing can effectively clean the ice of salt, nutrients, and other inclusions, which affect the albedo, conductivity and biogeochemical processes and thereby play a role in climate change. Advanced parameterizations for melt ponds are making their way into sea ice components of global climate models (e.g., Flocco et al., 2012; Hunke et al., 2013).

\subsubsection{New Components and Couplings: Emergence of Earth System Modelling}

\subsection{Carbon cycle}

The omission of internally consistent feedbacks among the physical, chemical and biogeochemical processes in the Earth's climate system is a limitation of AOGCMs. The conceptual issue is that the physical climate influences natural sources and sinks of $\mathrm{CO}_{2}$ and methane $\left(\mathrm{CH}_{4}\right)$, the two most important long-lived GHGs. ESMs incorporate many of the important biogeochemical processes, making it possible to simulate the evolution of these radiatively active species based on their emissions from natural and anthropogenic sources together with their interactions with the rest of the Earth system. Alternatively, when forced with specified concentrations, a model can be used to diagnose these sources with feedbacks included (Hibbard et al., 2007). Given the large natural sources and sinks of $\mathrm{CO}_{2}$ relative to anthropogenic emissions, and given the primacy of $\mathrm{CO}_{2}$ among anthropogenic $\mathrm{GHGs}$, some of the most important enhancements are the addition of terrestrial and oceanic carbon cycles. These cycles have been incorporated into many models (Christian et al., 2010; Tjiputra et al., 2010) used to make projections of climate change (Schurgers et al., 2008; Jungclaus et al., 2010). Several ESMs now include coupled carbon and nitrogen cycles (Thornton et al., 2007; Gerber et al., 2010; Zaehle and Dalmonech, 2011) in order to simulate the interactions of nitrogen compounds with ecosystem productivity, GHGs including nitrous oxide $\left(\mathrm{N}_{2} \mathrm{O}\right)$ and ozone $\left(\mathrm{O}_{3}\right)$, and global carbon sequestration (Zaehle and Dalmonech, 2011).

Oceanic uptake of $\mathrm{CO}_{2}$ is highly variable in space and time, and is determined by the interplay between the biogeochemical and physical processes in the ocean. About half of CMIP5 models make use of schemes that partition marine ecosystems into nutrients, plankton, zooplankton and detritus (hence called NPZD-type models) while others use a more simplified representation of ocean biogeochemistry (see Table 9.A.1). These NPZD-type models allow simulation of some of the important feedbacks between climate and oceanic $\mathrm{CO}_{2}$ uptake, but are limited by the lack of marine ecosystem dynamics. Some efforts have been made to include more plankton groups or plankton functional types in the models (Le Quere et al., 2005) with as-yet uncertain implications for Earth system response.

Ocean acidification and the associated decrease in calcification in many marine organisms provides a negative feedback on atmospheric $\mathrm{CO}_{2}$ increase (Ridgwell et al., 2007a). New-generation models therefore include various parameterizations of calcium carbonate $\left(\mathrm{CaCO}_{3}\right)$ 
production as a function of the saturation state of seawater with respect to calcite (Gehlen et al., 2007; Ridgwell et al., 2007a; Ilyina et al., 2009) or partial pressure $\mathrm{CO}_{2}\left(\mathrm{pCO}_{2}\right.$; Heinze, 2004). On centennial to multi-millennial scales, deep-sea carbonate sediments neutralize atmospheric $\mathrm{CO}_{2}$. Some CMIP5 models include the sediment carbon reservoir, and progress has been made toward refined sediment representation in the models (Heinze et al., 2009).

\subsection{Aerosol particles}

The treatment of aerosol particles has advanced since the AR4. Many AOGCMs and ESMs now include the basic features of the sulphur cycle and so represent both the direct effect of sulphate aerosol, along with some of the more complex indirect effects involving cloud droplet number and size. Further, several AOGCMs and ESMs are currently capable of simulating the mass, number, size distribution and mixing state of interacting multi-component aerosol particles (Bauer et al., 2008b; Liu et al., 2012b). The incorporation of more physically complete representations of aerosol often improves the simulated climate under historical and present-day conditions, including the mean pattern and interannual variability in continental rainfall (Rotstayn et al., 2010, 2011). However, despite the addition of aerosol-cloud interactions to many AOGCMs and ESMs since the AR4, the representation of aerosol particles and their interaction with clouds and radiative transfer remains an important source of uncertainty (see Sections 7.3.5 and 7.4). Additional aerosol-related topics that have received attention include the connection between dust aerosol and ocean biogeochemistry, the production of oceanic dimethylsulphide (DMS, a natural source of sulphate aerosol), and vegetation interactions with organic atmospheric chemistry (Collins et al., 2011).

\subsection{Methane cycle and permafrost}

In addition to $\mathrm{CO}_{2}$, an increasing number of ESMs and EMICs are also incorporating components of the $\mathrm{CH}_{4}$ cycle, for example, atmospheric $\mathrm{CH}_{4}$ chemistry and wetland emissions, to quantify some of the feedbacks from changes in $\mathrm{CH}_{4}$ sources and sinks under a warming climate (Stocker et al., 2012). Some models now simulate the evolution of the permafrost carbon stock (Khvorostyanov et al., 2008a, 2008b), and in some cases this is integrated with the representation of terrestrial and oceanic $\mathrm{CH}_{4}$ cycles (Volodin, 2008b; Volodin et al., 2010).

\subsection{Dynamic global vegetation models and wildland fires}

One of the potentially more significant effects of climate change is the alteration of the distribution, speciation and life cycle of vegetated ecosystems (Bergengren et al., 2001, 2011). Vegetation has a significant influence on the surface energy balance, exchanges of non- $\mathrm{CO}_{2}$ GHGs and the terrestrial carbon sink. Systematic shifts in vegetation, for example, northward migration of boreal forests, would therefore impose biogeophysical feedbacks on the physical climate system (Clark et al., 2011). In order to include these effects in projections of climate change, several dynamic global vegetation models (DGVMs) have been developed and deployed in ESMs (Cramer et al., 2001; Sitch et al., 2008; Ostle et al., 2009). Although agriculture and managed forests are not yet generally incorporated, DGVMs can simulate the interactions among natural and anthropogenic drivers of global warming, the state of terrestrial ecosystems and ecological feedbacks on further climate change. The incorporation of DGVMs has required considerable improvement in the physics of coupled models to produce stable and realistic distributions of flora (Oleson et al., 2008b). The improvements include better treatments of surface, subsurface and soil hydrological processes; the exchange of water with the atmosphere; and the discharge of water into rivers and streams. Whereas the first DGVMs have been coupled primarily to the carbon cycle, the current generation of DGVMs are being extended to include ecological sources and sinks of other non- $\mathrm{CO}_{2}$ trace gases including $\mathrm{CH}_{4}, \mathrm{~N}_{2} \mathrm{O}$, biogenic volatile organic compounds (BVOCs) and nitrogen oxides collectively known as $\mathrm{NO}_{x}$ (Arneth et al., 2010). BVOCs and $\mathrm{NO}_{\mathrm{x}}$ can alter the lifetime of some GHGs and act as precursors for secondary organic aerosols (SOAs) and ozone. Disturbance of the natural landscape by fire has significant climatic effects through its impact on vegetation and through its emissions of GHGs, aerosols and aerosol precursors. Because the frequency of wildland fires increases rapidly with increases in ambient temperature (Westerling et al., 2006), the effects of fires are projected to grow over the 21st century (Kloster et al., 2012). The interactions of fires with the rest of the climate system are now being introduced into ESMs (Arora and Boer, 2005; Pechony and Shindell, 2009; Shevliakova et al., 2009).

\subsection{Land use/land cover change}

The impacts of land use and land cover change on the environment and climate are explicitly included as part of the Representative Concentration Pathways (RCPs; cf. Chapters 1 and 12) used for climate projections to be assessed in later chapters (Moss et al., 2010). Several important types of land use and land cover change include effects of agriculture and changing agricultural practices, including the potential for widespread introduction of biofuel crops; the management of forests for preservation, wood harvest and production of woody biofuel stock; and the global trends toward greater urbanization. ESMs include increasingly detailed treatments of crops and their interaction with the landscape (Arora and Boer, 2010; Smith et al., 2010a, 2010b), forest management (Bellassen et al., 2010, 2011) and the interactions between urban areas and the surrounding climate systems (Oleson et al., 2008a).

\subsection{Chemistry-climate interactions and stratosphere- troposphere coupling}

Important chemistry-climate interactions such as the impact of the ozone hole and recovery on Southern Hemisphere (SH) climate or the radiative effects of stratospheric water vapour changes on surface temperature have been confirmed in multiple studies (SPARC-CCMVal, 2010; WMO, 2011). In the majority of the CMIP5 simulations stratospheric ozone is prescribed. The main advance since the AR4 is that time-varying rather than constant stratospheric ozone is now generally used. In addition, several CMIP5 models treat stratospheric chemistry interactively, thus prognostically calculating stratospheric ozone and other chemical constituents. Important chemistry-climate interactions such as an increased influx of stratospheric ozone in a warmer climate that results in higher ozone burdens in the troposphere have also been identified (Young et al., 2013). Ten of the CMIP5 models simulate tropospheric chemistry interactively whereas it is prescribed in the remaining models (see Table 9.1 and Eyring et al. (2013)). 
It is now widely accepted that in addition to the influence of tropospheric circulation and climate change on the stratosphere, stratospheric dynamics can in turn influence the tropospheric circulation and its variability (SPARC-CCMVal, 2010; WMO, 2011). As a result, many climate models now have the ability to include a fully resolved stratosphere with a model top above the stratopause, located at around 50 $\mathrm{km}$. The subset of CMIP5 models with high-top configurations is compared to the set of low-top models with a model top below the stratopause in several studies (Charlton-Perez et al., 2012; Hardiman et al., 2012; Wilcox et al., 2012), although other factors such as differences in tropospheric warming or ozone could affect the two sub-ensembles.

\subsection{Land ice sheets}

The rate of melt water release from the Greenland and Antarctic ice sheets in response to climate change remains a major source of uncertainty in projections of sea level rise (see Sections 13.4.3 and 13.4.4). Until recently, the long-term response of these ice sheets to alterations in the surrounding atmosphere and ocean has been simulated using offline models. Several ESMs currently have the capability to have ice sheet component models coupled to the rest of the climate system (Driesschaert et al., 2007; Charbit et al., 2008; Vizcaino et al., 2008; Huybrechts et al., 2011; Robinson et al., 2012) although these capabilities are not exercised for CMIP5.

\subsection{Additional features in ocean-atmosphere coupling}

Several features in the coupling between the ocean and the atmosphere have become more widespread since the AR4. The bulk formulae used to compute the turbulent fluxes of heat, water and momentum at the air-sea interface, have been revised. A number of models now consider the ocean surface current when calculating wind stress (e.g., Luo et al., 2005; Jungclaus et al., 2006). The coupling frequency has been increased in some cases to include the diurnal cycle, which was shown to reduce the SST bias in the tropical Pacific (Schmidt et al., 2006; Bernie et al., 2008; Ham et al., 2010). Several models now represent the coupling between the penetration of the solar radiation into the ocean and light-absorbing chlorophyll, with some implications on the representation of the mean climate and climate variability (Murtugudde et al., 2002; Wetzel et al., 2006). This coupling is achieved either by prescribing the chlorophyll distribution from observations, or by computing the chlorophyll distribution with an ocean biogeochemical model (e.g., Arora et al., 2009).

\subsubsection{Resolution}

The typical horizontal resolution (defined here as horizontal grid spacing) for current AOGCMs and ESMs is roughly 1 to 2 degrees for the atmospheric component and around 1 degree for the ocean (Table 9.1). The typical number of vertical layers is around 30 to 40 for the atmosphere and around 30 to 60 for the ocean (note that some 'high-top' models may have 80 or more vertical levels in the atmosphere). There has been some modest increase in model resolution since the AR4, especially for the near-term simulations (e.g., around 0.5 degree for the atmosphere in some cases), based on increased availability of more powerful computers. For the models used in long-term simulations with interactive biogeochemistry, the resolution has not increased substantially due to the trade-off against higher complexity in such models. Since the AR4, typical regional climate model resolution has increased from around $50 \mathrm{~km}$ to around $25 \mathrm{~km}$ (see Section 9.6.2.2), and the impact of this has been explored with multi-decadal regional simulations (e.g., Christensen et al., 2010). In some cases, RCMs are being run at $10 \mathrm{~km}$ resolution or higher (e.g., Kanada et al., 2008; Kusaka et al., 2010; van Roosmalen et al., 2010; Kendon et al., 2012).

Higher resolution can sometimes lead to a stepwise, rather than incremental, improvement in model performance (e.g., Roberts et al., 2004; Shaffrey et al., 2009). For example, ocean models undergo a transition from laminar to eddy-permitting when the computational grid contains more than one or two grid points per first baroclinic Rossby radius (i.e., finer than $50 \mathrm{~km}$ at low latitudes and $10 \mathrm{~km}$ at high latitudes) (Smith et al., 2000; McWilliams, 2008). Such mesoscale eddy-permitting ocean models better capture the large amount of energy contained in fronts, boundary currents, and time dependent eddy features (e.g., McClean et al., 2006). Models run at such resolution have been used for some climate simulations, though much work remains before they are as mature as the coarser models currently in use (Bryan et al., 2007; Bryan et al., 2010; Farneti et al., 2010; McClean et al., 2011; Delworth et al., 2012).

Similarly, atmospheric models with grids that allow the explicit representation of convective cloud systems (i.e., finer than a few kilometres) avoid employing a parameterization of their effects-a longstanding source of uncertainty in climate models. For example, Kendon et al. (2012) simulated the climate of the UK region over a 20-year period at $1.5 \mathrm{~km}$ resolution, and demonstrated several improvements of errors typical of coarser resolution models. Further discussion of this is provided in Section 7.2.2.

\subsection{Techniques for Assessing Model Performance}

Systematic evaluation of models through comparisons with observations is a prerequisite to applying them confidently. Several significant developments in model evaluation have occurred since the AR4 and are assessed in this section. This is followed by a description of the overall approach to evaluation taken in this chapter and a discussion of its known limitations.

\subsubsection{New Developments in Model Evaluation Approaches}

\subsubsection{Evaluating the Overall Model Results}

The most straightforward approach to evaluate models is to compare simulated quantities (e.g., global distributions of temperature, precipitation, radiation etc.) with corresponding observationally based estimates (e.g., Gleckler et al., 2008; Pincus et al., 2008; Reichler and Kim, 2008). A significant development since the AR4 is the increased use of quantitative statistical measures, referred to as performance metrics. The use of such metrics simplifies synthesis and visualization of model performance (Gleckler et al., 2008; Pincus et al., 2008; Waugh and Eyring, 2008; Cadule et al., 2010; Sahany et al., 2012) and enables the 
quantitative assessment of model improvements over time (Reichler and Kim, 2008). Recent work has addressed redundancy of multiple performance metrics through methods such as cluster analysis (Yokoi et al., 2011; Nishii et al., 2012).

\subsubsection{Isolating Processes}

To understand the cause of model errors it is necessary to evaluate the representation of processes both in the context of the full model and in isolation. A number of evaluation techniques to achieve both process and component isolation have been developed. One involves the so-called 'regime-oriented' approach to process evaluation. Instead of averaging model results in time (e.g., seasonal averages) or space (e.g. global averages), results are averaged within categories that describe physically distinct regimes of the system. Applications of this approach since the AR4 include the use of circulation regimes (Bellucci et al., 2010; Brown et al., 2010b; Brient and Bony, 2012; Ichikawa et al., 2012), cloud regimes (Williams and Brooks, 2008; Chen and Del Genio, 2009; Williams and Webb, 2009; Tsushima et al., 2013) and thermodynamic states (Sahany et al., 2012; Su, 2012). The application of new observations, such as vertically resolved cloud and water vapour information from satellites (Jiang et al., 2012a; Konsta et al., 2012; Quaas, 2012) and water isotopes (Risi et al., 2012a; Risi et al., 2012b), has also enhanced the ability to evaluate processes in climate models.

Another approach involves the isolation of model components or parameterizations in off-line simulations, such as Single Column Models of the atmosphere. Results of such simulations are compared to measurements from field studies or to results of more detailed process models (Randall et al., 2003). Numerous process evaluation data sets have been collected since the AR4 (Redelsperger et al., 2006; IIIingworth et al., 2007; Verlinde et al., 2007; May et al., 2008; Wood et al., 2011) and have been applied to the evaluation of climate model processes (Xie et al., 2008; Boone et al., 2009; Boyle and Klein, 2010; Hourdin et al., 2010). These studies are crucial to test the realism of the process formulations that underpin climate models.

\subsubsection{Instrument Simulators}

Satellites provide nearly global coverage, sampling across many meteorological conditions. This makes them powerful tools for model evaluation. The conventional approach has been to convert satellite-observed radiation information to 'model-equivalents' (Stephens and Kummerow, 2007), and these have been used in numerous studies (Allan et al., 2007; Gleckler et al., 2008; Li et al., 2008; Pincus et al., 2008; Waliser et al., 2009b; Li et al., 2011a, 2012a; Jiang et al., 2012a). A challenge is that limitations of the satellite sensors demand various assumptions in order to convert a satellite measurement into a 'model equivalent' climate variable.

An alternative approach is to calculate 'observation-equivalents' from models using radiative transfer calculations to simulate what the satellite would provide if the satellite system were 'observing' the model. This approach is usually referred to as an 'instrument simulator'. Microphysical assumptions (which differ from model to model) can be included in the simulators, avoiding inconsistencies. A simulator for cloud properties from the International Cloud Satellite Climatology
Project (ISCCP) (Yu et al., 1996; Klein and Jakob, 1999; Webb et al., 2001) has been widely used for model evaluation since the AR4 (Chen and Del Genio, 2009; Marchand et al., 2009; Wyant et al., 2009; Yokohata et al., 2010), often in conjunction with statistical techniques to separate model clouds into cloud regimes (e.g., Field et al., 2008; Williams and Brooks, 2008; Williams and Webb, 2009). New simulators for other satellite products have also been developed and are increasingly applied for model evaluation (Bodas-Salcedo et al., 2011). Although often focussed on clouds and precipitation, the simulator approach has also been used successfully for other variables such as upper tropospheric humidity (Allan et al., 2003; lacono et al., 2003; Ringer et al., 2003; Brogniez et al., 2005; Brogniez and Pierrehumbert, 2007; Zhang et al., 2008b; Bodas-Salcedo et al., 2011). Although providing an alternative to the use of model-equivalents from observations, instrument simulators have limitations (Pincus et al., 2012) and are best applied in combination with other model evaluation techniques.

\subsubsection{Initial Value Techniques}

To be able to forecast the weather a few days ahead, knowledge of the present state of the atmosphere is of primary importance. In contrast, climate predictions and projections simulate the statistics of weather seasons to centuries in advance. Despite their differences, both weather predictions and projections of future climate are performed with very similar atmospheric model components. The atmospheric component of climate models can be integrated as a weather prediction model if initialized appropriately (Phillips et al., 2004). This allows testing parameterized sub-grid scale processes without the complication of feedbacks substantially altering the underlying state of the atmosphere.

The application of these techniques since the AR4 has led to some new insights. For example, many of the systematic errors in the modelled climate develop within a few days of simulation, highlighting the important role of fast, parameterized processes (Klein et al., 2006; Boyle et al., 2008; Xie et al., 2012). Errors in cloud properties for example were shown to be present within a few days in a forecast in at least some models (Williams and Brooks, 2008), although this was not the case in another model (Boyle and Klein, 2010; Zhang et al., 2010b). Other studies have highlighted the advantage of such methodologies for the detailed evaluation of model processes using observations that are available only for limited locations and times (Williamson and Olson, 2007; Bodas-Salcedo et al., 2008; Xie et al., 2008; Hannay et al., 2009; Boyle and Klein, 2010), an approach that is difficult to apply to long-term climate simulations.

\subsubsection{Ensemble Approaches for Model Evaluation}

Ensemble methods are used to explore the uncertainty in climate model simulations that arise from internal variability, boundary conditions, parameter values for a given model structure or structural uncertainty due to different model formulations (Tebaldi and Knutti, 2007; Hawkins and Sutton, 2009; Knutti et al., 2010a). Since the AR4, techniques have been designed to specifically evaluate model performance of individual ensemble members. Although this is typically done to better characterize uncertainties, the methods and insights are applicable to model evaluation in general. The ensembles are generally 
of two types: Multi-model Ensembles (MMEs) and Perturbed Parameter (or Physics) Ensembles (PPEs).

\subsubsection{Multi-Model Ensembles}

The MME is created from existing model simulations from multiple climate modelling centres. MMEs sample structural uncertainty and internal variability. However, the sample size of MMEs is small, and is confounded because some climate models have been developed by sharing model components leading to shared biases (Masson and Knutti, 2011a). Thus, MME members cannot be treated as purely independent, which implies a reduction in the effective number of independent models (Tebaldi and Knutti, 2007; Jun et al., 2008; Knutti, 2010; Knutti et al., 2010a; Pennell and Reichler, 2011).

\subsubsection{Perturbed-Parameter Ensembles}

In contrast, PPEs are created to assess uncertainty based on a single model and benefit from the explicit control on parameter perturbations. This allows statistical methods to determine which parameters are the main drivers of uncertainty across the ensemble (e.g., Rougier et al., 2009). PPEs have been used frequently in simpler models such as EMICs (Xiao et al., 1998; Forest et al., 2002, 2006, 2008; Stott and Forest, 2007; Knutti and Tomassini, 2008; Sokolov et al., 2009; Loutre et al., 2011) and are now being applied to more complex models (Murphy et al., 2004; Annan et al., 2005; Stainforth et al., 2005; Collins et al., 2006a, 2007; Jackson et al., 2008a; Brierley et al., 2010; Klocke et al., 2011; Lambert et al., 2012). The disadvantage of PPEs is that they do not explore structural uncertainty, and thus the estimated uncertainty will depend on the underlying model that is perturbed (Yokohata et al., 2010) and may be too narrow (Sakaguchi et al., 2012). Several studies (Sexton et al., 2012; Sanderson, 2013) recognize the importance of sampling both parametric and structural uncertainty by combining information from both MMEs and PPEs. However, even these approaches cannot account for the effect on uncertainty of systematic errors.

\subsubsection{Statistical Methods Applied to Ensembles}

The most common approach to characterize MME results is to calculate the arithmetic mean of the individual model results, referred to as an unweighted multi-model mean. This approach of 'one vote per model' gives equal weight to each climate model regardless of (1) how many simulations each model has contributed, (2) how interdependent the models are or (3) how well each model has fared in objective evaluation. The multi-model mean will be used often in this chapter. Some climate models share a common lineage and so share common biases (Frame et al., 2005; Tebaldi and Knutti, 2007; Jun et al., 2008; Knutti, 2010; Knutti et al., 2010a, 2013; Annan and Hargreaves, 2011; Pennell and Reichler, 2011; Knutti and Sedlácek, 2013). As a result, collections such as the CMIP5 MME cannot be considered a random sample of independent models. This complexity creates challenges for how best to make quantitative inferences of future climate as discussed further in Chapter 12 (Knutti et al., 2010a; Collins et al., 2012; Stephenson et al., 2012; Sansom et al., 2013).

Annan and Hargreaves (2010) have proposed a 'rank histogram' approach to evaluate model ensembles as a whole, rather than individual models, by diagnosing whether observations can be considered statistically indistinguishable from a model ensemble. Studies based on this approach have suggested that MMEs (CMIP3/5) are 'reliable' in that they are not too narrow or too dispersive as a sample of possible models, but existing single-model-based ensembles tend to be too narrow (Yokohata et al., 2012, 2013). Although initial work has analysed the current mean climate state, further work is required to study the relationships between simulation errors and uncertainties in ensembles of future projections (Collins et al., 2012).

Bayesian methods offer insights into how to account for model inadequacies and combine information from several metrics in both MME and PPE approaches (Sexton and Murphy, 2012; Sexton et al., 2012), but they are complex. A simpler strategy of screening out some model variants on the basis of some observational comparison has been used with some PPEs (Lambert et al., 2012; Shiogama et al., 2012). Edwards et al. (2011) provided a statistical framework for 'pre-calibrating' out such poor model variants. Screening techniques have also been used with MMEs (Santer et al., 2009).

Additional Bayesian methods are applied to the MMEs so that past model performance is combined with prior distributions to estimate uncertainty from the MME (Furrer et al., 2007; Tebaldi and Knutti, 2007; Milliff et al., 2011). Similar to Bayesian PPE methods, common biases can be assessed within the MME to determine effective independence of the climate models (Knutti et al., 2013) (see Section 12.2.2 for a discussion of the assumptions in the Bayesian approaches).

\subsubsection{The Model Evaluation Approach Used in this Chapter and Its Limitations}

This chapter applies a variety of evaluation techniques ranging from visual comparison of observations and the multi-model ensemble and its mean, to application of quantitative performance metrics (see Section 9.2.2). No individual evaluation technique or performance measure is considered superior; rather, it is the combined use of many techniques and measures that provides a comprehensive overview of model performance.

Although crucial, the evaluation of climate models based on past climate observations has some important limitations. By necessity, it is limited to those variables and phenomena for which observations exist. Table 9.3 provides an overview of the observations used in this chapter. In many cases, the lack or insufficient quality of long-term observations, be it a specific variable, an important processes, or a particular region (e.g., polar areas, the upper troposphere/lower stratosphere (UTLS), and the deep ocean), remains an impediment. In addition, owing to observational uncertainties and the presence of internal variability, the observational record against which models are assessed is 'imperfect'. These limitations can be reduced, but not entirely eliminated, through the use of multiple independent observations of the same variable as well as the use of model ensembles.

The approach to model evaluation taken in the chapter reflects the need for climate models to represent the observed behaviour of past climate as a necessary condition to be considered a viable tool for future projections. This does not, however, provide an answer to 
the much more difficult question of determining how well a model must agree with observations before projections made with it can be deemed reliable. Since the AR4, there are a few examples of emergent constraints where observations are used to constrain multi-model ensemble projections. These examples, which are discussed further in Section 9.8.3, remain part of an area of active and as yet inconclusive research.

Table 9.3 Overview of observations that are used to evaluate climate models in this chapter. The quantity and CMIP5 output variable name are given along with references for the observations. Superscript (1) indicates this observations-based data set is obtained from atmospheric reanalysis. Superscript (D) indicates default reference; superscript (A) alternate reference.

\begin{tabular}{|c|c|c|c|c|}
\hline Quantity & $\begin{array}{l}\text { CMIP5 Output } \\
\text { Variable Name }\end{array}$ & $\begin{array}{c}\text { Observations } \\
\text { (Default/Alternates) }\end{array}$ & Reference & Figure and Section Number(s) \\
\hline \multicolumn{5}{|l|}{ ATMOSPHERE } \\
\hline $\begin{array}{l}\text { Zonal mean } \\
\text { wind }\left(\mathrm{m} \mathrm{s}^{-1}\right)\end{array}$ & $\begin{array}{c}\mathrm{Ua} \\
(200,850 \mathrm{hPa})\end{array}$ & $\begin{array}{l}\text { ERA-Interim }{ }^{1} \\
\text { NCEP-NCAR }^{1}\end{array}$ & $\begin{array}{l}\text { Dee et al. (2011) } \\
\text { Kalnay et al. (1996) }\end{array}$ & $\begin{array}{l}\text { Figure } 9.7^{D} \text {, Section 9.4.1 } \\
\text { Figure 9.7 }{ }^{A} \text {, Section 9.4.1 }\end{array}$ \\
\hline $\begin{array}{l}\text { Zonal wind } \\
\text { stress }\left(\mathrm{m} \mathrm{s}^{-1}\right)\end{array}$ & Tauu & $\begin{array}{l}\text { QuikSCAT satellite measurements } \\
\text { NCEP/NCAR reanalysis } \\
\text { ERA-Interim }\end{array}$ & $\begin{array}{l}\text { Risien and Chelton (2008) } \\
\text { Kalnay et al. (1996) } \\
\text { Dee et al. (2011) }\end{array}$ & $\begin{array}{l}\text { Figures } 9.19-9.20 \text {, Section } 9.4 .2 \\
\text { Figures } 9.19-9.20 \text {, Section } 9.4 .2 \\
\text { Figures } 9.19-9.20 \text {, Section } 9.4 .2\end{array}$ \\
\hline Meridional wind $\left(\mathrm{m} \mathrm{s}^{-1}\right)$ & $\begin{array}{c}V a \\
(200,850 \mathrm{hPa})\end{array}$ & $\begin{array}{l}\text { ERA-Interim }{ }^{1} \\
\text { NCEP-NCAR }{ }^{1}\end{array}$ & $\begin{array}{l}\text { Dee et al. (2011) } \\
\text { Kalnay et al. (1996) }\end{array}$ & $\begin{array}{l}\text { Figure 9.7D, Section 9.4.1 } \\
\text { Figure 9.7 }{ }^{A} \text {, Section 9.4.1 }\end{array}$ \\
\hline $\begin{array}{l}\text { Clear sky TOA short- } \\
\text { wave cloud radiative } \\
\text { effect }\left(W \mathrm{~m}^{-2}\right)\end{array}$ & $\begin{array}{l}\text { SW CRE } \\
\text { Derived from CMIP5 } \\
\text { rsut and rsutcs }\end{array}$ & $\begin{array}{l}\text { CERES EBAF } 2.6 \\
\text { CERES ES-4 ERBE }\end{array}$ & $\begin{array}{l}\text { Loeb et al. (2009) } \\
\text { Loeb et al. (2009) } \\
\text { Barkstrom (1984) }\end{array}$ & $\begin{array}{l}\text { Figures } 9.5^{D}, 9.6^{D}, 9.7^{D} \text {, Section } 9.4 .1 \\
\text { Figure } 9.5^{A} \text {, Section 9.4.1 } \\
\text { Figure 9.7 } \text {, Section 9.4.1 }\end{array}$ \\
\hline $\begin{array}{l}\text { Clear sky TOA long- } \\
\text { wave cloud radiative } \\
\text { effect }\left(\mathrm{W} \mathrm{m}^{-2}\right)\end{array}$ & $\begin{array}{l}\text { LW CRE } \\
\text { Derived from CMIP5 } \\
\text { rsut and rsutcs }\end{array}$ & $\begin{array}{l}\text { CERES EBAF } 2.6 \\
\text { CERES ES-4 ERBE }\end{array}$ & $\begin{array}{l}\text { Loeb et al. (2009) } \\
\text { Loeb et al. (2009) }\end{array}$ & $\begin{array}{l}\text { Figure } 9.9^{D} \text {, Section 9.4.1 } \\
\text { Figure 9.5 } \text {, Section 9.4.1 }\end{array}$ \\
\hline $\begin{array}{l}\text { Total precipitation } \\
\left(\mathrm{mm} \text { day }^{-1}\right)\end{array}$ & $\operatorname{Pr}$ & $\begin{array}{l}\text { GPCP } \\
\text { CMAP } \\
\text { CRU TS3.10.1 }\end{array}$ & $\begin{array}{l}\text { Adler et al. (2003) } \\
\text { Xie and Arkin (1997) } \\
\text { Mitchell and Jones (2005) }\end{array}$ & $\begin{array}{l}\text { Figures } 9.4,9.6^{D}, 9.7^{D} \text {, Section } 9.4 .1 \text {; } \\
\text { Figures } 9.38,9.40 \text {, Section } 9.6 .1 \\
\text { Figures } 9.6^{A}, 9.77^{A} \text {, Section } 9.4 .1 ; \\
\text { Figures } 9.38,9.40 \text {, Section } 9.6 .1 \\
\text { Figures } 9.38,9.39 \text {, Section } 9.6 .1\end{array}$ \\
\hline
\end{tabular}


Table 9.3 (continued)

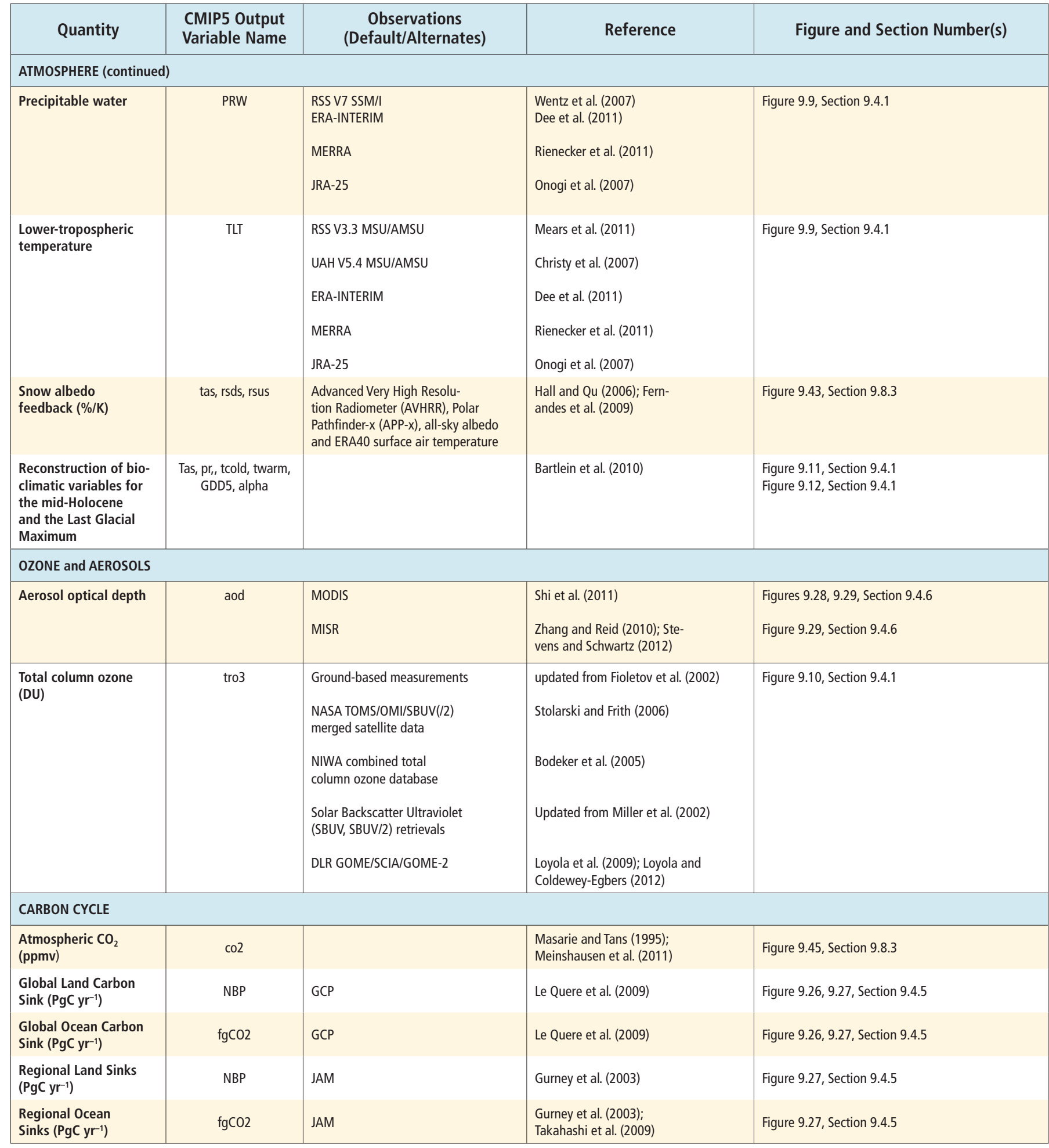


Table 9.3 (continued)

\begin{tabular}{|c|c|c|c|c|}
\hline Quantity & $\begin{array}{l}\text { CMIP5 Output } \\
\text { Variable Name }\end{array}$ & $\begin{array}{c}\text { Observations } \\
\text { (Default/Alternates) }\end{array}$ & Reference & Figure and Section Number(s) \\
\hline \multicolumn{5}{|l|}{ OCEAN } \\
\hline Annual mean salinity & so & & Antonov et al. (2010) & Figure 9.13 , Section 9.4.2 \\
\hline $\begin{array}{l}\text { Global ocean heat } \\
\text { content }(0 \text { to } 700 \mathrm{~m})\end{array}$ & $\mathrm{OHC}$ & $\begin{array}{l}\text { Levitus } \\
\text { Ishii } \\
\text { Domingues }\end{array}$ & $\begin{array}{l}\text { Levitus et al. (2009) } \\
\text { Ishii and Kimoto, 2009) } \\
\text { Domingues et al. (2008) }\end{array}$ & Figure 9.17, Section 9.4.2 \\
\hline $\begin{array}{l}\text { Annual mean tem- } \\
\text { perature and salinity }\end{array}$ & & $\begin{array}{l}\text { Palaeoclimate reconstruction } \\
\text { of temperature and salinity }\end{array}$ & Adkins et al. (2002) & Figure 9.18 , Section 9.4 .2 \\
\hline $\begin{array}{l}\text { Total area }\left(\mathbf{k m}^{2}\right) \text { of grid } \\
\text { cells where Sea Ice } \\
\text { Area Fraction }(\%) \text { is } \\
>15 \% \text {. Boundary of sea } \\
\text { ice where Sea Ice Area } \\
\text { Fraction }(\%) \text { is }>15 \%\end{array}$ & & $\begin{array}{l}\text { HadISST } \\
\text { NSIDC } \\
\text { NASA }\end{array}$ & $\begin{array}{l}\text { Rayner et al. (2003) } \\
\text { Fetterer et al. (2002) } \\
\text { Comiso and Nishio (2008) }\end{array}$ & $\begin{array}{l}\text { Figure } 9.22 \text {, Section } 9.4 .3 \\
\text { Figure } 9.23 \text {, Section } 9.4 .3 \\
\text { Figure } 9.24 \text {, Section } 9.4 .3\end{array}$ \\
\hline $\begin{array}{l}\text { 3-hour precipitation } \\
\text { fields }\end{array}$ & & $\begin{array}{l}15,000 \text { stations and cor- } \\
\text { rected Ta from COADS (Dai } \\
\text { and Deser, 1999; Dai, } 2001\end{array}$ & Dai (2006) & Figure 9.30, Section 9.5.2 \\
\hline $\begin{array}{l}\text { Absolute value of } \\
\text { MJJAS minus NDJFM } \\
\text { precipitation exceeding } \\
375 \mathrm{~mm}\end{array}$ & & $\begin{array}{l}\text { GPCP } \\
\text { (Adler et al., 2003) }\end{array}$ & Wang et al. (2011a) & Figure 9.32 , Section 9.5 .2 \\
\hline \multicolumn{5}{|l|}{ EXTREMES } \\
\hline $\begin{array}{l}\text { Daily maximum and } \\
\text { minimum surface air } \\
\text { temperature fields }\left({ }^{\circ} \mathrm{C}\right) \\
\text { Daily precipitation } \\
\text { fields (mm day-1) } \\
\text { for calculating } \\
\text { extremes indices }\end{array}$ & tas, precip & $\begin{array}{l}\text { ERA40 } \\
\text { ERA-Interim, } \\
\text { NCEP/NCAR Reanalysis 1, } \\
\text { NCEP-DOE, Reanalysis } 2\end{array}$ & $\begin{array}{l}\text { Uppala et al. (2005) } \\
\text { Dee et al. (2011) } \\
\text { Kistler et al. (2001) } \\
\text { Kanamitsu et al. (2002) } \\
\text { Calculation of indices is based } \\
\text { on Sillmann et al. (2013) }\end{array}$ & Figure 9.37 , Section 9.5.4 \\
\hline $\begin{array}{l}\text { Temperature extremes } \\
\text { indices based on } \\
\text { station observations }\end{array}$ & & HadEX2 & Donat et al. (2013) & Figure 9.37 , Section 9.5 .4 \\
\hline
\end{tabular}

Notes: 1 This observationally constrained data set is obtained from atmospheric reanalysis.

D Default reference.

A Alternate reference. 


\subsection{Experimental Strategies in Support of Climate Model Evaluation}

\subsubsection{The Role of Model Intercomparisons}

Systematic model evaluation requires a coordinated and well-documented suite of model simulations. Organized Model Intercomparison Projects (MIPs) provide this via standard or benchmark experiments that represent critical tests of a model's ability to simulate the observed climate. When modelling centres perform a common experiment, it offers the possibility to compare their results not just with observations, but with other models as well. This intercomparison enables researchers to explore the range of model behaviours, to isolate the various strengths and weaknesses of different models in a controlled setting, and to interpret, through idealized experiments, the inter-model differences. Benchmark MIP experiments offer a way to distinguish between errors particular to an individual model and those that might be more universal and should become priority targets for model improvement.

\subsubsection{Experimental Strategy for Coupled Model Intercomparison Project Phase 5}

\subsubsection{Experiments Utilized for Model Evaluation}

CMIP5 includes a much more comprehensive suite of model experiments than was available in the preceding CMIP3 results assessed in the AR4 (Meehl et al., 2007). In addition to a better constrained specification of historical forcing, the CMIP5 collection also includes initialized decadal-length predictions and long-term experiments using both ESMs and AOGCMs (Taylor et al., 2012b) (Figure 9.1). The $\mathrm{CO}_{2}$ forcing of these experiments is prescribed as a time series of either global mean concentrations or spatially resolved anthropogenic emissions (Section 9.3.2.2). The analyses of model performance in this chapter are based on the concentration-based experiments with the exception of the evaluation of the carbon cycle (see Section 9.4.5).

Most of the model diagnostics are derived from the historical simulations that span the period 1850-2005. In some cases, these historical simulations are augmented by results from a scenario run, either RCP4.5 or RCP8.5 (see Section 9.3.2.2), so as to facilitate comparison with more recent observations. CMIP5/Paleoclimate Modelling Intercomparison Project version 3 (PMIP3) simulations for the mid-Holocene and last glacial maximum are used to evaluate model response to palaeoclimatic conditions. Historical emissions-driven simulations are used to evaluate the prognostic carbon cycle. The analysis of global surface temperature variability is based in part on long pre-industrial control runs to facilitate calculation of variability on decadal to centennial time scales. Idealized simulations with $1 \%$ per year increases in $\mathrm{CO}_{2}$ are utilized to derive transient climate response. Equilibrium climate sensitivities are derived using results of specialized experiments, with fourfold $\mathrm{CO}_{2}$ increase, designed specifically for this purpose.

\subsubsection{Forcing of the Historical Experiments}

Under the protocols adopted for CMIP5 and previous assessments, the transient climate experiments are conducted in three phases. The first phase covers the start of the modern industrial period through to the present day, years 1850-2005 (van Vuuren et al., 2011). The second phase covers the future, 2006-2100, and is described by a collection of RCPs (Moss et al., 2010). As detailed in Chapter 12, the third phase is described by a corresponding collection of Extension Concentration
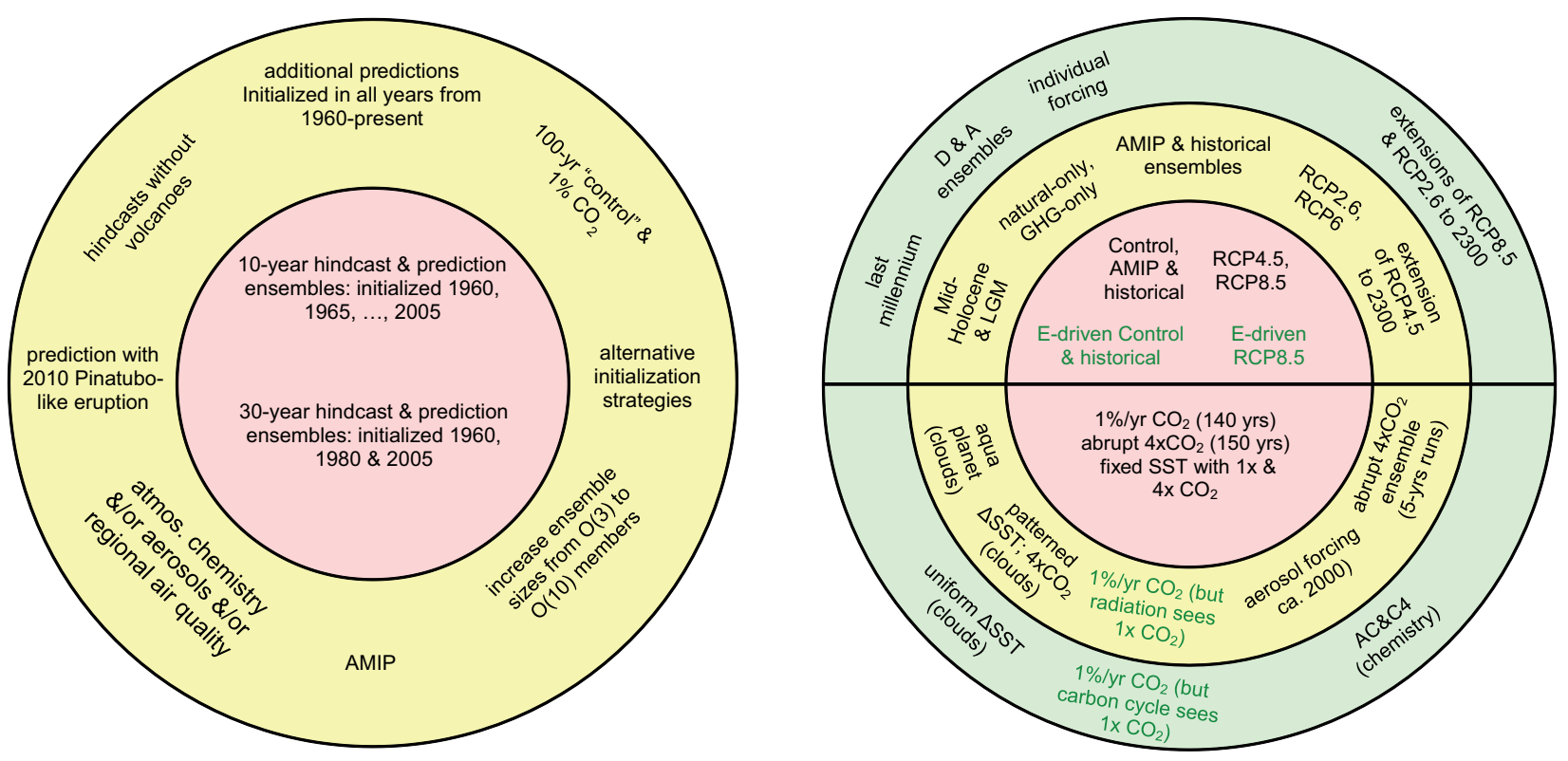

Figure 9.1 | Left: Schematic summary of CMIP5 short-term experiments with tier 1 experiments (yellow background) organized around a central core (pink background). (From Taylor et al., 2012b, their Figure 2). Right: Schematic summary of CMIP5 long-term experiments with tier 1 experiments (yellow background) and tier 2 experiments (green background) organized around a central core (pink background). Green font indicates simulations to be performed only by models with carbon cycle representations, and 'E-driven' means 'emission-driven'. Experiments in the upper semicircle either are suitable for comparison with observations or provide projections, whereas those in the lower semicircle are either idealized or diagnostic in nature, and aim to provide better understanding of the climate system and model behaviour. (From Taylor et al., 2012b, their Figure 3.) 
Pathways (Meinshausen et al., 2011). The forcings for the historical simulations evaluated in this section and are described briefly here (with more details in Annex II).

In the CMIP3 20th century experiments, the forcings from radiatively active species other than long-lived GHGs and sulphate aerosols were left to the discretion of the individual modelling groups (IPCC, 2007). By contrast, a comprehensive set of historical anthropogenic emissions and land use and land cover change data have been assembled for the CMIP5 experiments in order to produce a relatively homogeneous ensemble of historical simulations with common time series of forcing agents. Emissions of natural aerosols including soil dust, sea salt and volcanic species are still left to the discretion of the individual modelling groups.

For AOGCMs without chemical and biogeochemical cycles, the forcing agents are prescribed as a set of concentrations. The concentrations for GHGs and related compounds include $\mathrm{CO}_{2}, \mathrm{CH}_{4}, \mathrm{~N}_{2} \mathrm{O}$, all fluorinated gases controlled under the Kyoto Protocol (hydrofluorocarbons (HFCs), perfluorocarbons (PFCs), and sulphur hexafluoride $\left.\left(\mathrm{SF}_{6}\right)\right)$, and ozone-depleting substances controlled under the Montreal Protocol (chlorofluorocarbons (CFCs), hydrochlorofluorocarbons (HCFCs), Halons, carbon tetrachloride $\left(\mathrm{CCl}_{4}\right)$, methyl bromide $\left(\mathrm{CH}_{3} \mathrm{Br}\right)$, methyl chloride $\left.\left(\mathrm{CH}_{3} \mathrm{Cl}\right)\right)$. The concentrations for aerosol species include sulphate $\left(\mathrm{SO}_{4}\right)$, ammonium nitrate $\left(\mathrm{NH}_{4} \mathrm{NO}_{3}\right)$, hydrophobic and hydrophilic black carbon, hydrophobic and hydrophilic organic carbon, secondary organic aerosols (SOAs) and four size categories of dust and sea salt. For ESMs that include chemical and biogeochemical cycles, the forcing agents are prescribed both as a set of concentrations and as a set of emissions with provisions to separate the forcing by natural and anthropogenic $\mathrm{CO}_{2}$ (Hibbard et al., 2007). The emissions include time-dependent spatially resolved fluxes of $\mathrm{CH}_{4}, \mathrm{NO}_{x_{1}} \mathrm{CO}_{1} \mathrm{NH}_{3}$, black and organic carbon, and volatile organic compounds (VOCs). For models that treat the chemical processes associated with biomass burning, emissions of additional species such as $\mathrm{C}_{2} \mathrm{H}_{4} \mathrm{O}$ (acetaldehyde), $\mathrm{C}_{2} \mathrm{H}_{5} \mathrm{OH}$ (ethanol), $\mathrm{C}_{2} \mathrm{H}_{6} \mathrm{~S}$ (dimethylsulphide) and $\mathrm{C}_{3} \mathrm{H}_{6} \mathrm{O}$ (acetone) are also prescribed. Historical land use and land cover change is described in terms of the time-evolving partitioning of land surface area among cropland, pasture, primary land and secondary (recovering) land, including the effects of wood harvest and shifting cultivation, as well as land use changes and transitions from/to urban land (Hurtt et al., 2009). These emissions data are aggregated from empirical reconstructions of grassland and forest fires (Schultz et al., 2008; Mieville et al., 2010); international shipping (Eyring et al., 2010); aviation (Lee et al., 2009), sulphur (Smith et al., 2011b), black and organic carbon (Bond et al., 2007); and $\mathrm{NO}_{x_{1}} \mathrm{CO}, \mathrm{CH}_{4}$ and non methane volatile organic compounds (NMVOCs) (Lamarque et al., 2010) contributed by all other sectors.

For the natural forcings a recommended monthly averaged total solar irradiance time series was given, but there was no recommended treatment of volcanic forcing. Both integrated solar irradiance and its spectrum were available, but not all CMIP5 models used the spectral data. The data employed an 1850-2008 reconstruction of the solar cycle and its secular trend using observations of sunspots and faculae, the 10.7 $\mathrm{cm}$ solar irradiance measurements and satellite observations (Frohlich and Lean, 2004).For volcanic forcing CMIP5 models typically employed one of two prescribed volcanic aerosol data sets (Sato et al., 1993) or (Ammann et al., 2003) but at least one ESM employed interactive aerosol injection (Driscoll et al., 2012). The prescribed data sets did not incorporate injection from explosive volcanoes after 2000.

\subsubsection{Relationship of Decadal and Longer-Term Simulations}

The CMIP5 archive also includes a new class of decadal-prediction experiments (Meehl et al., 2009, 2013b) (Figure 9.1). The goal is to understand the relative roles of forced changes and internal variability in historical and near-term climate variables, and to assess the predictability that might be realized on decadal time scales. These experiments comprise two sets of hindcast and prediction ensembles with initial conditions spanning 1960 through 2005. The set of 10-year ensembles are initialized starting at 1960 in 1-year increments through the year 2005 while the 30-year ensembles are initialized at 1960, 1980 and 2005. The same physical models are often used for both the short-term and long-term experiments (Figure 9.1) despite the different initialization of these two sets of simulations. Results from the short-term experiments are described in detail in Chapter 11.

\subsection{Simulation of Recent and Longer-Term Records in Global Models}

\subsubsection{Atmosphere}

Many aspects of the atmosphere have been more extensively evaluated than other climate model components. One reason is the availability of near-global observationally based data for energy fluxes at the TOA, cloud cover and cloud condensate, temperature, winds, moisture, ozone and other important properties. As discussed in Box 2.3, atmospheric reanalyses have also enabled integrating independent observations in a physically consistent manner. In this section we use this diversity of data (see Table 9.3) to evaluate the large-scale atmospheric behaviour.

\subsubsection{Temperature and Precipitation Spatial Patterns of the Mean State}

Surface temperature is perhaps the most routinely examined quantity in atmospheric models. Many processes must be adequately represented in order for a model to realistically capture the observed temperature distribution. The dominant external influence is incoming solar radiation, but many aspects of the simulated climate play an important role in modulating regional temperature such as the presence of clouds and the complex interactions between the atmosphere and the underlying land, ocean, snow, ice and biosphere.

The annual mean surface air temperature (at $2 \mathrm{~m}$ ) is shown in Figure 9.2(a) for the mean of all available CMIP5 models, and the error, relative to an observationally constrained reanalysis (ECMWF reanalysis of the global atmosphere and surface conditions (ERA)-Interim; Dee et al., 2011) is shown in Figure 9.2(b). In most areas the multi-model mean agrees with the reanalysis to within $2^{\circ} \mathrm{C}$, but there are several locations where the biases are much larger, particularly at high elevations over the Himalayas and parts of both Greenland and Antarctica, near the ice edge in the North Atlantic, and over ocean upwelling regions 
off the west coasts of South America and Africa. Averaging the absolute error of the individual CMIP5 models (Figure 9.2c) yields similar magnitude as the multi-model mean bias (Figure 9.2b), implying that compensating errors across models is limited. The inconsistency across the three available global reanalyses (Figure 9.2d) that have assimilated temperature data at two metres (Onogi et al., 2007; Simmons et al., 2010) provides an indication of observational uncertainty. Although the reanalysis inconsistency is smaller than the mean absolute bias in almost all regions, areas where inconsistency is largest (typically where observations are sparse) tend to be the same regions where the CMIP5 models show largest mean absolute error.

Seasonal performance of models can be evaluated by examining the difference between means for December-January-February (DJF) and June-July-August (JJA). Figures 9.3(a) and (b) show the CMIP5 mean model seasonal cycle amplitude in surface air temperature (as measured by the difference between the DJF and JJA and the absolute value of this difference). The seasonal cycle amplitude is much larger over land where the thermal inertia is much smaller than over the oceans, and it is generally larger at higher latitudes as a result of the larger seasonal amplitude in insolation. Figures 9.3(c) and (d) show the mean model bias of the seasonal cycle relative to the ERA-Interim reanalysis (Dee et al., 2011). The largest biases correspond to areas of large seasonal amplitude, notably high latitudes over land, but relatively large biases are also evident in some lower latitude regions such as over northern India. Over most land areas the amplitude of the modelled seasonal cycle is larger than observed, whereas over much of the extratropical oceans the modelled amplitude is too small.

The simulation of precipitation is a more stringent test for models as it depends heavily on processes that must be parameterized. Challenges are compounded by the link to surface fields (topography, coastline, vegetation) that lead to much greater spatial heterogeneity at regional scales. Figure 9.4 shows the mean precipitation rate simulated by the CMIP5 multi-model ensemble, along with measures of error relative to precipitation analyses from the Global Precipitation Climatology Project (Adler et al., 2003). The magnitude of observational uncertainty for precipitation varies with region, which is why many studies make use (a) Multi Model Mean Surface Temperature

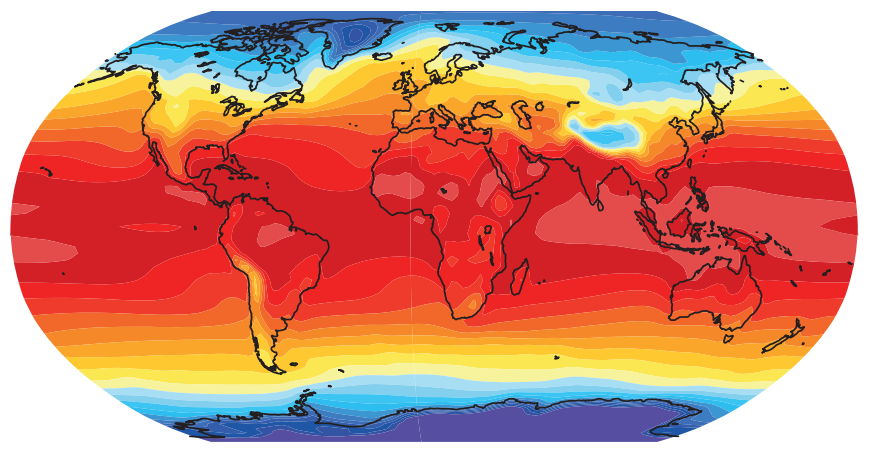

$\left({ }^{\circ} \mathrm{C}\right)$

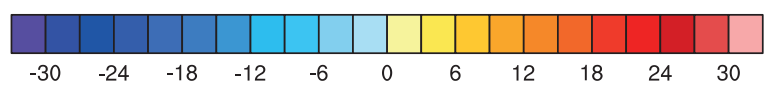

(c) Multi Model Mean of Absolute Error

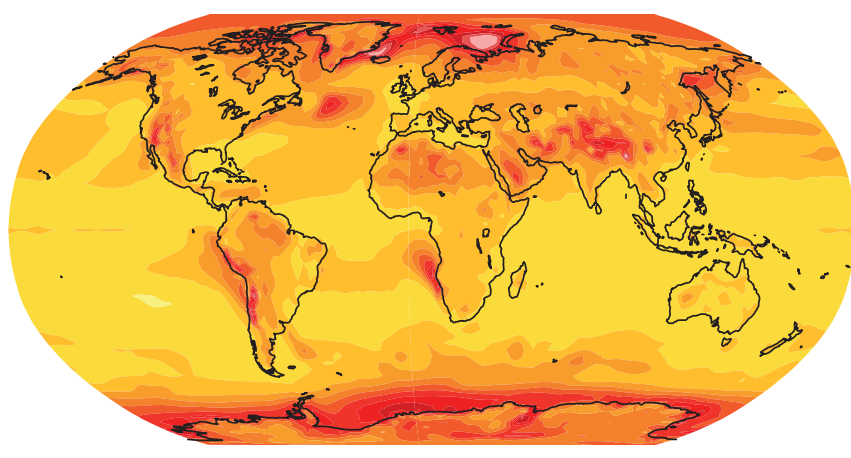

$\left({ }^{\circ} \mathrm{C}\right)$

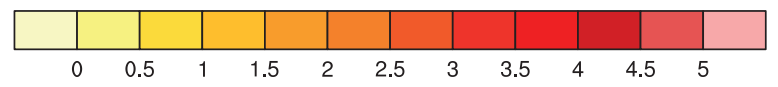

(b) Multi Model Mean Bias

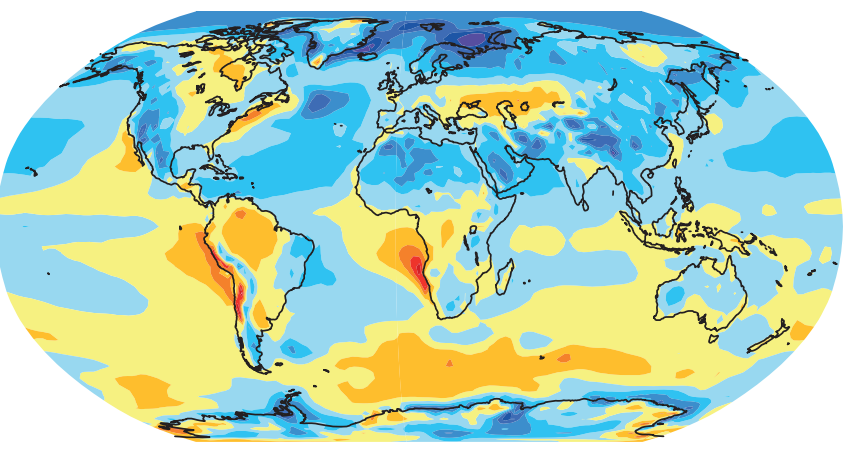

$\left({ }^{\circ} \mathrm{C}\right)$

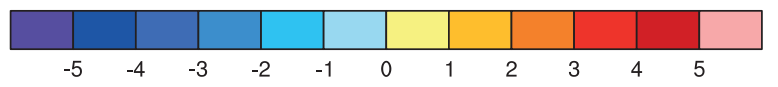

(d) Mean Reanalysis Inconsistency

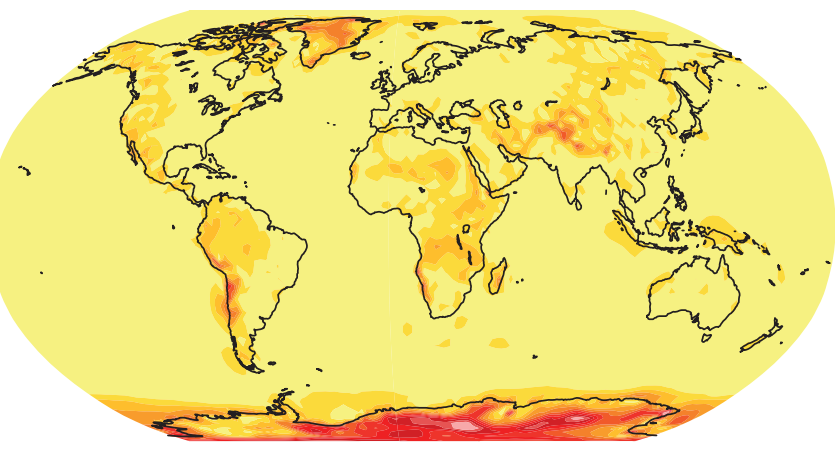

$\left({ }^{\circ} \mathrm{C}\right)$

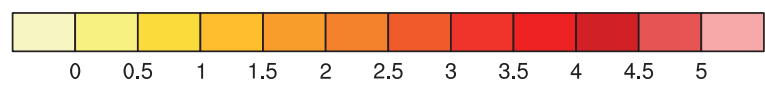

Figure 9.2 Annual-mean surface (2 $\mathrm{m}$ ) air temperature $\left({ }^{\circ} \mathrm{C}\right)$ for the period 1980-2005. (a) Multi-model (ensemble) mean constructed with one realization of all available models used in the CMIP5 historical experiment. (b) Multi-model-mean bias as the difference between the CMIP5 multi-model mean and the climatology from ECMWF reanalysis of the global atmosphere and surface conditions (ERA)-Interim (Dee et al., 2011); see Table 9.3. (c) Mean absolute model error with respect to the climatology from ERA-Interim. (d) Mean inconsistency between ERA-Interim, ERA 40-year reanalysis (ERA40) and Japanese 25-year ReAnalysis (JRA-25) products as the mean of the absolute pairwise differences between those fields for their common period (1979-2001). 
(a) Multi Model Mean Surface Temperature Seasonality

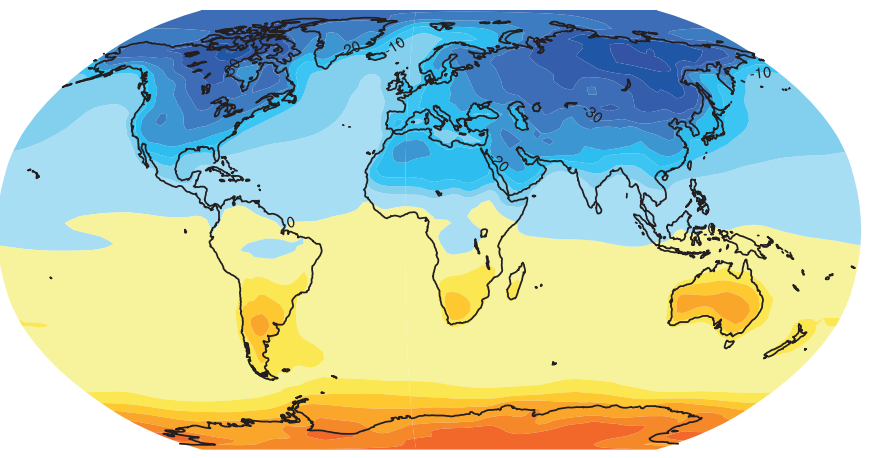

$\left({ }^{\circ} \mathrm{C}\right)$

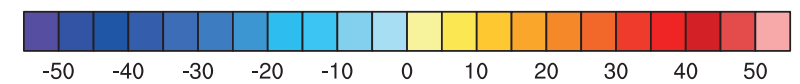

(c) Multi Model Mean Bias in Seasonality

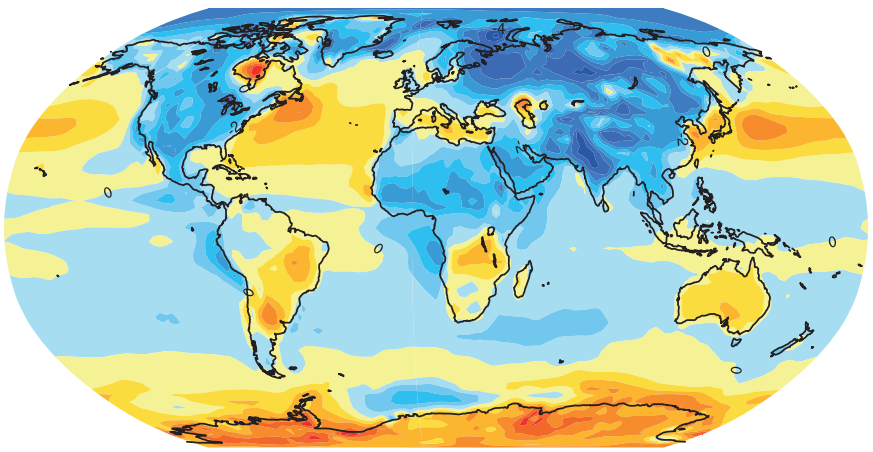

$\left({ }^{\circ} \mathrm{C}\right)$

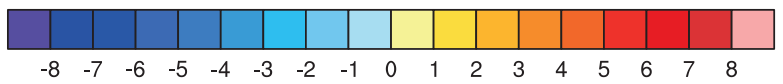

(b) Multi Model Mean of Absolute Seasonality

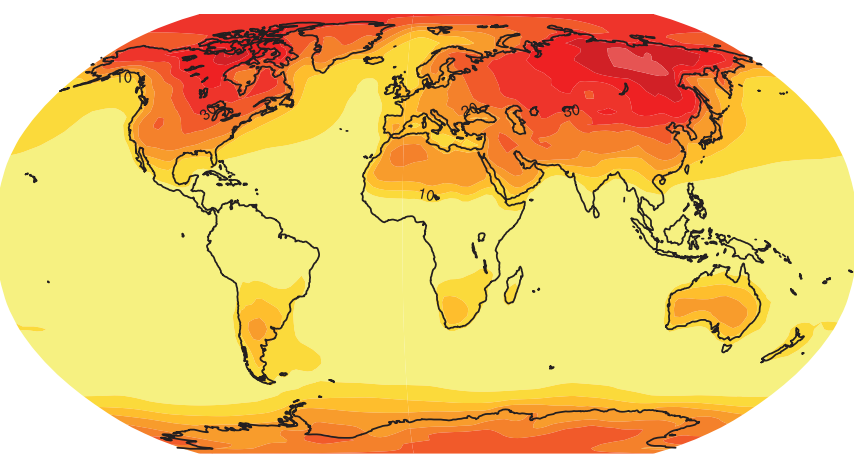

$\left({ }^{\circ} \mathrm{C}\right)$

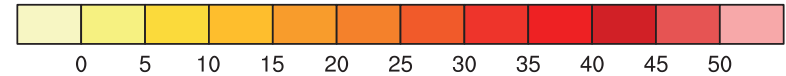

(d) Multi Model Mean Bias in Absolute Seasonality

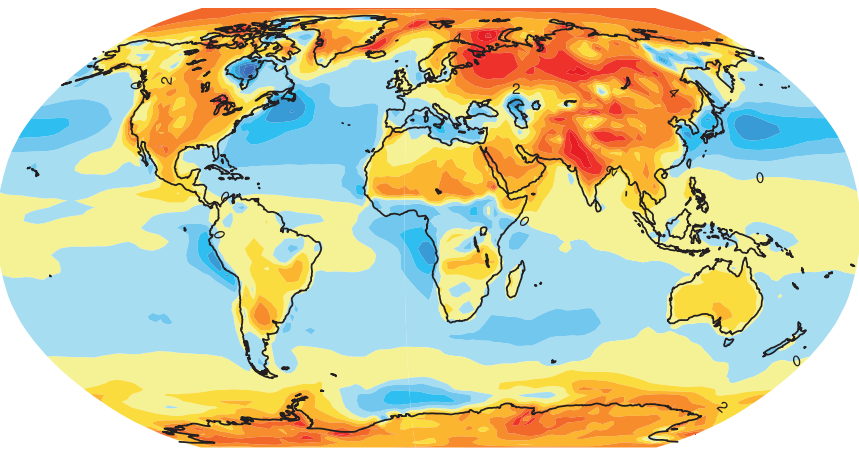

$\left({ }^{\circ} \mathrm{C}\right)$

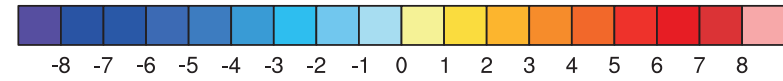

Figure 9.3 Seasonality (December-January-February minus June-July-August ) of surface $\left(2 \mathrm{~m}\right.$ ) air temperature $\left({ }^{\circ} \mathrm{C}\right)$ for the period 1980-2005. (a) Multi-model mean, calculated from one realization of all available CMIP5 models for the historical experiment. (b) Multi-model mean of absolute seasonality. (c) Difference between the multi-model mean and the ECMWF reanalysis of the global atmosphere and surface conditions (ERA)-Interim seasonality. (d) Difference between the multi-model mean and the ERA-Interim absolute seasonality.

of several estimates of precipitation. Known large-scale features are reproduced by the multi-model mean, such as a maximum precipitation just north of the equator in the central and eastern tropical Pacific, dry areas over the eastern subtropical ocean basins, and the minimum rainfall in Northern Africa (Dai, 2006). While many large-scale features of the tropical circulation are reasonably well simulated, there are persistent biases. These include too low precipitation along the equator in the Western Pacific associated with ocean-atmosphere feedbacks maintaining the equatorial cold tongue (Collins et al., 2010) and excessive precipitation in tropical convergence zones south of the equator in the Atlantic and the Eastern Pacific (Lin, 2007; Pincus et al., 2008). Other errors occurring in several models include an overly zonal orientation of the South-Pacific Convergence Zone (Brown et al., 2013) as well as an overestimate of the frequency of occurrence of light rain events (Stephens et al., 2010). Regional-scale precipitation simulation has strong parameter dependence (Rougier et al., 2009; Chen et al., 2010; Neelin et al., 2010), and in some models substantial improvements have been shown through increases in resolution (Delworth et al., 2012) and improved representations of sub-gridscale processes, particularly convection (Neale et al.,
2008). Judged by similarity with the spatial pattern of observations, the overall quality of the simulation of the mean state of precipitation in the CMIP5 ensemble is slightly better than in the CMIP3 ensemble (see FAQ 9.1 and Figure 9.6).

In summary, there is high confidence that large-scale patterns of surface temperature are well simulated by the CMIP5 models. In certain regions this agreement with observations is limited, particularly at elevations over the Himalayas and parts of both Greenland and Antarctica. The broad-scale features of precipitation as simulated by the CMIP5 models are in modest agreement with observations, but there are systematic errors in the Tropics.

\subsubsection{Atmospheric Moisture, Clouds and Radiation}

The global annual mean precipitable water is a measure of the total moisture content of the atmosphere. For the CMIP3 ensemble, the values of precipitable water agreed with one another and with multiple estimates from the National Centers for Environmental Prediction/ National Center for Atmospheric Research (NCEP/NCAR) and ECMWF 
(a) Multi Model Mean Precipitation

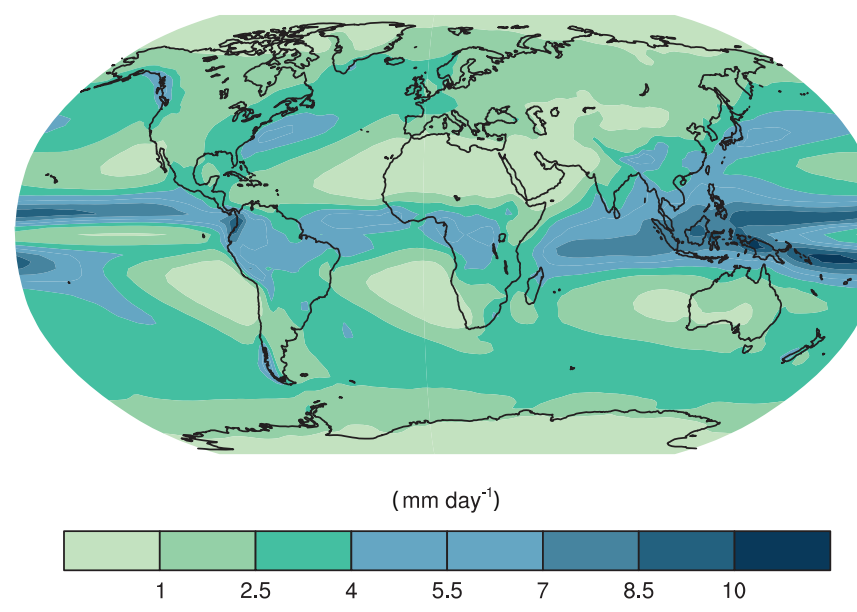

(c) Multi Model Mean of Absolute Error

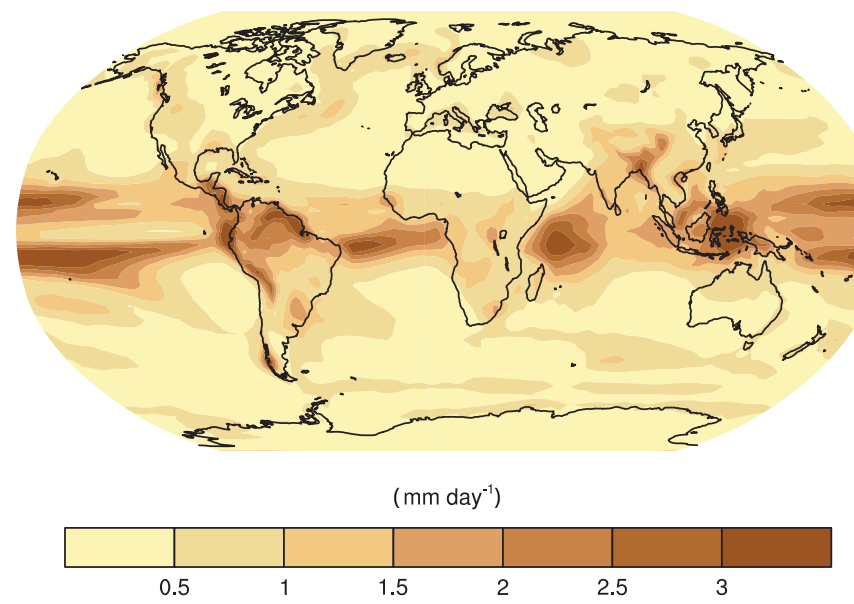

(b) Multi Model Mean Bias

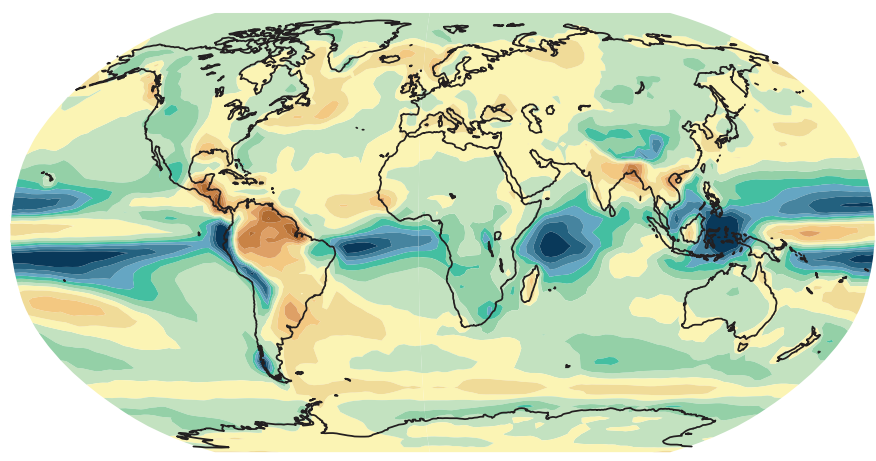

$\left(\mathrm{mm}\right.$ day $\left.^{-1}\right)$

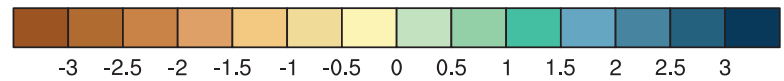

(d) Multi Model Mean of Relative Error

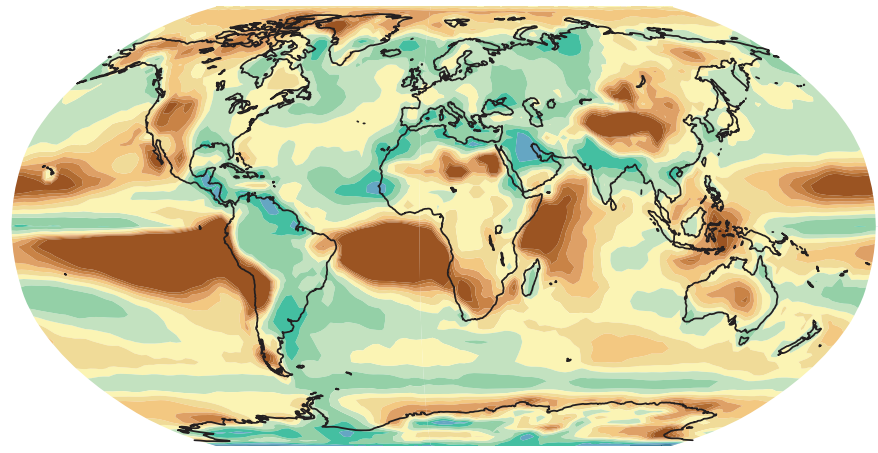

$(\%)$

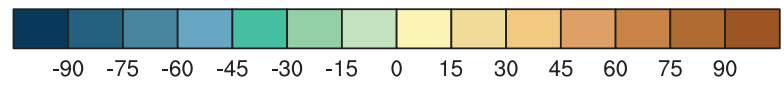

Figure 9.4 | Annual-mean precipitation rate (mm day ${ }^{-1}$ ) for the period 1980-2005. (a) Multi-model-mean constructed with one realization of all available AOGCMs used in the CMIP5 historical experiment. (b) Difference between multi-model mean and precipitation analyses from the Global Precipitation Climatology Project (Adler et al., 2003). (c) Multimodel-mean absolute error with respect to observations. (d) Multi-model-mean error relative to the multi-model-mean precipitation itself.

ERA40 meteorological reanalyses to within approximately 10\% (Waliser et al., 2007). Initial analysis of the CMIP5 ensemble shows the model results are within the uncertainties of the observations (Jiang et al., 2012a).

Modelling the vertical structure of water vapour is subject to greater uncertainty since the humidity profile is governed by a variety of processes. The CMIP3 models exhibited a significant dry bias of up to $25 \%$ in the boundary layer and a significant moist bias in the free troposphere of up to $100 \%$ (John and Soden, 2007). Upper tropospheric water vapour varied by a factor of three across the multi-model ensemble (Su et al., 2006). Many models have large biases in lower stratospheric water vapour (Gettelman et al., 2010), which could have implications for surface temperature change (Solomon et al., 2010). The limited number of studies available for the CMIP5 model ensemble broadly confirms the results from the earlier model generation. In tropical regions, the models are too dry in the lower troposphere and too moist in the upper troposphere, whereas in the extratropics they are too moist throughout the troposphere (Tian et al., 2013). However, many of the model values lie within the observational uncertainties.
Jiang et al. (2012a) show that the largest biases occur in the upper troposphere, with model values up to twice that observed, while in the middle and lower troposphere models simulate water vapour to within $10 \%$ of the observations.

The spatial patterns and seasonal cycle of the radiative fluxes at the TOA are fundamental energy balance quantities. Both the CMIP3 and CMIP5 model ensembles reproduce these patterns with considerable fidelity relative to the National Aeronautics and Space Adminsitration (NASA) Clouds and the Earth's Radiant Energy System (CERES) data sets (Pincus et al., 2008; Wang and Su, 2013). Globally averaged TOA shortwave and longwave components of the radiative fluxes in 12 atmosphere-only versions of the CMIP5 models were within $2.5 \mathrm{~W} \mathrm{~m}^{-2}$ of the observed values (Wang and Su, 2013).

Comparisons against surface components of radiative fluxes show that, on average, the CMIP5 models overestimate the global mean downward all-sky shortwave flux at the surface by $2 \pm 6 \mathrm{~W} \mathrm{~m}^{-2}(1 \pm$ $3 \%$ ) and underestimate the global downward longwave flux by $6 \pm 9$ W m-2 (2 $\pm 2 \%$ ) (Stephens et al., 2012). Although in tropical regions 
between 1 and $3 \mathrm{~W} \mathrm{~m}^{-2}$ of the bias may be due to systematic omission of precipitating and/or convective core ice hydrometeors (Waliser et al., 2011), the correlation between the biases in the all-sky and clear-sky downwelling fluxes suggests that systematic errors in clear-sky radiative transfer calculations may be a primary cause for these biases. This is consistent with an analysis of the global annual mean estimates of clear-sky atmospheric absorption from the CMIP3 ensemble and the systematic underestimation of clear-sky solar absorption by radiative transfer codes (Oreopoulos et al., 2012). The underestimation of absorption can be attributed to the omission or underestimation of (a) Shortwave cloud radiative effect - MOD-OBS

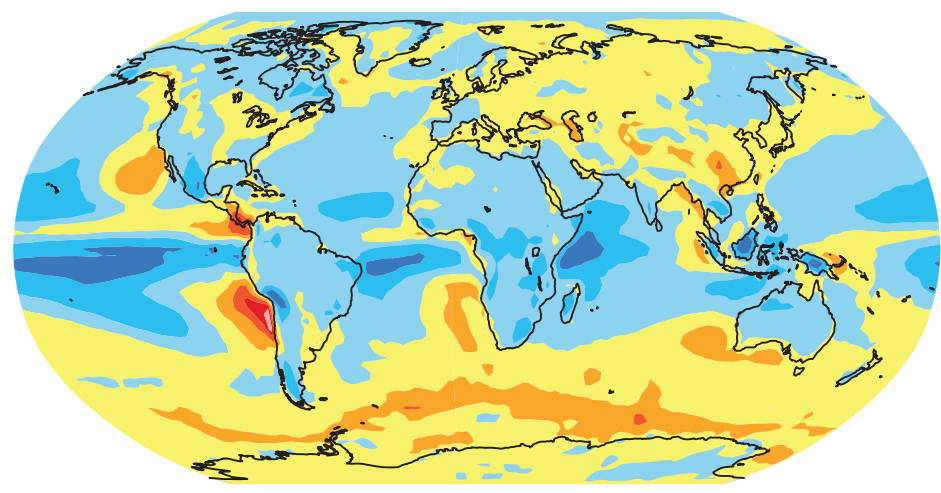

(b) Longwave cloud radiative effect - MOD-OBS

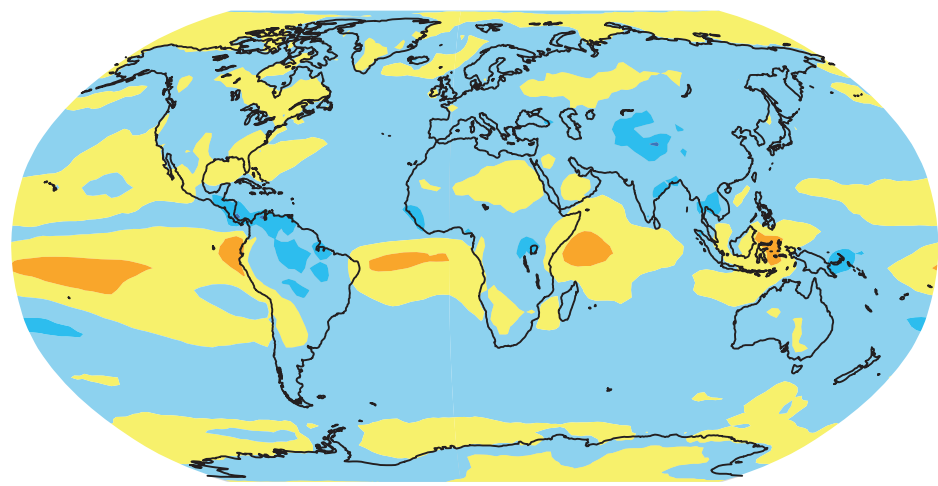

(c) Net cloud radiative effect - MOD-OBS

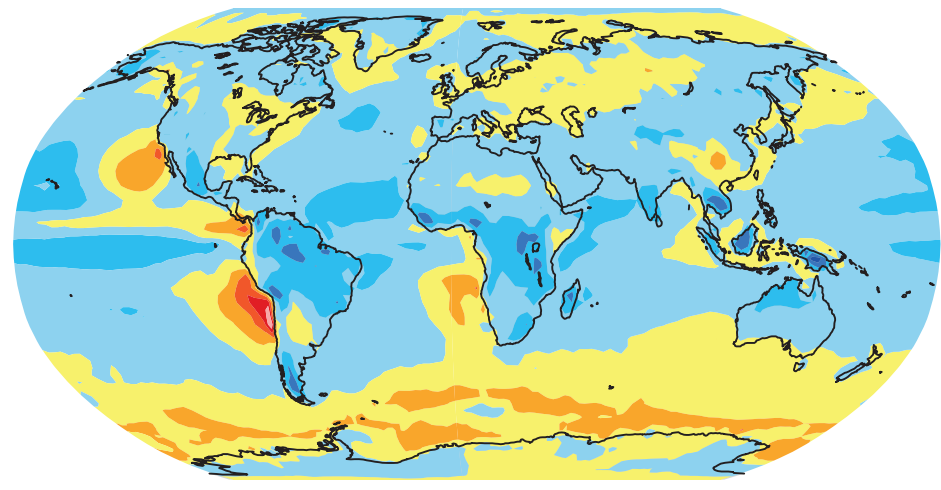

$\left(\mathrm{W} \mathrm{m}^{-2}\right)$

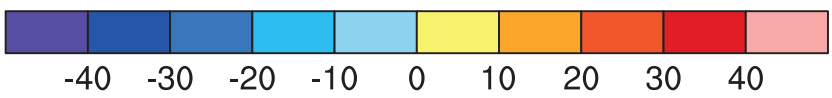

(d) zonal average of shortwave CRE

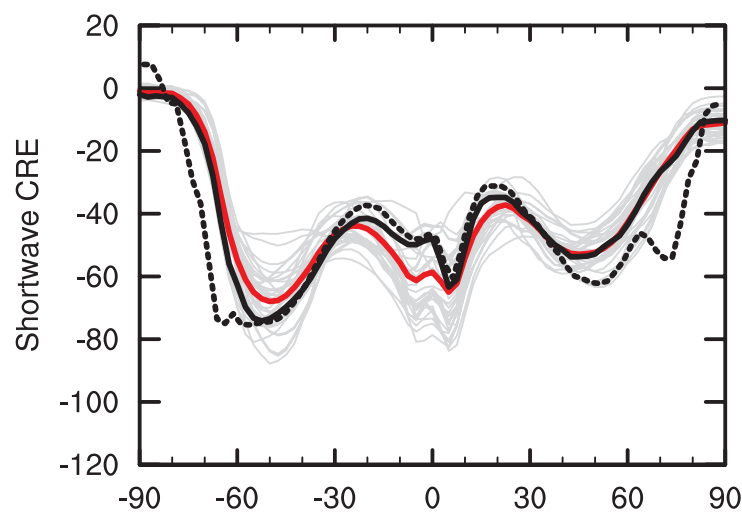

(e) zonal average of longwave CRE

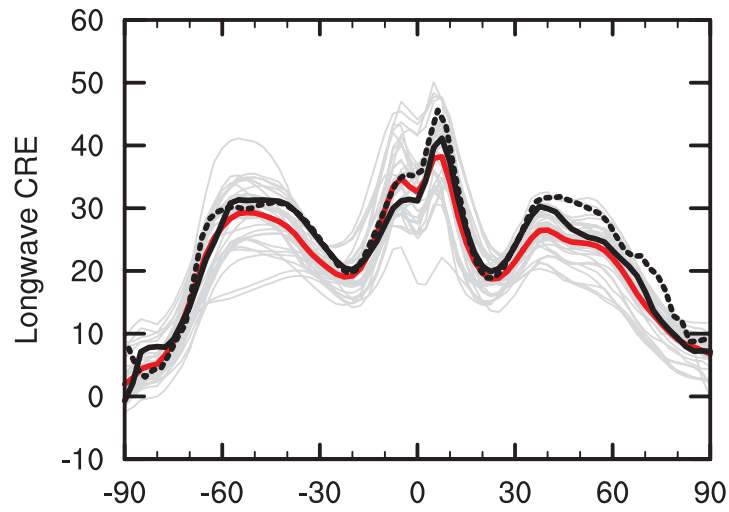

(f) zonal average of net CRE

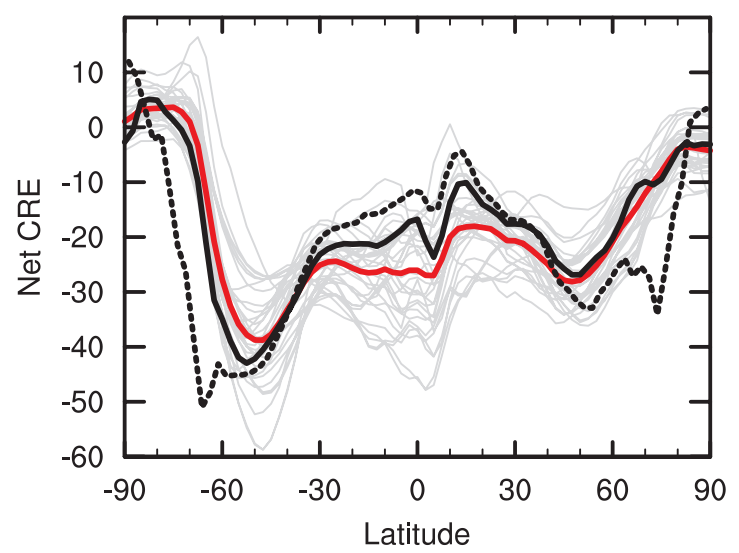

Figure 9.5 | Annual-mean cloud radiative effects of the CMIP5 models compared against the Clouds and the Earth's Radiant Energy System Energy Balanced and Filled 2.6 (CERES EBAF 2.6) data set (in $\mathrm{W} \mathrm{m}^{-2}$; top row: shortwave effect; middle row: longwave effect; bottom row: net effect). On the left are the global distributions of the multi-model-mean biases, and on the right are the zonal averages of the cloud radiative effects from observations (solid black: CERES EBAF 2.6; dashed black: CERES ES-4), individual models (thin grey lines), and the multi-model mean (thick red line). Model results are for the period 1985-2005, while the available CERES data are for 2001-2011. For a definition and maps of cloud radiative effect, see Section 7.2.1.2 and Figure 7.7. 
absorbing aerosols, in particular carbonaceous species (Kim and Ramanathan, 2008), or to the omission of weak-line (Collins et al., 2006b) or continuum (Ptashnik et al., 2011) absorption by water vapour (Wild et al., 2006).

One of the major influences on radiative fluxes in the atmosphere is the presence of clouds and their radiative properties. To measure the influence of clouds on model deficiencies in the TOA radiation budget, Figure 9.5 shows maps of deviations from observations in annual mean shortwave (top left), longwave (middle left) and net (bottom left) cloud radiative effect (CRE) for the CMIP5 multi-model mean. The figure (right panels) also shows zonal averages of the same quantities from two sets of observations, the individual CMIP5 models, and the multi-model average. The definition of CRE and observed mean fields for these quantities can be found in Chapter 7 (Section 7.2.1.2, Figure 7.7).

Models show large regional biases in CRE in the shortwave component, and these are particularly pronounced in the subtropics with too weak an effect (positive error) of model clouds on shortwave radiation in the stratocumulus regions and too strong an effect (negative error) in the trade cumulus regions. This error has been shown to largely result from an overestimation of cloud reflectance, rather than cloud cover (Nam et al., 2012). A too weak cloud influence on shortwave radiation is evident over the subpolar oceans of both hemispheres and the Northern Hemisphere (NH) land areas. It is evident in the zonal mean graphs that there is a wide range in both longwave and shortwave CRE between individual models. As is also evident, a significant reduction in the difference between models and observations has resulted from changes in the observational estimates of CRE, in particular at polar and subpolar as well as subtropical latitudes (Loeb et al., 2009).

Understanding the biases in CRE in models requires a more in-depth analysis of the biases in cloud properties, including the fractional coverage of clouds, their vertical distribution as well as their liquid water and ice content. Major progress in this area has resulted from both the availability of new observational data sets and improved diagnostic techniques, including the increased use of instrument simulators (e.g., Cesana and Chepfer, 2012; Jiang et al., 2012a). Many models have particular difficulties simulating upper tropospheric clouds (Jiang et al., 2012a), and low and mid-level cloud occurrence are frequently underestimated (Cesana and Chepfer, 2012; Nam et al., 2012; Tsushima et al., 2013). Global mean values of both simulated ice and liquid water path vary by factors of 2 to 10 between models (Jiang et al., 2012a; Li et al., 2012a). The global mean fraction of clouds that can be detected with confidence from satellites (optical thickness $>1.3$, Pincus et al. (2012)) is underestimated by 5 to $10 \%$ (Klein et al., 2013). Some of the above errors in clouds compensate to provide the global mean balance in radiation required by model tuning (Tsushima et al., 2013; Wang and Su, 2013; Box 9.1).

In-depth analysis of several global and regional models (Karlsson et al., 2008; Teixeira et al., 2011) has shown that the interaction of boundary layer and cloud processes with the larger scale circulation systems that ultimately drive the observed subtropical cloud distribution remains poorly simulated. Large errors in subtropical clouds have been shown to negatively affect SST patterns in coupled model simulations ( $\mathrm{Hu}$

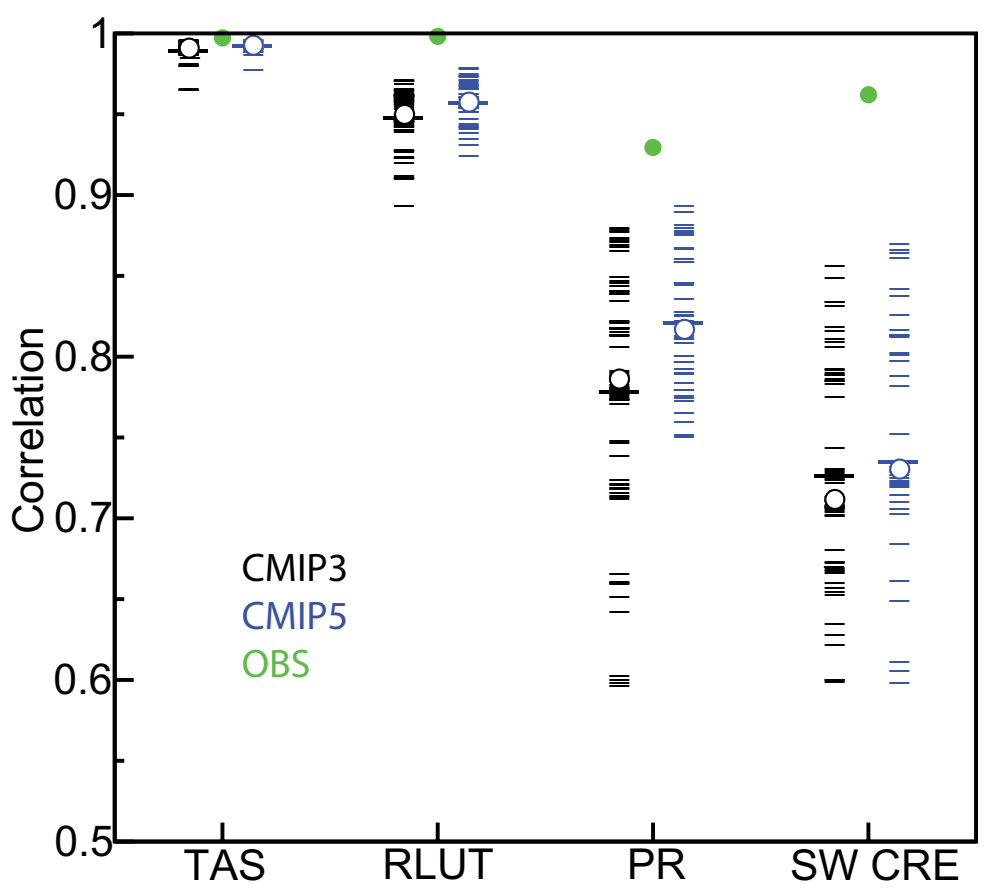

Figure 9.6 Centred pattern correlations between models and observations for the annual mean climatology over the period 1980-1999. Results are shown for individual CMIP3 (black) and CMIP5 (blue) models as thin dashes, along with the corresponding ensemble average (thick dash) and median (open circle). The four variables shown are surface air temperature (TAS), top of the atmosphere (TOA) outgoing longwave radiation (RLUT), precipitation (PR) and TOA shortwave cloud radiative effect (SW CRE). The observations used for each variable are the default products and climatological periods identified in Table 9.3. The correlations between the default and alternate (Table 9.3) observations are also shown (solid green circles). To ensure a fair comparison across a range of model resolutions, the pattern correlations are computed at a resolution of $4^{\circ}$ in longitude and $5^{\circ}$ in latitude. Only one realization is used from each model from the CMIP3 20C3M and CMIP5 historical simulations. 


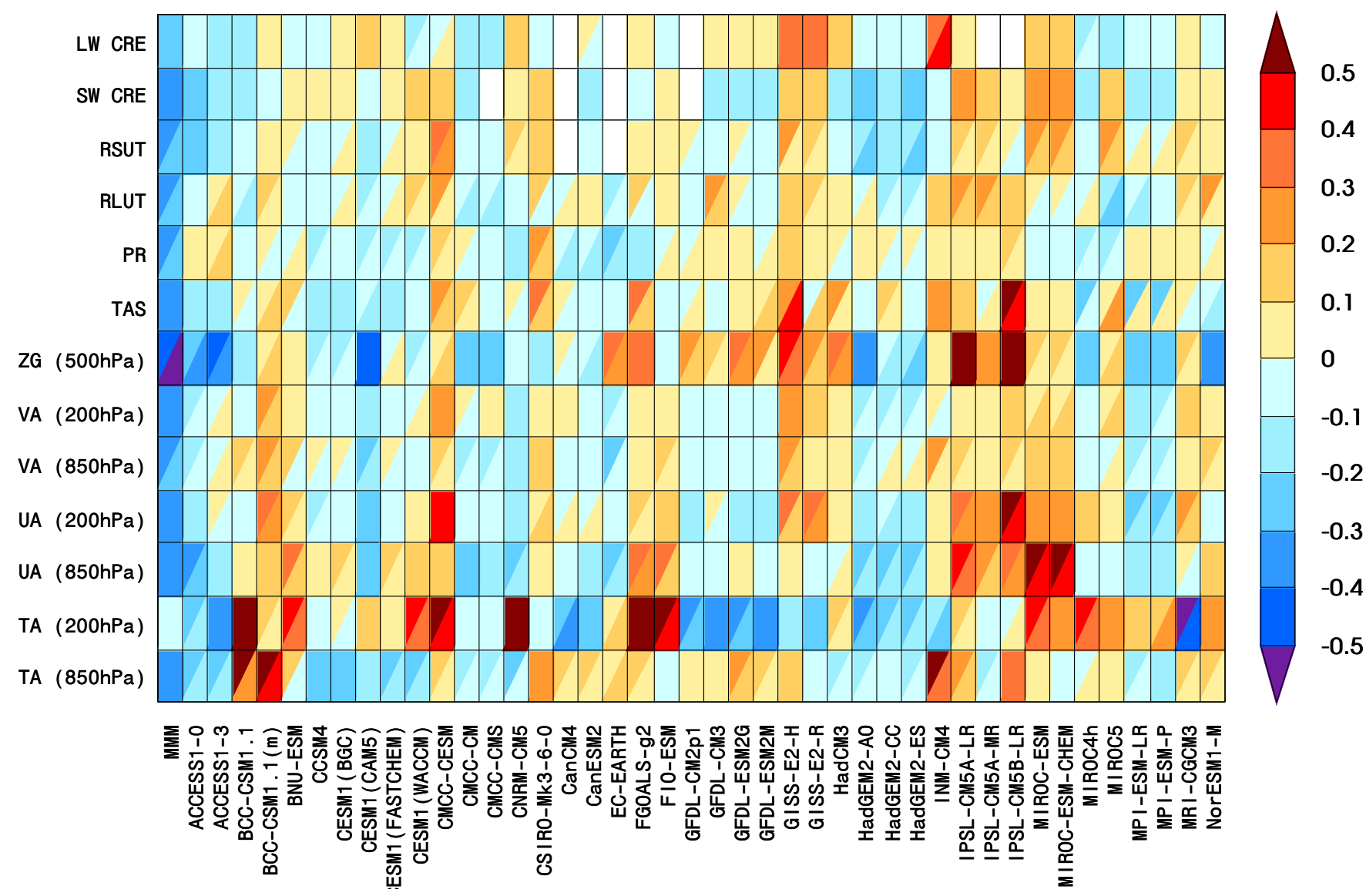

Figure 9.7| Relative error measures of CMIP5 model performance, based on the global seasonal-cycle climatology (1980-2005) computed from the historical experiments. Rows and columns represent individual variables and models, respectively. The error measure is a space-time root-mean-square error (RMSE), which, treating each variable separately, is portrayed as a relative error by normalizing the result by the median error of all model results (Gleckler et al., 2008). For example, a value of 0.20 indicates that a model's RMSE is $20 \%$ larger than the median CMIP5 error for that variable, whereas a value of -0.20 means the error is $20 \%$ smaller than the median error. No colour (white) indicates that model results are currently unavailable. A diagonal split of a grid square shows the relative error with respect to both the default reference data set (upper left triangle) and the alternate (lower right triangle). The relative errors are calculated independently for the default and alternate data sets. All reference data used in the diagram are summarized in Table 9.3 .

et al., 2011; Wahl et al., 2011). Several studies have highlighted the potential importance and poor simulation of subpolar clouds in the Arctic and Southern Oceans (Karlsson and Svensson, 2010; Trenberth and Fasullo, 2010b; Haynes et al., 2011; Bodas-Salcedo et al., 2012). A particular challenge for models is the simulation of the correct phase of the cloud condensate, although very few observations are available to evaluate models particularly with respect to their representation of cloud ice (Waliser et al., 2009b; Li et al., 2012a). Regime-oriented approaches to the evaluation of model clouds (see Section 9.2.1) have identified that compensating errors in the CRE are largely a result of misrepresentations of the frequency of occurrence of key observed cloud regimes, while the radiative properties of the individual regimes contribute less to the overall model deficiencies (Tsushima et al., 2013).

Several studies have identified progress in the simulation of clouds in the CMIP5 models compared to their CMIP3 counterparts. Particular examples include the improved simulation of vertically integrated ice water path (Jiang et al., 2012a; Li et al., 2012a) as well as a reduction of overabundant optically thick clouds in the mid-latitudes (Klein et al., 2013; Tsushima et al., 2013).
In summary, despite modest improvements there remain significant errors in the model simulation of clouds. There is very high confidence that these errors contribute significantly to the uncertainties in estimates of cloud feedbacks (see Section 9.7.2.3; Section 7.2.5, Figure 7.10) and hence the spread in climate change projections reported in Chapter 12.

\subsubsection{Quantifying Model Performance with Metrics}

Performance metrics were used to some extent in the Third Assessment Report (TAR) and the Fourth Assessment Report (AR4), and are expanded upon here because of their increased appearance in the recent literature. As a simple example, Figure 9.6 illustrates how the pattern correlation between the observed and simulated climatological annual mean spatial patterns depends very much on the quantity examined. All CMIP3 and CMIP5 models capture the mean surface temperature distribution quite well, with correlations above 0.95 , which are largely determined by the meridional temperature gradient. Correlations for outgoing longwave radiation are somewhat lower. For precipitation and the TOA shortwave cloud radiative effect, the correlations 
between models and observations are below 0.90 , and there is considerable scatter among model results. This example quantifies how some aspects of the simulated large-scale climate agree with observations better than others. Some of these differences are attributable to smoothly varying fields (e.g., temperature, water vapour) often agreeing better with observations than fields that exhibit fine structure (e.g., precipitation) (see also Section 9.6.1.1). Incremental improvement in each field is also evident in Figure 9.6, as gauged by the mean and median results in the CMIP5 ensemble having higher correlations than CMIP3. This multi-variate quantification of model improvement across development cycles is evident in several studies (e.g., Reichler and Kim, 2008; Knutti et al., 2013)

Figure 9.7 (following Gleckler et al., 2008) depicts the space-time rootmean-square error (RMSE) for the 1980-2005 climatological seasonal cycle of the historically forced CMIP5 simulations. For each of the fields examined, this 'portrait plot' depicts relative performance, with blue shading indicating performance being better, and red shading worse, than the median of all model results. In each case, two observations-based estimates are used to demonstrate the impact of the selection of reference data on the results. Some models consistently compare better with observations than others, some exhibit mixed performance and some stand out with relatively poor agreement with observations. For most fields, the choice of the observational data set does not substantially change the result for global error measures (e.g., between a state-of-the-art and an older-generation reanalysis), indicating that inter-model differences are substantially larger than the differences between the two reference data sets or the impact of two different climatological periods (e.g., for radiation fields: Earth Radiation Budget Experiment (ERBE) 1984-1988; CERES EBAF, 2001-2011). Nevertheless, it is important to recognize that different data sets often rely on the same source of measurements, and that the results in this figure can have some sensitivity to a variety of factors such as instrument uncertainty, sampling errors (e.g., limited record length of observations), the spatial scale of comparison, the domain considered and the choice of metric.

Another notable feature of Figure 9.7 is that in most cases the multi-model mean agrees more favourably with observations than any individual model. This has been long recognized to hold for surface temperature and precipitation (e.g., Lambert and Boer, 2001). However, since the AR4, it has become clear that this holds for a broad range of climatological fields (Gleckler et al., 2008; Pincus et al., 2008; Knutti et al., 2010a) and is theoretically better understood (Annan and Hargreaves, 2011). It is worth noting that when most models suffer from a common error, such as the cold bias at high latitudes in the upper troposphere (see TA $200 \mathrm{hPa}$ of Figure 9.7), individual models can agree better with observations than the multi-model mean.

Correlations between the relative errors for different quantities in Figure 9.7 are known to exist, reflecting physical relationships in the model formulations and in the real world. Cluster analysis methods have recently been used in an attempt to reduce this redundancy (e.g., Yokoi et al., 2011; Nishii et al., 2012), thereby providing more succinct summaries of model performance. Some studies have attempted an overall skill score by averaging together the results from multiple metrics (e.g., Reichler and Kim, 2008). Although this averaging process is largely arbitrary, combining the results of multiple metrics can reduce the chance that a poorer performing model will score well for the wrong reasons. Recent work (Nishii et al., 2012) has demonstrated that different methods used to produce a multi-variate skill measure for the CMIP3 models did not substantially alter the conclusions about the better and lesser performing models.

Large scale performance metrics are a typical first-step toward quantifying model agreement with observations, and summarizing broad characteristics of model performance that are not focussed on a particular application. More specialized performance tests target aspects of a simulation believed to be especially important for constraining model projections, although to date the connections between particular performance metrics and reliability of future projections are not well established. This important topic is addressed in Section 9.8.3, which highlights several identified relationships between model performance and projection responses.

\subsubsection{Long-Term Global-Scale Changes}

The comparison of observed and simulated climate change is complicated by the fact that the simulation results depend on both model formulation and the time-varying external forcings imposed on the models (Allen et al., 2000; Santer et al., 2007). De-convolving the importance of model and forcing differences in the historical simulations is an important topic that is addressed in Chapter 10; however, in this section a direct comparison is made to illustrate the ability of models to reproduce past changes.

\subsection{Global surface temperature}

Figure 9.8 compares the observational record of 20th century changes in global surface temperature to that simulated by each CMIP5 and EMIC model and the respective multi-model means. The inset on the right of the figure shows the climatological mean temperature for each model, averaged over the 1961-1990 reference period. Although biases in mean temperature are apparent, there is less confidence in observational estimates of climatological temperature than in variations about this mean (Jones et al. (1999). For the CMIP5 models, interannual variability in most of the simulations is qualitatively similar to that observed although there are several exceptions. The magnitude of interannual variations in the observations is noticeably larger than the multi-model mean because the averaging of multiple model results acts to filter much of the simulated variability. On the other hand, the episodic volcanic forcing that is applied to most models (see Section 9.3.2.2) is evident in the multi-model agreement with the observed cooling particularly noticeable after the 1991 Pinatubo eruption. The gradual warming evident in the observational record, particularly in the more recent decades, is also evident in the simulations, with the multi-model mean tracking the observed value closely over most of the century, and individual model results departing by less than about $0.5^{\circ} \mathrm{C}$. Because the interpretation of differences in model behaviour can be confounded by internal variability and forcing, some studies have attempted to identify and remove dominant factors such as El Niño-Southern Oscillation (ENSO) and the impacts of volcanic eruptions (e.g., Fyfe et al., 2010). Figure 9.8 shows the similar capability for EMICs to simulate the global-scale response to the 20th century forcings (Eby et al. 2013). These 
results demonstrate a level of consistency between the EMICs with both the observations and the CMIP5 ensemble.

In summary, there is very high confidence that models reproduce the general features of the global-scale annual mean surface temperature increase over the historical period, including the more rapid warming in the second half of the 20th century, and the cooling immediately following large volcanic eruptions. The disagreement apparent over the most recent 10 to 15 years is discussed in detail in Box 9.2. (a) Observed and CMIP5 simulated global mean surface air temperature

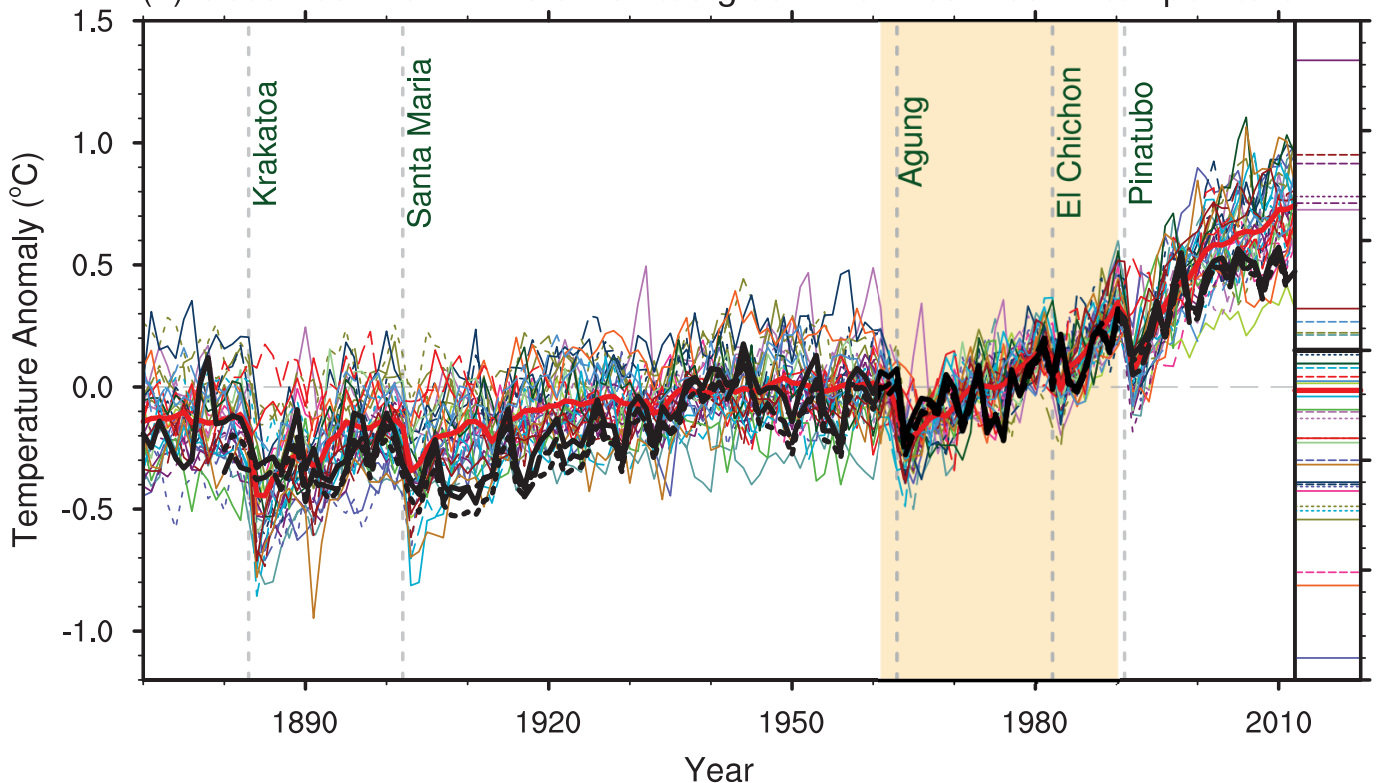

(b) Observed and EMICs simulated global mean surface air temperature

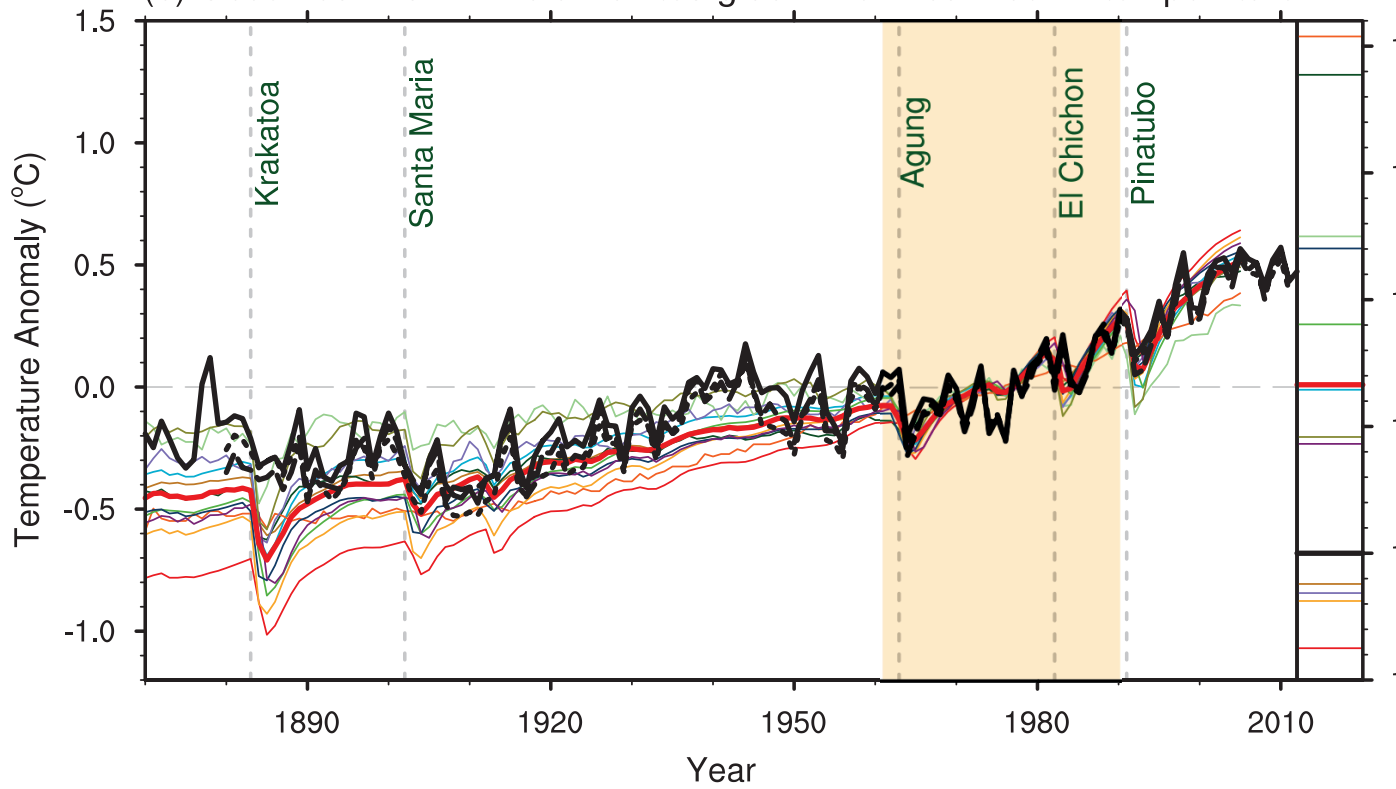

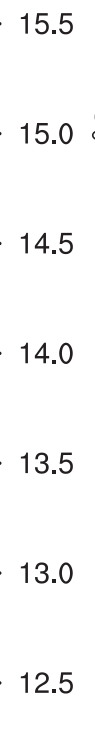

HadCRUT4

_. - MLOST

CMIP5 mean

- ACCESS1.3

- BCC-CSM1.1

BNU-ESM

CanESM2

CCSM4

- CESM1(BGC) CESM1(CAM5) CMCC-CM

- CMCC-CMS

CNRM-CM5

- CSIRO-Mk3.6.0

- FIO-ESM

- - GFDL-ESM2G

- - - - GFDL-ESM2M

GISS-E2-H

- — GISS-E2-H-CC

- - - GISS-E2-R

— - - GISS-E2-R-CC

- - HadGEM2-AO

- - - HadGEM2-CC HadGEM2-ES

IPSL-CM5A-LR

- IPSL-CM5A-MR

- - IPSL-CM5B-LR

MIROC5

- MIROC-ESM MIROC-ESM-CHEM

18.0

17.0 MPI-ESM-LR

- - MPI-ESM-MP

MRI-CGCM3

NorESM1-M

17.0

- _ NorESM1-ME

16.0

HadCRUT4

— - GISTEMP

-... MLOST

EMIC mean

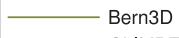

- CLIMBER2

15.0

CLIMBER

요 DCESS

क - GENIE

14.0

IGSM2

IAP RAS CM

LOVECLIM
MESMO

MIROC-lite

UMD

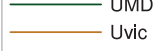

Figure 9.8 Observed and simulated time series of the anomalies in annual and global mean surface temperature. All anomalies are differences from the 1961-1990 time-mean of each individual time series. The reference period 1961-1990 is indicated by yellow shading; vertical dashed grey lines represent times of major volcanic eruptions. (a) Single simulations for CMIP5 models (thin lines); multi-model mean (thick red line); different observations (thick black lines). Observational data (see Chapter 2) are Hadley Centre/Climatic Research Unit gridded surface temperature data set 4 (HadCRUT4; Morice et al., 2012), Goddard Institute for Space Studies Surface Temperature Analysis (GISTEMP; Hansen et al., 2010) and Merged Land-Ocean Surface Temperature Analysis (MLOST; Vose et al., 2012) and are merged surface temperature (2 m height over land and surface temperature over the ocean). All model results have been sub-sampled using the HadCRUT4 observational data mask (see Chapter 10). Following the CMIP5 protocol (Taylor et al., 2012b), all simulations use specified historical forcings up to and including 2005 and use RCP4.5 after 2005 (see Figure 10.1 and note different reference period used there; results will differ slightly when using alternative RCP scenarios for the post-2005 period). (a) Inset: the global mean surface temperature for the reference period 1961-1990, for each individual model (colours), the CMIP5 multi-model mean (thick red), and the observations (thick black: Jones et al., 1999). (Bottom) Single simulations from available EMIC simulations (thin lines), from Eby et al. (2013). Observational data are the same as in (a). All EMIC simulations ended in 2005 and use the CMIP5 historical forcing scenario. (b) Inset: Same as in (a) but for the EMICS. 


\section{Box 9.2 | Climate Models and the Hiatus in Global Mean Surface Warming of the Past 15 Years}

The observed global mean surface temperature (GMST) has shown a much smaller increasing linear trend over the past 15 years than over the past 30 to 60 years (Section 2.4.3, Figure 2.20, Table 2.7; Figure 9.8; Box 9.2 Figure 1a, c). Depending on the observational data set, the GMST trend over 1998-2012 is estimated to be around one-third to one-half of the trend over 1951-2012 (Section 2.4.3, Table 2.7; Box 9.2 Figure 1a, c). For example, in HadCRUT4 the trend is $0.04^{\circ} \mathrm{C}$ per decade over $1998-2012$, compared to $0.11^{\circ} \mathrm{C}$ per decade over 1951-2012. The reduction in observed GMST trend is most marked in Northern Hemisphere winter (Section 2.4.3; Cohen et al., 2012). Even with this "hiatus" in GMST trend, the decade of the 2000s has been the warmest in the instrumental record of GMST (Section 2.4.3, Figure 2.19). Nevertheless, the occurrence of the hiatus in GMST trend during the past 15 years raises the two related questions of what has caused it and whether climate models are able to reproduce it.

Figure 9.8 demonstrates that 15-year-long hiatus periods are common in both the observed and CMIP5 historical GMST time series (see also Section 2.4.3, Figure 2.20; Easterling and Wehner, 2009; Liebmann et al., 2010). However, an analysis of the full suite of CMIP5 historical simulations (augmented for the period 2006-2012 by RCP4.5 simulations, Section 9.3.2) reveals that 111 out of 114 realizations show a GMST trend over 1998-2012 that is higher than the entire HadCRUT4 trend ensemble (Box 9.2 Figure 1a; CMIP5 ensemble mean trend is $0.21{ }^{\circ} \mathrm{C}$ per decade). This difference between simulated and observed trends could be caused by some combination of (a) internal climate variability, (b) missing or incorrect radiative forcing and (c) model response error. These potential sources of the difference, which are not mutually exclusive, are assessed below, as is the cause of the observed GMST trend hiatus.

\section{Internal Climate Variability}

Hiatus periods of 10 to 15 years can arise as a manifestation of internal decadal climate variability, which sometimes enhances and sometimes counteracts the long-term externally forced trend. Internal variability thus diminishes the relevance of trends over periods as short as 10 to 15 years for long-term climate change (Box 2.2, Section 2.4.3). Furthermore, the timing of internal decadal climate variability is not expected to be matched by the CMIP5 historical simulations, owing to the predictability horizon of at most 10 to 20 years (Section 11.2.2; CMIP5 historical simulations are typically started around nominally 1850 from a control run). However, climate models exhibit individual decades of GMST trend hiatus even during a prolonged phase of energy uptake of the climate system (e.g., Figure 9.8; Easterling and Wehner, 2009; Knight et al., 2009), in which case the energy budget would be balanced by increasing subsurface-ocean heat uptake (Meehl et al., 2011, 2013a; Guemas et al., 2013).

Owing to sampling limitations, it is uncertain whether an increase in the rate of subsurface-ocean heat uptake occurred during the past 15 years (Section 3.2.4). However, it is very likely ${ }^{2}$ that the climate system, including the ocean below $700 \mathrm{~m}$ depth, has continued to accumulate energy over the period 1998-2010 (Section 3.2.4, Box 3.1). Consistent with this energy accumulation, global mean sea level has continued to rise during 1998-2012, at a rate only slightly and insignificantly lower than during 1993-2012 (Section 3.7). The consistency between observed heat-content and sea level changes yields high confidence in the assessment of continued ocean energy accumulation, which is in turn consistent with the positive radiative imbalance of the climate system (Section 8.5.1; Section 13.3, Box 13.1). By contrast, there is limited evidence that the hiatus in GMST trend has been accompanied by a slower rate of increase in ocean heat content over the depth range 0 to $700 \mathrm{~m}$, when comparing the period 2003-2010 against 1971-2010. There is low agreement on this slowdown, since three of five analyses show a slowdown in the rate of increase while the other two show the increase continuing unabated (Section 3.2.3, Figure 3.2).

During the 15-year period beginning in 1998, the ensemble of HadCRUT4 GMST trends lies below almost all model-simulated trends (Box 9.2 Figure 1a), whereas during the 15-year period ending in 1998, it lies above 93 out of 114 modelled trends (Box 9.2 Figure $1 \mathrm{~b}$; HadCRUT4 ensemble-mean trend $0.26^{\circ} \mathrm{C}$ per decade, CMIP5 ensemble-mean trend $0.16^{\circ} \mathrm{C}$ per decade). Over the 62 -year period 1951-2012, observed and CMIP5 ensemble-mean trends agree to within $0.02^{\circ} \mathrm{C}$ per decade (Box 9.2 Figure $1 \mathrm{c}$; CMIP5 ensemble-mean trend $0.13^{\circ} \mathrm{C}$ per decade). There is hence very high confidence that the CMIP5 models show long-term GMST trends consistent with observations, despite the disagreement over the most recent 15-year period. Due to internal climate variability, in any given 15-year period the observed GMST trend sometimes lies near one end of a model ensemble (Box 9.2, Figure 1a, b; Easterling and Wehner, 2009), an effect that is pronounced in Box 9.2, Figure 1a, b because GMST was influenced by a very strong El Niño event in 1998.

(continued on next page)

\footnotetext{
In this Report, the following terms have been used to indicate the assessed likelihood of an outcome or a result: Virtually certain $99-100 \%$ probability, Very likely 90-100\%, Likely 66-100\%, About as likely as not 33-66\%, Unlikely 0-33\%, Very unlikely 0-10\%, Exceptionally unlikely 0-1\%. Additional terms (Extremely likely: 95-100\%, More likely than not $>50-100 \%$, and Extremely unlikely 0-5\%) may also be used when appropriate. Assessed likelihood is typeset in italics, e.g., very likely (see Section 1.4 and Box TS.1 for more details).
} 
Box 9.2 (continued)

Unlike the CMIP5 historical simulations referred to above, some CMIP5 predictions were initialized from the observed climate state during the late 1990s and the early 21st century (Section 11.1, Box 11.1; Section 11.2). There is medium evidence that these initialized predictions show a GMST lower by about $0.05^{\circ} \mathrm{C}$ to $0.1^{\circ} \mathrm{C}$ compared to the historical (uninitialized) simulations and maintain this lower GMST during the first few years of the simulation (Section 11.2.3.4, Figure 11.3 top left; Doblas-Reyes et al., 2013; Guemas et al., 2013). In some initialized models this lower GMST occurs in part because they correctly simulate a shift, around 2000, from a positive to a negative phase of the Interdecadal Pacific Oscillation (IPO, Box 2.5; e.g., Meehl and Teng, 2012; Meehl et al., 2013a). However, the improvement of this phasing of the IPO through initialization is not universal across the CMIP5 predictions (cf. Section 11.2.3.4). Moreover, while part of the GMST reduction through initialization indeed results from initializing at the correct phase of internal variability, another part may result from correcting a model bias that was caused by incorrect past forcing or incorrect model response to past forcing, especially in the ocean. The relative magnitudes of these effects are at present unknown (Meehl and Teng, 2012); moreover, the quality of a forecasting system cannot be evaluated from a single prediction (here, a 10-year prediction within the period 1998-2012; Section 11.2.3). Overall, there is medium confidence that initialization leads to simulations of GMST during 1998-2012 that are more consistent with the observed trend hiatus than are the uninitialized CMIP5 historical simulations, and that the hiatus is in part a consequence of internal variability that is predictable on the multi-year time scale.

\section{Radiative Forcing}

On decadal to interdecadal time scales and under continually increasing effective radiative forcing (ERF), the forced component of the GMST trend responds to the ERF trend relatively rapidly and almost linearly (medium confidence, e.g., Gregory and Forster, 2008; Held et al., 2010; Forster et al., 2013). The expected forced-response GMST trend is related to the ERF trend by a factor that has been estimated for the $1 \%$ per year $\mathrm{CO}_{2}$ increases in the CMIP5 ensemble as 2.0 [1.3 to 2.7] W m $\mathrm{m}^{-2}{ }^{\circ} \mathrm{C}^{-1}$ ( $90 \%$ uncertainty range; Forster et al., 2013). Hence, an ERF trend can be approximately converted to a forced-response GMST trend, permitting an assessment of how much of the change in the GMST trends shown in Box 9.2 Figure 1 is due to a change in ERF trend.

The AR5 best-estimate ERF trend over 1998-2011 is 0.22 [0.10 to 0.34$] \mathrm{W} \mathrm{m}^{-2}$ per decade ( $90 \%$ uncertainty range), which is substantially lower than the trend over 1984-1998 $(0.32$ [0.22 to 0.42$] \mathrm{W} \mathrm{m}^{-2}$ per decade; note that there was a strong volcanic eruption in 1982) and the trend over 1951-2011 (0.31 [0.19 to 0.40] W m-2 per decade; Box 9.2, Figure 1d-f; numbers based on Section 8.5.2, Figure 8.18; the end year 2011 is chosen because data availability is more limited than for GMST). The resulting forced-response GMST trend would approximately be 0.12 [0.05 to 0.29$]{ }^{\circ} \mathrm{C}$ per decade, 0.19 [0.09 to 0.39$]{ }^{\circ} \mathrm{C}$ per decade, and 0.18 [0.08 to 0.37$]{ }^{\circ} \mathrm{C}$ per decade for the periods 1998-2011, 1984-1998 and 1951-2011, respectively (the uncertainty ranges assume that the range of the conversion factor to GMST trend and the range of ERF trend itself are independent). The AR5 best-estimate ERF forcing trend difference between 1998-2011 and 1951-2011 thus might explain about one-half $\left(0.05^{\circ} \mathrm{C}\right.$ per decade) of the observed GMST trend difference between these periods ( 0.06 to $0.08^{\circ} \mathrm{C}$ per decade, depending on observational data set).

The reduction in AR5 best-estimate ERF trend over 1998-2011 compared to both 1984-1998 and 1951-2011 is mostly due to decreasing trends in the natural forcings, $-0.16[-0.27$ to -0.06$] \mathrm{W} \mathrm{m}^{-2}$ per decade over 1998-2011 compared to 0.01 [ -0.00 to 0.01$]$ W m ${ }^{-2}$ per decade over 1951-2011 (Section 8.5.2, Figure 8.19). Solar forcing went from a relative maximum in 2000 to a relative minimum in 2009, with a peak-to-peak difference of around $0.15 \mathrm{~W} \mathrm{~m}^{-2}$ and a linear trend over 1998-2011 of around $-0.10 \mathrm{~W} \mathrm{~m} \mathrm{~m}^{-2}$ per decade (cf. Section 10.3.1, Box 10.2). Furthermore, a series of small volcanic eruptions has increased the observed stratospheric aerosol loading after 2000, leading to an additional negative ERF linear-trend contribution of around $-0.06 \mathrm{~W} \mathrm{~m} \mathrm{~m}^{-2}$ per decade over 1998-2011 (cf. Section 8.4.2.2, Section 8.5.2, Figure 8.19; Box 9.2 Figure 1d, f). By contrast, satellite-derived estimates of tropospheric aerosol optical depth (AOD) suggests little overall trend in global mean AOD over the last 10 years, implying little change in ERF due to aerosol-radiative interaction (low confidence because of low confidence in AOD trend itself, Section 2.2.3; Section 8.5.1; Murphy, 2013). Moreover, because there is only low confidence in estimates of ERF due to aerosol-cloud interaction (Section 8.5.1, Table 8.5), there is likewise low confidence in its trend over the last 15 years.

For the periods 1984-1998 and 1951-2011, the CMIP5 ensemble-mean ERF trend deviates from the AR5 best-estimate ERF trend by only $0.01 \mathrm{~W} \mathrm{~m}^{-2}$ per decade (Box 9.2 Figure 1e, f). After 1998, however, some contributions to a decreasing ERF trend are missing in the CMIP5 models, such as the increasing stratospheric aerosol loading after 2000 and the unusually low solar minimum in 2009. Nonetheless, over 1998-2011 the CMIP5 ensemble-mean ERF trend is lower than the AR5 best-estimate ERF trend by $0.03 \mathrm{~W} \mathrm{~m} \mathrm{~m}^{-2}$ per decade (Box 9.2 Figure 1d). Furthermore, global mean AOD in the CMIP5 models shows little trend over 1998-2012, similar to the observations (Figure 9.29). Although the forcing uncertainties are substantial, there are no apparent incorrect or missing global mean forcings in the CMIP5 models over the last 15 years that could explain the model-observations difference during the warming hiatus.

(continued on next page) 
Box 9.2 (continued)

\section{Model Response Error}

The discrepancy between simulated and observed GMST trends during 1998-2012 could be explained in part by a tendency for some CMIP5 models to simulate stronger warming in response to increases in greenhouse gas (GHG) concentration than is consistent with observations (Section 10.3.1.1.3, Figure 10.4). Averaged over the ensembles of models assessed in Section 10.3.1.1.3, the bestestimate GHG and other anthropogenic (OA) scaling factors are less than one (though not significantly so, Figure 10.4), indicating that the model-mean GHG and OA responses should be scaled down to best match observations. This finding provides evidence that some CMIP5 models show a larger response to GHGs and other anthropogenic factors (dominated by the effects of aerosols) than the real world (medium confidence). As a consequence, it is argued in Chapter 11 that near-term model projections of GMST increase should be scaled down by about 10\% (Section 11.3.6.3). This downward scaling is, however, not sufficient to explain the model-mean overestimate of GMST trend over the hiatus period.

Another possible source of model error is the poor representation of water vapour in the upper atmosphere (Section 9.4.1.2). It has been suggested that a reduction in stratospheric water vapour after 2000 caused a reduction in downward longwave radiation and hence a surface-cooling contribution (Solomon et al., 2010), possibly missed by the models, However, this effect is assessed here to be small, because there was a recovery in stratospheric water vapour after 2005 (Section 2.2.2.1, Figure 2.5). (continued on next page)
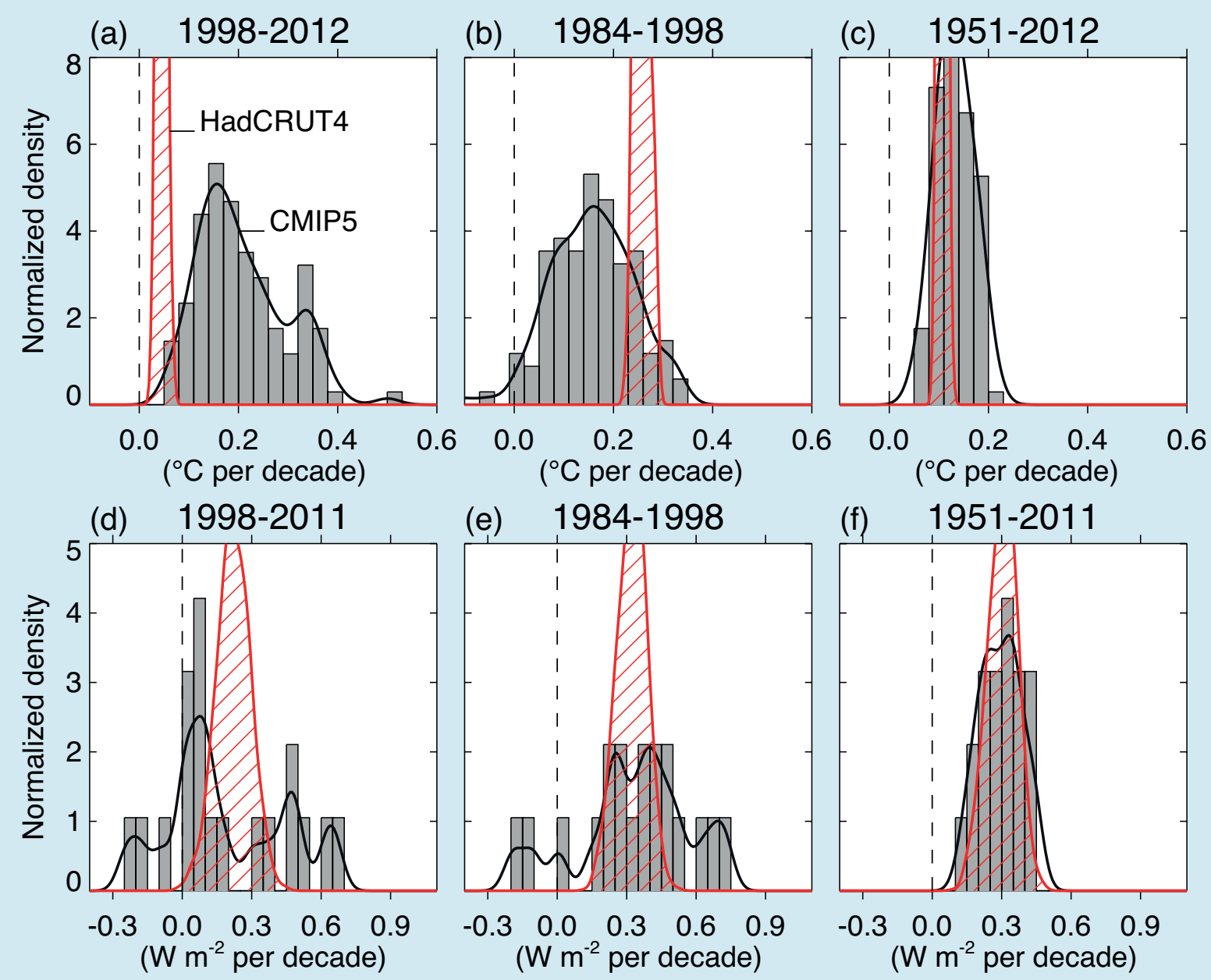

Box 9.2, Figure 1 | (Top) Observed and simulated global mean surface temperature (GMST) trends in degrees Celsius per decade, over the periods 1998-2012 (a), 1984-1998 (b), and 1951-2012 (c). For the observations, 100 realizations of the Hadley Centre/Climatic Research Unit gridded surface temperature data set 4 (HadCRUT4) ensemble are shown (red, hatched: Morice et al., 2012). The uncertainty displayed by the ensemble width is that of the statistical construction of the global average only, in contrast to the trend uncertainties quoted in Section 2.4.3, which include an estimate of internal climate variability. Here, by contrast, internal variability is characterized through the width of the model ensemble. For the models, all 114 available CMIP5 historical realizations are shown, extended after 2005 with the RCP4.5 scenario and through 2012 (grey, shaded: after Fyfe et al., 2010). (Bottom) Trends in effective radiative forcing (ERF, in W $\mathrm{m}^{-2}$ per decade) over the periods 1998-2011 (d), 1984-1998 (e), and 1951-2011 (f). The figure shows AR5 best-estimate ERF trends (red, hatched; Section 8.5.2, Figure 8.18) and CMIP5 ERF (grey, shaded: from Forster et al., 2013). Black lines are smoothed versions of the histograms. Each histogram is normalized so that its area sums up to one. 
Box 9.2 (continued)

In summary, the observed recent warming hiatus, defined as the reduction in GMST trend during 1998-2012 as compared to the trend during 1951-2012, is attributable in roughly equal measure to a cooling contribution from internal variability and a reduced trend in external forcing (expert judgment, medium confidence). The forcing trend reduction is primarily due to a negative forcing trend from both volcanic eruptions and the downward phase of the solar cycle. However, there is low confidence in quantifying the role of forcing trend in causing the hiatus, because of uncertainty in the magnitude of the volcanic forcing trend and low confidence in the aerosol forcing trend.

Almost all CMIP5 historical simulations do not reproduce the observed recent warming hiatus. There is medium confidence that the GMST trend difference between models and observations during 1998-2012 is to a substantial degree caused by internal variability, with possible contributions from forcing error and some CMIP5 models overestimating the response to increasing GHG and other anthropogenic forcing. The CMIP5 model trend in ERF shows no apparent bias against the AR5 best estimate over 1998-2012. However, confidence in this assessment of CMIP5 ERF trend is low, primarily because of the uncertainties in model aerosol forcing and processes, which through spatial heterogeneity might well cause an undetected global mean ERF trend error even in the absence of a trend in the global mean aerosol loading.

The causes of both the observed GMST trend hiatus and of the model-observation GMST trend difference during 1998-2012 imply that, barring a major volcanic eruption, most 15-year GMST trends in the near-term future will be larger than during 1998-2012 (high confidence; see 11.3.6.3. for a full assessment of near-term projections of GMST). The reasons for this implication are fourfold: first, anthropogenic greenhouse-gas concentrations are expected to rise further in all RCP scenarios; second, anthropogenic aerosol concentration is expected to decline in all RCP scenarios, and so is the resulting cooling effect; third, the trend in solar forcing is expected to be larger over most near-term 15-year periods than over 1998-2012 (medium confidence), because 1998-2012 contained the full downward phase of the solar cycle; and fourth, it is more likely than not that internal climate variability in the near-term will enhance and not counteract the surface warming expected to arise from the increasing anthropogenic forcing.

\subsection{Tropical tropospheric temperature trends}

Most climate model simulations show a larger warming in the tropical troposphere than is found in observational data sets (e.g., McKitrick et al., 2010; Santer et al., 2013). There has been an extensive and sometimes controversial debate in the published literature as to whether this difference is statistically significant, once observational uncertainties and natural variability are taken into account (e.g., Douglass et al., 2008; Santer et al., 2008, 2013; Christy et al., 2010; McKitrick et al., 2010; Bengtsson and Hodges, 2011; Fu et al., 2011; McKitrick et al., 2011; Thorne et al., 2011). For the period 1979-2012, the various observational data sets find, in the tropical lower troposphere (LT), a linear warming trend ranging from $0.06^{\circ} \mathrm{C}$ to $0.13^{\circ} \mathrm{C}$ per decade (Section 2.4.4, Figure 2.27). In the tropical middle troposphere (MT), the linear warming trend ranges from $0.02^{\circ} \mathrm{C}$ to $0.12^{\circ} \mathrm{C}$ per decade (Section 2.4.4, Figure 2.27). Uncertainty in these trend values arises from different methodological choices made by the groups deriving satellite products (Mears et al., 2011) and radiosonde compilations (Thorne et al., 2011), and from fitting a linear trend to a time series containing substantial interannual and decadal variability (Box 2.2; Section 2.4.4; (Santer et al., 2008; McKitrick et al., 2010)). Although there have been substantial methodological debates about the calculation of trends and their uncertainty, a $95 \%$ confidence interval of around $\pm 0.1^{\circ} \mathrm{C}$ per decade has been obtained consistently for both LT and MT (e.g., Section 2.4.4; McKitrick et al., 2010). In summary, despite unanimous agreement on the sign of the observed trends, there exists substantial disagreement between available estimates as to the rate of temperature changes in the tropical troposphere, and there is only low confidence in the rate of change and its vertical structure (Section 2.4.4).

For the 30-year period 1979-2009 (sometimes updated through 2010 or 2011), the CMIP3 models simulate a tropical warming trend ranging from $0.1^{\circ} \mathrm{C}$ to somewhat above $0.4^{\circ} \mathrm{C}$ per decade for both LT and MT (McKitrick et al., 2010), while the CMIP5 models simulate a tropical warming trend ranging from slightly below $0.15^{\circ} \mathrm{C}$ to somewhat above $0.4^{\circ} \mathrm{C}$ per decade for both LT and MT (Santer et al., 2013; see also PoChedley and Fu, 2012, who considered the period 1979-2005). Both model ensembles show trends that on average are higher than in the observational estimates, although both model ensembles overlap the observational ensemble. Because the differences between the various observational estimates are largely systematic and structural (Section 2.4.4; Mears et al., 2011), the uncertainty in the observed trends cannot be reduced by averaging the observations as if the differences between the data sets were purely random. Likewise, to properly represent internal climate variability, the full model ensemble spread must be used in a comparison against the observations (e.g., Box 9.2; Section 11.2.3.2; Raftery et al., 2005; Wilks, 2006; Jolliffe and Stephenson, 2011). The very high significance levels of model-observation discrepancies in LT and MT trends that were obtained in some studies (e.g., Douglass et al., 2008; McKitrick et al., 2010) thus arose to a substantial degree from using the standard error of the model ensemble mean as a measure of uncertainty, instead of the ensemble standard deviation or some other appropriate measure for uncertainty arising from internal climate 
variability (e.g., Box 9.2; Section 11.2.3.2; Raftery et al., 2005; Wilks, 2006; Jolliffe and Stephenson, 2011). Nevertheless, almost all model ensemble members show a warming trend in both LT and MT larger than observational estimates (McKitrick et al., 2010; Po-Chedley and Fu, 2012; Santer et al., 2013).

The CMIP3 models show a $1979-2010$ tropical SST trend of $0.19^{\circ} \mathrm{C}$ per decade in the multi-model mean, significantly larger than the various observational trend estimates ranging from $0.10^{\circ} \mathrm{C}$ to $0.14^{\circ} \mathrm{C}$ per decade (including the $95 \%$ confidence interval; Fu et al., 2011). As a consequence, simulated tropospheric temperature trends are also too large because models attempt to maintain static stability. By contrast, atmospheric models that are forced with the observed SST are in better agreement with observations, as was found in the CMIP3 model ECHAM5 (Bengtsson and Hodges, 2011) and the CMIP5 atmosphere-only runs. In the latter, the LT trend range for the period 19812008 is 0.13 to $0.19^{\circ} \mathrm{C}$ per decade-less than in the CMIP5 coupled models, but still an overestimate (Po-Chedley and Fu, 2012). The influence of SST trend errors on the analysis can be reduced by considering trends in tropospheric static stability, measured by the amplification of MT trends against LT trends; another approach is to consider the amplification of tropospheric trends against SST trends. The results of such analyses strongly depend on the time scale considered. Monthto-month variations are consistent between observations and models concerning amplification aloft against SST variations (Santer et al., 2005) and concerning amplification of MT against LT variations (PoChedley and Fu, 2012). By contrast, the 30-year trend in tropical static stability has been found to be larger than in the satellite observations for almost all ensemble members in both CMIP3 (Fu et al., 2011) and CMIP5 (Po-Chedley and Fu, 2012). However, if the radiosonde compilations are used for the comparison, the trends in static stability in the CMIP3 models agree much better with the observations, and inconsistency cannot be diagnosed unambiguously (Seidel et al., 2012). What caused the remaining trend overestimate in static stability is not clear but has been argued recently to result from an upward propagation of bias in the model climatology (O'Gorman and Singh, 2013).

In summary, most, though not all, CMIP3 and CMIP5 models overestimate the observed warming trend in the tropical troposphere during the satellite period 1979-2012. Roughly one-half to two-thirds of this difference from the observed trend is due to an overestimate of the SST trend, which is propagated upward because models attempt to maintain static stability. There is low confidence in these assessments, however, due to the low confidence in observed tropical tropospheric trend rates and vertical structure (Section 2.4.4).

\subsection{Extratropical circulation}

The AR4 concluded that models, when forced with observed SSTs, are capable of producing the spatial distribution of storm tracks, but generally show deficiencies in the numbers and depth of cyclones and the exact locations of the storm tracks. The ability to represent extratropical cyclones in climate models has been improving, partly due to increases in horizontal resolution.

Storm track biases over the North Atlantic have decreased in CMIP5 models compared to CMIP3 (Zappa et al., 2013) although models still produce too zonal a storm track in this region and most models underestimate cyclone intensity (Colle et al., 2013; Zappa et al., 2013). Chang et al. (2012) also find the storm tracks in the CMIP5 models to be too weak and too equatorwards in their position, similar to the CMIP3 models. The performance of the CMIP5 models in representing North Atlantic cyclones was found to be strongly dependent on model resolution (Colle et al., 2013). Studies based on individual models typically find that models capture the general characteristics of storm tracks and extratropical cyclones (Ulbrich et al., 2008; Catto et al., 2010) and their associated fronts (Catto et al., 2013) and show improvements over earlier model versions (Loptien et al., 2008). However, some models have deficiencies in capturing the location of storm tracks (Greeves et al., 2007; Catto et al., 2011), in part owing to problems related to the location of warm waters such as the Gulf Stream and Kuroshio Current (Greeves et al., 2007; Keeley et al., 2012). This is an important issue because future projections of storm tracks are sensitive to changes in SSTs (Catto et al., 2011; Laine et al., 2011; McDonald, 2011; Woollings et al., 2012). Some studies find that storm track and cyclone biases are strongly related to atmospheric processes and parameterizations (Bauer et al., 2008a; Boer and Lambert, 2008; Zappa et al., 2013). Representation of the Mediterranean storm track has been shown to be particularly dependent on model resolution (Pinto et al., 2006; Raible et al., 2007; Bengtsson et al., 2009; Ulbrich et al., 2009), as is the representation of storm intensity and associated extremes in this area (Champion et al., 2011). Most studies have focussed on NH storm tracks. However, recently two CMIP3 models were found to differ significantly in their simulation of extratropical cyclones affecting Australia (Dowdy et al., 2013) and only about a third of the CMIP3 models were able to capture the observed changes and trends in Southern Hemisphere (SH) baroclinicity responsible for a reduction in the growth rate of the leading winter storm track modes (Frederiksen et al., 2011). There is still a lack of information on SH storm track evaluation for the CMIP5 models.

\subsection{Tropical circulation}

Earlier assessments of a weakening Walker circulation (Vecchi et al., 2006; Vecchi and Soden, 2007; DiNezio et al., 2009) from models and reanalyses (Yu and Zwiers, 2010) have been tempered by subsequent evidence that tropical Pacific Trade winds may have strengthened since the early 1990s (e.g., Merrifield and Maltrud, 2011). Models suggest that the width of the Hadley cell should increase (Frierson et al., 2007; Lu et al., 2007), and there are indications that this has been observed over the past 25 years (Seidel et al., 2008) but at an apparent rate ( 2 to 5 degrees of latitude since 1979) that is faster than in the CMIP3 models (Johanson and Fu, 2009).

The tendency in a warming climate for wet areas to receive more precipitation and subtropical dry areas to receive less, often termed the 'rich-get richer' mechanism (Chou et al., 2006; Held and Soden, 2006) is simulated in CMIP3 models (Chou and Tu, 2008), and observational support for this is found from ocean salinity observations (Durack et al., 2012) and precipitation gauge data over land (Zhang et al., 2007). There is medium confidence that models are correct in simulating precipitation increases in wet areas and decreases in dry areas on broad spatial scales in a warming climate based on agreement among models and some evidence that this has been detected in observed trends (see Section 2.5.1). 
Several recent studies have examined the co-variability of tropical climate variables as a further means of evaluating climate models. Specifically, there are observed relationships between lower tropospheric temperature and total column precipitable water (Mears et al., 2007), and between surface temperature and relative humidity (Willett et al., 2010). Figure 9.9 (updated from Mears et al., 2007) shows the relationship between 25-year (1988-2012) linear trends in tropical precipitable water and lower tropospheric temperature for individual historical simulations (extended by appending RCP8.5 simulations after 2005, see Santer et al., 2013). As described by Mears et al. (2007), the ratio between changes in these two quantities is fairly tightly constrained in the model simulations and similar across a range of time scales, indicating that relative humidity is close to invariant in each model. In the updated figure, the Remote Sensing System (RSS) observations are in fairly good agreement with model expectations, and the University of Alabama in Huntsville (UAH) observations less so. The points associated with two of the reanalyses are also relatively far from the line, consistent with long-term changes in relative humidity. It is not known whether these discrepancies are due to remaining inhomogeneity in the observational data and/or reanalysis results, or due to problems with the climate simulations. All of the observational and reanalysis points lie at the lower end of the model distribution, consistent with the findings of (Santer et al., 2013).

\subsection{Ozone and lower stratospheric temperature trends}

Stratospheric ozone has been subject to a major perturbation since the late 1970s due to anthropogenic emissions of ozone-depleting substances (see also Section 2.2.2.2 and Figure 2.6). Since the AR4, there is increasing evidence that the ozone hole has led to a poleward shift and strengthening of the $\mathrm{SH}$ mid-latitude tropospheric jet during summer (Perlwitz et al., 2008; Son et al., 2008, 2010; SPARC-CCMVal, 2010; McLandress et al., 2011; Polvani et al., 2011; WMO, 2011; Swart and Fyfe, 2012b). These trends are well captured in both chemistry-climate models (CCMs) with interactive stratospheric chemistry and in CMIP3 models with prescribed time-varying ozone (Son et al., 2010; SPARC-CCMVal, 2010). However, around half of the CMIP3 models prescribe ozone as a fixed climatological value, and so these models are not able to simulate trends in surface climate attributable to changing stratospheric ozone amount (Karpechko et al., 2008; Son et al., 2008, 2010; Fogt et al., 2009). For CMIP5, a new time-varying ozone data set (Cionni et al., 2011) was developed and prescribed in the majority of models without interactive chemistry. This zonal mean data set is based on observations by Randel and Wu (2007) and CCM projections in the future (SPARC-CCMVal, 2010). Further, nine of the CMIP5 models include interactive chemistry and so compute their own ozone evolution. As a result, all CMIP5 models consider stratospheric ozone depletion and capture associated effects on SH surface climate, a significant advance over CMIP3. Figure 9.10 shows the global annual mean and Antarctic October mean of total column ozone in the CMIP5 models. The simulated trends in total column ozone are in medium agreement with observations, noting that some models that calculate ozone interactively show significant deviations from observation (Eyring et al., 2013). The multi-model mean agrees well with observations, and there is robust evidence that this constitutes a significant improvement over CMIP3, where around half of the models did not include stratospheric
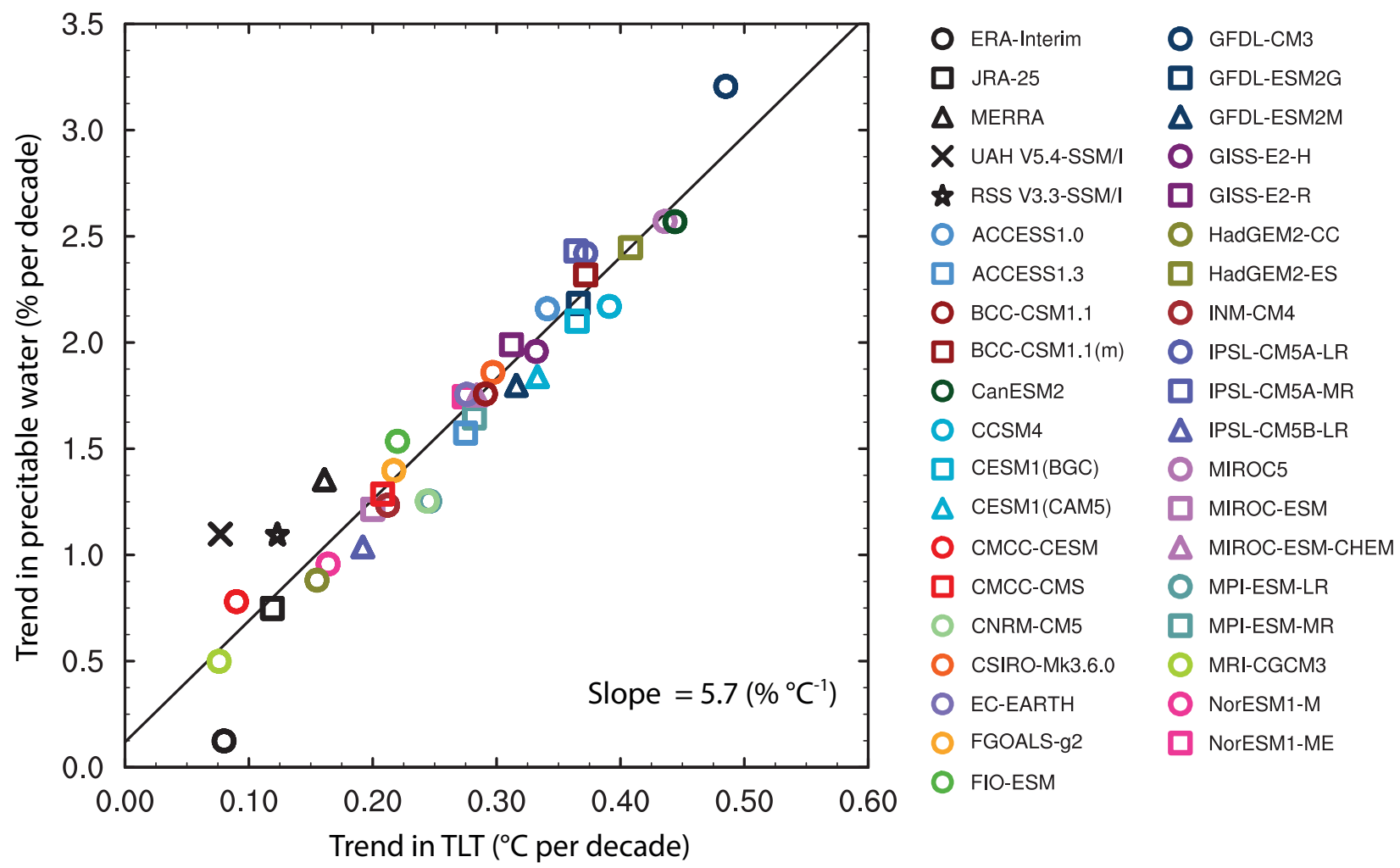

Figure 9.9 | Scatter plot of decadal trends in tropical $\left(20^{\circ} \mathrm{S}\right.$ to $\left.20^{\circ} \mathrm{N}\right)$ precipitable water as a function of trends in lower tropospheric temperature (TLT) over the world's oceans. Coloured symbols are from CMIP5 models; black symbols are from satellite observations or from reanalysis output. Trends are calculated over the 1988-2012 period, so CMIP5 historical runs, which typically end in December 2005, were extended using RCP8.5 simulations initialized using these historical runs. Figure updated from Mears et al. (2007). 
(a) Total Column Ozone ANN 90N-90S

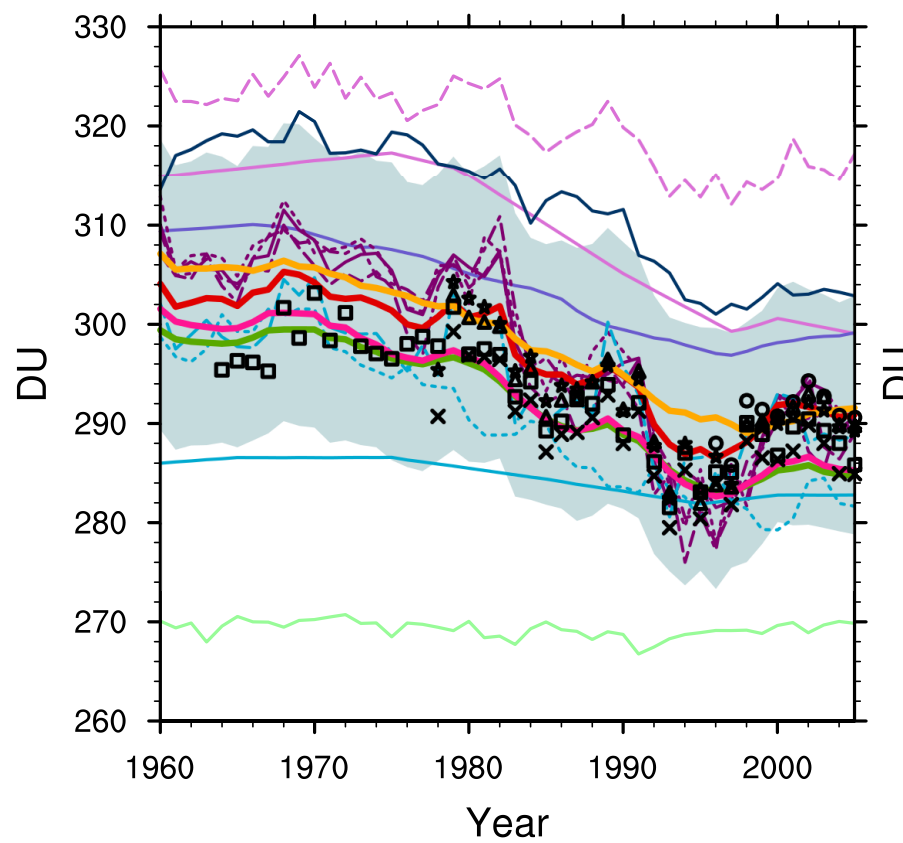

- DLR_GOME-SCIA-GOME2

$X$ NIWA

CCMVal-2

CCSM4

CNRM-CM5

- -- GISS-E2-H.p3

IPSL-CM5A-LR a Ground-based

* NOAA_SBUV

- CMIP5Chem

- -. CESM1(FASTCHEM)

- GFDL-CM3

..... GISS-E2-R.p2 MIROC4h (b) Total Column Ozone OCT 60S-90S

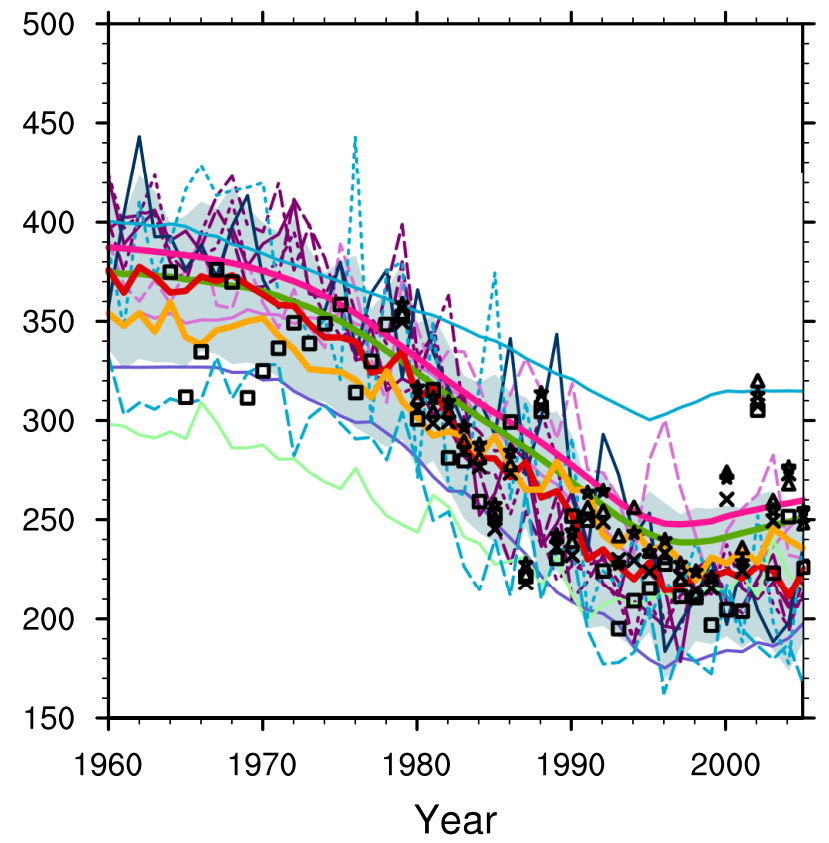

$\triangle$ NASA_TOMS-SBUV-OMI

- IGAC/SPARC

- CMIP5noChem

..... CESM1 (WACCM)

— GISS-ES-H.p2

- - - GISS-E2-R.p3

- - MIROC-ESM-CHEM

Figure 9.10 Time series of area-weighted total column ozone from 1960 to 2005 for (a) annual and global mean $\left(90^{\circ} \mathrm{S}\right.$ to $90^{\circ} \mathrm{N}$ ) and (b) Antarctic October mean (60요 $\mathrm{S}$ to $90^{\circ} \mathrm{S}$ ). Individual CMIP5 models with interactive or semi-interactive chemistry are shown in thin coloured lines, their multi-model mean (CMIP5Chem) in thick red and their standard deviation as the blue shaded area. Further shown are the multi-model mean of the CMIP5 models that prescribe ozone (CMIP5noChem, thick green), the International Global Atmospheric Chemistry/Stratospheric Processes and their Role in Climate (IGAC/SPARC) ozone database (thick pink), the Chemistry Climate Model Validation-2 (CCMVal-2) multimodel mean (thick orange), and observations from five different sources (black symbols). These sources include ground-based measurements (updated from Fioletov et al., 2002), National Aeronautics and Space Administration (NASA) Total Ozone Mapping Spectrometer/Ozone Monitoring Instrument/Solar Backscatter Ultraviolet(/2) (TOMS/OMI/SBUV(/2)) merged satellite data (Stolarski and Frith, 2006), the National Institute of Water and Atmospheric Research (NIWA) combined total column ozone database (Bodeker et al., 2005), Solar Backscatter Ultraviolet (SBUV, SBUV/2) retrievals (updated from Miller et al. 2002), and Deutsches Zentrum für Luft- und Raumfahrt/ Global Ozone Monitoring Experiment/ SCanning Imaging Absorption spectrometer for atmospheric chartography /GOME-2 (DLR GOME/SCIA/GOME-2; Loyola et al., 2009; Loyola and Coldewey-Egbers, 2012). Note that the IGAC/SPARC database over Antarctica (and thus the majority of the CMIP5noChem models) is based on ozonesonde measurements at the vortex edge (69 ${ }^{\circ}$ ) and as a result underestimates Antarctic ozone depletion compared to the observations shown. Ozone depletion was more pronounced after 1960 as equivalent stratospheric chlorine values steadily increased throughout the stratosphere. (Adapted from Figure 2 of Eyring et al., 2013.)

ozone trends. Correspondingly, there is high confidence that the representation of associated effects on high-latitude surface climate and lower stratospheric cooling trends has improved compared to CMIP3.

Lower stratospheric temperature change is affected by ozone, and since 1958 the change is characterized by a long-term global cooling trend interrupted by three 2-year warming episodes following large volcanic eruptions (Figure 2.24). During the satellite era (since 1979) the cooling occurred mainly in two step-like transitions in the aftermath of the El Chichón eruption in 1982 and the Mt Pinatubo eruption in 1991, with each cooling transition followed by a period of relatively steady temperatures (Randel et al., 2009; Seidel et al., 2011). This specific evolution of global lower stratosphere temperatures since 1979 is well captured in the CMIP5 models when forced with both natural and anthropogenic climate forcings, although the models tend to underes- timate the long-term cooling trend (Charlton-Perez et al., 2012; Eyring et al., 2013; Santer et al., 2013) (see Chapter 10).

Tropospheric ozone is an important GHG and as such needs to be well represented in climate simulations. In the historical period it has increased due to increases in ozone precursor emissions from anthropogenic activities (see Chapters 2 and 8). Since the AR4, a new emission data set has been developed (Lamarque et al., 2010), which has led to some differences in tropospheric ozone burden compared to previous studies, mainly due to biomass burning emissions (Lamarque et al., 2010; Cionni et al., 2011; Young et al., 2013). Climatological mean tropospheric ozone in the CMIP5 simulations generally agrees well with satellite observations and ozonesondes, although as in the stratosphere, biases exist for individual models (Eyring et al., 2013; Young et al., 2013) (see also Chapter 8). 


\subsubsection{Model Simulations of the Last Glacial Maximum and the Mid-Holocene}

Simulations of past climate can be used to test a model's response to forcings larger than those of the 20th century (see Chapter 5), and the CMIP5 protocol includes palaeoclimate simulations referred to as PMIP3 (Paleoclimate Model Intercomparison Project, version 3) (Taylor et al., 2012b). Specifically, the Last Glacial Maximum (LGM, 21000 years BP) allows testing of the modelled climate response to the presence of a large ice sheet in the $\mathrm{NH}$ and to lower concentrations of radiatively active trace gases, whereas the mid-Holocene $(\mathrm{MH}, 6000$ years $\mathrm{BP}$ ) tests the response to changes in seasonality of insolation in the NH (see Chapter 5). For these periods, palaeoclimate reconstructions allow quantitative model assessment (Braconnot et al., 2012).
In addition the CMIP5/PMIP3 simulations can compared to previous palaeoclimate intercomparisons (Joussaume and Taylor, 1995; Braconnot et al., 2007c).

Figure 9.11 compares model results to palaeoclimate reconstructions for both LGM (left) and MH (right). For most models the simulated LGM cooling is within the range of the climate reconstructions (Braconnot et al., 2007c; Izumi et al., 2013), however Hargreaves et al. (2011) find a global mean model warm bias over the ocean of about $1{ }^{\circ} \mathrm{C}$ for this period (Hargreaves et al., 2011). LGM simulations tend to overestimate tropical cooling and underestimate mid-latitude cooling (Kageyama et al., 2006; Otto-Bliesner et al., 2009). They thus underestimate polar amplification which is a feature also found for the mid-Holocene (Masson-Delmotte et al., 2006; Zhang et al., 2010a) and other climatic con- a

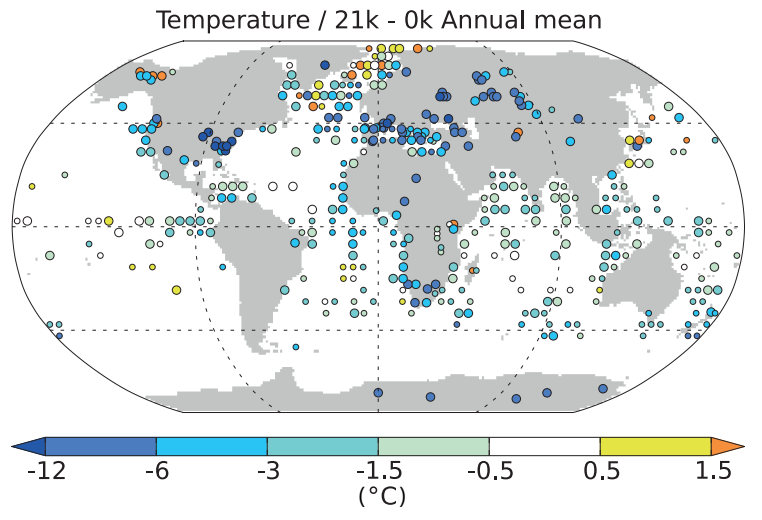

C

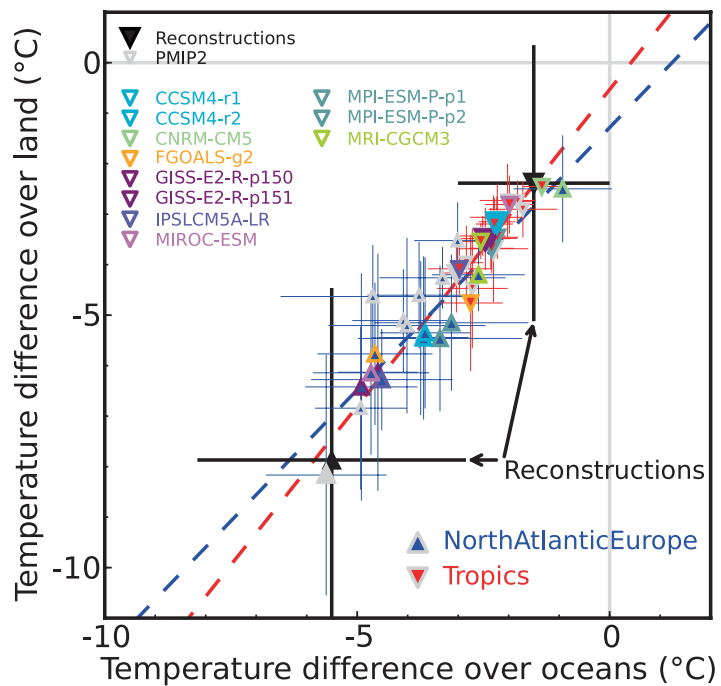

b

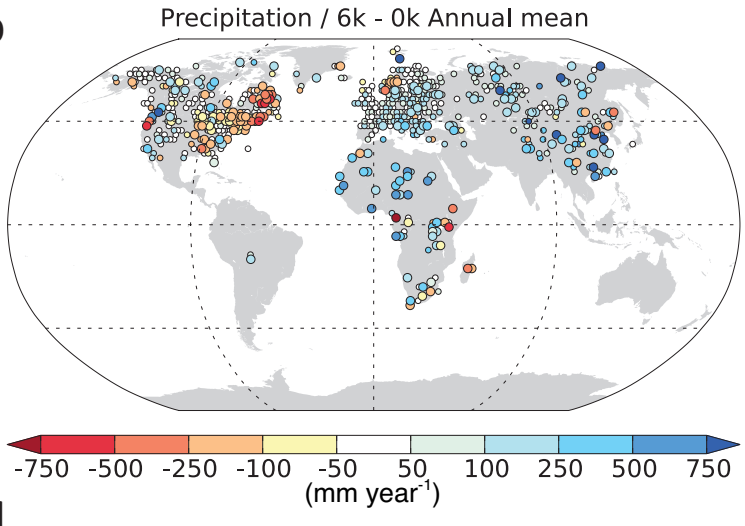

d

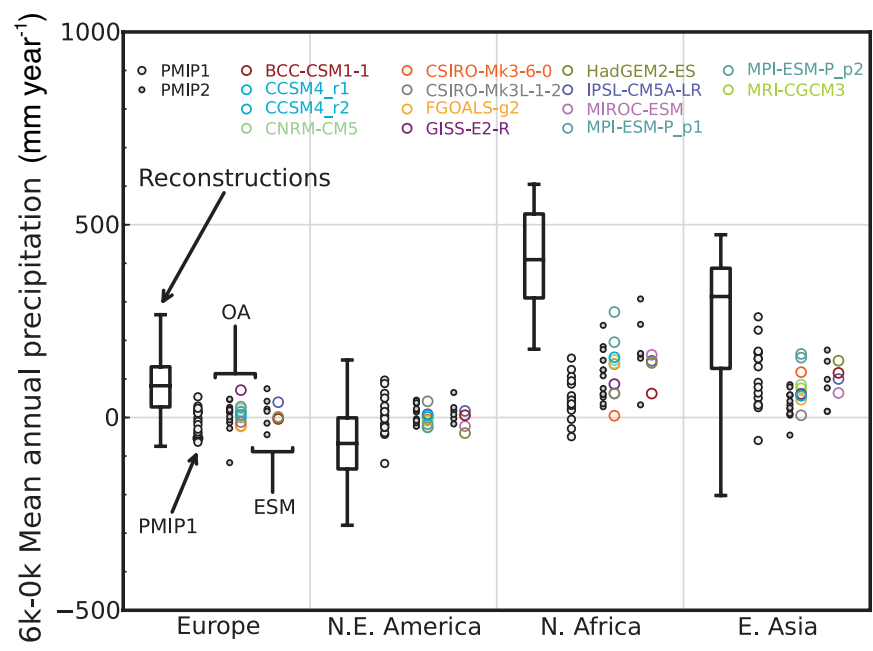

Figure 9.11 Reconstructed and simulated conditions for the Last Glacial Maximum (LGM, 21,000 years BP, left) and the mid-Holocene (MH, 6000 years BP, right). (a) LGM change in annual mean surface temperature $\left({ }^{\circ} \mathrm{C}\right.$ ) over land as shown by palaeo-environmental climate reconstructions from pollen, macrofossils, and ice cores (Bartlein et al., 2010; Braconnot et al., 2012), and in annual mean sea surface temperature $\left({ }^{\circ} \mathrm{C}\right.$ ) over the ocean from different type of marine records (Waelbroeck et al., 2009). (b) MH change in annual mean precipitation ( $\mathrm{mm} \mathrm{yr}^{-1}$ ) over land (Bartlein et al., 2010). In (a) and (b), the size of the dots is proportional to the uncertainties at the different sites as provided in the reconstructions. (c) Annual mean temperature changes over land against changes over the ocean, in the tropics (downward triangles) and over the North Atlantic and Europe (upward triangles). The mean and range of the reconstructions are shown in black, the Paleoclimate Modelling Intercomparison Project version 2 (PMIP2) simulations as grey triangles, and the CMIP5/ PMIP3 simulations as coloured triangles. The 5 to $95 \%$ model ranges are in red for the tropics and in blue for the North Atlantic/Europe. (d) Changes in annual mean precipitation in different data-rich regions. Box plots for reconstructions provide the range of reconstructed values for the region. For models, the individual model average over the region is plotted for PMIP2 (small grey circle) and CMIP5/PMIP3 simulations (coloured circles). Note that in PMIP2, 'ESM' indicates that vegetation is computed using a dynamical vegetation model, whereas in CMIP5/PMIP3 it indicates that models have an interactive carbon cycle with different complexity in dynamical vegetation (see Table 9.A.1). The limits of the boxes are as follows: Western Europe $\left(40^{\circ} \mathrm{N}\right.$ to $50^{\circ} \mathrm{N}, 10^{\circ} \mathrm{W}$ to $\left.30^{\circ} \mathrm{E}\right)$; northeast America $\left(35^{\circ} \mathrm{N}\right.$ to $60^{\circ} \mathrm{N}, 95^{\circ} \mathrm{W}$ to $\left.60^{\circ} \mathrm{W}\right)$; North Africa $\left(10^{\circ} \mathrm{N}\right.$ to $25^{\circ} \mathrm{N}, 20^{\circ} \mathrm{W}$ to $\left.30^{\circ} \mathrm{W}\right)$, and East Asia $\left(25^{\circ} \mathrm{N}\right.$ to $40^{\circ} \mathrm{N}, 75^{\circ} \mathrm{E}$ to $105^{\circ} \mathrm{E}$ ). (Adapted from Braconnot et al., 2012.) 
a Last Glacial Maximum

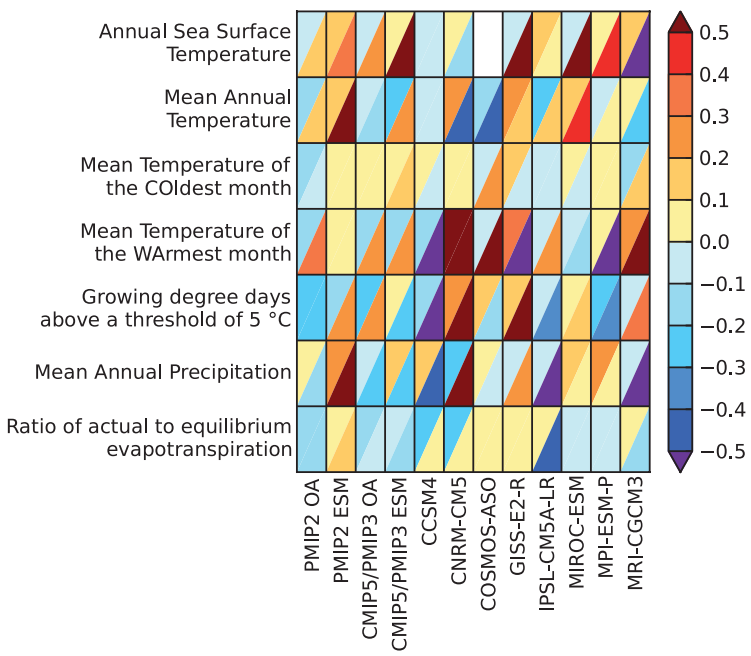

b Mid Holocene

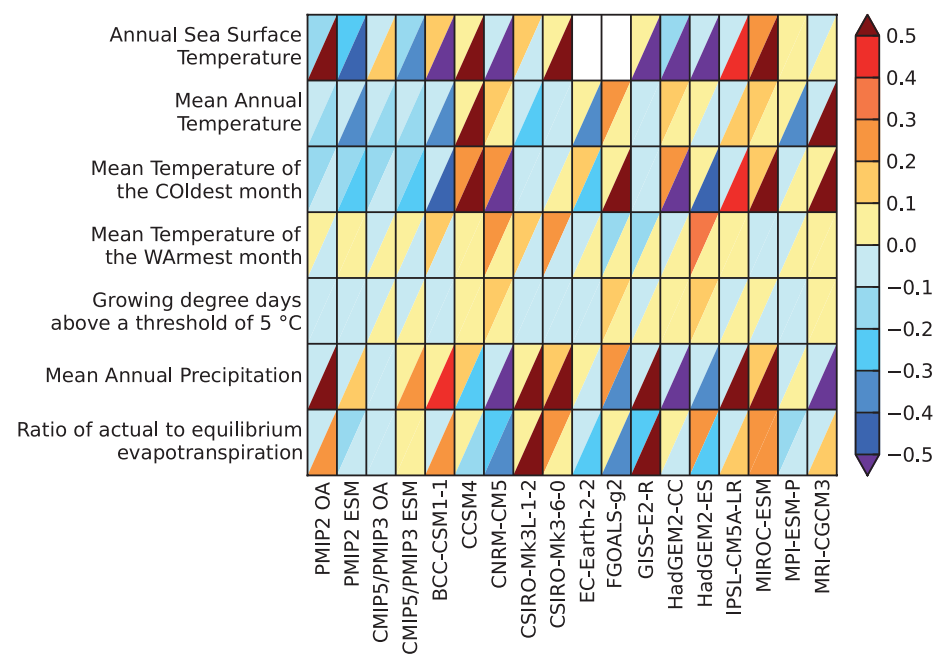

Figure 9.12 Relative model performance for the Last Glacial Maximum (LGM, about 21,000 yr BP) and the mid-Holocene (MH, about 6000 yr BP) for seven bioclimatic variables: annual mean sea surface temperature, mean annual temperature (over land), mean temperature of the coldest month, mean temperature of the warmest month, growing degree days above a threshold of $5^{\circ} \mathrm{C}$, and ratio of actual to equilibrium evapotranspiration. Model output is compared to the Bartlein et al. (2010) data set over land, including ice core data over Greenland and Antarctica (Braconnot et al., 2012) and the Margo data set (Waelbroeck et al., 2009) over the ocean. The CMIP5/Paleoclimate Modelling Intercomparison Project version 3 (PMIP3) ensemble of Ocean-Atmosphere (OA) and Earth System Model (ESM) simulations are compared to the respective PMIP2 ensembles in the first four columns of each panel. A diagonal divides each cell in two parts to show in the upper triangle a measure of the distance between model and data, taking into account the uncertainties in the palaeoclimate reconstructions (Guiot et al., 1999), and in the lower triangle the normalized mean-square error (NMSE) that indicates how well the spatial pattern is represented. In this graph all the values have been normalized following (Gleckler et al., 2008) using the median of the CMIP5/PMIP3 ensemble. The colour scale is such that blue colours mean that the result is better than the median CMIP5 model and red means that it is worse.

texts (Masson-Delmotte et al., 2010). Part of this can be attributed to uncertainties in the representation of sea ice and vegetation feedbacks that have been shown to amplify the response at the LGM and the MH in these latitudes (Braconnot et al., 2007b; Otto et al., 2009; O'ishi and Abe-Ouchi, 2011). Biases in the representation of the coupling between vegetation and soil moisture are also responsible for excessive continental drying at the LGM (Wohlfahrt et al., 2008) and uncertainties in vegetation feedback in monsoon regions (Wang et al., 2008; Dallmeyer et al., 2010). Nevertheless, the ratio between the simulated change in temperature over land and over the ocean (Figure 9.11c) is rather similar in different models, resulting mainly from simulation of the hydrological cycle over land and ocean (Sutton et al., 2007; Laine et al., 2009). At a regional scale, models tend to underestimate the changes in the north-south temperature gradient over Europe both at the LGM (Ramstein et al., 2007) and at the mid-Holocene (Brewer et al., 2007; Davis and Brewer, 2009).

The large-scale pattern of precipitation change during the MH (Figure $9.11 \mathrm{~d}$ ) is reproduced, but models tend to underestimate the magnitude of precipitation change in most regions. In the SH (not shown in the figure), the simulated change in atmospheric circulation is consistent with precipitation records in Patagonia and New Zealand, even though the differences between model results are large and the reconstructions have large uncertainties (Rojas et al., 2009; Rojas and Moreno, 2011).

A wider range of model performance metrics is provided in Figure 9.12 (Guiot et al., 1999; Brewer et al., 2007; Annan and Hargreaves, 2011; Izumi et al., 2013). Results for the MH are less reliable than for the
LGM, because the forcing is weaker and involves smaller scale responses over the continent (Hargreaves et al., 2013). As is the case for the simulations of present day climate, there is only modest improvement between the results of the more recent models (CMIP5/PMIP3) and those of earlier model versions (PMIP2) despite higher resolution and sophistication.

\subsubsection{Ocean}

Accurate simulation of the ocean in climate models is essential for the correct estimation of transient ocean heat uptake and transient climate response, ocean $\mathrm{CO}_{2}$ uptake, sea level rise, and coupled climate modes such as ENSO. In this section model performance is assessed for the mean state of ocean properties, surface fluxes and their impact on the simulation of ocean heat content and sea level, and aspects of importance for climate variability. Simulations of both the recent and more distant past are evaluated against available data. Following Chapter 3, ocean reanalyses are not used for model evaluation as many of their properties depend on the model used to build the reanalysis.

\subsubsection{Simulation of Mean Temperature and Salinity Structure}

Potential temperature and salinity are the main ocean state variables and their zonal distribution offers an evaluation of climate models in different parts of the ocean (upper ocean, thermocline, deep ocean). Over most latitudes, at depths ranging from $200 \mathrm{~m}$ to $2000 \mathrm{~m}$, the CMIP5 multi-model mean zonally averaged ocean temperature is too warm (Figure 9.13a), albeit with a cooler deep ocean. Similar biases were evident in the CMIP3 multi-model mean. Above 200 m, however, 

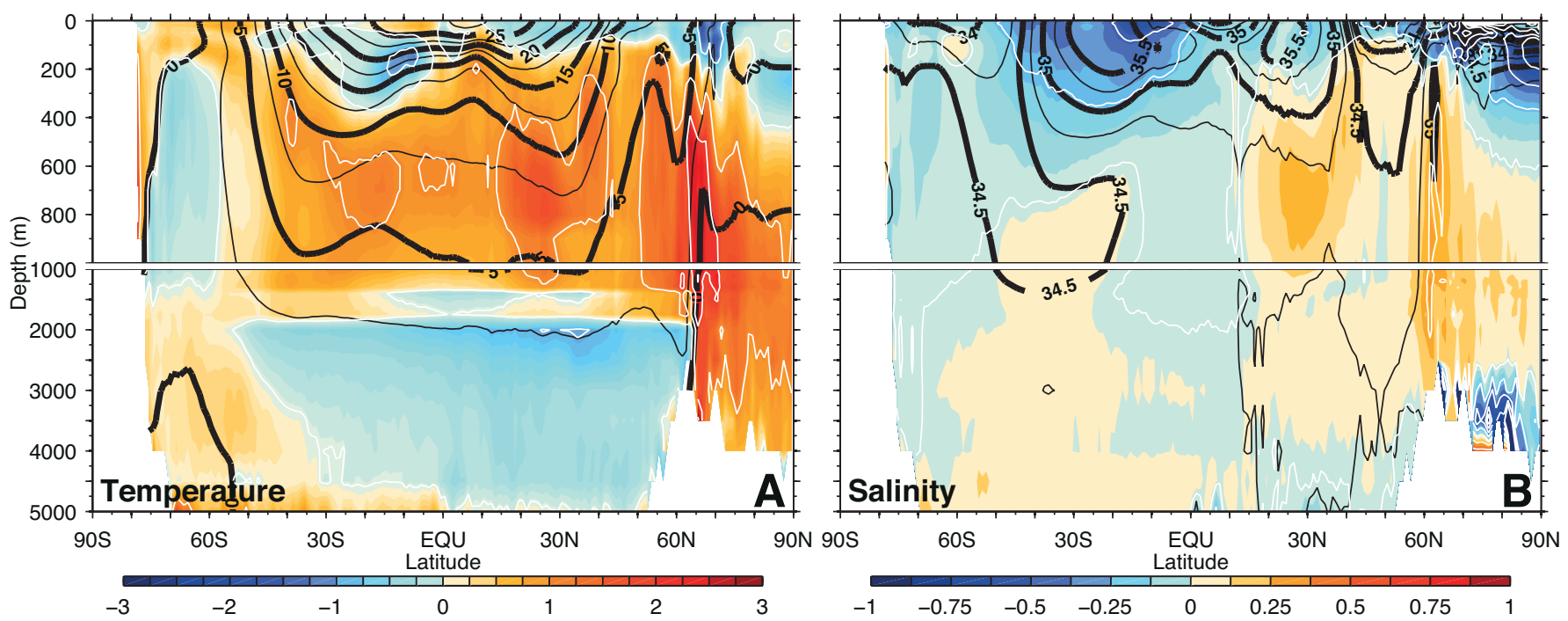

Figure 9.13 (a) Potential temperature (OC) and (b) salinity (PSS-78); shown in colour are the time-mean differences between the CMIP5 ensemble mean and observations, zonally averaged for the global ocean (excluding marginal and regional seas). The observed climatological values are sourced from the World Ocean Atlas 2009 (WOA09; Prepared by the Ocean Climate Laboratory, National Oceanographic Data Center, Silver Spring, MD, USA), and are shown as labelled black contours. White contours show regions in (a) where potential temperature differences exceed positive or negative 1,2 or $3^{\circ} \mathrm{C}$, and in (b) where salinity differences exceed positive or negative $0.25,0.5,0.75$ or 1 (PSS-78). The simulated annual mean climatologies are obtained for 1975 to 2005 from available historical simulations, whereas WOA09 synthesizes observed data from 1874 to 2008 in calculations of the annual mean; however, the median time for gridded observations most closely resembles the 1980-2010 period (Durack and Wijfels, 2012). Multiple realizations from individual models are first averaged to form a single-model climatology, before the construction of the multi-model ensemble mean. A total of 43 available CMIP5 models have contributed to the temperature panel (a) and 41 models to the salinity panel (b).

the CMIP5 (and CMIP3) multi-model mean is too cold, with maximum cold bias (more than $1{ }^{\circ} \mathrm{C}$ ) near the surface at mid-latitudes of the $\mathrm{NH}$ and near $200 \mathrm{~m}$ at $15^{\circ} \mathrm{S}$. Zonal salinity errors (Figure 9.13b) exhibit a different pattern from those of the potential temperature indicating that most do not occur via density compensation. Some near surface structures in the tropics and in the northern mid-latitude are indicative of density compensation and are presumably due to surface fluxes errors. At intermediate depths, errors in water mass formation translate into errors in both salinity and potential temperature.

In the AR4 it was noted that the largest errors in SST in CMIP3 were found in mid and high latitudes. While this is still the case in CMIP5, there is marginal improvement with fewer individual models exhibiting serious bias - the inter-model zonal mean SST error standard deviation is significantly reduced at all latitudes north of $40^{\circ} \mathrm{S}$ - even though the multi-model mean is only slightly improved (Figure 9.14a, c). Near the equator, the cold tongue error in the Pacific (see Section 9.4.2.5.1) is reduced by $30 \%$ in CMIP5; the Atlantic still exhibits serious errors and the Indian is still well simulated (Figure 9.14b,d). In the Tropics, Li and Xie (2012) have shown that SST errors could be classified into those exhibiting broad meridional structures that are due to cloud errors, and those associated with Pacific and Atlantic cold tongue errors that are due to thermocline depth errors.

Sea surface salinity (SSS) is more challenging to observe, even though the last decade has seen substantial improvements in the development of global salinity observations, such as those from the Array for Realtime Geostrophic Oceanography (ARGO) network (see Chapter 3). Whereas SST is strongly constrained by air-sea interactions, the sources of SSS variations (surface forcing via evaporation minus precipitation, sea ice formation/melt and river runoff) are only loosely related to the SSS itself, allowing errors to develop unchecked in coupled models. An analysis of CMIP3 models showed that, whereas the historical trend in global mean SSS is well captured by the models, regional SSS biases are as high as \pm 2.5 psu (Terray et al., 2012). Comparisons of modelled versus observed estimates of evaporation minus precipitation suggest that model biases in surface freshwater flux play a role in some regions (e.g., double Intertropical Convergence Zone (ITCZ) in the East Pacific; Lin, 2007) or over the Indian ocean (Pokhrel et al., 2012).

The performance of coupled climate models in simulating hydrographic structure and variability were assessed in two important regions, the Labrador and Irminger Seas and the Southern Ocean (de Jong et al., 2009) and (Sloyan and Kamenkovich, 2007). Eight CMIP3 models produce simulations of the intermediate and deep layers in the Labrador and Irminger Seas that are generally too warm and saline, with biases up to $0.7 \mathrm{psu}$ and $2.9^{\circ} \mathrm{C}$. The biases arise because the convective regime is restricted to the upper $500 \mathrm{~m}$; thus, intermediate water that in reality is formed by convection is, in the models, partly replaced by warmer water from the south. In the Southern Ocean, Subantarctic Mode Water (SAMW) and Antarctic Intermediate Water (AAIW), two water masses indicating very efficient ocean ventilation, are found to be well simulated in some CMIP3 and CMIP5 models but not in others, some having a significant fresh bias (Sloyan and Kamenkovich, 2007; Salle et al., 2013). McClean and Carman (2011) found biases in the properties of the North Atlantic mode waters and their formation rates in the CMIP3 models. Errors in Subtropical Mode Water (STMW) formation rate and volume produce a turnover time of 1 to 2 years, approximately half of that observed. Bottom water properties assessment in CMIP5 shows that about half of the models create dense water on 
(a) Zonal mean SST error CMIP5

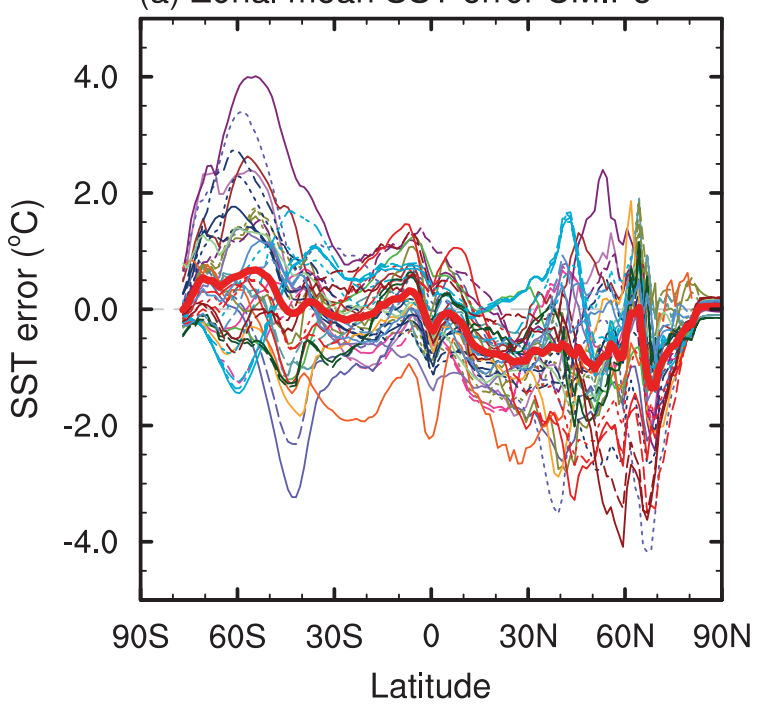

(c) Zonal mean SST error CMIP5 vs. CMIP3

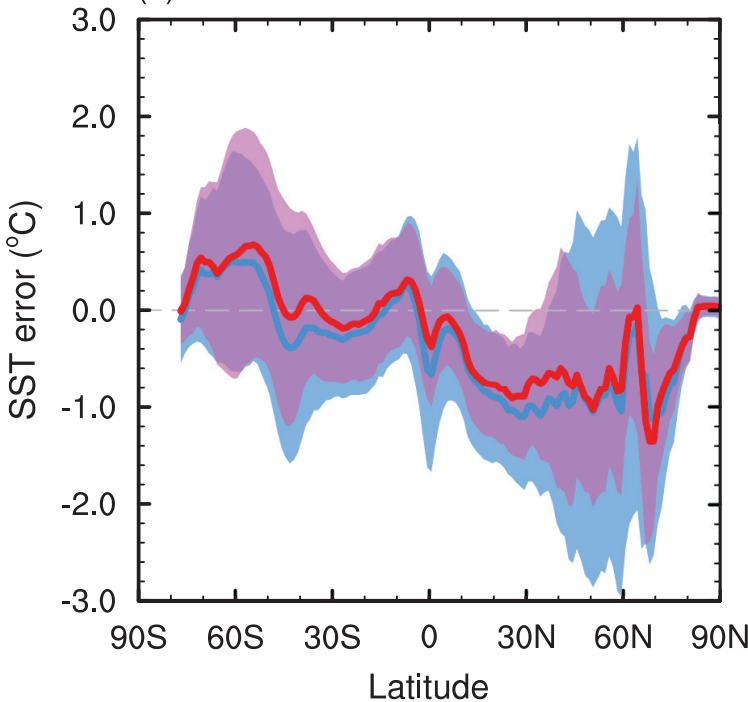

(b) Equatorial SST error CMIP5

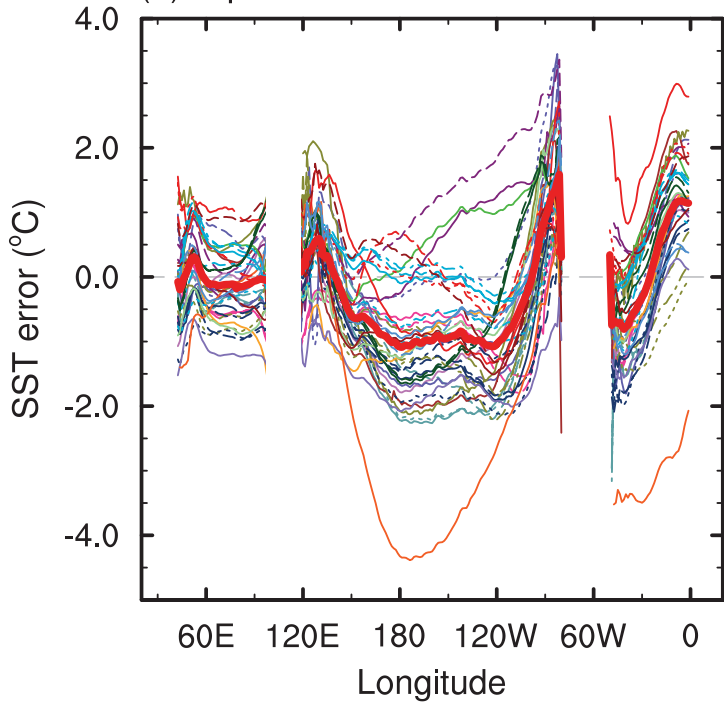

(d) Equatorial SST CMIP5 vs. CMIP3

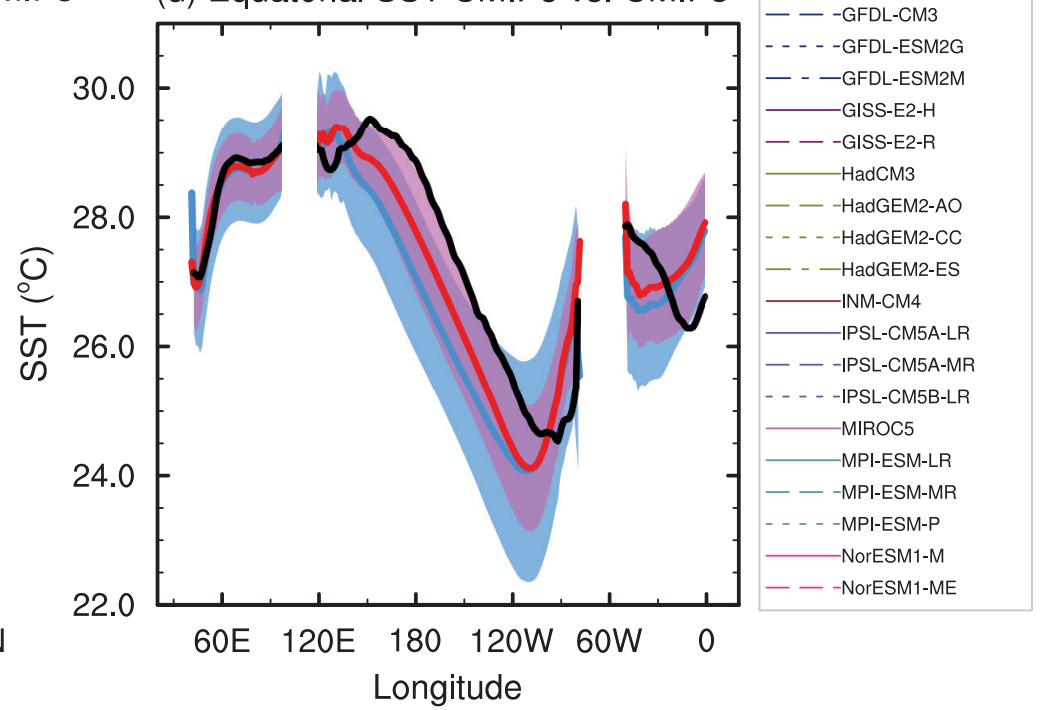

Figure 9.14 | (a) Zonally averaged sea surface temperature (SST) error in CMIP5 models. (b) Equatorial SST error in CMIP5 models. (c) Zonally averaged multi-model mean SST error for CMIP5 (red curve) and CMIP3 (blue curve), together with inter-model standard deviation (shading). (d) Equatorial multi-model mean SST in CMIP5 (red curve), CMIP3 (blue curve) together with inter-model standard deviation (shading) and observations (black). Model climatologies are derived from the 1979-1999 mean of the historical simulations. The Hadley Centre Sea Ice and Sea Surface Temperature (HadISST) (Rayner et al., 2003) observational climatology for 1979-1999 is used as reference for the error calculation (a), (b), and (c); and for observations in (d).

the Antarctic shelf, but it mixes with lighter water and is not exported as bottom water. Instead most models create deep water by open ocean deep convection, a process occurring rarely in reality (Heuzé et al., 2013) which leads to errors in deep water formation and properties in the Southern Ocean as shown in Figure 9.15.

Few studies have assessed the performance of models in simulating Mixed Layer Depth (MLD). In the North East Pacific region, Jang et al. (2011) found that the CMIP3 models exhibit the observed deep MLD in the Kuroshio Extension, though with a deep bias and only one large deep MLD region, rather than the observed two localized maxima. Other studies have noted MLD biases near sea ice edges (Capotondi et al., 2012).

\subsubsection{Simulation of Sea Level and Ocean Heat Content}

Steric and dynamic components of the mean dynamic topography (MDT) and sea surface height (SSH) patterns can be compared to observations (Maximenko et al., 2009). Pattern correlations between simulated and observed MDT are above 0.95 for all of the CMIP5 models (Figure 9.16), an improvement compared to CMIP3. MDT biases over tropical ocean regions are consistent with surface wind stress biases (Lee et al., 2013). Over the Antarctic Circumpolar Current, the parameterization of eddy-induced transports is essential for the models' density structure and thus MDT (Kuhlbrodt et al., 2012). High-resolution eddy resolving ocean models show improved SSH simulations over coarser resolution versions (McClean et al., 2006). Chapter 13 provides a more extensive 
assessment of sea level changes in CMIP5 simulations, including comparisons with century-scale historical records.

Ocean heat content $(\mathrm{OHC})$ depends only on ocean temperature, whereas absolute changes in sea level are also influenced by processes that are only now being incorporated into global models (e.g., mass loss from large ice sheets discussed in Section 9.1.3.2.7). However, global-scale changes in $\mathrm{OHC}$ are highly correlated with the thermosteric contribution to global SSH changes (Domingues et al., 2008). Approximately half of the historical CMIP3 simulations did not include the effects of volcanic eruptions, resulting in substantially greater than observed ocean heat uptake during the late 20th century (Gleckler et a)
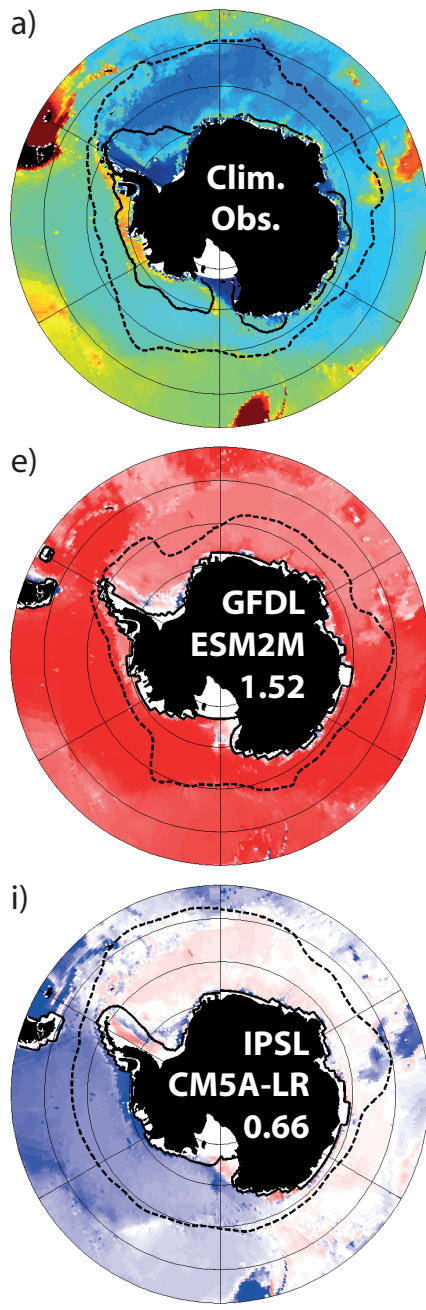

m)
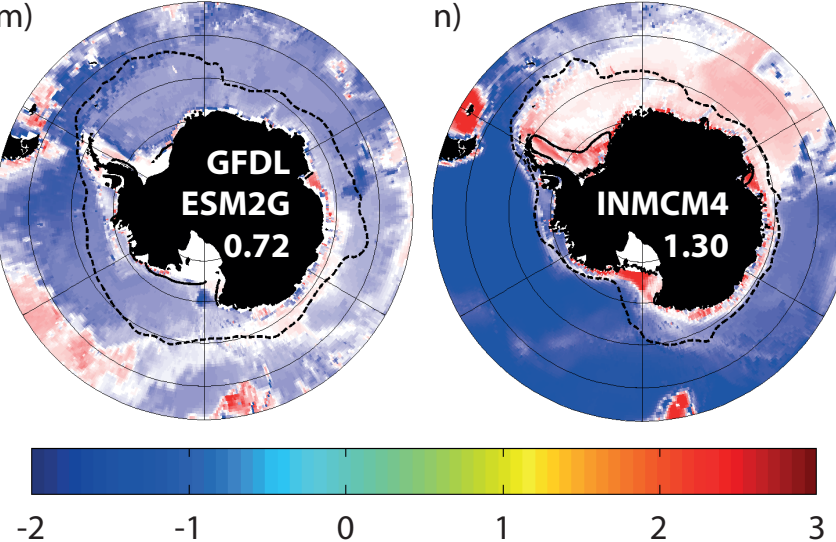
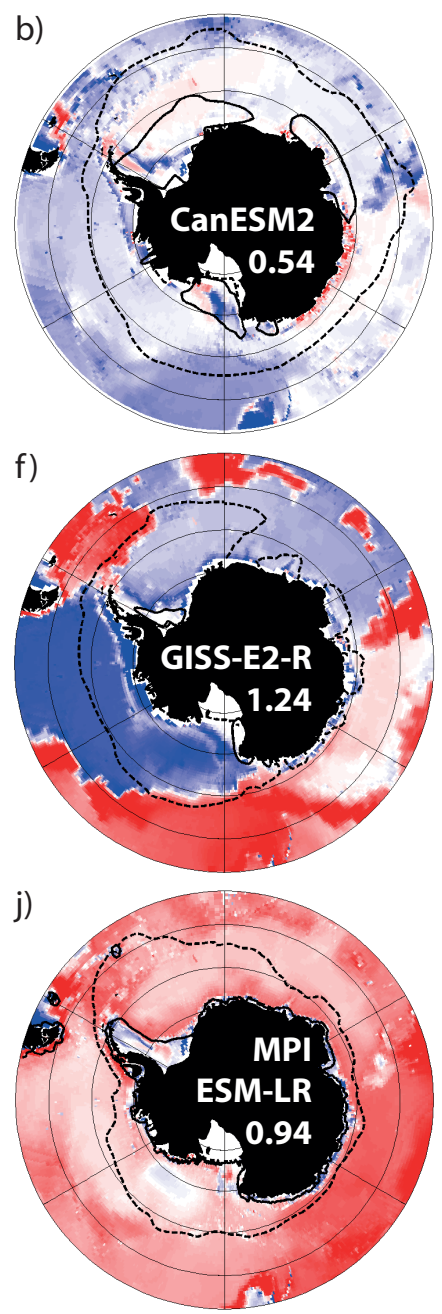

n)
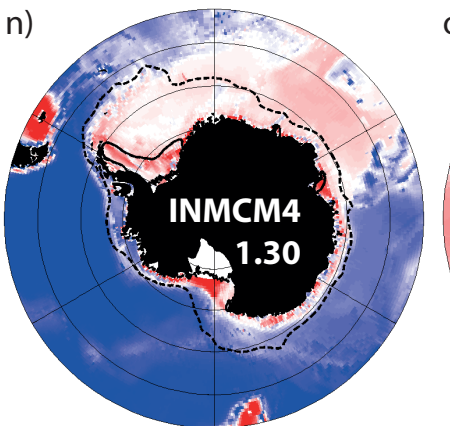

$\left({ }^{\circ} \mathrm{C}\right)$
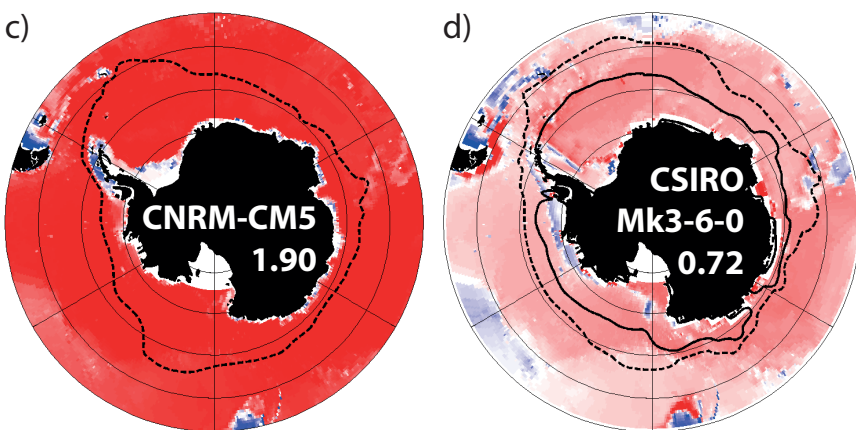

g)

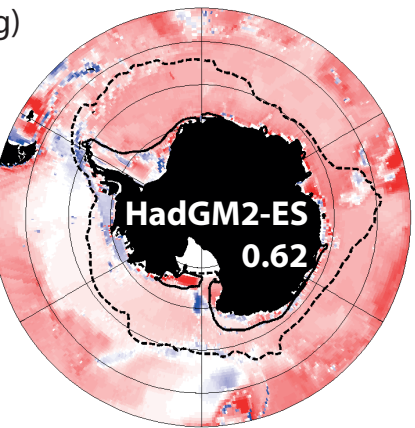

k)

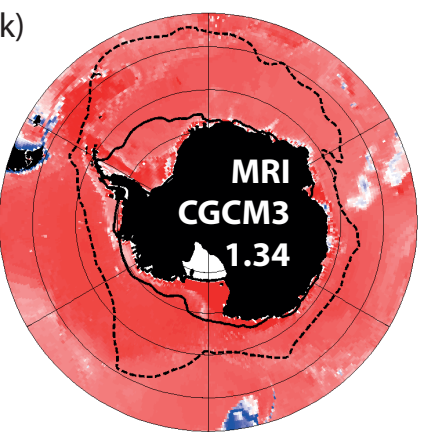

O)

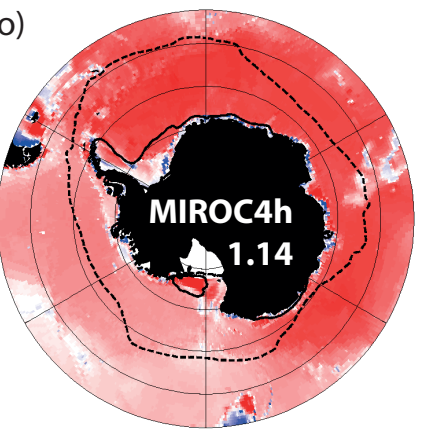

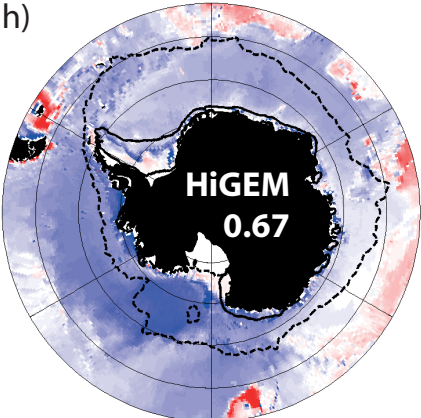

l)

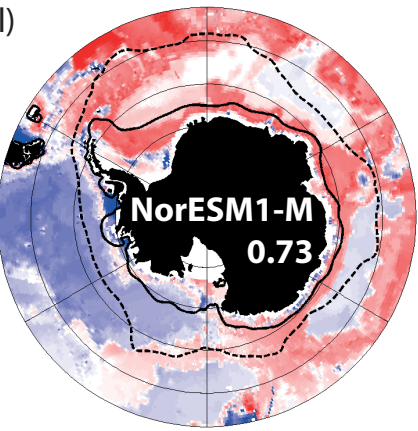

p)

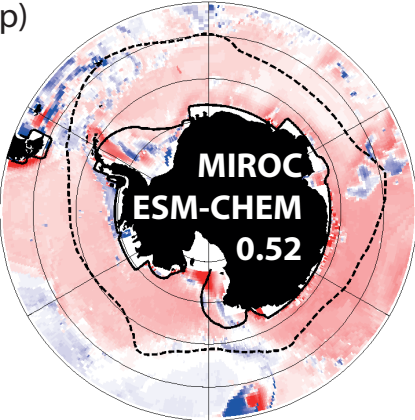

$-2$

$-1$

Figure 9.15 | Time-mean bottom potential temperature in the Southern Ocean, observed (a) and the differences between individual CMIP5 models and observations (b-p); left colour bar corresponds to the observations, right colour bar to the differences between model and observations (same unit). Thick dashed black line is the mean August sea ice extent (concentration >15\%); thick continuous black line is the mean February sea ice extent (concentration >15\%). Numbers indicate the area-weighted root-mean-square (RMS) error for all depths between the model and the climatology (unit ${ }^{\circ} \mathrm{C}$ ); mean RMS error $=0.97^{\circ} \mathrm{C}$. (After Heuzé et al., 2013.) 


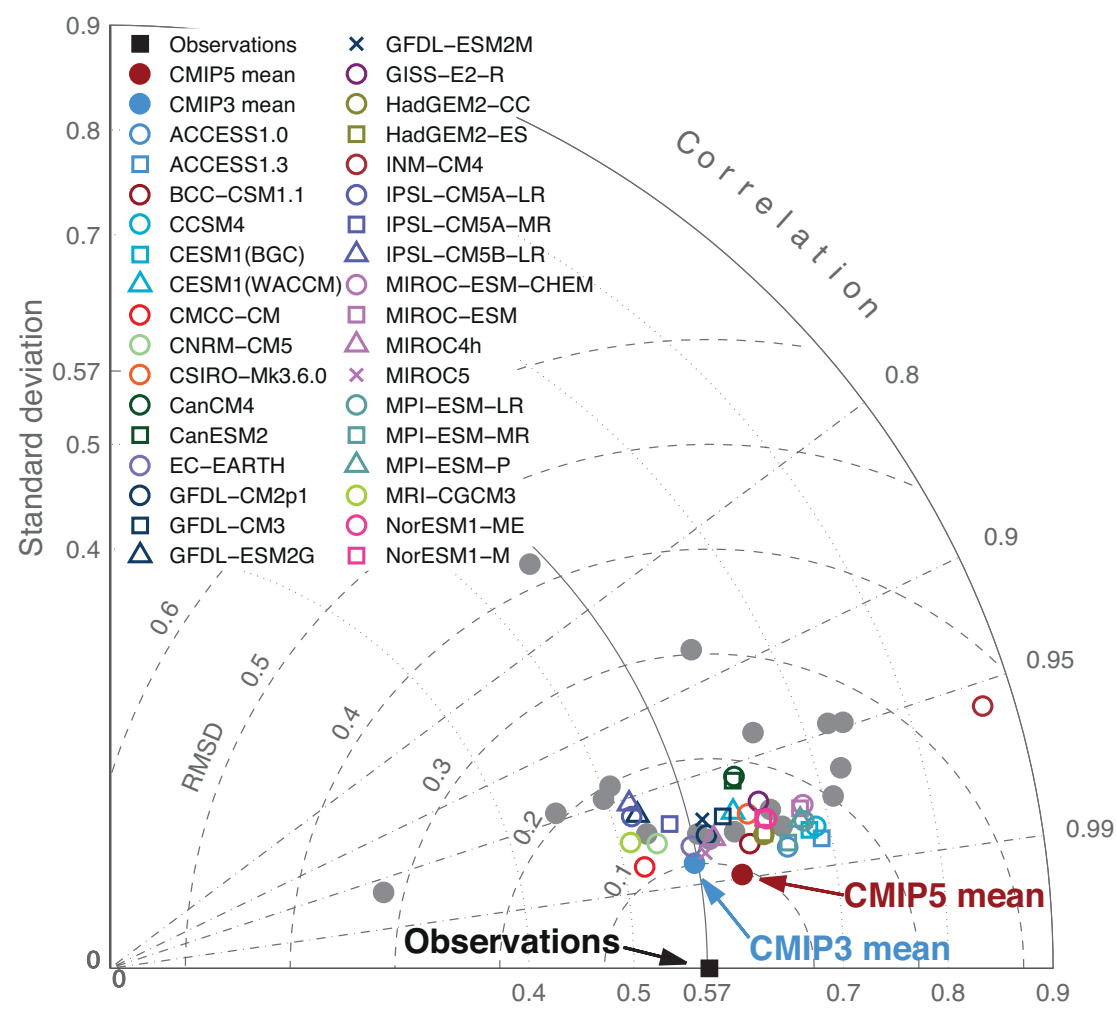

Figure 9.16 | Taylor diagram for the dynamic sea surface height climatology (1987-2000). The radial coordinate shows the standard deviation of the spatial pattern, normalized by the observed standard deviation. The azimuthal variable shows the correlation of the modelled spatial pattern with the observed spatial pattern. The root-mean square error with bias removed is indicated by the dashed grey circles about the observational point. Analysis is for the global ocean, $50^{\circ} \mathrm{S}$ to $50^{\circ} \mathrm{N}$. The reference data set is Archiving, Validation and Interpretation of Satellite Oceanographic data (AVISO), a merged satellite product (Ducet et al., 2000), which is described in Chapter 3. One realization per model is shown for each CMIP5 and CMIP3 model result. Grey filled circles are for individual CMIP3 models; other symbols as in legend.

al., 2006; Domingues et al., 2008). Figure 9.17 shows observed and simulated global 0 to $700 \mathrm{~m}$ and total $\mathrm{OHC}$ changes during the overlap period of the observational record and the CMIP5 historical experiment (1961-2005). Three upper-ocean observational estimates, assessed in Chapter 3, are also shown to indicate observational uncertainty. The CMIP5 multi-model mean falls within the range of observations for most of the period, and the intermodel spread is reduced relative to CMIP3 (Gleckler et al., 2006; Domingues et al., 2008). This may result from most CMIP5 models including volcanic forcings. When the deep ocean is included, the CMIP5 multi-model mean also agrees well with the observations, although the deeper ocean estimates are much more uncertain (Chapter 3). There is high confidence that many CMIP5 models reproduce the observed increase in ocean heat content since 1960.

EMIC results for changes in total $\mathrm{OHC}$ are also compared with observations in Figure 9.17. (Note: results in this figure are based on Eby et al. (2013) who show $\mathrm{OHC}$ changes for 0 to $2000 \mathrm{~m}$, whereas here the time-integrated net heat flux into the ocean surface is shown to compare with CMIP5 results (Figure 9.17b)). There is a tendency for the EMICs to overestimate total $\mathrm{OHC}$ changes and this could alter the temperature related feedbacks on the oceanic carbon cycle, and affect the long-term millennium projections in Chapter 12. However, it should be noted that high $\mathrm{OHC}$ changes can compensate for biases in climate sensitivity or RF so as to reproduce surface temperature changes over the 20th century. This will result in biased thermosteric sea level rise for millennial projections. Calibrated EMICs (Meinshausen et al., 2009; Sokolov et al., 2010) would remove such biases.

In idealized CMIP5 experiments $\left(\mathrm{CO}_{2}\right.$ increasing $\left.1 \% \mathrm{yr}^{-1}\right)$, the heat uptake efficiency of the CMIP5 models varies by a factor of two, explaining about $50 \%$ of the model spread (Kuhlbrodt and Gregory, 2012). Despite observational uncertainties, this recent work also provides limited evidence that in the upper $2000 \mathrm{~m}$, most CMIP5 models are less stratified (in the global mean) than is observed, which suggests that these models transport heat downwards more efficiently than the real ocean. These results are consistent with earlier studies (Forest et al., 2006, 2008; Boe et al., 2009a; Sokolov et al., 2010) that conclude the CMIP3 models may overestimate oceanic mixing efficiency and therefore underestimate the Transient Climate Response (TCR) and its impact on future surface warming. However, Kuhlbrodt and Gregory (2012) also find that this apparent bias explains very little of the model spread in TCR. Although some progress has been made in understanding mixing deficiencies in ocean models (Griffies and Greatbatch, 2012; llicak et al., 2012), this remains a key challenge in improving the representation of physical processes that impact the evolution of ocean heat content and thermal expansion. 
(a) CMIP5 0-700m ocean heat content change $\left(10^{22} \mathrm{~J}\right)$

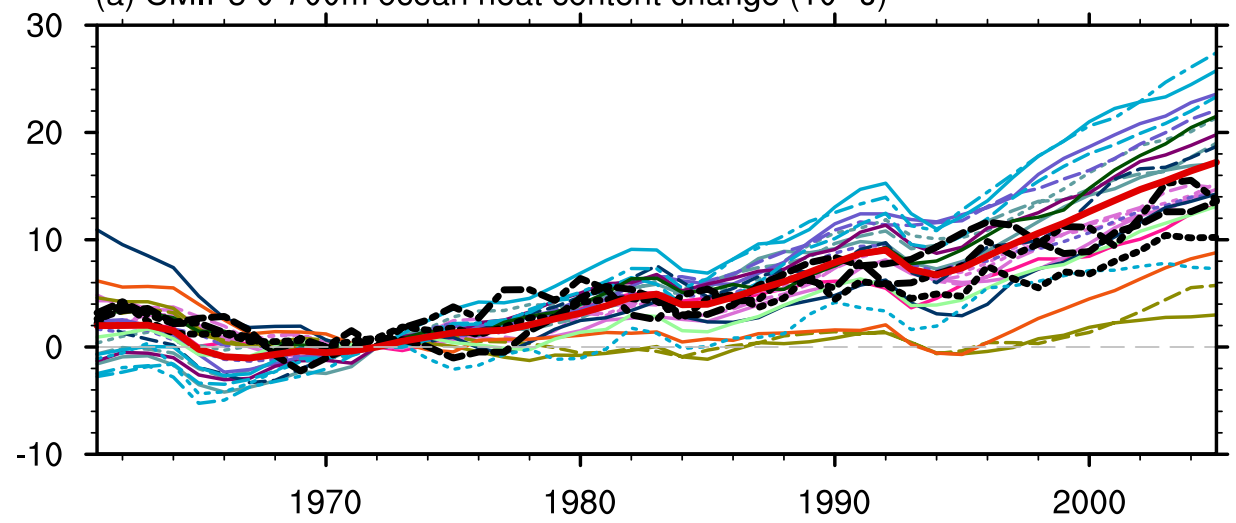

(b) CMIP5 total ocean heat content change $\left(10^{22} \mathrm{~J}\right)$

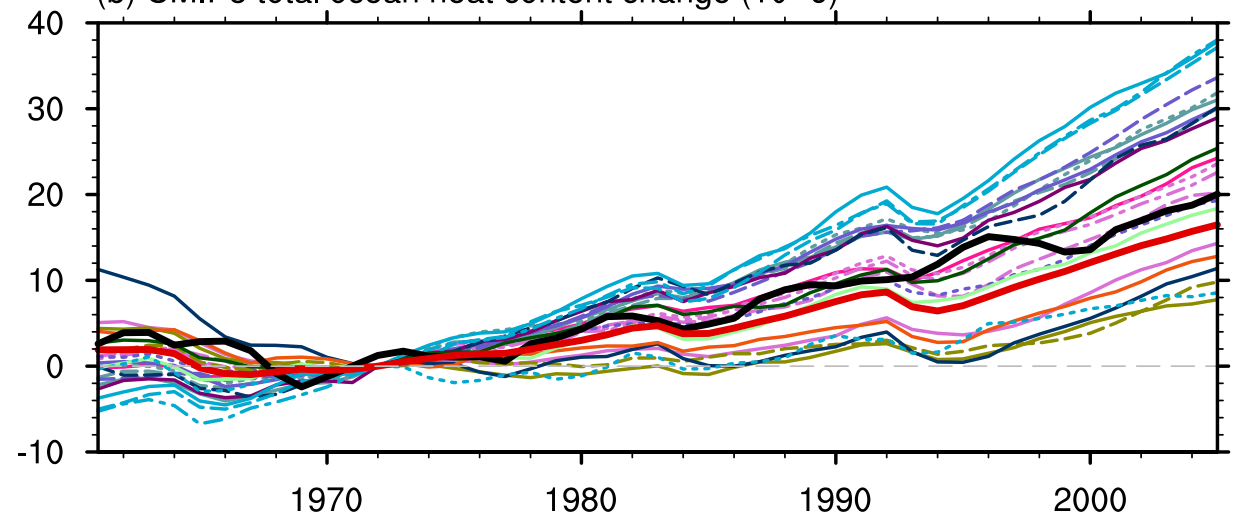

(c) EMIC total ocean heat content change $\left(10^{22} \mathrm{~J}\right)$

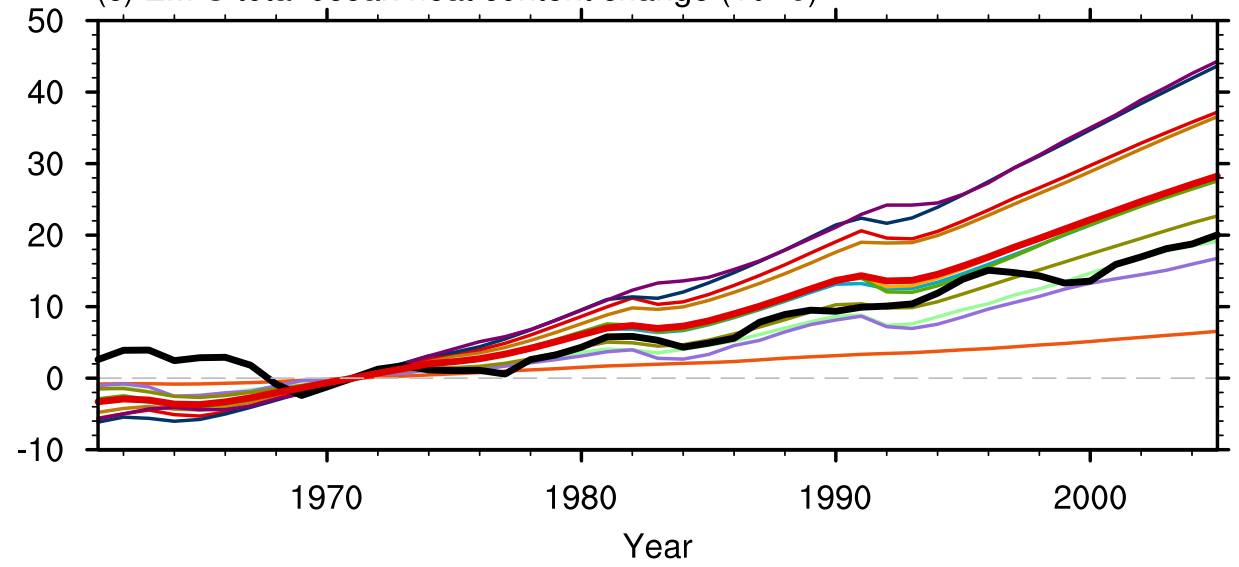

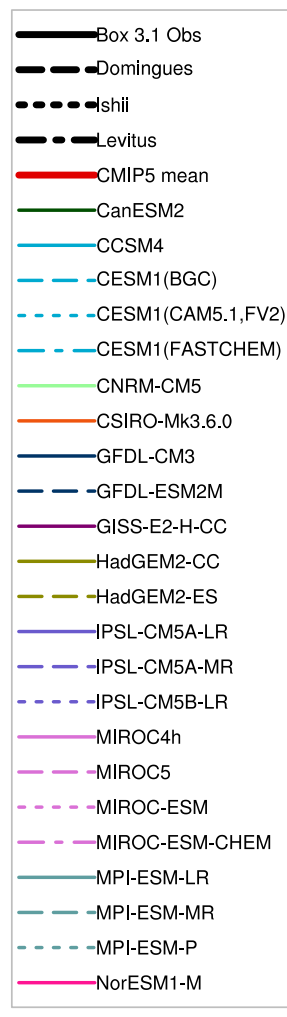

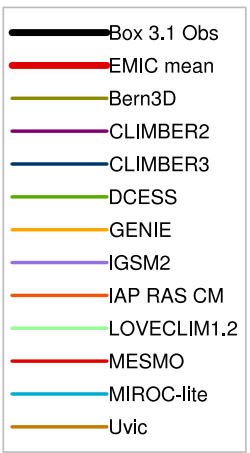

Figure 9.17 Time series of simulated and observed global ocean heat content anomalies (with respect to 1971). CMIP5 historical simulations and observations for both the upper 700 meters of the ocean (a) as well as for the total ocean heat content (b). Total ocean heat content results are also shown for EMICs and observations (c). EMIC estimates are based on time-integrated surface heat flux into the ocean. The 0 to $700 \mathrm{~m}$ and total heat content observational estimates (thick lines) are respectively described in Figure 3.2 and Box 3.1, Figure 1. Simulation drift has been removed from all CMIP5 runs with a contemporaneous portion of a quadratic fit to each corresponding pre-industrial control run (Gleckler et al., 2012). Units are $10^{22}$ Joules.

\subsubsection{Simulation of Circulation Features Important for Climate Response}

\subsection{Simulation of recent ocean circulation}

\section{Atlantic Meridional Overturning Circulation}

The Atlantic Meridional Overturning Circulation (AMOC) consists of northward transport of shallow warm water overlying a southward transport of deep cold water and is responsible for a considerable part of the northward oceanic heat transport. Long-term AMOC estimates have had to be inferred from hydrographic measurements sporadically available over the last decades (e.g., Bryden et al., 2005; Lumpkin et al., 2008, Chapter 3.6.3). Continuous AMOC monitoring at $26.5^{\circ} \mathrm{N}$ was started in 2004 (Cunningham et al., 2007) and now provides a 5-year mean value of $18.5 \mathrm{~Sv}$ with annual means having a standard deviation of 1 Sv (McCarthy et al., 2012). The ability of models to simulate this important circulation feature is tied to the credibility of simulated AMOC weakening during the 21 st century because the magnitude of 
the weakening is correlated with the initial AMOC strength (Gregory et al., 2005). The mean AMOC strength in CMIP5 models ranges from 15 to $30 \mathrm{~Sv}$ for the historical period which is comparable to the CMIP3 models (Weaver et al., 2012; see Figure 12.35). The variability of the AMOC is assessed in Section 9.5.3.3.1.

\section{Southern Ocean circulation}

The Southern Ocean is an important driver for the meridional overturning circulation and is closely linked to the zonally continuous Antarctic Circumpolar Current (ACC). Gupta et al. (2009) noted that relatively small deficiencies in the position of the ACC lead to more obvious biases in the SST in the models. The ability of CMIP3 models to adequately represent Southern Ocean circulation and water masses seems to be affected by several factors (Russell et al., 2006). The most important are the strength of the westerlies at the latitude of the Drake Passage, the heat flux gradient over this region, and the change in salinity with depth across the ACC. Kuhlbrodt et al. (2012) found that the strongest influence on ACC transport in the CMIP3 models was the Gent-McWilliams thickness diffusivity. The ACC has a typical transport through the Drake Passage of about 135 Sv (e.g., Cunningham et al., 2003). A comparison of CMIP5 models (Meijers et al., 2012) shows that, firstly, the ACC transport through Drake Passage is improved as compared to the CMIP3 models, and secondly, that the inter-model range in the zonal mean ACC position is smaller than in the CMIP3 ensemble (in CMIP5, the mean transport is $148 \mathrm{~Sv}$ and the standard deviation is $50 \mathrm{~Sv}$ across an ensemble of 21 models).

\section{Simulation of glacial ocean conditions}

Reconstructions of the last glacial maximum from sediment cores discussed in Chapter 5 indicate that the regions of deep water formation in the North Atlantic were shifted southward, that the boundary between North Atlantic Deep Water (NADW) and Antarctic Bottom Water (AABW) was substantially shallower than today, and that NADW formation was less intense (Duplessy et al., 1988; Dokken and Jansen, 1999; McManus et al., 2004; Curry and Oppo, 2005). This signal, although estimated from a limited number of sites, is robust (see Chapter 5). The AR4 reported that model simulations showed a wide range of AMOC response to LGM forcing (Weber et al., 2007), with some models exhibiting reduced strength of the $A M O C$ and its extension at depth and other showing no change or an increase. Figure 9.18 provides an update of the diagnosis proposed by Otto-Bliesner et al. (2007) to compare model results with the deep ocean data from Adkins et al. (2002) using PMIP2 and CMIP5/PMIP3 pre-industrial and LGM simulations (Braconnot et al., 2012). These models reproduce the modern deep ocean temperature-salinity (T-S) structure in the Atlantic basin, but most of them do not capture the cold and salty bottom

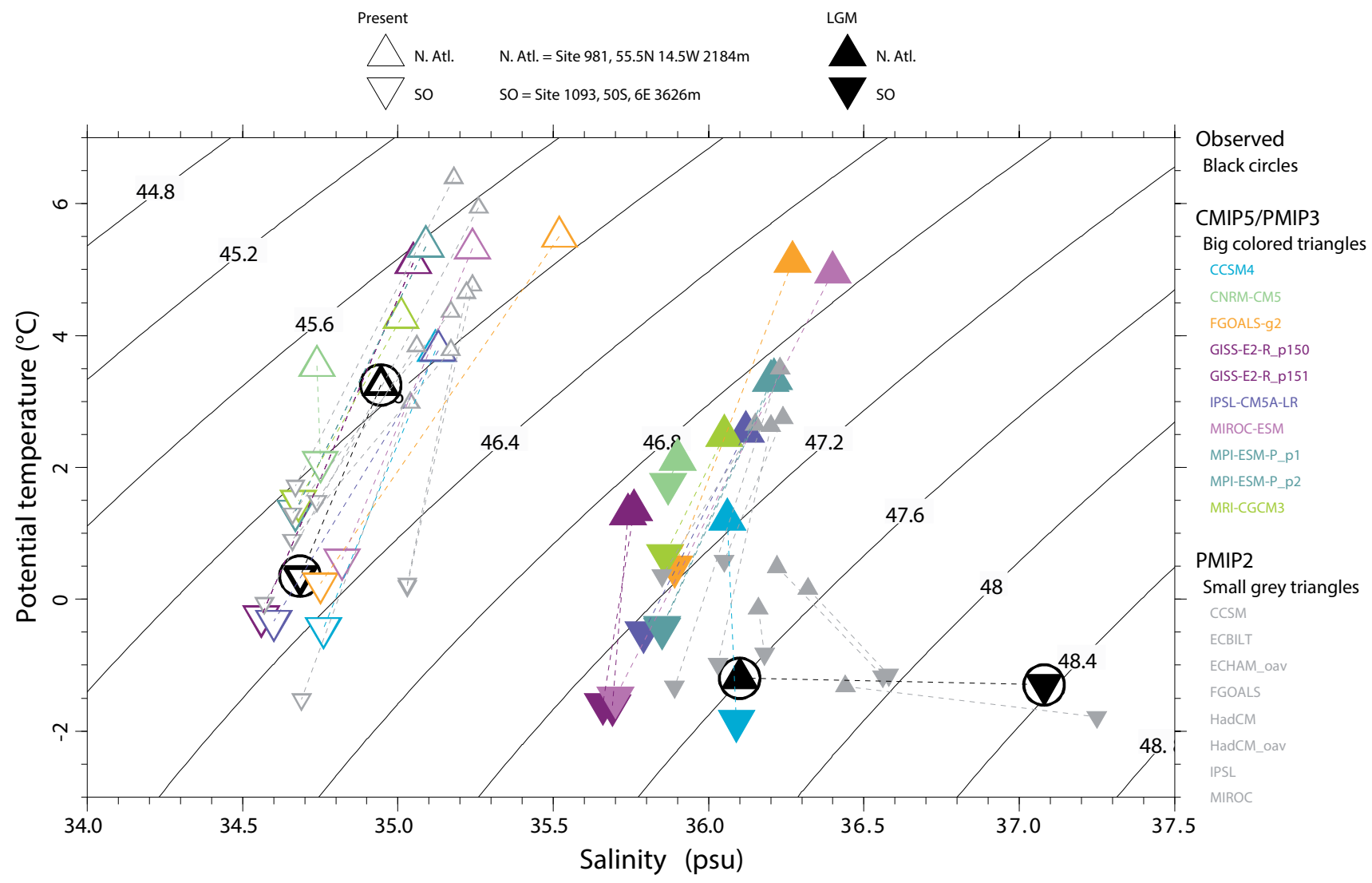

Figure 9.18 | Temperature and salinity for the modern period (open symbols) and the Last Glacial Maximum (LGM, filled symbols) as estimated from proxy data at Ocean Drilling Program (ODP) sites (black symbols, from Adkins et al., 2002) and simulated by the Paleoclimate Modelling Intercomparison Project version 2 (PMIP2, small triangles) and PMIP3/ CMIP5 (big triangles) models. The isolines represent lines of equal density. Site 981 (triangles) is located in the North Atlantic (Feni Drift, $55^{\circ} \mathrm{N}, 15^{\circ} \mathrm{W}, 2184 \mathrm{~m}$ ). Site 1093 (upsidedown triangles) is located in the South Atlantic (Shona Rise, 50 ${ }^{\circ} \mathrm{S}, 6^{\circ} \mathrm{E}, 3626 \mathrm{~m}$ ). In PMIP2, only Community Climate System Model (CCSM) included a 1 psu adjustment of ocean salinity at initialization to account for freshwater frozen into LGM ice sheets; the other PMIP2 model-simulated salinities have been adjusted to allow a comparison. In PMIP3, all simulations include the 1 psu adjustment as required in the PMIP2/CMIP5 protocol (Braconnot et al., 2012). The dotted lines allow a comparison of the values at the NH and SH sites for a same model. This figure is adapted from Otto-Bliesner et al. (2007). 
water suggested by the LGM reconstructions, providing evidence that processes responsible for such palaeoclimate changes may not be well reproduced in contemporary climate models. This is expected to also affect projected changes in deep ocean properties.

\subsubsection{Simulation of Surface Fluxes and Meridional Transports}

Surface fluxes play a large part in determining the fidelity of ocean simulations. As noted in the AR4, large uncertainties in surface heat and fresh water flux observations (usually obtained indirectly) do not allow useful evaluation of models. This is still the case and so the focus here is on an integrated quantity, meridional heat transport, which is less prone to errors. Surface wind stress is better observed and models are evaluated against observed products below.

The zonal component of wind stress is particularly important in driving ocean surface currents; modelled and observed values are shown in Figure 9.19. At middle to high latitudes, the model-simulated wind stress maximum lies 5 to 10 degree equatorward of that in the observationally based estimates, and so mid-latitude westerly winds are (a) CMIP5

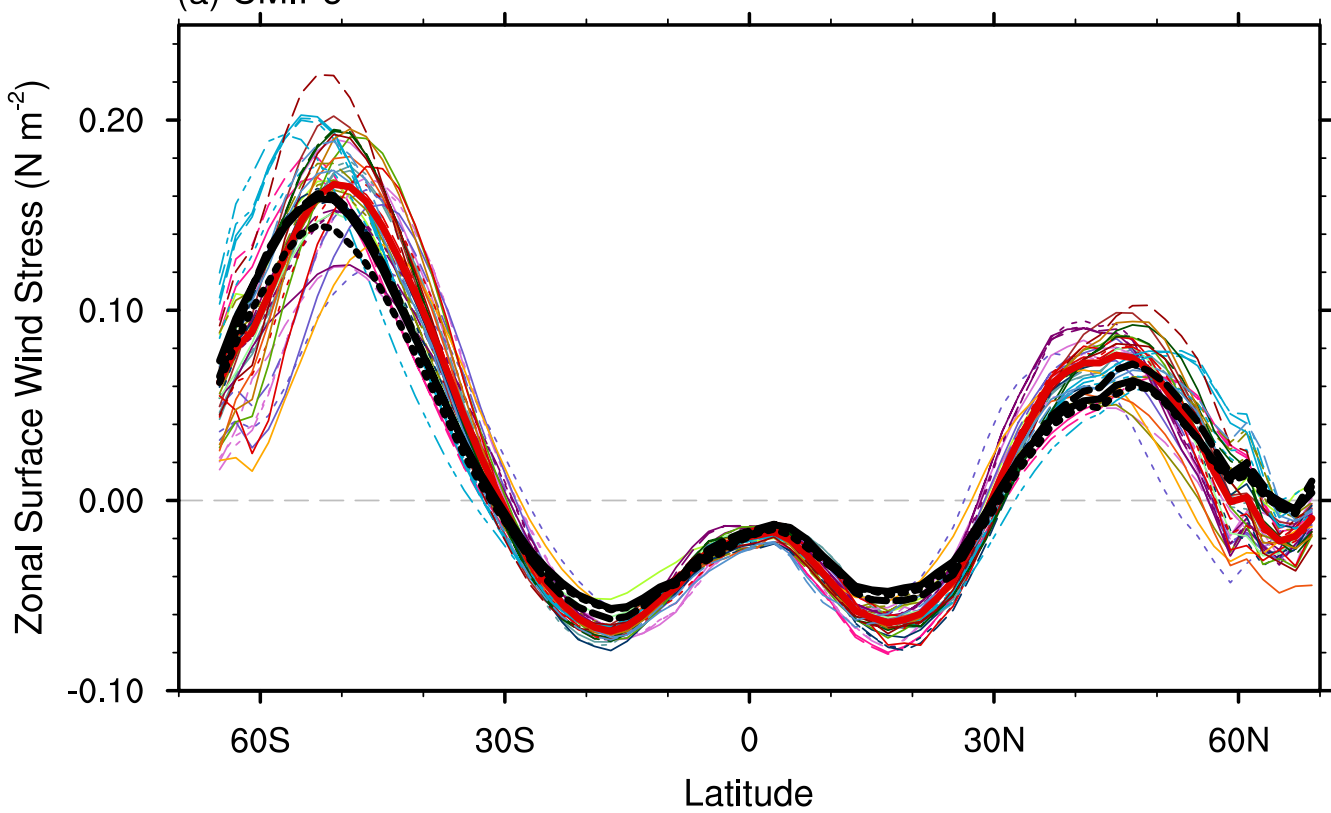

(b) CMIP5 and CMIP3

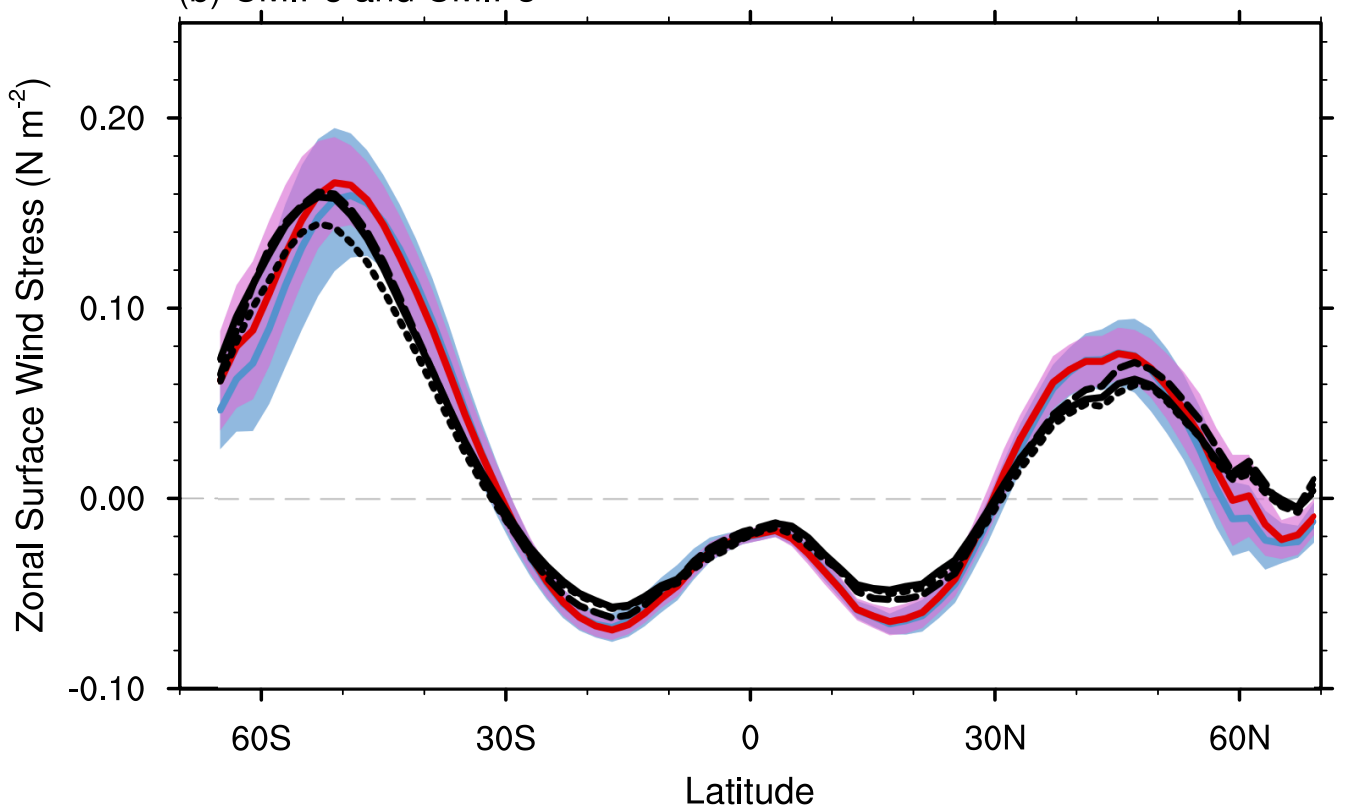

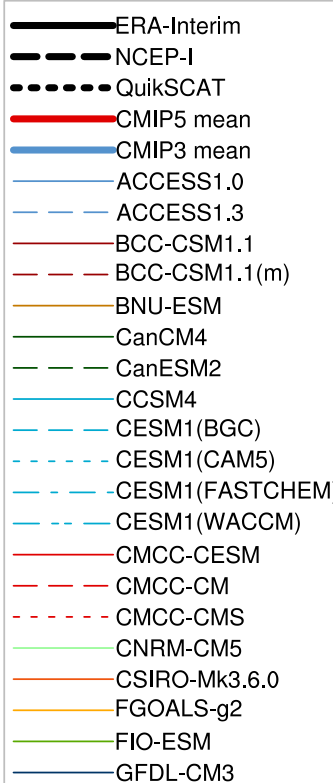

GFDL-CM3

- - - GFDL-ESM2G

- . - . - GFDL-ESM2M

GISS-E2-H

- - GISS-E2-H-CC

- - - - GISS-E2-R

- - - -GISS-E2-R-CC

HadCM3

- - HadGEM2-AO

- - - - HadGEM2-CC

- - - -HadGEM2-ES INM-CM4

IPSL-CM5A-LR

- - IPSL-CM5A-MR

- . - - - IPSL-CM5B-LR MIROC4h MIROC5 MIROC-ESM -MIROC-ESM-CHEM

MPI-ESM-LR

- - MPI-ESM-MR

MPI-ESM-P

MRI-CGCM3

NorESM1-M

NorESM1_ME

Figure 9.19 | Zonal-mean zonal wind stress over the oceans in (a) CMIP5 models and (b) multi-model mean comparison with CMIP3. Shown is the time-mean of the period 1970-1999 from the historical simulations. The black solid, dashed, and dotted curves represent ECMWF reanalysis of the global atmosphere and surface conditions (ERA)-Interim (Dee et al., 2011), National Centers for Environmental Prediction/National Center for Atmospheric Research (NCEP/NCAR) reanalysis I (Kalnay et al., 1996), and QuikSCAT satellite measurements (Risien and Chelton, 2008), respectively. In (b) the shading indicates the inter-model standard deviation. 
too strong in models. This equatorward shift in the southern ocean is slightly reduced in CMIP5 relative to CMIP3. At these latitudes, the largest near surface wind speed biases in CMIP5 are located over the Pacific sector and the smallest are in the Atlantic sector (Bracegirdle et al., 2013). Such wind stress errors may adversely affect oceanic heat and carbon uptake (Swart and Fyfe, 2012a). At middle to low latitudes, the CMIP3 and CMIP5 model spreads are smaller than at high latitudes, although near the equator this can occur through compensating errors (Figure 9.20). The simulated multi-model mean equatorial zonal wind stress is too weak in the Atlantic and Indian Oceans and too strong in the western Pacific, with no major improvement from CMIP3 to CMIP5.

The CMIP5 model simulations qualitatively agree with the various observational estimates on the most important features of ocean heat transport (Figure 9.21) and, in a multi-model sense, no major change from CMIP3 can be seen. All CMIP5 models are able to the represent the strong north-south asymmetry, with the largest values in the $\mathrm{NH}$, consistent with the observational estimates. At most latitudes the majority of CMIP5 model results fall within the range of observational estimates,

(a) CMIP5

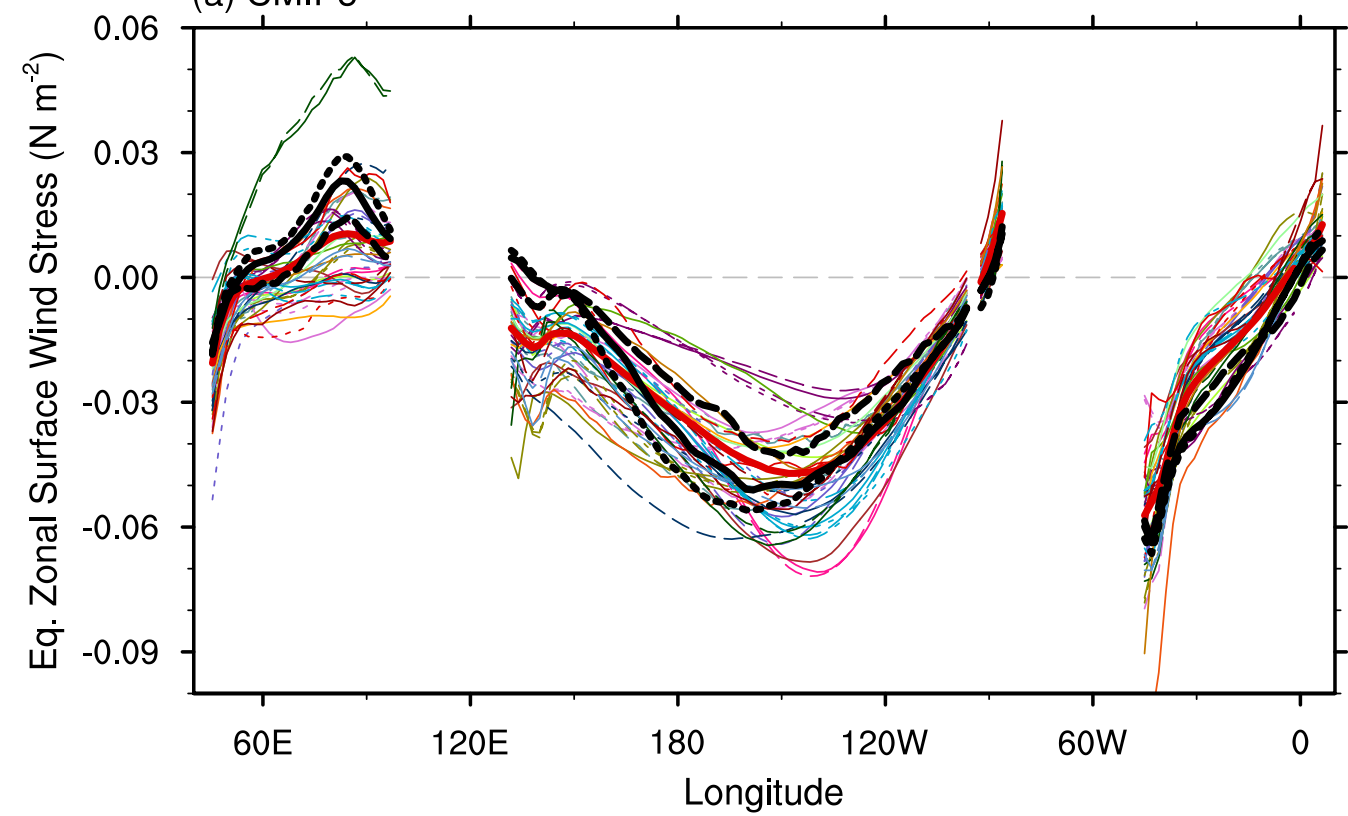

\begin{tabular}{l} 
ERA-Interim \\
\hdashline$\ldots$ NCEP-I \\
$\ldots-$ QuikSCAT
\end{tabular}

(b) CMIP5 and CMIP3

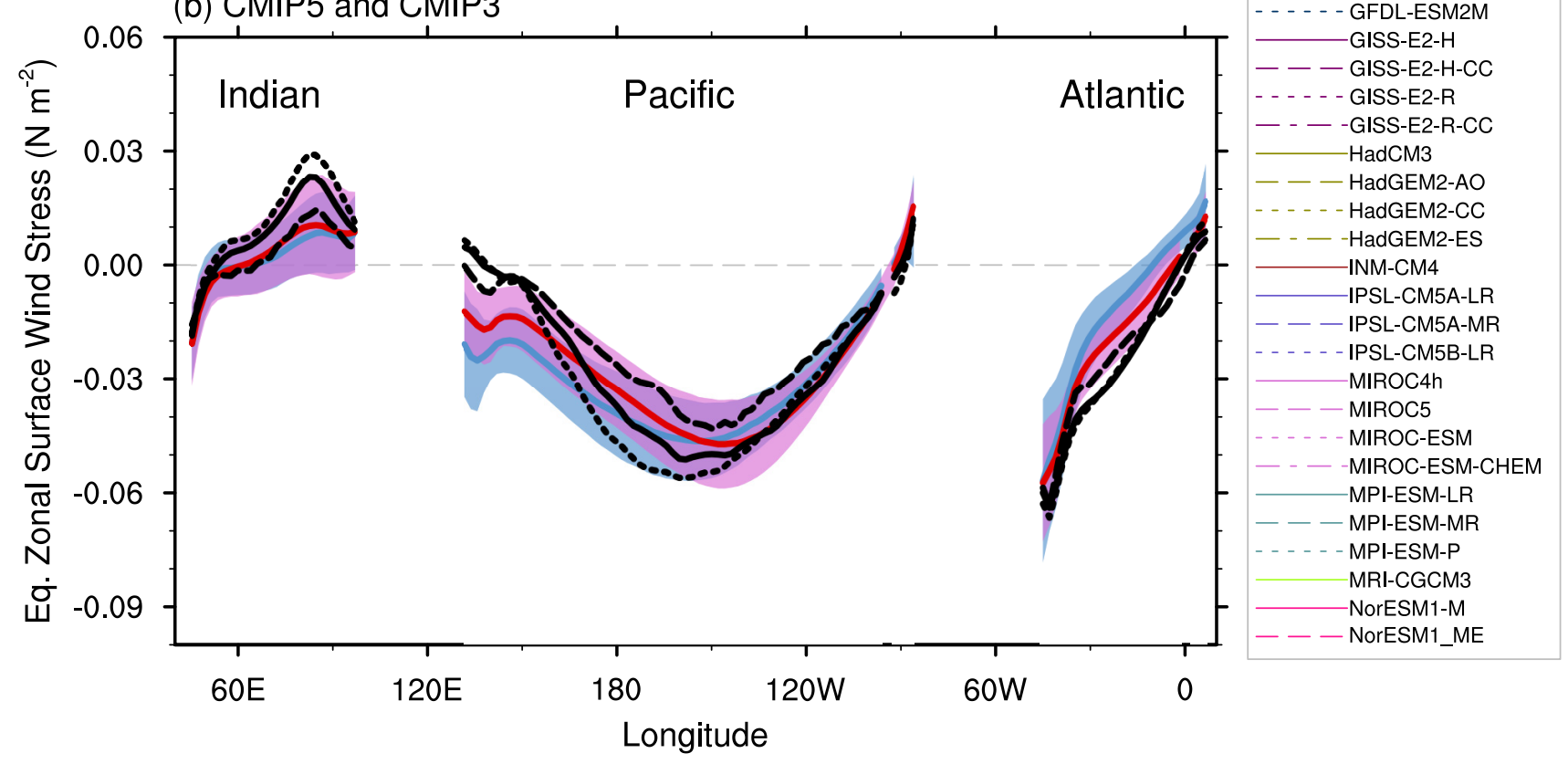

Figure 9.20 | Equatorial $\left(2^{\circ} \mathrm{S}\right.$ to $2^{\circ} \mathrm{N}$ averaged) zonal wind stress for the Indian, Pacific, and Atlantic oceans in (a) CMIP5 models and (b) multi-model mean comparison with CMIP3. Shown is the time-mean of the period 1970-1999 from the historical simulations. The black solid, dashed, and dotted curves represent ERA-Interim (Dee et al., 2011), National Centers for Environmental Prediction/National Center for Atmospheric Research (NCEP/NCAR) reanalysis I (Kalnay et al., 1996) and QuikSCAT satellite measurements (Risien and Chelton, 2008), respectively. In (b) the shading indicates the inter-model standard deviation. 


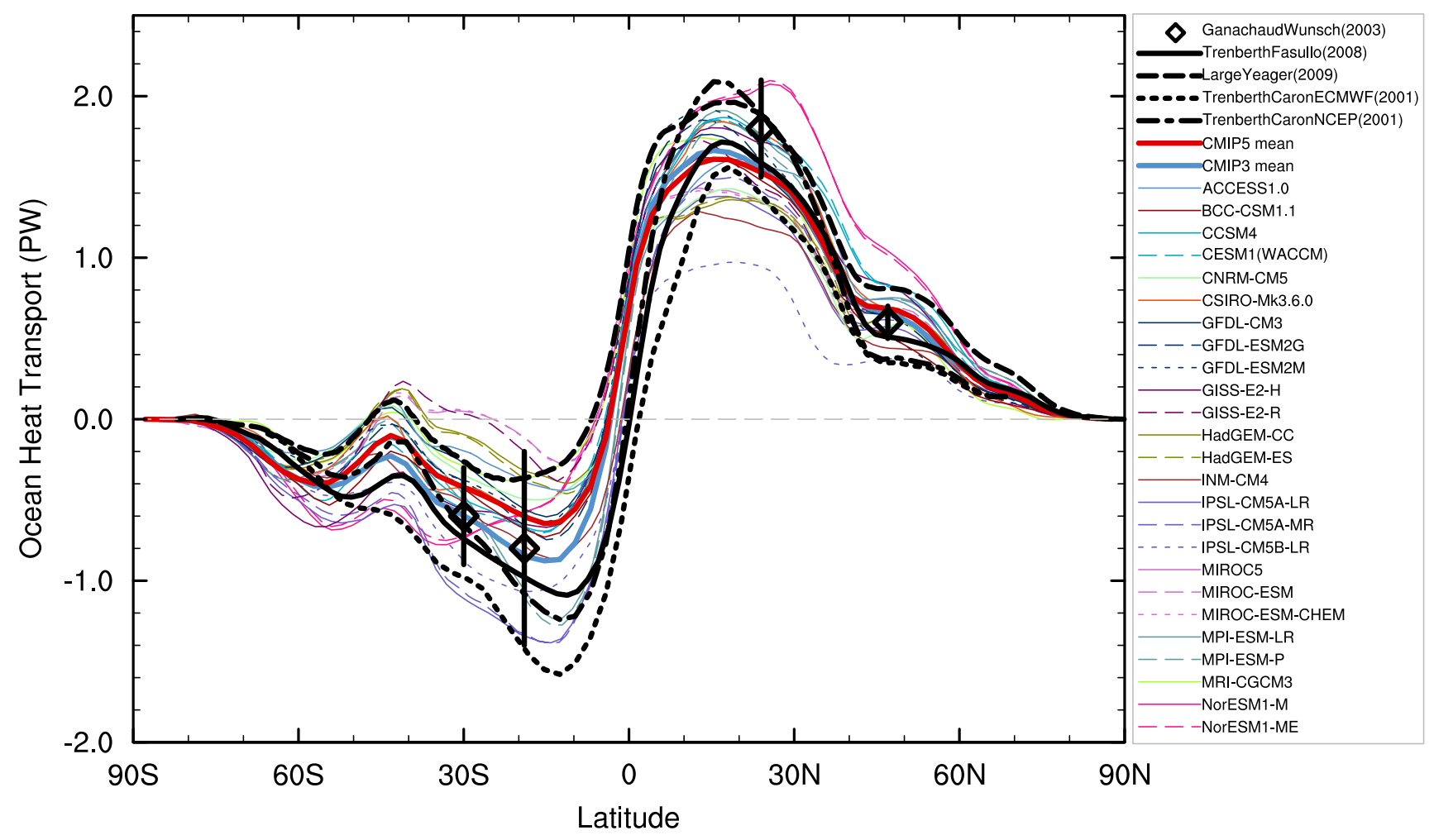

Figure 9.21 | Annual- and zonal-mean oceanic heat transport implied by net heat flux imbalances at the sea surface for CMIP5 simulations, under an assumption of negligible changes in oceanic heat content. Observational estimates include: the data set from Trenberth and Caron (2001) for the period February 1985 to April 1989, derived from reanalysis products from the National Centers for Environmental Prediction/National Center for Atmospheric Research (NCEP/NCAR; Kalnay et al., 1996; dash-dotted black) and European Centre for Medium Range Weather Forecasts 40-year reanalysis (ERA40; Uppala et al., 2005; short-dashed black), an updated version by Trenberth and Fasullo (2008) with improved top of the atmosphere (TOA) radiation data from the Clouds and Earth's Radiant Energy System (CERES) for March 2000 to May 2004, and updated NCEP reanalysis (Kistler et al., 2001) up to 2006 (solid black), the Large and Yeager (2009) analysis based on the range of annual mean transport estimated over the years 1984-2006, computed from air-sea surface fluxes adjusted to agree in the mean with a variety of satellite and in situ measurements (long-dashed black), and direct estimates by Ganachaud and Wunsch (2003) obtained from hydrographic sections during the World Ocean Circulation Experiment combined with inverse models (black diamonds). The model climatologies are derived from the years 1986 to 2005 in the historical simulations in CMIP5. The multi-model mean is shown as a thick red line. The CMIP3 multi-model mean is added as a thick blue line.

although there is some suggestion of modest underestimate between $15^{\circ} \mathrm{N}$ and $25^{\circ} \mathrm{N}$ and south of about $60^{\circ} \mathrm{S}$. Some models show an equatorward transport at Southern-Hemisphere mid-latitudes that is also featured in the observation estimate of Large and Yeager (2009). This highlights the difficulties in representing large-scale energy processes in the Southern ocean as discussed by Trenberth and Fasullo (2010b). Note that climate models should exhibit a vanishing net energy balance when long time averages are considered but unphysical sources and sinks lead to energy biases (Trenberth and Fasullo, 2009, 2010a; Lucarini and Ragone, 2011) that are also found in reanalysis constrained by observations (Trenberth et al., 2009). When correcting for the imperfect closure of the energy cycle, as done here, comparison between models and observational estimates become possible.

\subsubsection{Simulation of Tropical Mean State}

\subsection{Tropical Pacific Ocean}

Although the basic east-west structure of the tropical Pacific is well captured, models have shown persistent biases in important properties of the mean state (AchutaRao and Sperber, 2002; Randall et al., 2007; Guilyardi et al., 2009b) with severe local impacts (Brown et al., 2012). Among these biases are the mean thermocline depth and slope along the equator, the structure of the equatorial current system, and the excessive equatorial cold tongue (Reichler and Kim, 2008; Brown et al., 2010a; Zheng et al., 2012). Many reasons for these biases have been proposed, such as: too strong trade winds; a too diffusive thermocline; deficient horizontally isotropic mixing coefficients; insufficient penetration of solar radiation; and too weak tropical instability waves (Meehl et al., 2001; Wittenberg et al., 2006; Lin, 2007). It is noteworthy that CMIP5 models exhibit some improvements in the western equatorial Pacific when compared to CMIP3, with reduced SST and trade wind errors (Figures 9.14 and 9.20). Because of strong interactions between the processes involved, it is difficult to identify the ultimate source of these errors, although new approaches using the rapid adjustment of initialized simulations hold promise (Vannière et al., 2011).

A particular problem in simulating the seasonal cycle in the tropical Pacific arises from the 'double ITCZ', defined as the appearance of a spurious ITCZ in the SH associated with excessive tropical precipitation. Further problems are too strong a seasonal cycle in simulated SST and winds in the eastern Pacific and the appearance of a spurious semi-annual cycle. The latter has been attributed to meridional asymmetry in the background state that is too weak, possibly in conjunction with incorrect regional water vapour feedbacks (Li and Philander, 1996; Guilyardi, 2006; Timmermann et al., 2007; De Szoeke and Xie, 2008; Wu et al., 2008a; Hirota et al., 2011). 
A further persistent problem is insufficient marine stratocumulus cloud in the eastern tropical Pacific, caused presumably by weak coastal upwelling off South America leading to a warm SST bias (Lin, 2007). Although the problem persists, improvements are being made (AchutaRao and Sperber, 2006).

\subsection{Tropical Atlantic Ocean}

CMIP3 and CMIP5 models exhibit severe biases in the tropical Atlantic Ocean, so severe that some of the most fundamental features-the east-west SST gradient and the eastward shoaling thermocline along the equator-cannot be reproduced (Figure 9.14; (Chang et al., 2007; Chang et al., 2008; Richter and Xie, 2008; Richter et al., 2013). In many models, the warm SST bias along the Benguela coast is in excess of $5^{\circ} \mathrm{C}$ and the Atlantic warm pool in the western basin is grossly underestimated (Liu et al., 2013a). As in the Pacific, CMIP3 models suffer the double ITCZ error in the Atlantic. Hypotheses for the complex Atlantic bias problem tend to draw on the fact that the Atlantic Ocean has a far smaller basin, and thus encourages a tighter and more complex landatmosphere-ocean interaction. A recent study using a high-resolution coupled model suggests that the warm eastern equatorial Atlantic SST bias is more sensitive to the local rather than basin-wide trade wind bias and to a wet Congo basin instead of a dry Amazon-a finding that differs from previous studies (Patricola et al., 2012). Recent ocean model studies show that a warm subsurface temperature bias in the eastern equatorial Atlantic is common to virtually all ocean models forced with 'best estimated' surface momentum and heat fluxes, owing to problems in parameterization of vertical mixing (Hazeleger and Haarsma, 2005). Toniazzo and Woolnough (2013) show that among a variety of causes for the initial bias development, ocean-atmosphere coupling is key for their maintenance.

\subsection{Tropical Indian Ocean}

CMIP3 and CMIP5 models simulate equatorial Indian Ocean climate reasonably well (e.g., Figure 9.14), though most models produce weak westerly winds and a flat thermocline on the equator. The models show a large spread in the modelled depth of the $20^{\circ} \mathrm{C}$ isotherm in the eastern equatorial Indian Ocean (Saji et al., 2006). The reasons are unclear but may be related to differences in the various parameterizations of vertical mixing as well as the wind structure (Schott et al., 2009).

CMIP3 models generally simulate the Seychelles Chagos thermocline ridge in the Southwest Indian Ocean, a feature important for the Indian monsoon and tropical cyclone activity in this basin (Xie et al., 2002). The models, however, have significant problems in accurately representing its seasonal cycle because of the difficulty in capturing the asymmetric nature of the monsoonal winds over the basin, resulting in too weak a semi-annual harmonic in the local Ekman pumping over the ridge region compared to observations (Yokoi et al., 2009b). In about half of the models, the thermocline ridge is displaced eastward associated with the easterly wind biases on the equator (Nagura et al., 2013).

\subsubsection{Summary}

There is high confidence that the CMIP3 and CMIP5 models simulate the main physical and dynamical processes at play during transient ocean heat uptake, sea level rise, and coupled modes of variability. There is little evidence that CMIP5 models differ significantly from CMIP3, although there is some evidence of modest improvement. Many improvements are seen in individual CMIP5 ocean components (some now including interactive ocean biogeochemistry) and the number of relatively poor-performing models has been reduced (thereby reducing inter-model spread). New since the AR4, process-based model evaluation is now helping identify the cause of some specific biases, helping to overcome the limits set by the short observational records available.

\subsubsection{Sea Ice}

Evaluation of sea ice performance requires accurate information on ice concentration, thickness, velocity, salinity, snow cover and other factors. The most reliably measured characteristic of sea ice remains sea ice extent (usually understood as the area covered by ice with a concentration above 15\%). Caveats, however, exist related to the uneven reliability of different sources of sea ice extent estimates (e.g., satellite vs. pre-satellite observations; see Chapter 4), as well as to limitations of this characteristic as a metric of model performance (Notz et al., 2013).

(a) Arctic sea ice extent (1980-1999)

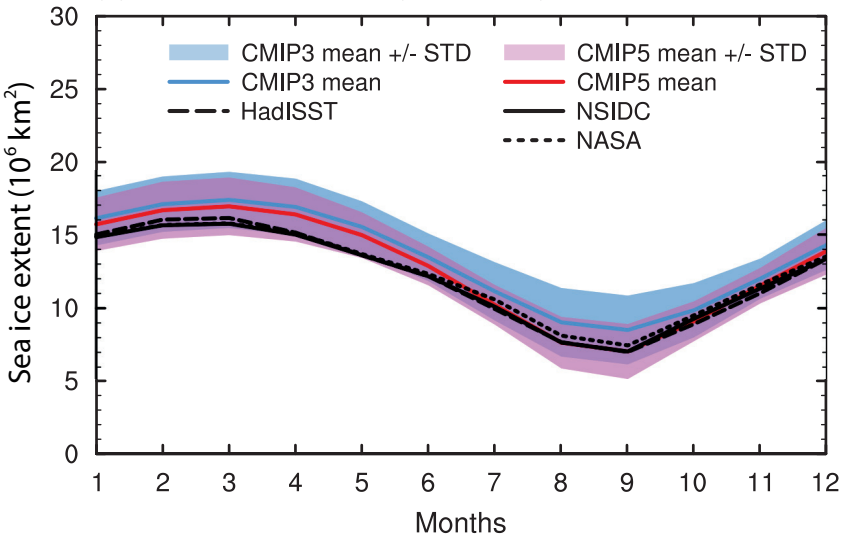

(b) Antarctic sea ice extent (1980-1999)

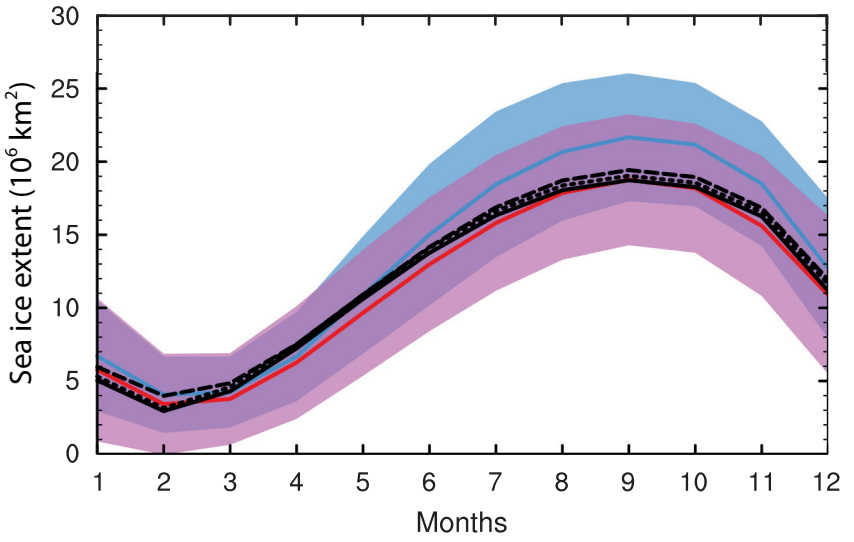

Figure 9.22 | Mean (1980-1999) seasonal cycle of sea ice extent (the ocean area with a sea ice concentration of at least 15\%) in the Northern Hemisphere (upper) and the Southern Hemisphere (lower) as simulated by 42 CMIP5 and 17 CMIP3 models. Each model is represented with a single simulation. The observed seasonal cycles (19801999) are based on the Hadley Centre Sea Ice and Sea Surface Temperature (HadISST; Rayner et al., 2003), National Aeronautics and Space Administration (NASA; Comiso and Nishio, 2008) and the National Snow and Ice Data Center (NSIDC; Fetterer et al., 2002) data sets. The shaded areas show the inter-model standard deviation for each ensemble. (Adapted from Pavlova et al., 2011.) 


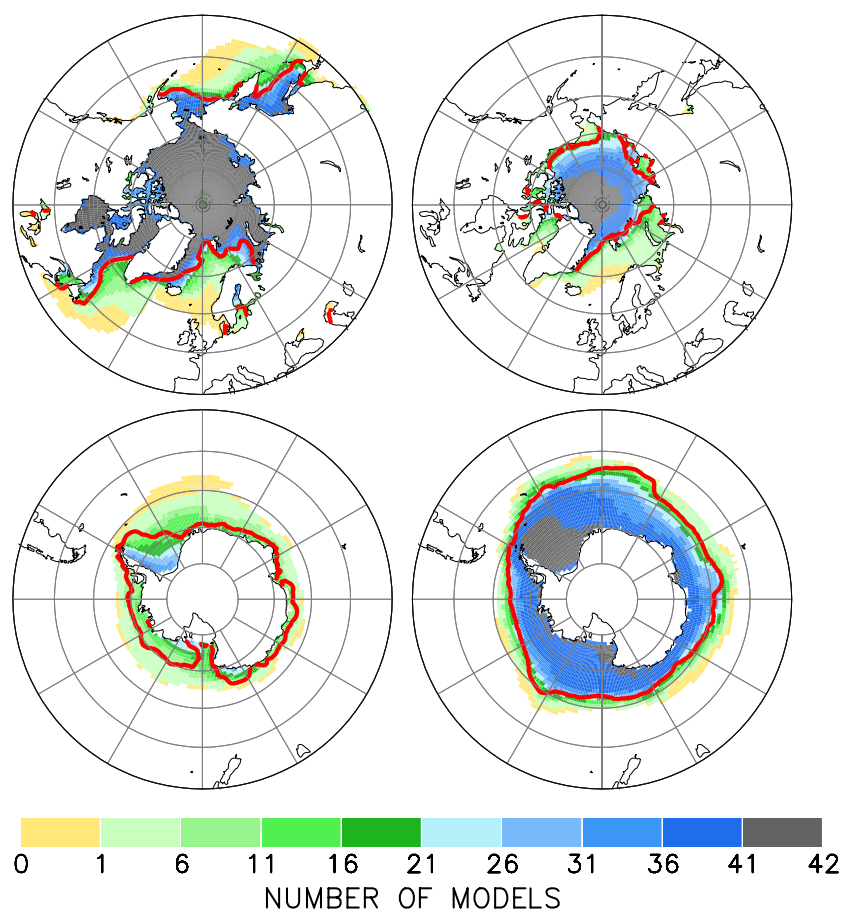

Figure 9.23 | Sea ice distribution (1986-2005) in the Northern Hemisphere (upper panels) and the Southern Hemisphere (lower panels) for February (left) and September (right). AR5 baseline climate (1986-2005) simulated by 42 CMIP5 AOGCMs. Each model is represented with a single simulation. For each $1^{\circ} \times 1^{\circ}$ longitude-latitude gric cell, the figure indicates the number of models that simulate at least $15 \%$ of the area covered by sea ice. The observed $15 \%$ concentration boundaries (red line) are based on the Hadley Centre Sea Ice and Sea Surface Temperature (HadISST) data set (Rayner et al., 2003). (Adapted from Pavlova et al., 2011.)

The CMIP5 multi-model ensemble exhibits improvements over CMIP3 in simulation of sea ice extent in the both hemispheres (Figure 9.22). In the Arctic, the multi-model mean error do not exceed $10 \%$ of the observationally based estimates for any given month. In the Antarctic, the corresponding multi-model mean error exceeds $10 \%$ (but is less than 20\%) near the annual minimum of sea ice extent; around the annual maximum, the CMIP5 multi-model mean shows a clear improvement over CMIP3.

In many models the regional distribution of sea ice concentration is poorly simulated, even if the hemispheric extent is approximately correct. In Figure 9.23, however, one can see that the median ice edge position (indicated by the colour at which half of the models have ice of $15 \%$ concentration) agrees reasonably well with observations in both hemispheres (except austral summer in Antarctica), as was the case for the CMIP3 models.

A widely discussed feature of the CMIP3 models as a group is a pronounced underestimation of the trend in the September (annual minimum) sea ice extent in the Arctic over the past several decades (e.g., Stroeve et al., 2007; Zhang, 2010; Rampal et al., 2011; Winton, 2011). Possible reasons for the discrepancy include variability inherent to high latitudes, model shortcomings, and observational uncertainties (e.g., Kattsov et al., 2010; Kay et al., 2011; Day et al., 2012). Compared to CMIP3, the CMIP5 models better simulate the observed trend of September Arctic ice extent (Figure 9.24). It has been suggested (Stroeve et al., 2012) that in some cases model improvements, such as new sea ice albedo parameterization schemes (e.g., Pedersen et al., 2009; Holland et al., 2012), have been responsible. (Holland et al., 2010) show that models with initially thicker ice generally retain more extensive ice throughout the 21st century, and indeed several of the CMIP5 models start the 20th century with rather thin winter ice cover promoting more rapid melt (Stroeve et al., 2012). Notz et al. (2013) caution, however, against direct comparison of modelled trends with observations unless the models' internal variability is carefully taken into account. Their analysis of the MPI-ESM ensemble shows that internal variability in the Arctic can result in individual model realizations exhibiting a range of trends (negative, or even positive) for the 29-year-long period starting in 1979, even if the background climate is warming. According to the distribution of sea ice extent trends over the period 1979-2010 obtained in an ensemble of simulations with individual CMIP5 models (Figure 9.24) about one quarter of the simulations shows a September trend in the Arctic as strong as, or stronger, than in observations.

The majority of CMIP5 (and CMIP3) models exhibit a decreasing trend in SH austral summer sea ice extent over the satellite era, in contrast to the observed weak but significant increase (see Chapter 4). A large spread in the modelled trends is present, and a comparison of multiple ensemble members from the same model suggests large internal variability during the late 20th century and the first decade of the 21st century (e.g., Landrum et al., 2012; Zunz et al., 2013). Compared to observations, CMIP5 models strongly overestimate the variability of sea ice extent, at least in austral winter (Zunz et al., 2013).Therefore, using the models to assess the potential role of the internal variability in the trend of sea ice extent in the Southern Ocean over the last three decades presents a significant challenge.

Sea ice is a product of atmosphere-ocean interaction. There are a number of ways in which sea ice is influenced by and interacts with the atmosphere and ocean, and some of these feedbacks are still poorly quantified. As noted in the AR4, among the primary causes of biases in simulated sea ice extent, especially its geographical distribution, are problems with simulating high-latitude winds, ocean heat advection and mixing. For example, Koldunov et al. (2010) have shown, for a particular CMIP3 model, that significant ice thickness errors originate from biases in the atmospheric component. Similarly, Melsom et al. (2009) note sea ice improvements associated with improved description of heat transport by ocean currents. Biases imparted on modelled sea ice, common to many models, may also be related to representation of high-latitude processes (e.g., polar clouds) or processes not yet commonly included in models (e.g., deposition of carbonaceous aerosols on snow and ice). Some CMIP5 models show improvements in simulation of sea ice that are connected to improvements in simulation of the atmosphere (e.g., Notz et al., 2013).

\subsubsection{Summary}

CMIP5 models reproduce the seasonal cycle of sea ice extent in both hemispheres. There is robust evidence that the downward trend in Arctic summer sea ice extent is better simulated than at the time of the AR4, with about one quarter of the simulations showing a trend as strong as, or stronger than, that observed over the satellite era. The performance improvements are not only a result of improvements in 
(a) Arctic sea ice extent in September (1900-2012)
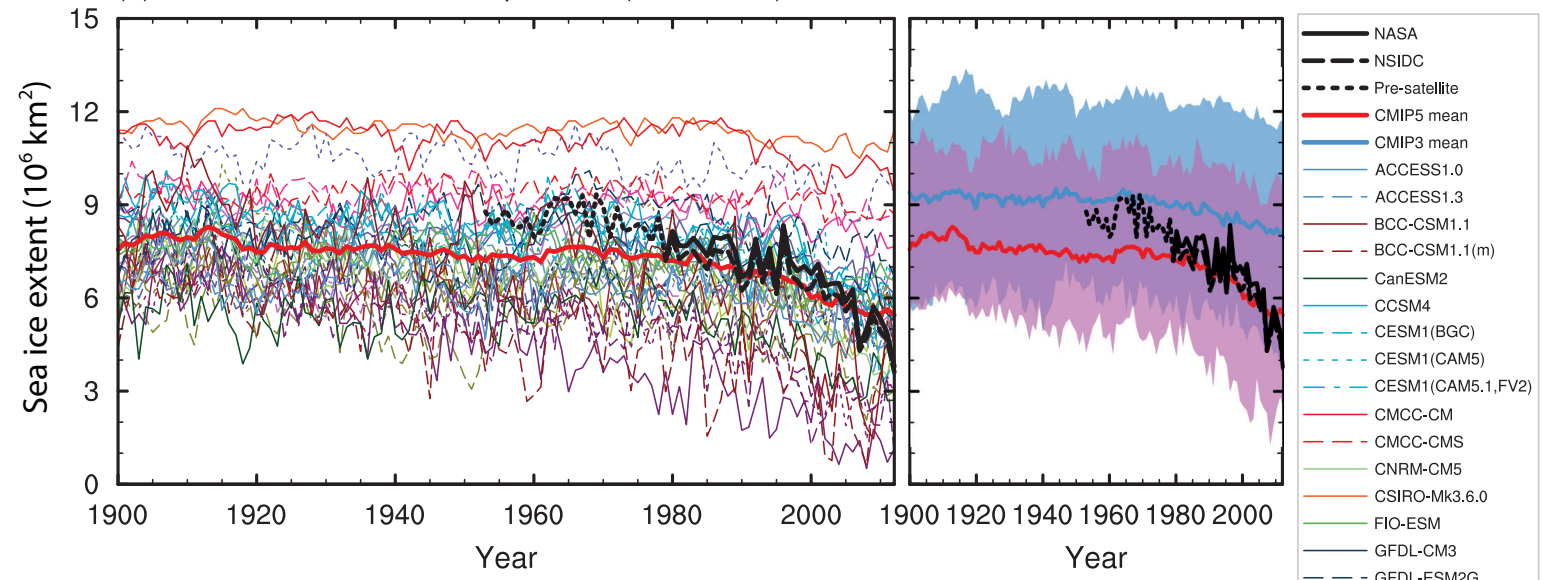

(b) Antarctic sea ice extent in February (1900-2012)
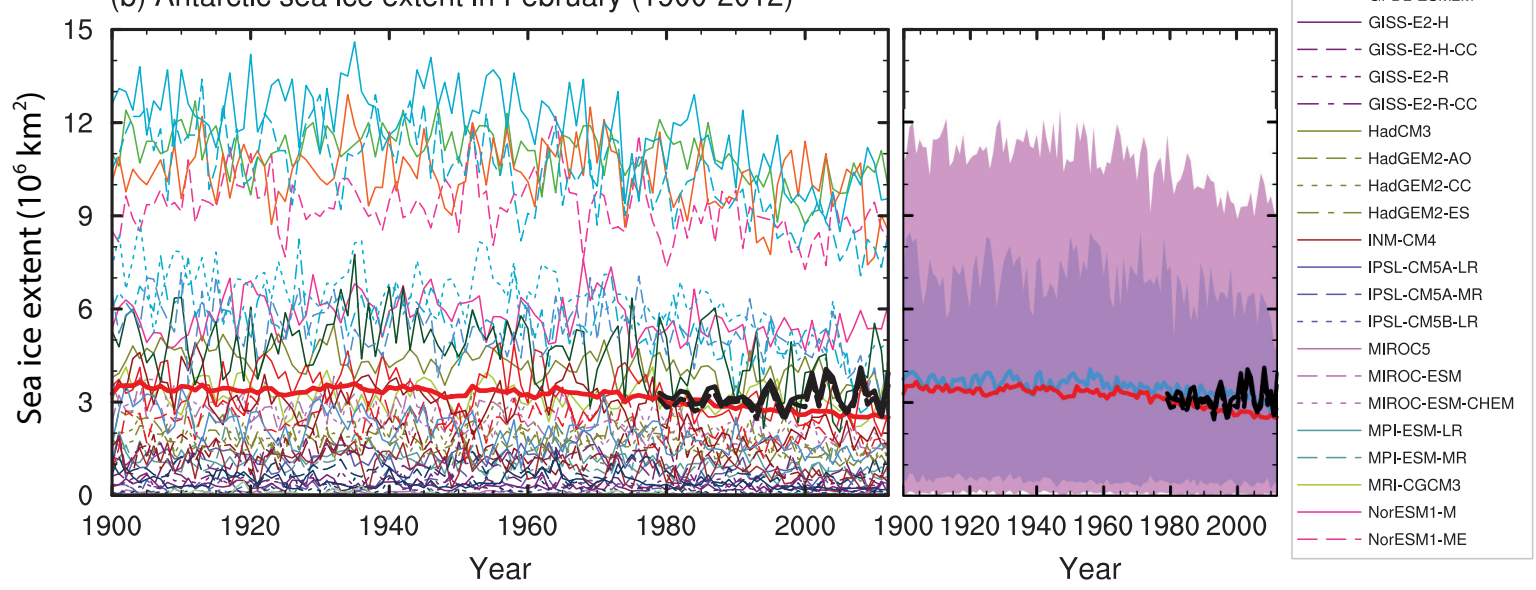

(c) CMIP5 Arctic ice extent September trends (1979- 2010)

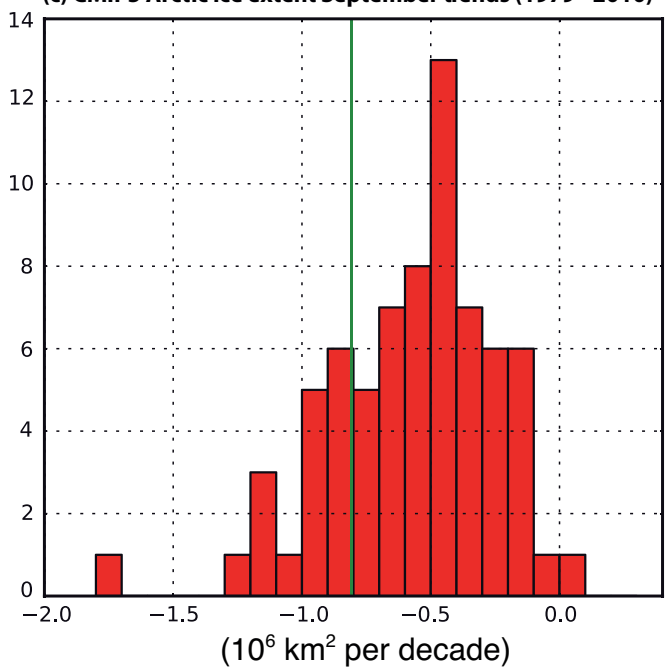

(d) CMIP5 Antarctic ice extent February trends (1979 - 2010)

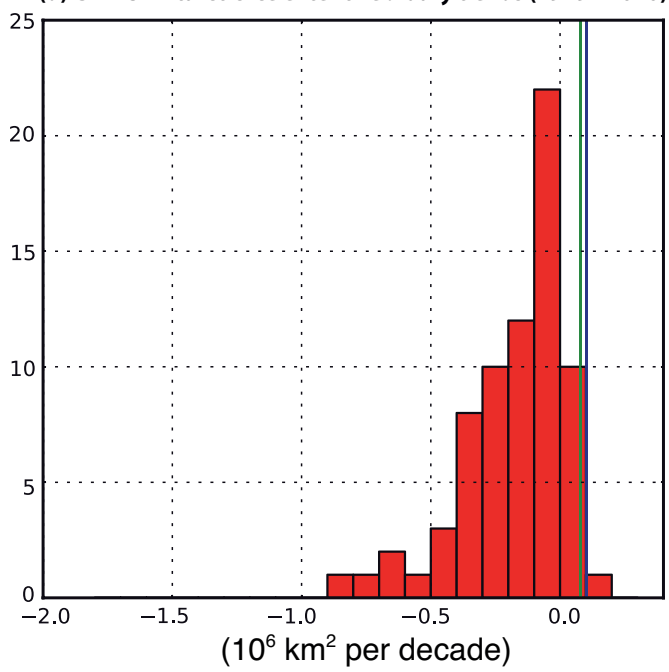

Figure 9.24 | (Top and middle rows) Time series of sea ice extent from 1900 to 2012 for (a) the Arctic in September and (b) the Antarctic in February, as modelled in CMIP5 (coloured lines) and observations-based (NASA; Comiso and Nishio, 2008) and NSIDC; (Fetterer et al., 2002), solid and dashed thick black lines, respectively). The CMIP5 multimodel ensemble mean (thick red line) is based on 37 CMIP5 models (historical simulations extended after 2005 with RCP4.5 projections). Each model is represented with a single simulation. The dotted black line for the Arctic in (a) relates to the pre-satellite period of observation-based time series (Stroeve et al., 2012). In (a) and (b) the panels on the right are based on the corresponding 37-member ensemble means from CMIP5 (thick red lines) and 12-model ensemble means from CMIP3 (thick blue lines). The CMIP3 12-model means are based on CMIP3 historical simulations extended after 1999 with Special Report on Emission Scenarios (SRES) A2 projections. The pink and light blue shadings denote the 5 to 95 percentile range for the corresponding ensembles. Note that these are monthly means, not yearly minima. (Adapted from Pavlova et al., 2011.) (Bottom row) CMIP5 sea ice extent trend distributions over the period 1979-2010 for (c) the Arctic in September and (d) the Antarctic in February. Altogether 66 realizations are shown from 26 different models (historical simulations extended after 2005 with RCP4.5 projections). They are compared against the observations-based estimates of the trends (green vertical lines in (c) and (d) from Comiso and Nishio (2008); blue vertical line in (d) from Parkinson and Cavalieri (2012)). In (c), the observations-based estimates (Cavalieri and Parkinson, 2012; Comiso and Nishio, 2008) coincide. 
sea ice components themselves but also in atmospheric circulation. Most CMIP5 models simulate a decrease in Antarctic sea ice extent over the past few decades compared to the small but significant increase observed.

\subsubsection{Land Surface, Fluxes and Hydrology}

The land surface determines the partitioning of precipitation into evapotranspiration and runoff, and the partitioning of surface net radiation into sensible and latent heat fluxes. Land surface processes therefore impact strongly on both the climate and hydrological resources. This subsection summarizes recent studies on the evaluation of land surface models, wherever possible emphasizing their performance in CMIP3 and CMIP5 climate models.

\subsubsection{Snow Cover and Near-Surface Permafrost}

The modelling of snow and near-surface permafrost (NSP) processes has received increased attention since the AR4, in part because of the recognition that these processes can provide significant feedbacks on climate change (e.g., Koven et al., 2011; Lawrence et al., 2011). The SnowMIP2 project compared results from 33 snowpack models of varying complexity, including some snow models that are used in AOGCMs, using driving data from five NH locations (Rutter et al., 2009). Most snow models were found to be consistent with observations at open sites, but there was much greater discrepancy at forested sites due to the complex interactions between plant canopy and snow cover. Despite these difficulties, the CMIP5 multi-model ensemble reproduces key features of the large-scale snow cover (Figure 9.25). In the $\mathrm{NH}_{\text {, }}$ models are able to simulate the seasonal cycle of snow cover over the northern parts of continents, with more disagreement in southerly regions where snow cover is sparse, particularly over China and Mongolia (Brutel-Vuilmet et al., 2013). The latter weaknesses are associated with incorrect timing of the snow onset and melt, and possibly with the choice of thresholds for diagnosing snow cover in the model output. In spite of the good performance of the multi-model mean, there is a significant inter-model scatter of spring snow cover extent in some regions. There is a strong linear correlation between Northern-Hemisphere spring snow cover extent and annual mean surface air temperature in the models, consistent with available observations. The recent negative trend in spring snow cover is underestimated by the CMIP5 (and CMIP3) models (Derksen and Brown, 2012), which is associated with an underestimate of the boreal land surface warming (Brutel-Vuilmet et al., 2013).

Some CMIP5 models now represent NSP and frozen soil processes (Koven et al., 2013), but this is not generally the case. Therefore it is difficult to make a direct quantitative evaluation of most CMIP5 models against permafrost observations. A less direct but more inclusive approach is to diagnose NSP extent using snow depths and skin temperatures generated by climate models to drive a stand-alone multi-layer permafrost model (Pavlova et al., 2007). Figure 9.25 shows the result of using this approach on the CMIP5 ensemble. The multi-model mean is able to simulate the approximate location of the NSP boundary (as indicated by the $0^{\circ} \mathrm{C}$ soil temperature isotherm). However, the range of present-day (1986-2005) NSP area inferred from individual models spans a factor of more than six $\left(\sim 4\right.$ to $\left.25 \times 10^{6} \mathrm{~km}^{2}\right)$ due

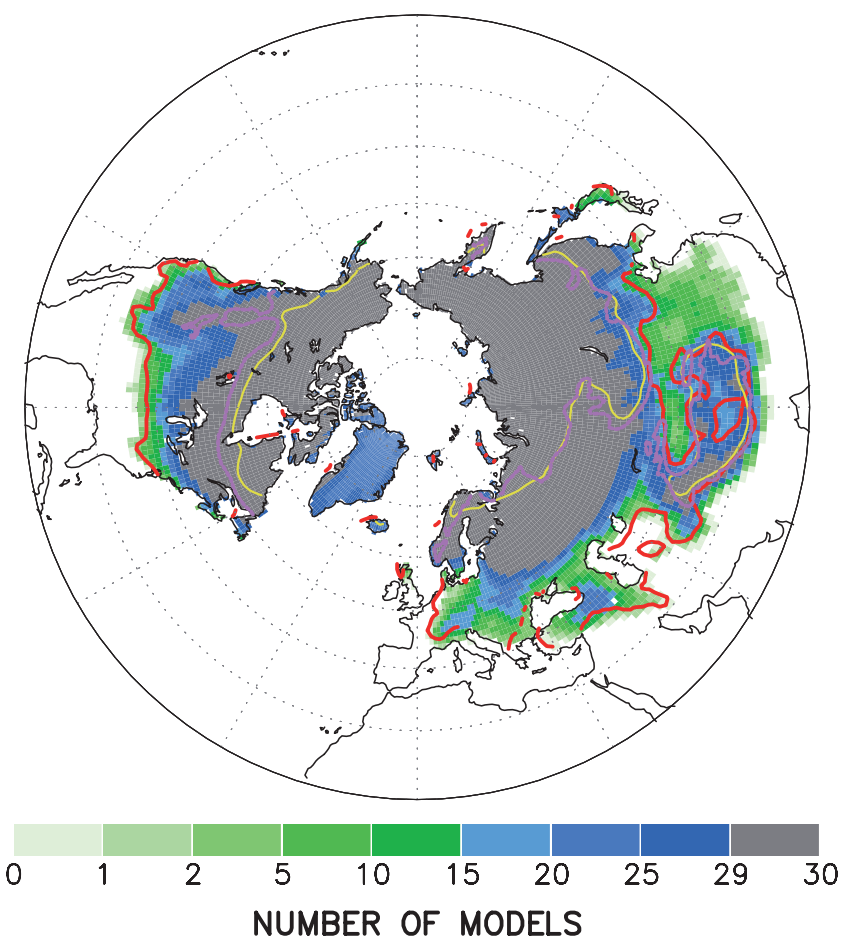

Figure 9.25 | Terrestrial snow cover distribution (1986-2005) in the Northern Hemisphere (NH) as simulated by 30 CMIP5 models for February, updated for CMIP5 from Pavlova et al. (2007). For each $1^{\circ} \times 1^{\circ}$ longitude-latitude grid cell, the figure indicates the number of models that simulate at least $5 \mathrm{~kg} \mathrm{~m}^{-2}$ of snow-water equivalent. The observations-based boundaries (red line) mark the territory with at least 20\% of the days per month with snow cover (Robinson and Frei, 2000) over the period 1986-2005. The annual mean $0^{\circ} \mathrm{C}$ isotherm at $3.3 \mathrm{~m}$ depth averaged across 24 CMIP5 models (yellow line) is a proxy for the near-surface permafrost boundary. Observed permafrost extent in the NH (magenta line) is based on Brown et al. $(1997,1998)$.

to differences in simulated surface climate and to varying abilities of the underlying land surface models. Even though many CMIP5 models include some representation of soil freezing in mineral soils, very few include key processes necessary to accurately model NSP changes, such as the distinct properties of organic soils, the existence of local water tables and the heat released by microbial respiration (Nicolsky et al., 2007; Wania et al., 2009; Koven et al., 2011, 2013).

Despite large differences in the absolute NSP area, the relationship between the decrease in NSP area and the warming air temperature over the present-day NSP region is similar, and approximately linear, in many models (Slater and Lawrence, 2013).

\subsubsection{Soil Moisture and Surface Hydrology}

The partitioning of precipitation into evapotranspiration and runoff is highly dependent on the moisture status of the land surface, especially the amount of soil moisture available for evapotranspiration, which in turn depends on properties of the land cover such as the rooting depth of plants.

There has been a long history of off-line evaluation of land surface schemes, aided more recently by the increasing availability of site-specific data (Friend et al., 2007; Blyth et al., 2010). Throughout this time, 
representations of the land surface have significantly increased in complexity, allowing the representation of key processes such as links between stomatal conductance and photosynthesis, but at the cost of increasing the number of poorly known internal model parameters. These more sophisticated land surface models are based on physical principles that should make them more appropriate for projections of future climate and increased $\mathrm{CO}_{2}$. However for specific data-rich sites, current land surface models still struggle to perform as well as statistical models in predicting year-to-year variations in latent and sensible heat fluxes (Abramowitz et al., 2008) and runoff (Materia et al., 2010).

There are few evaluations of the performance of land surface schemes in coupled climate models, but those that have been undertaken find major limitations associated with the atmospheric forcing rather than the land surface schemes themselves. For example, an evaluation of the soil moisture simulations of CMIP3 models found that long-term soil moisture trends could only be reproduced in models that simulated the reduction in solar radiation at the surface associated with 'global dimming' (Li et al., 2007). A comparison of simulated evapotranspiration fluxes from CMIP3 against large-scale observation-based estimates, showed underestimates in India and parts of eastern South America, and overestimates in the western USA, Australia and China (Mueller et al., 2011).

Land-atmosphere coupling determines the ability of climate models to simulate the influence of soil moisture anomalies on rainfall, droughts and high-temperature extremes (Fischer et al., 2007; Lorenz et al., 2012). The coupling strength depends both on the sensitivity of evapotranspiration to soil moisture, which is determined by the land surface scheme, and the sensitivity of precipitation to evapotranspiration, which is determined by the atmospheric model (Koster et al., 2004; Seneviratne et al., 2010). Comparison of climate model simulations to observations suggests that the models correctly represent the soil-moisture impacts on temperature extremes in southeastern Europe, but overestimate them in central Europe (Hirschi et al., 2011). The influence of soil moisture on rainfall varies significantly with region, and with the lead-time between a soil moisture anomaly and a rainfall event (Seneviratne et al., 2010). In some regions, such as the Sahel, enhanced precipitation can even be induced by dry anomalies (Taylor et al., 2011). Recent analyses of CMIP5 models reveals considerable spread in the ability of the models to reproduce observed correlations between precipitation and soil moisture in the tropics (Williams et al., 2012), and a systematic failure to simulate the positive impact of dry soil moisture anomalies on rainfall in the Sahel (Taylor et al., 2012a).

\subsubsection{Dynamic Global Vegetation and Nitrogen Cycling}

At the time of the AR4 very few climate models included dynamic vegetation, with vegetation being prescribed and fixed in all but a handful of coupled climate-carbon cycle models (Friedlingstein et al., 2006). Dynamic Global Vegetation Models (DGVMs) certainly existed at the time of the AR4 (Cramer et al., 2001) but these were not typically incorporated in climate models. Since the AR4 there has been continual development of offline DGVMs, and some climate models incorporate dynamic vegetation in at least a subset of the runs submitted to CMIP5 (also see Section 9.1.3.2.4), with likely consequences for climate model biases and regional climate projection (Martin and Levine, 2012).
DGVMs are designed to simulate the large-scale geographical distribution of plant functional types and how these patterns will change in response to climate change, $\mathrm{CO}_{2}$ increases, and other forcing factors (Cramer et al., 2001). These models typically include rather detailed representations of plant photosynthesis but less sophisticated treatments of soil carbon, with a varying number of soil carbon pools. In the absence of nitrogen limitations on $\mathrm{CO}_{2}$ fertilization, offline DGVMs agree qualitatively that $\mathrm{CO}_{2}$ increase alone will tend to enhance carbon uptake on the land while the associated climate change will tend to reduce it. There is also good agreement on the degree of $\mathrm{CO}_{2}$ fertilization in the case of no nutrient limitation (Sitch et al., 2008). However, under more extreme emissions scenarios the responses of the DGVMs diverge markedly. Large uncertainties are associated with the responses of tropical and boreal ecosystems to elevated temperatures and changing soil moisture status. Particular areas of uncertainty are the high-temperature response of photosynthesis (Galbraith et al., 2010), and the extent of $\mathrm{CO}_{2}$ fertilization (Rammig et al., 2010) in the Amazonian rainforest.

Most of the land surface models and DGVMs used in the CMIP5 models continue to neglect nutrient limitations on plant growth (see Section 6.4.6.2), even though these may significantly moderate the response of photosynthesis to $\mathrm{CO}_{2}$ (Wang and Houlton, 2009). Recent extensions of two land surface models to include nitrogen limitations improve the fit to 'Free-Air $\mathrm{CO}_{2}$ Enrichment Experiments', and suggest that models without these limitations are expected to overestimate the land carbon sink in the nitrogen-limited mid and high latitudes (Thornton et al., 2007; Zaehle et al., 2010).

\subsubsection{Land Use Change}

A major innovation in the land component of ESMs since the AR4 is the inclusion of the effects of land use change associated with the spread of agriculture, urbanization and deforestation. These affect climate by altering the biophysical properties of the land surface, such as its albedo, aerodynamic roughness and water-holding capacity (Bondeau et al., 2007; Bonan, 2008; Bathiany et al., 2010; Levis, 2010). Land use change has also contributed almost $30 \%$ of total anthropogenic $\mathrm{CO}_{2}$ emissions since 1850 (see Table 6.1), and affects emissions of trace gases, and VOCs such as isoprene. The latest ESMs used in CMIP5 attempt to model the $\mathrm{CO}_{2}$ emissions implied by prescribed land use change and many also simulate the associated changes in the biophysical properties of the land surface. This represents a major advance on the CMIP3 models which typically neglected land use change, aside from its assumed contribution to anthropogenic $\mathrm{CO}_{2}$ emissions.

However, the increasing sophistication of the modelling of the impacts of land use change has introduced additional spread in climate model projections. The first systematic model intercomparison demonstrated that large-scale land cover change can significantly affect regional climate (Pitman et al., 2009) and showed a large spread in the response of different models to the same imposed land cover change (de Noblet-Ducoudre et al., 2012). This uncertainty arises from the often counteracting effects of evapotranspiration and albedo changes (Boisier et al., 2012) and has consequences for the simulation of temperature and rainfall extremes (Pitman et al., 2012b). 


\subsubsection{Carbon Cycle}

An important development since the AR4 is the more widespread implementation of ESMs that include an interactive carbon cycle. Coupled climate-carbon cycle models are used extensively for the projections presented in Chapter 12. The evaluation of the carbon cycle within coupled models is discussed here, while the performance of the individual land and ocean carbon models, together with the detailed analysis of climate-carbon cycle feedbacks, is presented in Chapter 6 (Section 6.4 and Box 6.4).

The transition from AOGCMs to ESMs was motivated in part by the results from the first generation coupled climate-carbon cycle models, which suggested that feedbacks between the climate and the carbon cycle were uncertain but potentially very important in the context of 21st century climate change (Cox et al., 2000; Friedlingstein et al., 2001). The first-generation models used in the Coupled Climate Carbon Cycle Model Intercomparison Project ( $\left.C^{4} \mathrm{MIP}\right)$ included both extended AOGCMS and EMICs. The C ${ }^{4}$ MIP experimental design involved running each model under a common emission scenario (SRES A2) and calculating the evolution of the global atmospheric $\mathrm{CO}_{2}$ concentration interactively within the model. The impacts of climate-carbon cycle feedbacks were diagnosed by carrying out parallel "uncoupled" simulations in which increases in atmospheric $\mathrm{CO}_{2}$ did not influence climate. Analysis of the C4MIP runs highlighted a greater than $200 \mathrm{ppmv}$ range in the $\mathrm{CO}_{2}$ concentration by 2100 due to uncertainties in climate-carbon cycle feedbacks, and that the largest uncertainties were associated with the response of land ecosystems to climate and $\mathrm{CO}_{2}$ (Friedlingstein et al., 2006).

For CMIP5 a different experimental design was proposed in which the core simulations use prescribed RCPs of atmospheric $\mathrm{CO}_{2}$ and other GHGs (Moss et al., 2010). Under a prescribed $\mathrm{CO}_{2}$ scenario, ESMs calculate land and ocean carbon fluxes interactively, but these fluxes do not affect the evolution of atmospheric $\mathrm{CO}_{2}$. Instead the modelled land and ocean fluxes, along with the prescribed increase in atmospheric $\mathrm{CO}_{2}$, can be used to diagnose the 'compatible' emissions of $\mathrm{CO}_{2}$ consistent with the simulation (see Section 6.3; Miyama and Kawamiya, 2009; Arora et al., 2011). The compatible emissions for each model can then be evaluated against the best estimates of the actual historical $\mathrm{CO}_{2}$ emissions. Parallel model experiments in which the carbon cycle does not respond to the simulated climate change (which are equivalent to the 'uncoupled' simulations in ( 4 MIP) provide a means to diagnose climate-carbon cycle feedbacks in terms of their impact on the compatible emissions of $\mathrm{CO}_{2}$ (Hibbard et al., 2007).

Carbon cycle model evaluation is limited by the availability of direct observations at appropriately large spatial scales. Field studies and eddy-covariance flux measurements provide detailed information on the land carbon cycle over short-time scales and for specific locations, and ocean inventories are able to constrain the long-term uptake of anthropogenic $\mathrm{CO}_{2}$ by the ocean (Sabine et al., 2004; Takahashi et al., 2009). However the stores of carbon on the land are less well-known, even though these are important determinants of the $\mathrm{CO}_{2}$ fluxes from land use change. ESM simulations vary by a factor of at least six in global soil carbon (Anav et al., 2013; Todd-Brown et al., 2013) and by a factor of four in global vegetation carbon, although about two thirds of models are within $50 \%$ of the uncertain observational estimates (Anav et al., 2013).

Large-scale land-atmosphere and global atmosphere fluxes are not directly measured, but global estimates can be made from the carbon balance, and large-scale regional fluxes can be estimated from the inversion of atmospheric $\mathrm{CO}_{2}$ measurements (see Section 6.3.2). Figure 9.26 shows modelled annual mean ocean-atmosphere and net landatmosphere $\mathrm{CO}_{2}$ fluxes from the historical simulations in the CMIP5 archive (Anav et al., 2013). Also shown are estimates derived from offline ocean carbon cycle models, measurements of atmospheric $\mathrm{CO}_{2}$ and best estimates of the $\mathrm{CO}_{2}$ fluxes from fossil fuels and land use change (Le Quere et al., 2009). Uncertainties in these latter annual estimates are approximately $\pm 0.5 \mathrm{PgC}^{-1}$, arising predominantly from the uncertainty in the model-derived ocean $\mathrm{CO}_{2}$ uptake. The confidence limits for the ensemble mean are derived by assuming that the CMIP5 models form a $t$-distribution centred on the ensemble mean (Anav et al., 2013).

The evolution of the global ocean carbon sink is shown in the top panel of Figure 9.26. The CMIP5 ensemble mean global ocean uptake ( \pm standard deviation of the multi-model ensemble), computed using all the 23 models that reported ocean $\mathrm{CO}_{2}$ fluxes, increases from 0.47 $\pm 0.32 \mathrm{PgC} \mathrm{yr}^{-1}$ over the period $1901-1930$ to $1.53 \pm 0.36 \mathrm{PgC} \mathrm{yr}^{-1}$ for the period 1960-2005. For comparison, the Global Carbon Project (GCP) estimates a stronger ocean carbon sink of $1.92 \pm 0.3 \mathrm{PgC} \mathrm{yr}^{-1}$ for 1960-2005 (Anav et al., 2013). The bottom panel of Figure 9.26 shows the variability in global land carbon uptake evident in the GCP estimates, with the global land carbon sink being strongest during La Niña years and after volcanoes, and turning into a source during El Niño years. The CMIP5 models cannot be expected to precisely reproduce this year-to-year variability as these models will naturally simulate chaotic ENSO variability that is out of phase with the historical variability. However, the ensemble mean does successfully simulate a strengthening global land carbon sink during the 1990s, especially after the Mt Pinatubo eruption in 1991. The CMIP5 ensemble mean land-atmosphere flux ( \pm standard deviation of the multi-model ensemble) evolves from a small source of $-0.34 \pm 0.49 \mathrm{PgC}^{-1}$ over the period $1901-$ 1930 , predominantly due to land use change, to a sink of $0.47 \pm 0.72$ $\mathrm{PgC} \mathrm{yr}^{-1}$ in the period 1960-2005. The GCP estimates give a weaker sink of $0.36 \pm 1 \mathrm{PgC} \mathrm{yr}^{-1}$ for the 1960-2005 period.

Figure 9.27 shows the ocean-atmosphere fluxes (top panel) and mean land-atmosphere fluxes (bottom panel) simulated by ESMs and EMICs (Eby et al., 2013) for the period 1986-2005, and compares these to observation-based estimates from GCP and Atmospheric Tracer Transport Model Intercomparison Project (TRANSCOM3) atmospheric inversions (Gurney et al., 2003). Unlike Figure 9.26, only models that reported both land and ocean carbon fluxes are included in this figure. The atmospheric inversions results are taken from the Japanese Meteorological Agency (JMA) as this was the only TRANSCOM3 model that reported results for all years of the 1986-2005 reference period. The error bars on the observational estimates (red triangles) and the ESM simulations (black diamonds) represent the interannual variation in the form of the standard deviation of the annual fluxes. EMICs do not typically simulate interannual variability, so only mean values are shown for these models (green boxes). Here, as in Figure 9.26, the net 

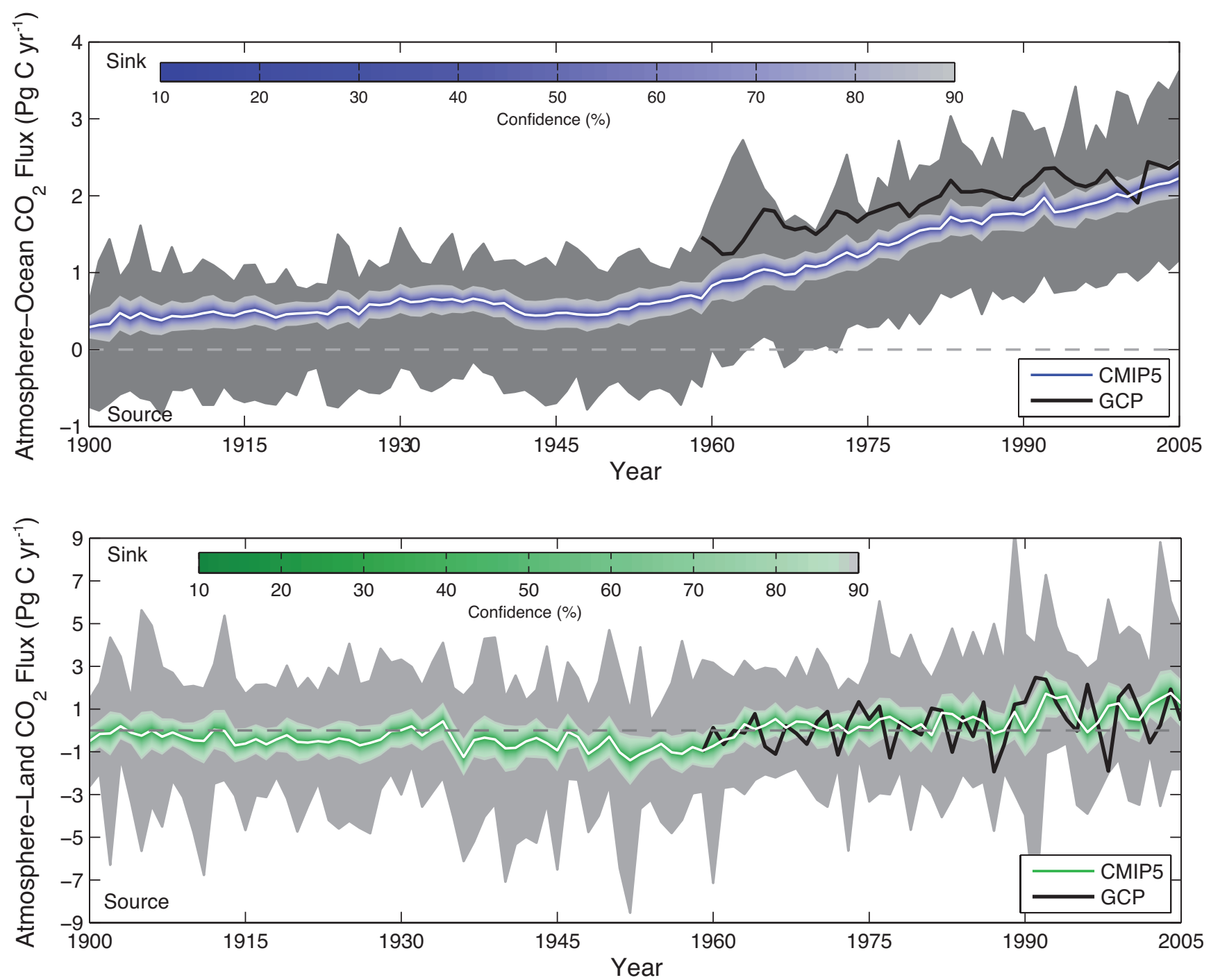

Figure 9.26| Ensemble-mean global ocean carbon uptake (top) and global land carbon uptake (bottom) in the CMIP5 ESMs for the historical period 1900-2005. For comparison, the observation-based estimates provided by the Global Carbon Project (Le Quere et al., 2009) are also shown (thick black line). The confidence limits on the ensemble mean are derived by assuming that the CMIP5 models come from a $t$-distribution. The grey areas show the range of annual mean fluxes simulated across the model ensemble. This figure includes results from all CMIP5 models that reported land $\mathrm{CO}_{2}$ fluxes, ocean $\mathrm{CO}_{2}$ fluxes, or both (Anav et al., 2013).

land-atmosphere flux is 'Net Biome Productivity (NBP)' which includes the net $\mathrm{CO}_{2}$ emissions from land use change as well as the changing carbon balance of undisturbed ecosystems.

For the period 1986-2005 the observation-based estimates of the global ocean carbon sink are 1.71 $\mathrm{PgC} \mathrm{yr}^{-1}$ (JMA), 2.19 $\mathrm{PgC} \mathrm{yr}^{-1}$ (GCP) and $2.33 \mathrm{PgC} \mathrm{yr}^{-1}$ (Takahashi et al., 2009). Taking into account the uncertainties in the mean values of these fluxes associated with interannual variability, the observationally constrained range is approximately 1.4 to $2.4 \mathrm{PgC} \mathrm{yr}^{-1}$. All of the ESMs, and all but one of the EMICs, simulate ocean sinks within this range. The observation-based estimates of GCP and JMA agree well on the mean global land carbon sink over the period 1986-2005, and most ESMs fit within the uncer-

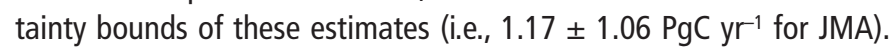
The exceptions are two ESMs sharing common atmosphere and land components (CESM1-BGC and NorESM1-ME) which model a net land carbon source rather than a sink over this period. The EMICs tend to systematically underestimate the contemporary land carbon sink (Eby et al., 2013). Some ESMs (notably GFDL-ESM2M and GFDL-ESM2G) significantly overestimate the interannual variation in the global landatmosphere $\mathrm{CO}_{2}$ flux, with a possible consequence being an overestimate of the vulnerability of tropical ecosystems to future climate change (Cox et al., 2013), and see Figure 9.45). All ESMs qualitatively simulate the expected pattern of ocean $\mathrm{CO}_{2}$ fluxes, with outgassing in the tropics and uptake in the mid and high latitudes (Anav et al., 2013). However, there are systematic differences between the ESMs and the JMA inversion estimates for the zonal land $\mathrm{CO}_{2}$ fluxes, with the ESMs tending to produce weaker uptake in the $\mathrm{NH}$, and simulating a net land carbon sink rather than a source in the tropics.

In summary, there is high confidence that CMIP5 ESMs can simulate the global mean land and ocean carbon sinks within the range of observation-based estimates. Overall, EMICs reproduce the recent global ocean $\mathrm{CO}_{2}$ fluxes uptake as well as ESMs, but estimate a lower 
a) Global Atmosphere-Ocean $\mathrm{CO}_{2}$ Flux

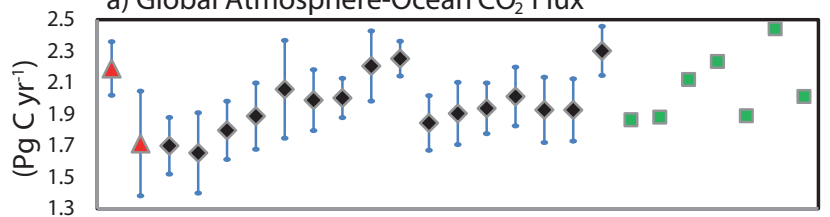

b) Global Atmosphere-Land $\mathrm{CO}_{2}$ Flux

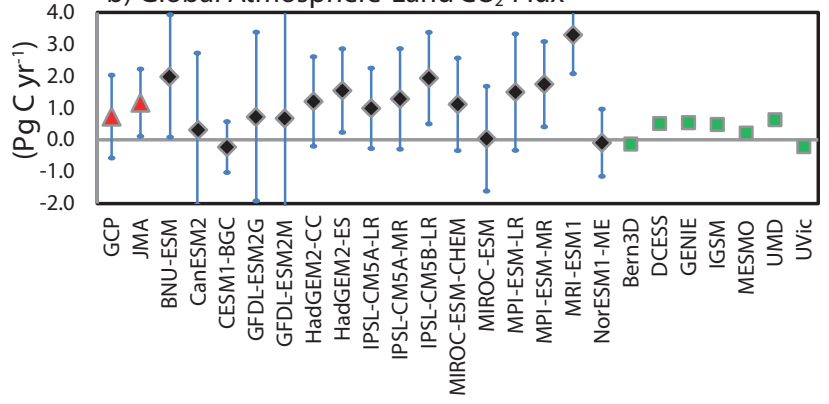

Figure 9.27 | Simulation of global mean (a) atmosphere-ocean $\mathrm{CO}_{2}$ fluxes ('fgCO2') and (b) net atmosphere-land $\mathrm{CO}_{2}$ fluxes ('NBP'), by ESMs (black diamonds) and EMICs (green boxes), for the period 1986-2005. For comparison, the observation-based estimates provided by Global Carbon Project (GCP; Le Quere et al., 2009), and the Japanese Meteorological Agency (JMA) atmospheric inversion (Gurney et al., 2003) are also shown as the red triangles. The error bars for the ESMs and observations represent interannual variability in the fluxes, calculated as the standard deviation of the annual means over the period 1986-2005.

land carbon sink compared with most ESMs while remaining consistent with the observations (Eby et al., 2013). With few exceptions, the CMIP5 ESMs also reproduce the large-scale pattern of ocean-atmosphere $\mathrm{CO}_{2}$ fluxes, with uptake in the Southern Ocean and northern mid-latitudes, and outgassing in the tropics. However, the geographical pattern of simulated land-atmosphere fluxes agrees much less well with inversion estimates, which suggest a larger sink in the northern mid-latitudes, and a net source rather than a sink in the tropics. While there are also inherent uncertainties in atmospheric inversions, discrepancies like this might be expected from known deficiencies in the CMIP5 generation of ESMs-namely the failure to correctly simulate nitrogen fertilization in the mid-latitudes, and a rudimentary treatment of the net $\mathrm{CO}_{2}$ emissions arising from land use change and forest regrowth.

\subsubsection{Aerosol Burdens and Effects on Insolation}

\subsubsection{Recent Trends in Global Aerosol Burdens and Effects on Insolation}

The ability of CMIP5 models to simulate the current burden of tropospheric aerosol and the decadal trends in this burden can be assessed using observations of aerosol optical depth (AOD, see Section 7.3.1.2). The historical data used to drive the CMIP5 20th century simulations reflect recent trends in anthropogenic $\mathrm{SO}_{2}$ emissions, and hence these trends should be manifested in the modelled and observed AOD. During the last three decades, anthropogenic emissions of $\mathrm{SO}_{2}$ from North America and Europe have declined due to the imposition of emission controls, while the emissions from Asia have increased. The combination of the European, North American, and Asians trends has yielded a global reduction in $\mathrm{SO}_{2}$ emissions of $20 \mathrm{Gg}\left(\mathrm{SO}_{2}\right)$, or $15 \%$ between 1970 and 2000 although emissions subsequently increased by $9 \mathrm{Gg}\left(\mathrm{SO}_{2}\right)$

between 2000 and 2005 (Smith et al., 2011b). For the period 2001 to 2005, CMIP5 models underestimate the mean AOD at $550 \mathrm{~nm}$ relative to satellite-retrieved AOD by at least $20 \%$ over virtually all land surfaces (Figure 9.28). The differences between the modelled and measured AODs exceed the errors in the Multi-angle Imaging Spectro-Radiometer (MISR) retrievals over land of \pm 0.05 or $0.2 \times A O D$ (Kahn et al., 2005) and the RMS errors in the corrected Moderate Resolution Imaging Spectrometer (MODIS) retrievals over ocean of 0.061 (Shi et al., 2011).

The effects of sulphate and other aerosol species on surface insolation through direct and indirect forcing appear to be one of the principal causes of the 'global dimming' between the 1960s and 1980s and subsequent 'global brightening' in the last two decades (see Section 2.3.3.1). This inference is supported by trends in aerosol optical depth and trends in surface insolation under cloud-free conditions. Thirteen out of 14 CMIP3 models examined by Ruckstuhl and Norris (2009) produce a transition from "dimming" to 'brightening' that is consistent with the timing of the transition from increasing to decreasing global anthropogenic aerosol emissions. The transition from 'dimming' to 'brightening' in both Europe and North America is well simulated with the HadGEM2 model (Haywood et al., 2011).

(a) Satellite AOD

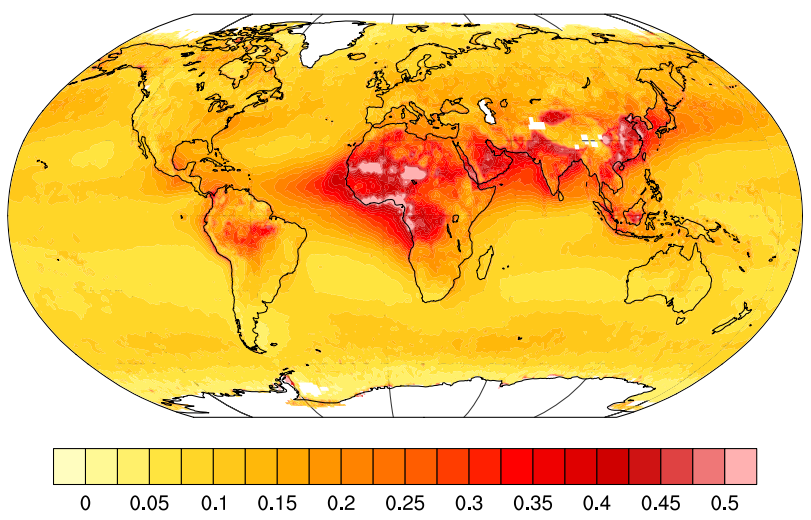

(b) Model minus satellite

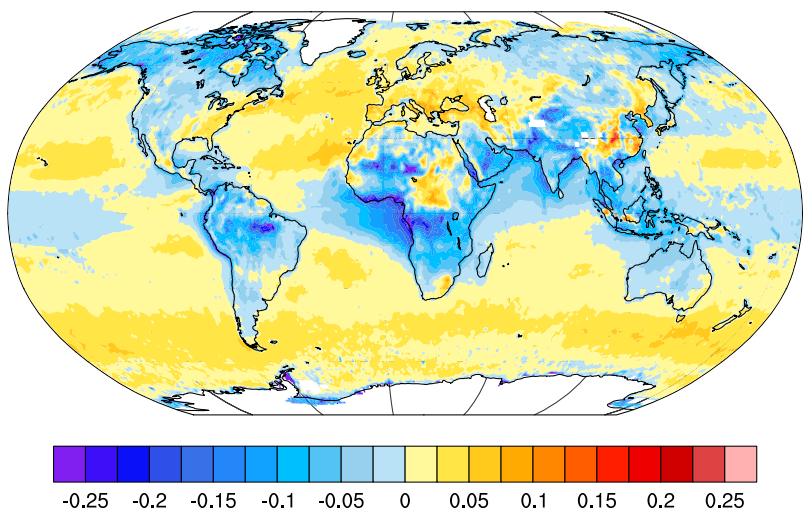

Figure 9.28 | (a): Annual mean visible aerosol optical depth (AOD) for 2001 through 2005 using the Moderate Resolution Imaging Spectrometer (MODIS) version 5 satellite retrievals for ocean regions (Remer et al., 2008) with corrections (Zhang et al., 2008a; Shi et al., 2011) and version 31 of MISR retrievals over land (Zhang and Reid, 2010; Stevens and Schwartz, 2012). (b): The absolute error in visible AOD from the median of a subset of CMIP5 models' historical simulations relative to the satellite retrievals of AOD shown in (a). The model outputs for 2001 through 2005 are from 21 CMIP5 models with interactive or semi-interactive aerosol representation. 


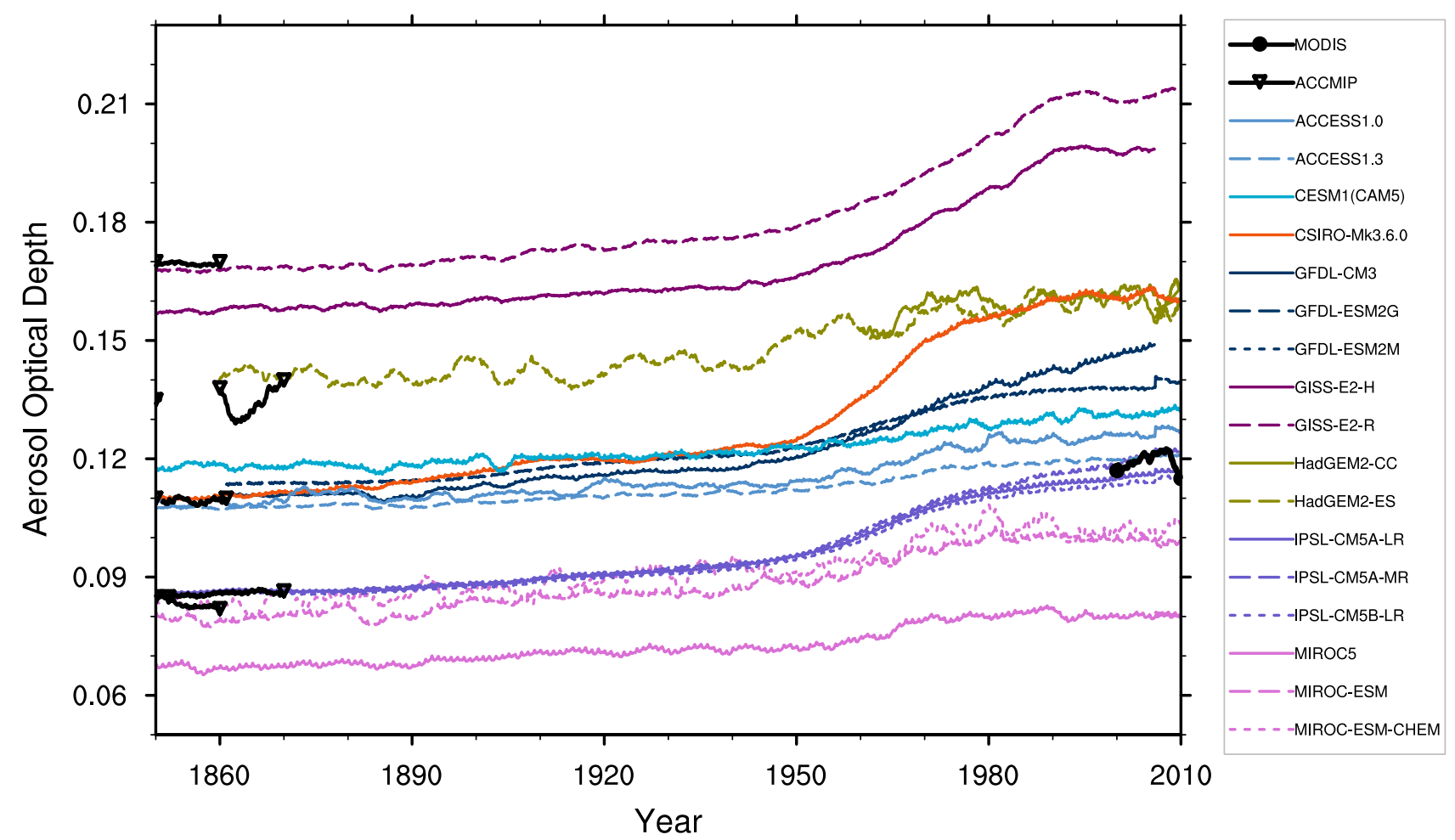

Figure 9.29 | Time series of global oceanic mean aerosol optical depth (AOD) from individual CMIP5 models' historical (1850-2005) and RCP4.5 (2006-2010) simulations, corrected Moderate Resolution Imaging Spectrometer (MODIS) satellite observations by Shi et al. (2011) and Zhang et al. (2008a), and the Atmospheric Chemistry and Climate Model Intercomparison Project (ACCMIP) simulations for the 1850s by Shindell et al. (2013b).

These recent trends are superimposed on a general upward trend in aerosol loading since 1850 reflected by an increase in global mean oceanic AODs from the CMIP5 historical and RCP 4.5 simulations from 1850 to 2010 (Figure 9.29). Despite the use of common anthropogenic aerosol emissions for the historical simulations (Lamarque et al., 2010), the simulated oceanic AODs for 2010 range from 0.08 to 0.215 , with nearly equal numbers of models over and underestimating the satellite retrieved AOD of 0.12 (Figure 9.29). This range in AODs results from differing estimates of the trends and of the initial global mean oceanic AOD at 1850 across the CMIP5 ensemble (Figure 9.29).

\subsubsection{Principal Sources of Uncertainty in Projections of Sulphate Burdens}

Natural sources of sulphate from oxidation of dimethylsulphide (DMS) emissions from the ocean surface are not specified under the RCP protocol and therefore represent a source of uncertainty in the sulphur cycle simulated by the CMIP5 ensemble. In simulations of present-day conditions, DMS emissions span a 5 to $95 \%$ confidence interval of 10.7 to $28.1 \mathrm{TgS} \mathrm{yr}^{-1}$ (Faloona, 2009). After chemical processing, DMS contributes between 18 and $42 \%$ of the global atmospheric sulphate burden and up to $80 \%$ of the sulphate burden over most the SH (Carslaw et al., 2010). Several CMIP5 models include prognostic calculation of the biogenic DMS source; however, the effects from differences in DMS emissions on modelled sulphate burdens remain to be quantified.

In contrast to CMIP3, the models in the CMIP5 ensemble are provided with a single internally consistent set of future anthropogenic $\mathrm{SO}_{2}$ emissions. The use of a single set of emissions removes an important, but not dominant, source of uncertainty in the AR5 simulations of the sulphur cycle. In experiments based on a single chemistry-climate model with perturbations to both emissions and sulphur-cycle processes, uncertainties in emissions accounted for $53.3 \%$ of the ensemble variance (Ackerley et al., 2009). The next largest source of uncertainty was associated with the wet scavenging of sulphate (see Section 7.3.2), which accounted for $29.5 \%$ of the intra-ensemble variance and represents the source/sink term with the largest relative range in the aerosol models evaluated by Faloona (2009). Similarly, simulations run with heterogeneous or harmonized emissions data sets yielded approximately the same intermodel standard deviation in sulphate burden of $25 \mathrm{Tg}$. These results show that a dominant source of the spread among the sulphate burdens is associated with differences in the treatment of chemical production, transport, and removal from the atmosphere (Liu et al., 2007; Textor et al., 2007). Errors in modelled aerosol burden systematically affect anthropogenic RF (Shindell et al., 2013b).

\subsection{Simulation of Variability and Extremes}

\subsubsection{Importance of Simulating Climate Variability}

The ability of a model to simulate the mean climate, and the slow, externally forced change in that mean state, was evaluated in the previous section. However, the ability to simulate climate variability, both unforced internal variability and forced variability (e.g., diurnal and seasonal cycles) is also important. This has implications for the signal- 
to-noise estimates inherent in climate change detection and attribution studies where low-frequency climate variability must be estimated, at least in part, from long control integrations of climate models (Section 10.2). It also has implications for the ability of models to make quantitative projections of changes in climate variability and the statistics of extreme events under a warming climate. In many cases, the impacts of climate change will be experienced more profoundly in terms of the frequency, intensity or duration of extreme events (e.g., heat waves, droughts, extreme rainfall events; see Section 12.4). The ability to simulate climate variability is also central to achieving skill in climate prediction by initializing models from the observed climate state (Sections 11.1 and 11.2).

Evaluating model simulations of climate variability also provides a means to explore the representation of certain processes, such as the coupled processes underlying the ENSO and other important modes of variability. A model's representation of the diurnal or seasonal cycleboth of which represent responses to external (rotational or orbital) forcing - may also provide some insight into a model's 'sensitivity' and by extension, the ability to respond correctly to $\mathrm{GHG}$, aerosol, volcanic and solar forcing.

\subsubsection{Diurnal-to-Seasonal Variability}

\subsubsection{Diurnal Cycles of Temperature and Precipitation}

The diurnally varying solar radiation received at a given location drives, through complex interactions with the atmosphere, land surface and upper ocean, easily observable diurnal variations in surface and near-surface temperature, precipitation, level stability and winds. The AR4 noted that climate models simulated the global pattern of the diurnal temperature range, zonally and annually averaged over the continent, but tended to underestimate its magnitude in many regions (Randall et al., 2007). New analyses over land indicate that model deficiencies in surface-atmosphere interactions and the planetary boundary layer are also expected to contribute to some of the diurnal cycle errors and that model agreement with observations depends on region, vegetation type and season (Lindvall et al., 2012). Analyses of CMIP3 simulations show that the diurnal amplitude of precipitation is realistic, but most models tend to start moist convection prematurely over land (Dai, 2006; Wang et al., 2011a). Many CMIP5 models also have peak precipitation several hours too early compared to surface observations and TRMM satellite observations (Figure 9.30). This and the so-called 'drizzling bias' (Dai, 2006) can have large adverse impacts on surface evaporation and runoff (Qian et al., 2006). Over the ocean, models often rain too frequently and underestimated the diurnal amplitude (Stephens et al., 2010). It has also been suggested that a weak diurnal cycle of surface air temperature is produced over the ocean because of a lack of diurnal variations in SST (Bernie et al., 2008), and most models have difficulty with this due to coarse vertical resolution and coupling frequency (Dai and Trenberth, 2004; Danabasoglu et al., 2006).

Improved representation of the diurnal cycle has been found with increased atmospheric resolution (Sato et al., 2009; Ploshay and Lau, 2010) or with improved representation of cloud physics (Khairoutdinov et al., 2005), but the reasons for these improvements remain poorly understood. Other changes such as the representation of entrainment in deep convection (Stratton and Stirling, 2012), improved coupling between shallow and deep convection, and inclusion of density currents (Peterson et al., 2009) have been shown to greatly improve the diurnal cycle of convection over tropical land and provide a good representation of the timing of convection over land in coupled oceanatmosphere simulations (Hourdin et al., 2013). Thanks to improvements like this, the best performing models in Figure 9.30 appear now to be able to capture the land and ocean diurnal phase and amplitude quite well.

\subsubsection{Blocking}

In the mid latitudes, climate is often characterized by weather regimes (see Chapter 2), amongst which blocking regimes play a role in the occurrence of extreme weather events (Buehler et al., 2011; Sillmann et al., 2011; Pfahl and Wernli, 2012). During blocking, the prevailing mid-latitude westerly winds and storm systems are interrupted by a local reversal of the zonal flow. Climate models in the past have universally underestimated the occurrence of blocking, in particular in the Euro-Atlantic sector (Scaife et al., 2010).

There are important differences in methods used to identify blocking (Barriopedro et al., 2010a), and the diagnosed blocking frequency can be very sensitive to details such as the choice of latitude (Barnes et al., 2012). Blocking indices can be sensitive to biases in the representation of mean state (Scaife et al., 2010) or in variability (Barriopedro et al., 2010b; Vial and Osborn, 2012). When blocking is measured via anomaly fields, rather than reversed absolute fields, model skill can be high even in relatively low-resolution models (e.g., Sillmann and Croci-Maspoli, 2009).

Recent work has shown that models with high horizontal (Matsueda, 2009; Matsueda et al., 2009, 2010) or vertical resolution (Anstey et al., 2013) are better able to simulate blocking. These improvement arise from increased representation of orography and atmospheric dynamics (Woollings et al., 2010b; Jung et al., 2012; Berckmans et al., 2013), as well as reduced ocean surface temperature errors in the extra tropics (Scaife et al., 2011). Improved physical parameterizations have also been shown to improve simulations of blocking (Jung et al., 2010). However, as in CMIP3 (Scaife et al., 2010; Barnes et al., 2012), most of the CMIP5 models still significantly underestimate winter Euro-Atlantic blocking (Anstey et al., 2013; Masato et al., 2012; Dunn-Sigouin and Son, 2013). These new results show that the representation of blocking events is improving in models, even though the overall quality of CMIP5 ensemble is medium. There is high confidence that model representation of blocking is improved through increases in model resolution.

\subsubsection{Madden-Julian Oscillation}

During the boreal winter the eastward propagating feature known as the Madden-Julian Oscillation (MJO; (Madden and Julian, 1972, 1994) predominantly affects the deep tropics, while during the boreal summer there is also northward propagation over much of southern Asia (Annamalai and Sperber, 2005). The MJO has received much attention given the prominent role it plays in tropical climate variability (e.g., monsoons, ENSO, and mid-latitudes; Lau and Waliser, 2011) 


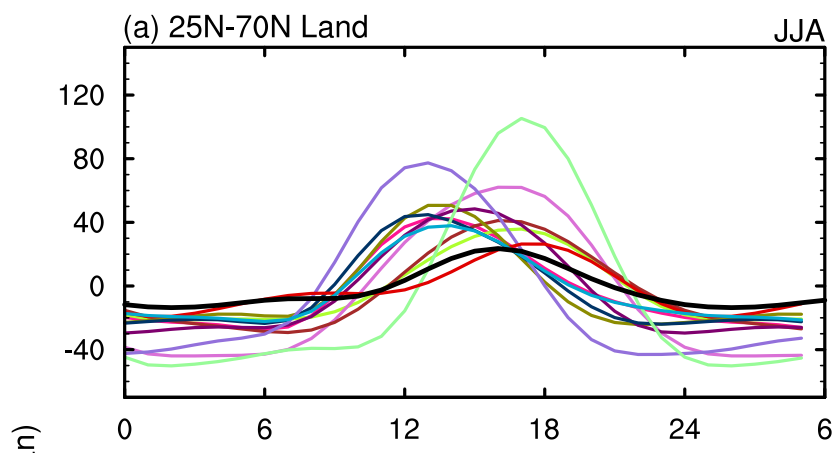

JJA

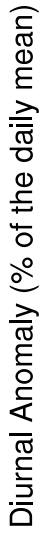

(b) $25 \mathrm{~S}-25 \mathrm{~N}$ Land

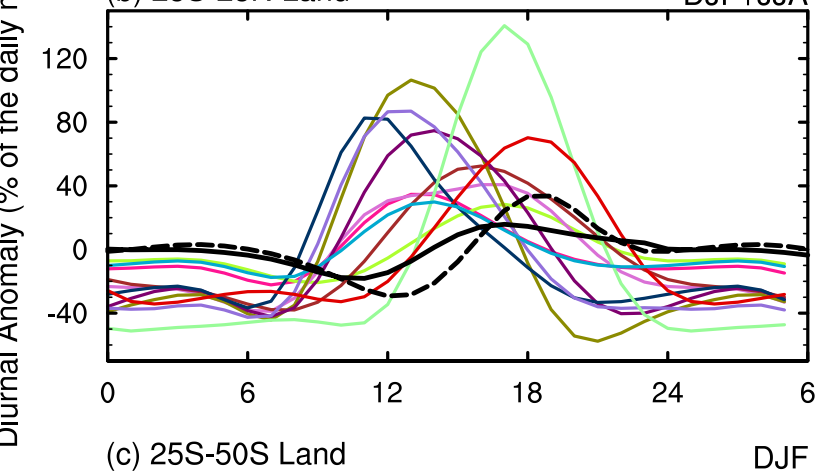

(c) 25S-50S Land

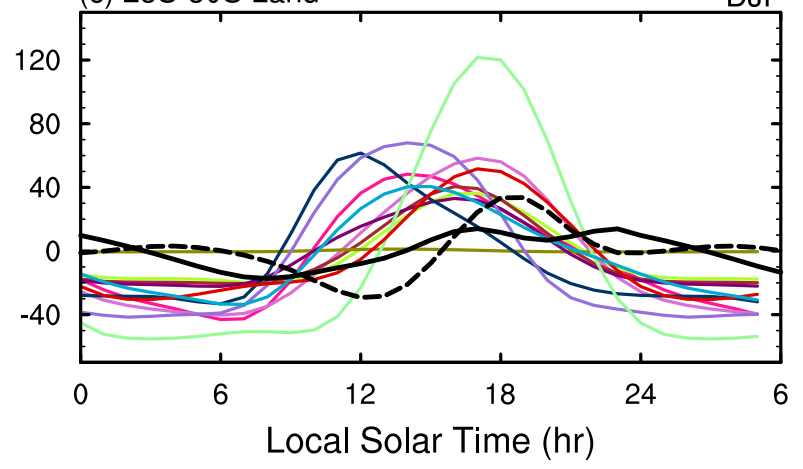

(d) $25 \mathrm{~N}-70 \mathrm{~N}$ Ocean

JJA

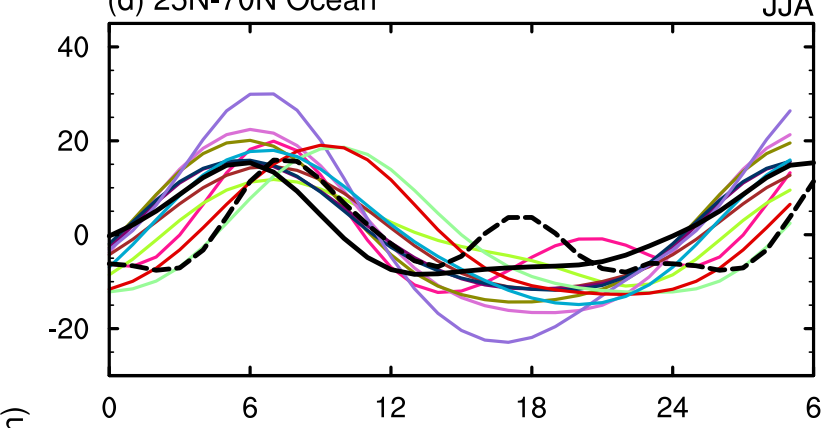

(e) $25 \mathrm{~S}-25 \mathrm{~N}$ Ocean

$D J F+J J A$

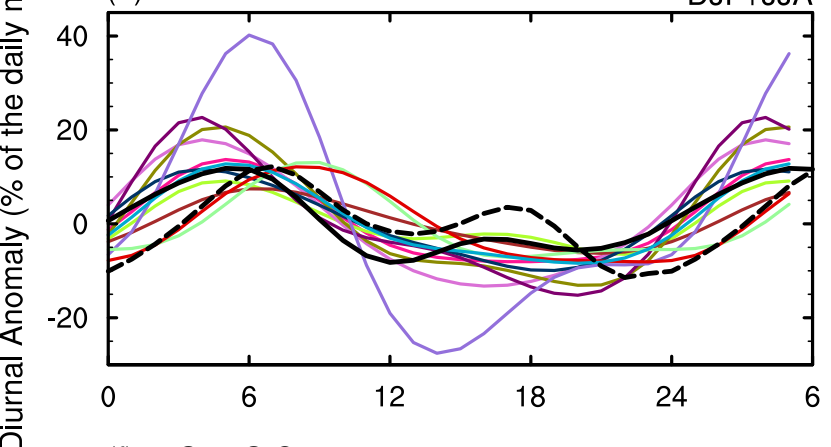

(f) $25 \mathrm{~S}-50 \mathrm{~S}$ Ocean

DJF

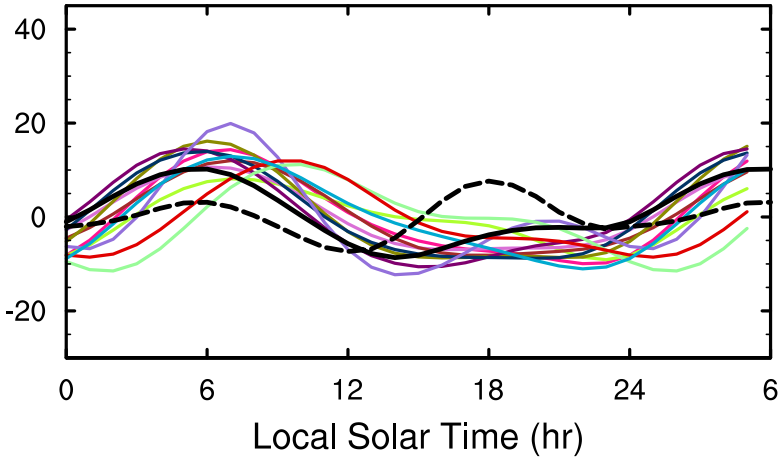

\begin{tabular}{lllll}
\hline OBS & --- TRMM & CCSM4 & CMCC-CM & CNRM-CM5 \\
EC-EARTH & GFDL-CM3 & GISS-E2-R & HadGEM2-ES & INM-CM4 \\
MIROC-ESM & MRI-CGCM3 & NorESM1-M &
\end{tabular}

Figure 9.30 Composite diurnal cycle of precipitation averaged over land (left) and ocean (right) for three different latitude bands at each local time and season (June-July-August (JJA), December-January-February (DJF), or their sum). For most of the CMIP5 models, data from 1980-2005 from the historical runs were averaged to derived the composite cycle; however, a few models had the required 3-hourly data only for 1990-2005 or 1996-2005. For comparison with the model results, a similar diagnosis from observations are shown (black solid line: surface-observed precipitation frequency; black dashed line: TRMM 3 B42 data set, 1998-2003 mean). (Update of Figure 17 of Dai, 2006.$)$

Phenomenological diagnostics (Waliser et al., 2009a) and process-oriented diagnostics (e.g., Xavier, 2012) have been used to evaluate MJO in climate models. An important reason for model errors in representing the MJO is that convection parameterizations do not provide sufficient build-up of moisture in the atmosphere for the large scale organized convection to occur (Kim et al., 2012; Mizuta et al., 2012). Biases in the model mean state also contribute to poor MJO simulation (Inness et al., 2003). High-frequency coupling with the ocean is also an important factor (Bernie et al., 2008). While new parameterizations of convection may improve the MJO (Hourdin et al., 2013), this sometimes occurs at the expense of a good simulation of the mean tropical climate (Kim et al., 2012). In addition, high resolution models with an improved diurnal cycle do not necessarily produce an improved MJO (Mizuta et al., 2012).

Most models underestimate the strength and the coherence of convection and wind variability (Lin et al., 2006; Lin and Li, 2008). The simplified metric shown in Figure 9.31 provides a synthesis of CMIP3 and CMIP5 model results (Sperber and Kim, 2012). It shows that simulation of the MJO is still a challenge for climate models (Lin et al., 2006; Kim et al., 2009; Xavier et al., 2010). Most models have weak coherence in their MJO propagation (smaller maximum positive correlation). Even so, relative to CMIP3 there has been improvement in CMIP5 in simulating the eastward propagation of boreal winter MJO convection from 
(a) EOF1

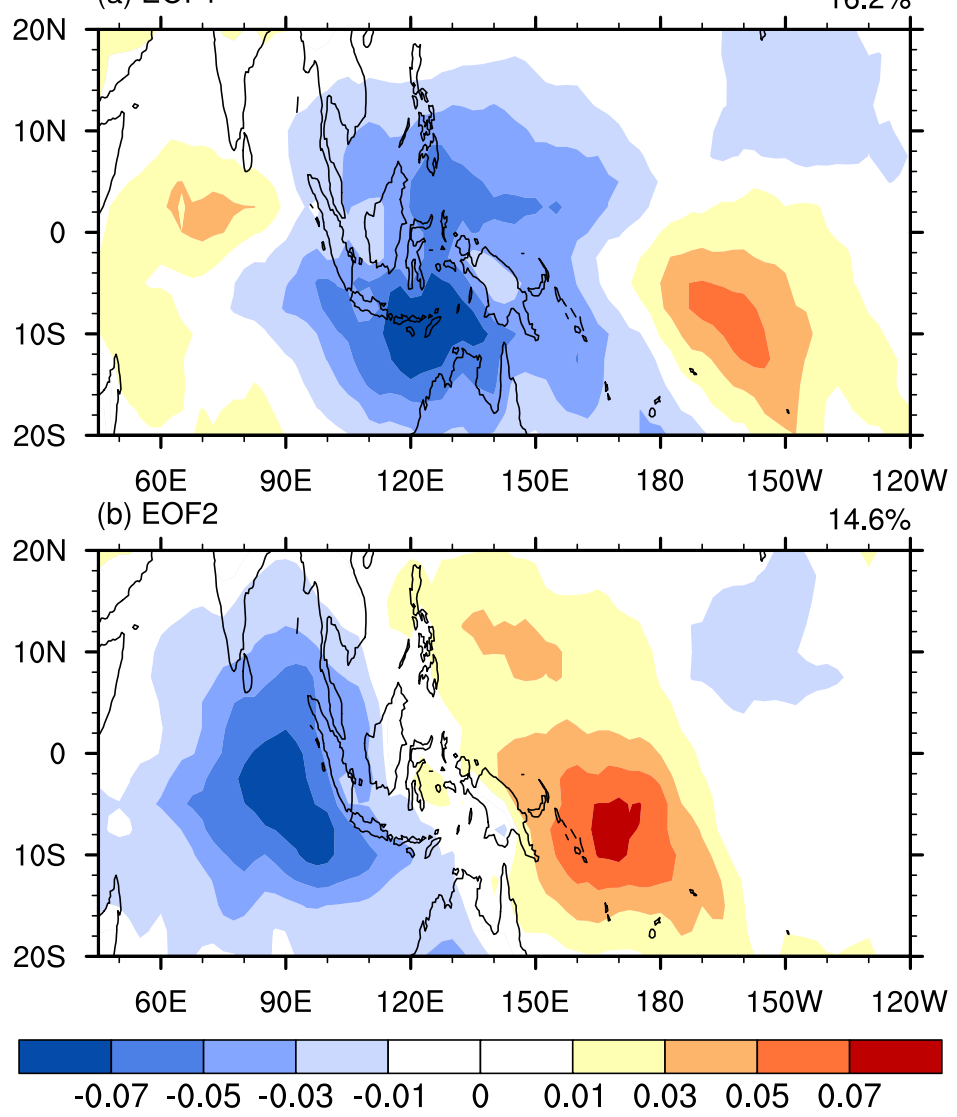

(c) Maximum Positive Correlation

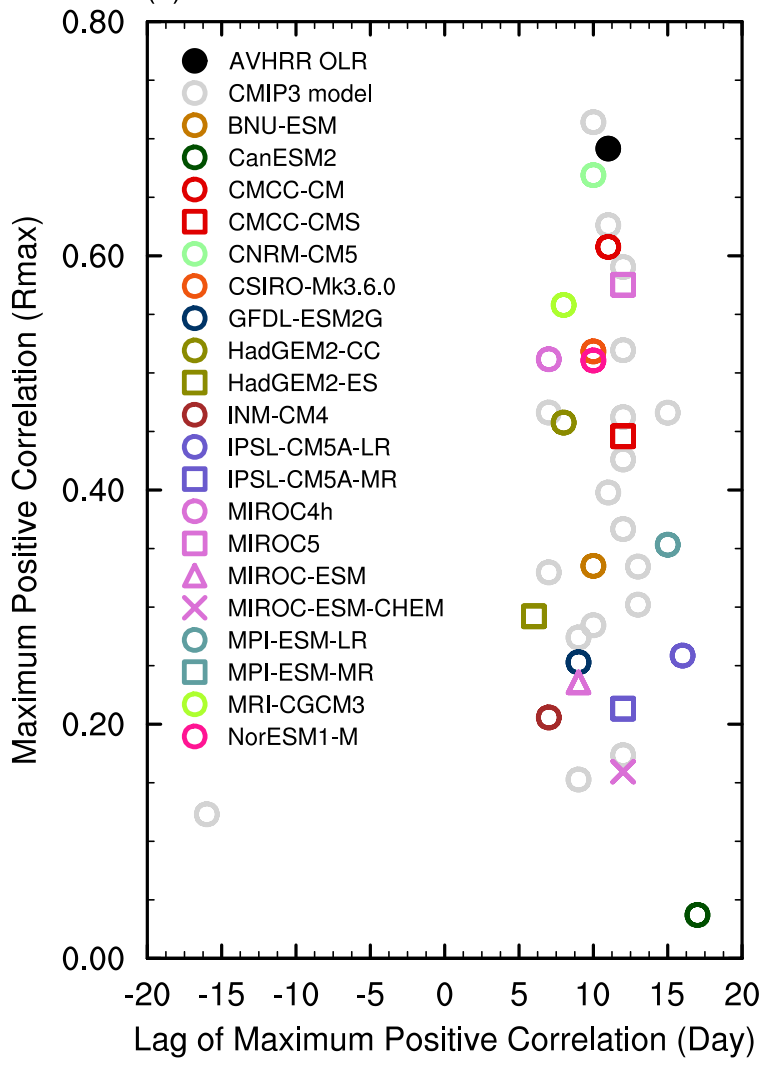

Figure 9.31 | (a, b) The two leading Empirical Orthogonal Functions (EOFs) of outgoing longwave radiation (OLR) from years of strong Madden-Julian Oscillation (MJO) variability computed following Sperber (2003). The 20- to 100-day filtered OLR from observations and each of the CMIP5 historical simulations and the CMIP3 simulations of 20th century climate is projected on these two leading EOFs to obtain MJO Principal Component time series. The scatterplot (c) shows the maximum positive correlation between the resulting MJO Principal Components and the time lag at which it occurred for all winters (November to March). The maximum positive correlation is an indication of the coherence with which the MJO convection propagates from the Indian Ocean to the Maritime Continent/western Pacific, and the time lag is approximately one fourth of the period of the MJO. (Constructed following Sperber and Kim, 2012.)

the Indian Ocean into the western Pacific (Hung et al., 2013) and northward propagation during boreal summer (Sperber et al., 2012). In addition there is evidence that models reproduce MJO characteristics in the east Pacific (Jiang et al., 2012b), and that, overall, there is improvement compared to previous generations of climate models (Waliser et al., 2003; Lin et al., 2006; Sperber and Annamalai, 2008).

\subsubsection{Large-Scale Monsoon Rainfall and Circulation}

Monsoons are the dominant modes of annual variation in the tropics (Trenberth et al., 2000; Wang and Ding, 2008), and affect weather and climate in numerous regions (Chapter 14). High-fidelity simulation of the mean monsoon and its variability is of great importance for simulating future climate impacts (Wang, 2006; Sperber et al., 2010; Colman et al., 2011; Turner and Annamalai, 2012). The monsoon is characterized by an annual reversal of the low level winds and well defined dry and wet seasons (Wang and Ding, 2008), and its variability is primarily connected to the MJO and ENSO (Section 9.5.3). The AR4 reported that most CMIP3 models poorly represent the characteristics of the monsoon and monsoon teleconnections (Randall et al., 2007), though improvement in CMIP5 has been noted for the mean climate, seasonal cycle, intraseasonal and interannual variability (Sperber et al., 2012).
The different monsoon systems are connected through the large-scale tropical circulation, offering the possibility to evaluate a models' representation of monsoon domain and intensity through the global monsoon concept (Wang and Ding, 2008; Wang et al., 2011a). The CMIP5 multi-model ensemble generally reproduces the observed spatial patterns but somewhat underestimates the extent and intensity, especially over Asia and North America (Figure 9.32). The best model has similar performance to the multi-model mean, whereas the poorest models fail to capture the monsoon precipitation domain and intensity over Asia and the western Pacific, Central America, and Australia. Fan et al. (2010) also show that CMIP3 simulations capture the observed trend of weakening of the South Asian summer circulation over the past half century, but are unable to reproduce the magnitude of the observed trend in precipitation. On longer time scales, mid-Holocene simulations show that even though models capture the sign of the monsoon precipitation changes, they tend to underestimate its magnitude (Braconnot et al., 2007b; Zhao and Harrison, 2012)

Poor simulation of the monsoon has been attributed to cold SST biases over the Arabian Sea (Levine and Turner, 2012), a weak meridional temperature gradient (Joseph et al., 2012), unrealistic development of the Indian Ocean dipole (Achuthavarier et al., 2012; Boschat et al., 
(a) GPCP

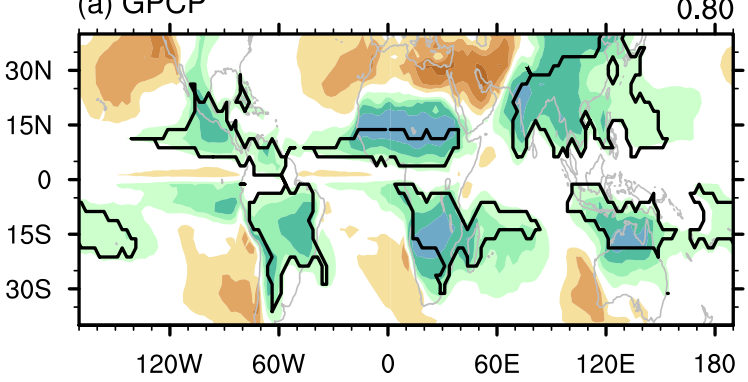

(c) Best Model

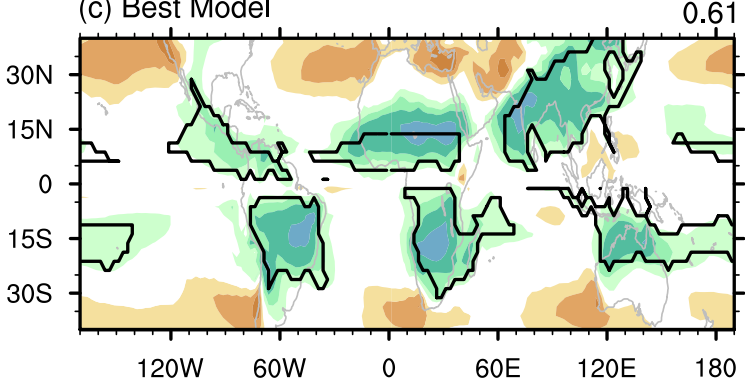

(b) CMIP5 MMM

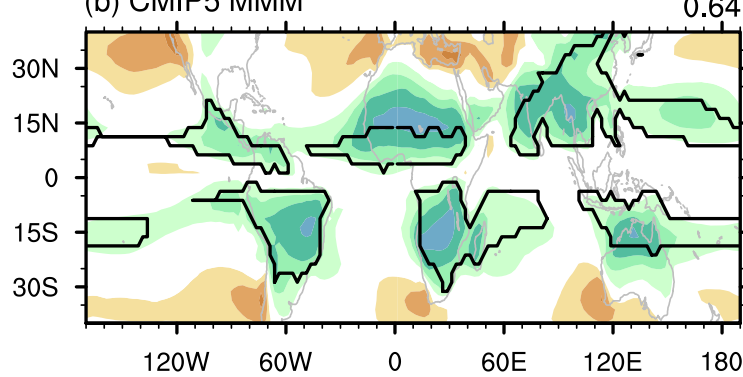

(d) Poorest Model

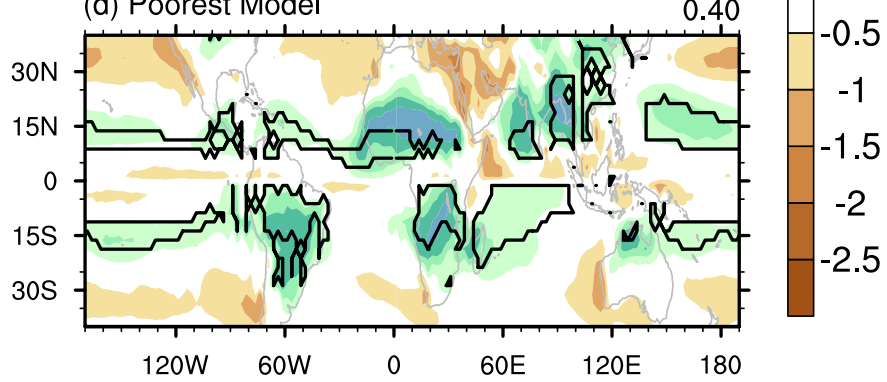

Figure 9.32 | Monsoon precipitation intensity (shading, dimensionless) and monsoon precipitation domain (lines) are shown for (a) observation-based estimates from Global Precipitation Climatology Project (GPCP), (b) the CMIP5 multi-model mean, (c) the best model and (d) the worst model in terms of the threat score for this diagnostic. These measures are based on the seasonal range of precipitation using hemispheric summer (May through September in the Northern Hemisphere (NH)) minus winter (November through March in the $\mathrm{NH}$ ) values. The monsoon precipitation domain is defined where the annual range is $>2.5 \mathrm{~mm}^{-1}{ }^{-1}$, and the monsoon precipitation intensity is the seasonal range divided by the annual mean. The threat scores (Wilks, 1995) indicate how well the models represent the monsoon precipitation domain compared to the GPCP data. The threat score in panel (a) is between GPCP and CMAP rainfall to indicate observational uncertainty, whereas in the other panel it is between the simulations and the GPCP observational data set. A threat score of 1.0 would indicate perfect agreement between the two data sets. See Wang and Ding (2008),Wang et al. (2011a), and Kim et al. (2011) for details of the calculations.

2012) and changes to the circulation through excessive precipitation over the southwest equatorial Indian Ocean (Bollasina and Ming, 2013). These biases lead to too weak inland moisture transport and an underestimate of monsoon precipitation over India. Similar SST biases contribute to model-data mismatch in the simulation of the mid-Holocene Asian monsoon (Ohgaito and Abe-Ouchi, 2009), even though the representation of atmospheric processes such as convection seems to dominate the model spread in this region (Ohgaito and Abe-Ouchi, 2009) or over Africa (Zheng and Braconnot, 2013). Factors that have contributed to improved representation of the monsoon in some CMIP5 models include better simulation of topography-related monsoon precipitation due to higher horizontal resolution (Mizuta et al., 2012), a more realistic ENSO-monsoon teleconnection (Meehl et al., 2012) and improved propagation of intraseasonal variations (Sperber and Kim, 2012). The impact of aerosols on monsoon precipitation and its variability is the subject of ongoing investigation (Lau et al., 2008).

These results provide robust evidence that CMIP5 models simulate more realistic monsoon climatology and variability than their CMIP3 predecessors, but they still suffer from biases in the representation of the monsoon domain and intensity leading to medium model quality at the global scale and declining quality at the regional scale.

\subsubsection{Interannual-to-Centennial Variability}

In addition to the annual, intra-seasonal and diurnal cycles described above, a number of other modes of variability arise on multi-annual to multi-decadal time scales (see also Box 2.5). Most of these modes have a particular regional manifestation whose amplitude can be larger than that of human-induced climate change. The observational record is usually too short to fully evaluate the representation of variability in models and this motivates the use of reanalysis or proxies, even though these have their own limitations.

\subsubsection{Global Surface Temperature Multi-Decadal Variability}

The AR4 concluded that modelled global temperature variance at decadal to inter-decadal time scales was consistent with 20th century observations. In addition, results from the last millennium suggest that simulated variability is consistent with indirect estimates (Hegerl et al., 2007).

Figure 9.33a shows simulated internal variability of mean surface temperature from CMIP5 pre-industrial control simulations. Model spread is largest in the tropics and mid to high latitudes (Jones et al., 2012), where variability is also large; however, compared to CMIP3, the spread is smaller in the tropics owing to improved representation of ENSO variability (Jones et al., 2012). The power spectral density of global mean temperature variance in the historical simulations is shown in Figure $9.33 \mathrm{~b}$ and is generally consistent with the observational estimates.

At longer time scale of the spectra estimated from last millennium simulations, performed with a subset of the CMIP5 models, can be assessed by comparison with different $\mathrm{NH}$ temperature proxy records (Figure 9.33c; see Chapter 5 for details). The CMIP5 millennium simulations include natural and anthropogenic forcings (solar, volcanic, GHGs, land use) (Schmidt et al., 2012). Significant differences between unforced and forced simulations are seen for time scale larger than 
(a) Standard deviation of zonal-mean surface temperature
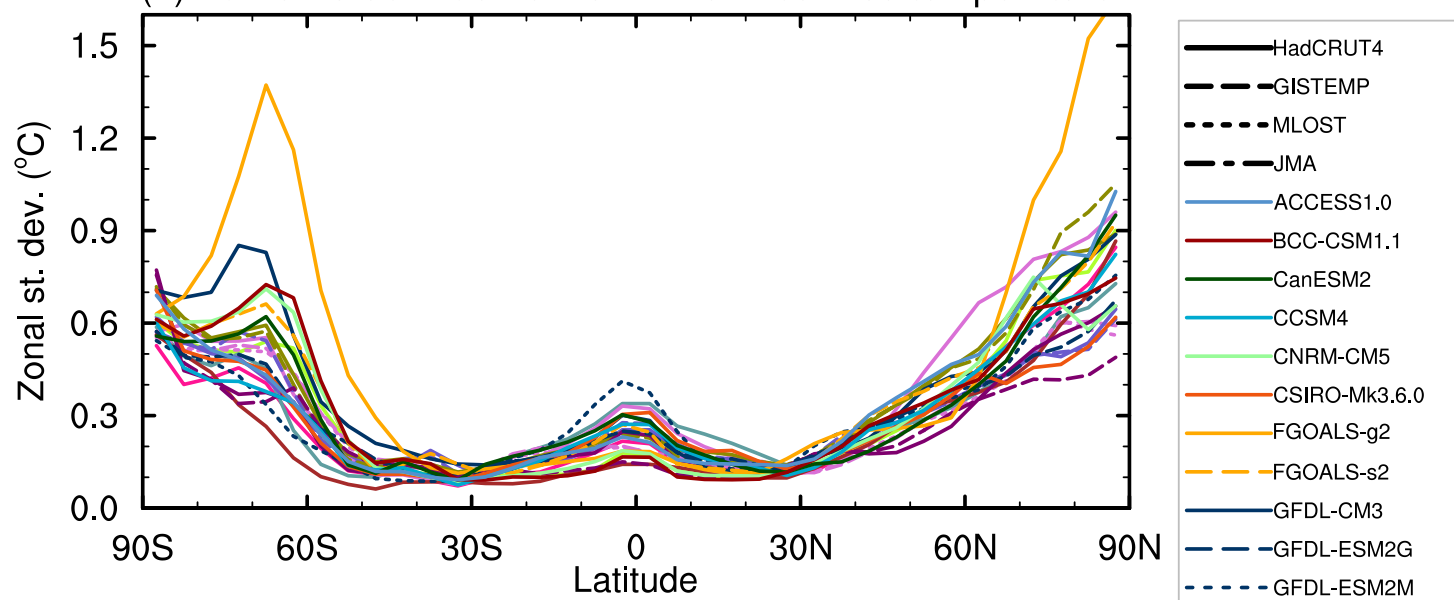

(b) Power spectra of 1901-2010 surface temperature

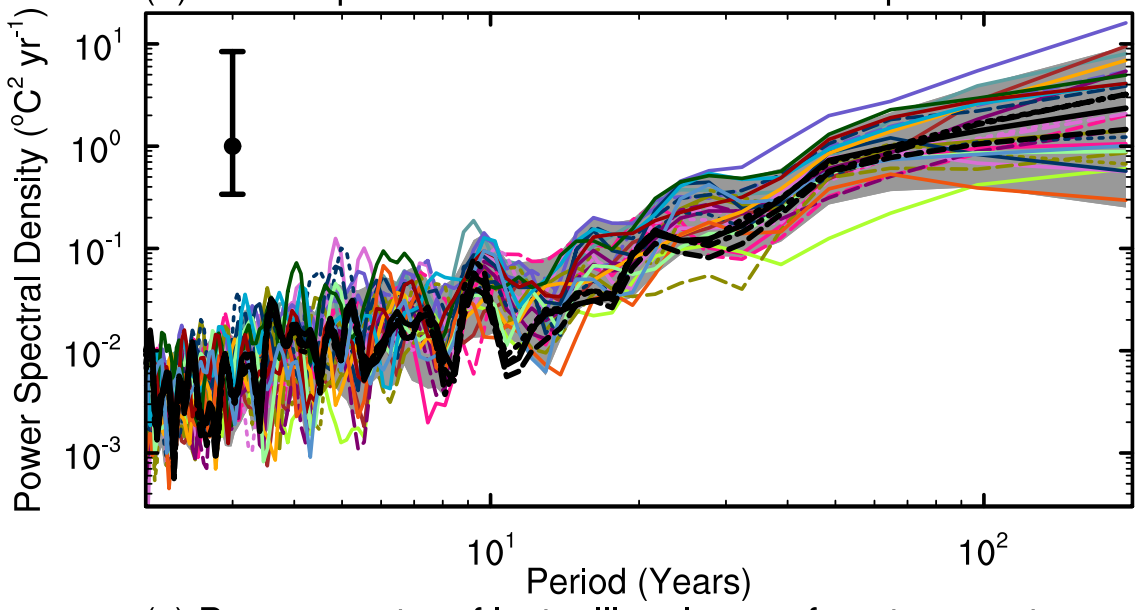

- - - - GFDL-ESM2M
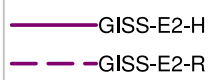

HadCM3

- - -HadGEM2-CC

- - - - HadGEM2-ES

-INM-CM4

IPSL-CM5A-LR

- -IPSL-CM5A-MR

MIROC5

- - -MIROC-ESM

- - - - MIROC-ESM-CHEM

MPI-ESM-LR

MRI-CGCM3

NorESM1-M

- - -NorESM1-ME

(c) Power spectra of last millennium surface temperature
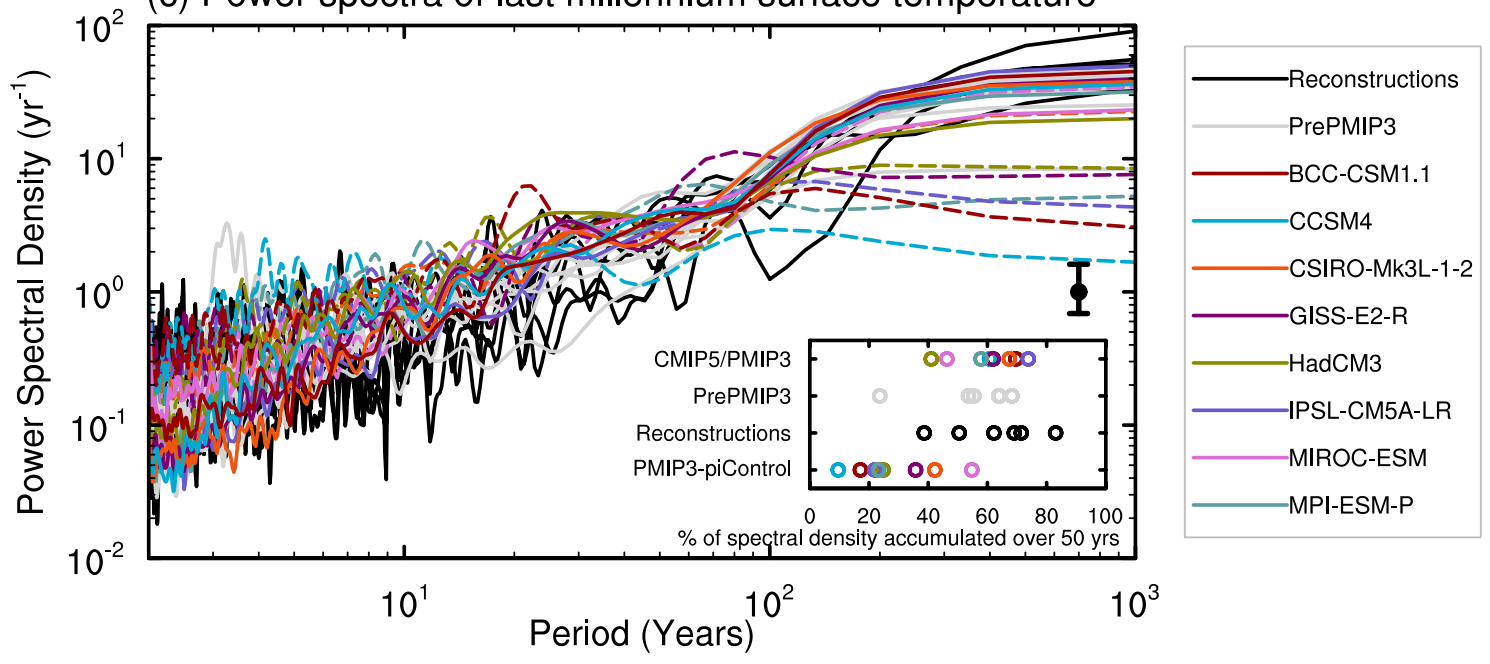

Figure 9.33 | Global climate variability as represented by: (a) Standard deviation of zonal-mean surface temperature of the CMIP5 pre-industrial control simulations (after Jones et al., 2012). (b) Power spectral density for 1901-2010 global mean surface temperature for both historical CMIP5 simulations and the observations (after Jones et al., 2012). The grey shading provides the 5 to $95 \%$ range of the simulations. (c) Power spectral density for Northern Hemisphere surface temperature from the CMIP5/ Paleoclimate Modelling Intercomparison Project version 3 (PMIP3) last-millennium simulations (colour, solid) using common external forcing configurations (Schmidt et al., 2012), together with the corresponding pre-industrial simulations (colour, dashed), previous last-millennium AOGCM simulations (black: Fernandez-Donado et al., 2013), and temperature reconstructions from different proxy records (see Section 5.3.5). For comparison between model results and proxy records, the spectra in (c) have been computed from normalized Northern Hemisphere time series. The small panel included in the bottom panel shows for the different models and reconstructions the percentage of spectral density cumulated for periods longer than 50 years, to highlight the differences between unforced (pre-industrial control) and forced (PMIP3 and pre-PMIP3) simulations, compared to temperature reconstruction for the longer time scales. In (b) and (c) the spectra have been computed using a Tukey-Hanning filter of width 97 and 100 years, respectively. The model outputs were not detrended, except for the MIROC-ESM millennium simulation. The 5 to $95 \%$ intervals (vertical lines) provide the accuracy of the power spectra estimated given a typical length of 110 years for (b) and 1150 years for (c). 
50 years, indicating the importance of forced variability at these time scales (Fernandez-Donado et al., 2013). It should be noted that a few models exhibit slow background climate drift which increases the spread in variance estimates at multi-century time scales. Nevertheless, the lines of evidence above suggest with high confidence that models reproduce global and $\mathrm{NH}$ temperature variability on a wide range of time scales.

\subsubsection{Extratropical Circulation, North Atlantic Oscillation and Other Related Dipolar and Annular Modes}

Based on CMIP3 models, Gerber et al. (2008) confirmed the AR4 assessment that climate models are able to capture the broad spatial and temporal features of the North Atlantic Oscillation (NAO), but there are substantial differences in the spatial patterns amongst individual models (Casado and Pastor, 2012; Handorf and Dethloff, 2012). Climate models tend to have patterns of variability that are more annular in character than observed (Xin et al., 2008). Models substantially overestimate persistence on sub-seasonal and seasonal time scales, and have difficulty simulating the seasonal cycle of annular mode time scales found in reanalyses (Gerber et al., 2008). The unrealistically long time scale of variability is worse in models with particularly strong equatorward biases in the mean jet location, a result which has been found to hold in the North Atlantic and in the SH (Barnes and Hartmann, 2010; Kidston and Gerber, 2010).

As described in the AR4, climate models have generally been unable to simulate changes as strong as the observed NAO trend over the period 1965-1995, either in coupled mode (Gillett, 2005; Stephenson et al., 2006; Stoner et al., 2009) or forced with observed boundary conditions (Scaife et al. (2009). However, there are a few exceptions to this (e.g., Selten et al., 2004; Semenov et al., 2008), so it is unclear to what extent the underestimation of late 20th century trends reflects model shortcomings versus internal variability. Further evidence has emerged of the coupling of NAO variability between the troposphere and the stratosphere, and even models with improved stratospheric resolution appear to underestimate the vertical coupling (Morgenstern et al., 2010) with consequences for the NAO response to anthropogenic forcing (Sigmond and Scinocca, 2010; Karpechko and Manzini, 2012; Scaife et al., 2012).

The Pacific basin analogue of the NAO, the North Pacific Oscillation (NPO) is a prominent pattern of wintertime atmospheric circulation variability characterized by a north-south dipole in sea level pressure (Linkin and Nigam, 2008). Although climate models simulate the main spatial features of the NPO, many of them are unable to capture the observed linkages with tropical variability and the ocean (Furtado et al., 2011).

Raphael and Holland (2006) showed that coupled models produce a clear Southern Annular Mode (SAM) but that there are relatively large differences between models in terms of the exact shape and orientation of this pattern. Karpechko et al. (2009) found that the CMIP3 models have problems representing linkages between the SAM and SST, surface air temperature, precipitation and particularly sea ice in the Antarctic region.

\subsubsection{Atlantic Modes}

\subsection{Atlantic Meridional Overturning Circulation variability}

Previous comparisons of the observed and simulated AMOC were restricted to its mean strength, as it had only been sporadically observed (see Chapter 3 and Section 9.4.2.3.1). Continuous AMOC time series now exist for latitudes $41^{\circ} \mathrm{N}$ (reconstructions since 1993) and $26.5^{\circ} \mathrm{N}$ (estimate based on direct observations since 2004) (Cunningham et al., 2010; Willis, 2010). At $26.5^{\circ} \mathrm{N}$, CMIP3 and CMIP5 model simulations show total $\mathrm{AMOC}$ variability that is within the observational uncertainty (Baehr et al., 2009; Marsh et al., 2009; Balan Sarojini et al., 2011; Msadek et al., 2013). However, the total AMOC is the sum of a wind-driven component and a mid-ocean geostrophic component. While both CMIP3 and CMIP5 models tend to overestimate the wind-driven variability, they tend to underestimate the mid-ocean geostrophic variability (Baehr et al., 2009; Balan Sarojini et al., 2011; Msadek et al., 2013). The latter is suggested to result from deficiencies in the simulation of the hydrographic characteristics (Baehr et al., 2009), specifically the Nordic Seas overflows (Yeager and Danabasoglu, 2012; Msadek et al., 2013).

\subsection{Atlantic multi-decadal variability/Atlantic Multi-decadal Oscillation}

The Atlantic Multi-decadal Variability (AMV), also known as Atlantic Multi-decadal Oscillation (AMO), is a mode of climate variability with an apparent period of about 70 years, and a pattern centred in the North Atlantic Ocean (see Section 14.7.6). In the AR4, it was shown that a number of climate models produced AMO-like multidecadal variability in the North Atlantic linked to variability in the strength of the AMOC. Subsequent analyses has confirmed this, however simulated time scales range from 40 to 60 years (Frankcombe et al., 2010; Park and Latif, 2010; Kavvada et al., 2013), to a century or more (Msadek and Frankignoul, 2009; Menary et al., 2012). In addition, the spatial patterns of variability related to the AMOC differ in many respects from one model to another as shown in Figure 9.34.

The presence of AMO-like variability in unforced simulations, and the fact that forced 20th century simulations in the CMIP3 multi-model ensemble produce AMO variability that is not in phase with that observed, implies the AMO is not predominantly a result of the forcings imposed on the models (Kravtsov and Spannagle, 2008; Knight, 2009; Ting et al., 2009). Results from the CMIP5 models also show a key role for internal variability, alongside a contribution from external forcings in recent decades (Terray, 2012). Historical AMO fluctuations have been better reproduced in a model with a more sophisticated aerosol treatment than was typically used in CMIP3 (Booth et al., 2012a), albeit at the expense of introducing other observational inconsistencies (Zhang et al., 2013). This could suggest that at least part of the AMO may in fact be forced, and that aerosols play a role. In addition to tropospheric aerosols, Otterå et al. (2010) showed the potential for simulated volcanic forcing to have influenced AMO fluctuations over the last 600 years. 


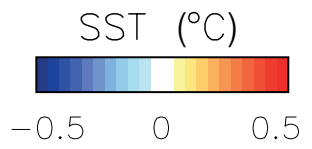

9
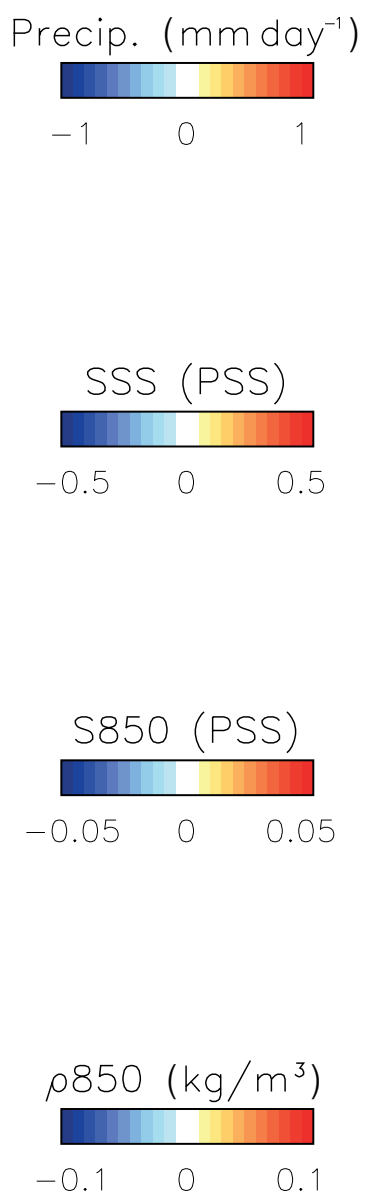
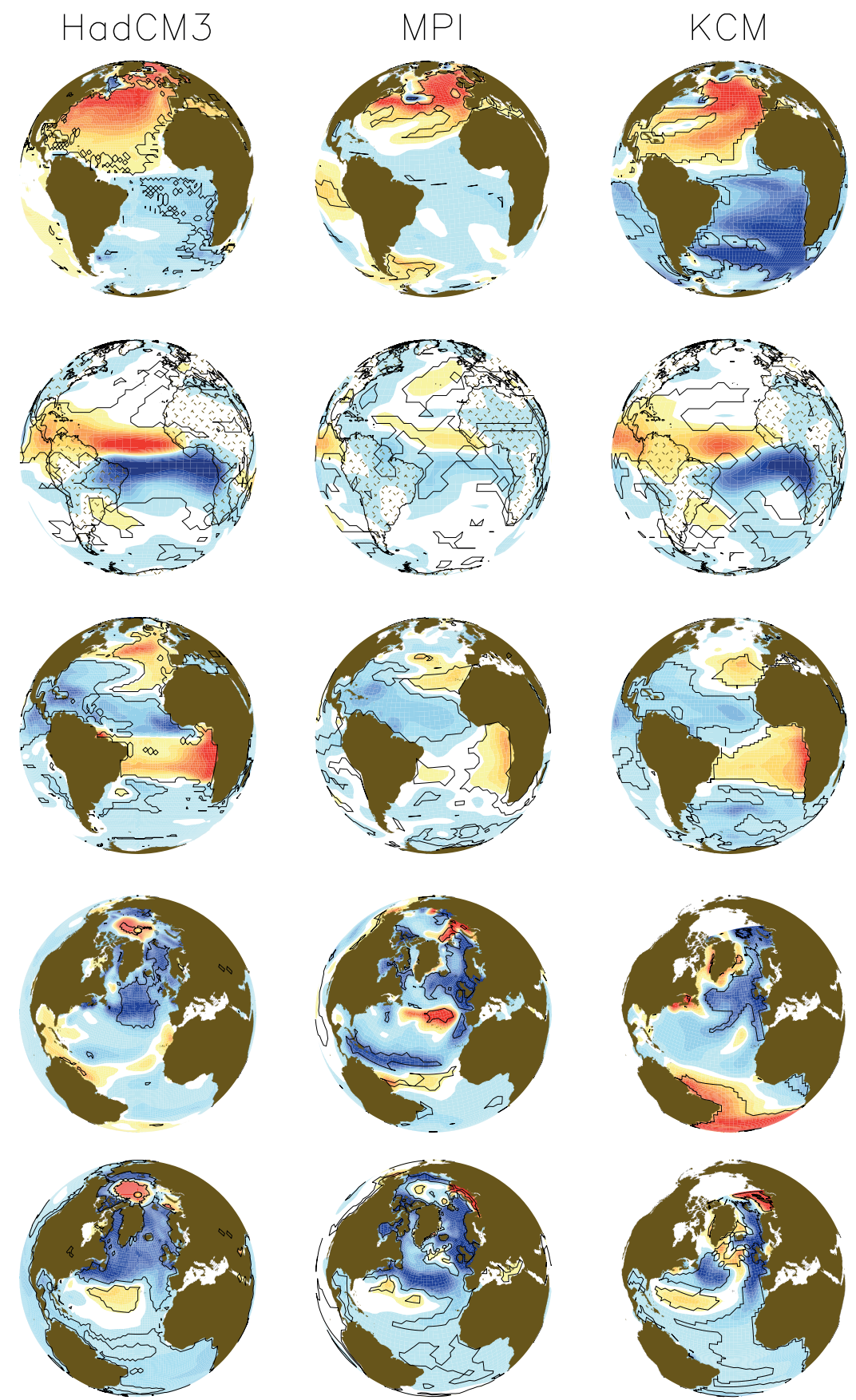

Figure 9.34 | Sequence of physical links postulated to connect Atlantic Meridional Overturning Circulation (AMOC) and Atlantic Multi-decadal Variability (AMV), and how they are represented in three climate models. Shown are regression patters for the following quantities (from top to bottom): sea surface temperature (SST) composites using AMOC time series; precipitation composites using cross-equatorial SST difference time series; equatorial salinity composites using Intertropical Convergence Zone (ITCZ)-strength time series; subpolar-gyre depth-averaged salinity (top 800 to $1000 \mathrm{~m}$ ) using equatorial salinity time series; subpolar gyre depth averaged density using subpolar gyre depth averaged salinity time series. From left to right: the two CMIP3 models HadCM3 and ECHAM/MPI-OM (MPI), and the non-CMIP model KCM. Black outlining signifies areas statistically significant at the $5 \%$ level for a two-tailed t test using the moving-blocks bootstrapping technique (Wilks, 1995). (Figure 3 from Menary et al., 2012.)

\subsection{Tropical zonal and meridional modes}

The Atlantic Meridional Mode (AMM) is the dominant mode of interannual variability in the tropical Atlantic, is characterized by an anomalous meridional shift in the ITCZ (Chiang and Vimont, 2004), and has impacts on hurricane tracks over the North Atlantic (Xie et al., 2005; Smirnov and Vimont, 2011). Virtually all CMIP models simulate AMMlike SST variability in their 20th century climate simulations. However, most models underestimate the SST variance associated with the AMM, and position the north tropical Atlantic SST anomaly too far equatorward. More problematic is the fact that the development of the AMM in many models is led by a zonal mode during boreal winter-a feature that is not observed in nature (Breugem et al., 2006). This spurious AMM behaviour in the models is expected to be associated with the severe model biases in simulating the ITCZ (see Section 9.4.2.5.2). 


\section{Atlantic Niño}

CMIP3 models have considerable difficulty simulating Atlantic Niño in their 20th century climate simulations. For many models the socalled 'Atl- $3^{\prime}$ SST index $\left(20^{\circ} \mathrm{W}\right.$ to $0^{\circ} \mathrm{W}, 3^{\circ} \mathrm{S}$ to $\left.3^{\circ} \mathrm{N}\right)$ displays the wrong seasonality, with the maximum value in either DJF or SON instead of JJA as is observed (Breugem et al., 2006). Despite large biases in the simulated climatology (Section 9.4.2.5.2), about one third of CMIP5 models capture some aspects of Atlantic Niño variability, including amplitude, spatial pattern and seasonality (Richter et al., 2013). This represents an improvement over CMIP3.

\subsubsection{Indo-Pacific Modes}

\subsection{El Niño-Southern Oscillation}

The ENSO phenomenon is the dominant mode of climate variability on seasonal to interannual time scales (see Wang and Picaut (2004) and Chapter 14). The representation of ENSO in climate models has steadily improved and now bears considerable similarity to observed ENSO properties (AchutaRao and Sperber, 2002; Randall et al., 2007; Guilyardi et al., 2009b). However, as was the case in the AR4, simulations (a) CMIP5

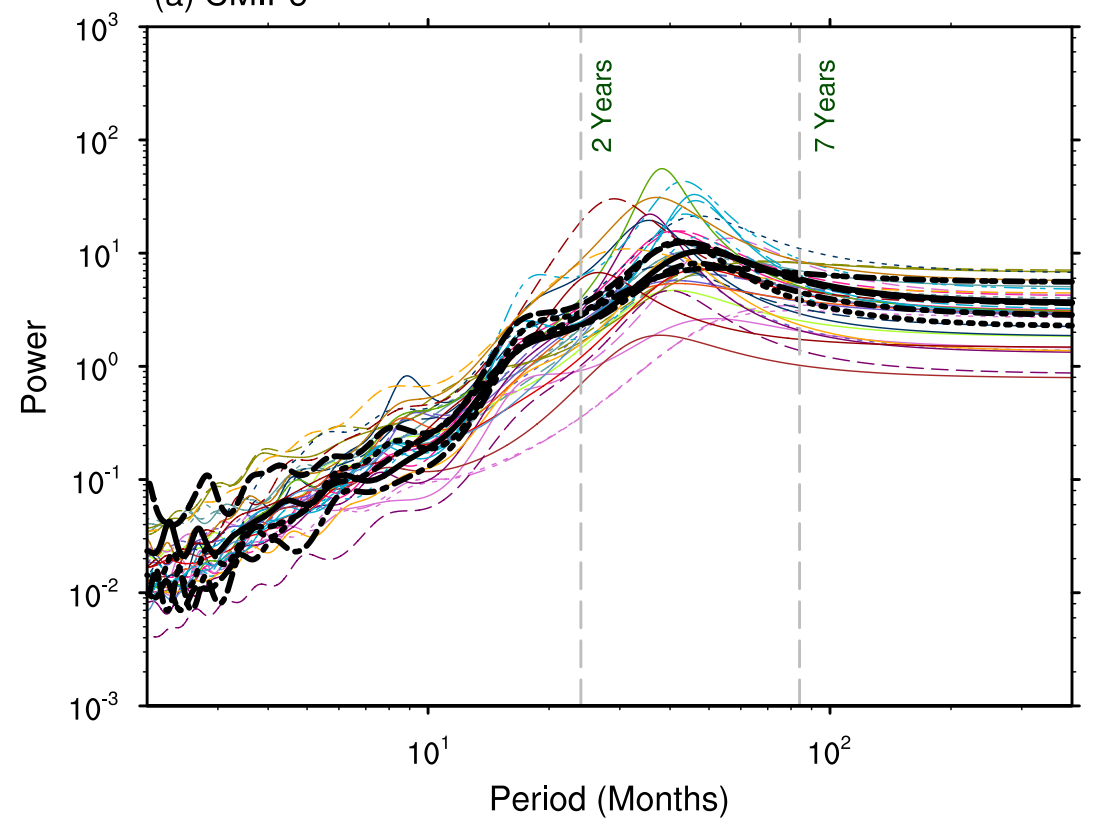

(b) CMIP3

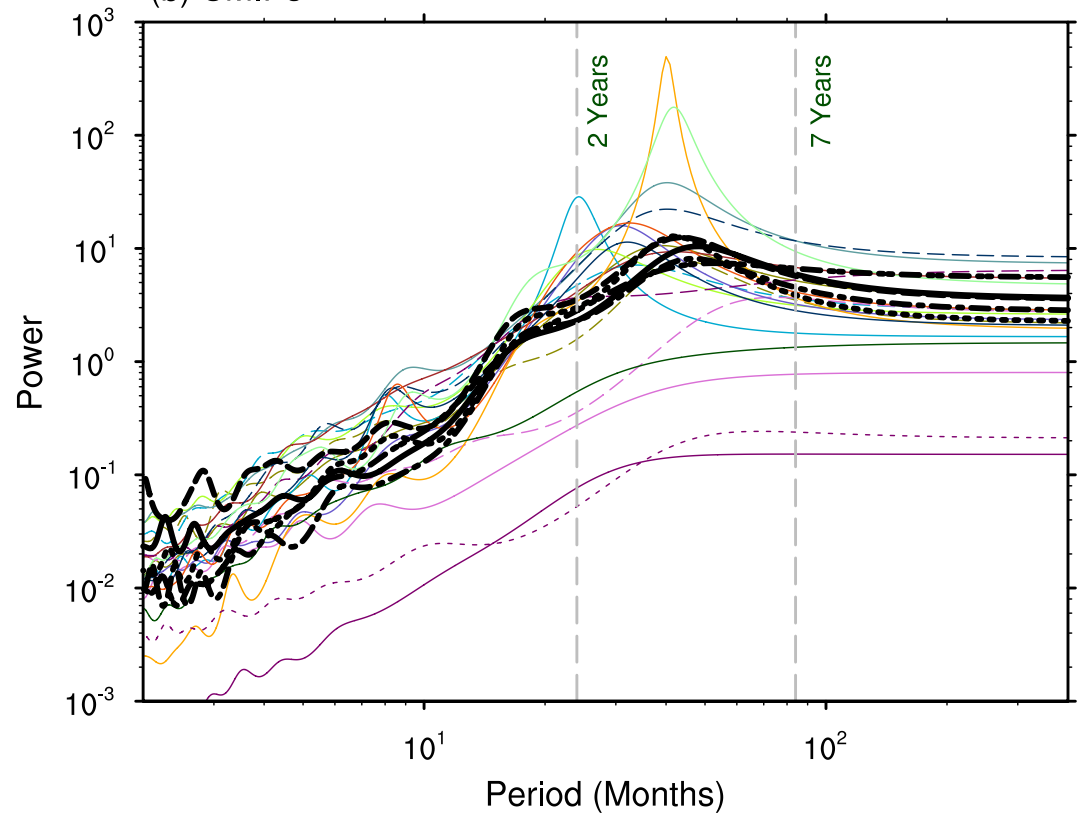

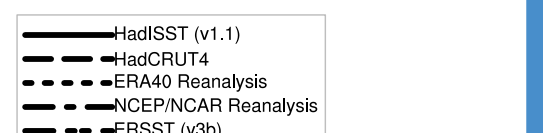

Figure 9.35 | Maximum entropy power spectra of surface air temperature averaged over the NINO3 region ( $5^{\circ} \mathrm{N}$ to $5^{\circ} \mathrm{S}, 150^{\circ} \mathrm{W}$ to $90^{\circ} \mathrm{W}$ ) for (a) the CMIP5 models and (b) the CMIP3 models. ECMWF reanalysis in (a) refers to the European Centre for Medium Range Weather Forecasts (ECMWF) 15-year reanalysis (ERA15). The vertical lines correspond to periods of two and seven years. The power spectra from the reanalyses and for SST from the Hadley Centre Sea Ice and Sea Surface Temperature (HadISST) version 1.1, Hadley Centre/Climatic Research Unit gridded surface temperature data set 4 (HadCRU 4), ECMWF 40-year reanalysis (ERA40) and National Centers for Environmental Prediction/National Center for Atmospheric Research (NCEP/NCAR) data set are given by the series of black curves. (Adapted from AchutaRao and Sperber, 2006.) 

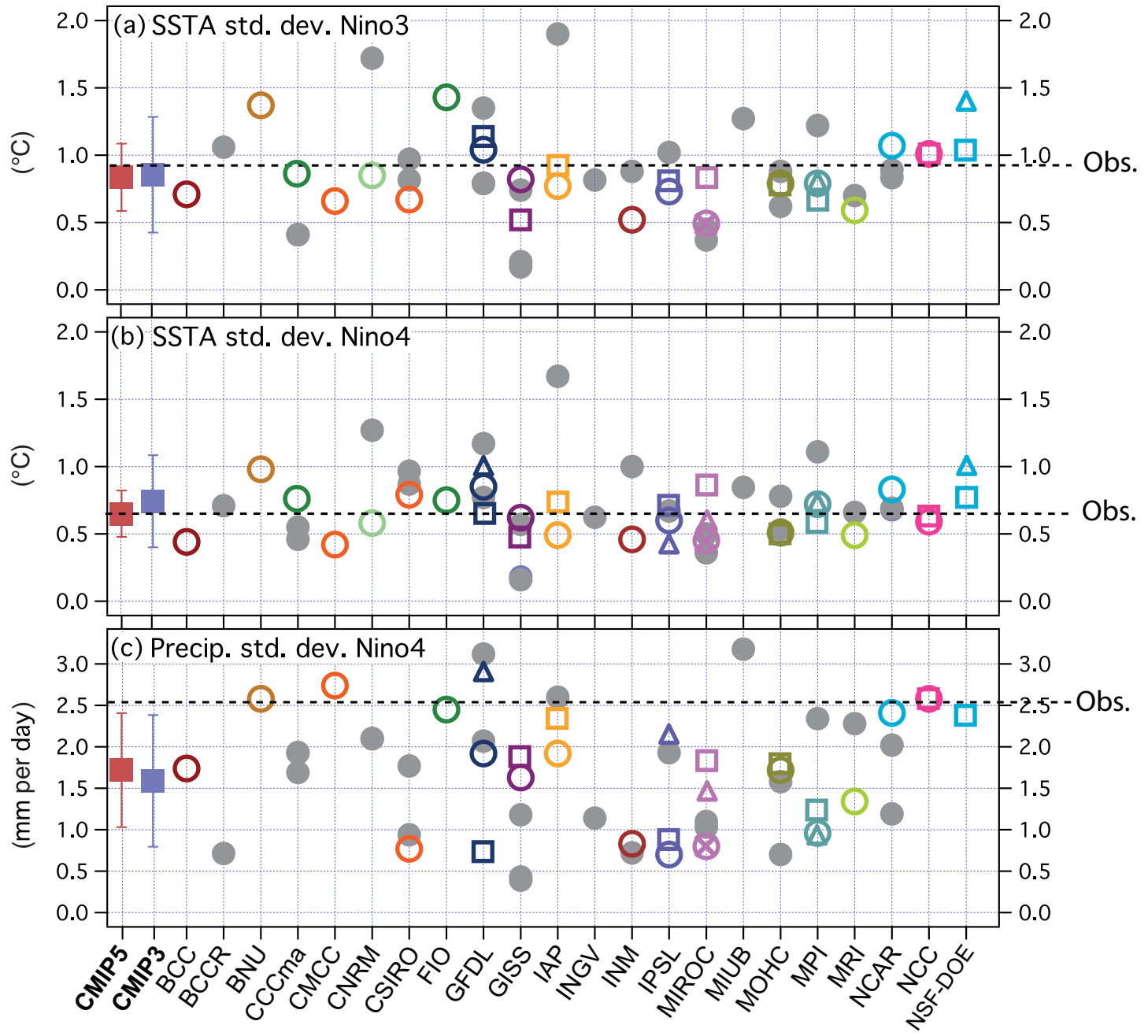

CMIP3 models

O BCC-CSM1.1

BNU-ESM

O CanESM2

C CSSM

$\square$ CESM1(FASTCHEM)

$\triangle$ CESM1(WACCM)

O CMCC-CM

CNRM-CM5

CSIRO-Mk3.6.0

FGOALS-g2

$\square$ FGOALS-s2

FIO-ESM

O GFDL-CM3

$\square$ GFDL-ESM2G

$\triangle$ GFDL-ESM2M

O GISS-E2-H

$\square$ GISS-E2-R

O HadGEM2-CC

$\square$ HadGEM2-ES

O INM-CM4

IPSL-CM5A-LR

$\square$ IPSL-CM5A-MR

$\triangle$ IPSL-CM5B-LR

MIROC4h

$\square$ MIROC5

$\triangle$ MIROC-ESM

$X$ MIROC-ESM-CHEM

O MPI-ESM-LR

$\square$ MPI-ESM-MR

$\triangle$ MPI-ESM-P

MRI-CGCM3

NorESM1-M

$\square$ NorESM1-ME

Figure 9.36 | ENSO metrics for pre-industrial control simulations in CMIP3 and CMIP5. (a) and (b): SST anomaly standard deviation $\left({ }^{\circ} \mathrm{C}\right)$ in Niño 3 and Niño 4 , respectively, (c) precipitation response (standard deviation, $\mathrm{mm} /$ day) in Niño4. Reference data sets, shown as dashed lines: Hadley Centre Sea Ice and Sea Surface Temperature (HadISST) version 1.1 for (a) and (b), CPC Merged Analysis of Precipitation (CMAP) for (c). The CMIP5 and CMIP3 multi-model means are shown as squares on the left of each panel with the whiskers representing the model standard deviation. Individual CMIP3 models shown as filled grey circles, and individual CMIP5 models are identified in the legend.

of both background climate (time mean and seasonal cycle, see Section 9.4.2.5.1) and internal variability exhibit serious systematic errors (van Oldenborgh et al., 2005; Capotondi et al., 2006; Guilyardi, 2006; Wittenberg et al., 2006; Watanabe et al., 2011; Stevenson et al., 2012; Yeh et al., 2012), many of which can be traced to the representation of deep convection, trade wind strength and cloud feedbacks, with little improvement from CMIP3 to CMIP5 (Braconnot et al., 2007a; L'Ecuyer and Stephens, 2007; Guilyardi et al., 2009a; Lloyd et al., 2009, 2010; Sun et al., 2009; Zhang and Jin, 2012).

While a number of CMIP3 models do not exhibit an ENSO variability maximum at the observed 2- to 7- year time scale, most CMIP5 models do have a maximum near the observed range and fewer models have the tendency for biennial oscillations (Figure 9.35; see also Stevenson, 2012). In CMIP3 the amplitude of El Niño ranged from less than half to more than double the observed amplitude (van Oldenborgh et al., 2005; AchutaRao and Sperber, 2006; Guilyardi, 2006; Guilyardi et al., 2009b). By contrast, the CMIP5 models show less inter-model spread (Figure 9.36; Kim and Yu, 2012). The CMIP5 models still exhibit errors in ENSO amplitude, period, irregularity, skewness, spatial patterns (Lin,
2007; Leloup et al., 2008; Guilyardi et al., 2009b; Ohba et al., 2010; Yu and Kim, 2011; Su and Jiang, 2012) or teleconnections (Watanabe et al., 2012; Weller and Cai, 2013a).

Since AR4, new analysis methods have emerged and are now being applied. For example, Jin et al. (2006) and Kim and Jin (2011a) identified five different feedbacks affecting the Bjerknes (or BJ) index, which in turn characterizes ENSO stability. Kim and Jin (2011b) applied this process-based analysis to the CMIP3 multi-model ensemble and demonstrated a significant positive correlation between ENSO amplitude and the $\mathrm{BJ}$ index. When respective components of the $\mathrm{BJ}$ index obtained from the coupled models were compared with those from observations, it was shown that most coupled models underestimated the negative thermal damping feedback (Lloyd et al., 2012; Chen et al., 2013) and the positive zonal advective and thermocline feedbacks.

Detailed quantitative evaluation of ENSO performance is hampered by the short observational record of key processes (Wittenberg, 2009; Li et al., 2011b; Deser et al., 2012) and the complexity and diversity of the processes involved (Wang and Picaut, 2004). While shortcomings 
remain (Guilyardi et al., 2009b), the CMIP5 model ensemble shows some improvement compared to CMIP3, but there has been no major breakthrough and the multi-model improvement is mostly due to a reduced number of poor-performing models.

\subsection{Indian Ocean basin and dipole modes}

Indian Ocean SST displays a basin-wide warming following El Niño (Klein et al., 1999). This Indian Ocean basin (IOB) mode peaks in boreal spring and persists through the following summer. Most CMIP5 models capture this IOB mode, an improvement over CMIP3 (Du et al., 2013). However, only about half the CMIP5 models capture its long temporal persistence, and these models tend to simulate ENSO-forced ocean Rossby waves in the tropical south Indian Ocean (Zheng et al., 2011).

The Indian Ocean zonal dipole mode (IOD) (Saji et al., 1999; Webster et al., 1999) appears to be part of a hemispheric response to tropical atmospheric forcing (Fauchereau et al., 2003; Hermes and Reason, 2005). Most CMIP3 models are able to reproduce the general features of the IOD, including its phase lock onto the July to November season (Saji et al., 2006). The modelled SST anomalies, however, tend to show too strong a westward extension along the equator in the eastern Indian Ocean. CMIP3 models exhibit considerable spread in IOD amplitude, some of which can be explained by differences in the strength of the simulated Bjerknes feedback (Liu et al., 2011; Cai and Cowan, 2013). No substantial change is seen in CMIP5 (Weller and Cai, 2013a).

Many models simulate the observed correlation between IOD and ENSO. The magnitude of this correlation varies substantially between models, but is apparently not tied to the amplitude of ENSO (Saji et al., 2006). A subset of CMIP3 models show a spurious correlation with ENSO following the decay of ENSO events, instead of during the ENSO developing phase, possibly due to erroneous representation of oceanic pathways connecting the equatorial Pacific and Indian Oceans (Cai et al., 2011).

\subsection{Tropospheric biennial oscillation}

The tropospheric biennial oscillation (TBO, Section 14.7.4) is a biennial tendency of many phenomena in the Indo-Pacific region that affects droughts and floods over large areas of south Asia and Australia (e.g., Chang and Li, 2000; Li et al., 2001; Meehl et al., 2003). The IOD involves regional patterns of SST anomalies in the TBO in the Indian Ocean during the northern fall season following the south Asian monsoon (Loschnigg et al., 2003). The TBO has been simulated in a number of global coupled climate model simulations (e.g., Ogasawara et al., 1999; Loschnigg et al., 2003; Nanjundiah et al., 2005; Turner et al., 2007; Meehl and Arblaster, 2011).

\subsubsection{Indo-Pacific Teleconnections}

Tropical SST variability provides a significant forcing of atmospheric teleconnections and drives a large portion of the climate variability over land (Goddard and Mason, 2002; Shin et al., 2010). Although local forcings and feedbacks can play an important role (Pitman et al., 2012a), the simulation of land surface temperatures and precipitation requires accurate predictions of SST patterns (Compo and
Sardeshmukh, 2009; Shin et al., 2010) as well as zonal wind variability patterns (Handorf and Dethloff, 2012). Teleconnections hence play a central role in regional climate change (see Chapter 14).

\subsection{Teleconnections affecting North America}

The Pacific North American (PNA) pattern is a wavetrain-like pattern in mid-level geopotential heights. The majority of CMIP3 models simulate the spatial structure of the PNA pattern in wintertime (Stoner et al., 2009). The PNA pattern has contributions from both internal atmospheric variability (Johnson and Feldstein, 2010) and ENSO and PDO teleconnections (Deser et al., 2004). The power spectrum of this temporal behaviour is generally captured by the CMIP3 models, although the level of year-to-year autocorrelation varies according to the strength of the simulated ENSO and PDO (Stoner et al., 2009).

\subsection{Tropical ENSO teleconnections}

These moist teleconnection pathways involve mechanisms related to those at play in the precipitation response to global warming (Chiang and Sobel, 2002; Neelin et al., 2003) and provide challenging test statistics for model precipitation response. Compared to earlier generation climate models, CMIP3 and CMIP5 models tend to do somewhat better (Neelin, 2007; Cai et al., 2009; Coelho and Goddard, 2009; Langenbrunner and Neelin, 2013) at precipitation reductions associated with El Niño over equatorial South America and the Western Pacific, although CMIP5 offers little further improvement over CMIP3 (see for instance the standard deviation of precipitation in the western Pacific in Figure 9.36). CMIP5 models simulate the sign of the precipitation change over broad regions, and do well at predicting the amplitude of the change (for a given SST forcing) (Langenbrunner and Neelin, 2013).

A regression of the West African monsoon precipitation index with global SSTs reveals two major teleconnections (Fontaine and Janicot, 1996). The first highlights the strong influence of ENSO, while the second reveals a relationship between the SST in the Gulf of Guinea and the northward migration of the monsoon rain belt over West Africa. Most CMIP3 models show a single dominant Pacific teleconnection, which is, however, of the wrong sign for half of the models (Joly et al., 2007). Only one model shows a significant second mode, emphasizing the difficulty in simulating the response of the African rain belt to Atlantic SST anomalies that are not synchronous with Pacific anomalies.

Both CMIP3 and CMIP5 models have been evaluated and found to vary in their abilities to represent both the seasonal cycle of correlations between the Niño 3.4 and North Australian SSTs (Catto et al., 2012a, 2012 b) with little change in quality from CMIP3 to CMIP5. Generally the models do not capture the strength of the negative correlations during the second half of the year. The models also still struggle to capture the SST evolution in the North Australian region during El Niño and La Niña. Teleconnection patterns from both ENSO and the Indian Ocean Dipole to precipitation over Australia are reasonably well simulated in the key September-November season (Cai et al., 2009; Weller and Cai, 2013b) in the CMIP3 and CMIP5 multi-model mean. 


\subsubsection{Pacific Decadal Oscillation and Interdecadal Pacific Oscillation}

The Pacific Decadal Oscillation (PDO) refers to a mode of variability involving SST anomalies over the North Pacific (north of $20^{\circ} \mathrm{N}$ ) (Mantua et al., 1997). Although the PDO time series exhibits considerable decadal variability, it is difficult to ascertain whether there are any robust spectral peaks given the relatively short observational record (Minobe, 1997, 1999; Pierce, 2001; Deser et al., 2004). The ability of climate models to represent the PDO has been assessed by Stoner et al. (2009) and Furtado et al. (2011). Their results indicate that approximately half of the CMIP3 models simulate the observed spatial pattern and temporal behaviour (e.g., enhanced variance at low frequencies); however, spectral peaks are consistently higher in frequency than those suggested by the short observational record. The modelled PDO correlation with SST anomalies in the tropical Indo-Pacific are strongly underestimated by the CMIP3 models (Wang et al., 2010; Deser et al., 2011; Furtado et al., 2011; Lienert et al., 2011). Climate models have been shown to simulate features of the closely related Interdecadal Pacific Oscillation (IPO, based on SSTs over the entire Pacific basin; see Section 14.7.3; Power and Colman, 2006; Power et al., 2006; Meehl et al., 2009), although deficiencies remain in the strength of the tropicalextratropical connections.

\subsubsection{The Quasi-Biennial Oscillation}

Significant progress has been made in recent years to model and understand the impacts of the Quasi-Biennial Oscillation (QBO; Baldwin et al., 2001). Many climate models have now increased their vertical domain and/or improved their physical parameterizations (see Tables 9.1 and 9.A.1), and some of these reproduce a QBO (e.g.,
HadGEM2, MPI-ESM-LR, MIROC). Many features of the QBO such as its width and phase asymmetry also appear spontaneously in these simulations due to internal dynamics (Dunkerton, 1991; Scaife et al., 2002; Haynes, 2006). Some of the QBO effects on the extratropical climate (Holton and Tan, 1980; Hamilton, 1998; Naoe and Shibata, 2010) as well as ozone (Butchart et al., 2003; Shibata and Deushi, 2005) are also reproduced in models.

\subsubsection{Summary}

In summary, most modes of interannual to interdecadal variability are now present in climate models. As in AR4, their assessment presents a varied picture and CMIP5 models only show a modest improvement over CMIP3, mostly due to fewer poor-performing models. New since the AR4, process-based model evaluation is now helping identify sources of specific biases, although the observational record is often too short or inaccurate to offer strong constraints. The assessment of modes and patterns is summarized in Table 9.4.

\subsubsection{Extreme Events}

Extreme events are realizations of the tail of the probability distribution of weather and climate variability. They are higher-order statistics and thus generally more difficult to realistically represent in climate models. Shorter time scale extreme events are often associated with smaller scale spatial structure, which may be better represented as model resolution increases. In the AR4, it was concluded that models could simulate the statistics of extreme events better than expected from the generally coarse resolution of the models at that time, especially for temperature extremes (Randall et al., 2007).

Table 9.4 | Summary of assessment of interannual to interdecadal variability in climate models. See also Figure 9.44.

\begin{tabular}{|c|c|c|c|c|c|c|c|}
\hline & $\begin{array}{l}\text { Short } \\
\text { Name }\end{array}$ & $\begin{array}{l}\text { Level of } \\
\text { Confidence }\end{array}$ & $\begin{array}{l}\text { Level of } \\
\text { Evidence for } \\
\text { Evaluation }\end{array}$ & $\begin{array}{l}\text { Degree of } \\
\text { Agreement }\end{array}$ & $\begin{array}{l}\text { Model } \\
\text { Quality }\end{array}$ & $\begin{array}{l}\text { Difference with AR4 } \\
\text { (including CMIP5 } \\
\text { vs. CMIP3) }\end{array}$ & Section \\
\hline $\begin{array}{l}\text { Global sea surface tem- } \\
\text { perature (SST) variability }\end{array}$ & SST-var & High & Robust & Medium & Medium & Slight improvement in the tropics & 9.5 .3 .1 \\
\hline $\begin{array}{l}\text { North Atlantic Oscillation } \\
\text { and Northern Annular Mode }\end{array}$ & NAO & Medium & Medium & Medium & High & No assessment & 9.5 .3 .2 \\
\hline Southern Annular Mode & SAM & Low & Limited & Medium & Medium & No assessment & 9.5 .3 .2 \\
\hline $\begin{array}{l}\text { Atlantic Meridional Overturn- } \\
\text { ing Circulation Variability }\end{array}$ & AMOC-var & Low & Limited & Medium & Medium & No improvement & 9.5 .3 .3 \\
\hline $\begin{array}{l}\text { Atlantic Multi-decadal } \\
\text { Variability }\end{array}$ & AMO & Low & Limited & Medium & Medium & No improvement & 9.5 .3 .3 \\
\hline Atlantic Meridional Mode & AMM & High & Medium & High & Low & No assessment & 9.5 .3 .3 \\
\hline Atlantic Niño & AN & Low & Limited & Medium & Low & Slight improvement & 9.5 .3 .3 \\
\hline El Niño Southern Oscillation & ENSO & High & Medium & High & Medium & Slight improvement & 9.5 .3 .4 \\
\hline Indian Ocean Basin mode & $\mathrm{IOB}$ & Medium & Medium & Medium & High & Slight improvement & 9.5 .3 .4 \\
\hline Indian Ocean Dipole & IOD & Medium & Medium & Medium & Medium & No improvement & 9.5 .3 .4 \\
\hline Pacific North American & PNA & High & Medium & High & Medium & Slight improvement & 9.5 .3 .5 \\
\hline $\begin{array}{l}\text { Tropical ENSO tele- } \\
\text { connections }\end{array}$ & ENSOtele & High & Robust & Medium & Medium & No improvement & 9.5.3.5 \\
\hline Pacific Decadal Oscillation & PDO & Low & Limited & Medium & Medium & No assessment & 9.5 .3 .6 \\
\hline $\begin{array}{l}\text { Interdecadal Pacific } \\
\text { Oscillation }\end{array}$ & IPO & Low & Limited & Medium & High & No assessment & 9.5 .3 .6 \\
\hline Quasi-Biennial Oscillation & QBO & Medium & Medium & Medium & High & No assessment & 9.5.3.7 \\
\hline
\end{tabular}


The IPCC has conducted an assessment of extreme events in the context of climate change- - the Special Report on Managing the Risks of Extreme Events and Disasters to Advance Climate Change Adaptation (SREX) (IPCC, 2012). Although there is no comprehensive climate model evaluation with respect to extreme events in SREX, there is some consideration of model performance taken into account in assessing uncertainties in projections.

\subsubsection{Extreme Temperature}

Since the AR4, evaluation of CMIP3 and CMIP5 models has been undertaken with respect to temperature extremes. Both model ensembles simulate present-day warm extremes, in terms of 20-year return values, reasonably well, with errors typically within a few degrees Celsius over most of the globe (Kharin et al., 2007; Kharin et al., 2012). The CMIP5 and CMIP3 models perform comparably for various temperature extreme indices, but with smaller inter-model spread in CMIP5.The inter-model range of simulated indices is similar to the spread amongst observationally based estimates in most regions (Sillmann et al., 2013). Figure 9.37 shows relative error estimates of available CMIP5 models for various extreme indices based on Sillmann et al. (2013). Although the relative performance of an individual model may depend on the choice of the reference data set (four different reanalyses are used), the mean and median models tend to outperform individual models. According to the standardized multi-model median errors $\left(\mathrm{RMSE}_{\mathrm{std}}\right)$ for CMIP3 and CMIP5 shown on the right side of Figure 9.37, the performance of the two ensembles is similar.

In terms of historical trends, CMIP3 and CMIP5 models generally capture observed trends in temperature extremes in the second half of the 20th century (Sillmann et al., 2013), as illustrated in Figure 9.37. The modelled trends are consistent with both reanalyses and station-based estimates. It is also clear in the figure that model-based indices respond coherently to major volcanic eruptions. Detection and attribution studies based on CMIP3 models suggest that models tend to overestimate the observed warming of warm temperature extremes and underestimate the warming of cold extremes in the second half of 20th century (Christidis et al., 2011; Zwiers et al., 2011) as noted in SREX (Seneviratne et al., 2012). See also Chapter 10. This is not as obvious in the CMIP5 model evaluation (Figure 9.37 and Sillmann et al. (2013)) and needs further investigation.

\subsubsection{Extreme Precipitation}

For extreme precipitation, observational uncertainty is much larger than for temperature, making model evaluation more challenging. Discrepancies between different reanalyses for extreme precipitation are substantial, whereas station-based observations have limited spatial coverage (Kharin et al., 2007, 2012; Sillmann et al., 2013). Moreover, a station-based observational data set, which is interpolated from station measurements, has a potential mismatch of spatial scale when compared to model results or reanalyses (Chen and Knutson, 2008). Uncertainties are especially large in the tropics. In the extratropics, precipitation extremes in terms of 20-year return values simulated by CMIP3 and CMIP5 models compared relatively well with the observational data sets, with typical discrepancies in the $20 \%$ range (Kharin et al., 2007, 2012). Figure 9.37 shows relative errors of CMIP5 models for five precipitation-related indices. Darker grey shadings in the RMSE columns for precipitation indicate larger discrepancies between models and reanalyses for precipitation extremes in general. Sillmann et al. (2013) found that the CMIP5 models tend to simulate more intense precipitation and fewer consecutive wet days than the CMIP3, and thus are closer to the observationally based indices.

It is known from sensitivity studies that simulated extreme precipitation is strongly dependent on model resolution. Growing evidence has shown that high-resolution models ( $50 \mathrm{~km}$ or finer in the atmosphere) can reproduce the observed intensity of extreme precipitation (Wehner et al., 2010; Endo et al., 2012; Sakamoto et al., 2012), though some of these results are based on models with observationally constrained surface or lateral boundary conditions (i.e., Atmospheric General Circulation Models (AGCMs) or Regional Climate Models (RCMs)).

In terms of historical trends, a detection and attribution study by Min et al. (2011) found consistency in sign between the observed increase in heavy precipitation over NH land areas in the second half of the 20th century and that simulated by CMIP3 models, but they found that the models tend to underestimate the magnitude of the trend (see also Chapter 10). Related to this, it has been pointed out from comparisons to satellite-based data sets that the majority of models underestimate the sensitivity of extreme precipitation intensity to temperature in the tropics (Allan and Soden, 2008; Allan et al., 2010; O'Gorman, 2012) and globally (Liu et al., 2009; Shiu et al., 2012). O'Gorman (2012) showed that this implies possible underestimation of the projected future increase in extreme precipitation in the tropics.

\subsubsection{Tropical Cyclones}

It was concluded in the AR4 that high-resolution AGCMs generally reproduced the frequency and distribution, but underestimated intensity of tropical cyclones (Randall et al., 2007). Since then, Mizuta et al. (2012) have shown that a newer version of the MRI-AGCM with improved parameterizations (at $20 \mathrm{~km}$ horizontal resolution) simulates tropical cyclones as intense as those observed with improved distribution as well. Another remarkable finding since the AR4 is that the observed year-to-year count variability of Atlantic hurricanes can be well simulated by modestly high resolution (100 km or finer) AGCMs forced by observed SST, though with less skill in other basins (Larow et al., 2008; Zhao et al., 2009; Strachan et al., 2013). Vortices that have some characteristics of tropical cyclones can also be detected and tracked in AOGCMs in CMIP3 and 5, but their intensities are generally too weak (Yokoi et al., 2009a; Yokoi et al., 2012; Tory et al., 2013; Walsh et al., 2013).

\subsubsection{Droughts}

Drought is caused by long time scale (months or longer) variability of both precipitation and evaporation. Sheffield and Wood (2008) found that models in the CMIP3 ensemble simulated large-scale droughts in the 20th century reasonably well, in the sense that multi-model spread includes the observational estimate in each of several regions. However, it should be noted that there are various definitions of drought (see Chapter 2 and Seneviratne et al., 2012) and the performance of simulated drought can depend on the definition. Moreover, different models 
CMIP5 global land 1981-2000

(a)
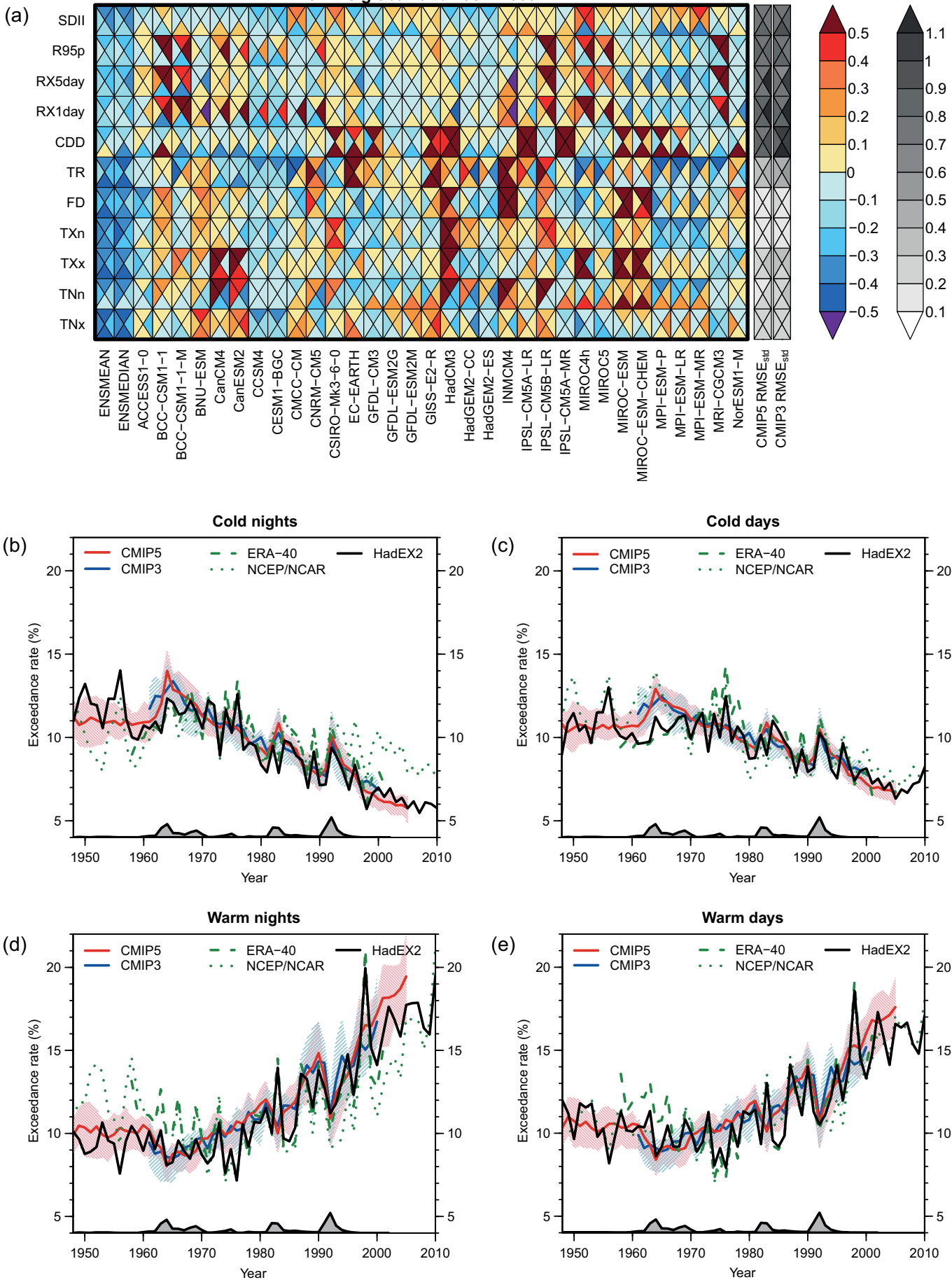

Figure 9.37 | (a) Portrait plot of relative error metrics for the CMIP5 temperature and precipitation extreme indices based on Sillmann et al. (2013). (b)-(e) Time series of global mean temperature extreme indices over land from 1948 to 2010 for CMIP3 (blue) and CMIP5 (red) models, ECMWF 40-year reanalysis (ERA40, green dashed) and National Centers for Environmental Prediction/National Center for Atmospheric Research (NCEP/NCAR, green dotted) reanalyses and HadEX2 station-based observational data set (black) based on Sillmann et al. (2013). In (a), reddish and bluish colours indicate, respectively, larger and smaller root-mean-square (RMS) errors for an individual model relative to the median model. The relative error is calculated for each observational data set separately. The grey-shaded columns on the right side indicate the RMS error for the multi-model median standardized by the spatial standard deviation of the index climatology in the reanalysis, representing absolute errors for CMIP3 and CMIP5 ensembles. Results for four different reference data sets, ERA-interim (top), ERA40 (left), NCEP/NCAR (right) and NCEP- Department of Energy (DOE) (bottom) reanalyses, are shown in each box. The analysis period is 1981-2000, and only land areas are considered. The indices shown are simple daily precipitation intensity index (SDII), very wet days (R95p), annual maximum 5-day/1-day precipitation (RX5day/ RX1day), consecutive dry days (CDD), tropical nights (TR), frost days (FD), annual minimum/maximum daily maximum surface air temperature (TXn/TXx), and annual minimum/ maximum daily minimum surface air temperature (TNn/TNx). See Box 2.4 for the definitions of indices. Note that only a small selection of the indices analysed in Sillmann et al. (2013) is shown, preferentially those that appear in Chapters 2, 10, 11, 12, 14. Also note that the NCEP/NCAR reanalysis has a known defect for TXx (Sillmann et al., 2013), but its impact on this figure is small. In (b)-(e), shading for model results indicates the 25th to 75th quantile range of inter-model spread. Grey shading along the horizontal axis indicates the evolution of globally averaged volcanic forcing according to Sato et al. (1993). The indices shown are the frequency of daily minimum/maximum surface air temperature below the 10th percentile (b: Cold nights/c: Cold days) and that above 90th percentile (d:Warm nights/e:Warm days) of the 1961-1990 base period. Note that, as these indices essentially represent changes relative to the base period, they are particularly suitable for being shown in time series and not straightforward for being shown in (a). 
can simulate drought with different mechanisms (McCrary and Randall, 2010; Taylor et al., 2012a). A comprehensive evaluation of CMIP5 models for drought is currently not available, although Sillmann et al. (2013) found that consecutive dry days simulated by CMIP5 models are comparable to observations in magnitude and distribution.

\subsubsection{Summary}

There is medium evidence (i.e., a few multi-model studies) and high agreement that the global distribution of temperature extremes are represented well by CMIP3 and CMIP5 models. The observed global warming trend of temperature extremes in the second half of the 20th century is reproduced in models, but there is medium evidence (a few CMIP3 studies) and medium agreement (not evident in a preliminary look at CMIP5) that models tend to overestimate the warming of warm temperature extremes and underestimate the warming of cold temperature extremes.

There is medium evidence (single multi-model study) and medium agreement (as inter-model difference is large) that CMIP5 models tend to simulate more intense and thus more realistic precipitation extremes than CMIP3, which could be partly due to generally higher horizontal resolution. There is medium evidence and high agreement that CMIP3 models tend to underestimate the sensitivity of extreme precipitation intensity to temperature. There is medium evidence and high agreement that high resolution (50 km or finer) AGCMs tend to simulate the intensity of extreme precipitation comparable to observational estimates.

There is medium evidence and high agreement that year-to-year count variability of Atlantic hurricanes can be well simulated by modestly high resolution (100 km or finer) AGCMs forced by observed SSTs. There is medium evidence and medium agreement (as inter-model difference is large) that the intensity of tropical cyclones is too weak in CMIP3 and CMIP5 models. Finally, there is medium evidence (a few multi-model studies) and medium agreement (as it might depend on definitions of drought) that models can simulate aspects of largescale drought.

\section{Box 9.3 | Understanding Model Performance}

This Box provides a synthesis of findings on understanding model performance based on the model evaluations discussed in this chapter.

\section{Uncertainty in Process Representation}

Some model errors can be traced to uncertainty in representation of processes (parameterizations). Some of these are long-standing issues in climate modelling, reflecting our limited, though gradually increasing, understanding of very complex processes and the inherent challenges in mathematically representing them. For the atmosphere, cloud processes, including convection and its interaction with boundary layer and larger-scale circulation, remain major sources of uncertainty (Section 9.4.1). These in turn cause errors or uncertainties in radiation which propagate through the coupled climate system. Distribution of aerosols is also a source of uncertainty arising from modelled microphysical processes and transport (Sections 9.4.1 and 9.4.6). Ocean models are subject to uncertainty in parameterizations of vertical and horizontal mixing and convection (Sections 9.4.2, 9.5.2 and 9.5.3), and ocean errors in turn affect the atmosphere through resulting SST biases. Simulation of sea ice is also affected by errors in both the atmosphere and the ocean as well as the parameterization of sea ice itself (Section 9.4.3). With respect to biogeochemical components in Earth System Models (ESMs), parameterizations of nitrogen limitation and forest fires are thought to be important for simulating the carbon cycle, but very few ESMs incorporate these so far (Sections 9.4.4 and 9.4.5).

\section{Error Propagation}

Causes of one model bias can sometimes be associated with another. Although the root cause of those biases is often unclear, knowledge of the causal chain or a set of interrelated biases can provide a key to further understanding and improvement of model performance. For example, biases in storm track position are partly due to a SST biases in the Gulf Stream and Kuroshio Current (Section 9.4.1). Some biases in variability or trend can be partly traced back to biases in mean states. The decreasing trend in September Arctic ice extent tends to be underestimated when sea ice thickness is overestimated (Section 9.4.3). In such cases, improvement of the mean state may improve simulated variability or trend.

\section{Sensitivity to Resolution}

Some phenomena or aspects of climate are found to be better simulated with models run at higher horizontal and/or vertical resolution. In particular, increased resolution in the atmosphere has improved, at least in some models, storm track and extratropical cyclones (Section 9.4.1), diurnal variation of precipitation over land (Section 9.5.2), extreme precipitation, and tropical cyclone intensity and structure (Section 9.5.4). Similarly, increased horizontal resolution in the ocean is shown to improve sea surface height variability, western boundary currents, tropical instability waves and coastal upwelling (Section 9.4.2), and variability of Atlantic meridional overturning circulation (Section 9.5.3). High vertical resolution and a high model top, as well as high horizontal resolution, are important for simulating lower stratospheric climate variability (Section 9.4.1), blocking (Section 9.5.2), the Quasi-Biennial Oscillation and the North Atlantic Oscillation (Section 9.5.3). (continued on next page) 
Box 9.3 (continued)

\section{Uncertainty in Observational Data}

In some cases, insufficient length or quality of observational data makes model evaluation challenging, and is a frequent problem in the evaluation of simulated variability or trends. This is evident for evaluation of upper tropical tropospheric temperature, tropical atmospheric circulation (Section 9.4.1), the Atlantic meridional overturning circulation, the North Atlantic Oscillation and the Pacific Decadal Oscillation (Section 9.5.3). Data quality has been pointed out as an issue for arctic cloud properties (Section 9.4.1), ocean heat content, heat and fresh water fluxes over the ocean (Section 9.4.2) and extreme precipitation (Section 9.5.4). Palaeoclimate reconstructions also have large inherent uncertainties (Section 9.5.2). It is clear therefore that updated or newly available data affect model evaluation conclusions.

\section{Other Factors}

Model evaluation can be affected by how models are forced. Uncertainties in specified greenhouse gases, aerosols emissions, land use change, etc. will all affect model results and hence evaluation of model quality. Different statistical methods used in model evaluation can also lead to subtle or substantive differences in the assessment of model quality.

\subsection{Downscaling and Simulation of Regional-Scale Climate}

Regional-scale climate information can be obtained directly from global models; however, their horizontal resolution is often too low to resolve features that are important at regional scales. High-resolution AGCMs, variable-resolution global models, and statistical and dynamical downscaling (i.e., regional climate modelling) are used to complement AOGCMs, and to generate region-specific climate information. These approaches are evaluated in the following.

\subsubsection{Global Models}

\subsubsection{Regional-Scale Simulation by Atmosphere-Ocean General Circulation Models}

A comparison of CMIP3 and CMIP5 seasonal cycles of temperature and precipitation for different regions (Figure 9.38) shows that temperature is generally better simulated than precipitation in terms of the amplitude and phase of the seasonal cycle. The multi-model mean is closer to observations than most of the individual models. The systematic difference between the CMIP5 and CMIP3 ensembles is small in most regions, although there is evident improvement in South Asia (SAS) and Tropical South America (TSA) in the rainy seasons. In some cases the spread amongst observational estimates can be of comparable magnitude to the model spread, e.g., winter in the Europe and Mediterranean (EUM) region.

There are as yet rather few published studies in which regional behaviour of the CMIP5 models is evaluated in great detail. Cattiaux et al. (2013) obtained results for Europe similar to those discussed above. Joetzjer et al. (2013) considered 13 models that participated in both CMIP3 and in CMIP5 and found that the seasonal cycle of precipitation over the Amazon improved in the latter.

Based on the CMIP archives, regional biases in seasonal and annual mean temperature and precipitation are shown for several land regions in Figure 9.39, and for polar and oceanic regions in Figure 9.40. The CMIP5 median temperature biases range from about $-3^{\circ} \mathrm{C}$ to $1.5^{\circ} \mathrm{C}$. Substantial cold biases over $\mathrm{NH}$ regions are more prevalent in winter (December to February) than summer (June to August). The median biases appear in most cases slightly less negative for CMIP5 than CMIP3. The spread amongst models, as characterized by the 25 to $75 \%$ and 5 to $95 \%$ ranges, is slightly reduced from CMIP3 to CMIP5 in a majority of the regions and is roughly $\pm 3^{\circ} \mathrm{C}$. The RMS error of individual CMIP5 models is smaller than that for CMIP3 in 24 of the 26 regions in Figure 9.39 in DJF, JJA and the annual mean. The absolute value of the ensemble mean bias has also been reduced in most cases. The inter-model spread remains large, particularly in high-latitude regions in winter and in regions with steep orography (such as CAS, SAS, TIB and WSA). The inter-model temperature spread has decreased from CMIP3 to CMIP5 over most of the oceans and over the Arctic and Antarctic land regions. The cold winter bias over the Arctic has been reduced. There is little systematic inter-ensemble difference in temperature over lower latitude oceans.

Biases in seasonal total precipitation are shown in the right column of Figures 9.39 and 9.40 for the NH winter (October to March) and summer (April to September) half years as well as the annual mean. The largest systematic biases over land regions occur in ALA, WSA and $\mathrm{TIB}$, where the annual precipitation exceeds that observed in all CMIP5 models, with a median bias on the order of $100 \%$. All these regions are characterized by high orography and / or a large fraction of solid precipitation, both of which are expected to introduce a negative bias in gauge-based precipitation (Yang and Ohata, 2001; Adam et al., 2006) that may amplify the model-observation discrepancy. A large negative relative bias in SAH occurs in October to March, but it is of negligible magnitude in absolute terms. In nearly all other seasonal and regional cases over land, the observational estimate falls within the range of the CMIP5 simulations. Compared with CMIP3, the CMIP5 median precipitation is slightly higher in most regions. In contrast with temperature, the seasonal and annual mean ensemble mean and the rootmean square precipitation biases are larger for CMIP5 than for CMIP3 in a slight majority of land regions (Figure 9.39) and in most of the 
(a) Temperature $\left({ }^{\circ} \mathrm{C}\right)$
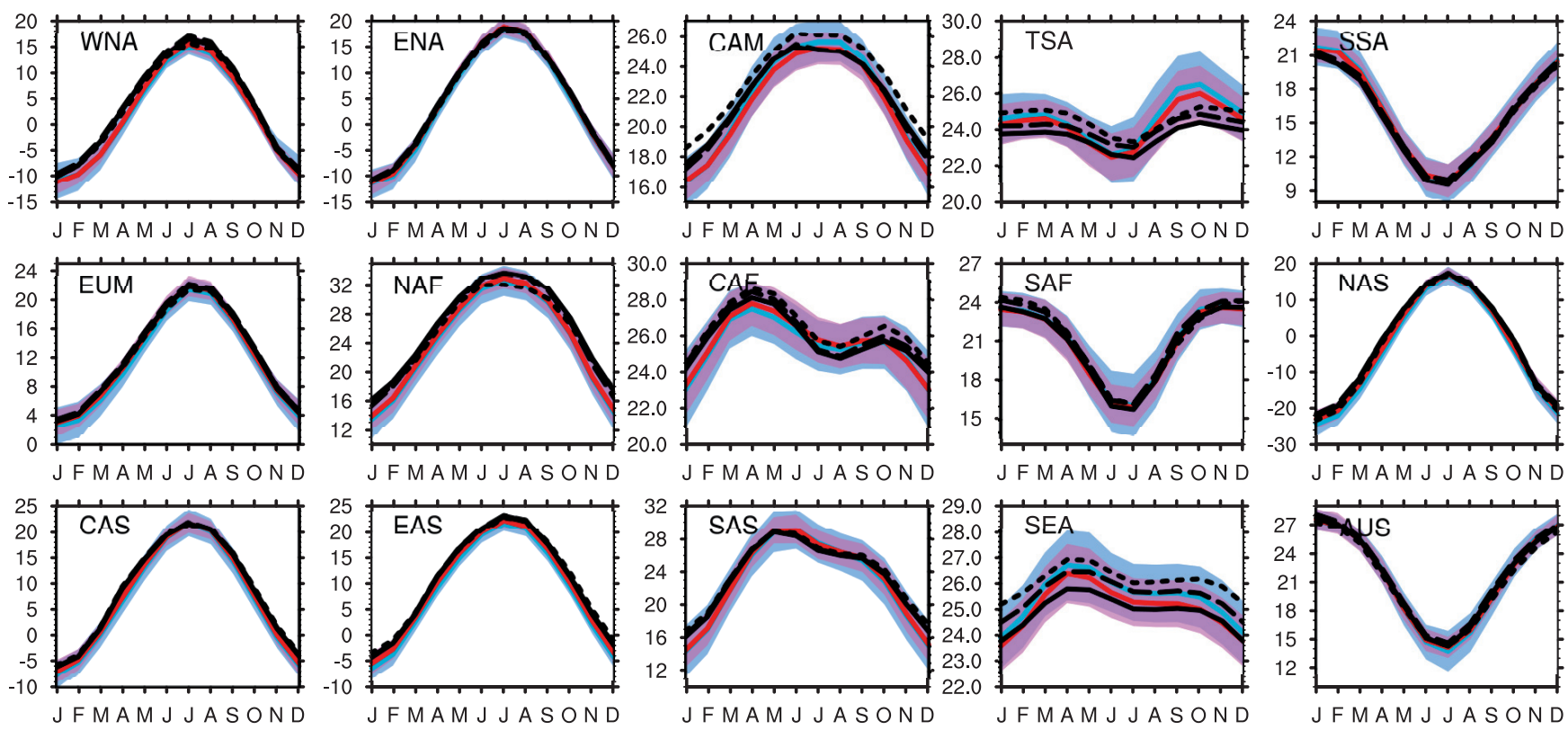

(b) Precipitation $\left(\mathrm{mm}\right.$ day $\left.^{-1}\right)$
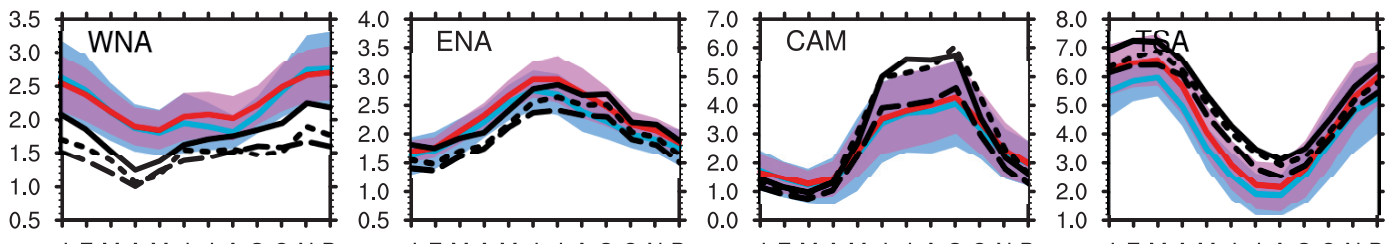

JFMAM J JASOND
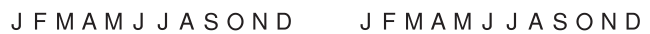

JFMAM J JASOND
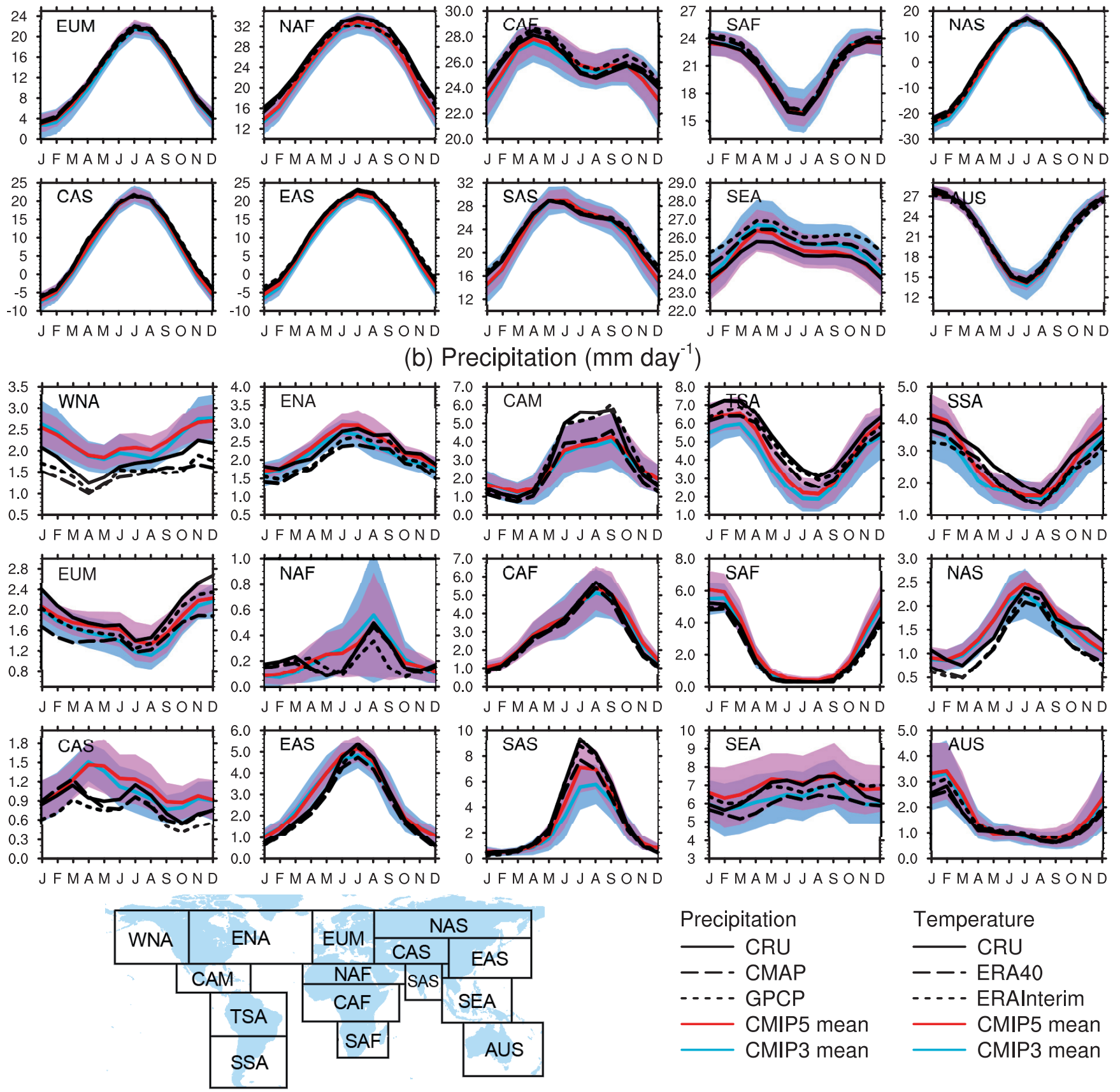

Precipitation

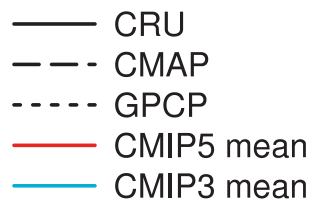

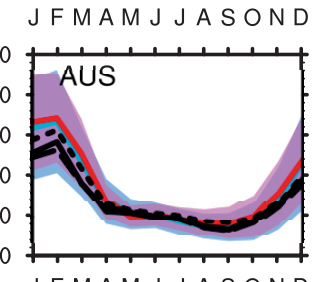

Temperature

CRU

- - ERA40

..... ERAInterim - CMIP5 mean - CMIP3 mean

Figure 9.38 Mean seasonal cycle of $(\mathrm{a})$ temperature $\left({ }^{\circ} \mathrm{C}\right)$ and $(\mathrm{b})$ precipitation $\left(\mathrm{mm}\right.$ day $\left.{ }^{-1}\right)$. The average is taken over land areas within the indicated regions, and over the period 1980-1999. The red line is the average over 45 CMIP5 models; the blue line is the average over 22 CMIP3 models. The standard deviation of the respective data set is indicated with shading. The different line styles in black refer to observational and reanalysis data: Climatic Research Unit (CRU) TS3.10, ECMWF 40-year reanalysis (ERA40) and ERA-Interim for temperature; CRU TS3.10.1, Global Precipitation Climatology Project (GPCP), and CPC Merged Analysis of Precipitation (CMAP) for precipitation. Note the different axis-ranges for some of the sub-plots. The 15 regions shown are: Western North America (WNA), Eastern North America (ENA), Central America (CAM), Tropical South America (TSA), Southern South America (SSA), Europe and Mediterranean (EUM), North Africa (NAF), Central Africa (CAF), South Africa (SAF), North Asia (NAS), Central Asia (CAS), East Asia (EAS), South Asia (SAS), Southeast Asia (SEA) and Australia (AUS). 
14 other regions (Figure 9.40). However, considering the observational uncertainty, the performance of the CMIP3 and CMIP5 ensembles is assessed to be broadly similar. The inter-model spreads are similar and typically largest in arid areas when expressed in relative terms.
Especially over the oceans and polar regions (Figure 9.40), the scarcity of observations and their uncertainty complicates the evaluation of simulated precipitation. Of two commonly used data sets, CMAP indicates systematically more precipitation than GPCP over low-latitude
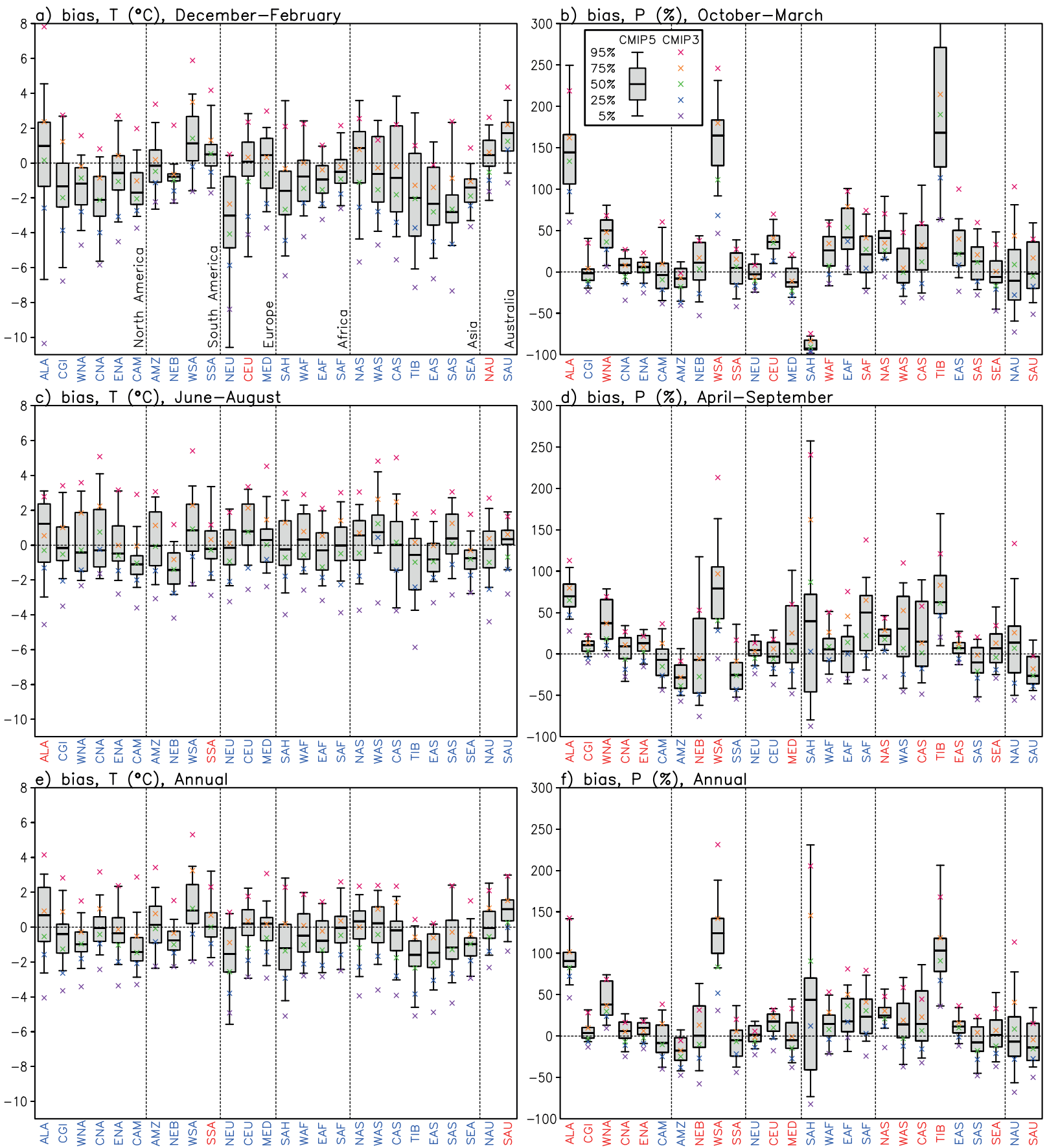

Figure 9.39 | Seasonal- and annual mean biases of (left) temperature $\left({ }^{\circ} \mathrm{C}\right)$ and (right) precipitation (\%) in the IPCC Special Report on Managing the Risks of Extreme Events and Disasters to Advance Climate Change Adaptation (SREX) land regions (cf. Seneviratne et al., 2012, p. 12. The region's coordinates can be found from their online Appendix 3.A). The 5th, 25th, 50th, 75th and 95th percentiles of the biases in 42 CMIP5 models are shown in box-and-whisker format, and corresponding values for 23 CMIP3 models with crosses. The CMIP3 20C3M simulations are complemented with the corresponding A1B runs for the 2001-2005 period. The biases are calculated over 1986-2005, using Climatic Research Unit (CRU) T3.10 as the reference for temperature and CRU TS 3.10.01 for precipitation. The regions are labelled with red when the root-mean-square error for the individual CMIP5 models is larger than that for CMIP3 and blue when it is smaller. The regions are: Alaska/NW Canada (ALA), Eastern Canada/Greenland/Iceland (CGI), Western North America (WNA), Central North America (CNA), Eastern North America (ENA), Central America/Mexico (CAM), Amazon (AMZ), NE Brazil (NEB), West Coast South America (WSA), SouthEastern South America (SSA), Northern Europe (NEU), Central Europe (CEU), Southern Europe/the Mediterranean (MED), Sahara (SAH), Western Africa (WAF), Eastern Africa (EAF), Southern Africa (SAF), Northern Asia (NAS), Western Asia (WAS), Central Asia (CAS), Tibetan Plateau (TIB), Eastern Asia (EAS), Southern Asia (SAS), Southeast Asia (SEA), Northern Australia (NAS) and Southern Australia/New Zealand (SAU). Note that the region WSA is poorly resolved in the models. 

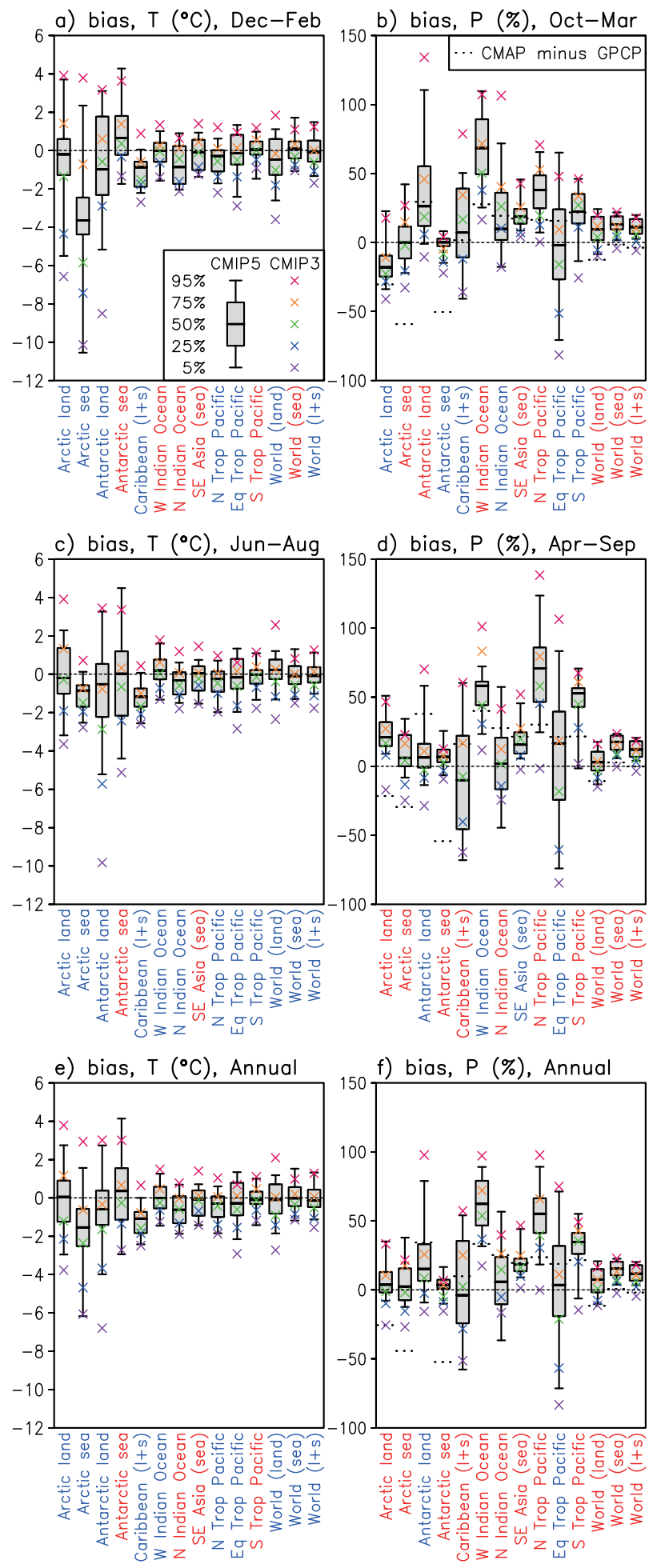

Figure 9.40 | As Figure 9.39, but for polar and ocean regions, with ECMWF reanalysis of the global atmosphere and surface conditions (ERA)-Interim reanalysis as the reference for temperature and Global Precipitation Climatology Project (GPCP) for precipitation. Global land, ocean and overall means are also shown. The regions are: Arctic: 67.5 to $90^{\circ} \mathrm{N}$, Caribbean (area defined by the following coordinates): $68.8^{\circ} \mathrm{W}, 11.4^{\circ} \mathrm{N}$; $85.8^{\circ} \mathrm{W}, 25^{\circ} \mathrm{N} ; 60^{\circ} \mathrm{W}, 25^{\circ} \mathrm{N}, 60^{\circ} \mathrm{W}, 11.44^{\circ} \mathrm{N}$; Western Indian Ocean: $25^{\circ} \mathrm{S}$ to $5^{\circ} \mathrm{N}, 52^{\circ} \mathrm{E}$ to $75^{\circ} \mathrm{E}$; Northern Indian Ocean: $5^{\circ} \mathrm{N}$ to $30^{\circ} \mathrm{N}, 60^{\circ} \mathrm{E}$ to $95^{\circ} \mathrm{E}$; Northern Tropical Pacific: $5^{\circ} \mathrm{N}$ to $25^{\circ} \mathrm{N}, 155^{\circ} \mathrm{E}$ to $150^{\circ} \mathrm{W}$; Equatorial Tropical Pacific: $5^{\circ} \mathrm{S}$ to $5^{\circ} \mathrm{N}, 155^{\circ} \mathrm{E}$ to $130^{\circ} \mathrm{W}$; Southern Tropical Pacific: $5^{\circ} \mathrm{S}$ to $25^{\circ} \mathrm{S}, 155^{\circ} \mathrm{E}$ to $130^{\circ} \mathrm{W}$; Antarctic: $50^{\circ} \mathrm{S}$ to $90^{\circ} \mathrm{S}$. The normalized difference between CPC Merged Analysis of Precipitation (CMAP) and GPCP precipitation is shown with dotted lines. oceans and less over many high-latitude regions (Yin et al., 2004; Shin et al., 2011). Over most low-latitude ocean regions, annual precipitation in most CMIP3 and CMIP5 models exceeds GPCP. The difference relative to CMAP is smaller although mostly of the same sign. In Arctic and Antarctic Ocean areas, simulated precipitation is much above CMAP, but more similar to GPCP. Over Antarctic land, precipitation in most models is below CMAP, but close to or above GPCP.

Continental to sub-continental mean values may not be representative for smaller-scale biases, as biases generally increase with decreasing spatial averaging (Masson and Knutti, 2011b; Raisanen and Ylhaisi, 2011). A typical order of magnitude for grid-box-scale annual mean biases in individual CMIP3 models was $2^{\circ} \mathrm{C}$ for temperature and $1 \mathrm{~mm}$ day $^{-1}$ for precipitation (Raisanen, 2007; Masson and Knutti, 2011b), with some geographical variation. This has been noted also in studies on how much spatial averaging would be needed in order to filter out the most unreliable small-scale features (e.g., Räisänen and Ylhäisi, 2011). In order to reduce such errors while still retaining information on small scales, Masson and Knutti (2011b) found, depending on the variable and the region, that smoothing needed to vary from the gridpoint scale to around $2000 \mathrm{~km}$.

On the whole, based on analysis of both ensemble means and inter-model spread, there is high confidence that the CMIP5 models simulate regional-scale temperature distributions somewhat better than the CMIP3 models did. This improvement is evident for most regions. For precipitation, there is medium confidence that there is no systematic change in model performance. In many regions, precipitation biases relative to CRU TS 3.10 .01 and GPCP (and CMAP) are larger for CMIP5 than for CMIP3, but given observational uncertainty, the two ensembles are broadly similar.

\subsubsection{Regional-Scale Simulation by Atmospheric General Circulation Models}

Stand-alone global atmospheric models (AGCMs) run at higher resolution than AOGCMs provide complementary regional-scale climate information, sometimes referred to as 'global downscaling'. One important example of this is the simulation of tropical cyclones (e.g., Zhao et al., 2009, 2012; Murakami and Sugi, 2010; Murakami et al., 2012). A number of advantages of high-resolution AGCMs have been identified, including improved regional precipitation (Zhao et al., 2009; Kusunoki et al., 2011) and blocking (Matsueda et al., 2009, 2010). As AGCMs do not simulate interactions with the ocean, their ability to capture some high-resolution phenomena, such as the cold wake in the surface ocean after a tropical cyclone, is limited (e.g., Hasegawa and Emori, 2007). As in lower-resolution models, performance is affected by the quality of physical parameterizations (Lin et al., 2012; Mizuta et al., 2012; Zhao et al., 2012).

\subsubsection{Regional-Scale Simulation by Variable-Resolution Global Climate Models}

An alternative to global high resolution is the use of variable resolution (so-called 'stretched grid') models with higher resolution over the region of interest. Some examples are Abiodun et al. (2011) who showed that such simulations improve the simulation of West African 
monsoon systems and African easterly jets, and White et al. (2013) who demonstrated improvements in temperature and precipitation related extreme indices. Fox-Rabinovitz et al. (2008) showed that regional biases in the high-resolution portion of a stretched grid model were similar to that of a global model with the same high resolution everywhere. Markovic et al. (2010) and Déqué (2010) reported similar results. Although not widely used, such methods can complement more conventional climate models.

\subsubsection{Regional Climate Downscaling}

Regional Climate Models (RCMs) are applied over a limited-area domain with boundary conditions either from global reanalyses or global climate model output. The use of RCMs for 'dynamical downscaling' has grown since the AR4, their resolution has increased, process-descriptions have developed further, new components have been added, and coordinated experimentation has become more widespread (Laprise, 2008; Rummukainen, 2010). Statistical downscaling (SD) involves deriving empirical relationships linking large-scale atmospheric variables (predictors) and local/regional climate variables (predictands). These relationships may then be applied to equivalent predictors from global models. SD methods have also been applied to RCM output (e.g., Boe et al., 2007; Déqué, 2007; Segui et al., 2010; Paeth, 2011; van Vliet et al., 2011). A significant constraint in a comprehensive evaluation of regional downscaling is that available studies often involve different methods, regions, periods and observational data for evaluation. Thus, evaluation results are difficult to generalize.

\subsubsection{Recent Developments of Statistical Methods}

The development of SD since the AR4 has been quite vigorous (e.g., Fowler et al., 2007; Maraun et al., 2010b), and many state-of-the-art approaches combine different methods (e.g., Vrac and Naveau, 2008; van Vliet et al., 2011). There is an increasing number of studies on extremes (e.g., Vrac and Naveau, 2008; Wang and Zhang, 2008), and on features such as hurricanes (Emanuel et al., 2008), river flow and discharge, sediment, soil erosion and crop yields (e.g., Zhang, 2007; Prudhomme and Davies, 2009; Lewis and Lamoureux, 2010). Techniques have also been developed to consider multiple climatic variables simultaneously in order to preserve some physical consistency (e.g., Zhang and Georgakakos, 2011). The methods used to evaluate SD approaches vary with the downscaled variable and include metrics related to intensities (e.g., Ning et al., 2011; Tryhorn and DeGaetano, 2011), temporal behaviour (e.g., May, 2007; Timbal and Jones, 2008; Maraun et al., 2010a; Brands et al., 2011), and physical processes (Lenderink and Van Meijgaard, 2008; Maraun et al., 2010a). SD capabilities are also examined through secondary variables like runoff, river discharge and stream flow (e.g., Boe et al., 2007; Teutschbein et al., 2011).

\subsubsection{Recent Developments of Dynamical Methods}

Since the AR4, typical RCM resolution has increased from around 50 $\mathrm{km}$ to around $25 \mathrm{~km}$ (e.g., Christensen et al., 2010). Long RCM runs at very high resolution are still, however, rather few (e.g., Yasutaka et al., 2008; Chan et al., 2012; Kendon et al., 2012). Coupled RCMs, with interactive ocean and, when appropriate, also sea ice have also been developed (Somot et al., 2008; Dorn et al., 2009; Artale et al., 2010;
Doscher et al., 2010). Smith et al. (2011a) added vegetation dynamicsecosystem biogeochemistry in an RCM.

At the time of the AR4, RCMs were typically used for time-slice experiments. Since then, multi-decadal and centennial RCM simulations have emerged in larger numbers (e.g., Diffenbaugh et al., 2011; Kjellstrom et al., 2011; de Elia et al., 2013). Coordinated RCM experiments and ensembles have also become much more common and today, with domains covering Europe (e.g., Christensen et al., 2010; Vautard et al., 2013), North America (e.g., Gutowski et al., 2010; Lucas-Picher et al., 2012a; Mearns et al., 2012), South America (e.g., Menendez et al., 2010; Chou et al., 2012; Krüger et al., 2012), Africa (e.g., Druyan et al., 2010; Ruti et al., 2011; Nikulin et al., 2012; Paeth et al., 2012; Hernández-Díaz et al., 2013), the Arctic (e.g., Inoue et al., 2006) and Asian regions (e.g., Feng and Fu, 2006; Shkolnik et al., 2007; Feng et al., 2011; Ozturk et al., 2012; Suh et al., 2012).

\subsubsection{Skill of Downscaling Methods}

Downscaling skill varies with location, season, parameter and boundary conditions (see Section 9.6.5) (e.g., Schmidli et al., 2007; Maurer and Hidalgo, 2008). Although there are indications that model skill increases with higher resolution, it does not do so linearly. Rojas (2006) found more improvement when increasing resolution from $135 \mathrm{~km}$ to $45 \mathrm{~km}$ than from $45 \mathrm{~km}$ to $15 \mathrm{~km}$. Walther et al. (2013) found that the diurnal precipitation cycle and light precipitation improved more when going from $12 \mathrm{~km}$ to $6 \mathrm{~km}$ resolution than when going from $50 \mathrm{~km}$ to $25 \mathrm{~km}$ or from $25 \mathrm{~km}$ to $12 \mathrm{~km}$. Higher resolution does enable better simulation of extremes (Seneviratne et al., 2012). For example, Pryor et al. (2012) noted that an increase in RCM resolution from $50 \mathrm{~km}$ to 6 $\mathrm{km}$ increased extreme wind speeds more than the mean wind speed. Kawazoe and Gutowski (2013) compared six RCMs and the two GCMs to high resolution observations, concluding that precipitation extremes were more representative in the RCMs than in the GCMs. Vautard et al. (2013) found that warm extremes in Europe were generally better simulated in RCMs with $12 \mathrm{~km}$ resolution compared to $50 \mathrm{~km}$. Kendon et al. (2012) and Chan et al. (2012) found mixed results in daily precipitation simulated at $12 \mathrm{~km}$ and $1.5 \mathrm{~km}$ resolution, although the latter had improved sub-daily features, perhaps as convection could be explicitly resolved.

Coupled RCMs, with an interactive ocean, offer further improvements. Döscher et al. (2010) reproduced empirical relationships between Arctic sea ice extent and sea ice thickness and NAO in a coupled RCM. Zou and Zhou (2013) found that a regional ocean-atmosphere model improved the simulation of precipitation over the western North Pacific compared to an uncoupled model. Samuelsson et al. (2010) showed that coupling a lake model with an RCM captured the effect of lakes on the air temperature over adjacent land. Lenaerts et al. (2012) added drifting snow in an RCM run for the Antarctica, which increased the area of ablation and improved the fit to observations. Smith et al. (2011a) added vegetation dynamics-ecosystem biogeochemistry into an RCM, and found some evidence of local feedback to air temperature.

Applying an RCM developed for a specific region to other regions exposes it to a wider range of conditions and therefore provides an 
opportunity for more rigorous evaluation. Transferability experiments target this by running RCMs for different regions while holding their process-descriptions constant (cf. Takle et al., 2007; Gbobaniyi et al., 2011; Jacob et al., 2012). Suh et al. (2012) noted that 10 RCMs run for Africa did well overall for average and maximum temperature, but systematically overestimated the daily minimum temperature. Precipitation was generally simulated betted for wet regions than for dry regions. Similarly, Nikulin et al. (2012) reported on 10 RCMs over Africa, run with boundary conditions from ERA-Interim, and evaluated against different observational data sets. Many of the RCMs simulated precipitation better than the ERA-Interim reanalysis itself.

Christensen et al. (2010) examined a range metrics related to simulation of extremes, mesoscale features, trends, aspects of variability and consistency with the driving boundary conditions. Only one of these metrics led to clear differentiation among RCMs (Lenderink, 2010). This may imply a general skilfulness of models, but may also simply indicate that the metrics were not very informative. Nevertheless, using some of these metrics, Coppola et al. (2010) and Kjellström et al. (2010) found that weighted sets of RCMs outperformed sets without weighting for both temperature and precipitation. Sobolowski and Pavelsky (2012) demonstrated a similar impact.

\subsubsection{Value Added through RCMs}

RCMs are regularly tested to evaluate whether they show improvements over global models (Laprise et al., 2008), that is, whether they do indeed 'add value'. In essence, added value is a measure of the extent to which the downscaled climate is closer to observations than the model from which the boundary conditions were obtained. Differences between RCM and GCM simulations are not always very obvious for time-averaged quantities on larger scales or in fairly homogeneous regions. RCM fields are, however, richer in spatial and temporal detail. Indeed, the added value of RCMs is mainly expected in the simulation of topography-influenced phenomena and extremes with relatively small spatial or short temporal character (e.g., Feser et al., 2011; Feser and Barcikowska, 2012; Shkol'nik et al., 2012). As an example, RCM downscaling led to better large-scale monsoon precipitation patterns (Gao et al., 2012) for East Asia than in the global models used for boundary conditions. In the few instances where RCMs have been interactively coupled to global models (i.e., 'two-way' coupling), the effects of improved small scales propagate to larger scales and this has been found to improve the simulation of larger scale phenomena (Lorenz and Jacob, 2005; Inatsu and Kimoto, 2009; Inatsu et al., 2012).

Other examples include improved simulation of convective precipitation (Rauscher et al., 2010), near-surface temperature (Feser, 2006), near-surface temperature and wind (Kanamaru and Kanamitsu, 2007), temperature and precipitation (Lucas-Picher et al., 2012b), extreme precipitation (Kanada et al., 2008), coastal climate features (Winterfeldt and Weisse, 2009; Winterfeldt et al., 2011; Kawazoe and Gutowski, 2013; Vautard et al., 2013), Atlantic hurricanes (Bender et al., 2010), European storm damage (Donat et al., 2010), strong mesoscale cyclones (Cavicchia and Storch, 2011), cutoff lows (Grose et al., 2012), polar lows (Zahn and von Storch, 2008) and higher statistical moments of the water budget (e.g., Bresson and Laprise, 2011).
In summary, there is high confidence that downscaling adds value to the simulation of spatial climate detail in regions with highly variable topography (e.g., distinct orography, coastlines) and for mesoscale phenomena and extremes. Regional downscaling is therefore complementary to results obtained directly from global climate models. These results are from a variety of distinct studies with different RCMs.

\subsubsection{Sources of Model Errors and Uncertainties}

In addition to issues related to resolution and model complexity (see Section 9.6.3), errors and uncertainties arise from observational uncertainty in evaluation data and parameterizations (see Box 9.3), choice of model domain and application of boundary conditions (driving data).

In the case of SD, sources of model errors and uncertainties depend on the choice of method, including the choice of the predictors, the estimation of empirical relationships between predictors and predictands from limited data sets, and also the data used to estimate the predictors (Frost et al., 2011). There are numerous different SD methods, and the findings are difficult to generalize.

Small domains allow less freedom for RCMs to generate the small-scale features that give rise to added value (e.g., Leduc and Laprise, 2009). Therefore large domains -covering entire continents- have become more common. Køltzow et al. (2008) found improvements with the use of a larger domain, but the RCM solution can become increasingly 'decoupled' from the driving data (e.g., Rockel et al., 2008), which can introduce inconsistencies. Large domains also introduce large internal variability, which can significantly contaminate interannual variability of seasonal means (Kanamitsu et al., 2010). Techniques such as spectral nudging (Misra, 2007; Separovic et al., 2012) can be used to constrain such inconsistencies (Feser et al., 2011). Winterfeldt and Weisse (2009) concluded that nudging improved the simulation of marine wind climate, while Otte et al. (2012) demonstrated improvements in temperature and precipitation. Nudging may, however, also lead to deterioration of features such as precipitation extremes (Alexandru et al., 2009; Kawazoe and Gutowski, 2013). Veljovic et al. (2010) showed that an RCM can in fact improve the large scales with respect to those inherent in the boundary conditions, and argued that nudging may be undesirable.

The quality of RCM results may vary according to the synoptic situation, season, and the geographic location of the lateral boundaries (Alexandru et al., 2007; Xue et al., 2007; Laprise et al., 2008; Separovic et al., 2008; Leduc and Laprise, 2009; Nikiema and Laprise, 2010; Rapaić et al., 2010). In addition to lateral boundary conditions, RCMs also need sea surface information. Few studies have explored the dependency of RCM results on the treatment of the SSTs and sea ice, although Koltzow et al. (2011) found that the specification of SSTs was less influential than was the domain or the lateral boundaries. Woollings et al. (2010a) investigated the effect of specified SST on the simulation of the Atlantic storm track and found that it was better simulated with high-resolution SSTs, whereas increasing temporal resolution gave mixed results.

As is the case in global models, RCM errors are directly related to shortcomings in process parameterizations. Examples include the 
representation of clouds, convection and land surface-atmosphere interactions, the planetary boundary layer, horizontal diffusion, and microphysics (Tjernstrom et al., 2008; Wyser et al., 2008; Lynn et al., 2009; Pfeiffer and Zängl, 2010; Axelsson et al., 2011; Crétat et al., 2012; Evans et al., 2012; Roy et al., 2012; Solman and Pessacg, 2012). The representation of land surface and atmosphere coupling is also important, particularly for simulating monsoon regions (Cha et al., 2008; Yhang and Hong, 2008; Boone et al., 2010; Druyan et al., 2010; van den Hurk and van Meijgaard, 2010).

\subsubsection{Relating Downscaling Performance to Credibility of Regional Climate Information}

A fundamental issue is how the performance of a downscaling method relates to its ability to provide credible future projections (Raisanen, 2007). This subject is discussed further in Section 9.8. The credibility of downscaled information of course depends on the quality of the downscaling method itself (e.g., Dawson et al., 2012; Déqué et al., 2012; Eum et al., 2012), and on the quality of the global climate models providing the large-scale boundary conditions (e.g., van Oldenborgh et al., 2009; Diaconescu and Laprise, 2013).

Specific to SD is the statistical stationarity hypothesis, that is, that the relationships inferred from historical data remain valid under a changing climate (Maraun, 2012). Vecchi et al. (2008) note that a statistical method that captures interannual hurricane variability gives very different results for projections compared to RCMs. Such results suggest that good performance of statistical downscaling as assessed against observations does not guarantee credible regional climate information. Some recent studies have proposed ways to evaluate SD approaches using RCM outputs (e.g., Vrac and Naveau, 2008; Driouech et al., 2010) or long series of observations (e.g. Schmith, 2008).

Giorgi and Coppola (2010) argued that regional-scale climate projections over land in the CMIP3 models were not sensitive to their temperature biases. For precipitation, the same was found for about two thirds of the global land area. However, there is some recent evidence that regional biases may be nonlinear for temperature extremes (Christensen et al., 2008; Boberg and Christensen, 2012; Christensen and Boberg, 2013) in both global and regional models. A mechanism at play may be that models tend to dry out the soil too effectively at high temperatures, which can lead to systematic biases in projected warm summertime conditions (Christensen et al., 2008; Kostopoulou et al., 2009). This is illustrated in Figure 9.41 for the Mediterranean region, which suggests a tendency in RCMs, CMIP3 and CMIP5 models towards an enhanced warm bias in the warmer months. The implication is that the typically large warming signal in these regions could be biased (Boberg and Christensen, 2012; Mearns et al., 2012). Findings such as these stress the importance of a thorough assessment of models' biases when they are applied for projections (e.g., de Elia and Cote, 2010; Boberg and Christensen, 2012; Christensen and Boberg, 2013).

Di Luca et al. (2012) analysed downscaled climate change projections from six RCMs run over North America. The climate change signals for seasonal precipitation and temperature were similar to those in the driving AOGCMs, and the spatial detail gained by downscaling was comparable in both present and future climate. Déqué et al. (2012) studied projections with several combinations of AOGCM and RCM for Europe. A larger part of the spread in winter temperature and (a) RCM

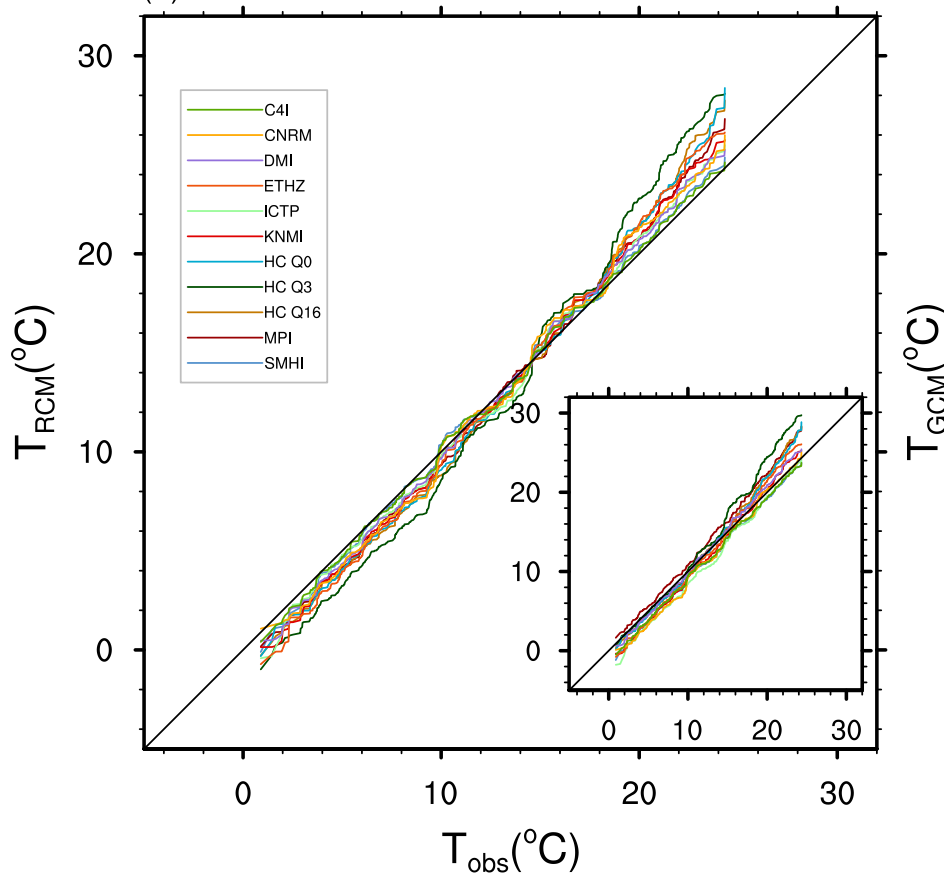

(b) CMIP5 (color) \& CMIP3 (grey)

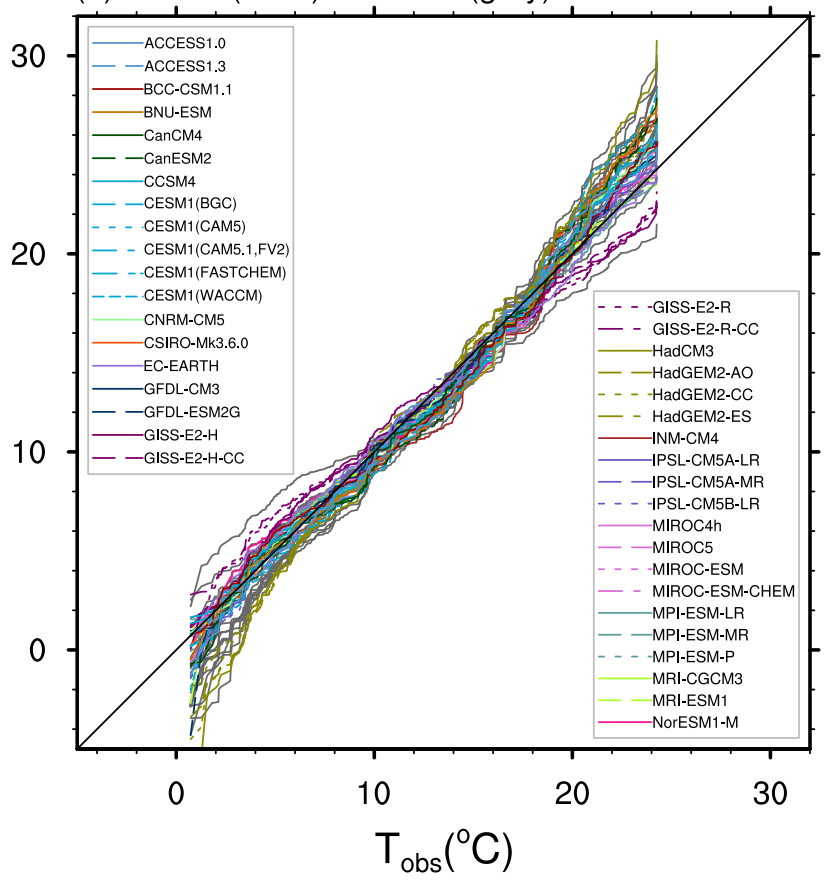

Figure 9.41 | Ranked modelled versus observed monthly mean temperature for the Mediterranean region for the 1961-2000 period. The Regional Climate Model (RCM) data (a) are from Christensen et al. (2008) and are adjusted to get a zero mean in model temperature with respect to the diagonal. The smaller insert shows uncentred data. The General Circulation Model (GCM) data (b) are from CMIP5 and CMIP3 and adjusted in the same way. (After Boberg and Christensen, 2012.) 
precipitation projections was explained by the differences in global model boundary conditions, although much of the spread in projected summer precipitation was explained by RCM. This underlines the importance of both the quality of the boundary conditions and the downscaling method.

\subsection{Climate Sensitivity and Climate Feedbacks}

An overall assessment of climate sensitivity and transient climate response is given in Box 12.2. Observational constraints based on observed warming over the last century are discussed in Section 10.8.2 and shown in Box 12.2, Figure 2.

\subsubsection{Equilibrium Climate Sensitivity, Idealized Radiative Forcing, and Transient Climate Response in the Coupled Model Intercomparison Project Phase 5 Ensemble}

Equilibrium climate sensitivity (ECS) is the equilibrium change in global and annual mean surface air temperature after doubling the atmospheric concentration of $\mathrm{CO}_{2}$ relative to pre-industrial levels. In the AR4, the range in equilibrium climate sensitivity of the CMIP3 models was $2.1^{\circ} \mathrm{C}$ to $4.4^{\circ} \mathrm{C}$, and the single largest contributor to this spread was differences among modelled cloud feedbacks. These assessments carry over to the CMIP5 ensemble without any substantial change (Table 9.5).

The method of diagnosing climate sensitivity in CMIP5 differs fundamentally from the method employed in CMIP3 and assessed in the AR4 (Randall et al., 2007). In CMIP3, an AGCM was coupled to a non-dynamic mixed-layer (slab) ocean model with prescribed ocean heat transport convergence. $\mathrm{CO}_{2}$ concentration was then instantaneously doubled, and the model was integrated to a new equilibrium with unchanged implied ocean heat transport. While computationally efficient, this method had the disadvantage of employing a different model from that used for the historical simulations and climate projections. However, in the few comparisons that were made, the resulting disagreement in ECS was less than about 10\% (Boer and Yu, 2003; Williams et al., 2008; Danabasoglu and Gent, 2009; Li et al., 2013a). In CMIP5, climate sensitivity is diagnosed directly from the AOGCMs following the approach of Gregory et al. (2004). In this case the $\mathrm{CO}_{2}$ concentration is instantaneously quadrupled and kept constant for 150 years of simulation, and both equilibrium climate sensitivity and RF are diagnosed from a linear fit of perturbations in global mean surface temperature to the instantaneous radiative imbalance at the TOA.

The transient climate response (TCR) is the change in global and annual mean surface temperature from an experiment in which the $\mathrm{CO}_{2}$ concentration is increased by $1 \% \mathrm{yr}^{-1}$, and calculated using the difference between the start of the experiment and a 20 -year period centred on the time of $\mathrm{CO}_{2}$ doubling. TCR is smaller than ECS because ocean heat uptake delays surface warming. TCR is linearly correlated with ECS in the CMIP5 ensemble (Figure 9.42), although the relationship may be nonlinear outside the range spanned in Table 9.5 (Knutti et al., 2005).

Based on the methods outlined above and explained in Section 9.7.2 below, Table 9.5 shows effective ERF, ECS, TCR and feedback strengths for the CMIP5 ensemble. The two estimates of ERF agree with each other to within $5 \%$ for six models (CanESM2, INM-CM4, IPSL-CM5A-LR, MIROC5, MPI-ESM-LR and MPI-ESM-P), although the deviation exceeds $10 \%$ for four models (CCSM4, CSIRO-Mk3-6-0, HadGEM2-ES, and MRI-CGCM3) and is indicative of deviations from the basic assumptions underlying one or both ERF estimation methods. However, the mean difference of $0.3 \mathrm{~W} \mathrm{~m}^{-2}$ between the two methods for diagnosing ERF is only about half of the ensemble standard deviation of $0.5 \mathrm{~W} \mathrm{~m}^{-2}$, or $15 \%$ of the mean value for ERF by $\mathrm{CO}_{2}$ using fixed SSTs. ECS and TCR vary across the ensemble by a factor of approximately 2 . The multi-model ensemble mean in ECS is $3.2^{\circ} \mathrm{C}$, a value nearly identical to that for CMIP3, while the CMIP5 ensemble range is $2.1^{\circ} \mathrm{C}$ to $4.7^{\circ} \mathrm{C}$, a spread which is also nearly indistinguishable from that for CMIP3. While every CMIP5 model whose heritage can
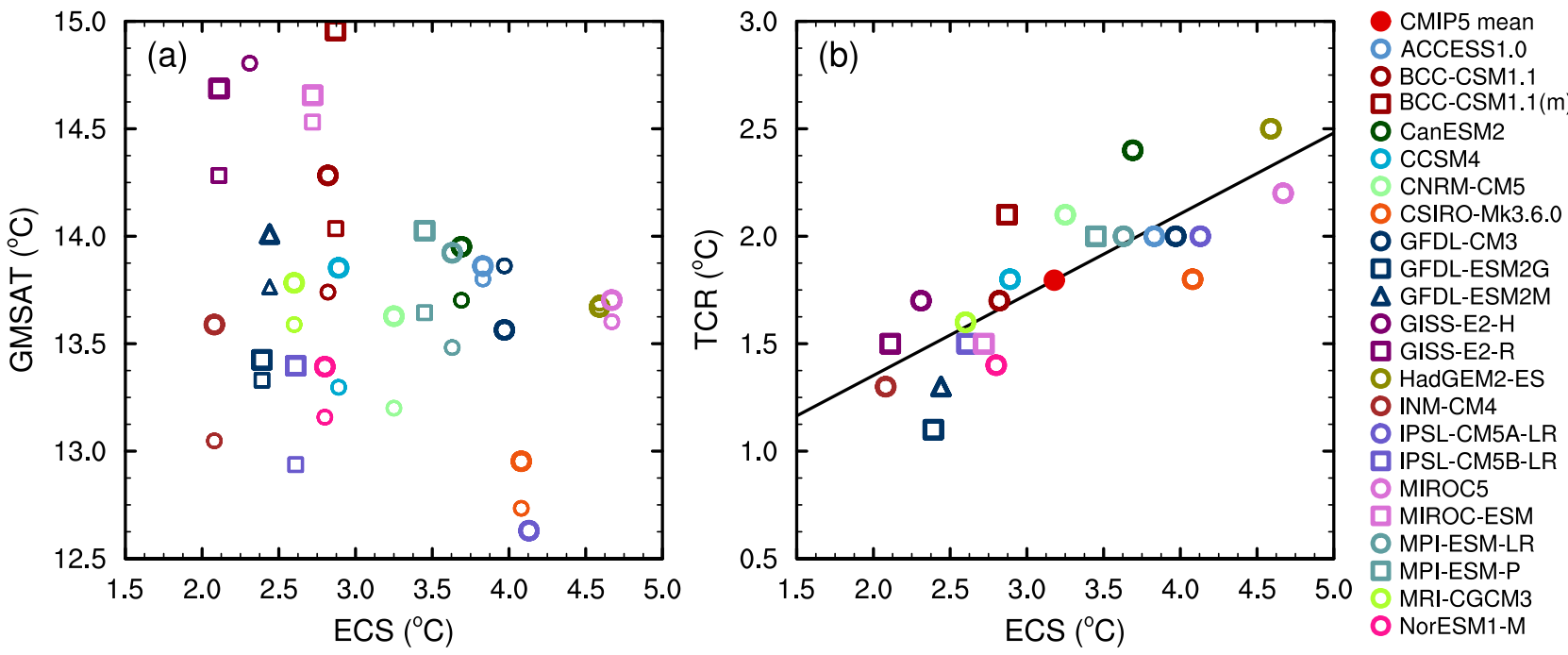

Figure 9.42 | (a) Equilibrium climate sensitivity (ECS) against the global mean surface temperature of CMIP5 models, both for the period $1961-1990$ (larger symbols, cf. Figure 9.8, Table 9.5) and for the pre-industrial control runs (smaller symbols). (b) Equilibrium climate sensitivity against transient climate response (TCR). The ECS and TCR information are based on Andrews et al. (2012) and Forster et al. (2013) and updated from the CMIP5 archive. 


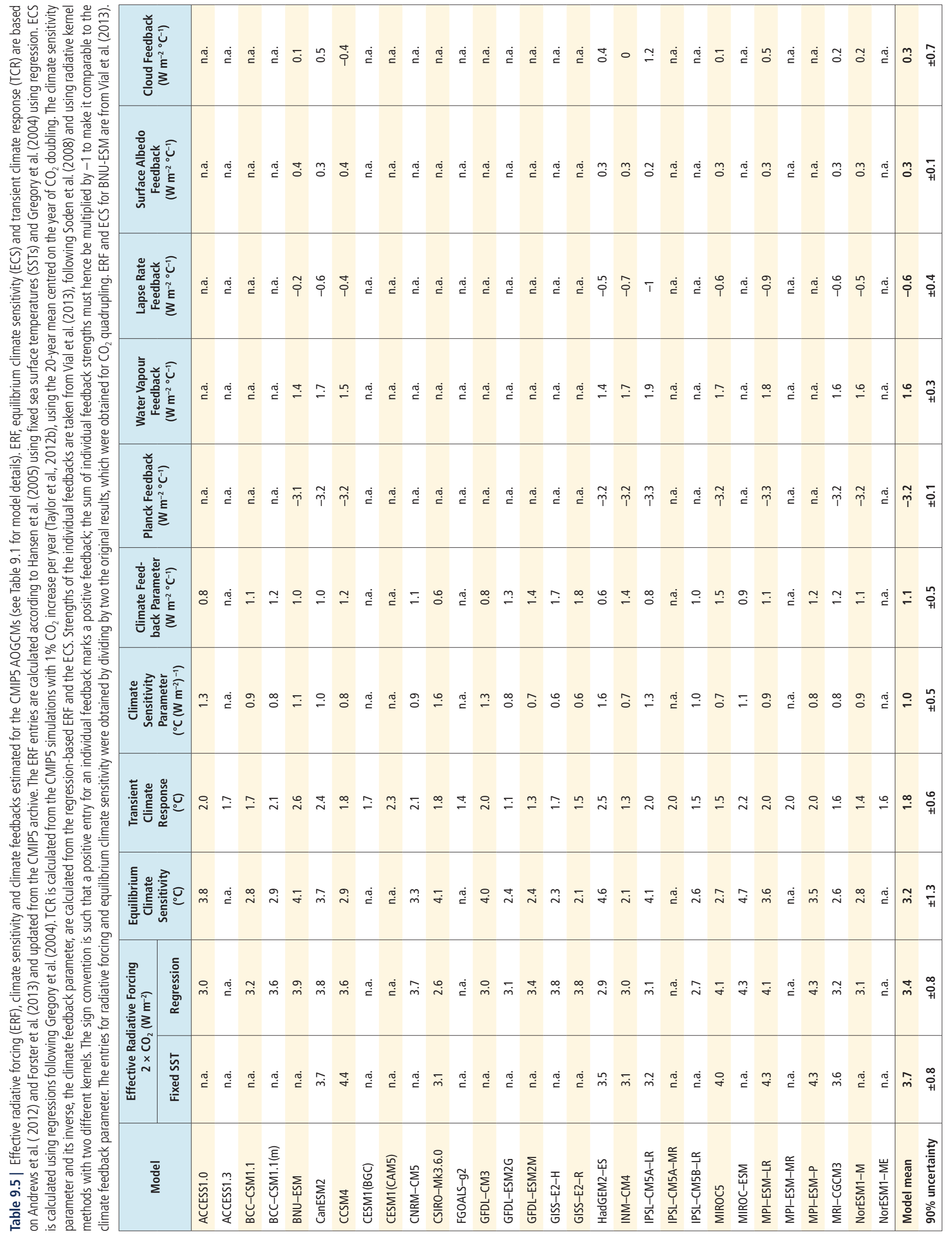



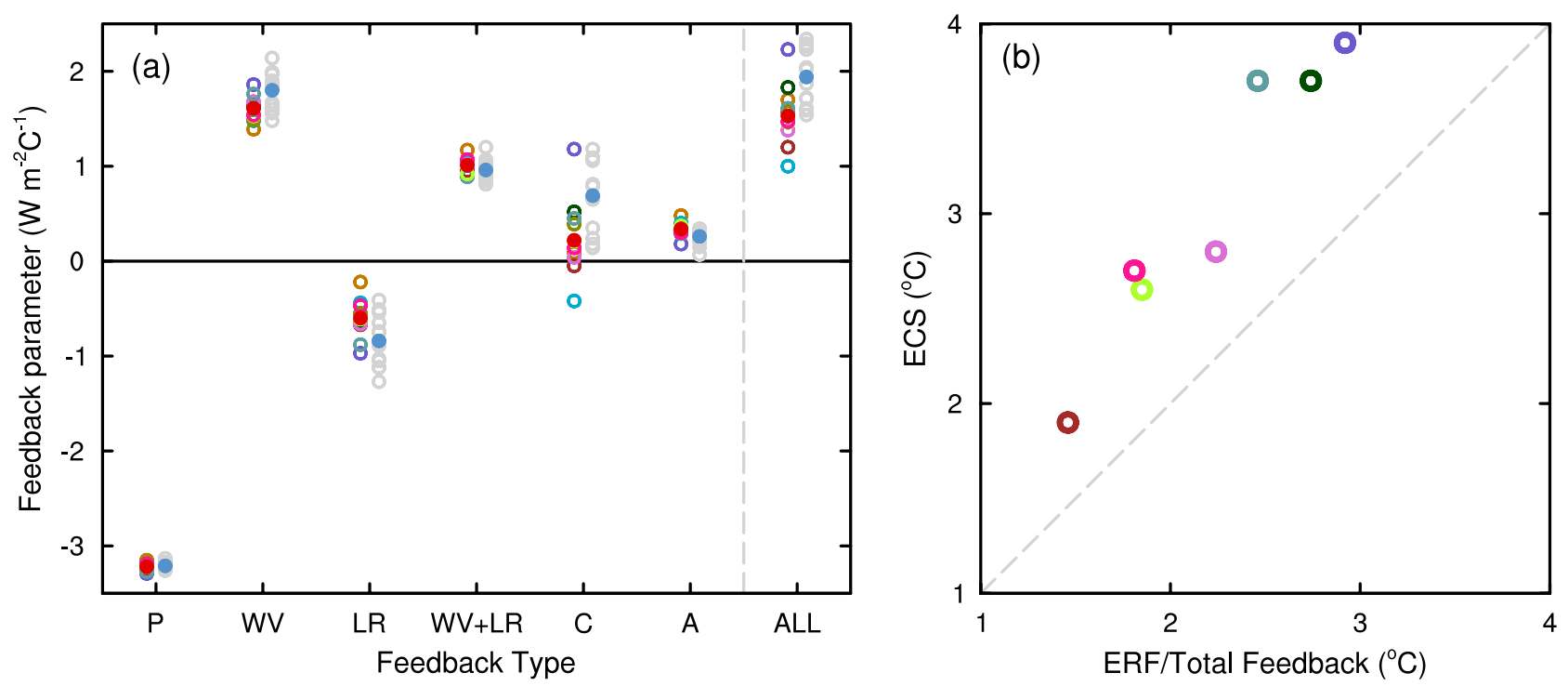

\begin{tabular}{|c|c|c|c|c|c|c|}
\hline CMIP5 mean & CMIP3 mean & CMIP3 models & OBNU-ESM & - CanESM2 & occsm4 & OHadGEM2 \\
\hline OINM-CM4 & OIPSL-CM5A-LR & MIROC5 & OMPI-ESM-LR & MRI-CGCM3 & ONorESM1-M & \\
\hline
\end{tabular}

Figure 9.43 | (a) Strengths of individual feedbacks for CMIP3 and CMIP5 models (left and right columns of symbols) for Planck (P), water vapour (WV), clouds (C), albedo (A), lapse rate (LR), combination of water vapour and lapse rate (WV+LR) and sum of all feedbacks except Planck (ALL), from Soden and Held (2006) and Vial et al. (2013), following Soden et al. (2008). CMIP5 feedbacks are derived from CMIP5 simulations for abrupt fourfold increases in $\mathrm{CO}_{2}$ concentrations $\left(4 \times \mathrm{CO}_{2}\right)$. (b) ECS obtained using regression techniques by Andrews et al. (2012) against ECS estimated from the ratio of $\mathrm{CO}_{2}$ ERF to the sum of all feedbacks. The $\mathrm{CO}_{2}$ ERF is one-half the $4 \times \mathrm{CO}_{2}$ forcings from Andrews et al. (2012), and the total feedback (ALL + Planck) is from Vial et al. (2013).

be traced to CMIP3 shows some change in ECS, there is no discernible systematic tendency. This broad similarity between CMIP3 and CMIP5 and the good agreement between different methods where they were applied to the same atmospheric GCM indicate that the uncertainty in methodology is minor compared to the overall spread in ECS. The change in TCR from CMIP3 to CMIP5 is generally of the same sign but of smaller magnitude compared to the change in ECS. The relationship between ECS and an estimates derived from total feedbacks are discussed in Section 9.7.2.

Although ECS can vary with global mean surface temperature owing to the temperature dependencies of the various feedbacks (Colman and McAvaney, 2009; cf. Section 9.7.2), Figure 9.42 shows no discernible correlation for the CMIP5 historical temperature ranges, a fact that suggests that ECS is less sensitive to errors in the current climate than to other sources of uncertainty.

\subsubsection{Understanding the Range in Model Climate Sensitivity: Climate Feedbacks}

The strengths of individual feedbacks for the CMIP3 and CMIP5 models are compared in Figure 9.43. The feedbacks are generally similar between CMIP3 and CMIP5, and the water vapour, lapse rate, and cloud feedbacks are assessed in detail in Chapter 7. The surface albedo feedback is assessed here to be likely positive. There is high confidence that the sum of all feedbacks (excluding the Planck feedback) is positive. Advances in estimating and understanding each of the feedback parameters in Table 9.5 are described in detail below (see also Chapters 7 and 8).

\subsubsection{Role of Humidity and Lapse Rate Feedbacks in Climate Sensitivity}

The compensation between the water vapour and lapse-rate feedbacks noted in the CMIP3 models is still present in the CMIP5 models, and possible explanations of the compensation have been developed (Ingram, 2010; Ingram, 2013). New formulations of the feedbacks, replacing specific with relative humidity, eliminate most of the cancellation between the water vapour and lapse rate feedbacks and reduce the inter-model scatter in the individual feedback terms (Held and Shell, 2012).

\subsubsection{Role of Surface Albedo in Climate Sensitivity}

Analysis of observed declines in sea ice and snow coverage from 1979 to 2008 suggests that the $\mathrm{NH}$ albedo feedback is between 0.3 and 1.1

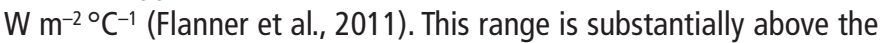
global feedback of $0.3 \pm 0.1 \mathrm{~W} \mathrm{~m}^{-2}{ }^{\circ} \mathrm{C}^{-1}$ of the CMIP5 models analysed in Table 9.5. One possible explanation is that the CMIP5 models underestimate the strength of the feedback as did the CMIP3 models based upon the systematic errors in simulated sea ice coverage decline relative to observed rates (Boe et al., 2009b).

\subsubsection{Role of Cloud Feedbacks in Climate Sensitivity}

Cloud feedbacks represent the main cause for the range in modelled climate sensitivity (Chapter 7). The spread due to inter-model differences in cloud feedbacks is approximately 3 times larger than the spread contributed by feedbacks due to variations in water vapour and lapse 
rate combined (Dufresne and Bony, 2008), and is a primary factor governing the range of climate sensitivity across the CMIP3 ensemble (Volodin, 2008a). Differences in equilibrium and effective climate sensitivity are due primarily to differences in the shortwave cloud feedback (Yokohata et al., 2008).

In perturbed ensembles of three different models, the primary cloud-related factor contributing to the spread in equilibrium climate sensitivity is the low-level shortwave cloud feedback (Yokohata et al., 2010; Klocke et al., 2011). Changes in the high-altitude clouds also induce climate feedbacks due to the large areal extent and significant longwave cloud radiative effects of tropical convective cloud systems. In experiments with perturbed physics ensembles of $\mathrm{AOGCMs}$, the parameterization of ice fall speed also emerges as one of the most important determinants of climate sensitivity (Sanderson et al., 2008a, 2010; Sexton et al., 2012). Other non-microphysical feedback mechanisms are detailed in Chapter 7.

Cloud feedbacks in AOGCMs are generally positive or near neutral (Shell et al., 2008; Soden et al., 2008), as evidenced by the net positive or neutral cloud feedbacks in all of the models examined in a multi-thousand member ensemble of AOGCMs constructed by parameter perturbations (Sanderson et al., 2010). The sign of cloud feedbacks in the current climate deduced from observed relationships between SSTs and TOA radiative fluxes are discussed further in Section 7.2.5.7.

\subsubsection{Relationship of Feedbacks to Modelled Climate Sensitivity}

The ECS can be estimated from the ratio of forcing to the total climate feedback parameter. This approach is applicable to simulations in which the net radiative balance is much smaller than the forcing and hence the modelled climate system is essentially in equilibrium. This approach can also serve to check the internal consistency of estimates of the ECS, forcing, and feedback parameters obtained using independent methods. The relationship between ECS from Andrews et al. (2012) and estimates of ECS obtained from the ratio of forcings to feedbacks is shown in Figure 9.43b. The forcings are estimated using both regression and fixed SST techniques (Gregory et al., 2004; Hansen et al., 2005) by Andrews et al. (2012) and the feedbacks are calculated using radiative kernels (Soden et al., 2008). On average, the ECS from forcing to feedback ratios underestimate the ECS from Andrews et al. (2012) by $25 \%$ and $35 \%$, or up to $50 \%$ for individual models, using fixed-SST and regression forcings, respectively.

\subsubsection{Relationship of Feedbacks to Uncertainty in Modelled Climate Sensitivity}

Objective methods for perturbing uncertain model parameters to optimize performance relative to a set of observational metrics have shown a tendency toward an increase in the mean and a narrowing of the spread of estimated climate sensitivity (Jackson et al., 2008a). This tendency is opposed by the effects of structural biases related to incomplete process representations in GCMs. If common structural biases are replicated across models in a MME (cf. Section 9.2.2.7), the most likely sensitivity for the MME tends to shift towards lower sensitivities while the possibility of larger sensitivities increases at the same time (Lemoine, 2010). Following Schlesinger and Mitchell (1987), Roe and Baker (2007) suggest that symmetrically distributed uncertainties in feedbacks lead to inherently asymmetrical uncertainties in climate sensitivity with increased probability in extreme positive values of the sensitivity. Roe and Baker (2007) conclude that this relationship makes it extremely difficult to reduce uncertainties in climate sensitivity through incremental improvements in the specification of feedback parameters. While subsequent analysis has suggested that this finding could be an artifact of the statistical formulation (Hannart et al., 2009) and linearization (Zaliapin and Ghil, 2010) of the relationship between feedback and sensitivity adopted by (Roe and Baker, 2007), these issues remain unsettled (Roe and Armour, 2011; Roe and Baker, 2011).

\subsubsection{Climate Sensitivity and Model Performance}

Despite the range in equilibrium sensitivity of $2.1^{\circ} \mathrm{C}$ to $4.4^{\circ} \mathrm{C}$ for CMIP3 models, they reproduce the global surface air temperature anomaly of $0.76^{\circ} \mathrm{C}$ over $1850-2005$ to within $25 \%$ relative error. The relatively small range of historical climate response suggests that there is another mechanism, for example a compensating non-GHG forcing, present in the historical simulations that counteracts the relatively large range in sensitivity obtained from idealized experiments forced only by increasing $\mathrm{CO}_{2}$. One possible mechanism is a systematic negative correlation across the multi-model ensemble between ECS and anthropogenic aerosol forcing (Kiehl, 2007; Knutti, 2008; Anderson et al., 2010). A second possible mechanism is a systematic overestimate of the mixing between the oceanic mixed layer and the full depth ocean underneath (Hansen et al., 2011). However, despite the same range of ECS in the CMIP5 models as in the CMIP3 models, there is no significant relationship across the CMIP5 ensemble between ECS and the 20th-century ERF applied to each individual model (Forster et al., 2013). This indicates a lesser role of compensating ERF trends from GHGs and aerosols in CMIP5 historical simulations than in CMIP3. Differences in ocean heat uptake also do not appreciably affect the spread in projected changes in global mean temperature by 2095 (Forster et al., 2013).

\subsubsection{Constraints on Climate Sensitivity from Earth System Models of Intermediate Complexity}

An EMIC intercomparison (Eby et al., 2013; Zickfeld et al., 2013) allows an assessment of model response characteristics, including ECS, TCR, and heat uptake efficiency (Table 9.6). In addition, Bayesian methods applied to PPE experiments using EMICs have estimated uncertainty in model response characteristics (see Box 12.2) based on simulated climate change in 20th century, past millennia, and LGM scenarios. Here, the range of response metrics (Table 9.6) described for default model configurations (Eby et al., 2013) indicates consistency with the CMIP5 ensemble.

\subsubsection{Climate Sensitivity During the Last Glacial Maximum}

Climate sensitivity can also be explored in another climatic context. The AR4 assessed attempts to relate simulated LGM changes in tropical SST to global climate sensitivity (Hegerl et al., 2007; Knutti and Hegerl, 2008). LGM temperature changes in the tropics (Hargreaves et al., 2007), but not in Antarctica (Hargreaves et al., 2012), have been 
Table 9.6 Model response metrics for EMICs in Table 9.2. $\mathrm{TCR}_{2 x_{1}} \mathrm{TCR}_{4 \mathrm{x}}$ and $\mathrm{ECS}_{4 \mathrm{x}}$ are the changes in global average model surface air temperature from the decades centred at years 70,140 and 995 respectively, from the idealized $1 \%$ increase to $4 \times \mathrm{CO}_{2}$ experiment. The ocean heat uptake efficiency, $\mathrm{\kappa}_{4 x}$, is calculated from the global average heat flux divided by $\mathrm{TCR}_{4 x}$ for the decade centred at year 140 , from the same idealized experiment. $\mathrm{ECS}_{2 \times}$ was calculated from the decade centred about year 995 from a $2 \times \mathrm{CO}_{2}$ pulse experiment. (Data from Eby et al., 2013.)

\begin{tabular}{|c|c|c|c|c|c|}
\hline Model & $\operatorname{TCR}_{2 x}\left({ }^{\circ} \mathrm{C}\right)$ & $\mathrm{ECS}_{2 \mathrm{x}}\left({ }^{\circ} \mathrm{C}\right)$ & $\operatorname{TCR}_{4 \mathrm{X}}\left({ }^{\circ} \mathrm{C}\right)$ & $\mathrm{ECS}_{4 \mathrm{X}}\left({ }^{\circ} \mathrm{C}\right)$ & $\kappa_{4 \mathrm{X}}\left(\mathrm{W} \mathrm{m}^{-2}{ }^{\circ} \mathrm{C}^{-1}\right)$ \\
\hline Bern3D & 2.0 & 3.3 & 4.6 & 6.8 & 0.58 \\
\hline CLIMBER2 & 2.1 & 3.0 & 4.7 & 5.8 & 0.84 \\
\hline DCESS & 2.1 & 2.8 & 3.9 & 4.8 & 0.72 \\
\hline FAMOUS & 2.3 & 3.5 & 5.2 & 8.0 & 0.55 \\
\hline IAP RAS CM & 1.6 & - & 3.7 & 4.3 & - \\
\hline IGSM2 & 1.5 & 1.9 & 3.7 & 4.5 & - \\
\hline LOVECLIM1.2 & 1.2 & 2.0 & 2.1 & 3.5 & 1.17 \\
\hline MESMO & 2.4 & 3.7 & 5.3 & 6.9 & 0.55 \\
\hline UMD & 1.6 & 2.2 & 3.2 & 4.3 & - \\
\hline Uvic & 1.9 & 3.5 & 4.3 & 6.6 & 0.92 \\
\hline EMIC mean & 1.8 & 3.0 & 4.0 & 5.6 & 0.8 \\
\hline EMIC range & $0.8-2.5$ & $1.9-4.0$ & $2.1-5.4$ & $3.5-8.0$ & $0.5-1.2$ \\
\hline
\end{tabular}

shown to scale well with climate sensitivity because the signal is mostly dominated by $\mathrm{CO}_{2}$ forcing in these regions (Braconnot et al., 2007b; Jansen et al., 2007). The analogy between the LGM climate sensitivity and future climate sensitivity is, however, not perfect (Crucifix, 2006). In a single-model ensemble of simulations, the magnitudes of the LGM cooling and the warming induced by a doubling of $\mathrm{CO}_{2}$ are nonlinear in the forcings applied (Hargreaves et al., 2007). Differences in the cloud radiative feedback are at the origin of this asymmetric response to equivalent positive and negative forcings (Yoshimori et al., 2009). There is thus still low confidence that the regional LGM model-data comparisons can be used to evaluate model climate sensitivity. However, even if the results do not scale perfectly with equilibrium or transient climate sensitivity, the LGM simulations allow the identification of the different feedback factors that contributed to the LGM global cooling (Yoshimori et al., 2011) and model spread in these feedbacks. The largest spread in LGM model feedbacks is found for the shortwave cloud feedback, just as for the modern climate. This correspondence between LGM and modern climates adds to the high confidence that the shortwave cloud feedback is the dominant source of model spread in climate sensitivity (cf. Section 5.3.3).

\subsubsection{Constraints on Equilibrium Climate Sensitivity from Climate-Model Ensembles and Observations}

The large scale climatological information available has so far been insufficient to constrain model behaviour to a range tighter than CMIP3, at least on a global scale. Sanderson and Knutti (2012) suggest that much of the available and commonly used large scale observations have already been used to develop and evaluate models and are therefore of limited value to further constrain climate sensitivity or TCR. The assessed literature suggests that the range of climate sensitivities and transient responses covered by CMIP3/5 cannot be narrowed significantly by constraining the models with observations of the mean climate and variability, consistent with the difficulty of constraining the cloud feedbacks from observations (see Chapter 7). Studies based on PPE and CMIP3 support the conclusion that a credible representation of the mean climate and variability is very difficult to achieve with equilibrium climate sensitivities below $2^{\circ} \mathrm{C}$ (Piani et al., 2005; Stainforth et al., 2005; Sanderson et al., 2008a, 2008b; Huber et al., 2011; Klocke et al., 2011; Fasullo and Trenberth, 2012). High climate sensitivity values above $5^{\circ} \mathrm{C}$ (in some cases above $10^{\circ} \mathrm{C}$ ) are found in the PPE based on HadAM/HadCM3. Several recent studies find that such high values cannot be excluded based on climatological constraints, but comparison with observations shows the smallest errors for many fields if ECS is between 3 and $4^{\circ} \mathrm{C}$ (Piani et al., 2005; Knutti et al., 2006; Rodwell and Palmer, 2007; Sanderson et al., 2008a, 2008b, 2010; Sanderson, 2011, 2013).

\subsection{Relating Model Performance to Credibility of Model Applications}

\subsubsection{Synthesis Assessment of Model Performance}

This chapter has assessed the performance of individual climate models as well as the multi-model mean. In addition, changes between models available now and those that were available at the time of the AR4 have been documented. The models display a range of abilities to 
simulate climate characteristics, underlying processes, and phenomena. No model scores high or low in all performance metrics, but some models perform substantially better than others for specific climate variables or phenomena. For a few climate characteristics, the assessment has shown that some classes of models, for example, those with higher horizontal resolution, higher model top or a more complete representation of the carbon cycle, aerosols or chemistry, agree better with observations, although this is not universally true.

Figure 9.44 provides a synthesis of key model evaluation results for AOGCMs and ESMs. The figure makes use of the calibrated language as defined in Mastrandrea et al. (2011). The $x$-axis refers to the level of confidence which increases towards the right as suggested by the increasing strength of shading. The level of confidence is a combination of the level of evidence and the degree of agreement. The level of

(a) Mean State

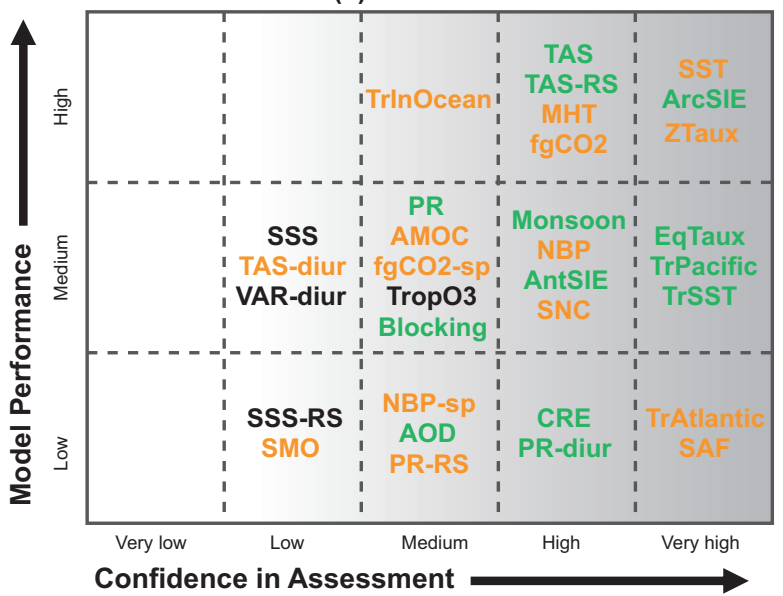

(c) Variability

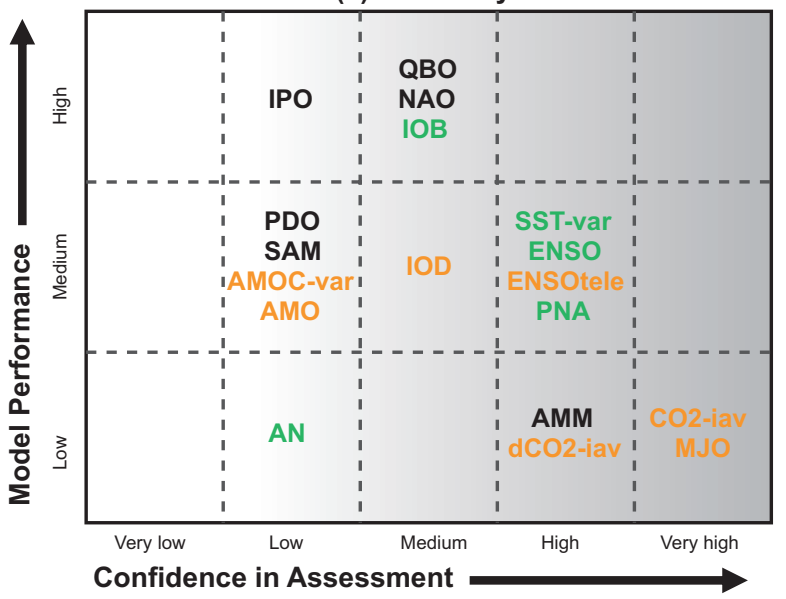

evidence includes the number of studies and quality of observational data. Generally, evidence is most robust when there are multiple, independent studies that evaluate multiple models using high-quality observations. The degree of agreement measures whether different studies come to the same conclusions or not. The figure shows that several important aspects of the climate are simulated well by contemporary models, with varying levels of confidence. The colour coding provides an indication of how model quality has changed from CMIP3 to CMIP5. For example, there is high confidence that the model performance for global mean surface air temperature (TAS) is high, and it is shown in green because there is robust evidence of improvement since CMIP3. By contrast, the diurnal cycle of global mean surface air temperature (TAS-diur) is simulated with medium performance, but there is low confidence in this assessment owing to as yet limited analyses. It should be noted that there are no instances in the figure for

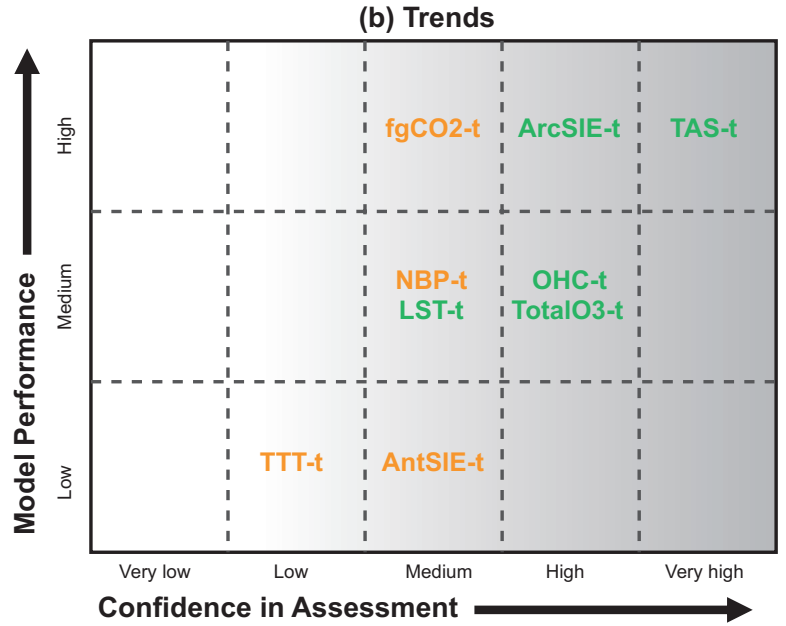

(d) Extremes

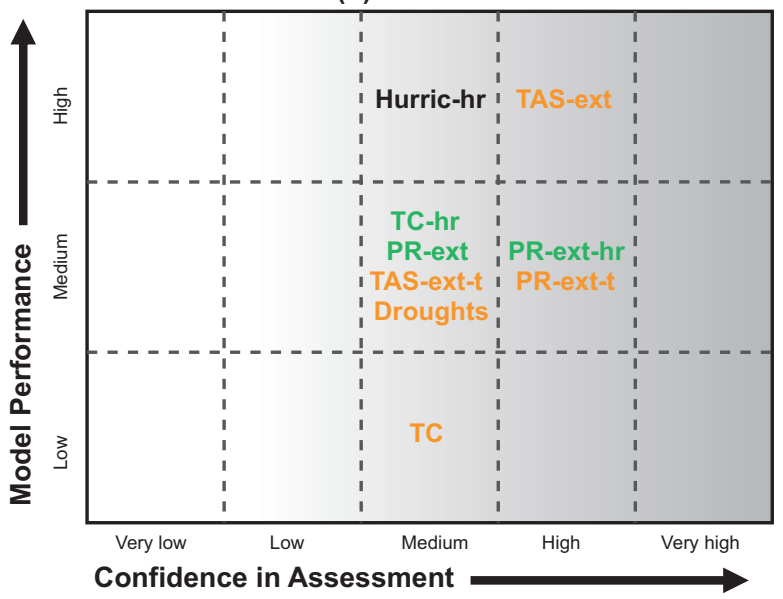

Degradation since CMIP3 No changes since CMIP3

Improvements since CMIP3

No relative assessment CMIP3 vs. CMIP5

Figure 9.44 | Summary of the findings of Chapter 9 with respect to how well the CMIP5 models simulate important features of the climate of the 20th century. Confidence in the assessment increases towards the right as suggested by the increasing strength of shading. Model performance improves from bottom to top. The colour coding indicates changes since CMIP3 (or models of that generation) to CMIP5. The assessment of model performance is expert judgment based on the agreement with observations of the multi-model mean and distribution of individual models around the mean, taking into account internal climate variability. Note that assessed model performance is simplified for representation in the figure and it is referred to the text for details of each assessment. The figure highlights the following key features, with the sections that back up the assessment added in parentheses: 


\section{PANEL a:}

AMOC

Atlantic Meridional Overturning Circulation mean

(Section 9.4.2.3)

AntSIE Seasonal cycle Antarctic sea ice extent (Section 9.4.3)

AOD Aerosol Optical Depth (Section 9.4.6)

ArctSIE Seasonal cycle Arctic sea ice extent (Section 9.4.3)

Blocking Blocking events (Section 9.5.2.2)

CRE Cloud radiative effects (Section 9.4.1.2)

EqTaux Equatorial zonal wind stress (Section 9.4.2.4)

fgCO2 Global ocean carbon sink (Section 9.4.5)

fgCO2-sp Spatial pattern of ocean-atmosphere $\mathrm{CO}_{2}$ fluxes (Section 9.4.5)
MHT

Monsoon

NBP

NBP-sp

PR

PR-diur

PR-RS

SAF

SMO

SNC

SSS

SSS-RS

SST

TAS

TAS-diur

TAS-RS

TrSST

Trop03

TrAtlantic

TrInOcean

TrPacific

VAR-diur

ZTaux

Meridional heat transport (Section 9.4.2.4)

Global monsoon (Section 9.5.2.4)

Global land carbon sink (Section 9.4.5)

Spatial pattern of land-atmosphere $\mathrm{CO}_{2}$ fluxes (Section 9.4.5)

Large scale precipitation (Sections 9.4.1.1, 9.4.1.3)

Diurnal cycle precipitation (Section 9.5.2.1)

Regional scale precipitation (Section 9.6.1.1)

Snow albedo feedbacks (Section 9.8.3)

Soil moisture (Section 9.4.4)

Snow cover (Section 9.4.4)

Sea surface salinity (Section 9.4.2.1)

Regional Sea surface salinity (Section 9.4.2.1)

Sea surface temperature (Section 9.4.2.1)

Large scale surface air temperature (Sections 9.4.1.1, 9.4.1.3)

Diurnal cycle surface air temperature (Section 9.5.2.1)

Regional scale surface air temperature (Section 9.6.1.1)

Tropical sea surface temperature (Section 9.4.2.1)

Tropospheric column ozone climatology (Section 9.4.1.4.5)

Tropical Atlantic mean state (Section 9.4.2.5)

Tropical Indian Ocean mean state (Section 9.4.2.5)

Tropical Pacific mean state (Section 9.4.2.5)

Diurnal cycle other variables (Section 9.5.2.1)

Zonal mean zonal wind stress (Section 9.4.2.4)

PANEL b (Trends)

AntSIE-t Trend in Antarctic sea ice extent (Section 9.4.3)

ArctSIE-t Trend in Arctic sea ice extent (Section 9.4.3)

fgCO2-t Global ocean carbon sink trends (Section 9.4.5)

LST-t Lower stratospheric temperature trends (Section 9.4.1.4.5)

NBP-t Global land carbon sink trends (Section 9.4.5)

OHC-t Global ocean heat content trends (Section 9.4.2.2)

Total03-t Total column ozone trends (Section 9.4.1.4.5)

TAS-t Surface air temperature trends (Section 9.4.1.4.1)

TTT-t Tropical tropospheric temperature trends (Section 9.4.1.4.2)
PANEL c (Variability):

$\begin{array}{ll}\text { AMM } & \text { Atlantic Meridional Mode (Section 9.5.3.3) } \\ \text { AMO } & \text { Atlantic Multi-decadal Variability (Section 9.5.3.3) } \\ \text { AMOC-var } & \text { Atlantic Meridional Overturning Circulation (Section 9.5.3.3) } \\ \text { AN } & \text { Atlantic Niño (Section 9.5.3.3) } \\ \text { CO2-iav } & \text { Interannual variability of atmospheric } \mathrm{CO}_{2} \text { (Section 9.8.3) } \\ \text { dCO2-iav } & \text { Sensitivity of } \mathrm{CO}_{2} \text { growth rate to tropical temperature } \\ & \text { (Section 9.8.3) } \\ \text { ENSO } & \text { El Niño Southern Oscillation (Section 9.5.3.4) } \\ \text { ENSOtele } & \text { Tropical ENSO teleconnections (Section 9.5.3.5) } \\ \text { IOB } & \text { Indian Ocean basin mode (Section 9.5.3.4) } \\ \text { IOD } & \text { Indian Ocean dipole (Section 9.5.3.4) } \\ \text { IPO } & \text { Interdecadal Pacific Oscillation (Section 9.5.3.6) } \\ \text { MJO } & \text { Madden-Julian Oscillation (Section 9.5.2.3) } \\ \text { NAO } & \text { North Atlantic Oscillation and Northern annular mode } \\ & \text { (Section 9.5.3.2) } \\ \text { PDO } & \text { Pacific Decadal Oscillation (Section 9.5.3.6) } \\ \text { PNA } & \text { Pacific North American (Section 9.5.3.5) } \\ \text { QBO } & \text { Quasi-Biennial Oscillation (Section 9.5.3.7) } \\ \text { SAM } & \text { Southern Annular Mode (Section 9.5.3.2) } \\ \text { SST-var } & \text { Global sea surface temperature variability (Section 9.5.3.1) }\end{array}$

PANEL d (Extremes):

Hurric-hr Year-to-year counts of Atlantic hurricanes in high-resolution AGCMs (Section 9.5.4.3)

PR-ext Global distributions of precipitation extremes (Section 9.5.4.2)

PR-ext-hr Global distribution of precipitation extremes in high-resolution AGCMs (Section 9.5.4.2)

PR-ext-t Global trends in precipitation extremes (Section 9.5.4.2)

TAS-ext Global distributions of surface air temperature extremes (Section 9.5.4.1)

TAS-ext-t Global trends in surface air temperature extremes (Section 9.5.4.1)

TC Tropical cyclone tracks and intensity (Section 9.5.4.3)

TC-hr Tropical cyclone tracks and intensity in high-resolution AGCMs (Section 9.5.4.3)

Droughts Droughts (Section 9.5.4.4) which CMIP5 models perform worse than CMIP3 models (something that would have been indicated by the red colour). A description that explains the expert judgment for each of the results presented in Figure 9.44 can be found in the body of this chapter, with a link to the specific sections given in the figure caption.

EMICs have also been evaluated to some extent in this chapter as they are used to provide long-term projections (in Chapter 12) beyond year 2300 , and to provide large ensembles emulating the response of more comprehensive ESMs and allowing probabilistic estimates. Results from the EMIC intercomparison project (Eby et al., 2013; Zickfeld et al., 2013) illustrate the ability to reproduce the large-scale climate changes in GMST (Figure 9.8) and OHC (Figure 9.17) during the 20th century. The models also estimate $\mathrm{CO}_{2}$ fluxes for land and oceans, which are as consistent with observations as are fluxes estimated by ESMs (Figure 9.27). This gives confidence that the EMICs, albeit limited in the scope and resolution of information they can provide, can be used for longterm projections compatible with those of ESMs (Plattner et al., 2008; Eby et al., 2013). Overall, these studies imply that EMICs are well suited for simulations extending beyond the CMIP5 ensemble. 
Frequently Asked Questions

\section{FAQ 9.1 | Are Climate Models Getting Better, and How Would We Know?}

Climate models are extremely sophisticated computer programs that encapsulate our understanding of the climate system and simulate, with as much fidelity as currently feasible, the complex interactions between the atmosphere, ocean, land surface, snow and ice, the global ecosystem and a variety of chemical and biological processes.

The complexity of climate models - the representation of physical processes like clouds, land surface interactions and the representation of the global carbon and sulphur cycles in many models-has increased substantially since the IPCC First Assessment Report in 1990, so in that sense, current Earth System Models are vastly 'better' than the models of that era. This development has continued since the Fourth Assessment, while other factors have also contributed to model improvement. More powerful supercomputers allow current models to resolve finer spatial detail. Today's models also reflect improved understanding of how climate processes work-understanding that has come from ongoing research and analysis, along with new and improved observations.

Climate models of today are, in principle, better than their predecessors. However, every bit of added complexity, while intended to improve some aspect of simulated climate, also introduces new sources of possible error (e.g., via uncertain parameters) and new interactions between model components that may, if only temporarily, degrade a model's simulation of other aspects of the climate system. Furthermore, despite the progress that has been made, scientific uncertainty regarding the details of many processes remains.

An important consideration is that model performance can be evaluated only relative to past observations, taking into account natural internal variability. To have confidence in the future projections of such models, historical climate-and its variability and change-must be well simulated. The scope of model evaluation, in terms of the kind and quantity of observations available, the availability of better coordinated model experiments, and the expanded use of various performance metrics, has provided much more quantitative information about model performance. But this alone may not be sufficient. Whereas weather and seasonal climate predictions can be regularly verified, climate projections spanning a century or more cannot. This is particularly the case as anthropogenic forcing is driving the climate system toward conditions not previously observed in the instrumental record, and it will always be a limitation.

Quantifying model performance is a topic that has featured in all previous IPCC Working Group I Reports. Reading back over these earlier assessments provides a general sense of the improvements that have been made. Past reports have typically provided a rather broad survey of model performance, showing differences between model-calculated versions of various climate quantities and corresponding observational estimates.

Inevitably, some models perform better than others for certain climate variables, but no individual model clearly emerges as 'the best' overall. Recently, there has been progress in computing various performance metrics, which synthesize model performance relative to a range of different observations according to a simple numerical score. Of course, the definition of such a score, how it is computed, the observations used (which have their

(continued on next page)
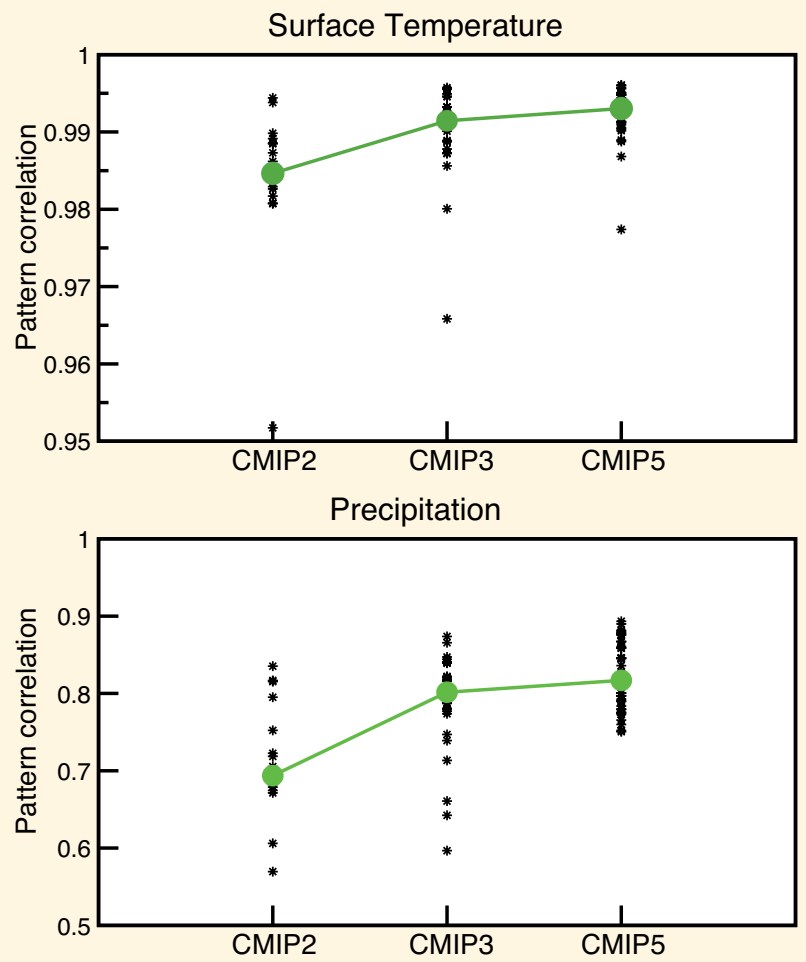

FAQ 9.1, Figure 1 | Model capability in simulating annual mean temperature and precipitation patterns as illustrated by results of three recent phases of the Coupled Model Intercomparison Project (CMIP2, models from about year 2000; CMIP3, models from about 2005; and CMIP5, the current generation of models). The figure shows the correlation (a measure of pattern similarity) between observed and modelled temperature (upper panel) and precipitation (lower panel). Larger values indicate better correspondence between modelled and observed spatial patterns. The black symbols indicate correlation coefficient for individual models, and the large green symbols indicate the median value (i.e., half of the model results lie above and the other half below this value). Improvement in model performance is evident by the increase in correlation for successive model generations. 
FAQ 9.1 (continued)

own uncertainties), and the manner in which various scores are combined are all important, and will affect the end result.

Nevertheless, if the metric is computed consistently, one can compare different generations of models. Results of such comparisons generally show that, although each generation exhibits a range in performance, the average model performance index has improved steadily between each generation. An example of changes in model performance over time is shown in FAQ 9.1, Figure 1, and illustrates the ongoing, albeit modest, improvement. It is interesting to note that both the poorest and best performing models demonstrate improvement, and that this improvement comes in parallel with increasing model complexity and an elimination of artificial adjustments to atmosphere and ocean coupling (so-called 'flux adjustment'). Some of the reasons for this improvement include increased understanding of various climate processes and better representation of these processes in climate models. More comprehensive Earth observations are also driving improvements.

So, yes, climate models are getting better, and we can demonstrate this with quantitative performance metrics based on historical observations. Although future climate projections cannot be directly evaluated, climate models are based, to a large extent, on verifiable physical principles and are able to reproduce many important aspects of past response to external forcing. In this way, they provide a scientifically sound preview of the climate response to different scenarios of anthropogenic forcing.

\subsubsection{Implications of Model Evaluation for Climate Change Detection and Attribution}

The evaluation of model simulations of historical climate is of direct relevance to detection and attribution (D\&A) studies (Chapter 10) since these rely on model-derived patterns (or 'fingerprints') of climate response to external forcing, and on the ability of models to simulate decadal and longer-time scale internal variability (Hegerl and Zwiers, 2011). Conversely, D\&A research contributes to model evaluation through estimation of the amplitude of modeled response to various forcings (Section 10.3.1.1.3). The estimated fingerprint for some variables such as water vapor is governed by basic physical processes that are well represented in models and are rather insensitive to model uncertainties (Santer et al., 2009). Figure 9.44 illustrates slight improvements in the representation of some of the modes of variability and climate phenomena discussed in Sections 9.5.2 and 9.5.3, suggesting with medium confidence that models now better reproduce internal variability. On the other hand, biases that affect D\&A studies remain. An example is the warm bias of lower-stratosphere temperature trends during the satellite period (Section 9.4.1.4.5) that can be linked to uncertainties in stratospheric ozone forcing (Solomon et al., 2012; Santer et al., 2013). Recent studies of climate extremes (Section 9.5.4) also provide evidence that models have reasonable skill in these important attributes of a changing climate; however, there is an indication that models have difficulties in reproducing the right balance between historical changes in cold and warm extremes. They also confirm that resolution affects the confidence that can be placed in the analyses of extreme in precipitation. D\&A studies focussed on extreme events are therefore constrained by current model limitations. Lastly, some D\&A studies have incorporated model quality results by repeating a multi-model analysis with only the models that agree best with observations (Santer et al., 2009). This model discrimination or weighting is less problematic for D\&A analysis than it is for model projections of future climate (Section 9.8.3), because D\&A research is focussed on historical and control-run simulations which can be directly evaluated against observations.

\subsubsection{Implications of Model Evaluation for Model Projections of Future Climate}

Confidence in climate model projections is based on physical understanding of the climate system and its representation in climate models, and on a demonstration of how well models represent a wide range of processes and climate characteristics on various spatial and temporal scales (Knutti et al., 2010b). A climate model's credibility is increased if the model is able to simulate past variations in climate, such as trends over the 20th century and palaeoclimatic changes. Projections from previous IPCC assessments can also be directly compared to observations (see Figures 1.4 and 1.5), with the caveat that these projections were not intended to be predictions over the short time scales for which observations are available to date. Unlike shorter lead forecasts, longer-term climate change projections push models into conditions outside the range observed in the historical period used for evaluation.

In some cases, the spread in climate projections can be reduced by weighting of models according to their ability to reproduce past observed climate. Several studies have explored the use of unequally weighted means, with the weights based on the models' performance in simulating past variations in climate, typically using some performance metric or collection of metrics (Connolley and Bracegirdle, 2007; Murphy et al., 2007; Waugh and Eyring, 2008; Pierce et al., 2009; Reifen and Toumi, 2009; Christensen et al., 2010; Knutti et al., 2010b; Raisanen et al., 2010; Abe et al., 2011; Shiogama et al., 2011; Watterson and Whetton, 2011; Tsushima et al., 2013). When applied to projections of Arctic sea ice, averages in which extra weight is given 
to models with the most realistic historical sea ice do give different results than the unweighted mean (Stroeve et al., 2007, 2012; Scherrer, 2011; Massonnet et al., 2012; Wang and Overland, 2012; Overland and Wang, 2013). Another frequently used approach is the re-calibration of model outputs to a given observed value (Boe et al., 2009b; Mahlstein and Knutti, 2012; Wang and Overland, 2012), see further discussion in Section 12.4.6.1. Some studies explicitly formulate a statistical frameworks that relate future observables to climate model output (reviewed in Knutti et al. (2010b) and Stephenson et al. (2012)). Such frameworks not only provide weights for the mean response but also allow the uncertainty in the predicted response to be quantified (Bracegirdle and Stephenson, 2012).

There are several encouraging examples of 'emergent constraints', which are relationships across an ensemble of models between some aspect of Earth System sensitivity and an observable trend or variation in the contemporary climate (Allen and Ingram, 2002; Hall and Qu, 2006; Eyring et al., 2007; Boe et al., 2009a, 2009b; Mahlstein and Knutti, 2010; Son et al., 2010; Huber et al., 2011; Schaller et al., 2011; Bracegirdle and Stephenson, 2012; Fasullo and Trenberth, 2012; O'Gorman, 2012). For example, analyzing the CMIP3 ensemble, Hall and Qu (2006) showed that inter-model variations of snow albedo feedback in the contemporary seasonal cycle strongly correlate with comparably large inter-model variations in this feedback under future climate change. An update of this analysis with CMIP5 models added is shown in Figure 9.45 (left panel). This relationship presumably arises from the fact that surface albedo values in areas covered by snow vary widely across the models, particularly in the heavily vegetated boreal forest zone. Models with higher surface albedos in these areas have a larger contrast between snow-covered and snow-free areas, and hence a stronger snow albedo feedback whether the context is the seasonal variation in sunshine or anthropogenic forcing. Comparison with an observational estimate of snow albedo feedback reveals a large spread with both high and low biases.

The right panel of Figure 9.45 shows another example of an emergent constraint, where the sensitivity of tropical land carbon to warming (i.e., without $\mathrm{CO}_{2}$ fertilization effects) is related to the sensitivity of the annual $\mathrm{CO}_{2}$ growth rate to tropical temperature anomalies (Cox et al., 2013) ). The horizontal axis is the regression of the atmospheric $\mathrm{CO}_{2}$ growth rate on the tropical temperature anomaly for each model. The strong statistical relationship between these two variables is consistent with the fact that interannual variability in the $\mathrm{CO}_{2}$ growth-rate is known to be dominated by the response of tropical land to climatic anomalies, associated particularly with ENSO. Thus the relationship has a physical as well as a statistical basis. The interannual sensitivity of the $\mathrm{CO}_{2}$ growth rate to tropical temperature can be estimated from observational data. Like the snow albedo feedback example, this intermodel relationship provides a credible means to reduce model spread in the sensitivity of tropical land carbon to tropical climate change.

On the other hand, many studies have failed to find strong relationships between observables and projections. Whetton et al. (2007) and Knutti et al. (2010a) found that correlations between local to regional climatological values and projected changes are small except for a few regions. Scherrer (2011) finds no robust relationship between the ability of the CMIP3 models to represent interannual variability of near-surface air temperature and the amplitude of future warming.Raisanen et al. (2010) report only small (10-20\%) reductions in cross-validation error of simulated 21 st century temperature changes when weighting the CMIP3 models based on their simulation of the present-day climatology. The main difficulties are sparse coverage in (a) Snow-albedo feedback

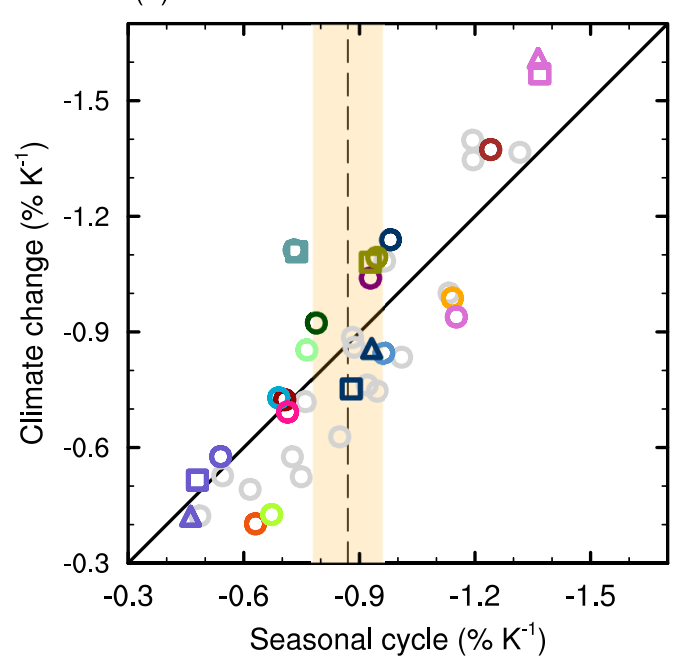

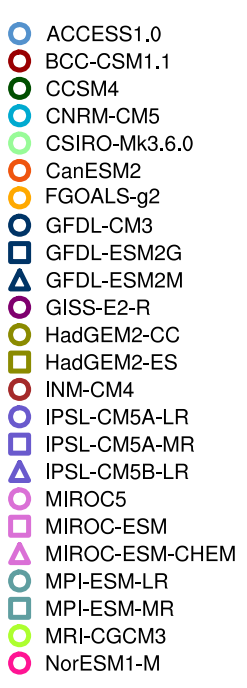

(b) Tropical Land carbon feedback

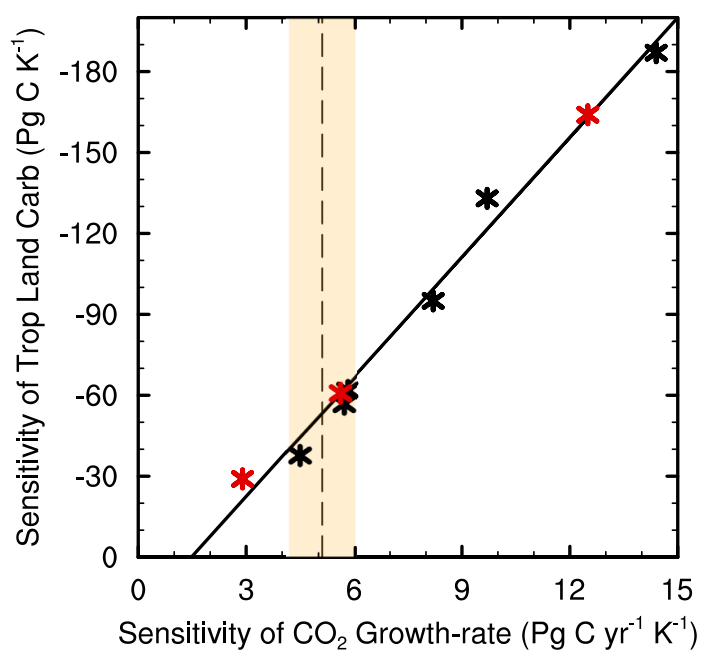

Figure 9.45 | (Left) Scatterplot of simulated springtime snow-albedo feedback $\left(\Delta \alpha_{s} / \Delta T_{s}\right)$ values in climate change $\left(y\right.$-axis) versus simulated springtime $\Delta \alpha_{s} / \Delta T_{s}$ values in the seasonal cycle ( $x$-axis) in transient climate change experiments from 17 CMIP3 (blue) and 24 CMIP5 models ( $\alpha_{s}$ and $T_{s}$ are surface albedo and surface air temperature, respectively). (Adapted from Hall and Qu, 2006.) (Right) Constraint on the climate sensitivity of land carbon in the tropics $\left(30^{\circ} \mathrm{N}\right.$ to $\left.30^{\circ} \mathrm{S}\right)$ from interannual variability in the growth rate of global atmospheric $\mathrm{CO}_{2}$ (Cox et al., 2013). This is based on results from Earth System Models (ESMs) with free-running $\mathrm{CO}_{2}$; Coupled Climate Carbon Cycle Model Intercomparison Project General Circulation Models (C4MIP GCMs, black labels; Friedlingstein et al., 2006), and three land carbon 'physics ensembles' with HadCM3 (red labels; Booth et al., $2012 \mathrm{~b})$. The values on the $y$-axis are calculated over the period 1960-2099 inclusive, and those on the $x$-axis over the period 1960-2010 inclusive. In both cases the temperature used is the mean (land+ocean) temperature over $30^{\circ} \mathrm{N}$ to $30^{\circ} \mathrm{S}$. The width of the vertical yellow bands in both (a) and (b) shows the observation-based estimate of the variable on the $x$-axis. 
many observed variables, short time series for observed trends, lack of correlation between observed quantities and projected past or future trends, and systematic errors in the models (Tebaldi and Knutti, 2007; Jun et al., 2008; Knutti, 2010; Knutti et al., 2010a), the ambiguity of possible performance metrics and the difficulty of associating them with predictive skill (Parker et al., 2007; Gleckler et al., 2008; Pincus et al., 2008; Reichler and Kim, 2008; Pierce et al., 2009; Knutti et al., 2010a).

Emergent constraints can be difficult to identify if climate models are structurally similar and share common biases, thereby reducing the effective ensemble size. Comparison of emergent constraints in MMEs from different modelling experiments can help reveal which constraints are robust (Massonnet et al., 2012; Bracegirdle and Stephenson, 2013). Another issue is that testing of large numbers of predictors will find statistically significant correlations that do not remain significant in a different ensemble. This is particularly important if many predictors are tested using only small ensembles like CMIP3 (DelSole and Shukla, 2009; Raisanen et al., 2010; Huber et al., 2011; Masson and Knutti, 2013). All of these potential pitfalls underscore the need for analysis of the mechanism underpinning the statistical relationship between current and future climate parameters for any proposed emergent constraint. 


\section{References}

Abe, M., H. Shiogama, T. Nozawa, and S. Emori, 2011: Estimation of future surface temperature changes constrained using the future-present correlated modes in inter-model variability of CMIP3 multimodel simulations. J. Geophys. Res. Atmos., 116, D18104.

Abiodun, B., W. Gutowski, A. Abatan, and J. Prusa, 2011: CAM-EULAG: A nonhydrostatic atmospheric climate model with grid stretching. Acta Geophys., 59, 1158-1167.

Abramowitz, G., R. Leuning, M. Clark, and A. Pitman, 2008: Evaluating the performance of land surface models. J. Clim., 21, 5468-5481.

AchutaRao, K., and K. Sperber, 2002: Simulation of the El Niño Southern Oscillation: Results from the coupled model intercomparison project. Clim. Dyn., 19, 191209.

AchutaRao, K., and K. Sperber, 2006: ENSO simulations in coupled ocean-atmosphere models: Are the current models better? Clim. Dyn., 27, 1-16.

Achuthavarier, D., V. Krishnamurthy, B. P. Kirtman, and B. H. Huang, 2012: Role of the Indian Ocean in the ENSO-Indian Summer Monsoon Teleconnection in the NCEP Climate Forecast System. J. Clim., 25, 2490-2508.

Ackerley, D., E. J. Highwood, and D. J. Frame, 2009: Quantifying the effects of perturbing the physics of an interactive sulfur scheme using an ensemble of GCMs on the climateprediction.net platform. J. Geophys. Res. Atmos., 114, D01203

Adachi, Y., et al., 2013: Basic performance of a new earth system model of the Meteorological Research Institute (MRI-ESM1). Papers Meteorol. Geophys. doi:10.2467/mripapers.64.1.

Adam, J., E. Clark, D. Lettenmaier, and E. Wood, 2006: Correction of global precipitation products for orographic effects. J. Clim., 19, 15-38.

Adkins, J. F., K. McIntyre, and D. P. Schrag, 2002: The salinity, temperature, and delta 0-18 of the glacial deep ocean. Science, 298, 1769-1773.

Adler, R. F., et al., 2003: The Version 2 Global Precipitation Climatology Project (GPCP) Monthly Precipitation Analysis (1979-Present). J. Hydrometeor., 4, 1147-1167.

Alekseev, V. A., E. M. Volodin, V. Y. Galin, V. P. Dymnikov, and V. N. Lykossov, 1998 Modeling of the present-day climate by the atmospheric model of INM RAS DNM GCM. Description of the model version A5421 and results of AMIP2 simulations. Institute of Numerical Mathematics, Moscow, Russia, 200 pp.

Alessandri, A., P. G. Fogli, M. Vichi, and N. Zeng, 2012: Strengthening of the hydrological cycle in future scenarios: Atmospheric energy and water balance perspective. Earth Syst. Dyn., 3, 199-212.

Alexander, M. J., et al., 2010: Recent developments in gravity-wave effects in climate models and the global distribution of gravity-wave momentum flux from observations and models. Q. J. R. Meteorol. Soc., 136, 1103-1124.

Alexandru, A., R. de Elia, and R. Laprise, 2007: Internal variability in regional climate downscaling at the seasonal scale. Mon. Weather Rev., 135 3221-3238.

Alexandru, A., R. de Elia, R. Laprise, L. Separovic, and S. Biner, 2009: Sensitivity study of regional climate model simulations to large-scale nudging parameters. Mon. Weather Rev., 137, 1666-1686.

Allan, R. P., and B. J. Soden, 2008: Atmospheric warming and the amplification of precipitation extremes. Science, 321, 1481-1484.

Allan, R. P., M. A. Ringer, and A. Slingo, 2003: Evaluation of moisture in the Hadley Centre climate model using simulations of HIRS water-vapour channel radiances. Q. J. R. Meteorol. Soc., 129, 3371-3389.

Allan, R. P., A. Slingo, S. F. Milton, and M. E. Brooks, 2007: Evaluation of the Met Office global forecast model using Geostationary Earth Radiation Budget (GERB) data. Q. J. R. Meteorol. Soc., 133, 1993-2010.

Allan, R. P., B. J. Soden, V. O. John, W. Ingram, and P. Good, 2010: Current changes in tropical precipitation. Environ. Res. Lett., 5, 025205.

Allen, M., P. Stott, J. Mitchell, R. Schnur, and T. Delworth, 2000: Quantifying the uncertainty in forecasts of anthropogenic climate change. Nature, 407, 617620.

Allen, M. R., and W. J. Ingram, 2002: Constraints on future changes in climate and the hydrologic cycle. Nature, 419, 224-232.

Ammann, C. M., G. A. Meehl, W. M. Washington, and C. S. Zender, 2003: A monthly and latitudinally varying volcanic forcing dataset in simulations of 20th century climate. Geophys. Res. Lett., 30, 1657.

Anav, A., et al., 2013: Evaluating the land and ocean components of the global carbon cycle in the CMIP5 Earth System Models. J. Clim., 26, 6801-6843.
Anderson, B. T., J. R. Knight, M. A. Ringer, C. Deser, A. S. Phillips, J. H. Yoon, and A. Cherchi, 2010: Climate forcings and climate sensitivities diagnosed from atmospheric global circulation models. Clim. Dyn., 35, 1461-1475.

Andrews, T., J. M. Gregory, M. J. Webb, and K. E. Taylor, 2012: Forcing, feedbacks and climate sensitivity in CMIP5 coupled atmosphere-ocean climate models. Geophys. Res. Lett., 39, L09712.

Annamalai, H., and K. R. Sperber, 2005: Regional heat sources and the active and break phases of boreal summer intraseasonal (30-50 day) variability. J. Atmos. Sci., 62, 2726-2748.

Annan, J, and J. Hargreaves, 2011: Understanding the CMIP3 Multimodel Ensemble. J. Clim., 24, 4529-4538.

Annan, J. D., and J. C. Hargreaves, 2010: Reliability of the CMIP3 ensemble. Geophys. Res. Lett., 37, L02703.

Annan, J. D., D. J. Lunt, J. C. Hargreaves, and P. J. Valdes, 2005: Parameter estimation in an atmospheric GCM using the Ensemble Kalman Filter. Nonlin. Proc. Geophys. $12,363-371$

Anstey, J. A., et al., 2013: Multi-model analysis of Northern Hemisphere winter blocking and its relation to the stratosphere. J. Geophys. Res. Atmos., 118, 3956-3971.

Antonov, J. I., et al., 2010: World Ocean Atlas 2009, Vol. 2: Salinity. [S. Levitus (eds.)]. NOAA Atlas NESDIS 69, U.S. Gov. Printing Office, Washington, D.C., 184 pp.

Archer, D. E., G. Eshel, A. Winguth, W. Broecker, R. Pierrehumbert, M. Tobis, and R. Jacob, 2000: Atmospheric PCO(2) sensitivity to the biological pump in the ocean. Global Biogeochem. Cycles, 14, 1219-1230.

Arneth, A., et al., 2010: From biota to chemistry and climate: Towards a comprehensive description of trace gas exchange between the biosphere and atmosphere. Biogeosciences, 7, 121-149.

Arora, V. K., and G. J. Boer, 2005: Fire as an interactive component of dynamic vegetation models. J. Geophys. Res.-Biogeosciences, 110, G02008.

Arora, V. K., and G. J. Boer, 2010: Uncertainties in the 20th century carbon budget associated with land use change. Global Change Biol., 16, 3327-3348.

Arora, V. K., et al., 2011: Carbon emission limits required to satisfy future representative concentration pathways of greenhouse gases. Geophys. Res. Lett., 38, L05805.

Arora, V. K., et al., 2009: The effect of terrestrial photosynthesis down regulation on the twentieth-century carbon budget simulated with the CCCma Earth System Model. J. Clim., 22, 6066-6088.

Artale, V., et al., 2010: An atmosphere-ocean regional climate model for the Mediterranean area: Assessment of a present climate simulation. Clim. Dyn., $35,721-740$

Arzhanov, M. M., P. F. Demchenko, A. V. Eliseev, and I. I. Mokhov, 2008: Simulation of characteristics of thermal and hydrologic soil regimes in equilibrium numerical experiments with a Climate Model of Intermediate Complexity. Izvestiya Atmos. Ocean. Phys., 44, 548-566.

Assmann, K. M., M. Bentsen, J. Segschneider, and C. Heinze, 2010: An isopycnic ocean carbon cycle model. Geosci. Model Dev., 3, 143-167.

Aumont, O., and L. Bopp, 2006: Globalizing results from ocean in situ iron fertilization studies. Global Biogeochem. Cycles, 20, Gb2017.

Aumont, O., E. Maier-Reimer, S. Blain, and P. Monfray, 2003: An ecosystem model of the global ocean including $\mathrm{Fe}, \mathrm{Si}, \mathrm{P}$ colimitations. Global Biogeochem. Cycles, $17,1060$.

Austin, J., and R. J. Wilson, 2006: Ensemble simulations of the decline and recovery of stratospheric ozone. J. Geophys. Res. Atmos., 111, D16314.

Axelsson, P., M. Tjernström, S. Söderberg, and G. Svensson, 2011: An ensemble of Arctic simulations of the AOE-2001 field experiment. Atmosphere, 2, 146-170.

Baehr, J., S. Cunnningham, H. Haak, P. Heimbach, T. Kanzow, and J. Marotzke, 2009: Observed and simulated estimates of the meridional overturning circulation at $26.5 \mathrm{~N}$ in the Atlantic. Ocean Sci., 5, 575-589.

Balan Sarojini, B., et al., 2011: High frequency variability of the Atlantic meridional overturning circulation. Ocean Science, 7, 471-486.

Baldwin, M. P., et al., 2001: The quasi-biennial oscillation. Rev. Geophys., 39, 179229.

Balsamo, G., P. Viterbo, A. Beljaars, B. van den Hurk, M. Hirschi, A. K. Betts, and K. Scipal, 2009: A revised hydrology for the ECMWF Model: Verification from field site to terrestrial water storage and impact in the Integrated Forecast System. J. Hydrometeorol., 10, 623-643. 
Bao, Q., G. Wu, Y. Liu, J. Yang, Z. Wang, and T. Zhou, 2010: An introduction to the coupled model FGOALS1.1-s and its performance in East Asia. Adv. Atmos. Sci., 27, 1131-1142.

Bao, Q., et al., 2013: The Flexible Global Ocean-Atmosphere-Land System model Version: FGOALS-s2. Adv. Atmos. Sci., doi:10.1007/s00376-012-2113-9.

Bao, Y., F. L. Qiao, and Z. Y. Song, 2012: Historical simulation and twenty-first century prediction of oceanic $\mathrm{CO}_{2}$ sink and pH change. Acta Ocean. Sin., 31, 87-97.

Barkstrom, B. R., 1984: The Earth Radiation Budget Experiment (ERBE). Bull. Am. Meteorol. Soc., 65, 1170-1185.

Barnes, E. A., and D. L. Hartmann, 2010: Influence of eddy-driven jet latitude on North Atlantic jet persistence and blocking frequency in CMIP3 integrations. Geophys. Res. Lett., 37, L23802.

Barnes, E. A., J. Slingo, and T. Woollings, 2012: A methodology for the comparison of blocking climatologies across indices, models and climate scenarios. Clim. Dyn., 38, 2467-2481.

Barnier, B., et al., 2006: Impact of partial steps and momentum advection schemes in a global ocean circulation model at eddy-permitting resolution. Ocean Dyn., 56, 543-567.

Barriopedro, D., R. Garcia-Herrera, and R. M. Trigo, 2010a: Application of blocking diagnosis methods to General Circulation Models. Part I: A novel detection scheme. Clim. Dyn., 35, 1373-1391.

Barriopedro, D., R. Garcia-Herrera, J. F. Gonzalez-Rouco, and R. M. Trigo, 2010b: Application of blocking diagnosis methods to General Circulation Models. Part II: Model simulations. Clim. Dyn., 35, 1393-1409.

Bartlein, P. J., et al., 2010: Pollen-based continental climate reconstructions at 6 and 21 ka: A global synthesis. Clim. Dyn., 37, 775-802.

Bathiany, S., M. Claussen, V. Brovkin, T. Raddatz, and V. Gayler, 2010: Combined biogeophysical and biogeochemical effects of large-scale forest cover changes in the MPI earth system model. Biogeosciences, 7, 1383-1399.

Bauer, H. S., V. Wulfmeyer, and L. Bengtsson, 2008a: The representation of a synopticscale weather system in a thermodynamically adjusted version of the ECHAM4 general circulation model. Meteorol. Atmos. Phys., 99, 129-153.

Bauer, S. E., D. Koch, N. Unger, S. M. Metzger, D. T. Shindell, and D. G. Streets, 2007: Nitrate aerosols today and in 2030: A global simulation including aerosols and tropospheric ozone. Atmos. Chem. Phys., 7, 5043-5059.

Bauer, S. E., et al., 2008b: MATRIX (Multiconfiguration Aerosol TRacker of mIXing state): An aerosol microphysical module for global atmospheric models. Atmos. Chem. Phys., 8, 6003-6035.

Beare, R., et al., 2006: An intercomparison of large-eddy simulations of the Stable Boundary Layer. Boundary-Layer Meteorol., 118, 247-272.

Bellassen, V., G. Le Maire, J. F. Dhote, P. Ciais, and N. Viovy, 2010: Modelling forest management within a global vegetation model Part 1: Model structure and general behaviour. Ecol. Model., 221, 2458-2474.

Bellassen, V., G. le Maire, O. Guin, J. F. Dhote, P. Ciais, and N. Viovy, 2011: Modelling forest management within a global vegetation model-Part 2: Model validation from a tree to a continental scale. Ecol. Model., 222, 57-75.

Bellouin, N., J. Rae, A. Jones, C. Johnson, J. Haywood, and O. Boucher, 2011: Aerosol forcing in the Climate Model Intercomparison Project (CMIP5) simulations by HadGEM2-ES and the role of ammonium nitrate. J. Geophys. Res., 116, 1-25.

Bellucci, A., S. Gualdi, and A. Navarra, 2010: The Double-ITCZ Syndrome in Coupled General Circulation Models: The role of large-scale vertical circulation regimes. J. Clim., 23, 1127-1145.

Bender, M. A., T. R. Knutson, R. E. Tuleya, J. J. Sirutis, G. A. Vecchi, S. T. Garner, and I. M. Held, 2010: Modeled impact of anthropogenic warming on the frequency of intense Atlantic hurricanes. Science, 327, 454-458.

Bengtsson, L., and K. Hodges, 2011: On the evaluation of temperature trends in the tropical troposphere. Clim. Dyn., 36, 419-430.

Bengtsson, L., K. I. Hodges, and N. Keenlyside, 2009: Will extratropical storms intensify in a warmer climate? J. Clim., 22, 2276-2301.

Berckmans, J., T. Woollings, M.-E. Demory, P.-L. Vidal, and M. Roberts, 2013: Atmospheric blocking in a high resolution climate model: Influences of mean state, orography and eddy forcing. Atmos. Sci. Lett., 14, 34-40.

Bergengren, J., D. Waliser, and Y. Yung, 2011: Ecological sensitivity: A biospheric view of climate change. Clim. Change, 107, 433-457.

Bergengren, J., S. Thompson, D. Pollard, and R. DeConto, 2001: Modeling global climate-vegetation interactions in a doubled $\mathrm{CO}_{2}$ world. Clim. Change, 50, $31-75$.
Bernie, D. J., E. Guilyardi, G. Madec, J. M. Slingo, S. Woolnough, and J. Cole, 2008: Impact of resolving the diurnal cycle in an ocean-atmosphere GCM. Part 2: A diurnally coupled CGCM. Clim. Dyn., 31, 909-925.

Bi, D., et al., 2013a:ACCESS-OM: The Ocean and Sea ice Core of the ACCESS Coupled Model. Aust. Meteorol. Oceanogr. J., 63, 213-232.

Bi, D., et al., 2013b: The ACCESS Coupled Model: Description, control climate and evaluation. Aust. Meteorol. Oceanogr. J., 63, 41-64.

Bitz, C. M., and W. H. Lipscomb, 1999: An energy-conserving thermodynamic sea ice model for climate study. J. Geophys. Res.. Oceans, 104, 15669-15677.

Blyth, E., J. Gash, A. Lloyd, M. Pryor, G. P. Weedon, and J. Shuttleworth, 2010: Evaluating the JULES Land Surface Model Energy Fluxes Using FLUXNET Data. J. Hydrometeorol., 11, 509-519.

Boberg, F., and J. H. Christensen, 2012: Overestimation of Mediterranean summer temperature projections due to model deficiencies. Nature Clim. Change, 2, 433-436.

Boccaletti, G., R. Ferrari, and B. Fox-Kemper, 2007: Mixed layer instabilities and restratification. J. Phys. Oceanogr., 37, 2228-2250.

Bodas-Salcedo, A., K. D. Williams, P. R. Field, and A. P. Lock, 2012: The surface downwelling solar radiation surplus over the Southern Ocean in the Met Office Model: The role of midlatitude cyclone clouds. J. Clim., 25, 7467-7486.

Bodas-Salcedo, A., M. Webb, M. Brooks, M. Ringer, K. Williams, S. Milton, and D. Wilson, 2008: Evaluating cloud systems in the Met Office global forecast model using simulated CloudSat radar reflectivities. J. Geophys. Res. Atmos., 113, D00A13.

Bodas-Salcedo, A., et al., 2011: COSP: Satellite simulation software for model assessment. Bull. Am. Meteorol. Soc., 92, 1023-1043.

Bodeker, G., H. Shiona, and H. Eskes, 2005: Indicators of Antarctic ozone depletion. Atmos. Chem. Phys., 5, 2603-2615.

Boe, J., A. Hall, and X. Qu, 2009a: Deep ocean heat uptake as a major source of spread in transient climate change simulations. Geophys. Res. Lett., 36, L22701.

Boe, J., L. Terray, F. Habets, and E. Martin, 2007: Statistical and dynamical downscaling of the Seine basin climate for hydro-meteorological studies. Int. J. Climatol. , 27, 1643-1655.

Boe, J. L., A. Hall, and X. Qu, 2009b: September sea-ice cover in the Arctic Ocean projected to vanish by 2100 . Nature Geosci., 2, 341-343.

Boer, G., and S. Lambert, 2008: The energy cycle in atmospheric models. Clim. Dyn., 30, 371-390.

Boer, G. J., and B. Yu, 2003: Climate sensitivity and climate state. Clim. Dyn., 21, 167-176.

Boisier, J.-P., et al., 2012: Attributing the biogeophysical impacts of land-use induced Land-Cover Changes on surface climate to specific causes. Results from the first LUCID set of simulations. J. Geophys. Res., 117, D12116.

Bollasina, M. A., and Y. Ming, 2013: The general circulation model precipitation bias over the southwestern equatorial Indian Ocean and its implications for simulating the South Asian monsoon. Clim. Dyn., 40, 823-838.

Bonan, G. B., 2008: Forests and climate change: Forcings, feedbacks, and the climate benefits of forests. Science, 320, 1444-1449.

Bond, T. C., et al., 2007: Historical emissions of black and organic carbon aerosol from energy-related combustion, 1850-2000. Global Biogeochem. Cycles, 21, GB2018.

Bondeau, A., P. C. Smith, S. Zaehle, S. Schaphoff, W. Lucht, W. Cramer, and D. Gerten, 2007: Modelling the role of agriculture for the 20th century global terrestrial carbon balance. Global Change Biol., 13, 679-706.

Boning, C. W., A. Dispert, M. Visbeck, S. R. Rintoul, and F. U. Schwarzkopf, 2008: The response of the Antarctic Circumpolar Current to recent climate change. Nature Geosci., 1, 864-869.

Boone, A., et al., 2009: THE AMMA Land Surface Model Intercomparison Project (ALMIP). Bull. Am. Meteorol. Soc., 90, 1865-1880.

Boone, A. A., I. Poccard-Leclercq, Y. K. Xue, J. M. Feng, and P. de Rosnay, 2010: Evaluation of the WAMME model surface fluxes using results from the AMMA land-surface model intercomparison project. Clim. Dyn., 35, 127-142.

Booth, B. B. B., N. J. Dunstone, P. R. Halloran, T. Andrews, and N. Bellouin, 2012a: Aerosols implicated as a prime driver of twentieth-century North Atlantic climate variability. Nature, 484, 228-232.

Booth, B. B. B., et al., 2012b: High sensitivity of future global warming to land carbon cycle processes. Environ. Res. Lett., 7, 024002.

Boschat, G., P. Terray, and S. Masson, 2012: Robustness of SST teleconnections and precursory patterns associated with the Indian summer monsoon. Clim. Dyn., 38, 2143-2165. 
Boyle, J., and S. A. Klein, 2010: Impact of horizontal resolution on climate mode forecasts of tropical precipitation and diabatic heating for the TWP-ICE period J. Geophys. Res., 115, D23113.

Boyle, J., S. Klein, G. Zhang, S. Xie, and X. Wei, 2008: Climate Model Forecast Experiments for TOGA COARE. Mon. Weather Rev., 136, 808-832.

Bracegirdle, T., et al., 2013: Assessment of surface winds over the Atlantic, Indian and Pacific Ocean sectors of the Southern Hemisphere in CMIP5 models: Historical bias, forcing response, and state dependence. J. Geophys. Res. Atmos., doi:10.1002/jgrd.50153.

Bracegirdle, T. J., and D. B. Stephenson, 2012: Higher precision estimates of regional polar warming by ensemble regression of climate model projections. Clim. Dyn. 39, 2805-2821.

Bracegirdle, T. J., and D. B. Stephenson, 2013: On the robustness of emergent constraints used in multi-model climate change projections of Arctic warming. J. Clim., 26, 669-678.

Braconnot, P., F. Hourdin, S. Bony, J. Dufresne, J. Grandpeix, and 0. Marti, 2007a Impact of different convective cloud schemes on the simulation of the tropical seasonal cycle in a coupled ocean-atmosphere model. Clim. Dyn., 29, 501-520.

Braconnot, P., et al., 2012: Evaluation of climate models using palaeoclimatic data. Nature Clim. Change, 2, 417-424.

Braconnot, P., et al., 2007b: Results of PMIP2 coupled simulations of the MidHolocene and Last Glacial Maximum - Part 2: Feedbacks with emphasis on the location of the ITCZ and mid- and high latitudes heat budget. Clim. Past, 3, 279-296.

Braconnot, P., et al., 2007c: Results of PMIP2 coupled simulations of the MidHolocene and Last Glacial Maximum - Part 1: Experiments and large-scale features. Clim. Past, 3, 261-277.

Brands, S., J. Taboada, A. Cofino, T. Sauter, and C. Schneider, 2011: Statistical downscaling of daily temperatures in the NW Iberian Peninsula from global climate models: Validation and future scenarios. Clim. Res., 48, 163-176.

Bresson, R., and R. Laprise, 2011: Scale-decomposed atmospheric water budget over North America as simulated by the Canadian Regional Climate Model for current and future climates. Clim. Dyn., 36, 365-384.

Breugem, W. P., W. Hazeleger, and R. J. Haarsma, 2006: Multimodel study of tropical Atlantic variability and change. Geophys. Res. Lett., 33, L23706.

Brewer, S., J. Guiot, and F. Torre, 2007: Mid-Holocene climate change in Europe: A data-model comparison. Clim. Past, 3, 499-512.

Briegleb, B. P., and B. Light, 2007: A Delta-Eddington multiple scattering parameterization for solar radiation in the sea ice component of the Community Climate System Model. NCAR Technical Note, National Center for Atmospheric Research, $100 \mathrm{pp}$.

Briegleb, B. P., C. M. Blitz, E. C. Hunke, W. H. Lipscomb, M. M. Holland, J. L. Schramm, and R. E. Moritz, 2004: Scientific description of the sea ice component in the Community Climate System Model, Version 3. NCAR Technical Note, National Center for Atmospheric Research, $70 \mathrm{pp}$.

Brient, F., and S. Bony, 2012: Interpretation of the positive low-cloud feedback predicted by a climate model under global warming. Clim. Dyn., doi:10.1007/ s00382-011-1279-7.

Brierley, C. M., M. Collins, and A. J. Thorpe, 2010: The impact of perturbations to ocean-model parameters on climate and climate change in a coupled model. Clim. Dyn., 34, 325-343.

Brogniez, H., and R. T. Pierrehumbert, 2007: Intercomparison of tropical tropospheric humidity in GCMs with AMSU-B water vapor data. Geophys. Res. Lett., 34, L17812

Brogniez, H., R. Roca, and L. Picon, 2005: Evaluation of the distribution of subtropical free tropospheric humidity in AMIP-2 simulations using METEOSAT water vapor channel data. Geophys. Res. Lett., 32, L19708.

Brovkin, V., J. Bendtsen, M. Claussen, A. Ganopolski, C. Kubatzki, V. Petoukhov, and A. Andreev, 2002: Carbon cycle, vegetation, and climate dynamics in the Holocene: Experiments with the CLIMBER-2 model. Global Biogeochem. Cycles, 16, 1139.

Brown, A., S. Milton, M. Cullen, B. Golding, J. Mitchell, and A. Shelly, 2012: Unified modeling and prediction of weather and climate: A 25-year journey. Bull. Am. Meteorol. Soc., 93, 1865-1877.

Brown, J., A. Fedorov, and E. Guilyardi, 2010a: How well do coupled models replicate ocean energetics relevant to ENSO? Clim. Dyn., 36, 2147-2158.

Brown, J., O. J. Ferrians, J. A. Heginbottom, and E. S. E.S. Melnikov, 1997: International Permafrost Association Circum-Arctic Map of Permafrost and Ground Ice Conditions. Geological Survey (U.S.), Denver, CO, USA.
Brown, J., O. J. Ferrians, J. A. Heginbottom, and E. S. E.S. Melnikov, 1998: Digital circum-arctic map of permafrost and ground ice conditions. In: Circumpolar Active-Layer Permafrost System (CAPS). CD-ROM. 1.0 ed., University of Colorado at Boulder National Snow and Ice Data Center. Boulder, CO, USA.

Brown, J. R., C. Jakob, and J. M. Haynes, 2010b: An evaluation of rainfall frequency and intensity over the Australian region in a Global Climate Model. J. Clim., 23, 6504-6525.

Brown, J. R., A. F. Moise, and R. A. Colman, 2013: The South Pacific Convergence Zone in CMIP5 simulations of historical and future climate. Clim. Dyn., doi:10.1007/ s00382-012-1591-X.

Brutel-Vuilmet, C., M. Menegoz, and G. Krinner, 2013: An analysis of present and future seasonal Northern Hemisphere land snow cover simulated by CMIP5 coupled climate models. Cryosphere, 7, 67-80.

Bryan, F. O., M. W. Hecht, and R. D. Smith, 2007: Resolution convergence and sensitivity studies with North Atlantic circulation models. Part I: The western boundary current system. Ocean Model., 16, 141-159.

Bryan, F. O., R. Tomas, J. M. Dennis, D. B. Chelton, N. G. Loeb, and J. L. McClean, 2010 Frontal scale air-sea interaction in high-resolution coupled climate models. $J$. Clim., 23, 6277-6291.

Bryan, K., and L. J. Lewis, 1979: Water mass model of the world ocean. J. Geophys. Res. Oceans Atmos., 84, 2503-2517.

Bryden, H. L., H. R. Longworth, and S. A. Cunningham, 2005: Slowing of the Atlantic meridional overturning circulation at $25^{\circ} \mathrm{N}$. Nature, 438, 655-657.

Buehler, T., C. C. Raible, and T. F. Stocker, 2011: The relationship of winter season North Atlantic blocking frequencies to extreme cold or dry spells in the ERA-40. Tellus A, 63, 212-222.

Butchart, N., A. A. Scaife, J. Austin, S. H. E. Hare, and J. R. Knight, 2003: Quasi-biennial oscillation in ozone in a coupled chemistry-climate model. J. Geophys. Res., 108, 4486.

Cadule, P., et al., 2010: Benchmarking coupled climate-carbon models against longterm atmospheric $\mathrm{CO}_{2}$ measurements. Global Biogeochem. Cycles, 24, Gb2016.

Cai, W., and T. Cowan, 2013: Why is the amplitude of the Indian Ocean Dipole overly large in CMIP3 and CMIP5 climate models? . Geophys. Res. Lett., doi:10.1002/ grl.50208.

Cai, W., A. Sullivan, and T. Cowan, 2011: Interactions of ENSO, the IOD, and the SAM in CMIP3 Models. J. Clim., 24, 1688-1704.

Cai, W. J., A. Sullivan, and T. Cowan, 2009: Rainfall teleconnections with Indo-Pacific variability in the WCRP CMIP3 models. J. Clim., 22, 5046-5071.

Calov, R., A. Ganopolski, V. Petoukhov, M. Claussen, and R. Greve, 2002: Large-scale instabilities of the Laurentide ice sheet simulated in a fully coupled climatesystem model. Geophys. Res. Lett., 29, 2216.

Cameron-Smith, P., J. F. Lamarque, P. Connell, C. Chuang, and F. Vitt, 2006: Toward an Earth system model: Atmospheric chemistry, coupling, and petascale computing. Scidac 2006: Scientific Discovery through Advanced Computing [W. M. Tang (ed.)]. Journal of Physics: Conference Series, Vol. 46, Denver, Colorado, USA.

Capotondi, A., A. Wittenberg, and S. Masina, 2006: Spatial and temporal structure of Tropical Pacific interannual variability in 20th century coupled simulations. Ocean Model., 15, 274-298.

Capotondi, A., M. A. Alexander, N. A. Bond, E. N. Curchitser, and J. D. Scott, 2012 Enhanced upper ocean stratification with climate change in the CMIP3 models. J. Geophys. Res. Oceans 117, C04031.

Cariolle, D., and H. Teyssedre, 2007: A revised linear ozone photochemistry parameterization for use in transport and general circulation models: Multiannual simulations. Atmos. Chem. Phys. , 7, 2183-2196.

Carslaw, K. S., O. Boucher, D. V. Spracklen, G. W. Mann, J. G. L. Rae, S. Woodward, and M. Kulmala, 2010: A review of natural aerosol interactions and feedbacks within the Earth system. Atmos. Chem. Phys., 10, 1701-1737.

Casado, M. J., and M. A. Pastor, 2012: Use of variability modes to evaluate AR4 climate models over the Euro-Atlantic region. Clim. Dyn., 38, 225-237.

Cattiaux, J., H. Douville, and Y. Peings, 2013: European temperatures in CMIP5: Origins of present-day biases and future uncertainties. Clim. Dyn., doi:10.1007/ s00382-013-1731-y.

Catto, J., N. Nicholls, and C. Jakob, 2012a: North Australian sea surface temperatures and the El Niño Southern Oscillation in observations and models. J. Clim., 25, 5011-5029.

Catto, J., N. Nicholls, and C. Jakob, 2012b: North Australian sea surface temperatures and the El Niño Southern Oscillation in the CMIP5 models. J. Clim., 25, 63756382. 
Catto, J. L., L. C. Shaffrey, and K. I. Hodges, 2010: Can climate models capture the structure of extratropical cyclones? J. Clim., 23, 1621-1635.

Catto, J. L., L. C. Shaffrey, and K. I. Hodges, 2011: Northern Hemisphere Extratropical cyclones in a warming climate in the HiGEM high-resolution climate Model. $J$. Clim., 24, 5336-5352.

Catto, J. L., C. Jakob, and N. Nicholls, 2013: A global evaluation of fronts and precipitation in the ACCESS model. Aust. Meteorol. Oceanogr. J., 63,191-203.

Cavalieri, D. J., and C. L. Parkinson, 2012: Arctic sea ice variability and trends, 1979 2010. Cryosphere, 6, 881-889.

Cavicchia, L., and H. von Storch, 2011: The simulation of medicanes in a highresolution regional climate model. Clim. Dyn., 39 2273-2290.

Cesana, G., and H. Chepfer, 2012: How well do climate models simulate cloud vertical structure? A comparison between CALIPSO-GOCCP satellite observations and CMIP5 models. Geophys. Res. Lett., 39, L20803.

Cha, D., D. Lee, and S. Hong, 2008: Impact of boundary layer processes on seasonal simulation of the East Asian summer monsoon using a Regional Climate Model. Meteorol. Atmos. Phys., 100, 53-72.

Champion, A. J., K. I. Hodges, L. O. Bengtsson, N. S. Keenlyside, and M. Esch, 2011: Impact of increasing resolution and a warmer climate on extreme weather from Northern Hemisphere extratropical cyclones. Tellus A, 63, 893-890.

Chan, S. C., E. J. Kendon, H. J. Fowler, S. Blenkinsop, C. A. T. Ferro, and D. B. Stephenson, 2012: Does increasing resolution improve the simulation of United Kingdom daily precipitation in a regional climate model? Clim. Dyn., doi:10.1007/s00382012-1568-9.

Chang, C. P., and T. Li, 2000: A theory for the tropical tropospheric biennial oscillation. J. Atmos. Sci, 57, 2209-2224.

Chang, C. Y., S. Nigam, and J. A. Carton, 2008: Origin of the springtime westerly bias in equatorial Atlantic surface winds in the Community Atmosphere Model version 3 (CAM3) simulation. J. Clim., 21, 4766-4778.

Chang, C. Y., J. A. Carton, S. A. Grodsky, and S. Nigam, 2007: Seasonal climate of the tropical Atlantic sector in the NCAR community climate system model 3: Error structure and probable causes of errors. J. Clim., 20, 1053-1070.

Chang, E. K. M., Y. Guo, and X. Xia, 2012: CMIP5 multi-model ensemble projection of storm track change under global warming. J. Geophys. Res., 117, D23118.

Charbit, S., D. Paillard, and G. Ramstein, 2008: Amount of $\mathrm{CO}_{2}$ emissions irreversibly leading to the total melting of Greenland. Geophys. Res. Lett., 35, L12503.

Charlton-Perez, A. J., et al., 2012: Mean climate and variability of the stratosphere in CMIP5 models. J. Geophys. Res., doi:10.1002/jgrd.50125.

Chen, C. T., and T. Knutson, 2008: On the verification and comparison of extreme rainfall indices from climate models. J. Clim., 21, 1605-1621.

Chen, H. M., T. J. Zhou, R. B. Neale, X. Q. Wu, and G. J. Zhang, 2010: Performance of the New NCAR CAM3.5 in East Asian summer monsoon simulations: Sensitivity to modifications of the Convection Scheme. J. Clim., 23, 3657-3675.

Chen, L., Y. Yu, and D. Sun, 2013: Cloud and water vapor feedbacks to the El Niño warming: Are they still biased in CMIP5 models? J. Clim., doi:10.1175/JCLI-D12-00575.1.

Chen, Y. H., and A. D. Del Genio, 2009: Evaluation of tropical cloud regimes in observations and a general circulation model. Clim. Dyn., 32, 355-369.

Chiang, J. C. H., and A. H. Sobel, 2002: Tropical tropospheric temperature variations caused by ENSO and their influence on the remote tropical climate. J. Clim., 15, 2616-2631.

Chiang, J. C. H., and D. J. Vimont, 2004: Analogous Pacific and Atlantic meridional modes of tropical atmosphere-ocean variability. J. Clim., 17, 4143-4158.

Chou, C., and J. Y. Tu, 2008: Hemispherical asymmetry of tropical precipitation in ECHAM5/MPI-OM during El Niño and under global warming. J. Clim., 21, 13091332.

Chou, C., J. D. Neelin, J. Y. Tu, and C. T. Chen, 2006: Regional tropical precipitation change mechanisms in ECHAM4/OPYC3 under global warming. J. Clim., 19, 4207-4223.

Chou, S., et al., 2012: Downscaling of South America present climate driven by 4-member HadCM3 runs. Clim. Dyn., 38, 635-653.

Christensen, J., F. Boberg, O. Christensen, and P. Lucas-Picher, 2008: On the need for bias correction of regional climate change projections of temperature and precipitation. Geophys. Res. Lett., 35, L20709.

Christensen, J., E. Kjellstrom, F. Giorgi, G. Lenderink, and M. Rummukainen, 2010: Weight assignment in regional climate models. Clim. Res., 44, 179-194.

Christensen, J. H., and F. Boberg, 2013: Temperature dependent climate projection deficiencies in CMIP5 models. Geophys. Res. Lett., 39, L24705.
Christian, J. R., et al., 2010: The global carbon cycle in the Canadian Earth system model (CanESM1): Preindustrial control simulation. J. Geophys. Res. Biogeosci., 115, G03014

Christidis, N., P.A. Stott, and S. J. Brown, 2011: The role of human activity in the recent warming of extremely warm daytime temperatures. J. Clim., 24, 1922-1930.

Christy, J. R., W. B. Norris, R. W. Spencer, and J. J. Hnilo, 2007: Tropospheric temperature change since 1979 from tropical radiosonde and satellite measurements. $J$. Geophys. Res. Atmos., 112, D06102.

Christy, J. R., et al., 2010: What do observational datasets say about modeled tropospheric temperature trends since 1979? Remote Sens., 2, 2148-2169.

Cimatoribus, A. A., S. S. Drijfhout, and H. A. Dijkstra, 2012: A global hybrid coupled model based on atmosphere-SST feedbacks. Clim. Dyn., 38, 745-760.

Cionni, I., et al., 2011: Ozone database in support of CMIP5 simulations: Results and corresponding radiative forcing. Atmos. Chem. Phys., 11, 11267-11292.

Clark, D. B., et al., 2011: The Joint UK Land Environment Simulator (JULES), model description - Part 2: Carbon fluxes and vegetation dynamics. Geosci. Model Dev., $4,701-722$.

Claussen, M., et al., 2002: Earth system models of intermediate complexity: Closing the gap in the spectrum of climate system models. Clim. Dyn., 18, 579-586.

Coelho, C. A. S., and L. Goddard, 2009: El Niño-induced tropical droughts in climate change projections. J. Clim., 22, 6456-6476.

Cohen, J. L., J. C. Furtado, M. Barlow, V. A. Alexeev, and J. E. Cherry, 2012:Asymmetric seasonal temperature trends. Geophys. Res. Lett., 39, L04705.

Collatz, G. J., M. Ribas-Carbo, and J. A. Berry, 1992: Coupled photosynthesis-stomatal conductance model for leaves of C4 Plants. Aust. J. Plant Physiol., 19, 519-538.

Collatz, G. J., J. T. Ball, C. Grivet, and J. A. Berry, 1991: Physiological and environmental regulation of stomatal conductance, photosynthesis and transpiration: A model that includes a laminar boundary layer. Agr. Forest Meteorol., 54, 107-136.

Colle, B. A., Z. Zhang, K. A. Lombardo, E. Chang, P. Liu, and M. Zhang, 2013: Historical evaluation and future prediction of eastern North America and western Atlantic extratropical cyclones in the CMIP5 models during the cool season. J. Clim., doi:10.1175/JCLI-D-12-00498.1.

Collins, M., S. Tett, and C. Cooper, 2001: The internal climate variability of HadCM3, a version of the Hadley Centre coupled model without flux adjustments. Clim. Dyn., 17, 61-81.

Collins, M., C. M. Brierley, M. MacVean, B. B. B. Booth, and G. R. Harris, 2007: The sensitivity of the rate of transient climate change to ocean physics perturbations. J. Clim., 20, 2315-2320.

Collins, M., B. B. B. Booth, G. R. Harris, J. M. Murphy, D. M. H. Sexton, and M. J. Webb, 2006a: Towards quantifying uncertainty in transient climate change. Clim. Dyn., 27, 127-147.

Collins, M., R. Chandler, P. Cox, J. Huthnance, J. Rougier, and D. Stephenson, 2012: Quantifying future climate change. Nature Clim. Change, 2, 403-409.

Collins, M., B. Booth, B. Bhaskaran, G. Harris, J. Murphy, D. Sexton, and M. Webb, 2010: Climate model errors, feedbacks and forcings: A comparison of perturbed physics and multi-model ensembles. Clim. Dyn., 36, 1737-1766.

Collins, W. D., J. M. Lee-Taylor, D. P. Edwards, and G. L. Francis, 2006b: Effects of increased near-infrared absorption by water vapor on the climate system. J. Geophys. Res. Atmos., 111, D18109.

Collins, W. D., et al., 2006c: The formulation and atmospheric simulation of the Community Atmosphere Model version 3 (CAM3). J. Clim., 19, 2144-2161.

Collins, W. D., et al., 2006d: The Community Climate System Model version 3 (CCSM3). J. Clim., 19, 2122-2143.

Collins, W. J., et al., 2011: Development and evaluation of an Earth-System modelHadGEM2. Geosci. Model Dev., 4, 1051-1075.

Colman, R., and B. McAvaney, 2009: Climate feedbacks under a very broad range of forcing. Geophys. Res. Lett., 36, L01702.

Colman, R. A., A. F. Moise, and L. I. Hanson, 2011: Tropical Australian climate and the Australian monsoon as simulated by 23 CMIP3 models. J. Geophys. Res. Atmos., 116, D10116.

Comiso, J. C., and F. Nishio, 2008: Trends in the sea ice cover using enhanced and compatible AMSR-E, SSM/I, and SMMR data. J. Geophys. Res. Oceans, 113, C02s07.

Compo, G. P., and P. D. Sardeshmukh, 2009: Oceanic influences on recent continental warming. Clim. Dyn., 32, 333-342.

Connolley, W., and T. Bracegirdle, 2007: An Antarctic assessment of IPCC AR4 coupled models. Geophys. Res. Lett., 34 L22505. 
Coon, M., R. Kwok, G. Levy, M. Pruis, H. Schreyer, and D. Sulsky, 2007: Arctic Ice Dynamics Joint Experiment (AIDJEX) assumptions revisited and found inadequate. J. Geophys. Res., 112, C11590.

Coppola, E., F. Giorgi, S. Rauscher, and C. Piani, 2010: Model weighting based on mesoscale structures in precipitation and temperature in an ensemble of regional climate models. Clim. Res., 44 121-134.

Cox, P., 2001: Description of the "TRIFFID" Dynamic Global Vegetation Model Hadley Centre, Met Office Hadley Centre, Berks, United Kingdom, $16 \mathrm{pp}$.

Cox, P. M., R. A. Betts, C. D. Jones, S. A. Spall, and I. J. Totterdell, 2000: Acceleration of global warming due to carbon-cycle feedbacks in a coupled climate model. Nature, 408, 184-187.

Cox, P. M., R. A. Betts, C. B. Bunton, R. L. H. Essery, P. R. Rowntree, and J. Smith, 1999: The impact of new land surface physics on the GCM simulation of climate and climate sensitivity. Clim. Dyn., 15, 183-203.

Cox, P. M., D. Pearson, B. B. B. Booth, P. Friedlingstein, C. Huntingford, C. D. Jones, and C. M. Luke, 2013: Sensitivity of tropical carbon to climate change constrained by carbon dioxide variability. Nature, 494, 341-344.

Cramer, W., et al., 2001: Global response of terrestrial ecosystem structure and function to $\mathrm{CO}_{2}$ and climate change: Results from six dynamic global vegetation models. Global Change Biol., 7, 357-373.

Crétat, J., B. Pohl, Y. Richard, and P. Drobinski, 2012: Uncertainties in simulating regional climate of Southern Africa: Sensitivity to physical parameterizations using WRF. Clim. Dyn., 38, 613-634.

Croft, B., U. Lohmann, and K. von Salzen, 2005: Black carbon ageing in the Canadian Centre for Climate modelling and analysis atmospheric general circulation model. Atmos. Chem. Phys., 5, 1931-1949.

Crucifix, M., 2006: Does the Last Glacial Maximum constrain climate sensitivity? Geophys. Res. Lett., 33, L18701.

Cunningham, S., et al., 2010: The present and future system for measuring the Atlantic meridional overturning circulation and heat transport. In: Proceedings of OceanObs'09: Sustained Ocean Observations and Information for Society (Vol. 2), Venice, Italy, 21-25 September 2009, ESA Publication.

Cunningham, S. A., S. G. Alderson, B. A. King, and M. A. Brandon, 2003: Transport and variability of the Antarctic Circumpolar Current in Drake Passage. J. Geophys. Res.-Oceans, 108, 8084.

Cunningham, S. A., et al., 2007: Temporal variability of the Atlantic meridional overturning circulation at $26.5^{\circ} \mathrm{N}$. Science, $317,935-938$.

Curry, W. B., and D. W. Oppo, 2005: Glacial water mass geometry and the distribution of delta $\mathrm{C}-13$ of sigma $\mathrm{CO}_{2}$ in the western Atlantic Ocean. Paleoceanography, 20, Pa1017.

Cuxart, J., et al., 2006: Single-column model intercomparison for a stably stratified atmospheric boundary layer. Boundary-Layer Meteorol., 118, 273-303.

Dai, A., 2001: Global precipitation and thunderstorm frequencies. Part II: Diurnal variations. J. Clim., 14, 1112-1128.

Dai, A., 2006: Precipitation characteristics in eighteen coupled climate models. J. Clim., 19, 4605-4630.

Dai, A., and C. Deser, 1999: Diurnal and semidiurnal variations in global surface wind and divergence fields. J. Geophys. Res. Atmos., 104, 31109-31125.

Dai, A., and K. E. Trenberth, 2004: The diurnal cycle and its depiction in the Community Climate System Model. J. Clim., 17, 930-951.

Dai, Y. J., R. E. Dickinson, and Y. P. Wang, 2004: A two-big-leaf model for canopy temperature, photosynthesis, and stomatal conductance. J. Clim., 17, 22812299.

Dai, Y. J., et al., 2003: The Common Land Model. Bull. Am. Meteorol. Soc., 84, 10131023.

Dallmeyer, A., M. Claussen, and J. Otto, 2010: Contribution of oceanic and vegetation feedbacks to Holocene climate change in monsoonal Asia. Clim. Past, 6, 195218.

Danabasoglu, G., and P. R. Gent, 2009: Equilibrium climate sensitivity: Is it accurate to use a Slab Ocean Model? J. Clim., 22, 2494-2499.

Danabasoglu, G., R. Ferrari, and J. C. McWilliams, 2008: Sensitivity of an ocean general circulation model to a parameterization of near-surface eddy fluxes. J. Clim., 21, 1192-1208.

Danabasoglu, G., W. G. Large, and B. P. Briegleb, 2010: Climate impacts of parameterized Nordic Sea overflows. J. Geophys. Res. Oceans, 115, C11005.

Danabasoglu, G., W. G. Large, J. J. Tribbia, P. R. Gent, B. P. Briegleb, and J. C. McWilliams, 2006: Diurnal coupling in the tropical oceans of CCSM3. J. Clim. $19,2347-2365$.
Danabasoglu, G., et al., 2012: The CCSM4 Ocean Component. J. Clim., 25, 13611389.

Davies, T., M. J. P. Cullen, A. J. Malcolm, M. H. Mawson, A. Staniforth, A. A. White, and $N$. Wood, 2005: A new dynamical core for the Met Office's global and regional modelling of the atmosphere. Q. J. R. Meteorol. Soc., 131, 1759-1782.

Davis, B. A. S., and S. Brewer, 2009: Orbital forcing and role of the latitudinal insolation/temperature gradient. Clim. Dyn., 32, 143-165.

Dawson, A., T. N. Palmer, and S. Corti, 2012: Simulating regime structures in weather and climate prediction models. Geophys. Res. Lett., 39, L21805.

Day, J. J., J. C. Hargreaves, J. D. Annan, and A. Abe-Ouchi, 2012: Sources of multidecadal variability in Arctic sea ice extent. Environ. Res. Lett., 7, 034011.

de Elia, R., and H. Cote, 2010: Climate and climate change sensitivity to model configuration in the Canadian RCM over North America. Meteorol. Z., 19, 325339.

de Elia, R., S. Biner, and A. Frigon, 2013: Interannual variability and expected regional climate change over North America. Clim. Dyn., doi:10.1007/s00382-013-17179.

de Jong, M. F., S. S. Drijfhout, W. Hazeleger, H. M. van Aken, and C. A. Severijns, 2009: Simulations of hydrographic properties in the Northwestern North Atlantic Ocean in Coupled Climate Models. J. Clim., 22, 1767-1786.

de Noblet-Ducoudre, N., et al., 2012: Determining robust impacts of land-use induced land-cover changes on surface climate over North America and Eurasia; Results from the first set of LUCID experiments. J. Clim., 25, 3261-3281.

De Szoeke, S. P., and S. P. Xie, 2008: The tropical eastern Pacific seasonal cycle: Assessment of errors and mechanisms in IPCC AR4 coupled ocean - atmosphere general circulation models. J. Clim., 21, 2573-2590.

Dee, D. P., et al., 2011: The ERA-Interim reanalysis: Configuration and performance of the data assimilation system. Q. J. R. Meteorol. Soc., 137, 553-597.

DelSole, T., and J. Shukla, 2009: Artificial skill due to predictor screening. J. Clim., $22,331-345$.

Delworth, T. L., et al., 2012: Simulated climate and climate change in the GFDL CM2.5 High-Resolution Coupled Climate Model. J. Clim., 25, 2755-2781.

Delworth, T. L., et al., 2006: GFDL's CM2 global coupled climate models. Part I: Formulation and simulation characteristics. J. Clim., 19, 643-674.

Déqué, M., 2007: Frequency of precipitation and temperature extremes over France in an anthropogenic scenario: Model results and statistical correction according to observed values. Global Planet. Change, 57, 16-26.

Déqué, M., 2010: Regional climate simulation with a mosaic of RCMs. Meteorol. Z., $19,259-266$.

Déqué, M., C. Dreveton, A. Braun, and D. Cariolle, 1994: The ARPEGE/IFS atmosphere model: A contribution to the French community climate modelling. Clim. Dyn., $10,249-266$

Déqué, M., S. Somot, E. Sanchez-Gomez, C. Goodess, D. Jacob, G. Lenderink, and O. Christensen, 2012: The spread amongst ENSEMBLES regional scenarios: Regional climate models, driving general circulation models and interannual variability. Clim. Dyn., 38, 951-964.

Derksen, C., and R. Brown, 2012: Spring snow cover extent reductions in the 2008-2012 period exceeding climate model projections. Geophys. Res. Lett., 39, L19504.

Deser, C., A. S. Phillips, and J. W. Hurrell, 2004: Pacific interdecadal climate variability: Linkages between the tropics and the North Pacific during boreal winter since 1900. J. Clim., 17, 3109-3124.

Deser, C., A. S. Phillips, V. Bourdette, and H. Teng, 2011: Uncertainty in climate change projections: The role of internal variability. Clim. Dyn., 38, 527-546.

Deser, C., et al., 2012: ENSO and Pacific decadal variability in Community Climate System Model Version 4. J. Clim., 25, 2622-2651.

Deushi, M., and K. Shibata, 2011: Development of a Meteorological Research Institute Chemistry-Climate Model version 2 for the Study of Tropospheric and Stratospheric Chemistry. Papers Meteorol. Geophys., 62, 1-46.

Di Luca, A., R. Elía, and R. Laprise, 2012: Potential for small scale added value of RCM's downscaled climate change signal. Clim. Dyn., 40, 601-618.

Diaconescu, E. P., and R. Laprise, 2013: Can added value be expected in RCMsimulated large scales? Clim. Dyn., doi:10.1007/s00382-012-1649-9.

Diffenbaugh, N., M. Ashfaq, and M. Scherer, 2011: Transient regional climate change: Analysis of the summer climate response in a high-resolution, centuryscale ensemble experiment over the continental United States. J. Geophys. Res. Atmos., 116, D24111.

DiNezio, P. N., A. C. Clement, G. A. Vecchi, B. J. Soden, and B. P. Kirtman, 2009: Climate response of the equatorial Pacific to global warming. J. Clim., 22, 4873-4892. 
Dix, M., et al., 2013: The ACCESS Coupled Model: Documentation of core CMIP5 simulations and initial results. Aust. Meteorol. Oceanogr. J., 63, 83-99.

Doblas-Reyes, F. J., et al., 2013: Initialized near-term regional climate change prediction. Nature Commun., 4, 1715.

Dokken, T. M., and E. Jansen, 1999: Rapid changes in the mechanism of ocean convection during the last glacial period. Nature, 401, 458-461.

Domingues, C., J. Church, N. White, P. Gleckler, S. Wijffels, P. Barker, and J. Dunn, 2008: Improved estimates of upper-ocean warming and multi-decadal sea-level rise. Nature, 453, 1090-1093.

Donat, M., G. Leckebusch, S. Wild, and U. Ulbrich, 2010: Benefits and limitations of regional multi-model ensembles for storm loss estimations. Clim. Res., 44, 211-225.

Donat, M. G., et al., 2013: Updated analyses of temperature and precipitation extreme indices since the beginning of the twentieth century: The HadEX2 dataset. J. Geophys. Res., doi:10.1002/2012JD018606.

Donner, L. J., et al., 2011: The dynamical core, physical parameterizations, and basic simulation characteristics of the atmospheric component AM3 of the GFDL Global Coupled Model CM3. J. Clim., 24, 3484-3519.

Dorn, W., K. Dethloff, and A. Rinke, 2009: Improved simulation of feedbacks between atmosphere and sea ice over the Arctic Ocean in a coupled regional climate model. Ocean Model., 29, 103-114.

Doscher, R., K. Wyser, H. E. M. Meier, M. W. Qian, and R. Redler, 2010: Quantifying Arctic contributions to climate predictability in a regional coupled ocean-iceatmosphere model. Clim. Dyn., 34, 1157-1176.

Douglass, D., J. Christy, B. Pearson, and S. Singer, 2008: A comparison of tropical temperature trends with model predictions. Int. J. Climatol. , 28, 1693-1701.

Dowdy, A. J., G. A. Mills, B. Timbal, and Y. Wang, 2013: Changes in the risk of extratropical cyclones in Eastern Australia. J. Clim., 26, 1403-1417.

Driesschaert, E., et al., 2007: Modeling the influence of Greenland ice sheet melting on the Atlantic meridional overturning circulation during the next millennia. Geophys. Res. Lett., 34, L10707.

Driouech, F., M. Deque, and E. Sanchez-Gomez, 2010: Weather regimes-Moroccan precipitation link in a regional climate change simulation. Global Planet. Change, 72, 1-10.

Driscoll, S., A. Bozzo, L. J. Gray, A. Robock, and G. Stenchikov, 2012: Coupled Model Intercomparison Project 5 (CMIP5) simulations of climate following volcanic eruptions. J. Geophys. Res. Atmos., 117, D17105.

Druyan, L. M., et al., 2010: The WAMME regional model intercomparison study. Clim. Dyn., 35, 175-192.

Du, Y., S.-P. Xie, Y.-L. Yang, X.-T. Zheng, L. Liu, and G. Huang, 2013: Indian Ocean variability in the CMIP5 multi-model ensemble: The basin mode. J. Clim., 26, 7240-7266.

Ducet, N., P. Y. Le Traon, and G. Reverdin, 2000: Global high-resolution mapping of ocean circulation from TOPEX/Poseidon and ERS-1 and-2. J. Geophys. Res. Oceans, 105, 19477-19498.

Dufresne, J.-L., et al., 2012: Climate change projections using the IPSL-CM5 Earth System Model: From CMIP3 to CMIP5. Clim. Dyn., doi:10.1007/s00382-0121636-1.

Dufresne, J. L., and S. Bony, 2008: An assessment of the primary sources of spread of global warming estimates from coupled atmosphere-ocean models. J. Clim., 21, 5135-5144.

Dunkerton, T. J., 1991: Nonlinear propagation of zonal winds in an atmosphere with Newtonian cooling and equatorial wavedriving. J. Atmos. Sci., 48, 236-263.

Dunn-Sigouin, E., and S.-W. Son, 2013: Northern Hemisphere blocking frequency and duration in the CMIP5 models. J. Geophys. Res., 118, 1179-1188.

Dunne, J. P., et al., 2013: GFDL's ESM2 global coupled climate-carbon Earth System Models Part II: Carbon system formulation and baseline simulation characteristics. J. Clim., doi:10.1175/JCLI-D-12-00150.1.

Dunne, J. P., et al., 2012: GFDL's ESM2 Global coupled climate-carbon Earth System models. Part I: Physical formulation and baseline simulation characteristics. J. Clim., 25, 6646-6665.

Duplessy, J. C., N. J. Shackleton, R. Fairbanks, L. Labeyrie, D. Oppo, and N. Kallel, 1988: Deep water source variation during the last climatic cycle and their impact on th global deep water circulation. Paleoceanography, 3, 343-360.

Durack, P. J., and S. E. Wijffels, 2010: Fifty-year trends in global ocean salinities and their relationship to broad-scale warming. J. Clim., 23, 4342-4362.

Durack, P. J., S. E. Wijffels, and R. J. Matear, 2012: Ocean salinities reveal strong global water cycle intensification during 1950 to 2000. Science, 336, 455-458.
Easterling, D. R., and M. F. Wehner, 2009: Is the climate warming or cooling? Geophys. Res. Lett., 36, L08706.

Eby, M., K. Zickfeld, A. Montenegro, D. Archer, K. J. Meissner, and A. J. Weaver, 2009: Lifetime of anthropogenic climate change: Millennial time scales of potential $\mathrm{CO}_{2}$ and surface temperature perturbations. J. Clim., 22, 2501-2511.

Eby, M., et al., 2013: Historical and idealized climate model experiments: An EMIC intercomparison. Clim. Past, 9, 1111-1140.

Edwards, N., and R. Marsh, 2005: Uncertainties due to transport-parameter sensitivity in an efficient 3-D ocean-climate model. Clim. Dyn., 24, 415-433.

Edwards, N. R., D. Cameron, and J. Rougier, 2011: Precalibrating an intermediate complexity climate model. Clim. Dyn., 37, 1469-1482.

Ek, M. B., et al., 2003: Implementation of Noah land surface model advances in the National Centers for Environmental Prediction operational mesoscale Eta model. J. Geophys. Res. Atmos., 108, 8851.

Eliseev, A. V., and I. I. Mokhov, 2011: Uncertainty of climate response to natural and anthropogenic forcings due to different land use scenarios. Adv. Atmos. Sci., 28, $1215-1232$.

Emanuel, K., R. Sundararajan, and J. Williams, 2008: Hurricanes and global warming - Results from downscaling IPCC AR4 simulations. Bull. Am. Meteorol. Soc., 89, 347-367.

Endo, H., A. Kitoh, T. Ose, R. Mizuta, and S. Kusunoki, 2012: Future changes and uncertainties in Asian precipitation simulated by multiphysics and multi-sea surface temperature ensemble experiments with high-resolution Meteorological Research Institute atmospheric general circulation models (MRI-AGCMs). J. Geophys. Res. Atmos., 117, D16118.

Essery, R. L. H., M. J. Best, R. A. Betts, P. M. Cox, and C. M. Taylor, 2003: Explicit representation of subgrid heterogeneity in a GCM land surface scheme. J. Hydrometeorol., 4, 530-543.

Eum, H., P. Gachon, R. Laprise, and T. Ouarda, 2012: Evaluation of regional climate model simulations versus gridded observed and regional reanalysis products using a combined weighting scheme. Clim. Dyn., 38, 1433-1457.

Evans, J. P., M. Ekstroem, and F. Ji, 2012: Evaluating the performance of a WRF physics ensemble over South-East Australia. Clim. Dyn., 39, 1241-1258.

Eyring, V., et al., 2010: Transport impacts on atmosphere and climate: Shipping. Atmos. Environ., 44, 4735-4771.

Eyring, V., et al., 2013: Long-term ozone changes and associated climate impacts in CMIP5 simulations. J. Geophys. Res., doi:10.1002/jgrd.50316.

Eyring, V., et al., 2007: Multimodel projections of stratospheric ozone in the 21st century. J. Geophys. Res. Atmos., 112, D16303.

Faloona, I., 2009: Sulfur processing in the marine atmospheric boundary layer: A review and critical assessment of modeling uncertainties. Atmos. Environ., 43, 2841-2854.

Fan, F. X., M. E. Mann, S. Lee, and J. L. Evans, 2010: Observed and modeled changes in the South Asian summer monsoon over the Historical Period. J. Clim., 23, 5193-5205.

Fanning, A. F., and A. J. Weaver, 1996: An atmospheric energy-moisture balance model: Climatology, interpentadal climate change, and coupling to an ocean general circulation model. J. Geophys. Res. Atmos., 101, 15111-15128.

Farneti, R., and P. R. Gent, 2011: The effects of the eddy-induced advection coefficient in a coarse-resolution coupled climate model. Ocean Model., 39, 135-145.

Farneti, R., T. L. Delworth, A. J. Rosati, S. M. Griffies, and F. R. Zeng, 2010: The role of mesoscale eddies in the rectification of the Southern Ocean response to climate change. J. Phys. Oceanogr., 40, 1539-1557.

Fasullo, J., and K. E. Trenberth, 2012: A less cloudy future: The role of subtropical subsidence in climate sensitivity. Science, 338, 792-794.

Fauchereau, N., S. Trzaska, Y. Richard, P. Roucou, and P. Camberlin, 2003: Sea-surface temperature co-variability in the southern Atlantic and Indian Oceans and its connections with the atmospheric circulation in the Southern Hemisphere. Int. J. Climatol. , 23, 663-677.

Felzer, B., D. Kicklighter, J. Melillo, C. Wang, Q. Zhuang, and R. Prinn, 2004: Effects of ozone on net primary production and carbon sequestration in the conterminous United States using a biogeochemistry model. Tellus $B, 56,230-248$.

Feng, J., and C. Fu, 2006: Inter-comparison of 10-year precipitation simulated by several RCMs for Asia. Adv. Atmos. Sci., 23 531-542.

Feng, J., et al., 2011: Comparison of four ensemble methods combining regional climate simulations over Asia. Meteorol. Atmos. Phys., 111, 41-53.

Fernandes, R., H. X. Zhao, X. J. Wang, J. Key, X. Qu, and A. Hall, 2009: Controls on Northern Hemisphere snow albedo feedback quantified using satellite Earth observations. Geophys. Res. Lett., 36, L21702. 
Fernandez-Donado, L., et al., 2013: Large-scale temperature response to external forcing in simulations and reconstructions of the last millennium. Clim. Past, 9, 393-421.

Ferrari, R., J. C. McWilliams, V. M. Canuto, and M. Dubovikov, 2008: Parameterization of eddy fluxes near oceanic boundaries. J. Clim., 21, 2770-2789.

Ferrari, R., S. M. Griffies, A. J. G. Nurser, and G. K. Vallis, 2010: A boundary-value problem for the parameterized mesoscale eddy transport. Ocean Model., 32 143-156.

Feser, F., 2006: Enhanced detectability of added value in limited-area model results separated into different spatial scales. Mon. Weather Rev., 134, 2180-2190.

Feser, F., and M. Barcikowska, 2012: The influence of spectral nudging on typhoon formation in regional climate models. Environ. Res. Lett., 7, 014024.

Feser, F., B. Rockel, H. von Storch, J. Winterfeldt, and M. Zahn, 2011: Regional climate models add value to global model data: A review and selected examples. Bull. Am. Meteorol. Soc., 92, 1181-1192.

Fetterer, F., K. Knowles, W. Meier, and M. Savoie, 2002: Sea Ice Index. National Snow and Ice Data Center. Boulder, CO, USA.

Fichefet, T., and M. A. M. Maqueda, 1997: Sensitivity of a global sea ice model to the treatment of ice thermodynamics and dynamics. J. Geophys. Res., 102, 12609-12646.

Fichefet, T., and M. A. M. Maqueda, 1999: Modelling the influence of snow accumulation and snow-ice formation on the seasonal cycle of the Antarctic sea-ice cover. Clim. Dyn., 15, 251-268.

Field, P. R., A. Gettelman, R. B. Neale, R. Wood, P. J. Rasch, and H. Morrison, 2008: Midlatitude cyclone compositing to constrain climate model behavior using satellite observations. J. Clim., 21, 5887-5903.

Fioletov, V., G. Bodeker, A. Miller, R. McPeters, and R. Stolarski, 2002: Global and zonal total ozone variations estimated from ground-based and satellite measurements: 1964-2000. J. Geophys. Res. Atmos., 107, 4647.

Fischer, E. M., S. I. Seneviratne, D. Lüthi, and C. Schär, 2007: Contribution of landatmosphere coupling to recent European summer heat waves. Geophys. Res. Lett., 34, L06707.

Flanner, M. G., K. M. Shell, M. Barlage, D. K. Perovich, and M. A. Tschudi, 2011 Radiative forcing and albedo feedback from the Northern Hemisphere cryosphere between 1979 and 2008. Nature Geosci. , 4, 151-155.

Flato, G., 2011: Earth system models: an overview. Wiley Interdisciplinary Reviews, Climate Change, 2, 783-800.

Flocco, D., D. Schroeder, D. L. Feltham, and E. C. Hunke, 2012: Impact of melt ponds on Arctic sea ice simulations from 1990 to 2007. J. Geophys. Res.Oceans, 117 C09032.

Fogli, P. G., et al., 2009: INGV-CMCC Carbon (ICC): A Carbon Cycle Earth System Model. CMCC Res. Papers. Euro-Mediterranean Center on Climate Change, Bologna, Italy, $31 \mathrm{pp}$.

Fogt, R. L., J. Perlwitz, A. J. Monaghan, D. H. Bromwich, J. M. Jones, and G. J. Marshall, 2009: Historical SAM variability. Part II: Twentieth-century variability and trends from reconstructions, observations, and the IPCC AR4 models. J. Clim., 22, $5346-5365$.

Fontaine, B., and S. Janicot, 1996: Sea surface temperature fields associated with West African rainfall anomaly types. J. Clim., 9, 2935-2940.

Forest, C. E., P. H. Stone, and A. P. Sokolov, 2006: Estimated PDFs of climate system properties including natural and anthropogenic forcings. Geophys. Res. Lett., 33 L01705.

Forest, C. E., P. H. Stone, and A. P. Sokolov, 2008: Constraining climate model parameters from observed 20th century changes. Tellus A, 60, 911-920.

Forest, C. E., P. H. Stone, A. P. Sokolov, M. R. Allen, and M. D. Webster, 2002: Quantifying uncertainties in climate system properties with the use of recent climate observations. Science, 295, 113-117.

Forster, P. M., T. Andrews, P. Good, J. M. Gregory, L. S. Jackson, and M. Zelinka, 2013 Evaluating adjusted forcing and model spread for historical and future scenarios in the CMIP5 generation of climate models. J. Geophys. Res. Atmos., 118, 11391150.

Fowler, H., S. Blenkinsop, and C. Tebaldi, 2007: Linking climate change modelling to impacts studies: Recent advances in downscaling techniques for hydrological modelling. Int. J. Climatol., 27, 1547-1578.

Fox-Kemper, B., R. Ferrari, and R. Hallberg, 2008: Parameterization of mixed layer eddies. Part I: Theory and diagnosis. J. Phys. Oceanogr., 38, 1145-1165.

Fox-Kemper, B., et al., 2011: Parameterization of mixed layer eddies. III: Implementation and impact in global ocean climate simulations. Ocean Model., $39,61-78$.
Fox-Rabinovitz, M., J. Cote, B. Dugas, M. Deque, J. McGregor, and A. Belochitski, 2008: Stretched-grid Model Intercomparison Project: Decadal regional climate simulations with enhanced variable and uniform-resolution GCMs. Meteorol. Atmos. Phys., 100, 159-177.

Frame, D., B. Booth, J. Kettleborough, D. Stainforth, J. Gregory, M. Collins, and M. Allen, 2005: Constraining climate forecasts: The role of prior assumptions. Geophys. Res. Lett., 32, L09702.

Frankcombe, L. M., A. von der Heydt, and H. A. Dijkstra, 2010: North Atlantic multidecadal climate variability: An investigation of dominant time scales and processes. J. Clim., 23, 3626-3638.

Frederiksen, C. S., J. S. Frederiksen, J. M. Sisson, and S. L. Osbrough, 2011: Australian winter circulation and rainfall changes and projections. Int. J. Clim. Change Strat. Manage., 3, 170-188.

Friedlingstein, P., et al., 2001: Positive feedback between future climate change and the carbon cycle. Geophys. Res. Lett., 28, 1543-1546.

Friedlingstein, P., et al., 2006: Climate-carbon cycle feedback analysis: Results from the (CMIP)-M-4 model intercomparison. J. Clim., 19, 3337-3353.

Friend, A. D., et al., 2007: FLUXNET and modelling the global carbon cycle. Global Change Biol., 13, 610-633.

Frierson, D. M. W., J. Lu, and G. Chen, 2007: Width of the Hadley cell in simple and comprehensive general circulation models. Geophys. Res. Lett., 34, L18804.

Frohlich, C., and J. Lean, 2004: Solar radiative output and its variability: Evidence and mechanisms. Astron. Astrophys. Rev., 12, 273-320.

Frost, A. J., et al., 2011: A comparison of multi-site daily rainfall downscaling techniques under Australian conditions. J. Hydrol., 408, 1-18.

Fu, Q., S. Manabe, and C. M. Johanson, 2011: On the warming in the tropical upper troposphere: Models versus observations. Geophys. Res. Lett., 38, L15704.

Furrer, R., R. Knutti, S. Sain, D. Nychka, and G. Meehl, 2007: Spatial patterns of probabilistic temperature change projections from a multivariate Bayesian analysis. Geophys. Res. Lett., 34, L06711.

Furtado, J., E. Di Lorenzo, N. Schneider, and N. A. Bond, 2011: North Pacific decadal variability and climate change in the IPCC AR4 models. J. Clim., 24, 3049-3067

Fyfe, J. C., N. P. Gillett, and D. W. J. Thompson, 2010: Comparing variability and trends in observed and modelled global-mean surface temperature. Geophys. Res. Lett., 37, L16802.

Fyke, J. G., A. J. Weaver, D. Pollard, M. Eby, L. Carter, and A. Mackintosh, 2011: A new coupled ice sheet/climate model: Description and sensitivity to model physics under Eemian, Last Glacial Maximum, late Holocene and modern climate conditions. Geosci. Model Dev., 4, 117-136.

Galbraith, D., P. E. Levy, S. Sitch, C. Huntingford, P. Cox, M. Williams, and P. Meir, 2010: Multiple mechanisms of Amazonian forest biomass losses in three dynamic global vegetation models under climate change. New Phytologist, 187, 647-665.

Ganachaud, A., and C. Wunsch, 2003: Large-scale ocean heat and freshwater transports during the World Ocean Circulation Experiment. J. Clim., 16, 696-705.

Gangsto, R., F. Joos, and M. Gehlen, 2011: Sensitivity of pelagic calcification to ocean acidification. Biogeosciences, 8, 433-458.

Gao, X., Y. Shi, D. Zhang, J. Wu, F. Giorgi, Z. Ji, and Y. Wang, 2012: Uncertainties in monsoon precipitation projections over China: Results from two high-resolution RCM simulations. Clim. Res., 52, 213-226.

Gates, W. L., et al., 1999: An overview of the results of the Atmospheric Model Intercomparison Project (AMIP I). Bull. Am. Meteorol. Soc., 80, 29-55.

Gbobaniyi, E. O., B. J. Abiodun, M. A. Tadross, B. C. Hewitson, and W. J. Gutowski, 2011: The coupling of cloud base height and surface fluxes: A transferability intercomparison. Theor. Appl. Climatol., 106, 189-210.

Gehlen, M., R. Gangsto, B. Schneider, L. Bopp, 0. Aumont, and C. Ethe, 2007: The fate of pelagic $\mathrm{CaCO}_{3}$ production in a high $\mathrm{CO}_{2}$ ocean: A model study. Biogeosciences, 4, 505-519.

Geller, M. A., et al., 2011: New gravity wave treatments for GISS climate models. J. Clim., 24, 3989-4002.

Gent, P. R., and J. C. McWilliams, 1990: Isopycnal mixing in ocean circulation models. J. Phys. Oceanogr., 20, 150-155.

Gent, P. R., and G. Danabasoglu, 2011: Response to increasing Southern Hemisphere winds in CCSM4. J. Clim., 24, 4992-4998.

Gent, P. R., J. Willebrand, T. J. McDougall, and J. C. McWilliams, 1995: Parameterizing eddy-induced tracer transports in ocean circulation models. J. Phys. Oceanogr., $25,463-474$

Gent, P. R., et al., 2011: The Community Climate System Model Version 4. J. Clim., 24, 4973-4991. 
Gerber, E. P., L. M. Polvani, and D. Ancukiewicz, 2008: Annular mode time scales in the Intergovernmental Panel on Climate Change Fourth Assessment Report models. Geophys. Res. Lett., 35, L22707.

Gerber, S., L. O. Hedin, M. Oppenheimer, S. W. Pacala, and E. Shevliakova, 2010: Nitrogen cycling and feedbacks in a global dynamic land model. Global Biogeochem. Cycles, 24, Gb1001.

Gettelman, A., et al., 2010: Multimodel assessment of the upper troposphere and lower stratosphere: Tropics and global trends. J. Geophys. Res. Atmos., 115, D00m08.

Ghan, S., X. Liu, R. Easter, P. Rasch, J. Yoon, and B. Eaton, 2012: Toward a minimal representation of aerosols in climate models: Comparative decomposition of aerosol direct, semi-direct and indirect radiative forcing. J. Clim., doi:10.1175/ JCLI-D-11-00650.1.

Gillett, N. P., 2005: Climate modelling -Northern Hemisphere circulation. Nature, 437, 496-496.

Giorgi, F., and E. Coppola, 2010: Does the model regional bias affect the projected regional climate change? An analysis of global model projections. Clim. Change, $100,787-795$.

Girard, L., J. Weiss, J. M. Molines, B. Barnier, and S. Bouillon, 2009: Evaluation of high-resolution sea ice models on the basis of statistical and scaling properties of Arctic sea ice drift and deformation. J. Geophys. Res., 114, C08015.

Gleckler, P., K. Taylor, and C. Doutriaux, 2008: Performance metrics for climate models. J. Geophys. Res. Atmos., 113, D06104.

Gleckler, P., K. AchutaRao, J. Gregory, B. Santer, K. Taylor, and T. Wigley, 2006: Krakatoa lives: The effect of volcanic eruptions on ocean heat content and thermal expansion. Geophys. Res. Lett., 33, L17702.

Gleckler, P. J., et al., 2012: Human-induced global ocean warming on multidecadal timescales, Nature Climate Change, 2, 524-529.

Gnanadesikan, A., S. M. Griffies, and B. L. Samuels, 2007: Effects in a climate model of slope tapering in neutral physics schemes. Ocean Model., 16, 1-16.

Goddard, L., and S. J. Mason, 2002: Sensitivity of seasonal climate forecasts to persisted SST anomalies. Clim. Dyn., 19, 619-631.

Golaz, J.-C., M. Salzmann, L. J. Donner, L. W. Horowitz, Y. Ming, and M. Zhao, 2011: Sensitivity of the aerosol indirect effect to subgrid variability in the cloud parameterization of the GFDL Atmosphere General Circulation Model AM3. J. Clim., 24, 3145-3160.

Goosse, H., and T. Fichefet, 1999: Importance of ice-ocean interactions for the global ocean circulation: A model study. J. Geophys. Res. Oceans, 104, 23337-23355.

Goosse, H., et al., 2010: Description of the Earth system model of intermediate complexity LOVECLIM version 1.2. Geosci. Model Dev., 3, 603-633.

Gordon, C., et al., 2000: The simulation of SST, sea ice extents and ocean heat transports in a version of the Hadley Centre coupled model without flux adjustments. Clim. Dyn., 16, 147-168.

Gordon, H., et al., 2010: The CSIRO Mk3.5 Climate Model. CAWCR Technical Report, 21, 1-74.

Gordon, H. B., et al., 2002: The CSIRO Mk3 Climate System Model. Technical Paper No. 60. CSIRO Atmospheric Research, Aspendale, Vic., Australia.

Greeves, C. Z., V. D. Pope, R. A. Stratton, and G. M. Martin, 2007: Representation of Northern Hemisphere winter storm tracks in climate models. Clim. Dyn., 28, 683-702.

Gregory, J. M., and P. M. Forster, 2008: Transient climate response estimated from radiative forcing and observed temperature change. J. Geophys. Res. Atmos., 113, D23105.

Gregory, J. M., et al., 2004: A new method for diagnosing radiative forcing and climate sensitivity. Geophys. Res. Lett., 31, L03205.

Gregory, J. M., et al., 2005: A model intercomparison of changes in the Atlantic thermohaline circulation in response to increasing atmospheric $\mathrm{CO}_{2}$ concentration. Geophys. Res. Lett., 32, L12703.

Griffies, S. M., 2009: Elements of MOM4p1. GFDL Ocean Group Technical Report No. 6. NOAA/GFDL. Princeton, USA, $371 \mathrm{pp}$.

Griffies, S. M., and R. J. Greatbatch, 2012: Physical processes that impact the evolution of global mean sea level in ocean climate models. Ocean Model., 51, $37-72$.

Griffies, S. M., M. J. Harrison, R. C. Pacanowski, and A. Rosati, 2004: A Technical Guide to MOM4. GFDL Ocean Group Technical Report No. 5, Princeton, USA, 337 pp.

Griffies, S. M., et al., 2005: Formulation of an ocean model for global climate simulations. Ocean Sci., 1, 45-79.

Griffies, S. M., et al., 2009: Coordinated Ocean-ice Reference Experiments (COREs). Ocean Model., 26, 1-46.
Grose, M., M. Pook, P. McIntosh, J. Risbey, and N. Bindoff, 2012: The simulation of cutoff lows in a regional climate model: Reliability and future trends. Clim. Dyn., $39,445-459$.

Guemas, V., F. J. Doblas-Reyes, I. Andreu-Burillo, and M. Asif, 2013: Retrospective prediction of the global warming slowdown in the last decade. Nature Clim. Change, doi:10.1038/nclimate1863.

Guilyardi, E., 2006: El Niño - mean state - seasonal cycle interactions in a multimodel ensemble. Clim. Dyn., 26, 229-348.

Guilyardi, E., P. Braconnot, F. F. Jin, S. T. Kim, M. Kolasinski, T. Li, and I. Musat, 2009a: Atmosphere feedbacks during ENSO in a coupled GCM with a modified atmospheric convection scheme. J. Clim., 22, 5698-5718.

Guilyardi, E., et al., 2009b: Understanding El Niño in ocean-atmosphere general circulation models: Progress and challenges. Bull. Am. Meteorol. Soc., 90, 325340.

Guiot, J., J. J. Boreux, P. Braconnot, F. Torre, and P. Participants, 1999: Data-model comparison using fuzzy logic in paleoclimatology. Clim. Dyn., 15, 569-581.

Gupta, A. S., A. Santoso, A. S. Taschetto, C. C. Ummenhofer, J. Trevena, and M. H. England, 2009: Projected changes to the Southern Hemisphere ocean and sea ice in the IPCC AR4 climate models. J. Clim., 22, 3047-3078.

Gurney, K. R., et al., 2003: TransCom $3 \mathrm{CO}_{2}$ inversion intercomparison: 1. Annual mean control results and sensitivity to transport and prior flux information. Tellus B, 55, 555-579.

Gutowski, W., et al., 2010: Regional extreme monthly precipitation simulated by NARCCAP RCMs. J. Hydrometeorol., 11, 1373-1379.

Hall, A., and X. Qu, 2006: Using the current seasonal cycle to constrain snow albedo feedback in future climate change. Geophys. Res. Lett., 33, L03502.

Hallberg, R., and A. Gnanadesikan, 2006: The role of eddies in determining the structure and response of the wind-driven southern hemisphere overturning: Results from the Modeling Eddies in the Southern Ocean (MESO) project. J. Phys. Oceanogr., 36, 2232-2252.

Hallberg, R., and A. Adcroft, 2009: Reconciling estimates of the free surface height in Lagrangian vertical coordinate ocean models with mode-split time stepping. Ocean Model., 29, 15-26.

Halloran, P. R., 2012: Does atmospheric $\mathrm{CO}_{2}$ seasonality play an important role in governing the air-sea flux of $\mathrm{CO}_{2}$ ? Biogeosciences, 9, 2311-2323.

Ham, Y.-G., J. S. Kug, I. S. Kang, F. F. Jin, and A. Timmermann, 2010: Impact of diurnal atmospher-ocean coupling on tropical climate simulations using a coupled GCM. Clim. Dyn., 34, 905-917.

Hamilton, K., 1998: Effects of an imposed Quasi-Biennial Oscillation in a comprehensive troposphere-stratosphere-mesosphere general circulation model. J. Atmos. Sci., 55, 2393- 2418.

Handorf, D., and K. Dethloff, 2012: How well do state-of-the-art atmosphere-ocean general circulation models reproduce atmospheric teleconnection patterns? Tellus A, 64, 19777.

Hannart, A., J. L. Dufresne, and P. Naveau, 2009: Why climate sensitivity may not be so unpredictable. Geophys. Res. Lett., 36, L16707.

Hannay, C., et al., 2009: Evaluation of Forecasted Southeast Pacific Stratocumulus in the NCAR, GFDL, and ECMWF Models. J. Clim., 22, 2871-2889.

Hansen, J., R. Ruedy, M. Sato, and K. Lo, 2010: Global surface temperature change. Rev. Geophys., 48, Rg4004.

Hansen, J., M. Sato, P. Kharecha, and K. von Schuckmann, 2011: Earth's energy imbalance and implications. Atmos. Chem. Phys., 11, 13421-13449.

Hansen, J., et al., 1983: Efficient Three-Dimensional Global Models for Climate Studies: Models I and II. Mon. Weath. Rev., 111, 609-662.

Hansen, J., et al., 1984: Climate Sensitivity: Analysis of Feedback Mechanisms. Clim. Proc. Clim. Sens. Geophys. Monogr., 29, 130-163.

Hansen, J., et al., 2005: Efficacy of climate forcings. J. Geophys. Res. Atmos., 110, D18104.

Hardiman, S. C., N. Butchart, T. J. Hinton, S. M. Osprey, and L. J. Gray, 2012: The effect of a well-resolved stratosphere on surface climate: Differences between CMIP5 simulations with high and low top versions of the Met Office Climate Model. J. Clim., 25, 7083-7099.

Hargreaves, J. C., A. Abe-Ouchi, and J. D. Annan, 2007: Linking glacial and future climates through an ensemble of GCM simulations. Clim. Past, 3, 77-87.

Hargreaves, J. C., J. D. Annan, M. Yoshimori, and A. Abe-Ouchi, 2012: Can the Last Glacial Maximum constrain climate sensitivity? Geophys. Res. Lett., 39, L24702.

Hargreaves, J. C., A. Paul, R. Ohgaito, A. Abe-Ouchi, and J. D. Annan, 2011: Are paleoclimate model ensembles consistent with the MARGO data synthesis? Clim. Past, 7, 917-933. 
Hargreaves, J. C., J. D. Annan, R. Ohgaito, A. Paul, and A. Abe-Ouchi, 2013: Skill and reliability of climate model ensembles at the Last Glacial Maximum and mid Holocene. Clim. Past, 9, 811-823.

Hasegawa, A., and S. Emori, 2007: Effect of air-sea coupling in the assessment of $\mathrm{CO}_{2}$-induced intensification of tropical cyclone activity. Geophys. Res. Lett., 34, L05701.

Hasumi, H., 2006: CCSR Ocean Component Model (COCO) Version 4.0. CCSR Report. Centre for Climate System Research, University of Tokyo, Tokyo, Japan, 68 pp.

Hasumi, H., and S. Emori, 2004: K-1 Coupled GCM (MIROC) Description. Center for Climate System Research, University of Tokyo, Tokyo, Japan, 34 pp.

Hawkins, E., and R. Sutton, 2009: The potential to narrow uncertainty in regional climate predictions. Bull. Am. Meteorol. Soc., 90, 1095-1107.

Haynes, J. M., C. Jakob, W. B. Rossow, G. Tselioudis, and J. Brown, 2011: Major characteristics of Southern Ocean cloud regimes and their effects on the energy budget. J. Clim., 24, 5061-5080.

Haynes, P. H., 2006: The latitudinal structure of the QBO. Q. J. R. Meteorol. Soc., 124, 2645-2670.

Haywood, J. M., N. Bellouin, A. Jones, O. Boucher, M. Wild, and K. P. Shine, 2011: The roles of aerosol, water vapor and cloud in future global dimming/brightening. J. Geophys. Res. Atmos., 116, D20203.

Hazeleger, W., and R. J. Haarsma, 2005: Sensitivity of tropical Atlantic climate to mixing in a coupled ocean-atmosphere model. Clim. Dyn., 25, 387-399.

Hazeleger, W., et al., 2012: EC-Earth V2.2: Description and validation of a new seamless earth system prediction model. Clim. Dyn., 39, 2611-2629.

Hegerl, G., and F. Zwiers, 2011: Use of models in detection and attribution of climate change. Clim. Change, 2, 570-591.

Hegerl, G. C., et al., 2007: Understanding and attributing climate change. In: Climate Change 2007: The Physical Science Basis. Contribution of Working Group I to the Fourth Assessment Report of the Intergovernmental Panel on Climate Change [Solomon, S., D. Qin, M. Manning, Z. Chen, M. Marquis, K. B. Averyt, M. Tignor and H. L. Miller (eds.)] Cambridge University Press, Cambridge, United Kingdom and New York, NY, USA, pp. 665-775.

Heinze, C., 2004: Simulating oceanic $\mathrm{CaCO}_{3}$ export production in the greenhouse. Geophys. Res. Lett., 31, L16308.

Heinze, C., I. Kriest, and E. Maier-Reimer, 2009: Age offsets among different biogenic and lithogenic components of sediment cores revealed by numerical modeling Paleoceanography, 24, PA4214.

Held, I. M., 2005: The gap between simulation and understanding in climate modeling. Bull. Am. Meteorol. Soc., 86, 1609-1614.

Held, I. M., and B. J. Soden, 2006: Robust responses of the hydrological cycle to global warming. J. Clim., 19, 5686-5699.

Held, I. M., and K. M. Shell, 2012: Using Relative Humidity as a State Variable in Climate Feedback Analysis. J. Clim., 25, 2578-2582.

Held, I. M., M. Winton, K. Takahashi, T. Delworth, F. R. Zeng, and G. K. Vallis, 2010: Probing the fast and slow components of global warming by returning abruptly to preindustrial forcing. J. Clim., 23, 2418-2427.

Henson, S. A., D. Raitsos, J. P. Dunne, and A. McQuatters-Gollop, 2009: Decadal variability in biogeochemical models: Comparison with a 50-year ocean colour dataset. Geophys. Res. Lett., 36, L21601.

Hermes, J. C., and C. J. C. Reason, 2005: Ocean model diagnosis of interannual coevolving SST variability in the South Indian and South Atlantic Oceans. J. Clim. $18,2864-2882$.

Hernández-Díaz, L., R. Laprise, L. Sushama, A. Martynov, K. Winger, and B. Dugas, 2013: Climate simulation over CORDEX Africa domain using the fifth-generation Canadian Regional Climate Model (CRCM5). Clim. Dyn., 40, 1415-1433.

Heuzé, C., K. J. Heywood, D. P. Stevens, and J. K. Ridley, 2013: Southern Ocean bottom water characteristics in CMIP5 models. Geophys. Res. Lett., doi:10.1002/ grl.50287.

Hewitt, H. T., et al., 2011: Design and implementation of the infrastructure of HadGEM3: The next-generation Met Office climate modelling system. Geosci. Model Dev., 4, 223-253.

Hibbard, K. A., G. A. Meehl, P. M. Cox, and P. Friedlingstein, 2007: A strategy for climate change stabilization experiments. Eos Trans. Am. Geophys. Union, 88, 217-221.

Hirai, M., T. Sakashita, H. Kitagawa, T. Tsuyuki, M. Hosaka, and M. Oh'Izumi, 2007: Development and validation of a new land surface model for JMA's operational global model using the CEOP observation dataset. J. Meteorol. Soc. Jpn., 85A $1-24$.
Hirota, N., Y. N. Takayabu, M. Watanabe, and M. Kimoto, 2011: Precipitation reproducibility over tropical oceans and its relationship to the double ITCZ problem in CMIP3 and MIROC5 climate models. J. Clim., 24, 4859-4873.

Hirschi, M., et al., 2011: Observational evidence for soil-moisture impact on hot extremes in southeastern Europe. Nature Geosci, 4, 17-21.

Hofmann, M., and M. A. Morales Maqueda, 2011: The response of Southern Ocean eddies to increased midlatitude westerlies: A non-eddy resolving model study. Geophys. Res. Lett., 38, L03605.

Holden, P. B., N. R. Edwards, D. Gerten, and S. Schaphoff, 2013: A model based constraint of $\mathrm{CO}_{2}$ fertilisation. Biogeosciences, 10, 339-355.

Holian, G. L., A. P. Sokolov, and R. G. Prinn, 2001: Uncertainty in atmospheric $\mathrm{CO}_{2}$ predictions from a parametric uncertainty analysis of a Global Ocean Carbon Cycle Model. Joint Program Report Series. MIT Joint Program on the Science and Policy of Global Change, Cambridge, MA, USA, 25 pp.

Holland, M., D. Bailey, B. Briegleb, B. Light, and E. Hunke, 2012: Improved sea ice shortwave radiation physics in CCSM4: The impact of melt ponds and aerosols on arctic aea ice. J. Clim., 25, 1413-1430.

Holland, M. M., M. C. Serreze, and J. Stroeve, 2010: The sea ice mass budget of the Arctic and its future change as simulated by coupled climate models. Clim. Dyn., doi:10.1007/s00382-008-0493-4.

Holton, J. R., and H. C. Tan, 1980: The influence of the equatorial Quasi-Biennial Oscillation on the global circulation at 50 mb. J. Atmos. Sci., 37, 2200-2208.

Horowitz, L. W., et al., 2003: A global simulation of tropospheric ozone and related tracers: Description and evaluation of MOZART, version 2. J. Geophys. Res. Atmos., 108, 4784

Hourdin, F., et al., 2012: Impact of the LMDZ atmospheric grid configuration on the climate and sensitivity of the IPSL-CM5A coupled model. Clim. Dyn., doi:10.1007/ s00382-012-1411-3.

Hourdin, F., et al., 2013: LMDZ5B: The atmospheric component of the IPSL climate model with revisited parameterizations for clouds and convection. Clim. Dyn., 40, 2193-2222.

Hourdin, F., et al., 2010: AMMA-Model Intercomparison Project. Bull. Am. Meteorol. Soc., 91, 95-104.

Hu, Z.-Z., B. Huang, Y.-T. Hou, W. Wang, F. Yang, C. Stan, and E. Schneider, 2011 Sensitivity of tropical climate to low-level clouds in the NCEP climate forecast system. Clim. Dyn., 36, 1795-1811.

Huang, C. J., F. Qiao, Q. Shu, and Z. Song, 2012: Evaluating austral summer mixedlayer response to surface wave-induced mixing in the Southern Ocean. $J$. Geophys. Res.-Oceans, 117, C00j18.

Huber, M., I. Mahlstein, M. Wild, J. Fasullo, and R. Knutti, 2011: Constraints on climate sensitivity from radiation patterns in climate models. J. Clim., 24, 1034-1052.

Hung, M., J. Lin, W. Wang, D. Kim, T. Shinoda, and S. Weaver, 2013: MJO and convectively coupled equatorial waves simulated by CMIP5 climate models. $J$. Clim., doi:10.1175/JCLI-D-12-00541.1.

Hunke, E. C., and J. K. Dukowicz, 1997: An elastic-viscous-plastic model for sea ice dynamics. J. Phys. Oceanogr., 27, 1849-1867.

Hunke, E. C., and W. H. Lipscomb, 2008: CICE: The Los Alamos Sea Ice ModelDocumentation and Software User's ManualVersion 4.1. Los Alamos National Laboratory, Los Alamos, NM, USA, 76 pp.

Hunke, E. C., W. H. Lipscomb, and A. K. Turner, 2010: Sea ice models for climate study: Retorspective and new directions. J. Glaciol., 56, 1162-1172.

Hunke, E. C., D. A. Hebert, and O. Lecomte, 2013: Level-ice melt ponds in the Los Alamos sea ice model, CICE. Ocean Model., doi:10.1016/j.ocemod.2012.11.008.

Hunke, E. C., D. Notz, A. K. Turner, and M. Vancoppenolle, 2011: The multiphase physics of sea ice : A review for model developers. Cryosphere, 5, 989-1009.

Hurrell, J., G. A. Meehl, D. Bader, T. L. Delworth, B. Kirtman, and B. Wielicki, 2009: A unified modeling approach to climate system prediction. Bull. Am. Meteorol. Soc., 90, 1819-1832.

Hurrell, J., et al., 2013: The Community Earth System Model: A framework for collaborative research. Bull. Am. Meteorol. Soc., doi:10.1175/BAMS-D-1200121.

Hurtt, G. C., et al., 2009: Harmonization of global land-use scenarios for the period 1500-2100 for IPCC-AR5. iLEAPS Newsl., 7, 6-8.

Hutchings, J. K., A. Roberts, C. A. Geiger, and J. Richter-Menge, 2011: Spatial and temporal characterization of sea-ice deformation. Ann. Glaciol., 52, 360-368.

Huybrechts, P., 2002: Sea-level changes at the LGM from ice-dynamic reconstructions of the Greenland and Antarctic ice sheets during the glacial cycles. Quat. Sci. Rev., 21, 203-231. 
Huybrechts, P., H. Goelzer, I. Janssens, E. Driesschaert, T. Fichefet, H. Goosse, and M. F. Loutre, 2011: Response of the Greenland and Antarctic ice sheets to multimillennial greenhouse warming in the Earth System Model of Intermediate Complexity LOVECLIM. Surv. Geophys., 32, 397-416.

Iacono, M. J., J. S. Delamere, E. J. Mlawer, and S. A. Clough, 2003: Evaluation of upper tropospheric water vapor in the NCAR Community Climate Model (CCM3) using modeled and observed HIRS radiances. J. Geophys. Res., 108, 4037.

Ichikawa, H., H. Masunaga, Y. Tsushima, and H. Kanzawa, 2012: Reproducibility by climate models of cloud radiative forcing associated with tropical convection. J. Clim., 25, 1247-1262.

Ilicak, M., A. J. Adcroft, S. M. Griffies, and R. W. Hallberg, 2012: Spurious dianeutral mixing and the role of momentum closure. Ocean Model., 45-46, 37-58.

Illingworth, A. J., et al., 2007: Cloudnet. Bull. Am. Meteor. Soc., 88, 883-898.

Ilyina, T., R. E. Zeebe, E. Maier-Reimer, and C. Heinze, 2009: Early detection of ocean acidification effects on marine calcification. Global Biogeochem. Cycles, 23, Gb1008.

Ilyina, T., K. Six, J. Segschneider, J. Maier-Reimer, H. Li, and I. Nunez-Riboni, 2013: The global ocean biogeochemistry model HAMOCC: Model architecture and performance as component of the MPI-Earth System Model in different CMIP5 experimental realizations. J. Adv. Model. Earth Syst., 5, 287-315.

Inatsu, M., and M. Kimoto, 2009: A scale interaction study on East Asian cyclogenesis using a General Circulation Model coupled with an Interactively Nested Regional Model. Mon. Weather Rev., 137, 2851-2868.

Inatsu, M., Y. Satake, M. Kimoto, and N. Yasutomi, 2012: GCM bias of the western Pacific summer monsoon and its correction by two-way nesting system. J. Meteorol. Soc. Jpn., 90B, 1-10.

Ingram, W., 2010: A very simple model for the water vapour feedback on climate change. Q. J. R. Meteorol. Soc., 136, 30-40.

Ingram, W., 2013: Some implications of a new approach to the water vapour feedback. Clim. Dyn., 40, 925-933.

Inness, P. M., J. M. Slingo, E. Guilyardi, and J. Cole, 2003: Simulation of the MaddenJulian oscillation in a coupled general circulation model. Part II: The role of the basic state. J. Clim., 16, 365-382.

Inoue, J., J. P. Liu, J. O. Pinto, and J. A. Curry, 2006: Intercomparison of Arctic Regional Climate Models: Modeling clouds and radiation for SHEBA in May 1998. J. Clim., 19, 4167-4178.

IPCC, 2007: Climate Change 2007: The Physical Science Basis. Contribution of Working Group I to the Fourth Assessment Report of the Intergovernmental Panel on Climate Change [Solomon, S., D. Qin, M. Manning, Z. Chen, M. Marquis, K. B. Averyt, M. Tignor and H. L. Miller (eds.)] Cambridge University Press, Cambridge, United Kingdom and New York, NY, USA, 996 pp.

IPCC, 2012: IPCC WGI/WGII Special Report on Managing the Risks of Extreme Events and Disasters to Advance Climate Change Adaptation (SREX). [Field, C.B., V. Barros, T.F. Stocker, D. Qin, D.J. Dokken, K.L. Ebi, M.D. Mastrandrea, K.J. Mach, G.-K. Plattner, S.K. Allen, M. Tignor, and P.M. Midgley (Eds.)]. Cambridge University Press, The Edinburgh Building, Shaftesbury Road, Cambridge CB2 8RU ENGLAND, $582 \mathrm{pp}$.

Ishii, M., and M. Kimoto, 2009: Reevaluation of historical ocean heat content variations with time-varying XBT and MBT depth bias corrections. J. Oceanogr., 65, 287-299.

Ito, A., and T. Oikawa, 2002: A simulation model of the carbon cycle in land ecosystems (Sim-CYCLE): A description based on dry-matter production theory and plot-scale validation. Ecol. Model., 151, 143-176.

Iversen, T., et al., 2013: The Norwegian Earth System Model, NorESM1-M. Part 2: Climate response and scenario projections. Geosci. Model Dev., 6, 1-27.

Izumi, K., P. J. Bartlein, and S. P. Harrison, 2013: Consistent large-scale temperature responses in warm and cold climates. Geophys. Res. Lett., doi:2013GL055097.

Jackson, C. S., M. K. Sen, G. Huerta, Y. Deng, and K. P. Bowman, 2008a: Error reduction and convergence in climate prediction. J. Clim., 21, 6698-6709.

Jackson, L., R. Hallberg, and S. Legg, 2008b: A parameterization of shear-driven turbulence for ocean climate models. J. Phys. Oceanogr., 38, 1033-1053.

Jacob, D., et al., 2012: Assessing the transferability of the Regional Climate Model REMO to Different COordinated Regional Climate Downscaling EXperiment (CORDEX) regions. Atmosphere, 3, 181-199.

Jakob, C., 2010: Accelerating progress in Global Atmospheric Model development through improved parameterizations: Challenges, opportunities, and strategies. Bull. Am. Meteorol. Soc., 91, 869-875.
Jang, C. J., J. Park, T. Park, and S. Yoo, 2011: Response of the ocean mixed layer depth to global warming and its impact on primary production: A case for the North Pacific Ocean. Ices J. Mar. Sci., 68, 996-1007.

Jansen, E., et al., 2007: Paleoclimate. In: Climate Change 2007: The Physical Science Basis. Contribution of Working Group I to the Fourth Assessment Report of the Intergovernmental Panel on Climate Change [Solomon, S., D. Qin, M. Manning, Z. Chen, M. Marquis, K. B. Averyt, M. Tignor and H. L. Miller (eds.)] Cambridge University Press, Cambridge, United Kingdom and New York, NY, USA, pp. 433498.

Jayne, S. R., 2009: The impact of abyssal mixing parameterizations in an ocean General Circulation Model. J. Phys. Oceanogr., 39, 1756-1775.

Ji, J., M. Huang, and K. Li, 2008: Prediction of carbon exchanges between China terrestrial ecosystem and atmosphere in 21 st century. Sci. China D, 51, 885-898.

Ji, J. J., 1995: A climate-vegetation interaction model: Simulating physical and biological processes at the surface. J. Biogeogr., 22, 445-451.

Jiang, J. H., et al., 2012a: Evaluation of cloud and water vapor simulations in CMIP5 climate models using NASA "A-Train" satellite observations. J. Geophys. Res., 117, D14105.

Jiang, X., et al., 2012b: Simulation of the intraseasonal variability over the Eastern Pacific ITCZ in climate models. Clim. Dyn., 39, 617-636.

Jin, F. F., S. T. Kim, and L. Bejarano, 2006:A coupled-stability index for ENSO. Geophys. Res. Let., 33, L23708.

Joetzjer, E., H. Douville, C. Delire, and P. Ciais, 2013: Present-day and future Amazonian precipitation in global climate models: CMIP5 versus CMIP3. Clim. Dyn., doi: 10.1007/s00382-012-1644-1.

Johanson, C. M., and Q. Fu, 2009: Hadley Cell Widening: Model Simulations versus Observations. J. Clim., 22, 2713-2725.

John, V., and B. Soden, 2007: Temperature and humidity biases in global climate models and their impact on climate feedbacks. Geophys. Res. Lett., 34, L18704.

Johns, T. C., et al., 2003: Anthropogenic climate change for 1860 to 2100 simulated with the HadCM3 model under updated emissions scenarios. Clim. Dyn., 20, 583-612.

Johns, T. C., et al., 2006: The new Hadley Centre Climate Model (HadGEM1): Evaluation of coupled simulations. J. Clim., 19, 1327-1353

Johnson, N. C., and S. B. Feldstein, 2010: The continuum of north Pacific sea level pressure patterns: Intraseasonal, interannual, and interdecadal variability. J. Clim., 23, 851-867.

Jolliffe, I. T., and D. B. Stephenson, 2011: Forecast Verification: A Practitioner's Guide in Atmospheric Science. 2nd ed. John Wiley \& Sons, Hoboken, NJ, 292 pp.

Joly, M., A. Voldoire, H. Douville, P. Terray, and J. F. Royer, 2007: African monsoon teleconnections with tropical SSTs: Validation and evolution in a set of IPCC4 simulations. Clim. Dyn., 29, 1-20.

Jones, A., D. L. Roberts, M. J. Woodage, and C. E. Johnson, 2001: Indirect sulphate aerosol forcing in a climate model with an interactive sulphur cycle. J. Geophys. Res. Atmos., 106, 20293-20310.

Jones, G. S., P. A. Stott, and N. Christidis, 2012: Attribution of observed historical near surface temperature variations to anthropogenic and natural causes using CMIP5 simulations. J. Geophys. Res., doi:10.1002/jgrd.50239.

Jones, P. D., M. New, D. E. Parker, S. Martin, and I. G. Rigor, 1999: Surface air temperature and its changes over the past 150 years. Rev. Geophys., 37, 173199.

Joseph, S., A. K. Sahai, B. N. Goswami, P. Terray, S. Masson, and J. J. Luo, 2012: Possible role of warm SST bias in the simulation of boreal summer monsoon in SINTEX-F2 coupled model. Clim. Dyn., 38, 1561-1576.

Joussaume, S., and K. E. Taylor, 1995: Status of the Paleoclimate Modeling Intercomparison Project. In: Proceedings of the first international AMIP scientific conference, WCRP-92, Monterey, USA, 425-430.

Jun, M., R. Knutti, and D. Nychka, 2008: Spatial analysis to quantify numerical model bias and dependence: How many climate models are there? J. Am. Stat. Assoc., 103, 934-947.

Jung, T., et al., 2010: The ECMWF model climate: Recent progress through improved physical parametrizations. Q. J. R. Meteorol. Soc., 136, 1145-1160.

Jung, T., et al., 2012: High-resolution global climate simulations with the ECMWF Model in Project Athena: Experimental design, model climate, and seasonal forecast skill. J. Clim., 25, 3155-3172.

Jungclaus, J. H., et al., 2006: Ocean circulation and tropical variability in the coupled model ECHAM5/MPI-OM. J. Clim., 19, 3952-3972. 
Jungclaus, J. H., et al., 2013: Characteristics of the ocean simulations in MPIOM the ocean componentof the MPI-Earth System Model. J. Adv. Model. Earth Syst., doi:10.1002/jame.20023.

Jungclaus, J. H., et al., 2010: Climate and carbon-cycle variability over the last millennium. Clim. Past, 6, 723-737.

Kageyama, M., et al., 2006: Last Glacial Maximum temperatures over the North Atlantic, Europe and western Siberia: A comparison between PMIP models, MARGO sea-surface temperatures and pollen-based reconstructions. Quat. Sci. Rev., 25, 2082-2102.

Kahn, R. A., B. J. Gaitley, J. V. Martonchik, D. J. Diner, K. A. Crean, and B. Holben, 2005 Multiangle Imaging Spectroradiometer (MISR) global aerosol optical depth validation based on 2 years of coincident Aerosol Robotic Network (AERONET) observations. J. Geophys. Res. Atmos., 110, D10s04.

Kalnay, E., et al., 1996: The NCEP/NCAR 40-year reanalysis project. Bull. Am. Meteorol. Soc., 77, 437-471.

Kanada, S., M. Nakano, S. Hayashi, T. Kato, M. Nakamura, K. Kurihara, and A. Kitoh, 2008: Reproducibility of Maximum Daily Precipitation Amount over Japan by a High-resolution Non-hydrostatic Model. Sola, 4, 105-108.

Kanamaru, H., and M. Kanamitsu, 2007: Fifty-seven-year California reanalysis downscaling at $10 \mathrm{~km}$ (CaRD10). Part II: Comparison with North American regional reanalysis. J. Clim., 20, 5572-5592.

Kanamitsu, M., K. Yoshimura, Y. B. Yhang, and S. Y. Hong, 2010: Errors of interannual variability and trend in dynamical downscaling of reanalysis. J. Geophys. Res. Atmos., 115, D17115.

Kanamitsu, M., W. Ebisuzaki, J. Woollen, S. K. Yang, J. J. Hnilo, M. Fiorino, and G. L. Potter, 2002: NCEP-DOE AMIP-II reanalysis (R-2). Bull. Am. Meteorol. Soc., 83 1631-1643.

Karlsson, J., and G. Svensson, 2010: The simulation of Arctic clouds and their influence on the winter surface temperature in present-day climate in the CMIP3 multi-model dataset. Clim. Dyn., 36, 623-635.

Karlsson, J., G. Svensson, and H. Rodhe, 2008: Cloud radiative forcing of subtropica low level clouds in global models. Clim. Dyn., 30, 779-788.

Karpechko, A., N. Gillett, G. Marshall, and A. Scaife, 2008: Stratospheric influence on circulation changes in the Southern Hemisphere troposphere in coupled climate models. Geophys. Res. Lett., 35, L20806.

Karpechko, A. Y., and E. Manzini, 2012: Stratospheric influence on tropospheric climate change in the Northern Hemisphere. J. Geophys. Res. Atmos., 117, D05133.

Karpechko, A. Y., N. P. Gillett, G. J. Marshall, and J. A. Screen, 2009: Climate impacts of the southern annular mode simulated by the CMIP3 models. J. Clim., 22, 6149-6150.

Kattsov, V. M., et al., 2010: Arctic sea-ice change: A grand challenge of climate science. J. Glaciol., 56, 1115-1121.

Kavvada, A., A. Ruiz-Barradas, and S. Nigam, 2013: AMO's structure and climate footprint in observations and IPCC AR5 climate simulations. Clim. Dyn. doi:10.1007/s00382-013-1712-1.

Kawazoe, S., and W. Gutowski, 2013: Regional, very heavy daily precipitation in NARCCAP simulations. J. Hydrometeorol., doi:10.1175/JHM-D-12-068.1.

Kay, J. E., M. M. Holland, and A. Jahn, 2011: Inter-annual to multi-decadal Arctic sea ice extent trends in a warming world. Geophys. Res. Lett., 38, L15708.

Keeley, S. P. E., R. T. Sutton, and L. C. Shaffrey, 2012: The impact of North Atlantic sea surface temperature errors on the simulation of North Atlantic European region climate. Q. J. R. Meteorol. Soc., doi:10.1002/qj.1912.

Kendon, E. J., N. M. Roberts, C. A. Senior, and M. J. Roberts, 2012: Realism of rainfall in a very high resolution regional climate model. J. Clim., 25, 5791-5806.

Khairoutdinov, M. F., D. A. Randall, and C. DeMott, 2005: Simulations of the Atmospheric general circulation using a cloud-resolving model as a superparameterization of physical processes. J. Atmos. Sci., 62, 2136-2154.

Kharin, V. V., F. W. Zwiers, X. B. Zhang, and G. C. Hegerl, 2007: Changes in temperature and precipitation extremes in the IPCC ensemble of global coupled model simulations. J. Clim., 20, 1419-1444.

Kharin, V. V., F. W. Zwiers, X. Zhang, and M. Wehner, 2012: Changes in temperature and precipitation extremes in the CMIP5 ensemble. Clim. Change, doi:10.1007/ s10584-013-0705-8.

Khvorostyanov, D. V., G. Krinner, P. Ciais, M. Heimann, and S. A. Zimov, 2008a: Vulnerability of permafrost carbon to global warming. Part I: Model description and role of heat generated by organic matter decomposition. Tellus $B, 60,250$ 264.
Khvorostyanov, D. V., P. Ciais, G. Krinner, S. A. Zimov, C. Corradi, and G. Guggenberger, 2008b: Vulnerability of permafrost carbon to global warming. Part II: Sensitivity of permafrost carbon stock to global warming. Tellus $B, 60,265-275$.

Kidston, J., and E. P. Gerber, 2010: Intermodel variability of the poleward shift of the austral jet stream in the CMIP3 integrations linked to biases in 20th century climatology. Geophys. Res. Lett., 37, L09708.

Kiehl, J. T., 2007: Twentieth century climate model response and climate sensitivity. Geophys. Res. Lett., 34.

Kim, D., and V. Ramanathan, 2008: Solar radiation budget and radiative forcing due to aerosols and clouds. J. Geophys. Res. Atmos., 113, D02203.

Kim, D., et al., 2012: The tropical subseasonal variability simulated in the NASA GISS general circulation model. J. Clim., 25, 4641-4659.

Kim, D., et al., 2009: Application of MJO simulation diagnostics to climate models. J. Clim., 22, 6413-6436.

Kim, H.-J., K. Takata, B. Wang, M. Watanabe, M. Kimoto, T. Yokohata, and T. Yasunari, 2011: Global monsoon, El Niño, and their interannual linkage simulated by MIROC5 and the CMIP3 CGCMs. J. Clim., 24, 5604-5618.

Kim, S., and F.-F. Jin, 2011a: An ENSO stability analysis. Part I: Results from a hybrid coupled model. Clim. Dyn., 36, 1593-1607.

Kim, S., and F.-F. Jin, 2011b: An ENSO stability analysis. Part II: Results from the twentieth and twenty-first century simulations of the CMIP3 models. Clim. Dyn. 36, 1609-1627.

Kim, S. T., and J.-Y.Yu, 2012: The two types of ENSO in CMIP5 models. Geophys. Res. Lett., 39, L11704.

Kirkevåg, K., et al., 2013: Aerosol-climate interactions in the Norwegian Earth System Model - NorESM1-M. Geophys. Model Dev., 6, 207-244.

Kistler, R., et al., 2001:The NCEP-NCAR 50-year reanalysis: Monthly means CD-ROM and documentation. Bull. Am. Meteorol. Soc., 82, 247-267.

Kjellstrom, E., G. Nikulin, U. Hansson, G. Strandberg, and A. Ullerstig, 2011: 21st century changes in the European climate: Uncertainties derived from an ensemble of regional climate model simulations. Tellus $A, 63,24-40$.

Kjellstrom, E., F. Boberg, M. Castro, J. Christensen, G. Nikulin, and E. Sanchez, 2010: Daily and monthly temperature and precipitation statistics as performance indicators for regional climate models. Clim. Res., 44 135-150.

Klein, P., and G. Lapeyre, 2009: The oceanic vertical pump induced by mesoscale and submesoscale turbulence. Annu. Rev. Mar. Sci., 1, 351-375.

Klein, S. A., and C. Jakob, 1999: Validation and sensitivities of frontal clouds simulated by the ECMWF model. Mon. Weather Rev., 127, 2514-2531.

Klein, S. A., B. J. Soden, and N. C. Lau, 1999: Remote sea surface temperature variations during ENSO: Evidence for a tropical atmospheric bridge. J. Clim., 12, 917-932.

Klein, S. A., X. Jiang, J. Boyle, S. Malyshev, and S. Xie, 2006: Diagnosis of the summertime warm and dry bias over the U.S. Southern Great Plains in the GFDL climate model using a weather forecasting approach. Geophys. Res. Lett., 33, L18805.

Klein, S. A., Y. Zhang, M. D. Zelinka, R. Pincus, J. S. Boyle, and P. J. Glecker, 2013: Are climate model simulations of clouds improving? An evaluation using the ISCCP simulator. J. Geophys. Res., doi:10.1002/jgrd.50141.

Klocke, D., R. Pincus, and J. Quaas, 2011: On constraining estimates of climate sensitivity with present-day observations through model weighting. J. Clim., 24, 6092-6099.

Kloster, S., N. M. Mahowald, J. T. Randerson, and P. J. Lawrence, 2012: The impacts of climate, land use, and demography on fires during the 21 st century simulated by CLM-CN. Biogeosciences, 9, 509-525.

Knight, J., et al., 2009: Do global temperature trends over the last decade falsify climate predictions? [In: State of the Climate in 2008]. Bull. Am. Meteorol. Soc., 90, S22-S23.

Knight, J. R., 2009: The Atlantic Multidecadal Oscillation inferred from the forced climate response in Coupled General Circulation Models. J. Clim., 22, 1610 1625.

Knutti, R., 2008: Why are climate models reproducing the observed global surface warming so well? Geophys. Res. Lett., 35, L18704

Knutti, R., 2010: The end of model democracy? Clim. Change, 102, 395-404.

Knutti, R., and G. C. Hegerl, 2008: The equilibrium sensitivity of the Earth's temperature to radiation changes. Nature Geosci., 1, 735-743.

Knutti, R., and L. Tomassini, 2008: Constraints on the transient climate response from observed global temperature and ocean heat uptake. Geophys. Res. Lett., 35, L09701. 
Knutti, R., and J. Sedlácek, 2013: Robustness and uncertainties in the new CMIP5 climate model projections. Nature Clim. Change, 3, 369-373.

Knutti, R., D. Masson, and A. Gettelman, 2013: Climate model genealogy: Generation CMIP5 and how we got there. Geophys. Res. Lett., 40, 1194-1199.

Knutti, R., G. A. Meehl, M. R. Allen, and D. A. Stainforth, 2006: Constraining climate sensitivity from the seasonal cycle in surface temperature. J. Clim., 19, 42244233.

Knutti, R., F. Joos, S. A. Muller, G. K. Plattner, and T. F. Stocker, 2005: Probabilistic climate change projections for $\mathrm{CO}_{2}$ stabilization profiles. Geophys. Res. Lett., 32, L20707.

Knutti, R., R. Furrer, C. Tebaldi, J. Cermak, and G. A. Meehl, 2010a: Challenges in combining projections from multiple climate models. J. Clim., 23, 2739-2758.

Knutti, R., G. Abramowitz, M. Collins, V. Eyring, P. J. Gleckler, B. Hewitson, and L. Mearns, 2010b: Good practice guidance paper on assessing and combining multi model climate projections. In: Meeting Report of the Intergovernmental Panel on Climate Change Expert Meeting on Assessing and Combining Multi Model Climate Projections [T. F. Stocker, T.F., D. Qin, G.-K. Plattner, M. Tignor, and P.M. Midgley (eds.)]. IPCC Working Group I Technical Support Unit, University of Bern, Bern, Switzerland.

Koch, D., et al., 2011: Coupled Aerosol-Chemistry-Climate Twentieth-Century Transient Model investigation: Trends in short-lived species and climate responses. J. Clim., 24, 2693-2714.

Koldunov, N. V., D. Stammer, and J. Marotzke, 2010: Present-day Arctic sea ice variability in the coupled ECHAM5/MPI-OM model. J. Clim., 23, 2520-2543.

Koltzow, M., T. Iversen, and J. Haugen, 2008: Extended Big-Brother experiments: The role of lateral boundary data quality and size of integration domain in regional climate modelling. Tellus $A, 60,398-410$.

Koltzow, M. A. O., T. Iversen, and J. E. Haugen, 2011: The importance of lateral boundaries, surface forcing and choice of domain size for dynamical downscaling of global climate simulations. Atmosphere, 2, 67-95.

Komuro, Y., et al., 2012: Sea-ice in twentieth-century simulations by new MIROC Coupled Models: A comparison between models with high resolution and with ice thickness distribution. J. Meteorol. Soc. Jpn, 90A, 213-232.

Konsta, D., H. Chepfer, and J.-L. Dufresne, 2012: A process oriented characterization of tropical oceanic clouds for climate model evaluation, based on a statistical analysis of daytime A-train observations. Clim. Dyn., 39, 2091-2108.

Koster, R., et al., 2004: Regions of strong coupling between soil moisture and precipitation. Science, 305, 1138-1140.

Kostopoulou, E., K. Tolika, I. Tegoulias, C. Giannakopoulos, S. Somot, C. Anagnostopoulou, and P. Maheras, 2009: Evaluation of a regional climate model using in situ temperature observations over the Balkan Peninsula. Tellus $A, 61$, 357-370.

Koven, C. D., W. J. Riley, and A. Stern, 2013: Analysis of permafrost thermal dynamics and response to climate change in the CMIP5 Earth System Models. J. Clim., 26, 1877-1900.

Koven, C. J., et al., 2011: Permafrost carbon-climate feedbacks accelerate global warming. Proc. Natl. Acad. Sci. U.S.A., 108, 14769-14774.

Kowalczyk, E. A., Y. P. Wang, R. M. Law, H. L. Davies, J. L. McGregor, and G. Abramowitz 2006: The CSIRO Atmosphere Biosphere Land Exchange (CABLE) model for use in climate models and as an offline model. CSIRO Marine and Atmospheric Research paper 013, Victoria, Australia, $37 \mathrm{pp}$.

Kowalczyk, E. A., et al., 2013: The land surface model component of ACCESS: Description and impact on the simulated surface climatology. Aust. Meteorol. Oceanogr. J., 63, 65-82.

Kravtsov, S., and C. Spannagle, 2008: Multidecadal climate variability in observed and modeled surface temperatures. J. Clim., 21, 1104-1121.

Krinner, G., et al., 2005: A dynamic global vegetation model for studies of the coupled atmosphere-biosphere system. Global Biogeochem. Cycles, 19, GB1015.

Krüger, L., R. da Rocha, M. Reboita, and T. Ambrizzi, 2012: RegCM3 nested in HadAM3 scenarios A2 and B2: Projected changes in extratropical cyclogenesis, temperature and precipitation over the South Atlantic Ocean. Clim. Change, $113,599-621$.

Kuhlbrodt, T., and J. Gregory, 2012: Ocean heat uptake and its consequences for the magnitude of sea level rise and climate change. Geophys. Res. Lett., doi:10.1029/2012GL052952.

Kuhlbrodt, T., R. S. Smith, Z. Wang, and J. M. Gregory, 2012: The influence of eddy parameterizations on the transport of the Antarctic Circumpolar Current in coupled climate models. Ocean Model., 52-53, 1-8.
Kusaka, H., T. Takata, and Y. Takane, 2010: Reproducibility of regional climate in central Japan using the 4-km Resolution WRF Model. Sola, 6, 113-116.

Kusunoki, S., R. Mizuta, and M. Matsueda, 2011: Future changes in the East Asian rain band projected by global atmospheric models with $20-\mathrm{km}$ and $60-\mathrm{km}$ grid size. Clim. Dyn., 37, 2481-2493.

L'Ecuyer, T., and G. Stephens, 2007: The tropical atmospheric energy budget from the TRMM perspective. Part II: Evaluating GCM representations of the sensitivity of regional energy and water cycles to the 1998-99 ENSO Cycle. J. Clim., 20, 4548-4571.

Laine, A., G. Lapeyre, and G. Riviere, 2011: A quasigeostrophic model for moist storm tracks. J. Atmos. Sci., 68, 1306-1322.

Laine, A., M. Kageyama, P. Braconnot, and R. Alkama, 2009: Impact of greenhouse gas concentration changes on surface energetics in IPSL-CM4: Regional warming patterns, land-sea warming ratios, and glacial-interglacial differences. J. Clim., 22, 4621-4635.

Lamarque, J. F., et al., 2012: CAM-chem: Description and evaluation of interactive atmospheric chemistry in the Community Earth System Model. Geosci. Model Dev., 5, 369-411.

Lamarque, J. F., et al., 2010: Historical (1850-2000) gridded anthropogenic and biomass burning emissions of reactive gases and aerosols: Methodology and application. Atmos. Chem. Phys., 10, 7017-7039.

Lambert, F. H., G. R. Harris, M. Collins, J. M. Murphy, D. M. H. Sexton, and B. B. B. Booth, 2012: Interactions between perturbations to different Earth system components simulated by a fully-coupled climate model. Clim. Dyn., doi:10.1007/ s00382-012-1618-3.

Lambert, S., and G. Boer, 2001: CMIP1 evaluation and intercomparison of coupled climate models. Clim. Dyn., 17, 83-106.

Landrum, L., M. M. Holland, D. P. Schneider, and E. Hunke, 2012: Antarctic sea ice climatology, variability and late 20th century change in CCSM4. J. Clim., 25, 4817-4838.

Langenbrunner, B., and J. D. Neelin, 2013: Analyzing ENSO teleconnections in CMIP models as a measure of model fidelity in simulating precipitation. J. Clim. doi:10.1175/JCLI-D-12-00542.1.

Laprise, R., 2008: Regional climate modelling. J. Comput. Phys., 227, 3641-3666.

Laprise, R., et al., 2008: Challenging some tenets of regional climate modelling. Meteorol. Atmos. Phys., 100, 3-22.

Large, W., and S. Yeager, 2009: The global climatology of an interannually varying air-sea flux data set. Clim. Dyn., 33, 341-364.

Larow, T. E., Y. K. Lim, D. W. Shin, E. P. Chassignet, and S. Cocke, 2008: Atlantic basin seasonal hurricane simulations. J. Clim., 21, 3191-3206.

Lau, K. M., et al., 2008: The Joint Aerosol-Monsoon Experiment -A new challenge for monsoon climate research. Bull. Am. Meteorol. Soc., 89, 369-383.

Lau, W. K. M., and D. E. Waliser, 2011: Intraseasonal Variability of the AtmosphereOcean Climate System. Springer Science+Business Media, New York, NY, USA, and Heidelberg, Germany.

Lawrence, D. M., et al., 2012: The CCSM4 Land Simulation, 1850-2005: Assessment of surface climate and new capabilities. J. Clim., 25, 2240-2260.

Lawrence, D. M., et al., 2011: Parameterization improvements and functional and structural advances in version 4 of the Community Land Model. J. Adv. Model. Earth Syst., 3, $2011 \mathrm{MS} 000045$.

Le Quere, C., et al., 2005: Ecosystem dynamics based on plankton functional types for global ocean biogeochemistry models. Global Change Biol., 11, 2016-2040.

Le Quere, C., et al., 2009: Trends in the sources and sinks of carbon dioxide. Nature Geosci., 2, 831-836.

Lecomte, O., T. Fichefet, M. Vancoppenolle, and M. Nicolaus, 2011: A new snow thermodynamic scheme for large-scale sea-ice models. Ann. Glaciol., 52, $337-$ 346.

Leduc, M., and R. Laprise, 2009: Regional climate model sensitivity to domain size. Clim. Dyn., 32, 833-854.

Lee, D. S., et al., 2009: Aviation and global climate change in the 21st century. Atmos. Environ., 43, 3520-3537.

Lee, T., D. E. Waliser, J.-L. F. Li, F. W. Landerer, and M. M. Gierach, 2013: Evaluation of CMIP3 and CMIP5 wind stress climatology using satellite measurements and atmospheric reanalysis products. J. Clim., doi:10.1175/JCLI-D-12-00591.1.

Legg, S., L. Jackson, and R. W. Hallberg, 2008: Eddy-resolving modeling of overflows. In: Eddy Resolving Ocean Models, 177 ed. [M. Hecht, and H. Hasumi (eds.)]. American Geophysical Union, Washington, DC, pp. 63-82. 
Legg, S., et al., 2009: Improving oceanic overflow representation in climate models: The Gravity Current Entrainment Climate Process Team. Bull. Am. Meteorol. Soc., $90,657-670$.

Leloup, J., M. Lengaigne, and J.-P. Boulanger, 2008: Twentieth century ENSO characteristics in the IPCC database. Clim. Dyn., 30, 277-291.

Lemoine, D. M., 2010: Climate sensitivity distributions dependence on the possibility that models share biases. J. Clim., 23, 4395-4415.

Lenaerts, J., M. van den Broeke, S. Dery, E. van Meijgaard, W. van de Berg, S. Palm, and J. Rodrigo, 2012: Modeling drifting snow in Antarctica with a regional climate model: 1. Methods and model evaluation. J. Geophys. Res. Atmos., 117, D05108.

Lenderink, G., 2010: Exploring metrics of extreme daily precipitation in a large ensemble of regional climate model simulations. Clim. Res., 44 151-166.

Lenderink, G., and E. Van Meijgaard, 2008: Increase in hourly precipitation extremes beyond expectations from temperature changes. Nature Geosci., 1, 511-514.

Levine, R. C., and A. G. Turner, 2012: Dependence of Indian monsoon rainfall on moisture fluxes across the Arabian Sea and the impact of coupled model sea surface temperature biases. Clim. Dyn., 38, 2167-2190.

Levis, S., 2010: Modeling vegetation and land use in models of the Earth System. Clim. Change, 1, 840-856.

Levitus, S., J. I. Antonov, T. P. Boyer, R. A. Locarnini, H. E. Garcia, and A. V. Mishonov, 2009: Global ocean heat content 1955-2008 in light of recently revealed instrumentation problems. Geophys. Res. Lett., 36, L07608

Levy, H., L. W. Horowitz, M. D. Schwarzkopf, Y. Ming, J.-C. Golaz, V. Naik, and V. Ramaswamy, 2013: The roles of aerosol direct and indirect effects in past and future climate change. J. Geophys. Res., doi:10.1002/jgrd.50192.

Lewis, T., and S. Lamoureux, 2010: Twenty-first century discharge and sediment yield predictions in a small high Arctic watershed. Global Planet. Change, 71, 27-41.

Li, C., J.-S. von Storch, and J. Marotzke, 2013a: Deep-ocean heat uptake and equilibrium climate response. Clim. Dyn., 40, 1071-1086.

Li, G., and S.-P. Xie, 2012: Origins of tropical-wide SST biases in CMIP multi-model ensembles. Geophys. Res. Lett., 39, L22703.

Li, H. B., A. Robock, and M. Wild, 2007: Evaluation of Intergovernmental Panel on Climate Change Fourth Assessment soil moisture simulations for the second half of the twentieth century. J. Geophys. Res. Atmos., 112, D06106

Li, J.-L. F., D. E. Waliser, and J. H. Jiang, 2011a: Correction to "Comparisons of satellites liquid water estimates to ECMWF and GMAO analyses, 20th century IPCC AR4 climate simulations, and GCM simulations". Geophys. Res. Lett., 38, L24807.

Li, J.-L. F., et al., 2008: Comparisons of satellites liquid water estimates to ECMWF and GMAO analyses, 20th century IPCC AR4 climate simulations, and GCM simulations. Geophys. Res. Lett., 35, L19710.

Li, J., S.-P. X. and A. Mestas-Nunez, E. R. C. and Gang Huang, R. D'Arrigo, F. Liu, J. Ma, and X. Zheng, 2011b: Interdecadal modulation of ENSO amplitude during the last millennium. Nature Clim. Change, 1, 114-118.

Li, J. L. F., et al., 2012a: An observationally-based evaluation of cloud ice water in CMIP3 and CMIP5 GCMs and contemporary reanalyses using contemporary satellite data. J. Geophys. Res., 117, D16105.

Li, L., et al., 2013b: Development and Evaluation of Grid-point Atmospheric Model of IAP LASG, Version 2.0 (GAMIL 2.0). Adv. Atmos. Sci., 30, 855-867.

Li, L., et al., 2012b: The Flexible Global Ocean-Atmosphere-Land System Model: Gridpoint Version 2: FGOALS-g2. Adv. Atmos. Sci., doi:10.1007/s00376-012-2140-6.

$\mathrm{Li}$, T., and G. H. Philander, 1996: On the annual cycle in the eastern equatorial Pacific J. Clim., 9, 2986-2998.

Li, T., C. W. Tham, and C. P. Chang, 2001: A coupled air-sea-monsoon oscillator for the tropospheric biennial oscillation. J. Clim., 14, 752-764.

Liebmann, B., R. M. Dole, C. Jones, I. Blade, and D. Allured, 2010: Influence of choice of time period on global surface temperature trend wstimates. Bull. Am. Meteorol. Soc., 91, 1485-1491.

Lienert, f., J. C. Fyfe, and W. J. Merryfield, 2011: Do climate models capture the tropical influences on North Pacific sea surface temperature variability? J. Clim. 24, 6203-6209.

Lin, A. L., and T. Li, 2008: Energy spectrum characteristics of Boreal Summer Intraseasonal Oscillations: Climatology and variations during the ENSO developing and decaying phases. J. Clim., 21, 6304-6320.

Lin, J.-L., 2007: The double-ITCZ problem in IPCC AR4 Coupled GCMs: Oceanatmosphere feedback analysis. J. Clim., 20, 4497-4525.

Lin, J. L., et al., 2006: Tropical intraseasonal variability in 14 IPCC AR4 climate models. Part I: Convective signals. J. Clim., 19, 2665-2690.

Lin, P., Y. Yongqiang, and H. Liu, 2013: Long-term stability and oceanic mean state simulated by the coupled model FGOALS-s2. Adv. Atmos. Sci., 30, 175-192.
Lin, Y., et al., 2012: TWP-ICE global atmospheric model intercomparison: Convection responsiveness and resolution impact. J. Geophys. Res., 117, D09111.

Lindvall, J., G. Svensson, and C. Hannay, 2012: Evaluation of near-surface parameters in the two versions of the atmospheric model in CESM1 using flux station observations. J. Clim., 26 26-44.

Linkin, M., and S. Nigam, 2008: The north pacific oscillation-west Pacific teleconnection pattern: Mature-phase structure and winter impacts. J. Clim., 21, 1979-1997.

Liu, H., C. Wang, S. K. Lee, and D. Enfield, 2013a: Atlantic Warm Pool Variability in the CMIP5 Simulations. J. Clim., doi:10.1175/JCLI-D-12-00556.1.

Liu, H. L., P. F. Lin, Y. Q. Yu, and X. H. Zhang, 2012a: The baseline evaluation of LASG/ IAP Climate system Ocean Model (LICOM) version 2.0. Acta Meteorol. Sin., 26, 318-329.

Liu, J., 2010: Sensitivity of sea ice and ocean simulations to sea ice salinity in a coupled global climate model. Science China Earth Sci., 53, 911-918.

Liu, L., W. Yu, and T. Li, 2011: Dynamic and thermodynamic air-sea coupling associated with the Indian Ocean dipole diagnosed from 23 WCRP CMIP3 Models. J. Clim., 24, 4941-4958.

Liu, S. C., C. B. Fu, C. J. Shiu, J. P. Chen, and F. T. Wu, 2009: Temperature dependence of global precipitation extremes. Geophys. Res. Lett., 36, L17702.

Liu, X., et al., 2012b: Toward a minimal representation of aerosols in climate models: Description and evaluation in the Community Atmosphere Model CAM5. Geophys. Model Dev., 5, 709-739.

Liu, X. H., et al., 2007: Uncertainties in global aerosol simulations: Assessment using three meteorological data sets. J. Geophys. Res. Atmos., 112, D11212

Liu, Y., 1996: Modeling the emissions of nitrous oxide and methane from the terrestrial biosphere to the atmosphere. In: Joint Program Report Series. MIT Joint Program on the Science and Policy of Global Change, Cambridge, MA, USA, $219 \mathrm{pp}$

Liu, Y., J. Hu, B. He, Q. Bao, A. Duan, and G. X. Wu, 2013b: Seasonal evolution of subtropical anticyclones in the Climate System Model FGOALS-s2. Adv. Atmos. Sci., 30, 593-606.

Lloyd, J., E. Guilyardi, and H. Weller, 2010: The role of atmosphere feedbacks during ENSO in the CMIP3 models. Part II: Using AMIP runs to understand the heat flux feedback mechanisms. Clim. Dyn., 37, 1271-1292.

Lloyd, J., E. Guilyardi, and H. Weller, 2012: The role of atmosphere feedbacks during ENSO in the CMIP3 Models. Part III: The Shortwave Flux Feedback. J. Clim., 25, 4275-4293.

Lloyd, J., E. Guilyardi, H. Weller, and J. Slingo, 2009: The role of atmosphere feedbacks during ENSO in the CMIP3 models. Atmos. Sci. Lett., 10, 170-176.

Loeb, N. G., et al., 2009: Toward optimal closure of the Earth's top-of-atmosphere radiation budget. J. Clim., 22, 748-766.

Lohmann, U., K. von Salzen, N. McFarlane, H. G. Leighton, and J. Feichter, 1999: Tropospheric sulfur cycle in the Canadian general circulation model. J. Geophys. Res. Atmos., 104, 26833-26858.

Long, M. C., K. Lindsay, S. Peacock, J. K. Moore, and S. C. Doney, 2012: Twentiethcentury oceanic carbon uptake and storage in CESM1 (BGC). J. Clim., doi:10.1175/ JCLI-D-12-00184.1.

Loptien, U., O. Zolina, S. Gulev, M. Latif, and V. Soloviov, 2008: Cyclone life cycle characteristics over the Northern Hemisphere in coupled GCMs. Clim. Dyn., 31, 507-532.

Lorenz, P., and D. Jacob, 2005: Influence of regional scale information on the global circulation: A two-way nesting climate simulation. Geophys. Res. Lett., 32, L18706.

Lorenz, R., E. L. Davin, and S. I. Seneviratne, 2012: Modeling land-climate coupling in Europe: Impact of land surface representation on climate variability and extremes. J. Geophys. Res., 117, doi:10.1029/2012JD017755.

Losch, M., D. Menemenlis, J.-M. Campin, P. Heimbach, and C. Hill, 2010: On the formulation of sea-ice models. Part 1: Effects of different solver implementations and parameterizations. Ocean Model., 33, 129-144.

Loschnigg, J., G. A. Meehl, P. J. Webster, J. M. Arblaster, and G. P. Compo, 2003: The Asian monsoon, the tropospheric biennial oscillation, and the Indian Ocean zonal mode in the NCAR CSM. J. Clim., 16, 1617-1642.

Loutre, M. F., A. Mouchet, T. Fichefet, H. Goosse, H. Goelzer, and P. Huybrechts, 2011: Evaluating climate model performance with various parameter sets using observations over the recent past. Clim. Past, 7, 511-526.

Loyola, D., and M. Coldewey-Egbers, 2012: Multi-sensor data merging with stacked neural networks for the creation of satellite long-term climate data records. Eurasip J. Adv. Signal Proc., doi:10.1186/1687-6180-2012-91. 
Loyola, D., et al., 2009: Global long-term monitoring of the ozone layer-a prerequisite for predictions. Int. J. Remote Sens., 30, 4295-4318.

Lu, J., G. A. Vecchi, and T. Reichler, 2007: Expansion of the Hadley cell under global warming. Geophys. Res. Lett., 34, L06805.

Lu, J. H., and J. J. Ji, 2006: A simulation and mechanism analysis of long-term variations at land surface over arid/semi-arid area in north China. J. Geophys. Res. Atmos., 111, D09306.

Lucarini, V., and F. Ragone, 2011: Energetics of climate models: Net energy balance and meridional enthalpy transport. Rev. Geophys., 49, RG1001.

Lucas-Picher, P., S. Somot, M. Déqué, B. Decharme, and A. Alias, 2012a: Evaluation of the regional climate model ALADIN to simulate the climate over North America in the CORDEX framework. Clim. Dyn., doi:10.1007/s00382-012-1613-8.

Lucas-Picher, P., M. Wulff-Nielsen, J. Christensen, G. Adalgeirsdottir, R. Mottram, and S. Simonsen, 2012b: Very high resolution regional climate model simulations over Greenland: Identifying added value. J. Geophys. Res. Atmos., 117, D02108.

Lumpkin, R., K. G. Speer, and K. P. Koltermann, 2008: Transport across $48^{\circ} \mathrm{N}$ in the Atlantic Ocean. J. Phys. Oceanogr., 38, 733-752.

Luo, J. J., S. Masson, E. Roeckner, G. Madec, and T. Yamagata, 2005: Reducing climatology bias in an ocean-atmosphere CGCM with improved coupling physics. J. Clim., 18, 2344-2360.

Lynn, B., R. Healy, and L. Druyan, 2009: Quantifying the sensitivity of simulated climate change to model configuration. Clim. Change, 92, 275-298.

MacKinnon, J., et al., 2009: Using global arrays to investigate internal-waves and mixing. In: OceanObs09: Sustained Ocean Observations and Information for Society, Venice, Italy, ESA.

Madden, R. A., and P. R. Julian, 1972: Description of global-scale circulation ells in tropics with a 40-50 day period. J. Atmos. Sci., 29, 1109-1123.

Madden, R. A., and P. R. Julian, 1994: Observations of the 40-50-Day Tropical Oscillation-a Review. Mon. Weather Rev., 122, 814-837.

Madec, G., 2008: NEMO ocean engine. Technical Note. Institut Pierre-Simon Laplace (IPSL), France, 300pp.

Madec, G., P. Delecluse, M. Imbard, and C. Levy, 1998: OPA 8.1 ocean general circulation model reference manual. IPSL Note du Pole de Modelisation, Institut Pierre-Simon Laplace (IPSL), France, 91 pp.

Mahlstein, I., and R. Knutti, 2010: Regional climate change patterns identified by cluster analysis. Clim. Dyn., 35, 587-600.

Mahlstein, I., and R. Knutti, 2012: September Arctic sea ice predicted to disappear near 2C global warming above present. J. Geophys. Res., 117, D06104.

Maier-Reimer, E., I. Kriest, J. Segschneider, and P. Wetze, 2005: The HAMburg Ocean Carbon Cycle Model HAMOCC 5.1-Technical Description Release 1.1. Tech. Rep. 14, Rep. Earth Syst. Sci., Max Planck Institute for Meteorology, Hamburg, Germany, $50 \mathrm{pp}$.

Mantua, N. J., S. R. Hare, Y. Zhang, J. M. Wallace, and R. C. Francis, 1997: A Pacific interdecadal climate oscillation with impacts on salmon production. Bull. Am. Meteorol. Soc., 78, 1069-1079.

Manzini, E., C. Cagnazzo, P. G. Fogli, A. Bellucci, and W. A. Muller, 2012: Stratospheretroposphere coupling at inter-decadal time scales: Implications for the North Atlantic Ocean. Geophys. Res. Lett., 39, L05801.

Maraun, D., 2012: Nonstationarities of regional climate model biases in European seasonal mean temperature and precipitation sums. Geophys. Res. Lett., 39, L06706.

Maraun, D., H. Rust, and T. Osborn, 2010a: Synoptic airflow and UK daily precipitation extremes: Development and validation of a vector generalised linear model. Extremes, 13, 133-153.

Maraun, D., et al., 2010b: Precipitation downscaling under climate change: Recent developments to bridge the gap between dynamical models and the end user. Rev. Geophys., 48, RG3003.

Marchand, R., N. Beagley, and T. P. Ackerman, 2009: Evaluation of hydrometeor occurrence profiles in the Multiscale Modeling Framework Climate Model using atmospheric classification. J. Clim., 22, 4557-4573.

Markovic, M., H. Lin, and K. Winger, 2010: Simulating global and North American climate using the Global Environmental Multiscale Model with a VariableResolution Modeling Approach. Mon. Weather Rev., 138, 3967-3987.

Marsh, R., S. A. Mueller, A. Yool, and N. R. Edwards, 2011: Incorporation of the C-GOLDSTEIN efficient climate model into the GENIE framework: "eb_go_gs" configurations of GENIE. Geophys. Model Dev., 4, 957-992.

Marsh, R., et al., 2009: Recent changes in the North Atlantic circulation simulated with eddy-permitting and eddy-resolving ocean models. Ocean Model., 28, 226-239.
Marsland, S. J., et al., 2013: Evaluation of ACCESS Climate Model ocean diagnostics in CMIP5 simulations. Aust. Meteorol. and Oceanogr. J., 63,101-119.

Martin, G. M., and R. C. Levine, 2012: The influence of dynamic vegetation on the present-day simulation and future projectons of the South Asian summer monsoon in the HadGEM2 family. Earth Syst. Dyn., 2, 245-261.

Martin, G. M., et al., 2011: The HadGEM2 family of Met Office Unified Model climate configurations. Geophys. Model Dev., 4, 723-757.

Masarie, K. A., and P. P. Tans, 1995: Extension and integration of atmospheric carbon dioxide data into a globally consistent measurement record. J. Geophys. Res. Atmos., 100, 11593-11610.

Masato, G., B. Hoskins, and T. Woollings, 2012: Winter and summer Northern Hemisphere blocking in CMIP5 models. J. Clim., doi:10.1175/JCLI-D-12-00466.1.

Masson-Delmotte, V., et al., 2010: EPICA Dome C record of glacial and interglacial intensities. Quat. Sci. Rev., 29, 113-128.

Masson-Delmotte, V., et al., 2006: Past and future polar amplification of climate change: Climate model intercomparisons and ice-core constraints. Clim. Dyn., 27, 437-440.

Masson, D., and R. Knutti, 2011 a: Climate model genealogy. Geophys. Res. Lett., 38, L08703.

Masson, D., and R. Knutti, 2011b: Spatial-scale dependence of climate model performance in the CMIP3 ensemble. J. Clim., 24, 2680-2692.

Masson, D., and R. Knutti, 2013: Predictor screening, calibration and observational constraints in climate model ensembles: An illustration using climate sensitivity. J. Clim., 26, 887-898.

Massonnet, F., T. Fichefet, H. Goosse, C. M. Bitz, G. Philippon-Berthier, M. M. Holland, and P.-Y. Barriat, 2012: Constraining projections of summer Arctic sea ice. Cryosphere, 6, 1383-1394.

Mastrandrea, M. D., et al., 2011: Guidance Note for Lead Authors of the IPCC Fifth Assessment Report on Consistent Treatment of Uncertainties. Intergovernmental Panel on Climate Change (IPCC). IPCC guidance note, Jasper Ridge, CA, USA, 7 pp.

Materia, S., P. A. Dirmeyer, Z. C. Guo, A. Alessandri, and A. Navarra, 2010: The sensitivity of simulated river discharge to land surface representation and meteorological forcings. J. Hydrometeorol., 11, 334-351.

Matsueda, M., 2009: Blocking predictability in operational medium-range ensemble forecasts. Sola, 5, 113-116.

Matsueda, M., R. Mizuta, and S. Kusunoki, 2009: Future change in wintertime atmospheric blocking simulated using a 20-km-mesh atmospheric global circulation model. J. Geophys. Res. Atmos., 114, D12114.

Matsueda, M., H. Endo, and R. Mizuta, 2010: Future change in Southern Hemisphere summertime and wintertime atmospheric blockings simulated using a 20-km-mesh AGCM. Geophys. Res. Lett., 37, L02803.

Matsumoto, K., K. S. Tokos, A. R. Price, and S. J. Cox, 2008: First description of the Minnesota Earth System Model for Ocean biogeochemistry (MESMO 1.0). Geophys. Model Dev., 1, 1-15.

Maurer, E., and H. Hidalgo, 2008: Utility of daily vs. monthly large-scale climate data: An intercomparison of two statistical downscaling methods. Hydrol. Earth Syst. Sci., 12, 551-563.

Mauritsen, T., et al., 2012: Tuning the climate of a global model. J. Adv. Model. Earth Syst., 4, M00A01.

Maximenko, N., et al., 2009: Mean dynamic topography of the ocean derived from satellite and drifting buoy data using three different techniques. J. Atmos. Ocean. Technol., 26, 1910-1919.

May, P. T., J. H. Mather, G. Vaughan, K. N. Bower, C. Jakob, G. M. McFarquhar, and G. G. Mace, 2008: The Tropical Warm Pool International Cloud Experiment. Bull. Am. Meteorol. Soc., 89, 629-645.

May, W., 2007: The simulation of the variability and extremes of daily precipitation over Europe by the HIRHAM regional climate model. Global Planet. Change, $57,59-82$.

McCarthy, G., et al., 2012: Observed interannual variability of the Atlantic meridional overturning circulation at 26.5 degrees N. Geophys. Res. Lett., 39, L19609.

McClean, J. L., and J. C. Carman, 2011: Investigation of IPCC AR4 coupled climate model North Atlantic modewater formation. Ocean Model., 40, 14-34.

McClean, J. L., M. E. Maltrud, and F. O. Bryan, 2006: Measures of the fidelity of eddying ocean models. Oceanography, 19, 104-117.

McClean, J. L., et al., 2011: A prototype two-decade fully-coupled fine-resolution CCSM simulation. Ocean Model. 39, 10-30. 
McCormack, J. P., S. D. Eckermann, D. E. Siskind, and T. J. McGee, 2006: CHEM2D-OPP: A new linearized gas-phase ozone photochemistry parameterization for highaltitude NWP and climate models. Atmos. Chem. Phys., 6, 4943-4972.

McCrary, R. R., and D. A. Randall, 2010: Great plains drought in simulations of the twentieth century. J. Clim., 23, 2178-2196.

McDonald, R. E., 2011: Understanding the impact of climate change on Northern Hemisphere extra-tropical cyclones. Clim. Dyn., 37, 1399-1425.

McDougall, T. J., and P. C. McIntosh, 2001: The temporal-residual-mean velocity. Part II: Isopycnal interpretation and the tracer and momentum equations. J. Phys. Oceanogr., 31, 1222-1246.

McKitrick, R., S. McIntyre, and C. Herman, 2010: Panel and multivariate methods for tests of trend equivalence in climate data series. Atmos. Sci. Lett., 11, 270-277.

McKitrick, R., S. McIntyre, and C. Herman, 2011: Panel and multivariate methods for tests of trend equivalence in climate data series. Atmos. Sci. Lett. 12, 386-388.

McLandress, C., T. Shepherd, J. Scinocca, D. Plummer, M. Sigmond, A. Jonsson, and M. Reader, 2011: Separating the dynamical effects of climate change and ozone depletion. Part II Southern Hemisphere troposphere. J. Clim., 24, 1850-1868.

McLaren, A. J., et al., 2006: Evaluation of the sea ice simulation in a new coupled atmosphere-ocean climate model (HadGEM1). J. Geophys. Res. Oceans, 111, C12014.

McManus, J. F., R. Francois, J. M. Gherardi, L. D. Keigwin, and S. Brown-Leger, 2004: Collapse and rapid resumption of Atlantic meridional circulation linked to deglacial climate changes. Nature, 428, 834-837.

McWilliams, J. C., 2008: The nature and consequences of oceanic eddies. In: Ocean Modeling in an Eddying Regime [M. Hecht and H. Hasumi (eds.)]. American Geophysical Union, Washington, DC, pp. 5-15.

Mearns, L. O., et al., 2012: The North American Regional Climate Change Assessment Program: Overview of Phase I Results. Bull. Am. Meteorol. Soc., 93, 1337-1362.

Mears, C. A., F. J. Wentz, P. Thorne, and D. Bernie, 2011: Assessing uncertainty in estimates of atmospheric temperature changes from MSU and AMSU using a Monte-Carlo estimation technique. J. Geophys. Res., 116, D08112.

Mears, C. A., B. D. Santer, F. J. Wentz, K. E. Taylor, and M. F. Wehner, 2007: Relationship between temperature and precipitable water changes over tropical oceans. Geophys. Res. Lett., 34, L24709.

Meehl, G. A., and J. M. Arblaster, 2011: Decadal variability of Asian-Australian Monsoon-ENSO-TBO relationships. J. Clim., 24, 4925-4940.

Meehl, G. A., and H. Teng, 2012: Case studies for initialized decadal hindcasts and predictions for the Pacific region. Geophys. Res. Lett., 39, L22705.

Meehl, G. A., J. M. Arblaster, and J. Loschnigg, 2003: Coupled ocean-atmosphere dynamical processes in the tropical Indian and Pacific Oceans and the TBO. J. Clim., 16, 2138-2158.

Meehl, G. A., J. M. Arblaster, J. T. Fasullo, A. X. Hu, and K. E. Trenberth, 2011: Modelbased evidence of deep-ocean heat uptake during surface-temperature hiatus periods. Nature Clim. Change, 1, 360-364.

Meehl, G. A., A. Hu, J. Arblaster, J. Fasullo, and K. E. Trenberth, 2013a: Externally forced and internally generated decadal climate variability associated with the Interdecadal Pacific Oscillation. J. Clim., doi:10.1175/JCLI-D-12-00548.1.

Meehl, G. A., P. R. Gent, J. M. Arblaster, B. L. Otto-Bliesner, E. C. Brady, and A. Craig, 2001: Factors that affect the amplitude of El Niño in global coupled climate models. Clim. Dyn., 17, 515-526.

Meehl, G. A., J. M. Arblaster, J. M. Caron, H. Annamalai, M. Jochum, A. Chakraborty, and R. Murtugudde, 2012: Monsoon regimes and processes in CCSM4. Part I: The Asian-Australian Monsoon. J. Clim., 25, 2583-2608.

Meehl, G. A., et al., 2007: The WCRP CMIP3 multimodel dataset -A new era in climate change research. Bull. Am. Meteorol. Soc., 88, 1383-1394.

Meehl, G. A., et al., 2009: Decadal prediction: Can it be skillful? Bull. Am. Meteorol. Soc., 90, 1467-1485.

Meehl, G. A., et al., 2013b: Decadal climate prediction: An update from the trenches. Bull. Am. Meteorol. Soc., doi:10.1175/BAMS-D-12-00241.1.

Meijers, A., E. Shuckburgh, N. Bruneau, J.-B. Sallee, T. Bracegirdle, and Z. Wang, 2012: Representation of the Antarctic Circumpolar Current in the CMIP5 climate models and future changes under warming scenarios. J. Geophys. Res. Oceans, $117, \mathrm{C} 12008$

Meinshausen, M., et al., 2009: Greenhouse-gas emission targets for limiting global warming to 2 degrees C. Nature, 458, 1158-1162.

Meinshausen, M., et al., 2011: The RCP greenhouse gas concentrations and their extensions from 1765 to 2300. Clim. Change, 109, 213-241.
Meissner, K. J., A. J. Weaver, H. D. Matthews, and P. M. Cox, 2003: The role of land surface dynamics in glacial inception: a study with the UVic Earth System Model. Clim. Dyn., 21, 515-537.

Melillo, J. M., A. D. McGuire, D. W. Kicklighter, B. Moore, C. J. Vorosmarty, and A. L. Schloss, 1993: Global climate-change and terrestrial net primary production. Nature, 363, 234-240.

Melsom, A., V. Lien, and W. P. Budgell, 2009: Using the Regional Ocean Modeling System (ROMS) to improve the ocean circulation from a GCM 20th century simulation. Ocean Dyn., 59, 969-981.

Menary, M., W. Park, K. Lohmann, M. Vellinga, D. Palmer, M. Latif, and J. H. Jungclaus, 2012: A multimodel comparison of centennial Atlantic meridional overturning circulation variability. Clim. Dyn., 38, 2377-2388.

Menendez, C., M. de Castro, A. Sorensson, J. Boulanger, and C. M. Grp, 2010: CLARIS Project: Towards climate downscaling in South America. Meteorol. Z., 19, 357362.

Menon, S., D. Koch, G. Beig, S. Sahu, J. Fasullo, and D. Orlikowski, 2010: Black carbon aerosols and the third polar ice cap. Atmos. Chem. Phys., 10, 4559-4571.

Mercado, L. M., C. Huntingford, J. H. C. Gash, P. M. Cox, and V. Jogireddy, 2007: Improving the representation of radiation interception and photosynthesis for climate model applications. Tellus B, 59, 553-565.

Merrifield, M. A., and M. E. Maltrud, 2011: Regional sea level trends due to a Pacific trade wind intensification. Geophys. Res. Lett., 38, L21605.

Merryfield, W. J., et al., 2013: The Canadian Seasonal to Interannual Prediction System. Part I: Models and Initialization. Mon. Weather Rev., doi:10.1175/MWRD-12-00216.1.

Mieville, A., et al., 2010: Emissions of gases and particles from biomass burning during the 20th century using satellite data and an historical reconstruction. Atmos. Environ., 44, 1469-1477.

Miller, A., et al., 2002: A cohesive total ozone data set from the SBUV(/2) satellite system. J. Geophys. Res. Atmos., 107, 4701.

Milliff, R., A. Bonazzi, C. Wikle, N. Pinardi, and L. Berliner, 2011: Ocean ensemble forecasting. Part I: Ensemble Mediterranean winds from a Bayesian hierarchical model. Q. J. R. Meteorol. Soc., 137, 858-878.

Milly, P. C. D., and A. B. Shmakin, 2002: Global modeling of land water and energy balances. Part I: the land dynamics (LaD) model. J. Hydrometeorol., 3, 283-299.

Min, S. K., X. B. Zhang, F. W. Zwiers, and G. C. Hegerl, 2011: Human contribution to more-intense precipitation extremes. Nature, 470, 376-379.

Minobe, S., 1997: A 50-70 year climatic oscillation over the North Pacific and North America. Geophys. Res. Lett., 24, 683-686.

Minobe, S., 1999: Resonance in bidecadal and pentadecadal climate oscillations over the North Pacific: Role in climatic regime shifts. Geophys. Res. Lett., 26, $855-858$.

Misra, V., 2007: Addressing the issue of systematic errors in a regional climate model. J. Clim., 20, 801-818.

Mitchell, T. D., and P. D. Jones, 2005: An improved method of constructing a database of monthly climate observations and associated high-resolution grids. Int. J. Climatol. , 25, 693-712.

Miyama, T., and M. Kawamiya, 2009: Estimating allowable carbon emission for CO(2) concentration stabilization using a GCM-based Earth system model. Geophys. Res. Lett., 36, L19709.

Mizuta, R., et al., 2012: Climate simulations using MRI-AGCM3.2 with 20-km grid. J. Meteorol. Soc. Jpn., 90A, 233-258.

Molteni, F., 2003: Atmospheric simulations using a GCM with simplified physical parameterizations. I: Model climatology and variability in multi-decadal experiments. Clim. Dyn., 20, 175-191.

Montoya, M., A. Griesel, A. Levermann, J. Mignot, M. Hofmann, A. Ganopolski, and S. Rahmstorf, 2005: The earth system model of intermediate complexity CLIMBER-3 alpha. Part 1: description and performance for present-day conditions. Clim. Dyn., 25, 237-263.

Morgenstern, 0., et al., 2010: Anthropogenic forcing of the Northern Annular Mode in CCMVal-2 models. J. Geophys. Res., 115, D00M03.

Morice, C. P., J. J. Kennedy, N. A. Rayner, and P. D. Jones, 2012: Quantifying uncertainties in global and regional temperature change using an ensemble of observational estimates: The HadCRUT4 data set. J. Geophys. Res. Atmos., 117, D08101.

Moss, R. H., et al., 2010: The next generation of scenarios for climate change research and assessment. Nature, 463, 747-756. 
Mouchet, A., and L. M. François, 1996: Sensitivity of a global oceanic carbon cycle model to the circulation and the fate of organic matter: Preliminary results. Phys. Chem. Earth, 21, 511-516.

Msadek, R., and C. Frankignoul, 2009: Atlantic multidecadal oceanic variability and its influence on the atmosphere in a climate model. Clim. Dyn., 33, 45-62.

Msadek, R., W. E. Johns, S. G. Yeager, G. Danabasoglu, T. Delworth, and T. Rosati, 2013: The Atlantic meridional heat transport at $26.5^{\circ} \mathrm{N}$ and its relationship with the MOC in the RAPID-array and GFDL and NCAR coupled models. J. Clim., doi:10.1175/JCLI-D-12-00081.1.

Mueller, B., et al., 2011: Evaluation of global observations-based evapotranspiraion datasets and IPCC AR4 simulations. Geophys. Res. Lett., 38, L06402.

Muller, S. A., F. Joos, N. R. Edwards, and T. F. Stocker, 2006: Water mass distribution and ventilation time scales in a cost-efficient, three-dimensional ocean model. J. Clim., 19, 5479-5499.

Murakami, H., and M. Sugi, 2010: Effect of model resolution on tropical cyclone climate projections. Sola, 6, 73-76.

Murakami, H., et al., 2012: Future changes in tropical cyclone activity projected by the new high-resolution MRI-AGCM. J. Clim., 25, 3237-3260.

Murphy, D. M., 2013: Little net clear-sky radiative forcing from recent regional redistribution of aerosols. Nature Geosci, 6, 258-262.

Murphy, J., B. Booth, M. Collins, G. Harris, D. Sexton, and M. Webb, 2007: A methodology for probabilistic predictions of regional climate change from perturbed physics ensembles. Philos. Trans. R. Soc. London A, 365 1993-2028.

Murphy, J. M., D. M. H. Sexton, D. N. Barnett, G. S. Jones, M. J. Webb, M. Collins, and D. A. Stainforth, 2004: Quantification of modelling uncertainties in a large ensemble of climate change simulations. Nature, 430, 768-772.

Murtugudde, R., J. Beauchamp, C. R. McClain, M. Lewis, and A. J. Busalacchi, 2002: Effects of penetrative radiation on the upper tropical ocean circulation. J. Clim., $15,470-486$

Muryshev, K. E., A. V. Eliseev, I. I. Mokhov, and N. A. Diansky, 2009: Validating and assessing the sensitivity of the climate model with an ocean general circulation model developed at the Institute of Atmospheric Physics, Russian Academy of Sciences. Izvestiya Atmos. Ocean. Phys., 45, 416-433.

Nagura, M., W. Sasaki, T. Tozuka, J.-J. Luo, S. K. Behera, and T. Yamagata, 2013: Longitudinal biases in the Seychelles Dome simulated by 35 ocean-atmosphere coupled general circulation models. J. Geophys. Res., doi:10.1029/2012JC008352.

Nakano, H., H. Tsujino, M. Hirabara, T. Yasuda, T. Motoi, M. Ishii, and G. Yamanaka, 2011: Uptake mechanism of anthropogenic $\mathrm{CO}_{2}$ in the Kuroshio Extension region in an ocean general circulation model. J. Oceanogr., 67, 765-783.

Nam, C., S. Bony, J. L. Dufresne, and H. Chepfer, 2012: The 'too few, too bright' tropical low-cloud problem in CMIP5 models. Geophys. Res. Lett., 39, L21801.

Nanjundiah, R. S., V. Vidyunmala, and J. Srinivasan, 2005: The impact of increase in $\mathrm{CO}_{2}$ on the simulation of tropical biennial oscillations (TBO) in 12 coupled general circulation models. Atmos. Sci. Lett., 6, 183-191.

Naoe, H., and K. Shibata, 2010: Equatorial quasi-biennial oscillation influence on northern winter extratropical circulation. J. Geophys. Res. Atmos., 115, D19102.

Neale, R. B., J. H. Richter, and M. Jochum, 2008: The Impact of Convection on ENSO: From a delayed oscillator to a series of events. J. Clim., 21, 5904-5924.

Neale, R. B., J. Richter, S. Park, P. H. Lauritzen, S. J. Vavrus, P. J. Rasch, and M. Zhang, 2013: The Mean Climate of the Community Atmosphere Model (CAM4) in forced SST and fully coupled experiments. J. Clim., doi:10.1175/JCLI-D-12-00236.1.

Neale, R. B., et al., 2010: Description of the NCAR Community Atmosphere Model (CAM 4.0). NCAR Technical Note NCAR/TN-486+STR, National Center for Atmopsheric Research, Boulder, CO, $268 \mathrm{pp}$.

Neelin, J. D., 2007: Moist dynamics of tropical convection zones in monsoons, teleconnections and global warming. In: The Global Circulation of the Atmosphere [T. Schneider and A. Sobel (eds.)]. Princeton University Press, Princeton, NJ. 385 pp.

Neelin, J. D., and N. Zeng, 2000: A quasi-equilibrium tropical circulation modelFormulation. J. Atmos. Sci., 57, 1741-1766.

Neelin, J. D., C. Chou, and H. Su, 2003: Tropical drought regions in global warming and El Niño teleconnections. Geophys. Res. Lett., 30, 2275.

Neelin, J. D., A. Bracco, H. Luo, J. C. McWilliams, and J. E. Meyerson, 2010: Considerations for parameter optimization and sensitivity in climate models. Proc. Nat. Acad. Sci. U.S.A., 107, 21349-21354.

Neggers, R. A. J., 2009: A dual mass flux framework for boundary layer convection. Part II: Clouds. J. Atmos. Sci., 66, 1489-1506.

Neggers, R. A. J., M. Kohler, and A. C. M. Beljaars, 2009: A dual mass flux framework for boundary layer convection. Part I: Transport. J. Atmos. Sci., 66, 1465-1487.
Nicolsky, D. J., V. E. Romanovsky, V. A. Alexeev, and D. M. Lawrence, 2007: Improved modeling of permafrost dynamics in a GCM land-surface scheme. J. Geophys. Res., 34, L08501.

Nikiema, O., and R. Laprise, 2010: Diagnostic budget study of the internal variability in ensemble simulations of the Canadian RCM. Clim. Dyn., 36 2313-2337.

Nikulin, G., et al., 2012: Precipitation climatology in an ensemble of CORDEX-Africa regional climate simulations. J. Clim., doi:10.1175/jcli-d-11-00375.1.

Ning, L., M. E. Mann, R. Crane, and T. Wagener, 2011: Probabilistic projections of climate change for the Mid-Atlantic region of the United States-Validation of precipitation downscaling during the Historical Era. J. Clim., 25, 509-526.

Nishii, K., et al., 2012: Relationship of the reproducibility of multiple variables among Global Climate Models. J. Meteorol. Soc. Jpn., 90A, 87-100.

Notz, D., F. A. Haumann, H. Haak, J. H. Jungclaus, and J. Marotzke, 2013: Sea-ice evolution in the Arctic as modeled by MPI-ESM. J. Adv. Model. Earth Syst., doi:10.1002/jame.20016.

O'Connor, F. M., C. E. Johnson, O. Morgenstern, and W. J. Collins, 2009: Interactions between tropospheric chemistry and climate model temperature and humidity biases. Geophys. Res. Lett., 36, L16801.

O'Farrell, S. P., 1998: Investigation of the dynamic sea ice component of a coupled atmosphere sea ice general circulation model. J. Geophys. Res.-Oceans, 103, 15751-15782.

O'Gorman, P. A., 2012: Sensitivity of tropical precipitation extremes to climate change. Nature Geosci., 5, 697-700.

O'Gorman, P. A., and M. S. Singh, 2013: Vertical structure of warming consistent with an upward shift in the middle and upper troposphere. Geophys. Res. Lett., doi:10.1002/grl.50328.

O'ishi, R., and A. Abe-Ouchi, 2011: Polar amplification in the mid-Holocene derived from dynamical vegetation change with a GCM. Geophys. Res. Lett., 38, L14702.

Ogasawara, N., A. Kitoh, T. Yasunari, and A. Noda, 1999: Tropospheric biennia oscillation of ENSO-monsoon system in the MRI coupled GCM. J. Meteorol. Soc. Jpn., 77, 1247-1270.

Ohba, M., D. Nohara, and H. Ueda, 2010: Simulation of asymmetric ENSO transition in WCRP CMIP3 Multimodel Experiments. J. Clim., 23, 6051-6067.

Ohgaito, R., and A. Abe-Ouchi, 2009: The effect of sea surface temperature bias in the PMIP2 AOGCMs on mid-Holocene Asian monsoon enhancement. Clim. Dyn., 33, 975-983.

Oka, A., E. Tajika, A. Abe-Ouchi, and K. Kubota, 2011: Role of the ocean in controlling atmospheric $\mathrm{CO}_{2}$ concentration in the course of global glaciations. Clim. Dyn., 37, 1755-1770.

Oleson, K. W., 2004: Technical description of the Community Land Model (CLM). NCAR Technical Note NCAR/TN-461+STR, National Center for Atmospheric Research, Boulder, CO, $174 \mathrm{pp}$.

Oleson, K. W., G. B. Bonan, J. Feddema, M. Vertenstein, and C. S. B. Grimmond, 2008a: An urban parameterization for a global climate model. Part I: Formulation and evaluation for two cities. J. Appl. Meteorol. Climatol., 47, 1038-1060.

Oleson, K. W., et al., 2010: Technical Description of version 4.0 of the Community Land Model (CLM) NCAR Technical Note NCAR/TN-478+STR, National Center for Atmospheric Research, Boulder, CO, $257 \mathrm{pp}$.

Oleson, K. W., et al., 2008b: Improvements to the Community Land Model and their impact on the hydrological cycle. J. Geophys. Res. Biogeosci., 113, G01021

Onogi, K., et al., 2007: The JRA-25 reanalysis. J. Meteorol. Soc. Jpn., 85, 369-432.

Opsteegh, J. D., R. J. Haarsma, F. M. Selten, and A. Kattenberg, 1998: ECBILT: A dynamic alternative to mixed boundary conditions in ocean models. Tellus $A$, 50, 348-367.

Oreopoulos, L., et al., 2012: The continual intercomparison of radiation codes: Results from Phase I. J. Geophys. Res. Atmos., 117, D06118.

Ostle, N. J., et al., 2009: Integrating plant-soil interactions into global carbon cycle models. J. Ecol., 97, 851-863.

Otte, T. L., C. G. Nolte, M. J. Otte, and J. H. Bowden, 2012: Does nudging squelch the extremes in Regional Climate Modeling? J. Clim., 25, 7046-7066.

Ottera, O. H., M. Bentsen, H. Drange, and L. Suo, 2010: External forcing as a metronome for Atlantic multidecadal variability. Nature Geosci., 3, 688-694.

Otto-Bliesner, B. L., et al., 2007: Last Glacial Maximum ocean thermohaline circulation: PMIP2 model intercomparisons and data constraints. Geophys. Res. Lett., 34, L12706.

Otto-Bliesner, B. L., et al., 2009: A comparison of PMIP2 model simulations and the MARGO proxy reconstruction for tropical sea surface temperatures at last glacial maximum. Clim. Dyn., 32, 799-815. 
Otto, J., T. Raddatz, M. Claussen, V. Brovkin, and V. Gayler, 2009: Separation of atmosphere-ocean-vegetation feedbacks and synergies for mid-Holocene climate. Global Biogeochem. Cycles, 23, L09701.

Overland, J. E., and M. Wang, 2013: When will the summer Arctic be nearly sea ice free? Geophys. Res. Lett., doi:10.1002/grl.50316, doi:10.1002/grl.50316.

Ozturk, T., H. Altinsoy, M. Turkes, and M. Kurnaz, 2012: Simulation of temperature and precipitation climatology for the central Asia CORDEX domain using RegCM 4.0. Clim. Res., 52, 63-76.

Paeth, H., 2011: Postprocessing of simulated precipitation for impact research in West Africa. Part I: Model output statistics for monthly data. Clim. Dyn., 36 1321-1336.

Paeth, H., et al., 2012: Progress in regional downscaling of west African precipitation. Atmos. Sci. Lett., 12, 75-82.

Palmer, J. R., and I. J. Totterdell, 2001: Production and export in a global ocean ecosystem model. Deep-Sea R. Pt. I, 48, 1169-1198.

Parekh, P., F. Joos, and S. A. Muller, 2008: A modeling assessment of the interplay between aeolian iron fluxes and iron-binding ligands in controlling carbon dioxide fluctuations during Antarctic warm events. Paleoceanography, 23, Pa4202.

Park, S., and C. S. Bretherton, 2009: The University of Washington Shallow Convection and Moist Turbulence schemes and their impact on climate simulations with the Community Atmosphere Model. J. Clim., 22, 3449-3469.

Park, W., and M. Latif, 2010: Pacific and Atlantic multidecadal variability in the Kiel Climate Model. Geophys. Res. Lett., 37, L24702.

Parker, D., C. Folland, A. Scaife, J. Knight, A. Colman, P. Baines, and B. W. Dong, 2007: Decadal to multidecadal variability and the climate change background. $J$. Geophys. Res. Atmos., 112, D18115.

Parkinson, C. L., and D. J. Cavalieri, 2012: Antarctic sea ice variability and trends, 1979-2010. Cryosphere, 6, 871-880.

Patricola, C. M., M. Li, Z. Xu, P. Chang, R. Saravanan, and J.-S. Hsieh, 2012: An investigation of tropical Atlantic bias in a high-resolution Coupled Regional Climate Model. Clim. Dyn., doi:10.1007/s00382-012-1320-5.

Pavlova, T. V., V. M. Kattsov, and V. A. Govorkova, 2011: Sea ice in CMIP5 models: Closer to reality? Trudy GGO (MGO Proc., in Russian), 564, 7-18.

Pavlova, T. V., V. M. Kattsov, Y. D. Nadyozhina, P. V. Sporyshev, and V. A. Govorkova, 2007: Terrestrial cryosphere evolution through the 20th and 21st centuries as simulated with the new generation of global climate models. Earth Cryosphere (in Russian), 11, 3-13.

Pechony, O., and D. T. Shindell, 2009: Fire parameterization on a global scale. J. Geophys. Res. Atmos., 114, D16115

Pedersen, C. A., E. Roeckner, M. Lüthje, and J. Winther, 2009: A new sea ice albedo scheme including melt ponds for ECHAM5 general circulation model. J. Geophys. Res., 114, D08101.

Pennell, C., and T. Reichler, 2011: On the effective number of climate models. J. Clim., 24 2358-2367

Perlwitz, J., S. Pawson, R. Fogt, J. Nielsen, and W. Neff, 2008: Impact of stratospheric ozone hole recovery on Antarctic climate. Geophys. Res. Lett., 35, L08714.

Peterson, T. C., et al., 2009: State of the Climate in 2008. Bull. Am. Meteorol. Soc., 90, S1-S196

Petoukhov, V., I. I. Mokhov, A. V. Eliseev, and V. A. Semenov, 1998: The IAP RAS global climate model. Dialogue-MSU, Moscow, Russia.

Petoukhov, V., A. Ganopolski, V. Brovkin, M. Claussen, A. Eliseev, C. Kubatzki, and S. Rahmstorf, 2000: CLIMBER-2: a climate system model of intermediate complexity. Part I: Model description and performance for present climate. Clim. Dyn., 16, 1-17.

Petoukhov, V., et al., 2005: EMIC Intercomparison Project (EMIP-CO2): Comparative analysis of EMIC simulations of climate, and of equilibrium and transient responses to atmospheric $\mathrm{CO}_{2}$ doubling. Clim. Dyn., 25, 363-385.

Pfahl, S., and H. Wernli, 2012: Quantifying the relevance of atmospheric blocking for co-located temperature extremes in the Northern Hemisphere on (sub-)daily time scales. Geophys. Res. Lett., 39, L12807.

Pfeiffer, A., and G. Zängl, 2010: Validation of climate-mode MM5-simulations for the European Alpine Region. Theor. Appl. Climatol., 101, 93-108.

Phillips, T. J., et al., 2004: Evaluating parameterizations in General Circulation Models: Climate simulation meets weather prediction. Bull. Am. Meteorol. Soc. 85, 1903-1915.

Piani, C., D. J. Frame, D. A. Stainforth, and M. R. Allen, 2005: Constraints on climate change from a multi-thousand member ensemble of simulations. Geophys. Res. Lett., 32, L23825.
Pierce, D. W., 2001: Distinguishing coupled ocean-atmosphere interactions from background noise in the North Pacific. Prog. Oceanogr., 49, 331-352.

Pierce, D. W., T. P. Barnett, B. D. Santer, and P. J. Gleckler, 2009: Selecting global climate models for regional climate change studies. Proc. Natl. Acad. Sci. U.S.A., $106,8441-8446$

Pincus, R., C. P. Batstone, R. J. P. Hofmann, K. E. Taylor, and P. J. Glecker, 2008: Evaluating the present-day simulation of clouds, precipitation, and radiation in climate models. J. Geophys. Res. Atmos., 113, D14209

Pincus, R., S. Platnick, S. A. Ackerman, R. S. Hemler, and R. J. P. Hofmann, 2012: Reconciling simulated and observed views of clouds: MODIS, ISCCP, and the limits of instrument simulators. J. Clim., 25, 4699-4720.

Pinto, J. G., T. Spangehl, U. Ulbrich, and P. Speth, 2006: Assessment of winter cyclone activity in a transient ECHAM4-OPYC3 GHG experiment. Meteorol. Z., 15, 279-291.

Piot, M., and R. von Glasow, 2008: The potential importance of frost flowers, recycling on snow, and open leads for ozone depletion events. Atmos. Chem. Phys., 8, 2437-2467.

Pitman, A., A. Arneth, and L. Ganzeveld, 2012a: Regionalizing global climate models. Int. J. Climatol. , 32, 321-337.

Pitman, A. J., 2003: The evolution of, and revolution in, land surface schemes designed for climate models. Int. J. Climatol. , 23, 479-510.

Pitman, A. J., et al., 2012b: Effects of land cover change on temperature and rainfall extremes in multi-model ensemble simulations. Earth Syst. Dyn., 13, 213-231.

Pitman, A. J., et al., 2009: Uncertainties in climate responses to past land cover change: First results from the LUCID intercomparison study. Geophys. Res. Lett., 36, L14814.

Plattner, G. K., et al., 2008: Long-term climate commitments projected with climatecarbon cycle models. J. Clim., 21, 2721-2751.

Ploshay, J. J., and N.-C. Lau, 2010: Simulation of the diurnal cycle in tropical rainfall and circulation during Boreal Summer with a high-resolution GCM. Mon. Weather Rev., 138, 3434-3453.

Po-Chedley, S., and Q. Fu, 2012: Discrepancies in tropical upper tropospheric warming between atmospheric circulation models and satellites. Environ. Res. Lett., 7, 044018

Pokhrel, S., H. Rahaman, A. Parekh, S. K. Saha, A. Dhakate, H. S. Chaudhari, and R. M. Gairola, 2012: Evaporation-precipitation variability over Indian Ocean and its assessment in NCEP Climate Forecast System (CFSv2). Clim. Dyn., 39, 25852608.

Polvani, L., D. Waugh, G. Correa, and S. Son, 2011: Stratospheric ozone depletion: The main driver of twentieth-century atmospheric circulation changes in the Southern Hemisphere. J. Clim., 24, 795-812.

Pope, V. D., M. L. Gallani, P. R. Rowntree, and R. A. Stratton, 2000: The impact of new physical parametrizations in the Hadley Centre climate model: HadAM3. Clim. Dyn., 16, 123-146.

Power, S., and R. Colman, 2006: Multi-year predictability in a coupled general circulation model. Clim. Dyn., 26, 247-272

Power, S., M. Haylock, R. Colman, and X. D. Wang, 2006: The predictability of interdecadal changes in ENSO activity and ENSO teleconnections. J. Clim., 19, 4755-4771.

Prudhomme, C., and H. Davies, 2009: Assessing uncertainties in climate change impact analyses on the river flow regimes in the UK. Part 1: Baseline climate. Clim. Change, 93, 177-195.

Pryor, S., G. Nikulin, and C. Jones, 2012: Influence of spatial resolution on regional climate model derived wind climates. J. Geophys. Res. Atmos., 117, D03117.

Ptashnik, I. V., R. A. McPheat, K. P. Shine, K. M. Smith, and R. G. Williams, 2011: Water vapor self-continuum absorption in near-infrared windows derived from laboratory measurements. J. Geophys. Res. Atmos., 116, D16305.

Qian, T. T., A. Dai, K. E. Trenberth, and K. W. Oleson, 2006: Simulation of global land surface conditions from 1948 to 2004. Part I: Forcing data and evaluations. J. Hydrometeorol., 7, 953-975.

Qiao, F., Y. Yuan, Y. Yang, Q. Zheng, C. Xia, and J. Ma, 2004: Wave-induced mixing in the upper ocean: Distribution and application to a global ocean circulation model. Geophys. Res. Lett., 31, L11303.

Quaas, J., 2012: Evaluating the "critical relative humidity" as a measure of subgrid-scale variability of humidity in general circulation model cloud cover parameterizations using satellite data. J. Geophys. Res. Atmos., 117, D09208.

Raftery, A. E., T. Gneiting, F. Balabdaoui, and M. Polakowski, 2005: Using Bayesian model averaging to calibrate forecast ensembles. Mon. Weather Rev., 133, 1155-1174. 
Raible, C. C., M. Yoshimori, T. F. Stocker, and C. Casty, 2007: Extreme midlatitude cyclones and their implications for precipitation and wind speed extremes in simulations of the Maunder Minimum versus present day conditions. Clim. Dyn., $28,409-423$.

Raisanen, J., 2007: How reliable are climate models? Tellus A, 59, 2-29.

Raisanen, J., and J. S. Ylhaisi, 2011: How much should climate model output be smoothed in space? J. Clim., 24, 867-880.

Raisanen, J., L. Ruokolainen, and J. Ylhaisi, 2010: Weighting of model results for improving best estimates of climate change. Clim. Dyn., 35, 407-422.

Rammig, A., et al., 2010: Estimating the risk of Amazonian forest dieback. New Phytologist, 187, 694-706.

Rampal, P., J. Weiss, C. Dubois, and J. M. Campin, 2011: IPCC climate models do not capture Arctic sea ice drift acceleration: Consequences in terms of projected sea ice thinning and decline. J. Geophys. Res. Oceans, 116, C00d07.

Ramstein, G., M. Kageyama, J. Guiot, H. Wu, C. Hely, G. Krinner, and S. Brewer, 2007: How cold was Europe at the Last Glacial Maximum? A synthesis of the progress achieved since the first PMIP model-data comparison. Clim. Past, 3, 331-339.

Randall, D. A., M. F. Khairoutdinov, A. Arakawa, and W. W. Grabowski, 2003: Breaking the cloud parameterization deadlock. Bull. Am. Meteorol. Soc., 84, 1547-1564.

Randall, D. A., et al., 2007: Climate models and their evaluation. In: Climate Change 2007: The Physical Science Basis. Contribution of Working Group I to the Fourth Assessment Report of the Intergovernmental Panel on Climate Change [Solomon, S., D. Qin, M. Manning, Z. Chen, M. Marquis, K. B. Averyt, M. Tignor and H. L. Miller (eds.)] Cambridge University Press, Cambridge, United Kingdom and New York, NY, USA, pp. 589-662.

Randel, W., and F. Wu, 2007: A stratospheric ozone profile data set for 1979-2005: Variability, trends, and comparisons with column ozone data. J. Geophys. Res. Atmos., 12, D06313.

Randel, W. J., et al., 2009: An update of observed stratospheric temperature trends. J. Geophys. Res. Atmos., 114, D02107.

Rapaić, M., M. Leduc, and R. Laprise, 2010: Evaluation of the internal variability and estimation of the downscaling ability of the Canadian Regional Climate Model for different domain sizes over the north Atlantic region using the Big-Brother experimental approach. Clim. Dyn., 36 1979-2001.

Raphael, M. N., and M. M. Holland, 2006: Twentieth century simulation of the Southern Hemisphere climate in coupled models. Part 1: Large scale circulation variability. Clim. Dyn., 26, 217-228.

Rashid, H. A., A. C. Hirst, and M. Dix, 2013: Atmospheric circulation features in the ACCESS model simulations for CMIP5: Historical simulation and future projections Aust. Meteorol. Oceanogr. J., 63, 145-160.

Rauscher, S. A., E. Coppola, C. Piani, and F. Giorgi, 2010: Resolution effects on regional climate model simulations of seasonal precipitation over Europe. Clim. Dyn., 35, 685-711.

Rayner, N. A., et al., 2003: Global analysis of sea surface temperature, sea ice, and night marine air temperature since the late ninteeth century. J. Geophys. Res., $108,4407$.

Redelsperger, J.-L., C. D. Thorncroft, A. Diedhiou, T. Lebel, D. J. Parker, and J. Polcher, 2006: African Monsoon Multidisciplinary Analysis: An international research project and field campaign. Bull. Am. Meteorol. Soc., 87, 1739-1746.

Redi, M. H., 1982: Oceanic isopycnal mixing by coordinate rotation. J. Phys. Oceanogr., 12, 1154-1158.

Reichler, T., and J. Kim, 2008: How well do coupled models simulate today's climate? Bull. Am. Meteorol. Soc., 89, 303-311.

Reick, C. H., T. Raddatz, V. Brovkin, and V. Gayler, 2013: The representation of natural and anthropogenic land cover change in MPI-ESM. J. Adv. Model. Earth Syst., doi:10.1002/jame.20022.

Reifen, C., and R. Toumi, 2009: Climate projections: Past performance no guarantee of future skill? Geophys. Res. Lett., 36, L13704.

Remer, L. A., et al., 2008: Global aerosol climatology from the MODIS satellite sensors. J. Geophys. Res. Atmos., 113, D14s07.

Richter, I., and S.-P. Xie, 2008: On the origin of equatorial Atlantic biases in coupled general circulation models. Clim. Dyn., 31, 587-598.

Richter, I., S.-P. Xie, S. K. Behera, T. Doi, and Y. Masumoto, 2013: Equatorial Atlantic variability and its relation to mean state biases in CMIP5. Clim. Dyn., doi:10.1007/s00382-012-1624-5.

Richter, J. H., F. Sassi, and R. R. Garcia, 2010: Toward a physically based gravity wave source parameterization in a General Circulation Model. J. Atmos. Sci., 67, 136-156.
Ridgwell, A., and J. C. Hargreaves, 2007: Regulation of atmospheric CO(2) by deepsea sediments in an Earth system model. Global Biogeochem. Cycles, 21, Gb2008.

Ridgwell, A., I. Zondervan, J. C. Hargreaves, J. Bijma, and T. M. Lenton, 2007a: Assessing the potential long-term increase of oceanic fossil fuel $\mathrm{CO}_{2}$ uptake due to $\mathrm{CO}_{2}$-calcification feedback. Biogeosciences, 4, 481-492.

Ridgwell, A., et al., 2007b: Marine geochemical data assimilation in an efficient Earth System Model of global biogeochemical cycling. Biogeosciences, 4, 87-104.

Rienecker, M. M., et al., 2011: MERRA: NASA's modern-era retrospective analysis for research and applications. J. Clim., 24, 3624-3648.

Ringer, M. A., J. M. Edwards, and A. Slingo, 2003: Simulation of satellite channel radiances in the Met Office Unified Model. Q. J. R. Meteorol. Soc., 129, 11691190.

Rio, C., and F. Hourdin, 2008: A thermal plume model for the convective boundary layer: Representation of cumulus clouds. J. Atmos. Sci, 65, 407-425.

Rio, C., F. Hourdin, F. Couvreux, and A. Jam, 2010: Resolved versus parametrized boundary-layer plumes. Part II: Continuous formulations of mixing rates for mass-flux schemes. Boundary-Layer Meteorol., 135, 469-483.

Risi, C., et al., 2012a: Process-evaluation of tropospheric humidity simulated by general circulation models using water vapor isotopic observations: 2 . Using isotopic diagnostics to understand the mid and upper tropospheric moist bias in the tropics and subtropics. J. Geophys. Res. Atmos., 117, D05304.

Risi, C., et al., 2012b: Process-evaluation of tropospheric humidity simulated by general circulation models using water vapor isotopologues: 1. Comparison between models and observations. J. Geophys. Res. Atmos., 117, D05303.

Risien, C. M., and D. B. Chelton, 2008: A global climatology of surface wind and wind stress fields from eight years of QuikSCAT Scatterometer data. J. Phys. Oceanogr., 38, 2379-2413.

Ritz, S. P., T. F. Stocker, and F. Joos, 2011: A coupled dynamical ocean-energy balance atmosphere model for paleoclimate studies. J. Clim., 24, 349-375.

Roberts, M. J., et al., 2004: Impact of an eddy-permitting ocean resolution on control and climate change simulations with a global coupled GCM. J. Clim., 17, 3-20.

Robinson, A., R. Calov, and A. Ganopolski, 2012: Multistability and critical thresholds of the Greenland ice sheet. Nature Clim. Change, 2, 429-432.

Robinson, D. A., and A. Frei, 2000: Seasonal variability of northern hemisphere snow extent using visible satellite data. Prof. Geograph., 51, 307-314.

Rockel, B., C. L. Castro, R. A. Pielke, H. von Storch, and G. Leoncini, 2008: Dynamical downscaling: Assessment of model system dependent retained and added variability for two different regional climate models. J. Geophys. Res. Atmos. 113, D21107.

Rodwell, M., and T. Palmer, 2007: Using numerical weather prediction to assess climate models. Q. J. R. Meteorol. Soc., 133, 129-146.

Roe, G. H., and M. B. Baker, 2007: Why is climate sensitivity so unpredictable? Science, 318, 629-632.

Roe, G. H., and K. C. Armour, 2011: How sensitive is climate sensitivity? Geophys. Res. Lett., 38, L14708.

Roe, G. H., and M. B. Baker, 2011: Comment on "Another look at climate sensitivity" by Zaliapin and Ghil (2010). Nonlin.Proc. Geophys., 18, 125-127.

Roeckner, E., et al., 2006: Sensitivity of simulated climate to horizontal and vertical resolution in the ECHAM5 atmosphere model. J. Clim., 19, 3771-3791.

Rojas, M., 2006: Multiply nested regional climate simulation for southern South America: Sensitivity to model resolution. Mon. Weather Rev., 134, 2208-2223.

Rojas, M., and P. I. Moreno, 2011: Atmospheric circulation changes and neoglacial conditions in the Southern Hemisphere mid-latitudes: Insights from PMIP2 simulations at 6 kyr. Clim. Dyn., 37, 357-375.

Rojas, M., et al., 2009: The Southern Westerlies during the last glacial maximum in PMIP2 simulations. Clim. Dyn., 32, 525-548.

Romanou, A., et al., 2013: Natural air-sea flux of $\mathrm{CO}_{2}$ in simulations of the NASAGISS climate model: Sensitivity to the physical ocean model formulation. Ocean Model., doi:10.1016/j.ocemod.2013.01.008.

Rotstayn, L. D., and U. Lohmann, 2002: Simulation of the tropospheric sulfur cycle in a global model with a physically based cloud scheme. J. Geophys. Res., 107, 4592.

Rotstayn, L. D., M. A. Collier, R. M. Mitchell, Y. Qin, S. K. Campbell, and S. M. Dravitzki, 2011: Simulated enhancement of ENSO-related rainfall variability due to Australian dust. Atmos. Chem. Phys., 11, 6575-6592. 
Rotstayn, L. D., S. J. Jeffrey, M. A. Collier, S. M. Dravitzki, A. C. Hirst, J. I. Syktus, and K. K. Wong, 2012: Aerosol- and greenhouse gas-induced changes in summer rainfall and circulation in the Australasian region: A study using single-forcing climate simulations. Atmos. Chem. Phys., 12, 6377-6404.

Rotstayn, L. D., et al., 2010: Improved simulation of Australian climate and ENSOrelated rainfall variability in a global climate model with an interactive aerosol treatment. Int. J. Climatol. , 30, 1067-1088.

Rougier, J., D. M. H. Sexton, J. M. Murphy, and D. Stainforth, 2009: Analyzing the climate sensitivity of the HadSM3 climate model using ensembles from different but related experiments. J. Clim., 22, 3540-3557.

Roy, P., P. Gachon, and R. Laprise, 2012: Assessment of summer extremes and climate variability over the north-east of North America as simulated by the Canadian Regional Climate Model. Int. J. Climatol. , 32 1615-1627.

Ruckstuhl, C., and J. R. Norris, 2009: How do aerosol histories affect solar "dimming" and "brightening" over Europe?: IPCC-AR4 models versus observations. J. Geophys. Res. Atmos., 114, D00d04.

Rummukainen, M., 2010: State-of-the-art with regional climate models. Clim. Change, 1, 82-96.

Russell, J. L., R. J. Stouffer, and K. W. Dixon, 2006: Intercomparison of the Southern Ocean circulations in IPCC coupled model control simulations. J. Clim., 19, 4560-4575.

Ruti, P. M., et al., 2011: The West African climate system: A review of the AMMA model inter-comparison initiatives. Atmos. Sci. Lett., 12 116-122

Rutter, N., et al., 2009: Evaluation of forest snow processes models (SnowMIP2). J. Geophys. Res. Atmos., 114, D06111.

Sabine, C. L., et al., 2004: The oceanic sink for anthropogenic $\mathrm{CO}_{2}$. Science, 305 367-371.

Saha, S., et al., 2010: The NCEP Climate Forecast System Reanalysis. Bull. Am. Meteorol. Soc., 91, 1015-105.

Sahany, S., J. D. Neelin, K. Hales, and R. B. Neale, 2012: Temperature-moisture dependence of the Deep Convective Transition as a constraint on entrainment in climate models. J. Atmos. Sci., 69, 1340-1358.

Saji, N. H., S. P. Xie, and T. Yamagata, 2006: Tropical Indian Ocean variability in the IPCC twentieth-century climate simulations. J. Clim., 19, 4397-4417.

Saji, N. H., B. N. Goswami, P. N. Vinayachandran, and T. Yamagata, 1999: A dipole mode in the tropical Indian Ocean. Nature, 401, 360-363.

Sakaguchi, K., X. B. Zeng, and M. A. Brunke, 2012: The hindcast skill of the CMIP ensembles for the surface air temperature trend. J. Geophys. Res. Atmos., 117, D16113.

Sakamoto, T. T., et al., 2012: MIROC4h - a new high-resolution atmosphere-ocean coupled general circulation model. J. Meteorol. Soc. Jpn., 90, 325-359.

Salas-Melia, D., 2002: A global coupled sea ice-ocean model. Ocean Model., 4, 137-172

Salle, J. B., E. Shuckburgh, N. Bruneau, A. J. S. Meijers, T. J. Bracegirdle, Z. Wang, and T. Roy, 2013: Assessment of Southern Ocean water mass circulation and characteristics in CMIP5 models: Historical bias and forcing response. J. Geophys. Res. Oceans, doi:10.1002/jgrc.20135.

Samuelsson, P., E. Kourzeneva, and D. Mironov, 2010: The impact of lakes on the European climate as simulated by a regional climate model. Boreal Environ. Res. $15,113-129$.

Sander, S. P., 2006: Chemical Kinetics and Photochemical Data for Use in Atmospheric Studies. Evaluation 15. JPL Publications, Pasadena, CA, USA, 523 pp.

Sanderson, B. M., 2011: A multimodel study of parametric uncertainty in predictions of climate response to rising greenhouse gas concentrations. J. Clim., 25, 1362 1377.

Sanderson, B. M., 2013: On the estimation of systematic error in regressionbased predictions of climate sensitivity. Clim. Change, doi:10.1007/s10584-0120671-6.

Sanderson, B. M., and R. Knutti, 2012: On the interpretation of constrained climate model ensembles. Geophys. Res. Lett., 39, L16708.

Sanderson, B. M., K. M. Shell, and W. Ingram, 2010: Climate feedbacks determined using radiative kernels in a multi-thousand member ensemble of AOGCMs. Clim. Dyn., 35, 1219-1236.

Sanderson, B. M., C. Piani, W. J. Ingram, D. A. Stone, and M. R. Allen, 2008a: Towards constraining climate sensitivity by linear analysis of feedback patterns in thousands of perturbed-physics GCM simulations. Clim. Dyn., 30, 175-190.

Sanderson, B. M., et al., 2008b: Constraints on model response to greenhouse gas forcing and the role of subgrid-scale processes. J. Clim., 21, 2384-2400.
Sansom, P. G., D. B. Stephenson, C. A. T. Ferro, G. Zappa, and L. Shaffrey, 2013: Simple uncertainty frameworks for selecting weighting schemes and interpreting multimodel ensemble climate change experiments doi:10.1175/JCLI-D-12-00462.1.

Santer, B., et al., 2009: Incorporating model quality information in climate change detection and attribution studies. Proc. Natl. Acad. Sci. U.S.A., 106, 1477814783.

Santer, B., et al., 2007: Identification of human-induced changes in atmospheric moisture content. Proc. Natl. Acad. Sci. U.S.A., 104, 15248-15253.

Santer, B., et al., 2008: Consistency of modelled and observed temperature trends in the tropical troposphere. Int. J. Climatol. , 28, 1703-1722.

Santer, B., et al., 2005: Amplification of surface temperature trends and variability in the tropical atmosphere. Science, 309, 1551-1556.

Santer, B. D., et al., 2013: Identifying human influences on atmospheric temperature. Proc. Natl. Acad. Sci. U.S.A, 110, 26-33.

Sato, M., J. E. Hansen, M. P. McCormick, and J. B. Pollack, 1993: Stratospheric aerosol optical depth, 1850-1990. J. Geophys. Res. Atmos., 98, 22987-22994

Sato, T., H. Miura, M. Satoh, Y. N. Takayabu, and Y. Q. Wang, 2009: Diurnal cycle of precipitation in the Tropics simulated in a global cloud-resolving model. J. Clim., 22, 4809-4826.

Scaife, A. A., N. Butchart, C. D. Warner, and R. Swinbank, 2002: Impact of a spectral gravity wave parameterization on the stratosphere in the met office unified model. J. Atmos. Sci., 59, 1473-1489.

Scaife, A. A., T. Woollings, J. Knight, G. Martin, and T. Hinton, 2010: Atmospheric blocking and mean biases in climate models. J. Clim., 23, 6143-6152.

Scaife, A. A., et al., 2011: Improved Atlantic winter blocking in a climate model. Geophys. Res. Lett., 38, L23703.

Scaife, A. A., et al., 2012: Climate change and stratosphere-troposphere interaction. Clim. Dyn., 38, 2089-2097.

Scaife, A. A., et al., 2009: The CLIVAR C20C project: Selected twentieth century climate events. Clim. Dyn., 33, 603-614.

Schaller, N., I. Mahlstein, J. Cermak, and R. Knutti, 2011: Analyzing precipitation projections: A comparison of different approaches to climate model evaluation. J. Geophys. Res. Atmos., 116, D10118.

Scherrer, S., 2011: Present-day interannual variability of surface climate in CMIP3 models and its relation to future warming. Int. J. Climatol. , 31, 1518-1529.

Schlesinger, M. E., and J. F. B. Mitchell, 1987: Climate model simulations of the equilibrium climatic response to increased carbon-dioxide. Rev. Geophys., 25, 760-798.

Schmidli, J., C. Goodess, C. Frei, M. Haylock, Y. Hundecha, J. Ribalaygua, and T. Schmith, 2007: Statistical and dynamical downscaling of precipitation: An evaluation and comparison of scenarios for the European Alps. J. Geophys. Res. Atmos., 112, D04105.

Schmidt, G. A., et al., 2012: Climate forcing reconstructions for use in PMIP simulations of the Last Millennium (v1.1). Geophys. Model Dev., 5, 185-191.

Schmidt, G. A., et al., 2006: Present day atmospheric simulations using GISS ModelE: Comparison to in-situ, satellite and reanalysis data. J. Clim., 19, 153-192.

Schmith, T., 2008: Stationarity of regression relationships: Application to empirical downscaling. J. Clim., 21, 4529-4537.

Schmittner, A., A. Oschlies, X. Giraud, M. Eby, and H. L. Simmons, 2005: A global model of the marine ecosystem for long-term simulations: Sensitivity to ocean mixing, buoyancy forcing, particle sinking, and dissolved organic matter cycling. Global Biogeochem. Cycles, 19, Gb3004.

Schott, F. A., S.P. Xie, and J. P. McCreary, Jr., 2009: Indian Ocean circulation and climate variability. Rev. Geophys., 47, RG1002.

Schramm, J. L., M. M. Holland, J. A. Curry, and E. E. Ebert, 1997: Modeling the thermodynamics of a sea ice thickness 1 . Sensitivity to ice thickness resolution. J. Geophys. Res., 102, 23079-23091.

Schultz, M. G., et al., 2008: Global wildland fire emissions from 1960 to 2000. Global Biogeochem. Cycles, 22, GB2002.

Schurgers, G., U. Mikolajewicz, M. Groger, E. Maier-Reimer, M. Vizcaino, and A. Winguth, 2008: Long-term effects of biogeophysical and biogeochemical interactions between terrestrial biosphere and climate under anthropogenic climate change. Global Planet. Change, 64, 26-37.

Scoccimarro, E., et al., 2011: Effects of tropical cyclones on ocean heat transport in a high resolution Coupled General Circulation Model. J. Clim., 24, 4368-4384.

Séférian, R., et al., 2013: Skill assessment of three earth system models with common marine biogeochemistry. Clim. Dyn., 40, 2549-2573. 
Segui, P., A. Ribes, E. Martin, F. Habets, and J. Boe, 2010: Comparison of three downscaling methods in simulating the impact of climate change on the hydrology of Mediterranean basins. J. Hydrol., 383, 111-124.

Seidel, D. J., M. Free, and J. S. Wang, 2012: Reexamining the warming in the tropical upper troposphere: Models versus radiosonde observations. Geophys. Res. Lett., 39, L22701.

Seidel, D. J., Q. Fu, W. J. Randel, and T. J. Reichler, 2008: Widening of the tropical belt in a changing climate. Nature Geosci., 1, 21-24.

Seidel, D. J., N. P. Gillett, J. R. Lanzante, K. P. Shine, and P.W. Thorne, 2011: Stratospheric temperature trends: Our evolving understanding. Clim. Change, 2, 592-616.

Selten, F. M., G. W. Branstator, H. A. Dijkstra, and M. Kliphuis, 2004: Tropical origins for recent and future Northern Hemisphere climate change. Geophys. Res. Lett., 31, L21205.

Semenov, V. A., M. Latif, J. H. Jungclaus, and W. Park, 2008: Is the observed NAO variability during the instrumental record unusual? Geophys. Res. Lett., 35, L11701.

Seneviratne, S., et al., 2012: Changes in climate extremes and their impacts on the natural physical environment. In: IPCC WGI/WGII Special Report on Managing the Risks of Extreme Events and Disasters to Advance Climate Change Adaptation (SREX), [Field, C.B., V. Barros, T.F. Stocker, D. Qin, D.J. Dokken, K.L. Ebi, M.D. Mastrandrea, K.J. Mach, G.-K. Plattner, S.K. Allen, M. Tignor, and P.M. Midgley (Eds.)]. Cambridge University Press, The Edinburgh Building, Shaftesbury Road, Cambridge CB2 8RU ENGLAND, pp. 109-230.

Seneviratne, S. I., D. Luethi, M. Litschi, and C. Schaer, 2006: Land-atmosphere coupling and climate change in Europe. Nature, 443, 205-209.

Seneviratne, S. I., et al., 2010: Investigating soil moisture-climate interactions in a changing climate: A review. Earth Sci. Rev., 99, 125-161.

Separovic, L., R. De Elia, and R. Laprise, 2008: Reproducible and irreproducible components in ensemble simulations with a Regional Climate Model. Mon. Weather Rev., 136, 4942-4961.

Separovic, L., R. Elía, and R. Laprise, 2012: Impact of spectral nudging and domain size in studies of RCM response to parameter modification. Clim. Dyn., 38, 1325-1343.

Severijns, C. A., and W. Hazeleger, 2010: The efficient global primitive equation climate model SPEEDO V2.0. Geophys. Model Dev., 3, 105-122.

Sexton, D. M. H., and J. M. Murphy, 2012: Multivariate probabilistic projections using imperfect climate models. Part II: robustness of methodological choices and consequences for climate sensitivity. Clim. Dyn., 38, 2543-2558.

Sexton, D. M. H., J. M. Murphy, M. Collins, and M. J. Webb, 2012: Multivariate probabilistic projections using imperfect climate models part I: Outline of methodology. Clim. Dyn., 38, 2513-2542.

Shaffer, G., and J. L. Sarmiento, 1995: Biogeochemical cycling in the global ocean. 1. A new, analytical model with continuous vertical resolution and high-latitude dynamics. J. Geophys. Res. Oceans, 100, 2659-2672.

Shaffer, G., S. M. Olsen, and J. O. P. Pedersen, 2008: Presentation, calibration and validation of the low-order, DCESS Earth System Model (Version 1). Geophys. Model Dev., 1, 17-51.

Shaffrey, L. C., et al., 2009: UK HiGEM: The new UK High-Resolution Global Environment Model-Model description and basic evaluation. J. Clim., 22, 1861-1896.

Sheffield, J., and E. F. Wood, 2008: Projected changes in drought occurrence under future global warming from multi-model, multi-scenario, IPCC AR4 simulations. Clim. Dyn., 31, 79-105.

Shell, K. M., J. T. Kiehl, and C. A. Shields, 2008: Using the radiative kernel technique to calculate climate feedbacks in NCAR's Community Atmospheric Model. J. Clim., 21, 2269-2282.

Shevliakova, E., et al., 2009: Carbon cycling under 300 years of land use change: Importance of the secondary vegetation sink. Global Biogeochem. Cycles, 23, GB2022

Shi, Y., J. Zhang, J. S. Reid, B. Holben, E. J. Hyer, and C. Curtis, 2011: An analysis of the collection 5 MODIS over-ocean aerosol optical depth product for its implication in aerosol assimilation. Atmos. Chem. Phys., 11, 557-565.

Shibata, K., and M. Deushi, 2005: Radiative effect of ozone on the quasi-biennial oscillation in the equatorial stratosphere. Geophys. Res. Lett., 32, L24802.

Shin, D., J. Kim, and H. Park, 2011: Agreement between monthly precipitation estimates from TRMM satellite, NCEP reanalysis, and merged gauge-satellite analysis. J. Geophys. Res. Atmos., 116, D16105.
Shin, S. I., D. Sardeshmukh, and K. Pegion, 2010: Realism of local and remote feedbacks on tropical sea surface temperatures in climate models. J. Geophys. Res. Atmos., 115, D21110.

Shindell, D. T., et al., 2013a: Interactive ozone and methane chemistry in GISSE2 historical and future climate simulations Atmos. Chem. Phys., 13, 2653-2689.

Shindell, D. T., et al., 2013b: Radiative forcing in the ACCMIP historical and future climate simulations. Atmos. Chem. Phys., 13, 2939-2974.

Shiogama, H., S. Emori, N. Hanasaki, M. Abe, Y. Masutomi, K. Takahashi, and T. Nozawa, 2011: Observational constraints indicate risk of drying in the Amazon basin. Nature Commun., 2, 253.

Shiogama, H., et al., 2012: Perturbed physics ensemble using the MIROC5 coupled atmosphere-ocean GCM without flux corrections: Experimental design and results. Clim. Dyn., 39, 3041-3056.

Shiu, C.-J., S. C. Liu, C. Fu, A. Dai, and Y. Sun, 2012: How much do precipitation extremes change in a warming climate? Geophys. Res. Lett., 39, L17707.

Shkol'nik, I., V. Meleshko, S. Efimov, and E. Stafeeva, 2012: Changes in climate extremes on the territory of Siberia by the middle of the 21st century: An ensemble forecast based on the MGO regional climate model. Russ. Meteorol. Hydrol., 37, 71-84.

Shkolnik, I., V. Meleshko, and V. Kattsov, 2007: The MGO climate model for Siberia. Russ. Meteorol. Hydrol., 32, 351-359.

Siebesma, A. P., P. M. M. Soares, and J. Teixeira, 2007: A combined eddy-diffusivity mass-flux approach for the convective boundary layer. J. Atmos. Sci, , 64, 1230 1248.

Sigmond, M., and J. F. Scinocca, 2010: The influence of the basic state on the Northern Hemisphere circulation response to climate change. J. Clim., 23, 1434-1446.

Sillmann, J., and M. Croci-Maspoli, 2009: Present and future atmospheric blocking and its impact on European mean and extreme climate. Geophys. Res. Lett., 36, L10702.

Sillmann, J., M. Croci-Maspoli, M. Kallache, and R. W. Katz, 2011: Extreme cold winter temperatures in Europe under the influence of North Atlantic atmospheric blocking. J. Clim., 24, 5899-5913.

Sillmann, J., V. V. Kharin, X. Zhang, and F. W. Zwiers, 2013: Climate extreme indices in the CMIP5 multi-model ensemble. Part 1: Model evaluation in the present climate. J. Geophys. Res., doi:10.1029/2012JD018390.

Simmons, A. J., K. M. Willett, P. D. Jones, P. W. Thorne, and D. P. Dee, 2010: Lowfrequency variations in surface atmospheric humidity, temperature, and precipitation: Inferences from reanalyses and monthly gridded observational data sets. J. Geophys. Res. Atmos., 115, D01110.

Simmons, H. L., S. R. Jayne, L. C. St Laurent, and A. J. Weaver, 2004: Tidally driven mixing in a numerical model of the ocean general circulation. Ocean Model., 6, 245-263.

Sitch, S., et al., 2003: Evaluation of ecosystem dynamics, plant geography and terrestrial carbon cycling in the LPJ dynamic global vegetation model. Global Change Biol., 9, 161-185.

Sitch, S., et al., 2008: Evaluation of the terrestrial carbon cycle, future plant geography and climate-carbon cycle feedbacks using five Dynamic Global Vegetation Models (DGVMs). Global Change Biol., 14, 2015-2039.

Six, K. D., and E. Maier-Reimer, 1996: Effects of plankton dynamics on seasonal carbon fluxes in an Ocean General Circulation Model. Global Biogeochem. Cycles, 10, 559-583.

Slater, A. G., and D. M. Lawrence, 2013: Diagnosing present and future permafrost from climate models. J. Clim., doi:10.1175/JCLI-D-12-00341.1.

Sloyan, B. M., and I. V. Kamenkovich, 2007: Simulation of Subantarctic Mode and Antarctic Intermediate Waters in climate models. J. Clim., 20, 5061-5080.

Smirnov, D., and D. J. Vimont, 2011: Variability of the Atlantic Meridional Mode during the Atlantic Hurricane Season. J. Clim., 24, 1409-1424.

Smith, B., P. Samuelsson, A. Wramneby, and M. Rummukainen, 2011a: A model of the coupled dynamics of climate, vegetation and terrestrial ecosystem biogeochemistry for regional applications. Tellus $A, 63,87-106$.

Smith, P. C., N. De Noblet-Ducoudre, P. Ciais, P. Peylin, N. Viovy, Y. Meurdesoif, and A. Bondeau, 2010a: European-wide simulations of croplands using an improved terrestrial biosphere model: Phenology and productivity. J. Geophys. Res. Biogeosci, 115, G01014.

Smith, P. C., P. Ciais, P. Peylin, N. De Noblet-Ducoudre, N. Viovy, Y. Meurdesoif, and A. Bondeau, 2010b: European-wide simulations of croplands using an improved terrestrial biosphere model: 2 . Interannual yields and anomalous $\mathrm{CO}_{2}$ fluxes in 2003. J. Geophys. Res. Biogeosci, 115, G04028 
Smith, R. D., M. E. Maltrud, F. O. Bryan, and M. W. Hecht, 2000: Numerical simulation of the North Atlantic Ocean at 1/10 degrees. J. Phys. Oceanogr., 30, 1532-1561.

Smith, R. S., J. M. Gregory, and A. Osprey, 2008: A description of the FAMOUS (version XDBUA) climate model and control run. Geophys. Model Dev., 1, 53-68.

Smith, S. J., J. van Aardenne, Z. Klimont, R. J. Andres, A. Volke, and S. D. Arias, 2011b: Anthropogenic sulfur dioxide emissions: 1850-2005. Atmos. Chem. Phys., 11, $1101-1116$.

Sobolowski, S., and T. Pavelsky, 2012: Evaluation of present and future North American Regional Climate Change Assessment Program (NARCCAP) regional climate simulations over the southeast United States. J. Geophys. Res. Atmos. 117, D01101.

Soden, B. J., and I. M. Held, 2006: An assessment of climate feedbacks in coupled ocean-atmosphere models. J. Clim., 19, 3354-3360.

Soden, B. J., I. M. Held, R. Colman, K. M. Shell, J. T. Kiehl, and C. A. Shields, 2008 : Quantifying climate feedbacks using radiative kernels. J. Clim., 21, 3504-3520.

Sokolov, A. P., and P. H. Stone, 1998: A flexible climate model for use in integrated assessments. Clim. Dyn., 14, 291-303.

Sokolov, A. P., C. E. Forest, and P. H. Stone, 2010: Sensitivity of climate change projections to uncertainties in the estimates of observed changes in deep-ocean heat content. Clim. Dyn., 34, 735-745.

Sokolov, A. P., et al., 2009: Probabilistic forecast for twenty-first-century climate based on uncertainties in emissions (without policy) and climate parameters. J. Clim., 22, 5175-5204.

Sokolov, A. P., et al., 2005: The MIT Integrated Global System Model (IGSM) Version 2: Model description and baseline evaluation. MIT JP Report 124. MIT, Cambridge, MA.

Solman, S., and N. Pessacg, 2012: Regional climate simulations over South America: Sensitivity to model physics and to the treatment of lateral boundary conditions using the MM5 model. Clim. Dyn., 38, 281-300.

Solomon, S., P. J. Young, and B. Hassler, 2012: Uncertainties in the evolution of stratospheric ozone and implications for recent temperature changes in the tropical lower stratosphere. Geophys. Res. Lett., 39 L17706.

Solomon, S., K. H. Rosenlof, R. W. Portmann, J. S. Daniel, S. M. Davis, T. J. Sanford, and G. K. Plattner, 2010: Contributions of stratospheric water vapor to decada changes in the rate of global warming. Science, 327, 1219-1223.

Somot, S., F. Sevault, M. Deque, and M. Crepon, 2008: 21st century climate change scenario for the Mediterranean using a coupled atmosphere-ocean regional climate model. Global Planet. Change, 63 112-126.

Son, S., et al., 2008: The impact of stratospheric ozone recovery on the Southern Hemisphere westerly jet. Science, 320, 1486-1489.

Son, S., et al., 2010: Impact of stratospheric ozone on Southern Hemisphere circulation change: A multimodel assessment. J. Geophys. Res. Atmos., 115, D00M07.

Song, Z., F. Qiao, and Y. Song, 2012: Response of the equatorial basin-wide SST to non-breakingsurface wave-induced mixing in a climate model: An amendment to tropical bias. J. Geophys. Res., doi:10.1029/2012JC007931.

SPARC-CCMVal, 2010: SPARC Report on the Evaluation of Chemistry-Climate Models [V. Eyring, T.G. Shepherd, D.W. Waugh (eds.)], SPARC Report No. 5, WCRP-132, WMO/TD-No. 1526

Sperber, K., and D. Kim, 2012: Simplified metrics for the identification of the MaddenJulian oscillation in models. Atmos. Sci. Let., doi:10.1002/asl.378.

Sperber, K., et al., 2012: The Asian summer monsoon: An intercomparison of CMIP-5 vs. CMIP-3 simulations of the late 20th century. Clim. Dyn., doi:10.1007/s00382012-1607-6.

Sperber, K. R., 2003: Propagation and the vertical structure of the Madden-Julian oscillation. Mon. Weather Rev., 131, 3018-3037.

Sperber, K. R., and H. Annamalai, 2008: Coupled model simulations of boreal summer intraseasonal (30-50 day) variability, Part 1: Systematic errors and caution on use of metrics. Clim. Dyn., 31, 345-372.

Sperber, K. R., et al., 2010: Monsoon Fact Sheet: CLIVAR Asian-Australian Monsoon Panel.

Stainforth, D. A., et al., 2005: Uncertainty in predictions of the climate response to rising levels of greenhouse gases. Nature, 433, 403-406.

Stephens, G. L., and C. D. Kummerow, 2007: The remote sensing of clouds and precipitation from space: A review. J. Atmos. Sci., 64, 3742-3765.

Stephens, G. L., et al., 2010: Dreary state of precipitation in global models. J. Geophys. Res., 115, D24211.

Stephens, G. L., et al., 2012: An Update on the Earth's energy balance in light of new global observations. Nature Geosci., 5, 691-696.
Stephenson, D. B., M. Collins, J. C. Rougier, and R. E. Chandler, 2012: Statistical problems in the probabilistic prediction of climate change. Environmetrics, 23 , 364-372.

Stephenson, D. B., V. Pavan, M. Collins, M. M. Junge, R. Quadrelli, and C. M. G. Participating, 2006: North Atlantic Oscillation response to transient greenhouse gas forcing and the impact on European winter climate: A CMIP2 multi-model assessment. Clim. Dyn., 27, 401-420.

Stevens, B., and S. E. Schwartz, 2012: Observing and modeling Earth's energy flows. Surv. Geophys., 33, 779-816.

Stevens, B., et al., 2012: The atmospheric component of the MPI-M Earth System Model: ECHAM6. J. Adv. Model. Earth Syst., doi:10.1002/jame.20015.

Stevenson, S., 2012: Significant changes to ENSO strength and impacts in the twenty-first century: Results from CMIP5. Geophys. Res. Lett., doi:10.1029/2012GL052759.

Stevenson, S., B. Fox-Kemper, M. Jochum, R. Neale, C. Deser, and G. Meehl, 2012: Will there be a significant change to El Niño in the twenty-first century? J. Clim., 25, 2129-2145.

Stocker, B. D., K. Strassmann, and F. Joos, 2011: Sensitivity of Holocene atmospheric $\mathrm{CO}_{2}$ and the modern carbon budget to early human land use: Analyses with a process-based model. Biogeosciences, 8, 69-88.

Stocker, B. D., et al., 2012: Multiple greenhouse gas feedbacks from the land biosphere under future climate change scenarios. Nature Clim. Change, doi:10.1038/nclimate1864.

Stolarski, R., and S. Frith, 2006: Search for evidence of trend slow-down in the longterm TOMS/SBUV total ozone data record: The importance of instrument drift uncertainty. Atmos. Chem. Phys. , 6, 4057-4065.

Stoner, A. M. K., K. Hayhoe, and D. J. Wuebbles, 2009: Assessing General Circulation Model simulations of atmospheric teleconnection patterns. J. Clim., 22, 43484372.

Stott, P. A., and C. E. Forest, 2007: Ensemble climate predictions using climate models and observational constraints. Philos. R. Soc. London A, 365, 2029-2052.

Strachan, J., P. L. Vidale, K. Hodges, M. Roberts, and M.-E. Demory, 2013: Investigating global tropical cyclone activity with a hierarchy of AGCMs: The role of model resolution. J. Clim., 26, 133-152.

Strassmann, K. M., F. Joos, and G. Fischer, 2008: Simulating effects of land use changes on carbon fluxes: Past contributions to atmospheric $\mathrm{CO}_{2}$ increases and future commitments due to losses of terrestrial sink capacity. Tellus $B, 60$, 583-603.

Stratton, R. A., and A. J. Stirling, 2012: Improving the diurnal cycle of convection in GCMs. Q. J. R. Meteorol. Soc., 138, 1121-1134.

Stroeve, J., M. Holland, W. Meier, T. Scambos, and M. Serreze, 2007: Arctic sea ice decline: Faster than forecast. Geophys. Res. Lett., 34, L09501.

Stroeve, J. C., V. Kattsov, A. Barrett, M. Serreze, T. Pavlova, M. Holland, and W. N. Meier, 2012: Trends in Arctic sea ice extent from CMIP5, CMIP3 and observations. Geophys. Res. Lett., 39, L16502.

$\mathrm{Su}, \mathrm{H}$. , and J. H. Jiang, 2012: Tropical clouds and circulation changes during the 2006-07 and 2009-10 El Niños. J. Clim., doi:10.1175/JCLI-D-1200152.1.

Su, H., D. E. Waliser, J. H. Jiang, J. L. Li, W. G. Read, J. W. Waters, and A. M. Tompkins, 2006: Relationships of upper tropospheric water vapor, clouds and SST: MLS observations, ECMWF analyses and GCM simulations. Geophys. Res. Lett., 33, L22802

$\mathrm{Su}, \mathrm{H}$., et al. , 2012: Diagnosis of regime-dependent cloud simulation errors in CMIP5 models using "A-Train" satellite observations and reanalysis data. J. Geophys. Res., doi:10.1029/2012JD018575.

Sudo, K., M. Takahashi, J. Kurokawa, and H. Akimoto, 2002: CHASER: A global chemical model of the troposphere - 1. Model description. J. Geophys. Res. Atmos., 107, 4339.

Suh, M., S. Oh, D. Lee, D. Cha, S. Choi, C. Jin, and S. Hong, 2012: Development of new ensemble methods based on the performance skills of regional climate models over South Korea. J. Clim., 25, 7067-7082.

Sun, D.-Z., Y. Yu, and T. Zhang, 2009: Tropical water vapor and cloud feedbacks in climate models: A further assessment using coupled simulations. J. Clim., 22, 1287-1304.

Sutton, R. T., B. W. Dong, and J. M. Gregory, 2007: Land/sea warming ratio in response to climate change: IPCC AR4 model results and comparison with observations. Geophys. Res. Lett., 34, L02701

Svensson, G., and A. Holtslag, 2009: Analysis of model results for the turning of the wind and related momentum fluxes in the stable boundary layer. BoundaryLayer Meteorol., 132, 261-277. 
Svensson, G., et al., 2011: Evaluation of the diurnal cycle in the atmospheric boundary layer over land as represented by a variety of single-column models: The Second GABLS Experiment. Boundary-Layer Meteorol., 140, 177-206.

Swart, N. C., and J. C. Fyfe, 2012a: Ocean carbon uptake and storage influenced by wind bias in global climate models. Nature Clim. Change, 2, 47-52.

Swart, N. C., and J. C. Fyfe, 2012b: Observed and simulated changes in the Southern Hemisphere surface westerly wind-stress. Geophys. Res. Lett., doi:10.1029/2012GL052810.

Tachiiri, K., J. C. Hargreaves, J. D. Annan, A. Oka, A. Abe-Ouchi, and M. Kawamiya, 2010: Development of a system emulating the global carbon cycle in Earth system models. Geophys. Model Dev., 3, 365-376.

Takahashi, T., et al., 2009: Climatological mean and decadal change in surface ocean pCO(2), and net sea-air $\mathrm{CO}_{2}$ flux over the global oceans. Deep-Sea Res. Pt., 56, 554-577.

Takata, K., S. Emori, and T. Watanabe, 2003: Development of the minimal advanced treatments of surface interaction and runoff. Global Planet. Change, 38, 209222.

Takemura, T., T. Nakajima, O. Dubovik, B. N. Holben, and S. Kinne, 2002: Singlescattering albedo and radiative forcing of various aerosol species with a global three-dimensional model. J. Clim., 15, 333-352.

Takemura, T., T. Nozawa, S. Emori, T. Y. Nakajima, and T. Nakajima, 2005: Simulation of climate response to aerosol direct and indirect effects with aerosol transportradiation model. J. Geophys. Res. Atmos., 110, D02202.

Takemura, T., H. Okamoto, Y. Maruyama, A. Numaguti, A. Higurashi, and T. Nakajima, 2000: Global three-dimensional simulation of aerosol optical thickness distribution of various origins. J. Geophys. Res. Atmos., 105, 17853-17873.

Takemura, T., M. Egashira, K. Matsuzawa, H. Ichijo, R. O'Ishi, and A. Abe-Ouchi, 2009: A simulation of the global distribution and radiative forcing of soil dust aerosols at the Last Glacial Maximum. Atmos. Chem. Phys., 9, 3061-3073.

Takle, E. S., et al., 2007: Transferability intercomparison—An opportunity for new insight on the global water cycle and energy budget. Bull. Am. Meteorol. Soc., $88,375-384$.

Taylor, C. M., R. A. M. de Jeu, F. Guichard, P. P. Harris, and W. A. Dorigo, 2012a: Afternoon rain more likely over drier soils. Nature, 489, 423-426.

Taylor, C. M., A. Gounou, F. Guichard, P. P. Harris, R. J. Ellis, F. Couvreux, and M. De Kauwe, 2011: Frequency of Sahelian storm initiation enhanced over mesoscale soil-moisture patterns. Nature Geosci., 4, 430-433.

Taylor, K. E., R. J. Stouffer, and G. A. Meehl, 2012b: An overview of CMIP5 and the experiment design. Bull. Am. Meteorol. Soc., 93, 485-498.

Tebaldi, C., and R. Knutti, 2007: The use of the multi-model ensemble in probabilistic climate projections. Philos. Trans. R. Soc. London A, 365 2053-2075.

Teixeira, J., et al., 2008: Parameterization of the atmospheric boundary layer. Bull. Am. Meteorol. Soc., 89, 453-458.

Teixeira, J., et al., 2011: Tropical and subtropical cloud transitions in weather and climate prediction models: The GCSS/WGNE Pacific Cross-Section Intercomparison (GPCI). J. Clim., 24, 5223-5256.

Terray, L., 2012: Evidence for multiple drivers of North Atlantic multi-decadal climate variability. Geophys. Res. Lett., 39, L19712.

Terray, L., L. Corre, S. Cravatte, T. Delcroix, G. Reverdin, and A. Ribes, 2012: Nearsurface salinity as nature's rain gauge to detect human influence on the tropical water cycle. J. Clim., 25, 958-977.

Teutschbein, C., F. Wetterhall, and J. Seibert, 2011: Evaluation of different downscaling techniques for hydrological climate-change impact studies at the catchment scale. Clim. Dyn., 37, 2087-2105.

Textor, C., et al., 2007: The effect of harmonized emissions on aerosol properties in global models—an AeroCom experiment. Atmos. Chem. Phys., 7, 4489-4501.

Thorndike, A. S., D. A. Rothrock, G. A. Maykut, and R. Colony, 1975: Thickness distribution of sea ice J. Geophys. Res. Oceans Atmos., 80, 4501-4513.

Thorne, P. W., et al., 2011: A quantification of uncertainties in historical tropical tropospheric temperature trends from radiosondes. J. Geophys. Res., 116, D12116.

Thornton, P. E., J. F. Lamarque, N. A. Rosenbloom, and N. M. Mahowald, 2007: Influence of carbon-nitrogen cycle coupling on land model response to $\mathrm{CO}_{2}$ fertilization and climate variability. Global Biogeochem. Cycles, 21, GB4018.

Tian, B., E. J. Fetzer, B. H. Kahn, J. Teixeira, E. Manning, and T. Hearty, 2013: Evaluating CMIP5 models using AIRS tropospheric air temperature and specific humidity climatology. J. Geophys. Res. Atmos., 118, 114-134.

Timbal, B., and D. Jones, 2008: Future projections of winter rainfall in southeast Australia using a statistical downscaling technique. Clim. Change, 86, 165-187.
Timmermann, A., S. Lorenz, S.-I. An, A. Clement, and S.-P. Xie, 2007: The effect of orbital forcing on the mean climate and variability of the tropical Pacific. J. Clim., 20, 4147-4159.

Timmermann, R., H. Goosse, G. Madec, T. Fichefet, C. Ethe, and V. Duliere, 2005: On the representation of high latitude processes in the ORCA-LIM global coupled sea ice-ocean model. Ocean Model., 8, 175-201.

Ting, M., Y. Kushnir, R. Seager, and C. Li, 2009: Forced and internal twentieth-century SST trends in the north Atlantic. J. Clim., 22, 1469-1481.

Tjernstrom, M., J. Sedlar, and M. Shupe, 2008: How well do regional climate models reproduce radiation and clouds in the Arctic? An evaluation of ARCMIP simulations. J. Appl. Meteorol. Climatol., 47, 2405-2422.

Tjiputra, J. F., K. Assmann, M. Bentsen, I. Bethke, O. H. Ottera, C. Sturm, and C. Heinze, 2010: Bergen Earth system model (BCM-C): Model description and regional climate-carbon cycle feedbacks assessment. Geophys. Model Dev., 3, 123-141.

Tjiputra, J. F., et al., 2013: Evaluation of the carbon cycle components inthe Norwegian Earth System Model (NorESM). Geophys. Model Dev., 6, 301-325.

Todd-Brown, K. E. O., J. T. Randerson, W. M. Post, F. M. Hoffman, C. Tarnocai, E. A. G. Schuur, and S. D. Allison, 2013: Causes of variation in soil carbon simulations from CMIP5 Earth system models and comparison with observations. Biogeosciences, 10, 1717-1736.

Toniazzo, T., and S. Woolnough, 2013: Development of warm SST errors in the southern tropical Atlantic in CMIP5 decadal hindcasts. Clim. Dyn., doi:10.1007/ s00382-013-1691-2.

Tory, K., S. Chand, R. Dare, and J. McBride, 2013: An assessment of a model-, gridand basin-independent tropical cyclone detection scheme in selected CMIP3 global climate models. J. Clim., doi:10.1175/JCLI-D-12-00511.1.

Trenberth, K. E., and J. M. Caron, 2001: Estimates of meridional atmosphere and ocean heat transports. J. Clim., 14, 3433-3443.

Trenberth, K. E., and J. T. Fasullo, 2008: An observational estimate of inferred ocean energy divergence. J. Phys. Oceanogr., 38, 984-999.

Trenberth, K. E., and J. T. Fasullo, 2009: Global warming due to increasing absorbed solar radiation. Geophys. Res. Lett., 36, L07706.

Trenberth, K. E., and J. T. Fasullo, 2010a: Climate change: Tracking Earth's energy. Science, 328, 316-317.

Trenberth, K. E., and J. T. Fasullo, 2010b: Simulation of present-day and twenty-firstcentury energy budgets of the Southern Oceans. J. Clim., 23, 440-454.

Trenberth, K. E., D. P. Stepaniak, and J. M. Caron, 2000: The global monsoon as seen through the divergent atmospheric circulation. J. Clim., 13, 3969-3993.

Trenberth, K. E., J. T. Fasullo, and J. Kiehl, 2009: Earth's global energy budget. Bull. Am. Meteorol. Soc., 90, 311-323.

Tryhorn, L., and A. DeGaetano, 2011: A comparison of techniques for downscaling extreme precipitation over the northeastern United States. Int. J. Climatol. , 31, 1975-1989.

Tschumi, T., F. Joos, and P. Parekh, 2008: How important are Southern Hemisphere wind changes for low glacial carbon dioxide? A model study. Paleoceanography, 23, PA4208.

Tschumi, T., F. Joos, M. Gehlen, and C. Heinze, 2011: Deep ocean ventilation, carbon isotopes, marine sedimentation and the deglacial $\mathrm{CO}(2)$ rise. Clim. Past, 7, $771-800$.

Tsigaridis, K., and M. Kanakidou, 2007: Secondary organic aerosol importance in the future atmosphere. Atmos. Environ., 41, 4682-4692.

Tsujino, H., M. Hirabara, H. Nakano, T. Yasuda, T. Motoi, and G. Yamanaka, 2011: Simulating present climate of the global ocean-ice system using the Meteorological Research Institute Community Ocean Model (MRI. COM): Simulation characteristics and variability in the Pacific sector. J. Oceanogr., 67 449-479.

Tsushima, Y., M. Ringer, M. Webb, and K. Williams, 2013: Quantitative evaluation of the seasonal variations in climate model cloud regimes. Clim. Dyn., doi:10.1007/ s00382-012-1609-4.

Turner, A. G., and H. Annamalai, 2012: Climate change and the south Asian summer monsoon. Nature Clim. Change, 2, 1-9.

Turner, A. G., P. M. Inness, and J. M. Slingo, 2007: The effect of doubled $\mathrm{CO}_{2}$ and model basic state biases on the monsoon-ENSO system. II: Changing ENSO regimes. Q. J. R. Meteorol. Soc., 133, 1159-1173.

Ulbrich, U., G. C. Leckebusch, and J. G. Pinto, 2009: Extra-tropical cyclones in the present and future climate: a review. Theor. Appl. Climatol., 96, 117-131.

Ulbrich, U., J. G. Pinto, H. Kupfer, G. C. Leckebusch, T. Spangehl, and M. Reyers, 2008: Changing northern hemisphere storm tracks in an ensemble of IPCC climate change simulations. J. Clim., 21, 1669-1679. 
UNESCO, 1981: Tenth report of the joint panel on oceanographic tables and standards UNESCO.

Uotila, P., S. O'Farrell, S. J. Marsland, and D. Bi, 2012: A sea-ice sensitivity study with a global ocean-ice model. Ocean Model., 51, 1-18.

Uotila, P., S. O'Farrell, S. J. Marsland, and D. Bi, 2013: The sea-ice performance of the Australian climate models participating in the CMIP5. Aust. Meteorol. Oceanogr. J., 63, 121-143.

Uppala, S. M., et al., 2005: The ERA-40 re-analysis. Q. J. R. Meteorol. Soc., 131, 2961-3012.

van den Hurk, B., and E. van Meijgaard, 2010: Diagnosing land-atmosphere interaction from a regional climate model simulation over West Africa. J. Hydrometeorol., 11, 467-481.

van Oldenborgh, G., et al., 2009: Western Europe is warming much faster than expected. Clim. Past, 5, 1-12.

van Oldenborgh, G. J., S. Y. Philip, and M. Collins, 2005: El Niño in a changing climate: A multi-model study. Ocean Sci., 1, 81-95.

van Roosmalen, L., J. H. Christensen, M. B. Butts, K. H. Jensen, and J. C. Refsgaard, 2010: An intercomparison of regional climate model data for hydrological impact studies in Denmark. J. Hydrol., 380, 406-419.

van Vliet, M., S. Blenkinsop, A. Burton, C. Harpham, H. Broers, and H. Fowler, 2011: A multi-model ensemble of downscaled spatial climate change scenarios for the Dommel catchment, Western Europe. Clim. Change, 111, 249-277.

van Vuuren, D., et al., 2011: The representative concentration pathways: An overview. Clim. Change, 109, 5-31.

Vancoppenolle, M., T. Fichefet, H. Goosse, S. Bouillon, G. Madec, and M. A. M. Maqueda, 2009: Simulating the mass balance and salinity of Arctic and Antarctic sea ice. 1. Model description and validation. Ocean Model., 27, 33-53.

Vancoppenolle, M., H. Goosse, A. de Montety, T. Fichefet, B. Tremblay, and J. L. Tison, 2010: Interactions between brine motion, nutrients and primary production in sea ice. J. Geophys. Res., 115, C02005.

Vannière, B., E. Guilyardi, G. Madec, F. J. Doblas-Reyes, and S. Woolnough, 2011 Using seasonal hindcasts to understand the origin of the equatorial cold tongue bias in CGCMs and its impact on ENSO. Clim. Dyn., 40, 963-981.

Vautard, R., et al., 2013: The simulation of European heat waves from an ensemble of regional climate models within the EURO-CORDEX project. Clim. Dyn. doi:10.1007/s00382-013-1714-z.

Vecchi, G. A., and B. J. Soden, 2007: Global warming and the weakening of the tropical circulation. J. Clim., 20, 4316-4340.

Vecchi, G. A., K. L. Swanson, and B. J. Soden, 2008: Climate Change:Whither hurricane activity? Science, 322, 687-689.

Vecchi, G. A., B. J. Soden, A. T. Wittenberg, I. M. Held, A. Leetmaa, and M. J. Harrison 2006:Weakening of tropical Pacific atmospheric circulation due to anthropogenic forcing. Nature, 327, 216--219.

Veljovic, K., B. Rajkovic, M. J. Fennessy, E. L. Altshuler, and F. Mesinger, 2010: Regional climate modeling: Should one attempt improving on the large scales? Latera boundary condition scheme: Any impact? Meteorol. Z., 19, 237-246.

Verlinde, J., et al., 2007: The Mixed-Phase Arctic Cloud Experiment. Bull. Am. Meteorol. Soc., 88, 205-221.

Verseghy, D. L., 2000: The Canadian Land Surface Scheme (CLASS): Its history and future. Atmos. Ocean, 38, 1-13

Vial, J., and T. J. Osborn, 2012: Assessment of atmosphere-ocean general circulation model simulations of winter northern hemisphere atmospheric blocking. Clim. Dyn., 39, 95-112.

Vial, J., J.-L. Dufresne, and S. Bony, 2013: On the interpretation of inter-model spread in CMIP5 climate sensitivity estimates. Clim. Dyn., doi:10.1007/s00382-013 1725-9.

Vichi, M., S. Masina, and A. Navarra, 2007: A generalized model of pelagic biogeochemistry for the global ocean ecosystem. Part II: Numerical simulations. J. Mar. Syst., 64, 110-134.

Vichi, M., et al., 2011: Global and regional ocean carbon uptake and climate change: Sensitivity to a substantial mitigation scenario. Clim. Dyn., 37, 1929-1947.

Vizcaino, M., U. Mikolajewicz, M. Groger, E. Maier-Reimer, G. Schurgers, and A. M. E. Winguth, 2008: Long-term ice sheet-climate interactions under anthropogenic greenhouse forcing simulated with a complex Earth System Model. Clim. Dyn. 31, 665-690

Voldoire, A., et al., 2013: The CNRM-CM5.1 global climate model : Description and basic evaluation. Clim. Dyn., 40, 2091-2121.
Volodin, E. M., 2007: Atmosphere-ocean general circulation model with the carbon cycle. Izvestiya Atmos. Ocean. Phys. , 43, 298-313.

Volodin, E. M., 2008a: Relation between temperature sensitivity to doubled carbon dioxide and the distribution of clouds in current climate models. Izvestiya Atmos. Ocean. Phys., 44, 288-299.

Volodin, E. M, 2008b: Methane cycle in the INM RAS climate model. Izvestiya Atmos. Ocean. Phys., 44, 153-159.

Volodin, E. M., and V. N. Lykosov, 1998: Parametrization of heat and moisture transfer in the soil-vegetation system for use in atmospheric general circulation models: 1. Formulation and simulations based on local observational data. Izvestiya Akad. Nauk Fizik. Atmosf. Okean., 34, 453-465.

Volodin, E. M., N. A. Dianskii, and A. V. Gusev, 2010: Simulating present-day climate with the INMCM4.0 coupled model of the atmospheric and oceanic general circulations. Izvestiya Atmos. Ocean. Phys., 46, 414-431.

von Salzen, K., et al., 2013: The Canadian Fourth Generation Atmospheric Globa Climate Model (CanAM4). Part I: Representation of physical processes. Atmos. Ocean, 51, 104-125.

Vose, R. S., et al., 2012: NOAA'S merged land-ocean surface temperature analysis. Bull. Am. Meteorol. Soc., 93, 1677-1685.

Vrac, M., and P. Naveau, 2008: Stochastic downscaling of precipitation: From dry events to heavy rainfall Water Resour. Res. , 43, W07402.

Waelbroeck, C., et al., 2009: Constraints on the magnitude and patterns of ocean cooling at the Last Glacial Maximum. Nature Geosci., 2, 127-132.

Wahl, S., M. Latif, W. Park, and N. Keenlyside, 2011: On the Tropical Atlantic SST warm bias in the Kiel Climate Model. Clim. Dyn., 36, 891-906.

Waliser, D., K. W. Seo, S. Schubert, and E. Njoku, 2007: Global water cycle agreement in the climate models assessed in the IPCC AR4. Geophys. Res. Lett., 34, L16705

Waliser, D., et al., 2009a: MJO simulation diagnostics. J. Clim., 22, 3006-3030.

Waliser, D. E., J. L. F. Li, T. S. L'Ecuyer, and W. T. Chen, 2011: The impact of precipitating ice and snow on the radiation balance in global climate models. Geophys. Res. Lett., 38, L06802.

Waliser, D. E., et al., 2003: AGCM simulations of intraseasonal variability associated with the Asian summer monsoon. Clim. Dyn., 21, 423-446.

Waliser, D. E., et al., 2009b: Cloud ice: A climate model challenge with signs and expectations of progress. J. Geophys. Res., 114, D00A21.

Walsh, K., S. Lavender, E. Scoccimarro, and H. Murakami, 2013: Resolution dependence of tropical cyclone formation in CMIP3 and finer resolution models. Clim. Dyn., 40, 585-599.

Walther, A., J.-H. Jeong, G. Nikulin, C. Jones, and D. Chen, 2013: Evaluation of the warm season diurnal cycle of precipitation over Sweden simulated by the Rossby Centre regional climate model RCA3. Atmos. Res., 119, 131-139.

Wang, B., 2006: The Asian Monsoon. Springer Science+Business Media, Praxis, New York, NY, USA, 787 pp.

Wang, B., and Q. H. Ding, 2008: Global monsoon: Dominant mode of annual variation in the tropics. Dyn. Atmos. Oceans, 44, 165-183.

Wang, B., H. J. Kim, K. Kikuchi, and A. Kitoh, 2011a: Diagnostic metrics for evaluation of annual and diurnal cycles. Clim. Dyn., 37, 941-955.

Wang, B., H. Wan, Z. Z. Ji, X. Zhang, R. C. Yu, Y. Q. Yu, and H. T. Liu, 2004: Design of a new dynamical core for global atmospheric models based on some efficient numerical methods. Sci. China $A, 47,4-21$.

Wang, C., and J. Picaut, 2004: Understanding ENSO physics -A review. In: Earth's Climate: The Ocean-Atmosphere Interaction [C. Wang, S.-P. Xie and J.A. Carton (eds.)]. American Geophysical Union, Washington, DC, pp. 21-48.

Wang, H., and W. Su, 2013: Evaluating and understanding top of the atmosphere cloud radiative effects. In Intergovernmental Panel on Climate Change (IPCC) Fifth Assessment Report (AR5) Coupled Model Intercomparison Project Phase 5 (CMIP5) models using satellite observations. J. Geophys. Res. Atmos., 118, 683-699.

Wang, J., Q. Bao, N. Zeng, Y. Liu, G. Wu, and D. Ji, 2013: The Earth System Mode FGOALS-s2: Coupling a dynamic global vegetation and terrestrial carbon mode with the physical climate. Adv. Atmos. Sci., doi:10.1007/s00376-013-2169-1.

Wang, J. F., and X. B. Zhang, 2008: Downscaling and projection of winter extreme daily precipitation over North America. J. Clim., 21, 923-937.

Wang, M., and J. E. Overland, 2012: A sea ice free summer Arctic within 30 years: An update from CMIP5 models. Geophys. Res. Lett., 39, L18501.

Wang, M., J. E. Overland, and N. A. Bond, 2010: Climate projections for selected large marine ecosystems. J. Mar. Syst., 79, 258-266. 
Wang, Y., M. Notaro, Z. Liu, R. Gallimore, S. Levis, and J. E. Kutzbach, 2008: Detecting vegetation-precipitation feedbacks in mid-Holocene North Africa from two climate models. Clim. Past, 4, 59-67.

Wang, Y. P., and B. Z. Houlton, 2009: Nitrogen constraints on terrestrial carbon uptake: Implications for the global carbon-climate feedback. Geophys. Res. Lett., 36, L24403.

Wang, Y. P., et al., $2011 \mathrm{~b}$ : Diagnosing errors in a land surface model (CABLE) in the time and frequency domains. J. Geophys. Res. Biogeosci., 116, G01034.

Wania, R., I. Ross, and I. C. Prentice, 2009: Integrating peatlands and permafrost into a dynamic global vegetation model: 1 . Evaluation and sensitivity of physical land surface processes. Global Biogeochem. Cycles, 23, Gb3014.

Watanabe, M., 2008: Two regimes of the equatorial warm pool. Part I: A simple tropical climate model. J. Clim., 21, 3533-3544.

Watanabe, M., M. Chikira, Y. Imada, and M. Kimoto, 2011: Convective control of ENSO simulated in MIROC. J. Clim., 24, 543-562.

Watanabe, M., J. S. Kug, F. F. Jin, M. Collins, M. Ohba, and A. T. Wittenberg, 2012: Uncertainty in the ENSO amplitude change from the past to the future. Geophys. Res. Lett., 39, L20703.

Watanabe, M., et al., 2010: Improved climate simulation by MIROC5: Mean states, variability, and climate sensitivity. J. Clim., 23, 6312-6335.

Watanabe, S., Y. Kawatani, Y. Tomikawa, K. Miyazaki, M. Takahashi, and K. Sato, 2008: General aspects of a T213L256 middle atmosphere general circulation model. J. Geophys. Res. Atmos., 113, D12110.

Watterson, I., and P. Whetton, 2011: Distributions of decadal means of temperature and precipitation change under global warming. J. Geophys. Res. Atmos., 116, D07101.

Waugh, D., and V. Eyring, 2008: Quantitative performance metrics for stratosphericresolving chemistry-climate models. Atmos. Chem. Phys., 8, 5699-5713.

Weaver, A. J., et al., 2001: The UVic Earth System Climate Model: Model description, climatology, and applications to past, present and future climates. Atmos. Ocean, 39, 361-428.

Weaver, A. J., et al., 2012: Stability of the Atlantic meridional overturning circulation: A model intercomparison. Geophys. Res. Lett., 39, L20709.

Webb, M., C. Senior, S. Bony, and J. J. Morcrette, 2001: Combining ERBE and ISCCP data to assess clouds in the Hadley Centre, ECMWF and LMD atmospheric climate models. Clim. Dyn., 17, 905-922.

Weber, S. L., et al., 2007: The modern and glacial overturning circulation in the Atlantic Ocean in PMIP coupled model simulations. Clim. Past, 3, 51-64.

Webster, P. J., A. M. Moore, J. P. Loschnigg, and R. R. Leben, 1999: Coupled oceanatmosphere dynamics in the Indian Ocean during 1997-98. Nature, 401, 356360.

Wehner, M. F., R. L. Smith, G. Bala, and P. Duffy, 2010: The effect of horizontal resolution on simulation of very extreme US precipitation events in a global atmosphere model. Clim. Dyn., 34, 241-247.

Weller, E., and W. Cai, 2013a: Realism of the Indian Ocean Dipole in CMIP5 models: The implication for 1 climate projections. J. Clim., doi:10.1175/JCLI-D12-00807.1.

Weller, E., and W. Cai, 2013b: Asymmetry in the IOD and ENSO teleconnection in a CMIP5 model ensemble and its relevance to regional rainfall. J. Clim., doi:10.1175/JCLI-D-12-00789.1.

Wentz, F. J., L. Ricciardulli, K. Hilburn, and C. Mears, 2007: How much more rain will global warming bring? Science, 317, 233-235.

Westerling, A. L., H. G. Hidalgo, D. R. Cayan, and T. W. Swetnam, 2006: Warming and earlier spring increase western US forest wildfire activity. Science, 313, 940-943.

Wetzel, P., E. Maier-Reimer, M. Botzet, J. H. Jungclaus, N. Keenlyside, and M. Latif, 2006: Effects of ocean biology on the penetrative radiation in a Coupled Climate Model. J. Clim., 19, 3973-3987.

Whetton, P., I. Macadam, J. Bathols, and J. O'Grady, 2007: Assessment of the use of current climate patterns to evaluate regional enhanced greenhouse response patterns of climate models. Geophys. Res. Lett., 34, L14701.

White, C. J., et al., 2013: On regional dynamical downscaling for the assessment and projection of temperature and precipitation extremes across Tasmania, Australia. Clim. Dyn., doi:10.1007/s00382-013-1718-8.

Wilcox, L. J., A. J. Charlton-Perez, and L. J. Gray, 2012: Trends in Austral jet position in ensembles of high- and low-top CMIP5 models. J. Geophys. Res., 117, D13115.

Wild, M., C. N. Long, and A. Ohmura, 2006: Evaluation of clear-sky solar fluxes in GCMs participating in AMIP and IPCC-AR4 from a surface perspective. J. Geophys. Res. Atmos., 111, D01104
Wilks, D. S., 1995: Statistical Methods in the Atmospheric Sciences. Vol. 59, Academic Press, San Diego, CA, USA, 467 pp.

Wilks, D. S., 2006: Statistical Methods in the Atmospheric Sciences. Vol. 91, Academic Press, Elsevier, San Diego, CA, USA, 627 pp.

Willett, K., P. Jones, P. Thorne, and N. Gillett, 2010: A comparison of large scale changes in surface humidity over land in observations and CMIP3 general circulation models. Environ. Res. Lett., 5, 025210.

Williams, C. J. R., R. P. Allan, and D. R. Kniveton, 2012: Diagnosing atmosphere-land feedbacks in CMIP5 climate models. Environ. Res. Lett., 7, 044003.

Williams, K., and M. Webb, 2009: A quantitative performance assessment of cloud regimes in climate models. Clim. Dyn., 33 141-157.

Williams, K. D., and M. E. Brooks, 2008: Initial tendencies of cloud regimes in the Met Office unified model. J. Clim., 21, 833-840.

Williams, K. D., W. J. Ingram, and J. M. Gregory, 2008: Time variation of effective climate sensitivity in GCMs. J. Clim., 21, 5076-5090.

Williamson, D. L., and J. G. Olson, 2007: A comparison of forecast errors in CAM2 and CAM3 at the ARM Southern Great Plains site. J. Clim., 20, 4572-4585.

Williamson, M. S., T. M. Lenton, J. G. Shepherd, and N. R. Edwards, 2006: An efficient numerical terrestrial scheme (ENTS) for Earth system modelling. Ecol. Model., 198, 362-374.

Willis, J. K., 2010: Can in situ floats and satellite altimeters detect long-term changes in Atlantic Ocean overturning? Geophys. Res. Lett., 37, L06602.

Winterfeldt, J., and R. Weisse, 2009: Assessment of value added for surface marine wind speed obtained from two regional climate models. Mon. Weather Rev., 137, 2955-2965.

Winterfeldt, J., B. Geyer, and R. Weisse, 2011: Using QuikSCAT in the added value assessment of dynamically downscaled wind speed. Int. J. Climatol. , 31, 10281039.

Winton, M., 2000: A reformulated three-layer sea ice model. J. Atmos. Ocean. Technol., 17, 525-531.

Winton, M., 2011: Do climate models underestimate the sensitivity of Northern Hemisphere sea ice cover? J. Clim., 24, 3924-3934.

Wittenberg, A. T., 2009: Are historical records sufficient to constrain ENSO simulations? Geophys. Res. Lett., 36, L12702.

Wittenberg, A. T., A. Rosati, N. C. Lau, and J. J. Ploshay, 2006: GFDL's CM2 Global Coupled Climate Models. Part III: Tropical Pacific climate and ENSO. J. Clim., 19, 698-722.

WMO, 2011: Scientific Assessment of Ozone Depletion: 2010. Global Ozone Research and Monitoring Project-Report. World Meteorological Organisation, Geneva, Switzerland.

Wohlfahrt, J., et al., 2008: Evaluation of coupled ocean-atmosphere simulations of the mid-Holocene using palaeovegetation data from the northern hemisphere extratropics. Clim. Dyn., 31, 871-890.

Wood, R., et al., 2011: The VAMOS Ocean-Cloud-Atmosphere-Land Study Regional Experiment (VOCALS-REx): Goals, platforms, and field operations. Atmos. Chem. Phys., 11, 627-654.

Woollings, T., B. Hoskins, M. Blackburn, D. Hassell, and K. Hodges, 2010a: Storm track sensitivity to sea surface temperature resolution in a regional atmosphere model. Clim. Dyn., 35, 341-353.

Woollings, T., A. Charlton-Perez, S. Ineson, A. G. Marshall, and G. Masato, 2010b: Associations between stratospheric variability and tropospheric blocking. J. Geophys. Res. Atmos., 115, D06108.

Woollings, T., J. M. Gregory, J. G. Pinto, M. Reyers, and D. J. Brayshaw, 2012: Response of the North Atlantic storm track to climate change shaped by ocean-atmosphere coupling. Nature Geosci., 5, 313-317.

Wright, D. G., and T. F. Stocker, 1992: Sensitivities of a zonally averaged Global Ocean Circulation Model.. J. Geophys. Res. Oceans, 97, 12707-12730.

Wu, Q. G., D. J. Karoly, and G. R. North, 2008a: Role of water vapor feedback on the amplitude of season cycle in the global mean surface air temperature. Geophys. Res. Lett., 35, L08711.

Wu, T., 2012: A mass-flux cumulus parameterization scheme for large-scale models: Description and test with observations. Clim. Dyn., 38, 725-744.

Wu, T., R. Yu, and F. Zhang, 2008b:A modified dynamic framework for the atmospheric spectral model and its application. J. Atmos. Sci, 65, 2235-2253.

Wu, T., et al., 2010a: Erratum-The Beijing Climate Center atmospheric general circulation model: Description and its performance for the present-day climate. Clim. Dyn., 34, 149-150. 
Wu, T., et al., 2010b: The Beijing Climate Center atmospheric general circulation model: Description and its performance for the present-day climate. Clim. Dyn., 34, 123-147.

Wyant, M. C., C. S. Bretherton, and P. N. Blossey, 2009: Subtropical low cloud response to a warmer climate in a superparameterized climate model. Part I: Regime sorting and physical mechanisms. J. Adv. Model. Earth Syst., 1, 7.

Wyser, K., et al., 2008: An evaluation of Arctic cloud and radiation processes during the SHEBA year: Simulation results from eight Arctic regional climate models. Clim. Dyn., 30, 203-223.

Xavier, P. K., 2012: Intraseasonal convective moistening in CMIP3 models. J. Clim., 25, 2569-2577.

Xavier, P. K., J. P. Duvel, P. Braconnot, and F. J. Doblas-Reyes, 2010: An evaluation metric for intraseasonal variability and its application to CMIP3 twentiethcentury simulations. J. Clim., 23, 3497-3508.

Xiao, X., et al., 1998: Transient climate change and net ecosystem production of the terrestrial biosphere. Global Biogeochem. Cycles, 12, 345-360.

Xie, L., T. Z. Yan, L. J. Pietrafesa, J. M. Morrison, and T. Karl, 2005: Climatology and interannual variability of North Atlantic hurricane tracks. J. Clim., 18, 5370-5381.

Xie, P., and P. A. Arkin, 1997: Global Precipitation: A 17-year monthly analysis based on gauge observations, satellite estimates, and numerical model outputs. Bull. Am. Meteorol. Soc., 78, 2539-2558.

Xie, S., J. Boyle, S. A. Klein, X. Liu, and S. Ghan, 2008: Simulations of Arctic mixedphase clouds in forecasts with CAM3 and AM2 for M-PACE. J. Geophys. Res., 113, D04211.

Xie, S., H.-Y. Ma, J. S. Boyle, S. A. Klein, and Y. Zhang, 2012: On the correspondence between short- and long- timescale systematic errors in CAM4/CAM5 for the years of tropical convection. J. Clim., 25, 7937-7955.

Xie, S. P., H. Annamalai, F. A. Schott, and J. P. McCreary, 2002: Structure and mechanisms of South Indian Ocean climate variability. J. Clim., 15, 864-878.

Xin, X.-G., T.-J. Zhou, and R.-C. Yu, 2008: The Arctic Oscillation in coupled climate models. Chin. J. Geophys. Chinese Edition, 51, 337-351.

Xin, X., L. Zhang, J. Zhang, T. Wu, and Y. Fang, 2013: Climate change projections over East Asia with BCC_CSM1.1 climate model under RCP scenarios. J. Meteorol. Soc. Jpn., 91, 413-429.

Xin, X., T. Wu, J. Li, Z. Wang, W. Li, and F. Wu, 2012: How well does BCC_CSM1.1 reproduce the 20th century climate change over China? . Atmos. Ocean. Sci. Lett., 6, 21-26.

Xu, Y. F., Y. Huang, and Y. C. Li, 2012: Summary of recent climate change studies on the carbon and nitrogen cycles in the terrestrial ecosystem and ocean in China. Adv. Atmos. Sci. , 29, 1027-1047.

Xue, Y. K., R. Vasic, Z. Janjic, F. Mesinger, and K. E. Mitchell, 2007: Assessment of dynamic downscaling of the continental US regional climate using the Eta/SSiB regional climate model. J. Clim., 20, 4172-4193.

Yakovlev, N. G., 2009: Reproduction of the large-scale state of water and sea ice in the Arctic Ocean in 1948-2002: Part I. Numerical model. Izvestiya Atmos. Ocean. Phys., 45, 628-641.

Yang, D., and T. Ohata, 2001: A bias-corrected Siberian regional precipitation climatology. J. Hydrometeorol, 2, 122-139.

Yasutaka, W., N. Masaomi, K. Sachie, and M. Chiashi, 2008: Climatologica reproducibility evaluation and future climate projection of extreme precipitation events in the Baiu season using a high-resolution non-hydrostatic RCM in comparison with an AGCM. J. Meteorol. Soc. Jpn., 86, 951-967.

Yeager, S., and G. Danabasoglu, 2012: Sensitivity of Atlantic Meridional Overturning Circulation variability to parameterized Nordic Sea overflows in CCSM4. J. Clim. 25, 2077-2103.

Yeh, S. W., Y. G. Ham, and J. Y. Lee, 2012: Changes in the tropical Pacific SST trend from CMIP3 to CMIP5 and its implication of ENSO. J. Clim., 25, 7764-7771.

Yhang, Y. B., and S. Y. Hong, 2008: Improved physical processes in a regional climate model and their impact on the simulated summer monsoon circulations over East Asia. J. Clim., 21, 963-979.

Yin, X., A. Gruber, and P. Arkin, 2004: Comparison of the GPCP and CMAP merged gauge-satellite monthly precipitation products for the period 1979-2001. J. Hydrometeorol., 5, 1207-1222.

Yokohata, T., M. J. Webb, M. Collins, K. D. Williams, M. Yoshimori, J. C. Hargreaves, and J. D. Annan, 2010: Structural similarities and differences in climate responses to $\mathrm{CO}_{2}$ increase between two perturbed physics ensembles. J. Clim., 23, 13921410.
Yokohata, T., J. Annan, M. Collins, C. Jackson, M. Tobis, M. Webb, and J. Hargreaves, 2012: Reliability of multi-model and structurally different single-model ensembles. Clim. Dyn., 39, 599-616.

Yokohata, T., et al., 2013: Reliability and importance of structural diversity of climate model ensembles. Clim. Dyn., doi:10.1007/s00382-013-1733-9.

Yokohata, T., et al., 2008: Comparison of equilibrium and transient responses to $\mathrm{CO}_{2}$ increase in eight state-of-the-art climate models. Tellus A, 60, 946-961.

Yokoi, S., Y. N. Takayabu, and J. C. L. Chan, 2009a: Tropical cyclone genesis frequency over the western North Pacific simulated in medium-resolution coupled general circulation models. Clim. Dyn., 33, 665-683.

Yokoi, S., C. Takahashi, K. Yasunaga, and R. Shirooka, 2012: Multi-model projection of tropical cyclone genesis frequency over the western North Pacific: CMIP5 results. Sola, 8, 137-140.

Yokoi, S., et al., 2011: Application of cluster analysis to climate model performance metrics. J. Appl. Meteorol. Climatol., 50, 1666-1675.

Yokoi, T., T. Tozuka, and T. Yamagata, 2009b: Seasonal variations of the Seychelles Dome simulated in the CMIP3 models. J. Phys. Oceanogr., 39, 449-457.

Yoshimori, M., T. Yokohata, and A. Abe-Ouchi, 2009: A comparison of climate feedback strength between $\mathrm{CO}_{2}$ doubling and LGM experiments. J. Clim., 22, 3374-3395.

Yoshimori, M., J. C. Hargreaves, J. D. Annan, T. Yokohata, and A. Abe-Ouchi, 2011: Dependency of feedbacks on forcing and climate state in physics parameter ensembles. J. Clim., 24, 6440-6455.

Young, P. J., et al., 2013: Pre-industrial to end 21st century projections of tropospheric ozone from the Atmospheric Chemistry and Climate Model Intercomparison Project (ACCMIP). Atmos. Chem. Phys., 13, 2063-2090.

Yu, B., and F. W. Zwiers, 2010: Changes in equatorial atmospheric zonal circulations in recent decades. Geophys. Res. Lett., 37, L05701.

Yu, J.-Y., and S. T. Kim, 2011: Reversed spatial asymmetries between El Niño and La Niña and their linkage to decadal ENSO modulation in CMIP3 models. J. Clim., 24, 5423-5434.

Yu, W., M. Doutriaux, G. Seze, H. LeTreut, and M. Desbois, 1996: A methodology study of the validation of clouds in GCMs using ISCCP satellite observations. Clim. Dyn., 12, 389-401.

Yukimoto, S., et al., 2011: Meteorological Research Institute-Earth System Model v1 (MRI-ESM1) - Model Description. Technical Report of MRI. Ibaraki, Japan, 88 pp.

Yukimoto, S., et al., 2012: A new global climate model of the Meteorological Research Institute: MRI-CGCM3-Model description and basic performance. J. Meteorol. Soc. Jpn., 90A, 23-64.

Zaehle, S., and D. Dalmonech, 2011: Carbon-nitrogen interactions on land at global scales: Current understanding in modelling climate biosphere feedbacks. Curr. Opin. Environ. Sustain., 3, 311-320.

Zaehle, S., P. Friedlingstein, and A. D. Friend, 2010: Terrestrial nitrogen feedbacks may accelerate future climate change. Geophys. Res. Lett., 37, L01401.

Zahn, M., and H. von Storch, 2008: A long-term climatology of North Atlantic polar lows. Geophys. Res. Lett., 35, L22702.

Zalesny, V. B., et al., 2010: Numerical simulation of large-scale ocean circulation based on the multicomponent splitting method. Russ. J. Numer. Anal. Math. Model., 25, 581-609.

Zaliapin, I., and M. Ghil, 2010: Another look at climate sensitivity. Nonlin. Proc. Geophys., 17, 113-122.

Zappa, G., L. C. Shaffrey, and K. I. Hodges, 2013: The ability of CMIP5 models to simulate North Atlantic extratropical cyclones. J. Clim., doi:10.1175/JCLI-D-1200501.1.

Zeng, N., 2003: Glacial-interglacial atmospheric $\mathrm{CO}_{2}$ change-The glacial burial hypothesis. Adv. Atmos. Sci., 20, 677-693.

Zeng, N., 2006: Quasi-100ky glacial-interglacial cycles triggered by subglacial burial carbon release. Clim. Past, 2, 371-397.

Zeng, N., J. D. Neelin, and C. Chou, 2000: A quasi-equilibrium tropical circulation model-Implementation and simulation. J. Atmos. Sci., 57, 1767-1796.

Zeng, N., A. Mariotti, and P. Wetzel, 2005: Terrestrial mechanisms of interannual $\mathrm{CO}_{2}$ variability. Global Biogeochem. Cycles, 19, GB1016.

Zeng, N., H. F. Qian, E. Munoz, and R. lacono, 2004: How strong is carbon cycleclimate feedback under global warming? Geophys. Res. Lett., 31, L20203.

Zhang, F., and A. Georgakakos, 2011: Joint variable spatial downscaling. Clim. Change, 111, 945-972

Zhang, J., and J. S. Reid, 2010: A decadal regional and global trend analysis of the aerosol optical depth using a data-assimilation grade over-water MODIS and Level 2 MISR aerosol products. Atmos. Chem. Phys., 10, 10949-10963. 
Zhang, J., J. S. Reid, D. L. Westphal, N. L. Baker, and E. J. Hyer, 2008a: A system for operational aerosol optical depth data assimilation over global oceans. J. Geophys. Res. Atmos., 113, D10208.

Zhang, Q., H. S. Sundqvist, A. Moberg, H. Kornich, J. Nilsson, and K. Holmgren, 2010a: Climate change between the mid and late Holocene in northern high latitudesPart 2: Model-data comparisons. Clim. Past, 6, 609-626.

Zhang, R., et al., 2013: Have aerosols caused the observed Atlantic multidecadal variability? J. Atmos. Sci, doi:10.1175/JAS-D-12-0331.1.

Zhang, W., and F.-F. Jin, 2012: Improvements in the CMIP5 simulations of ENSO-SSTA meridional width. Geophys. Res. Lett., 39, L23704.

Zhang, X., 2007: A comparison of explicit and implicit spatial downscaling of GCM output for soil erosion and crop production assessments. Clim. Change, 84, 337-363.

Zhang, X., 2010: Sensitivity of arctic summer sea ice coverage to global warming forcing: towards reducing uncertainty in arctic climate change projections. Tellus A, 62, 220-227.

Zhang, X., et al., 2007: Detection of human influence on twentieth-century precipitation trends. Nature, 448, 461-465.

Zhang, Y., S. A. Klein, J. Boyle, and G. G. Mace, 2010b: Evaluation of tropical cloud and precipitation statistics of Community Atmosphere Model version 3 using CloudSat and CALIPSO data. J. Geophys. Res., 115, D12205.

Zhang, Y., et al., 2008b: On the diurnal cycle of deep convection, high-level cloud, and upper troposphere water vapor in the Multiscale Modeling Framework. J. Geophys. Res., 113, D16105.

Zhao, M., I. M. Held, and S.-J. Lin, 2012: Some counterintuitive dependencies of tropical cyclone frequency on parameters in a GCM. J. Atmos. Sci., 69, 22722283.

Zhao, M., I. M. Held, S. J. Lin, and G. A. Vecchi, 2009: Simulations of global hurricane climatology, interannual variability, and response to global warming using a 50-km resolution GCM. J. Clim., 22, 6653-6678.

Zhao, T. L., et al., 2008: A three-dimensional model study on the production of $\mathrm{BrO}$ and Arctic boundary layer ozone depletion. J. Geophys. Res., 113, D24304.

Zhao, Y., and S. P. Harrison, 2012: Mid-Holocene monsoons: A multi-model analysis of the inter-hemispheric differences in the responses to orbital forcing and ocean feedbacks. Clim. Dyn., 39, 1457-1487.

Zheng, W. P., and P. Braconnot, 2013: Characterization of model spread in PMIP2 Mid-Holocene simulations of the African monsoon. J. Clim., 26, 1192-1210.

Zheng, X.-T., S.-P. Xie, and Q. Liu, 2011: Response of the Indian Ocean basin mode and its capacitor effect to global warming. J. Clim., 24, 6146-6164.

Zheng, Y., J.-L. Lin, and T. Shinoda, 2012: The equatorial Pacific cold tongue simulated by IPCC AR4 coupled GCMs: Upper ocean heat budget and feedback analysis. J. Geophys. Res. Oceans, 117, C05024.

Zickfeld, K., et al., 2013: Long-term climate change commitment and reversibility:An EMIC intercomparison. J. Clim., doi:10.1175/JCLI-D-12-00584.1.

Zou, L., and T. Zhou, 2013: Can a regional ocean-atmosphere coupled model improve the simulation of the interannual variability of the western North Pacific summer monsoon? J. Clim., 26, 2353-2367.

Zunz, V., H. Goosse, and F. Massonnet, 2013: How does internal variability influence the ability of CMIP5 models to reproduce the recent trend in Southern Ocean sea ice extent? Cryosphere, 7, 451-468.

Zwiers, F. W., X. Zhang, and Y. Feng, 2011: Anthropogenic influence on long return period daily temperature extremes at regional scales. J. Clim., 24, 881-892. 


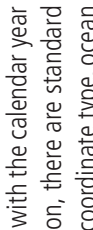

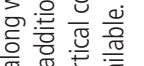
要

空

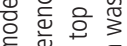

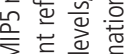

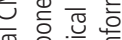

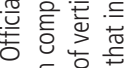

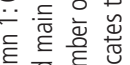

흥 믕 흘

空㖺言

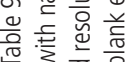

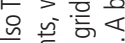

要类

\begin{tabular}{|c|c|c|c|c|}
\hline 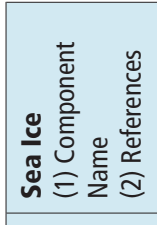 & 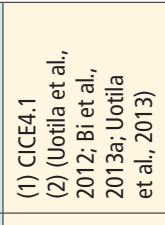 & 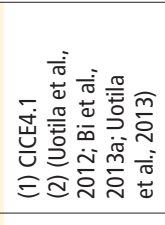 & 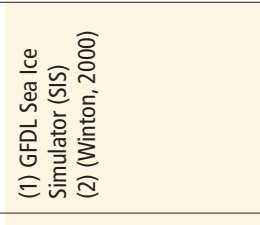 & 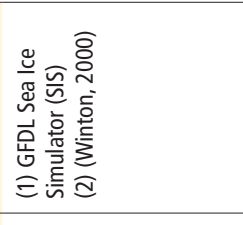 \\
\hline 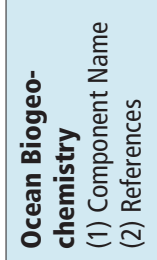 & 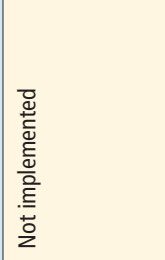 & 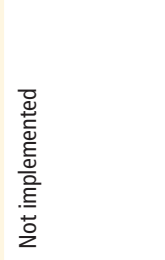 & 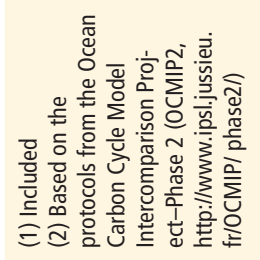 & 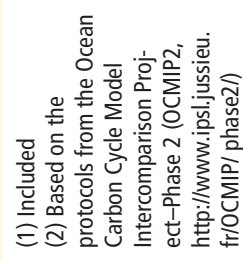 \\
\hline
\end{tabular}

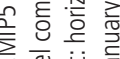

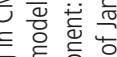

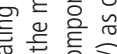

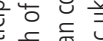

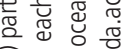

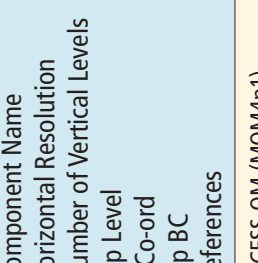

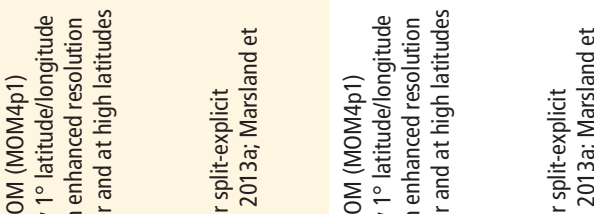

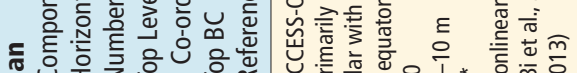

ฐ

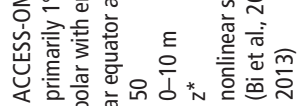

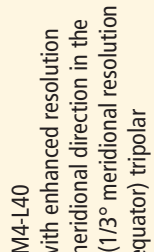

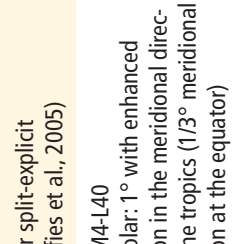

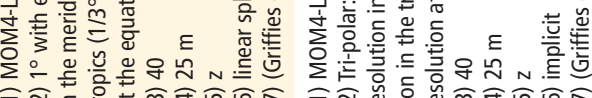

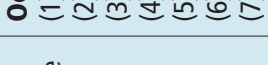

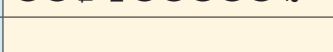

\section{(1)}

为

\begin{tabular}{|c|c|}
\hline 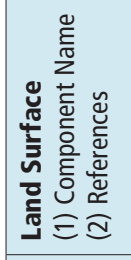 & 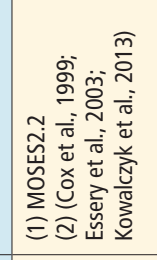 \\
\hline 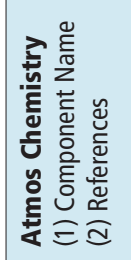 & 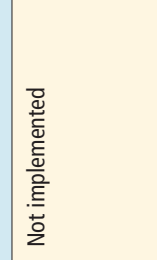 \\
\hline
\end{tabular}

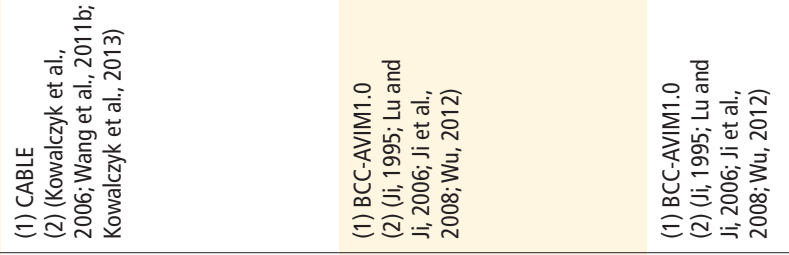

인

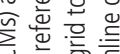

\begin{tabular}{|c|c|}
\hline 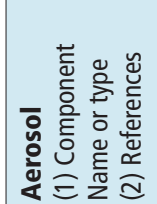 & 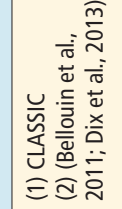 \\
\hline
\end{tabular}

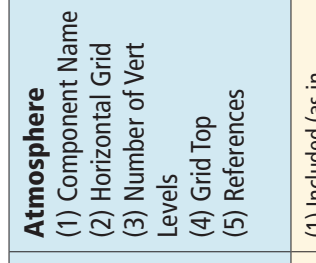

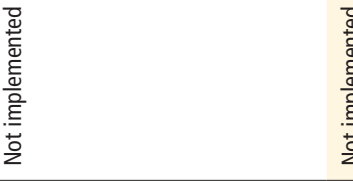

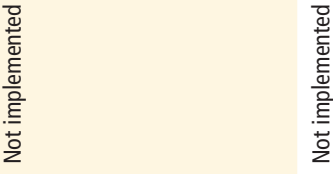

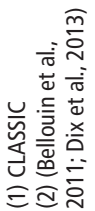

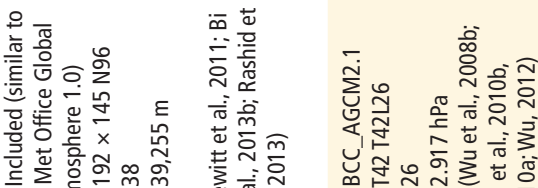

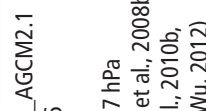

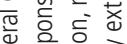

过

둥 들

응 은

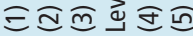

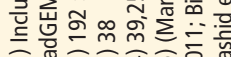

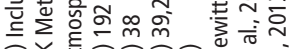

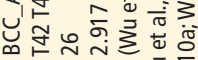

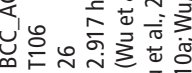

\begin{tabular}{|c|c|c|c|c|}
\hline 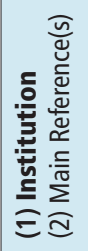 & 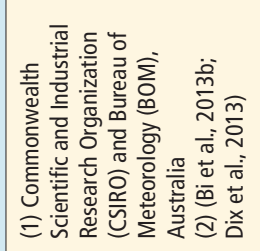 & 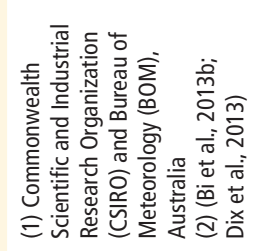 & 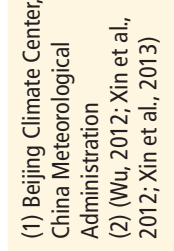 & 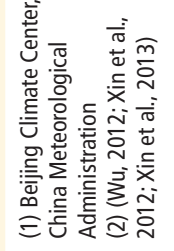 \\
\hline 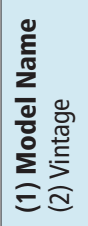 & 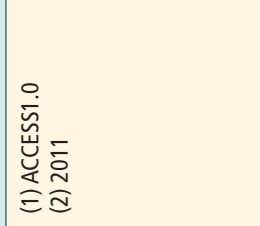 & 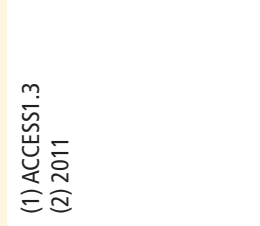 & 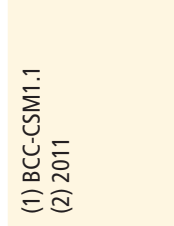 & 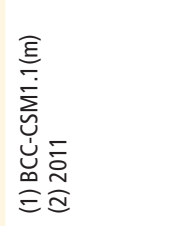 \\
\hline
\end{tabular}




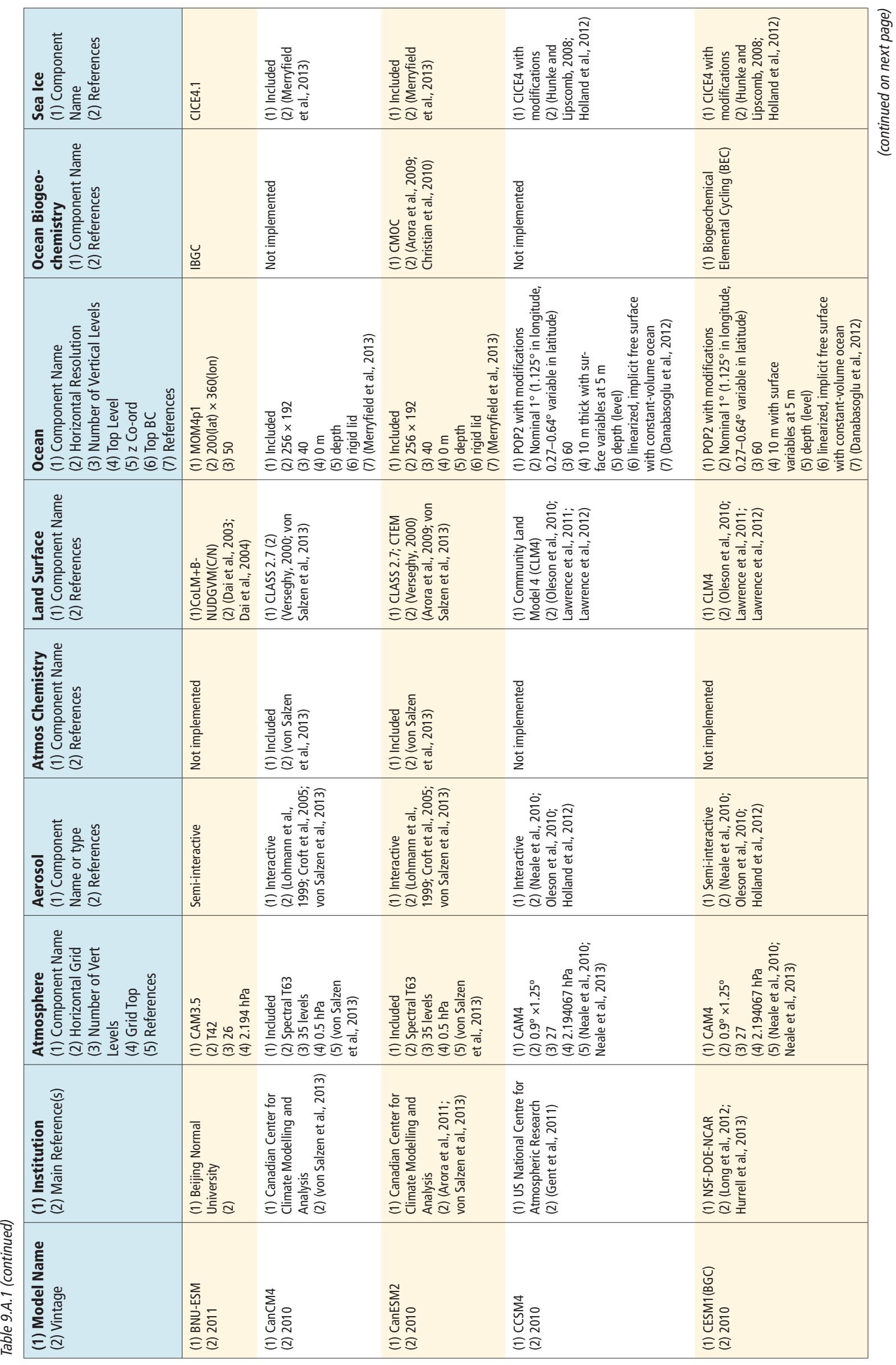



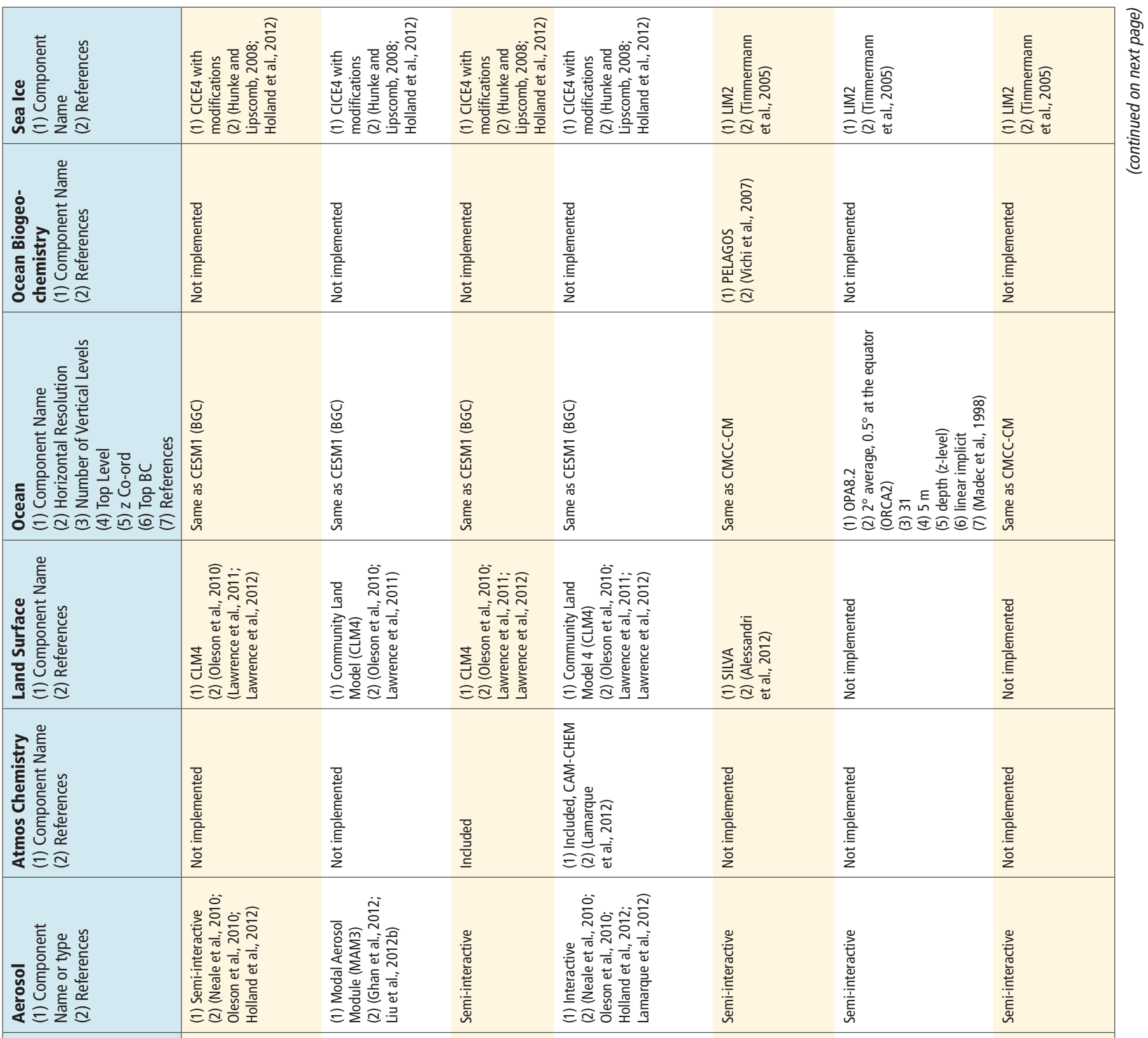

\begin{tabular}{|c|c|c|c|c|c|c|c|}
\hline 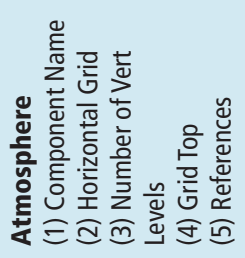 & 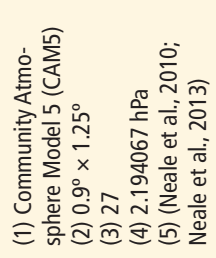 & 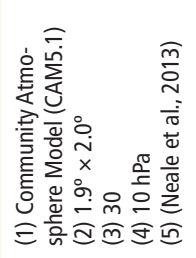 & 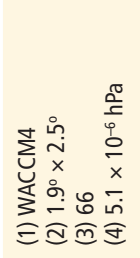 & 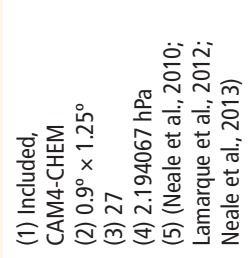 & 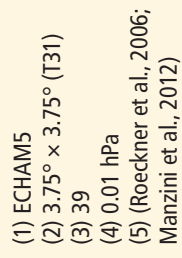 & 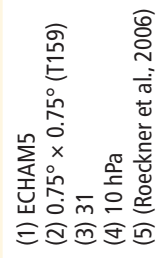 & 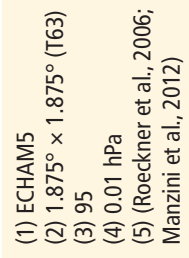 \\
\hline 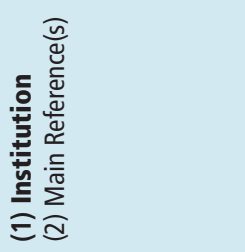 & 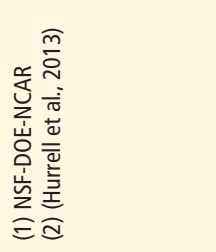 & 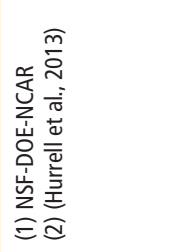 & 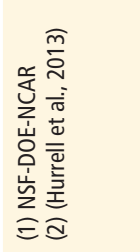 & 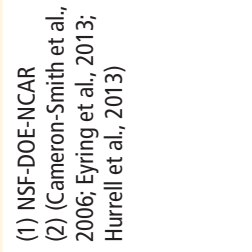 & 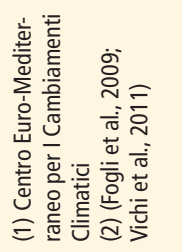 & 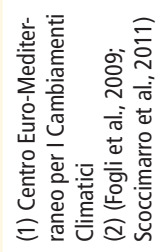 & 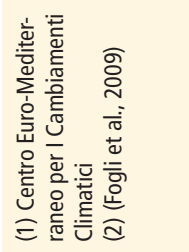 \\
\hline 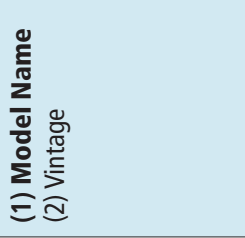 & 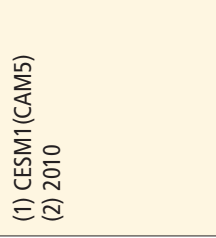 & 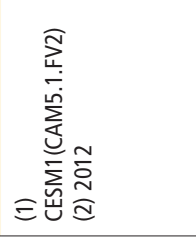 & 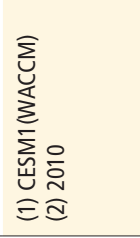 & 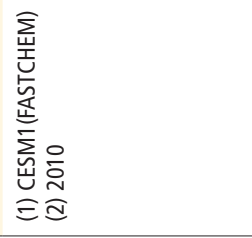 & 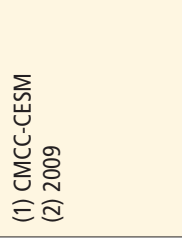 & 总 & 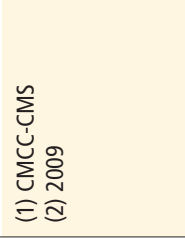 \\
\hline
\end{tabular}




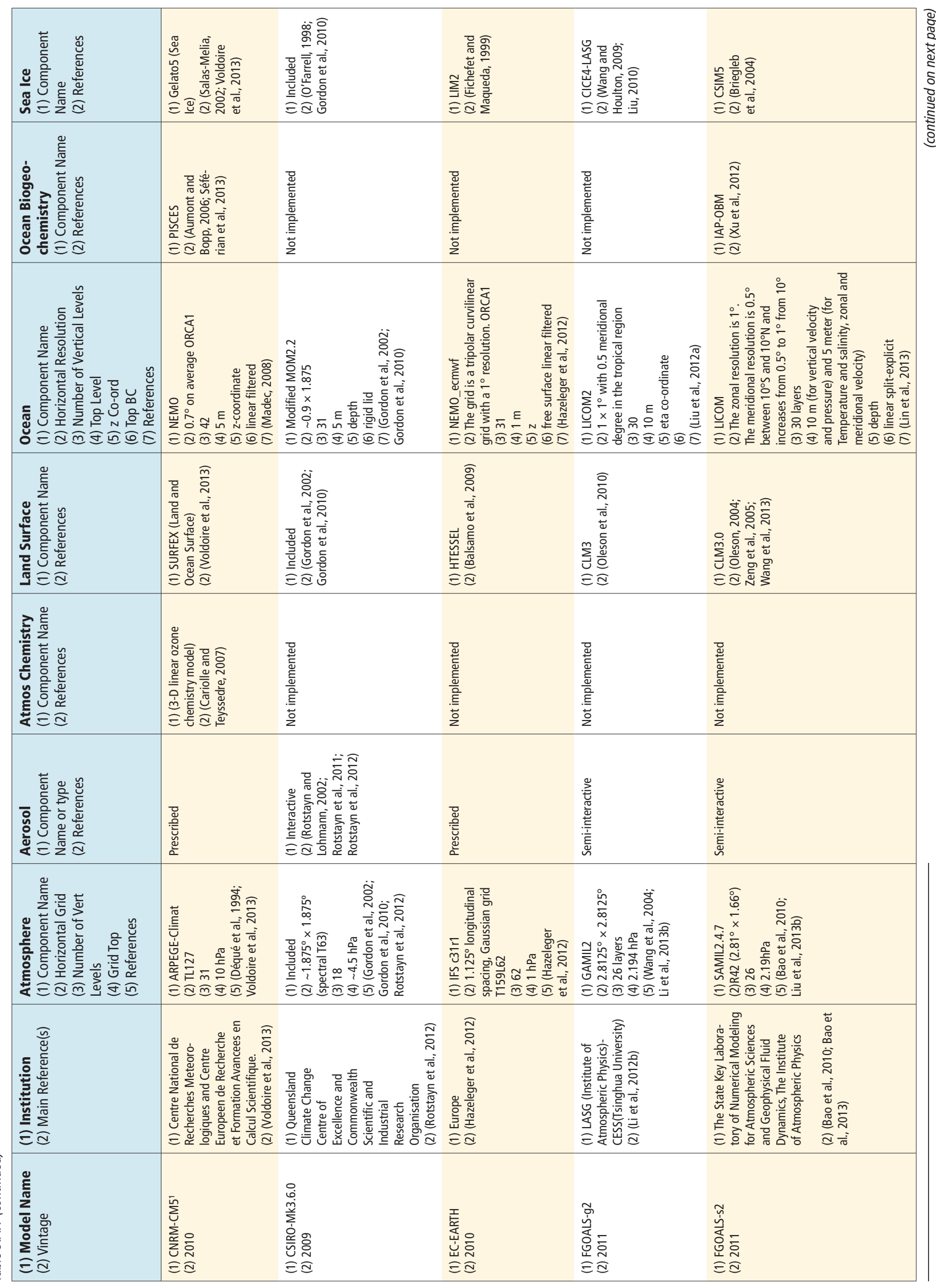




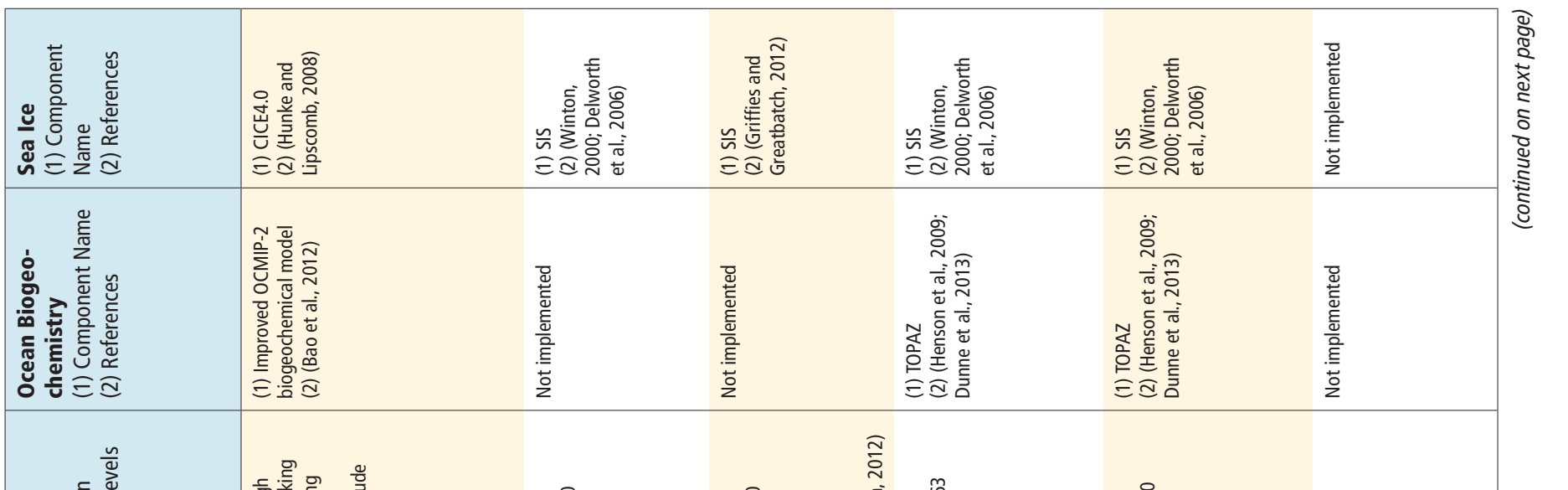

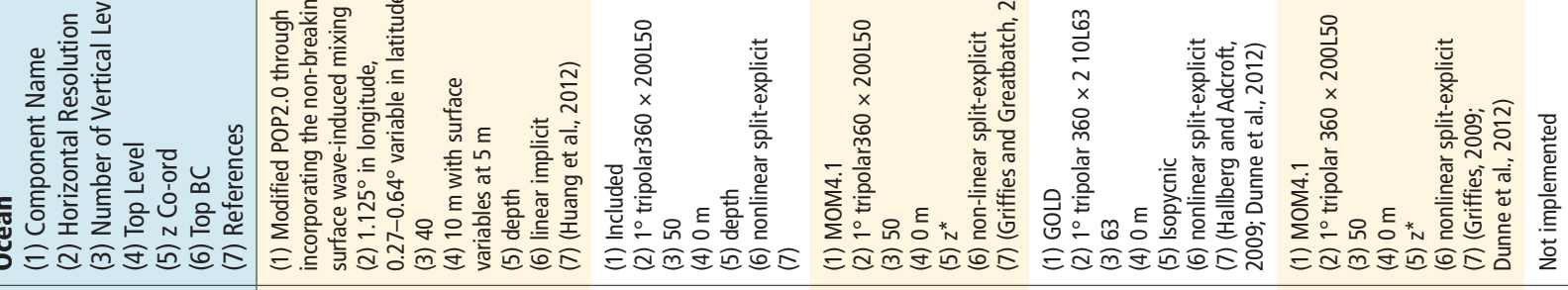
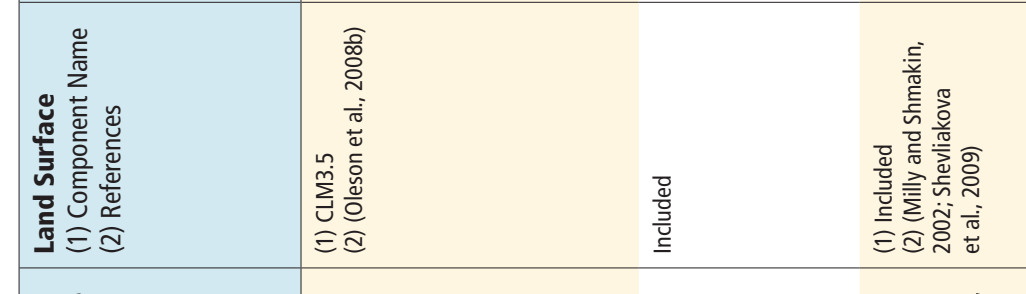

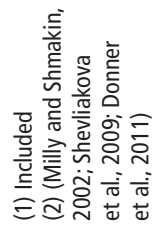

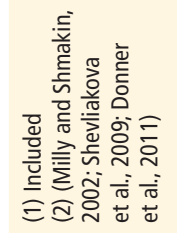
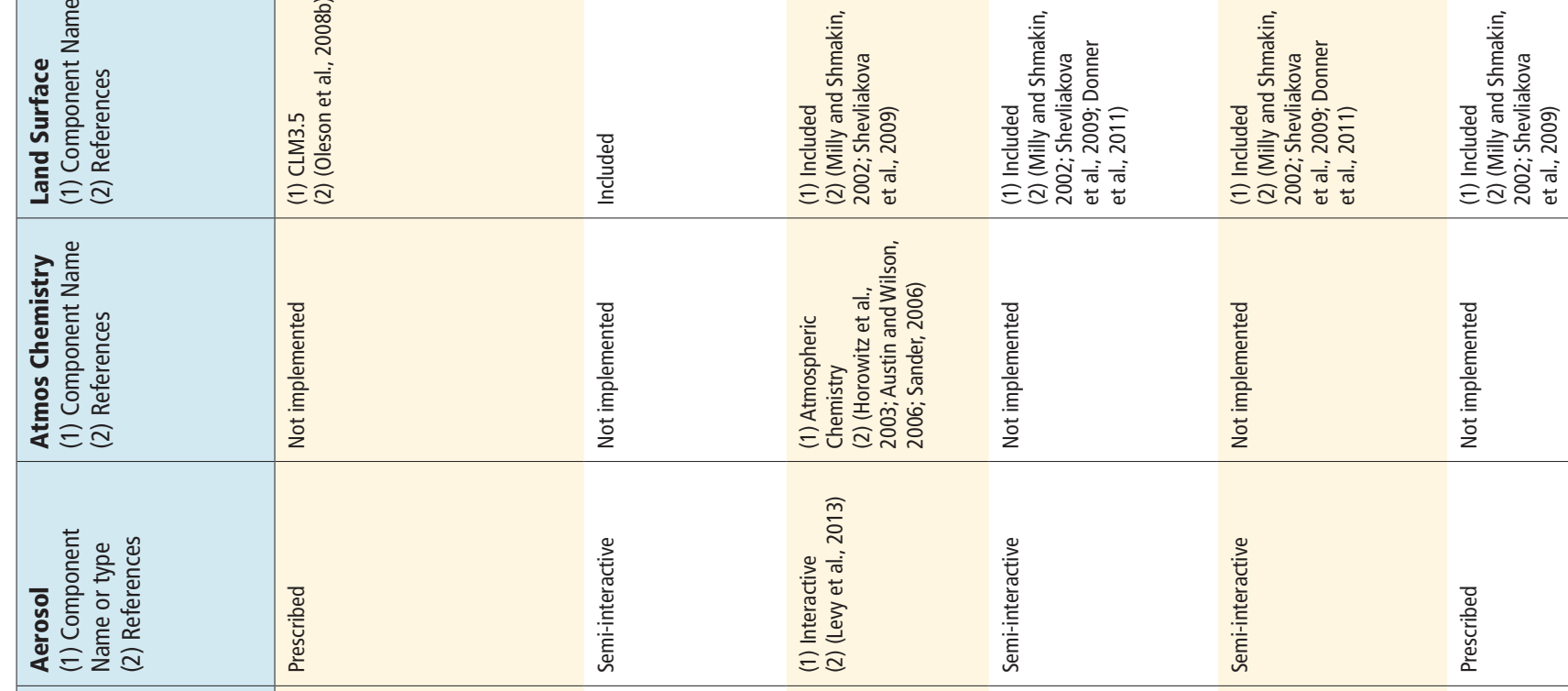

总

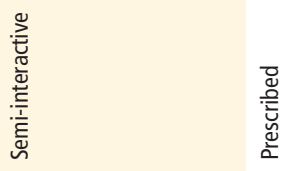

\begin{tabular}{|c|c|c|c|c|c|c|}
\hline 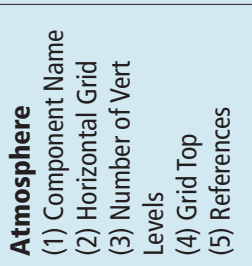 & 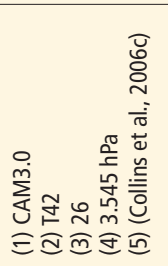 & 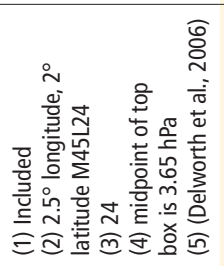 & 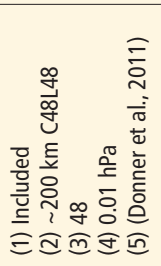 & 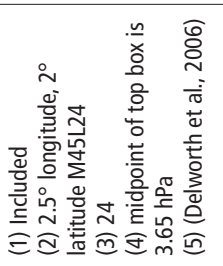 & 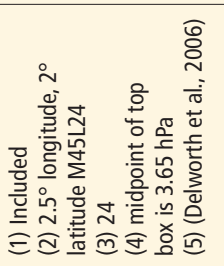 & 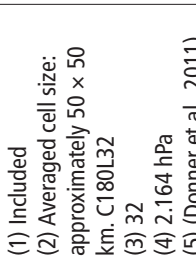 \\
\hline 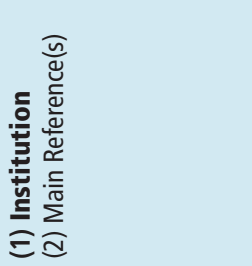 & 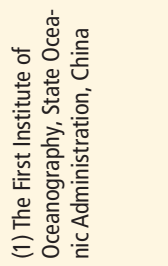 & 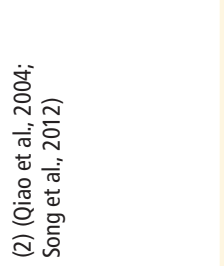 & 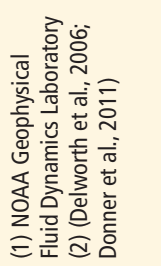 & 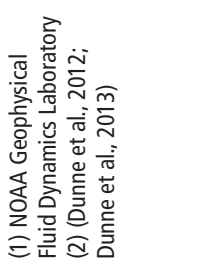 & 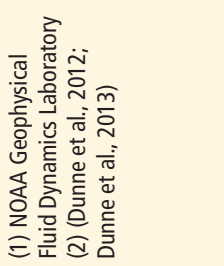 & 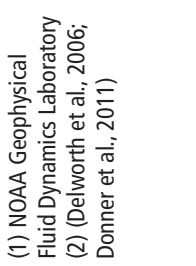 \\
\hline 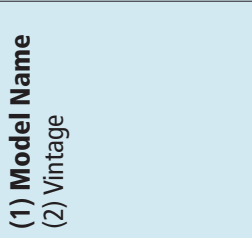 & 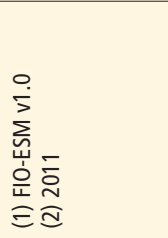 & 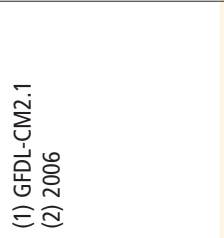 & 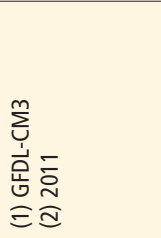 & 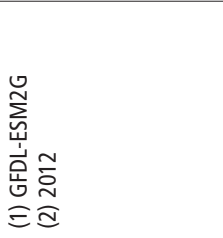 & 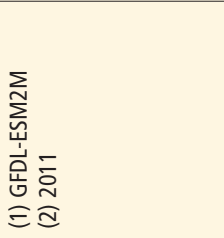 & 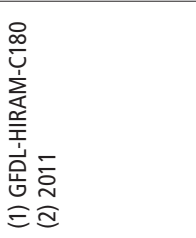 \\
\hline
\end{tabular}




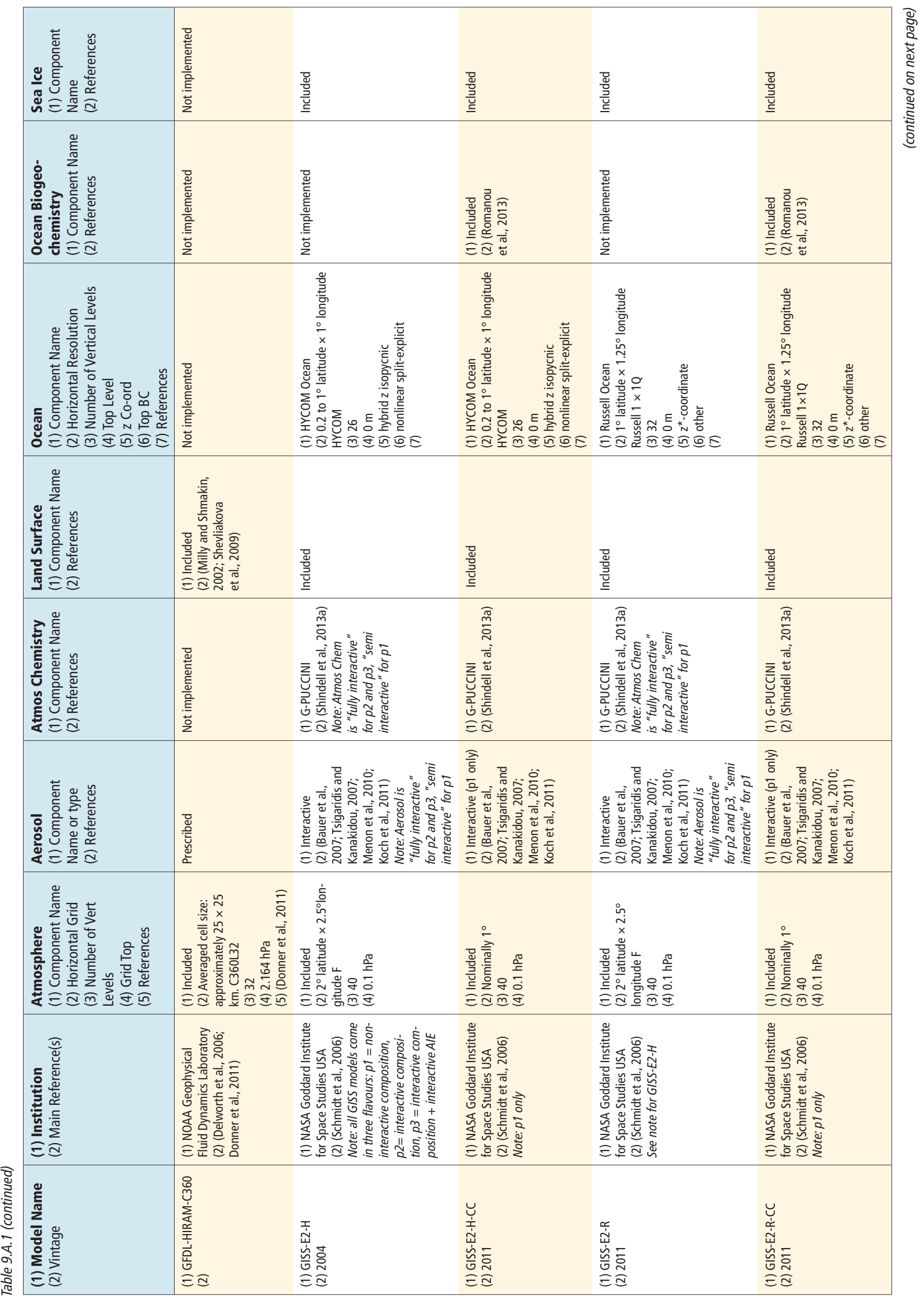



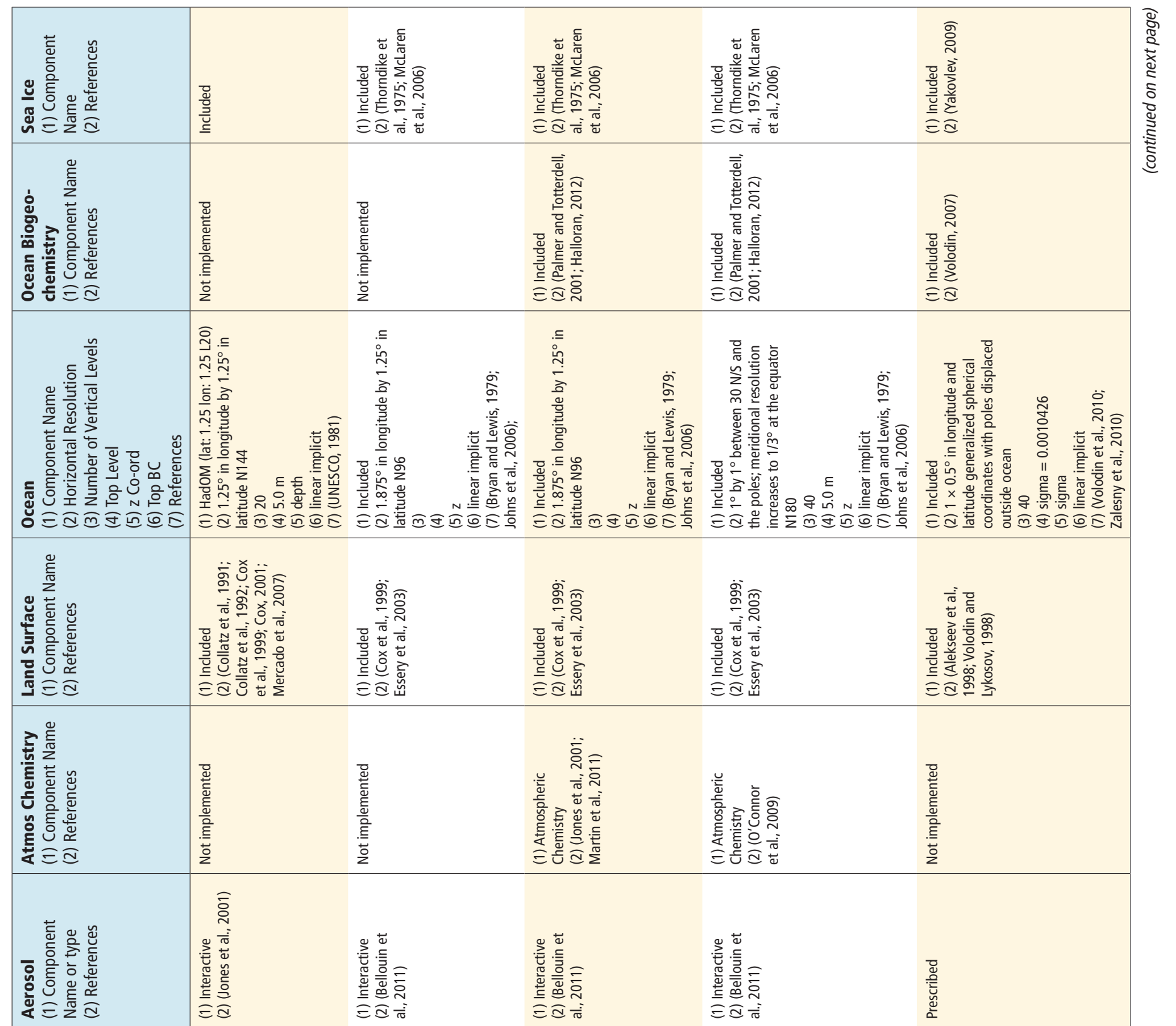

\begin{tabular}{|c|c|c|c|c|c|}
\hline 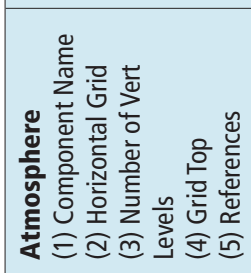 & 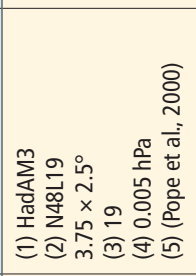 & 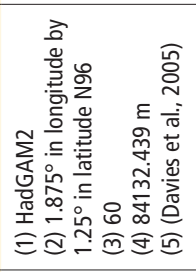 & 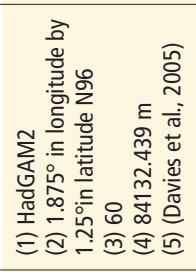 & 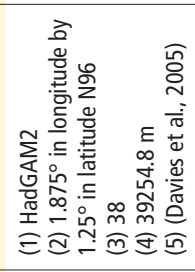 & 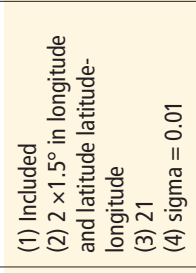 \\
\hline 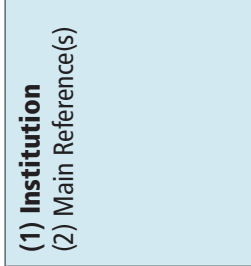 & 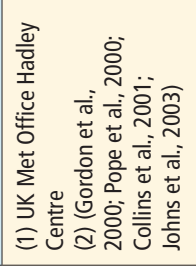 & 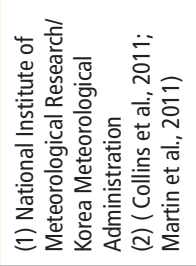 & 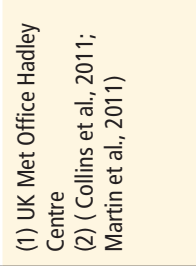 & 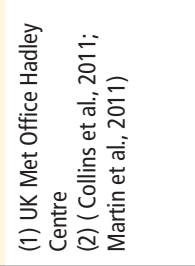 & 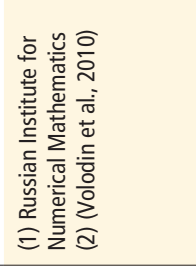 \\
\hline 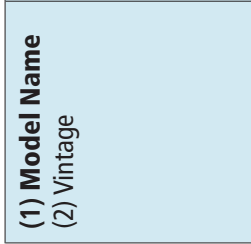 & 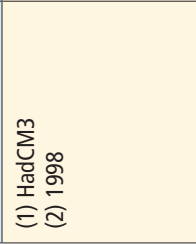 & 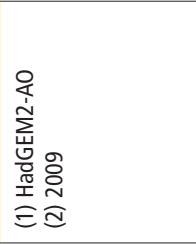 & 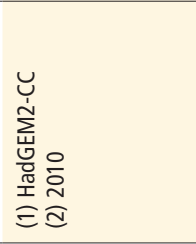 & 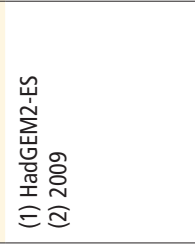 & 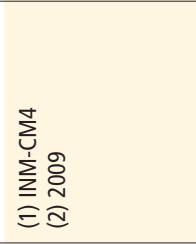 \\
\hline
\end{tabular}




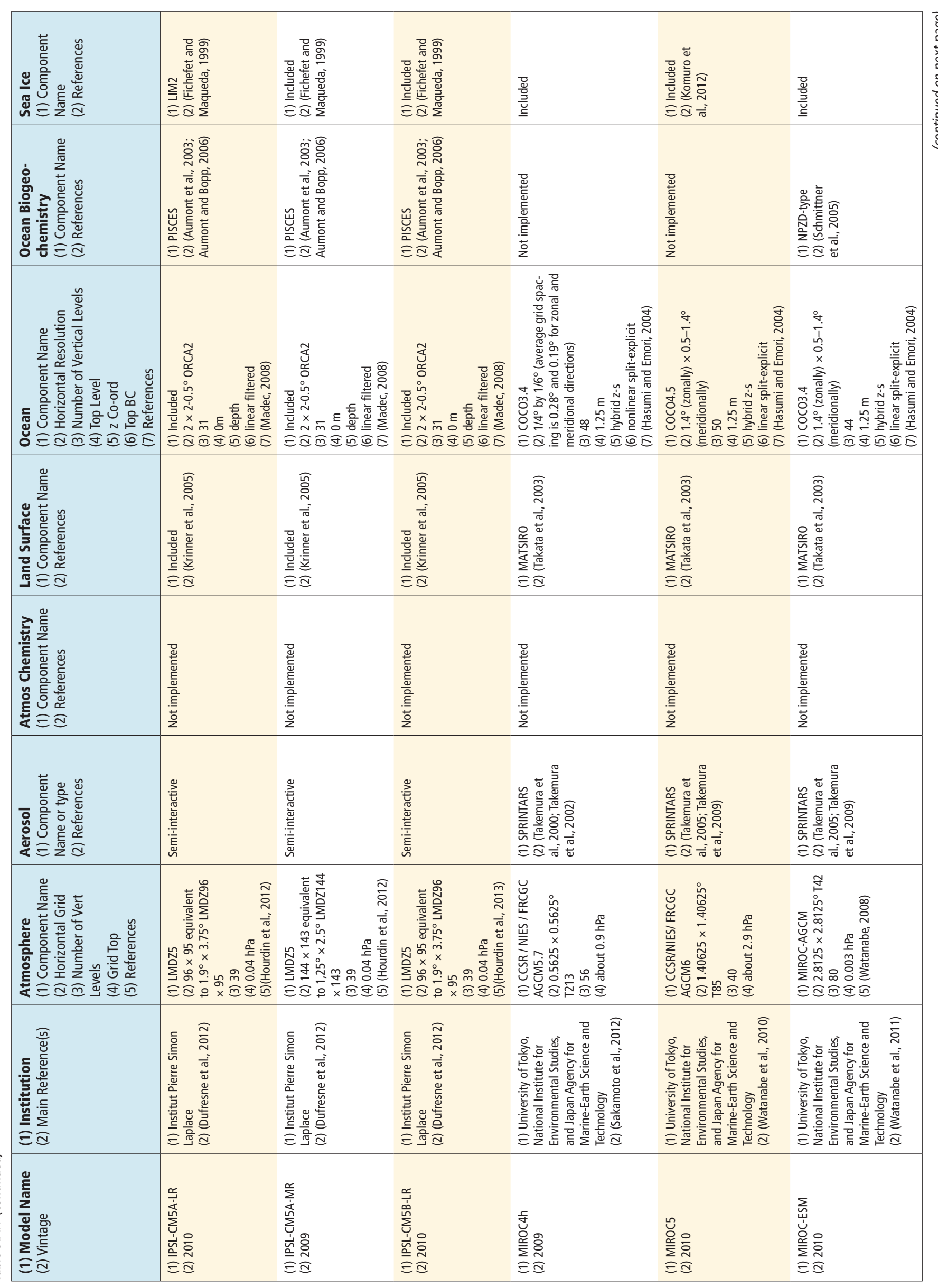




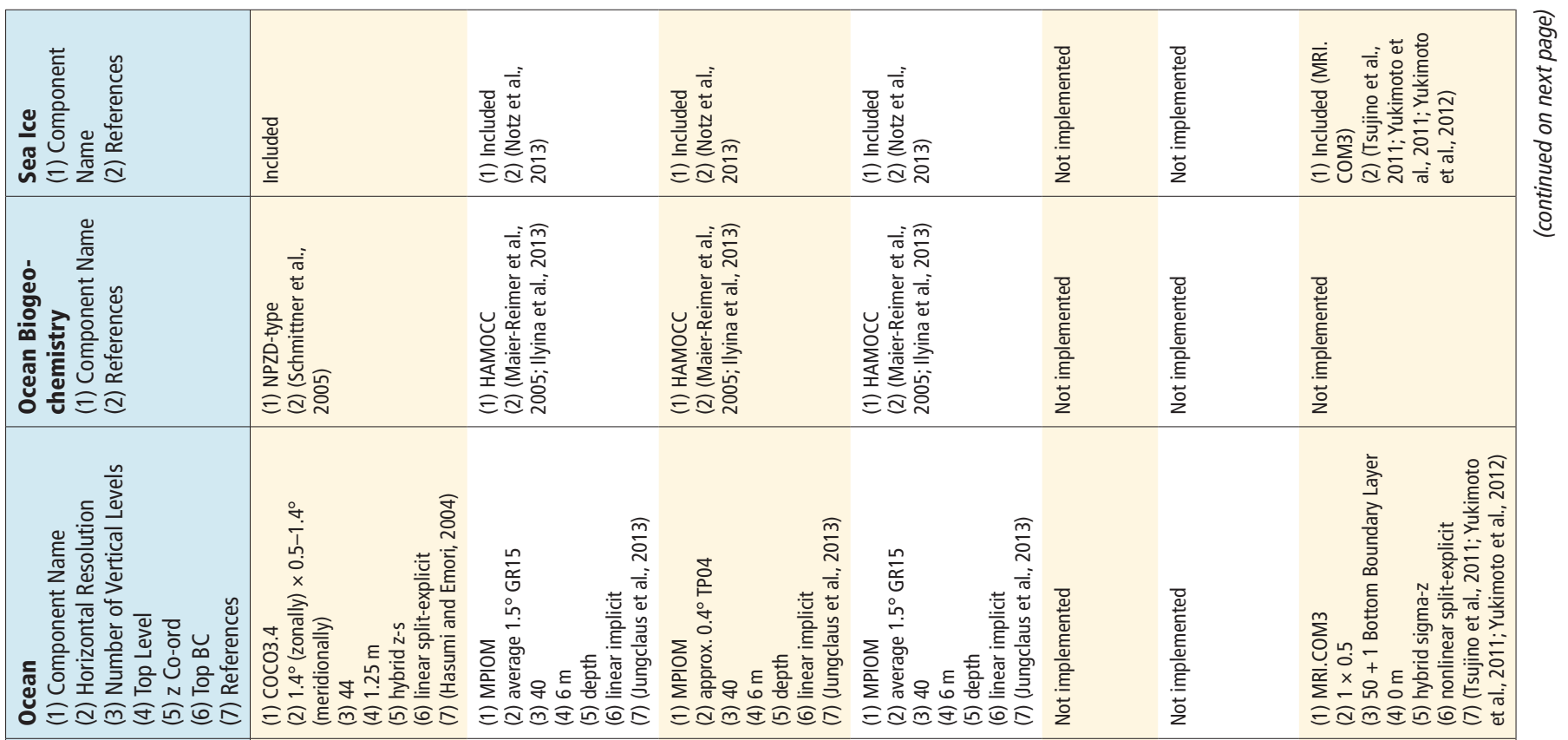

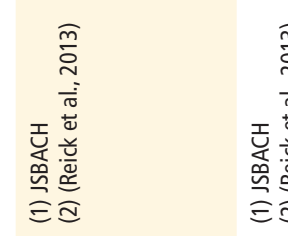

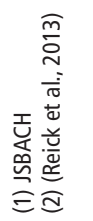

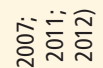

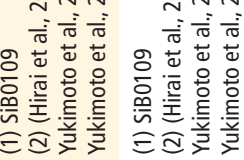

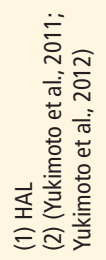

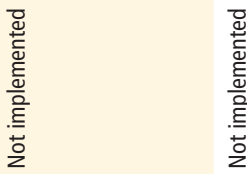
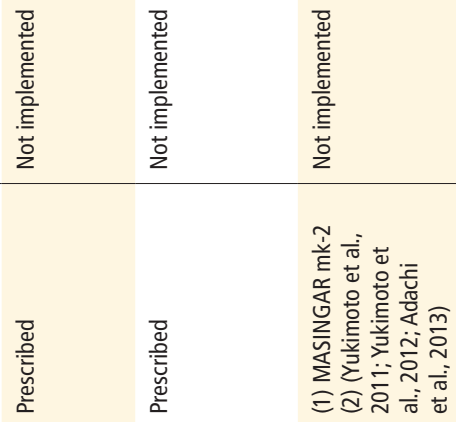
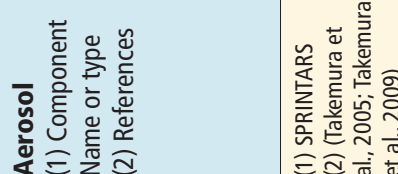

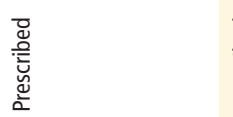

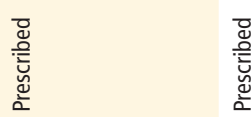

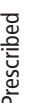

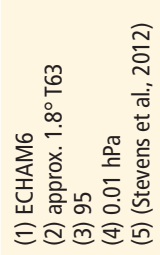

音高

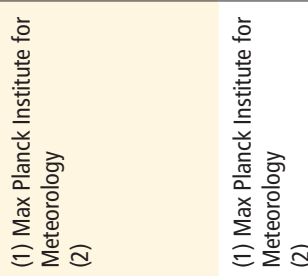

离

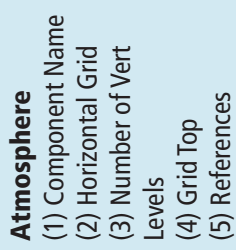

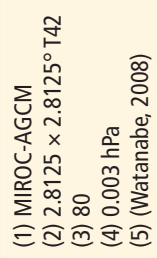
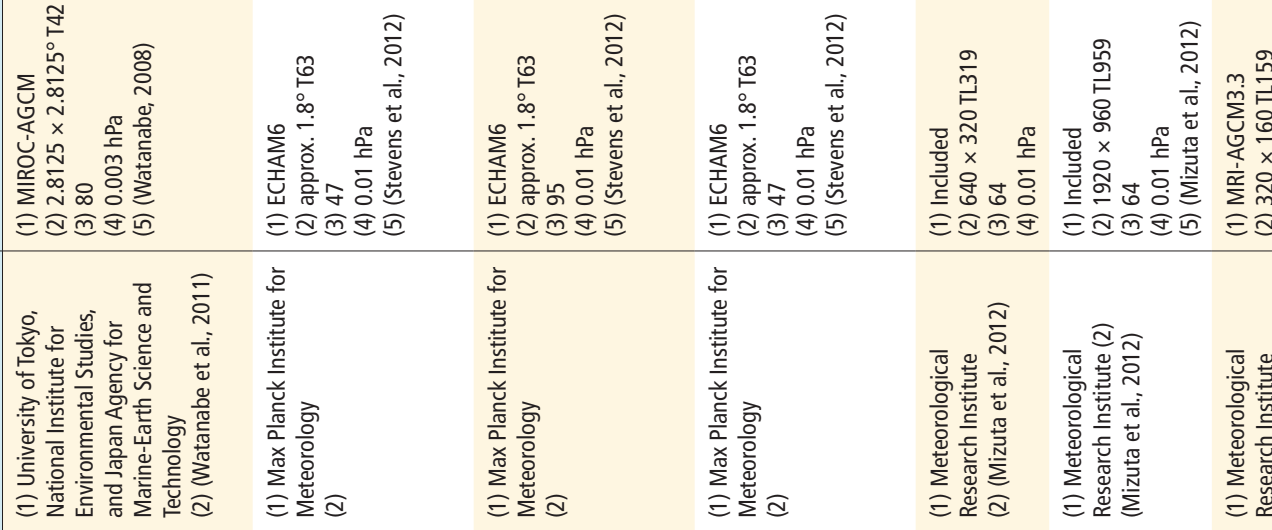

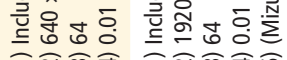
$\frac{1}{\cos 2018}$

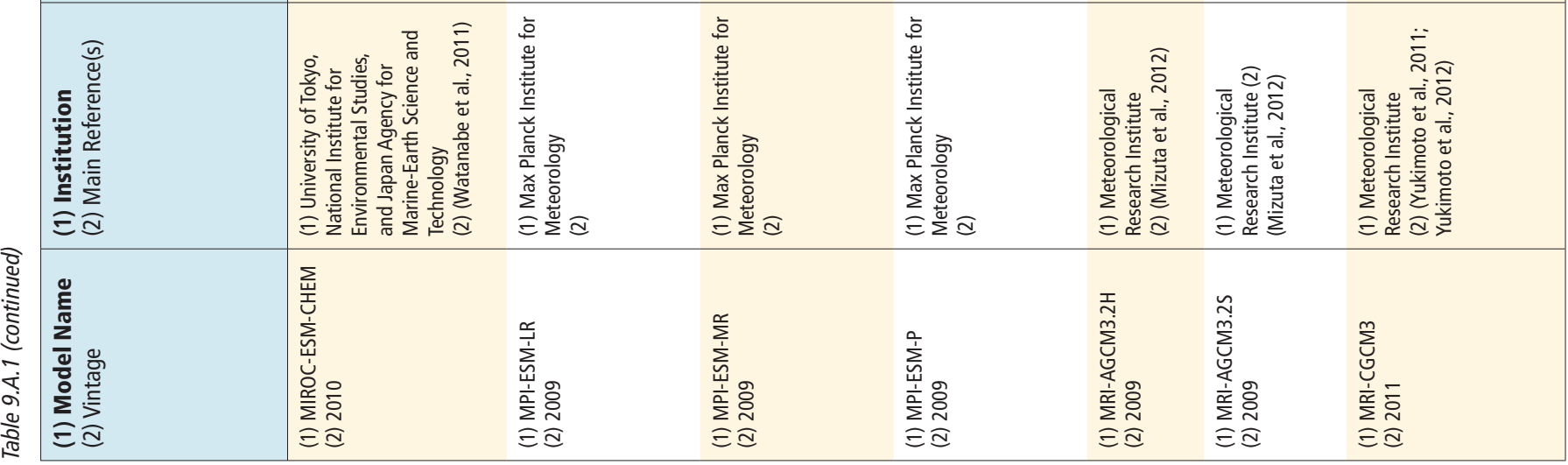




\begin{tabular}{|c|c|c|c|c|}
\hline 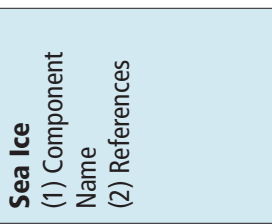 & 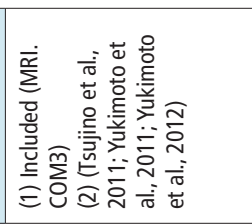 & 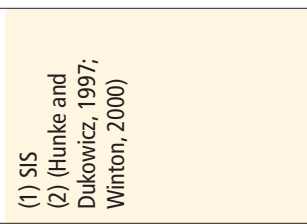 & 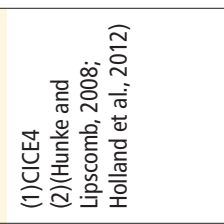 & 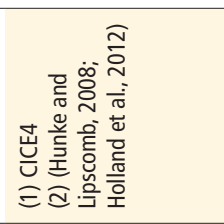 \\
\hline 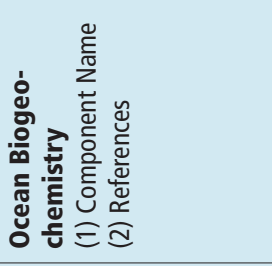 & 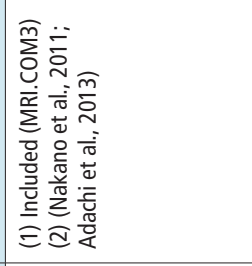 & 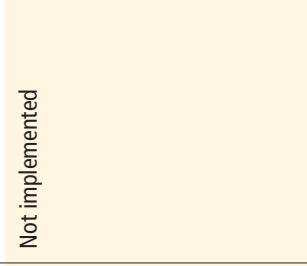 & 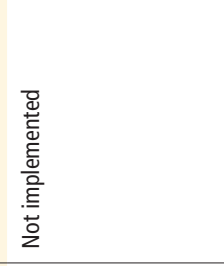 & 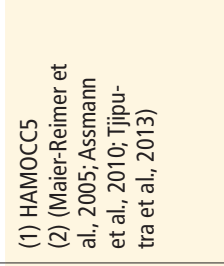 \\
\hline 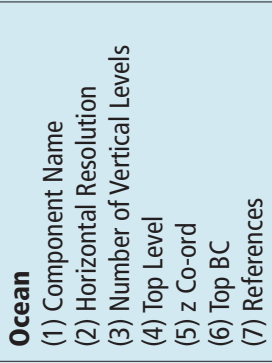 & 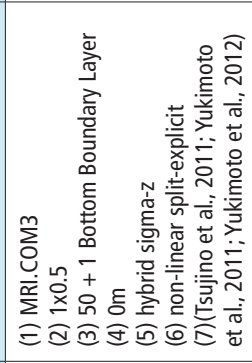 & 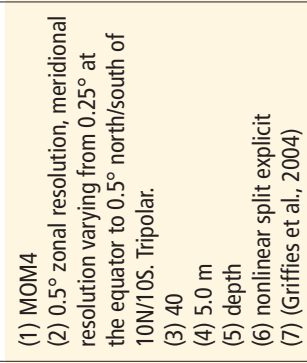 & 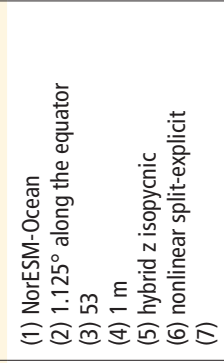 & 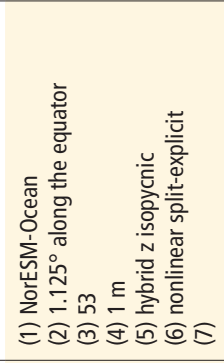 \\
\hline 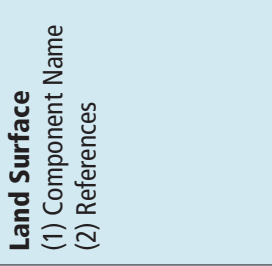 & 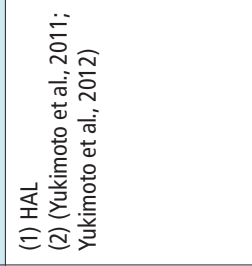 & 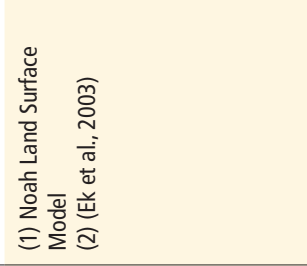 & 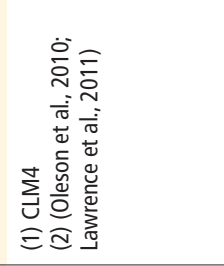 & 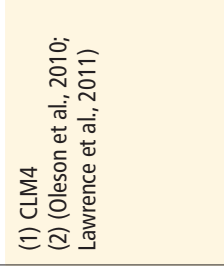 \\
\hline 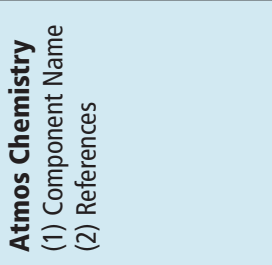 & 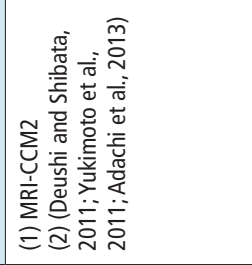 & 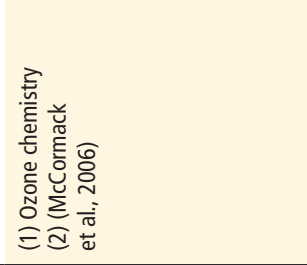 & 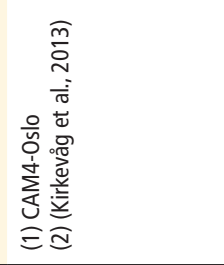 & 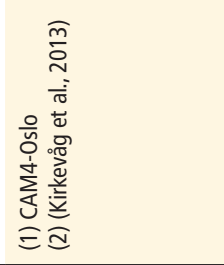 \\
\hline 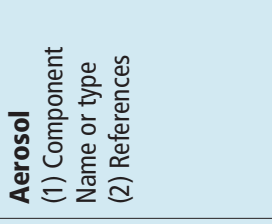 & 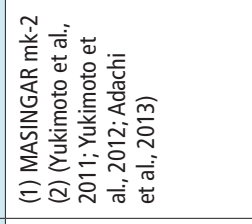 & 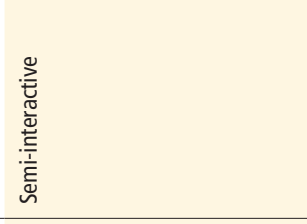 & 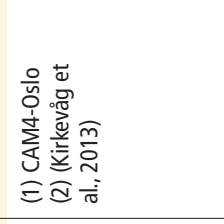 & 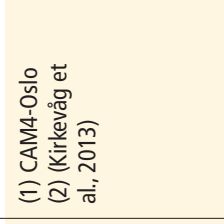 \\
\hline 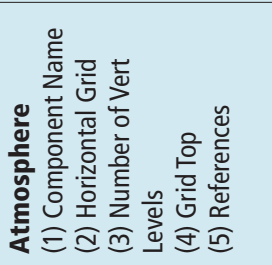 & 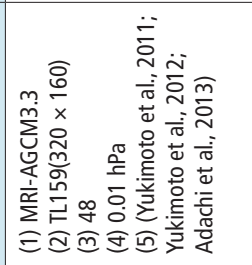 & 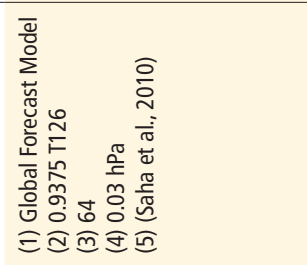 & 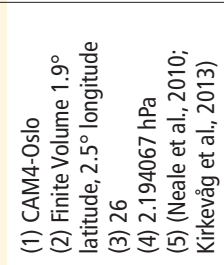 & 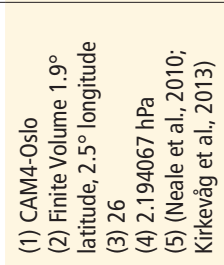 \\
\hline 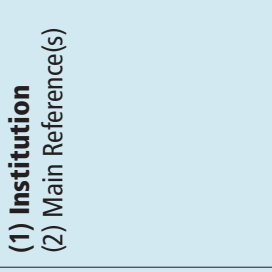 & 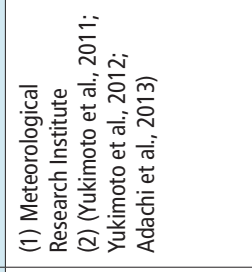 & 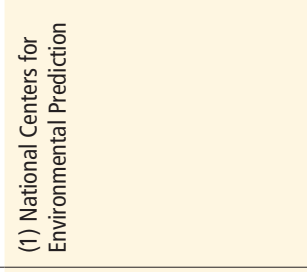 & 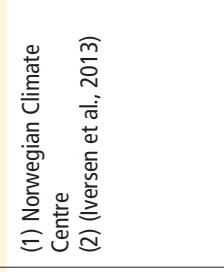 & 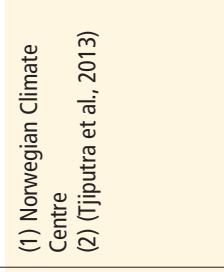 \\
\hline 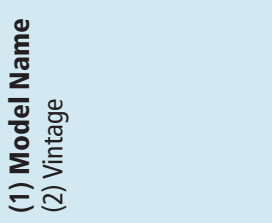 & 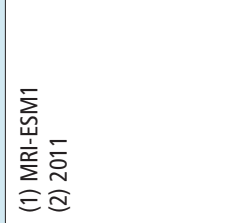 & 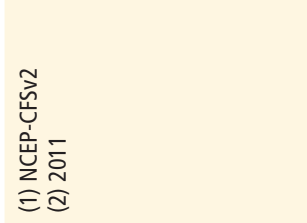 & 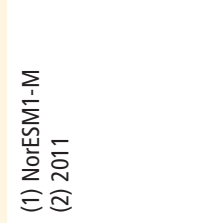 & 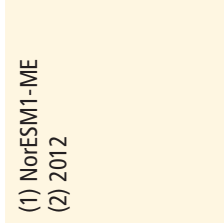 \\
\hline
\end{tabular}




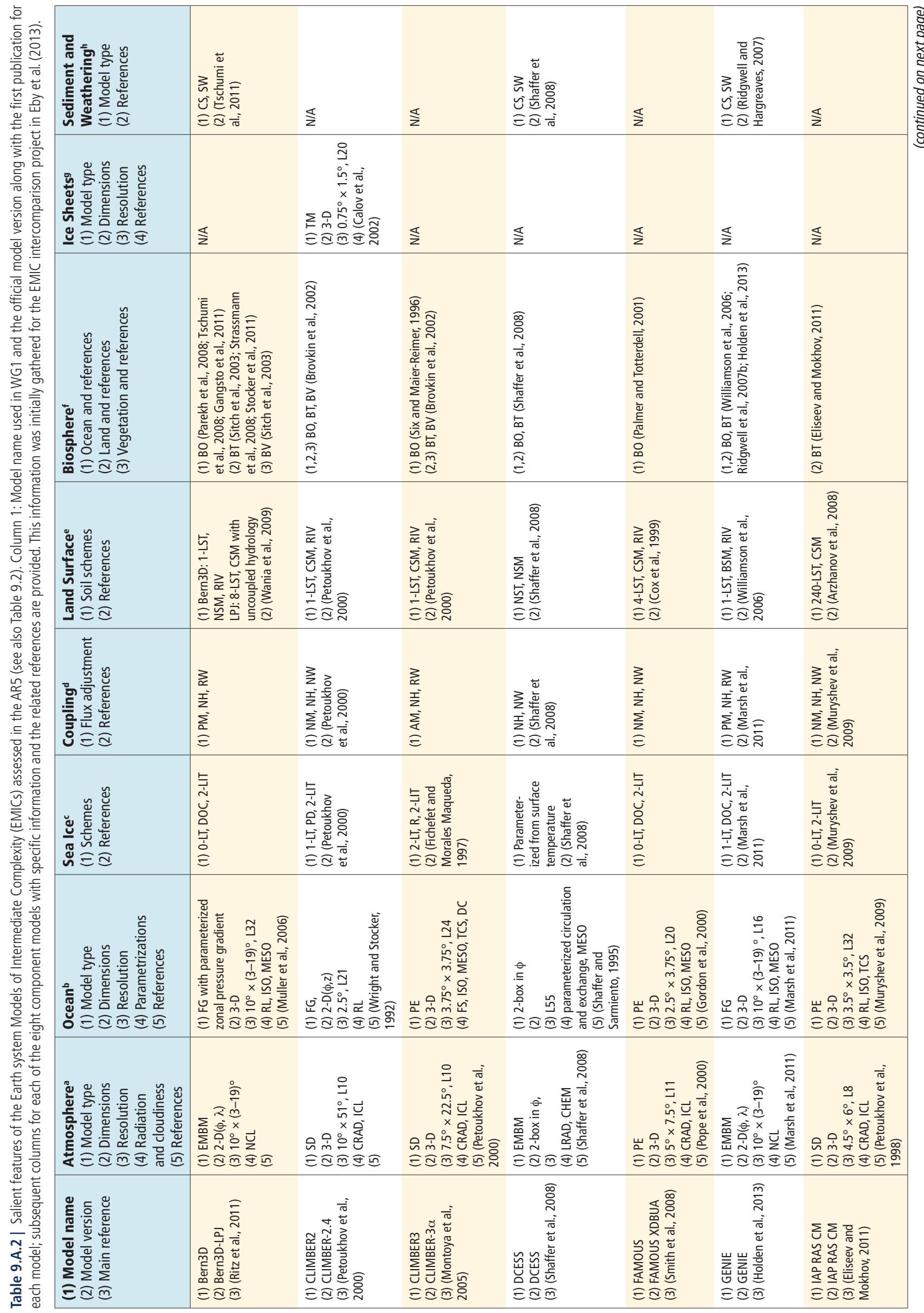




\begin{tabular}{|c|c|c|c|c|c|c|c|}
\hline 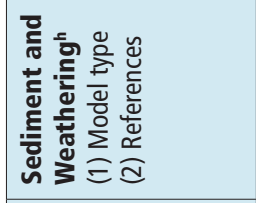 & $\stackrel{\bar{z}}{\mathbf{z}}$ & $\stackrel{\mathbb{z}}{z}$ & $\underline{z}$ & 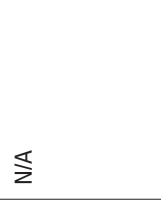 & $\stackrel{\nwarrow}{z}$ & $\stackrel{\mathbb{z}}{z}$ & $\frac{\hat{z}}{z}$ \\
\hline 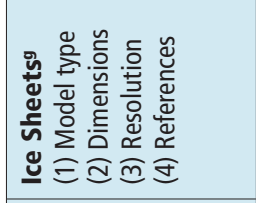 & $\stackrel{1}{z}$ & 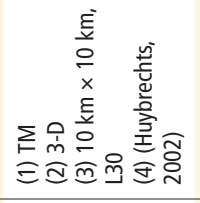 & $\frac{\pi}{2}$ & $\stackrel{\mathbb{1}}{z}$ & $\stackrel{\leq}{z}$ & 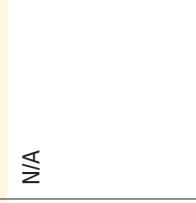 & 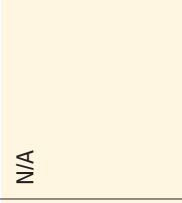 \\
\hline 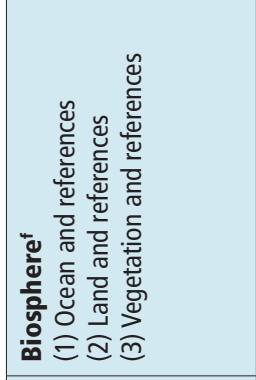 & 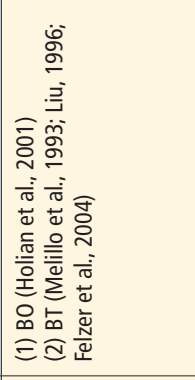 & 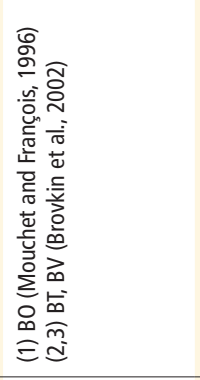 & 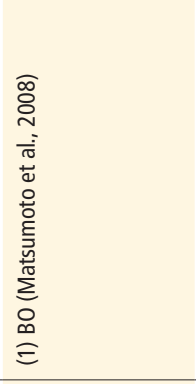 & 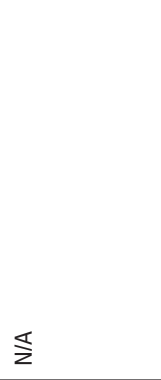 & 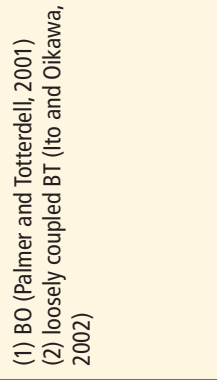 & $\stackrel{\Sigma}{z}$ & 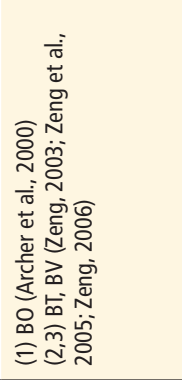 \\
\hline 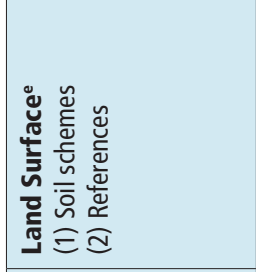 & 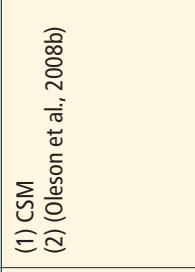 & 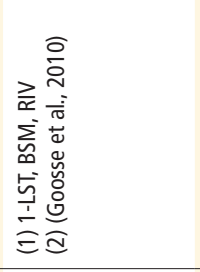 & 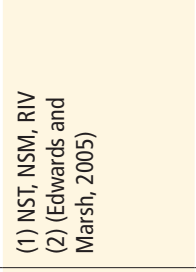 & 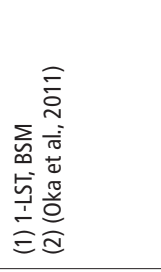 & 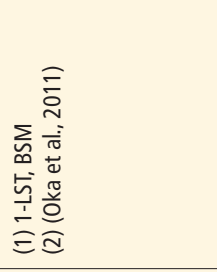 & 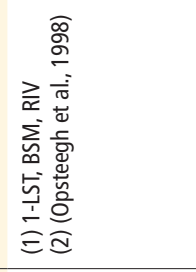 & 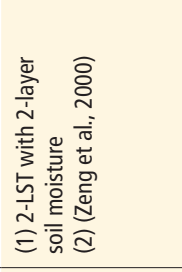 \\
\hline 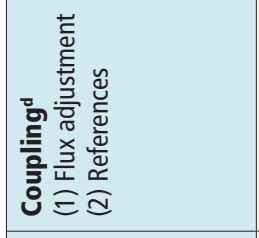 & 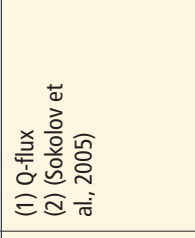 & 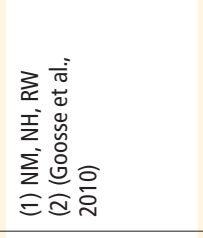 & 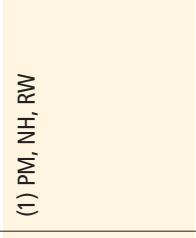 & 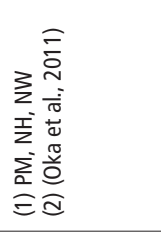 & 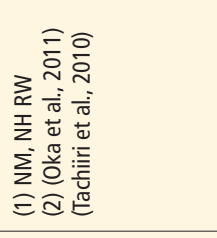 & 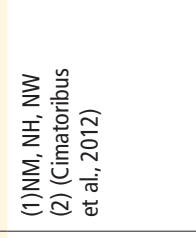 & 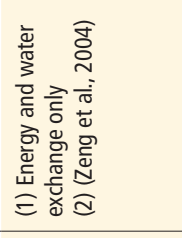 \\
\hline 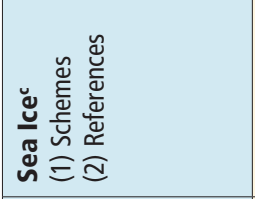 & 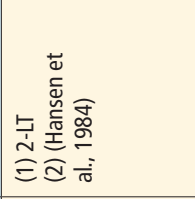 & 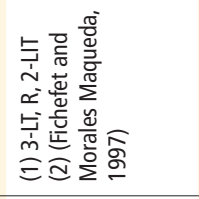 & 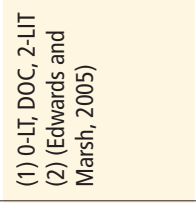 & 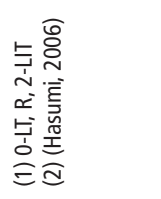 & 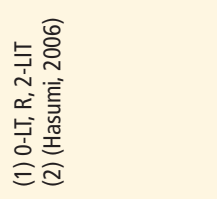 & 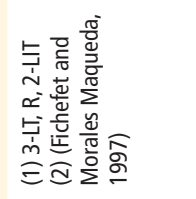 & 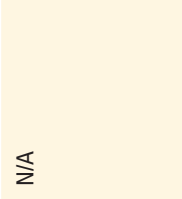 \\
\hline 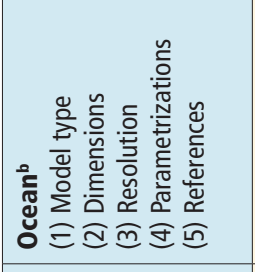 & 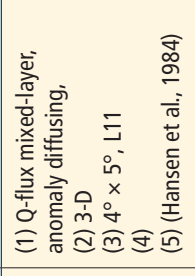 & 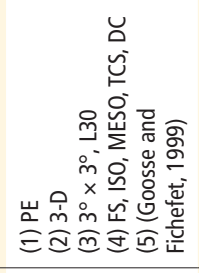 & 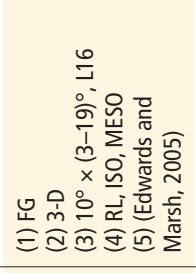 & 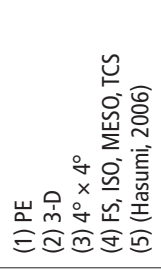 & 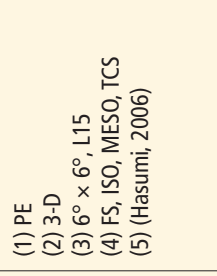 & 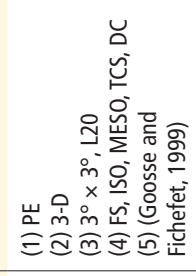 & 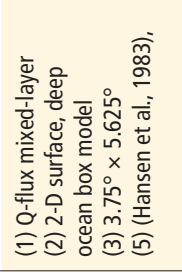 \\
\hline 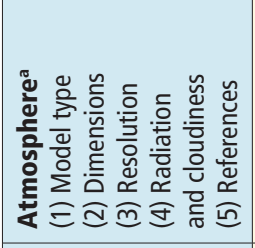 & 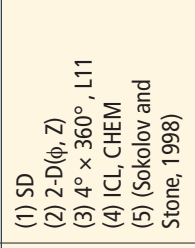 & 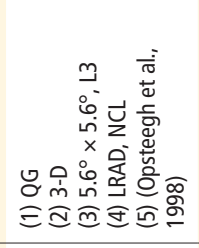 & 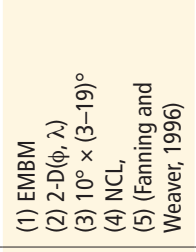 & 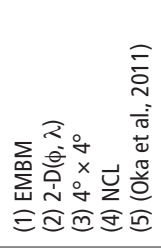 & 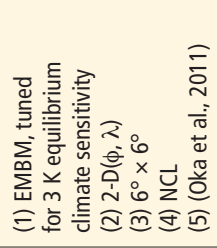 & 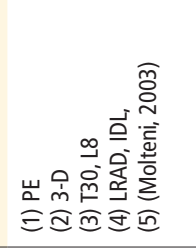 & 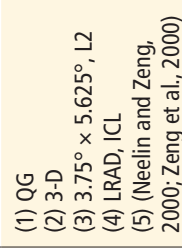 \\
\hline 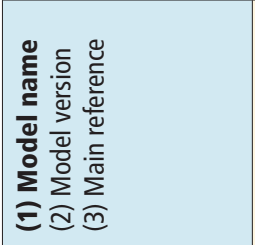 & 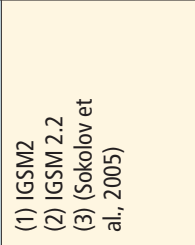 & 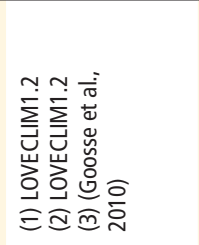 & 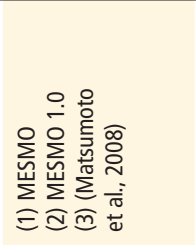 & 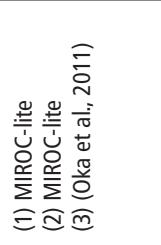 & 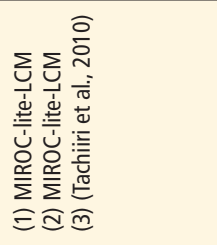 & 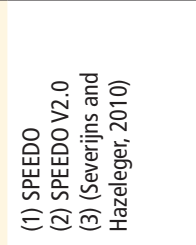 & 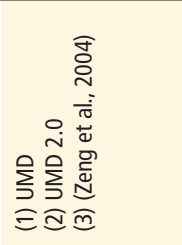 \\
\hline
\end{tabular}




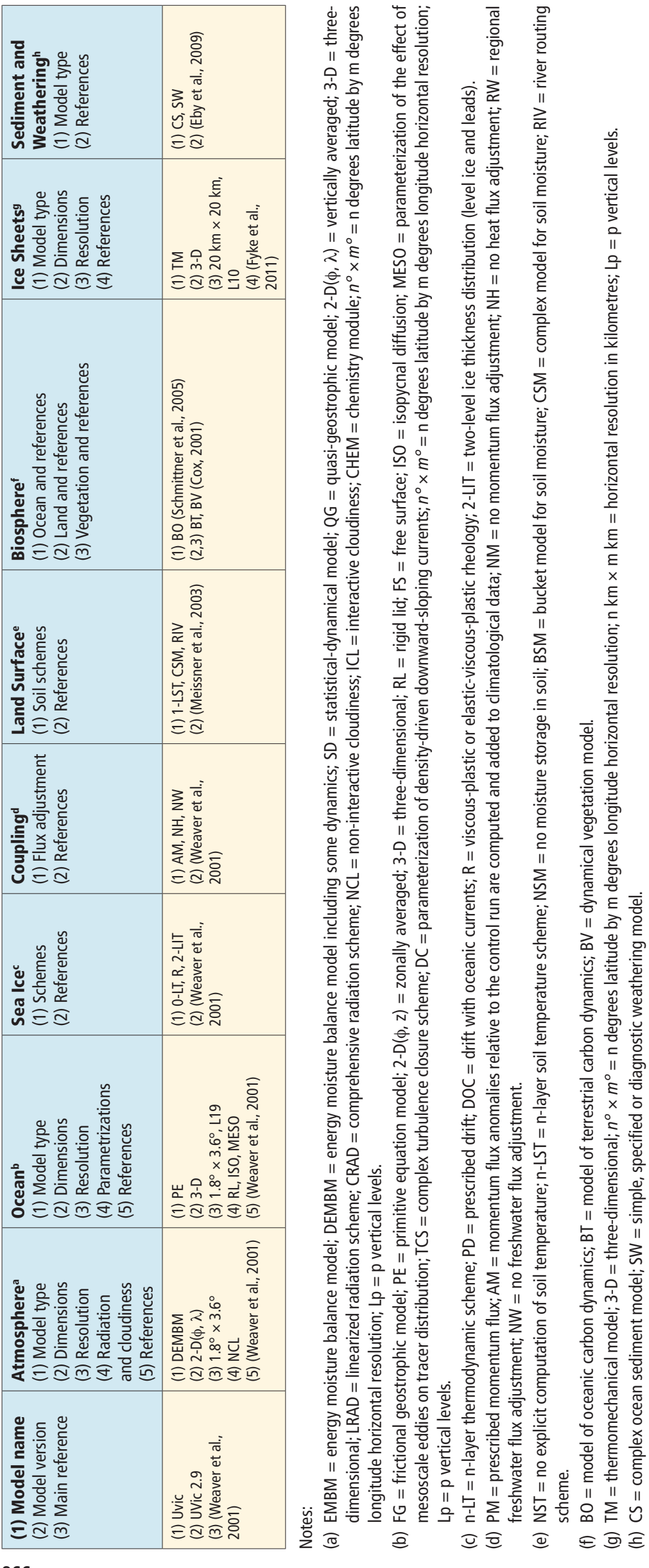

